\title{
Geologic Studies in Alaska by the U.S. Geological Survey, 1997
}

U.S. Geological Survey Professional Paper 1614

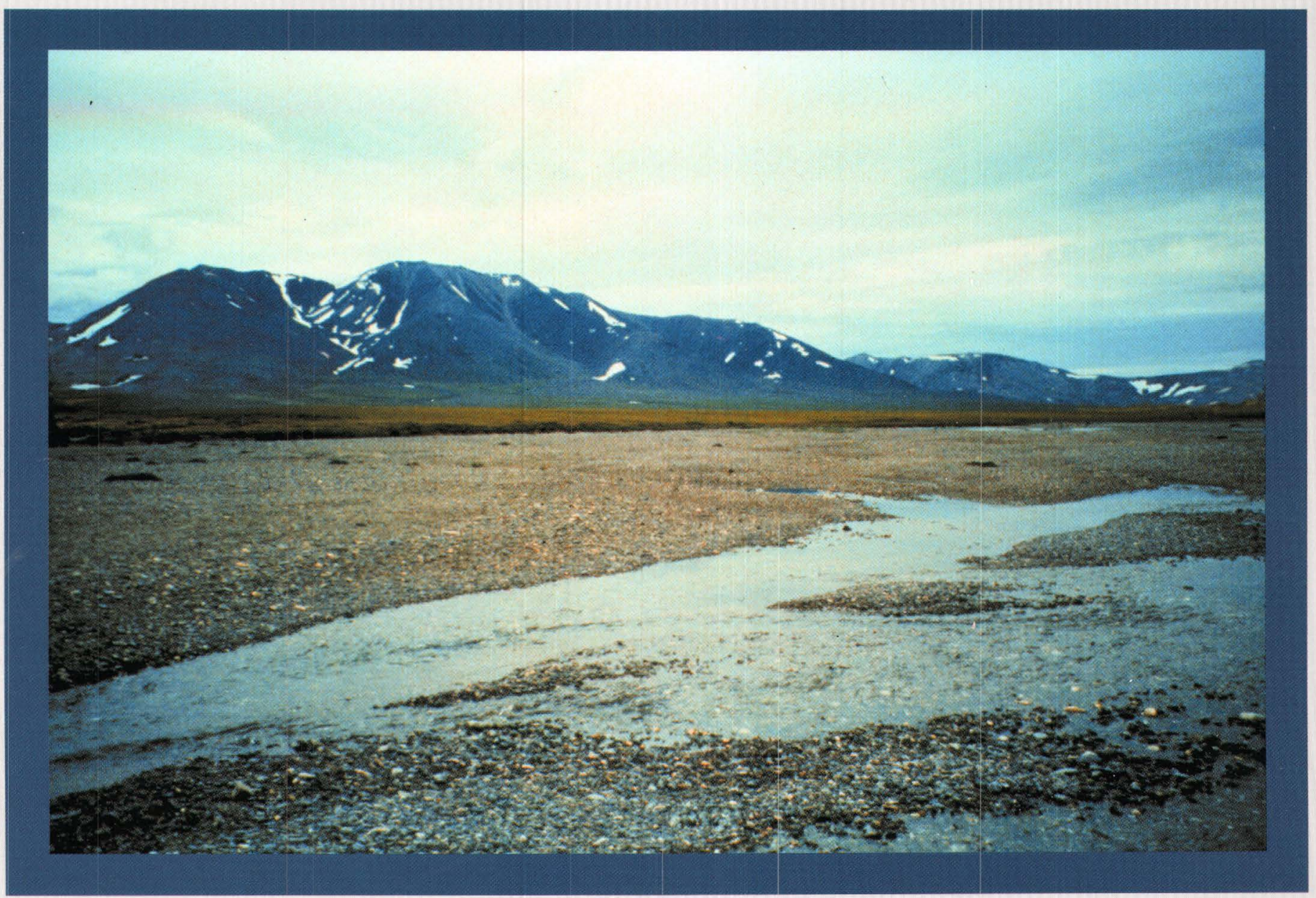


Cover. The Mint River is one of many drainages in the northwestern Seward Peninsula that provide surface runoff and sediment load into Lopp Lagoon and the Bering Sea. Recent geochemical studies (see article by Parnow and others, this volume) indicate that stream sediments collected from some drainages in this area contain anomalous concentrations of arsenic and tin, reflecting the presence of known tin-bearing granites and associated mineral deposits in this area. Although detrital arsenic and tin may be naturally entering the nearshore marine environment, the data suggest that solution transport of metals is extremely insignificant. 


\section{Geologic Studies in Alaska by the U.S. Geological Survey, 1997}

Edited by Karen D. Kelley

U.S. Geological Survey Professional Paper 1614

U.S. Department of the Interior

U.S. Geological Survey 


\section{U.S. Department of the Interior \\ Bruce Babbitt, Secretary}

\section{U.S. Geological Survey}

Charles G. Groat, Director

For sale by U.S. Geological Survey, Information Services Box 25286, Federal Center

Denver, CO 80225

This publication is also available online at: http://greenwood.cr.usgs.gov/pub/ppapers/p1614/

\footnotetext{
Any use of trade, product, or firm names in this publication is for descriptive purposes only and

does not imply endorsement by the U.S. Government
}

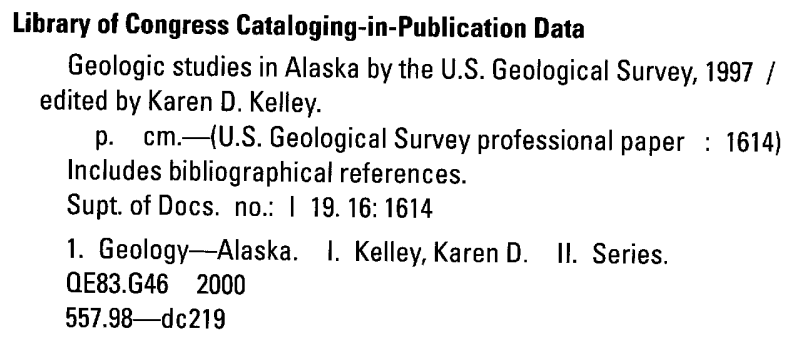




\section{Contents}

Introduction

By Karen D. Kelley 1

\section{RESOURCES}

Geologic setting of Mississippian vein-breccias at the Kady $\mathrm{Zn}-\mathrm{Pb}-\mathrm{Cu}-\mathrm{Ag}$ prospect: Plumbing system for a failed Sedex deposit?

By Melanie B. Werdon. 5

Chemical and isotopic data for rocks and ores from the Upper Triassic Greens Creek and Woewodski Island volcanogenic massive sulfide deposits, southeastern Alaska

By Rainer J. Newberry and David A. Brew .35

Core lithofacies analysis and fluvio-tidal environments in the AK 94 CBM-1 well, near Wasilla, Alaska

By Romeo M. Flores, Mark D. Myers, Gary D. Stricker, and Julie A. Houle. 57

\section{GEOLOGIC FRAMEWORK}

Lower Paleozoic deep-water facies of the Medfra area, central Alaska

By Julie A. Dumoulin, Dwight C. Bradley, Anita G. Harris, and John E. Repetski. 73

Alagogshak Volcano: A Pleistocene andesite-dacite stratovolcano in Katmai National Park

By Wes Hildreth, Judy Fierstein, Marvin A. Lanphere, and David F. Siems 105

Gravity changes during the 26 years following the 1964 Alaskan earthquake

By David F. Barnes 115

\section{ENVIRONMENT AND CLIMATE}

Metal cycling along the northwestern Seward Peninsula, Alaska: A possible natural cause of metal contamination in the Arctic

By Cynthia C. Parnow, Richard J. Goldfarb, Karen D. Kelley, and Geoff S.York 123

Major-element, trace-element, and strontium-isotope systematics of natural waters in the Fairbanks mining district: Constraints from local geology

By Richard J. Goldfarb, G. Lang Farmer, Barrett A. Cieutat, and Allen L. Meier 139 


\section{BIBLIOGRAPHIES}

U.S. Geological Survey reports on Alaska released in 1997

Compiled by John P. Galloway and Susan Toussaint 151

Reports on Alaska in non-USGS publications released in 1997 that include USGS authors

Compiled by John P. Galloway and Susan Toussaint 157 


\section{Contributors to this Professional Paper}

Anchorage

Bradley, Dwight C.

Dumoulin, Julie A.

U.S. Geological Survey

4200 University Drive

Anchorage, Alaska 99508

York, Geoff S.

U.S. Geological Survey

Alaska Biological Science Center

1011 Tudor Road

Anchorage, Alaska 99503

Houle, Julie A.

Meyers, Mark D.

Alaska Department of Natural Resources

Division of Oil and Gas

Anchorage, Alaska 99503-5948

\section{Denver}

Flores, Romeo M., Mail Stop 939

Goldfarb, Richard J., Mail Stop 964

Kelley, Karen D., Mail Stop 964

Meier, Allen L., Mail Stop 973

Parnow, Cynthia C., Mail Stop 964

Siems, David F., Mail Stop 973

Stricker, Gary D., Mail Stop 939

U.S. Geological Survey

P.O. Box 25046, Mail Stop

Denver Federal Center

Denver, Colorado 80225

\section{Fairbanks}

Werdon, Melanie B.

Alaska Division of Geological and Geophysical Surveys

794 University Ave., Suite 200

Fairbanks, Alaska 99709-3645

Newberry, Rainer J.

Department of Geology

University of Alaska

Fairbanks, Alaska 99775
Menlo Park

Barnes, David F., Mail Stop 989

Brew, David A., Mail Stop 901

Fierstein, Judy, Mail Stop 910

Hildreth, Wes, Mail Stop 910

Lanphere, Marvin A., Mail Stop 937

Galloway, John P., Mail Stop 904

Toussaint, Susan, Mail Sop 955

U.S. Geological Survey

345 Middlefield Road, Mail Stop

Menlo Park, California 94025

\section{Reston}

Harris, Anita G., Mail Stop 926A

Repetski, John E., Mail Stop 926A

U.S. Geological Survey

National Center, Mail Stop

12201 Sunrise Valley Drive

Reston, Virginia 20192

\section{Others}

Cieutat, Barrett A.

ERM Southwest

3501 North Causeway Boulevard, Suite 200

Metairie, Louisiana 70002

Farmer, G. Lang

Department of Geology

University of Colorado

Boulder, Colorado 80302 



\title{
Geologic Studies in Alaska by the U.S. Geological Survey, 1997
}

\author{
Edited by Karen D. Kelley
}

\section{Introduction}

\section{ByKaren D. Kelley}

The eight papers that follow continue the series ${ }^{1}$ of U.S. Geological Survey (USGS) reports on investigations in the geologic sciences in Alaska. The series presents new and sometimes preliminary findings that are of interest to earth scientists in academia, government, and industry; to land and resource managers; and to the general public. Reports presented in Geologic Studies in Alaska cover a broad spectrum of topics from all parts of the State (fig. 1), which serves to emphasize the diversity of USGS efforts to meet the Nation's needs for earth-science information in Alaska.

The papers in this volume are organized under the topics Resources, Geologic Framework, and Environment and Climate. Such an organization is intended to reflect the scope and objectives of USGS programs currently active in Alaska. Resource papers include one that presents detailed observations from a Mississippian $\mathrm{Zn}-\mathrm{Pb}-\mathrm{Cu}-\mathrm{Ag}$ occurrence in the Brooks Range (Werdon). Mineralogic, chemical, and isotopic data provide the basis for a proposed relationship between this vein-breccia deposit and the shale-hosted massive sulfide deposit type, which includes the active Red Dog mine in the western Brooks Range. Also included under the topic of Resources is a paper that presents geochemical and isotopic data from the Greens Creek and Woewodski Island volcanogenic massive sulfide deposits in southeastern Alaska (Newberry and Brew). The depositional environment and sedimentological setting of Tertiary coal beds in the Matanuska and Susitna Valleys is the focus of a third paper under the topic of Resources (Flores and others).

Geologic Framework studies provide background information that is the scientific basis for present and future studies of the environment, mineral and energy resources, paleoclimate, and hazards in Alaska. One paper presents the results of sedimentologic and paleontologic comparisons of lower Paleozoic, deep-water-facies rock units in central Alaska (Dumoulin and others). The authors show which of these units are likely to correlate with one another, suggest likely source regions, and provide a structural restoration of units that have been fragmented by large fault motions. A second framework paper provides a map, rock descriptions, and chemical compositions of volcanic rocks in a newly recognized, geologically young volcanic center in the Aleutian volcanic arc (Hildreth and others). A third paper presents an interesting summary of gravity changes that occurred in south-central Alaska during the great earthquake of 1964 and for the following 25 years (Barnes). Gravity changes correlate with land-elevation changes in some cases, but not in others, which means that different processes are responsible for the gravity changes.

\footnotetext{
1 From 1975 through 1988, the Geologic Studies in Alaska series was published as USGS Circulars, which were initially titled "The United States Geological Survey in Alaska: Accomplishments during 19xx." From 1989 to 1994, the series was published as the more formal USGS Bulletins. As a result of reorganization of USGS publications, beginning in 1995 the series has been published as USGS Professional Papers.
} 


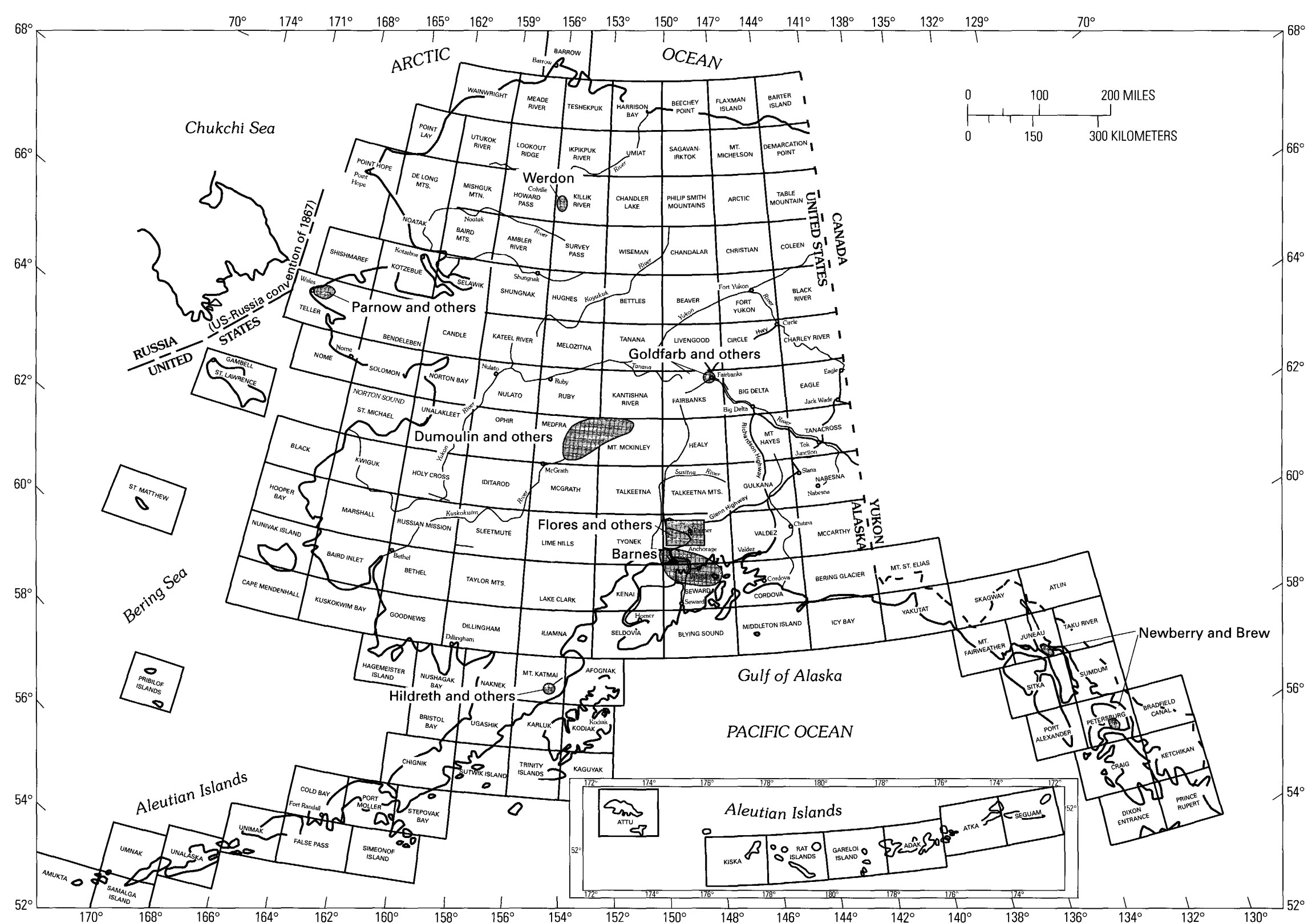

Figure 1. Index map of Alaska showing 1:250,000-scale quadrangles and locations of study areas discussed in this book. 
Environmental studies are the focus of two papers. One study discusses the nature and origin of uncommonly high arsenic and heavy-metal concentrations in stream sediments of the northwestern Seward Peninsula, a region of traditional subsistence living (Parnow and others). A second study evaluates the chemical characteristics of natural waters north of Fairbanks, an area known for atypically high arsenic concentrations in ground waters (Goldfarb and others).

Two bibliographies at the end of the volume present reports about Alaskan earth sciences in USGS publications during 1997, and reports about Alaska by USGS authors in nonUSGS publications during the same period. 



\title{
Geologic Setting of Mississippian Vein-Breccias at the Kady Zn-Pb-Cu-Ag Prospect: Plumbing System for a Failed Sedex Deposit?
}

\author{
By Melanie B. Werdon
}

\section{Abstract}

The north-central and northwestern Brooks Range of Alaska hosts widespread $\mathrm{Zn}$ - $\mathrm{Pb}$ - $\mathrm{Ag}$ shale-hosted massive sulfide (Sedex), vein-breccia, and disseminated sulfide deposits. The vein-breccia and disseminated occurrences show no obvious igneous association and are hosted by a deformed, but only weakly metamorphosed, package of Upper Devonian to Lower Mississippian mixed continental and marine clastic rocks (the Endicott Group). The Sedex deposits (i.e., Red Dog, Drenchwater) are hosted by black siliceous carbonaceous shale and mudstone of the Mississippian to Pennsylvanian Kuna Formation. Previous studies have suggested that the vein-breccia zones are either Carboniferous or were formed along Mesozoic or postMesozoic faults. This study documents a Mississippian age for the Kady vein-breccia prospect and investigates the physical and chemical controls on ore formation.

Early diagenetic and hydrothermal features at the Kady Zn$\mathrm{Pb}-\mathrm{Cu}-\mathrm{Ag}$ vein-breccia prospect provide insights into Carboniferous basinal dewatering of the Upper Devonian to Lower Mississippian Endicott Group in the northern Brooks Range, Alaska. Sulfides at Kady occur in several subparallel, quartzdominated, linear vein-breccia zones (1-35 m wide, with a minimum strike length of about $800 \mathrm{~m}$ ), which are offset by Mesozoic structures, and as permeability-controlled, strata-bound disseminated cements in gray sandstone in a $5-\mathrm{km}$ by $3-\mathrm{km}$ area. Sphalerite $\left(\left(\mathrm{Zn}_{81.5-99.7}, \mathrm{Fe}_{0.1-17.9}, \mathrm{Cd}_{0.1-0.9}\right) \mathrm{S}\right)$ is the dominant sulfide mineral, followed by galena, chalcopyrite, minor pyrite, trace bravoite and $\mathrm{Ag}+\mathrm{Sb} \pm \mathrm{Pb}$ sulfosalts, and extremely rare pyrrhotite. The relative proportion and volumetric abundance of the primary minerals varies widely within and between veinbreccia zones. Main stage quartz+sulfide ( \pm late calcite) veinbreccia zones cut rare early ankerite and siderite veins. Sulfides and quartz precipitated during a steep drop in temperature, possibly during the change from lithostatic to hydrostatic pressure during extensional faulting, from a relatively low temperature ( $\leq$ $250^{\circ} \mathrm{C}$ ), slightly acidic, carbon-destructive hydrothermal fluid. Textural and mineralogical data indicate that sulfides and quartz were deposited under evolving chemical conditions (i.e., increasing $\mathrm{pH}$, fugacity of oxygen, and fugacity of sulfur).
The lack of known Sedex mineralization in the northcentral Brooks Range suggests that Kady represents the hydrothermal fluid pathway below a failed or nonexistent Sedex system. Although early diagenetic processes within the Endicott Group were favorable for the mineral-destructive release of base metals from detrital minerals, base metals were not transported and deposited in overlying shales but, rather, were deposited within the Endicott Group. Unfavorable conditions may have existed for Sedex deposit formation above the Kady prospect including one or more combinations of the following: boiling above the level of the vein-breccia system leading to rapid precipitation of base metal sulfides in the vein-breccias; dispersion of buoyant, low-salinity, metal-bearing fluids (if they reached the sea floor) such that metals would not be deposited and accumulated in massive amounts; and a short-lived, lowtemperature system.

\section{Introduction}

The Kady $\mathrm{Zn}-\mathrm{Pb}-\mathrm{Cu}-\mathrm{Ag}$ vein-breccia and disseminated sulfide prospect is located in the southwestern part of the Killik River quadrangle in the north-central Brooks Range, Alaska (fig. 1). Kady is located within Gates of the Arctic National Park near the headwaters of a tributary to Outwash Creek at lat $68^{\circ} 12^{\prime} \mathrm{N}$. and long $154^{\circ} 58^{\prime} \mathrm{W}$. in T. 32 N., R. 17 E.

Numerous $\mathrm{Zn}-\mathrm{Pb}-\mathrm{Ag}$ vein-breccia and disseminated sulfide occurrences were discovered in the northern Brooks Range during reconnaissance studies by the U.S. Bureau of Mines and U.S. Geological Survey in the 1970's to 1990's (e.g., Jansons and Parke, 1981; Jansons, 1982; Meyer and Kurtak, 1992; Kelley and others, 1997). These vein-breccia and disseminated sulfide occurrences are hosted by arenaceous, mixed continental-marine clastic rocks of the Upper Devonian to Lower Mississippian Endicott Group and extend from the western Brooks Range, near Red Dog, eastward to the Killik River quadrangle. The Kady prospect was discovered in 1985 by tracing stream-sediment geochemical anomalies to their source (Duttweiler, 1987). 


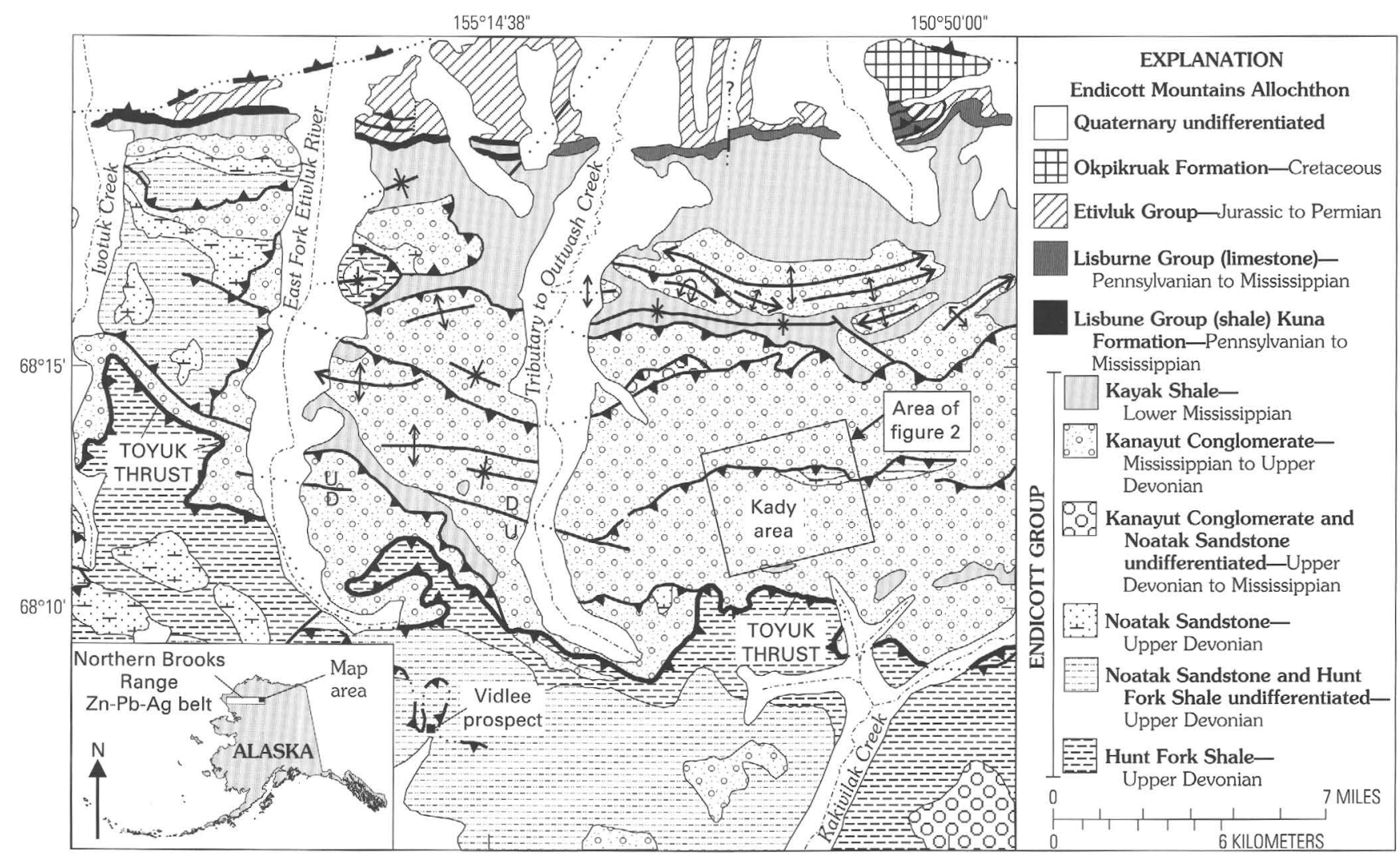

Figure 1. Geologic map of the southwest part of the Killik River quadrangle, north-central Brooks Range, Alaska. Geology modified from Mull and others (1994) and Mull and Werdon (1994).

Mineralized rocks at Kady occur within the Endicott Mountains allochthon, a regionally extensive package of predominantly sedimentary rocks of Devonian to Cretaceous age, which are part of the Brooks Range fold-and-thrust belt. Two tectonic events of significance have been recognized within (or have affected rocks of) the Endicott Mountains allochthon: (1) Mississippian to Pennsylvanian(?) extension, and (2) compression during the Late Jurassic to Early Cretaceous Brookian orogeny. Extension during Mississippian to Pennsylvanian(?) time in the northern Brooks Range is suggested by abrupt facies changes and inferred normal faults (Moore and others, 1986), shalehosted stratiform or sedimentary exhalative (Sedex) massive sulfide deposits (Moore and others, 1986; Forrest, 1983), and regionally extensive, but volumetrically minor, volcanic tuffs and intrusions within Mississippian- to Pennsylvanian-age units (Werdon, 1996; Mull and Werdon, 1994). Alkaline volcanic rocks at the shale-hosted Red Dog and Drenchwater massive sulfide deposits have ${ }^{40} \mathrm{Ar}{ }^{\beta 9} \mathrm{Ar}$ plateau ages ranging from 343 Ma to $336 \mathrm{Ma}$ (Werdon and others, 1998). Northward emplacement of allochthons during the Mesozoic Brookian orogeny is related to convergence between the continental Arctic Alaska terrane and the interior of Alaska (Moore and others, 1994).

A reconnaissance study by Ellersieck and others (1990) has suggested that the vein-breccia occurrences may either be feeder zones to Carboniferous Sedex deposits or may have formed along Mesozoic or post-Mesozoic faults in the Brooks Range. Although the $\mathrm{Zn}-\mathrm{Pb}-\mathrm{Ag}$ geochemical signature and relative stratigraphic position of the vein-breccia occurrences suggests that they may be genetically related to the Sedex hydrothermal event, previous studies have not provided evidence documenting a Carboniferous age for the vein-breccia occurrences. The timing of Sedex and vein-breccia mineralization in the northern Brooks Range has recently been investigated by the ${ }^{40} \mathrm{Ar} /{ }^{39} \mathrm{Ar}$ laser step-heating method (Werdon and others, 1998). Volcanic tuffs, breccias, and small intrusions at Drenchwater have been cut and partially replaced by sulfide mineralization (Werdon, 1996). Biotite from the intrusions provides an estimate of the maximum age for the Sedex mineralizing event. A 3-m-thick, hydrothermal white mica altered igneous sill(?), which intrudes the Ikalukrok unit of the Upper Mississippian to Pennsylvanian(?) Kuna Formation, was also dated from the south side of the main Red Dog deposit. The igneous sill(?) consists of intergrown fine-grained white mica, clay(?), and granular quartz, and it is cut by hydrothermal quartz \pm sphaleriite \pm pyrite \pm galena \pm white mica veins. The plateau age of this whole-rock sample records the time at which temperatures cooled below the argon closure temperature of hydrothermally produced white mica (interpreted as representing the late stage of the hydrothermal system). The biotite from Drenchwater and the white mica from the sill(?) at Red Dog suggest that the maximum and minimum age limits for the Sedex mineralizing event are approximately $337 \mathrm{Ma}$ and about $314 \mathrm{Ma}$, respectively (Werdon and others, 1998).

Recrystallized detrital white mica from sandstone adjacent to a quartz-sulfide vein at Kady was also dated by the ${ }^{40} \mathrm{Ar} /{ }^{39} \mathrm{Ar}$ laser step-heating method (Werdon and others, 1998). Plateau ages of $324 \pm 2 \mathrm{Ma}$ and $325 \pm 2 \mathrm{Ma}$, and an isochron age of $324 \pm 3$ Ma were obtained from three whole-rock sandstone chips from 
the same hand specimen. Although these dates fall within the ${ }^{40} \mathrm{Ar} /{ }^{39} \mathrm{Ar}$ timing constraints for the Carboniferous Sedex mineralizing event, it could be argued that the ages represent a regional heating event if the field and petrographic data suggest that mineralization at Kady did not occur during Carboniferous time. In addition, lead isotopic compositions of galena at Kady (Gaccetta and Church, 1989; Werdon, 1998) are statistically indistinguishable at $2 \sigma$ from those of galena in the shale-hosted Red Dog, Lik, and Drenchwater massive sulfide deposits (Gaccetta and Church, 1989). The isotopic similarity suggests a similar source for the lead in these deposits, and it suggests, but does not require, that these deposits are the same age because lead isotopic ratios do not change when physically remobilized.

The data presented in this paper document that the mineralization at the Kady vein-breccia prospect is Carboniferous in age. The physical and chemical controls on ore formation are also discussed. Unlike most vein-breccia occurrences in the northern Brooks Range, the Kady prospect is well exposed and the geologic and structural relationships are clear. The lack of extensive weathering allowed detailed examination of the mineralized zones and determination of the composition, cementation, diagenetic history, and hydrothermal alteration of the host rocks. Investigation of the host rocks was undertaken to (1) evaluate the local Endicott Group's potential as a source of $\mathrm{Zn}, \mathrm{Pb}$ and $\mathrm{Cu},(2)$ characterize wallrock alteration adjacent to the veinbreccia zones, and (3) investigate the stratigraphic controls of both disseminated and vein-breccia mineralization. The lack of known Sedex mineralization in the Killik River quadrangle suggests that the Kady prospect represents the hydrothermal fluid pathway below a failed or nonexistent Sedex system. Kady provides a good opportunity to examine why Sedex mineralization may not have formed.

\section{Methods}

Geologic and structural mapping of the Kady area was conducted at a scale of 1:15,840 during 19 days in the field. The size, texture(s), and mineralogy of each vein-breccia and disseminated sulfide zone was recorded. Forty-five doubly-polished thin sections of vein- and breccia-textured ore were examined by transmitted- and reflected-light petrography. Eight doubly-polished, 30- $\mu \mathrm{m}$ thin sections of sphalerite were quantitatively analyzed for $\mathrm{Zn}, \mathrm{Fe}, \mathrm{Cd}, \mathrm{Mn}$, and S using the University of AlaskaFairbanks Cameca electron microprobe. Standards used for calibration include: $\mathrm{MnS}(\mathrm{Mn}, \mathrm{S}), \mathrm{Fe}_{40} \mathrm{Zn}_{60} \mathrm{~S}(\mathrm{Fe})$, and $\mathrm{CdSZnS}(\mathrm{Cd}$, $\mathrm{Zn})$. Analytical operating conditions include an accelerating potential of $25 \mathrm{keV}$, a beam current of $20 \mathrm{nA}$, and a beam diameter of $1 \mu \mathrm{m}$. Weight-percent analyses were converted to mole percent. Additionally, the sphalerite color for each point was recorded.

Selected hand samples of visibly mineralized rocks at Kady were analyzed for major and trace elements by inductively coupled plasma atomic emission spectroscopy (ICP-AES), and rocks suspected of containing elevated levels of $\mathrm{Ag}, \mathrm{Zn}, \mathrm{Pb}$, and $\mathrm{Cu}$ were analyzed by fire assay (Meyer and Kurtak, 1992). All $\mathrm{Au}$ analyses were determined by the fire-assay method. Several pulps from this data set were also analyzed for trace elements by $\mathrm{X}$-ray fluorescence (XRF) to confirm suspected interference problems (discussed in text). Interference problems in fire-assay analyses for Au occurred in samples that contain greater than 2 percent $\mathrm{Pb}$.

Sphalerite, galena, chalcopyrite, and pyrite separates were made by cutting out selected areas with a trim saw, crushing to between 30 and 100 mesh, washing, and then hand-picking grains under high magnification to minimize contamination between phases. Sulfur isotopic analyses for sphalerite, galena, chalcopyrite, and ore-stage pyrite are reported as per mil variations with respect to the Canyon Diablo iron meteorite. Samples were analyzed by Geochron Laboratories, Inc., Cambridge, Mass., and have analytical uncertainties of $\pm 0.2 \%$ at $2 \sigma$.

Hydrothermal quartz separates were made by cutting out selected pieces of quartz from both vein- and breccia-textured, sulfide-bearing samples. Oxygen isotopic compositions obtained from hydrothermal quartz are reported as per mil (\%o) variations of ${ }^{18} \mathrm{O} /{ }^{16} \mathrm{O}$ with respect to standard mean ocean water (SMOW). Samples were analyzed by Dr. K.L. Shelton, University of Missouri-Columbia, and the analytical precision is generally better than $\pm 0.1 \%$ at $1 \sigma$.

Approximately 115 clastic rocks and their cements at Kady were examined petrographically, by electron microprobe energy dispersive spectroscopy (EDS), and by cathodoluminescence (CL) (operated at 20-25 keV). The results are summarized in the Appendix.

Fluid-inclusion temperature and salinity data were obtained from vein quartz at Kady. The individual analyses are presented in Werdon (1998), along with a complete description of the samples, sample locations, and analytical methods.

\section{Geology and Structure of the Kady Area}

Stratigraphic units in the Kady area are part of the Lower Mississippian to Upper Devonian Endicott Group, which includes the Hunt Fork Shale, Noatak Sandstone, Kanayut Conglomerate, and Kayak Shale. The Hunt Fork Shale is exposed along a fault separating a south-dipping upright section of Noatak Sandstone to the south from an overturned, southdipping section of the Noatak Sandstone to the north (fig. 2). The Hunt Fork Shale consists of brown, fine-grained, carbonatecemented sandstone interbedded with variably micaceous, grayish-black, compact to fissile shale. The Hunt Fork Shale served as a large-scale detachment surface in the Kady area; therefore, stratigraphically underlying units are not present. The thickness of the Hunt Fork Shale is difficult to determine because it is typically structurally thickened or thinned, but it is estimated to be greater than $500 \mathrm{~m}$ thick in the Kady area (Mull and others, 1994).

The lower part of the Noatak Sandstone is exposed in the core of several anticlines (fig. 2). The lower part of the Noatak Sandstone in the Kady area consists of interbedded red-brown to black shale; red-brown siltstone; brown, fine- to mediumgrained calcareous sandstone; and lesser orange-brown sandy limestone. It is characterized by distinctive, manganese-oxidecoated, carbonate-rip-up-clast layers (intra-formational lithoclast conglomerates) within calcareous sandstones, and small (less than $2 \mathrm{~cm}$ ) brachiopods. The brachiopods are similar to brachiopods in Devonian units elsewhere in the northern Brooks 


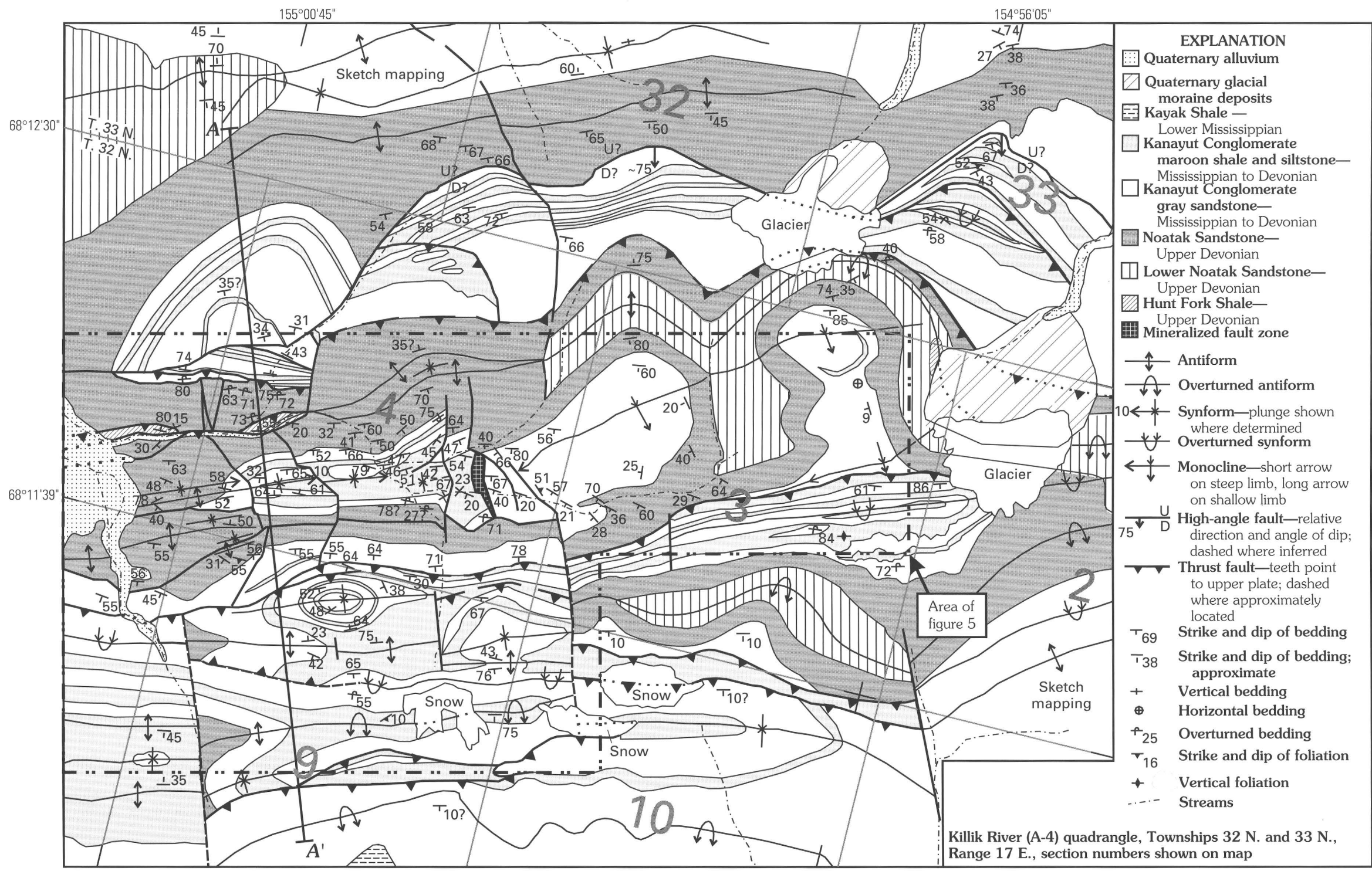

Figure 2. Geologic map of the Kady area. 
Range (R. Blodgett, oral commun., 1993). The thickness of the lower part of the Noatak Sandstone is estimated to be approximately $50 \mathrm{~m}$, and it is depositionally overlain by the upper part of the Noatak Sandstone.

The upper part of the Noatak Sandstone in the Kady area is a light greenish brown, medium-grained, thin wavy-bedded to crossbedded to massive, partially to completely carbonatecemented sandstone (fig. $3 A$ ). It is characterized by patchy poikilitic carbonate cement, calcareous concretions (as large as $30 \mathrm{~cm}$ in diameter) (fig. $3 B$ ), thin black shale flasers, variably micaceous partings, and small patches of disseminated pyrite (fig. 3 C). The thickness of the upper part of the Noatak Sandstone is estimated to be about $170 \mathrm{~m}$. The carbonate cement content decreases as the Noatak Sandstone grades upward into the Kanayut Conglomerate.

The Kanayut Conglomerate in the Kady area consists of interbedded gray sandstone, red-brown siltstone and shale, and minor calcareous sandstone and quartz-chert-pebble conglomerate. It contains occasional limonite rip-up clasts, carbonaceous plant fragments, thin coal beds, and intergranular carbonaceous material that highlights bedding within sandstone. The gray sandstone at the base of the Kanayut Conglomerate is a distinctive marker bed; it forms massive, prominently outcropping ridges covered by black lichen. The gray sandstone is dominantly quartz cemented (generally less than or equal to 5 percent carbonate cement) and contains fining-upward sequences (fig. $3 D$ ), trough crossbedding, planar bedding, and minor, thin conglomerate beds (pebbles as large as $4 \mathrm{~cm}$ in diameter; average less than or equal to $2 \mathrm{~cm}$ ) (fig. $3 E$ ). Thinner, erosion-resistant, gray sandstone layers are interbedded with reddish-brown siltstone, maroon to black shale, and conglomerate in the upper half of the Kanayut Conglomerate. The thickness of the Kanayut Conglomerate is estimated to be about 300 m. The Kayak Shale depositionally overlies the Kanayut Conglomerate, but it was not examined as part of this study.

Sedimentary rocks of the Endicott Group are complexly and spectacularly folded and faulted in the Kady area. The initial stages of Mesozoic compression produced kilometer-scale folds (open and overturned antiforms and synforms) (figs. 2, 3F). The axes of the folds trend roughly N. $75^{\circ} \mathrm{E}$., and their axial planes
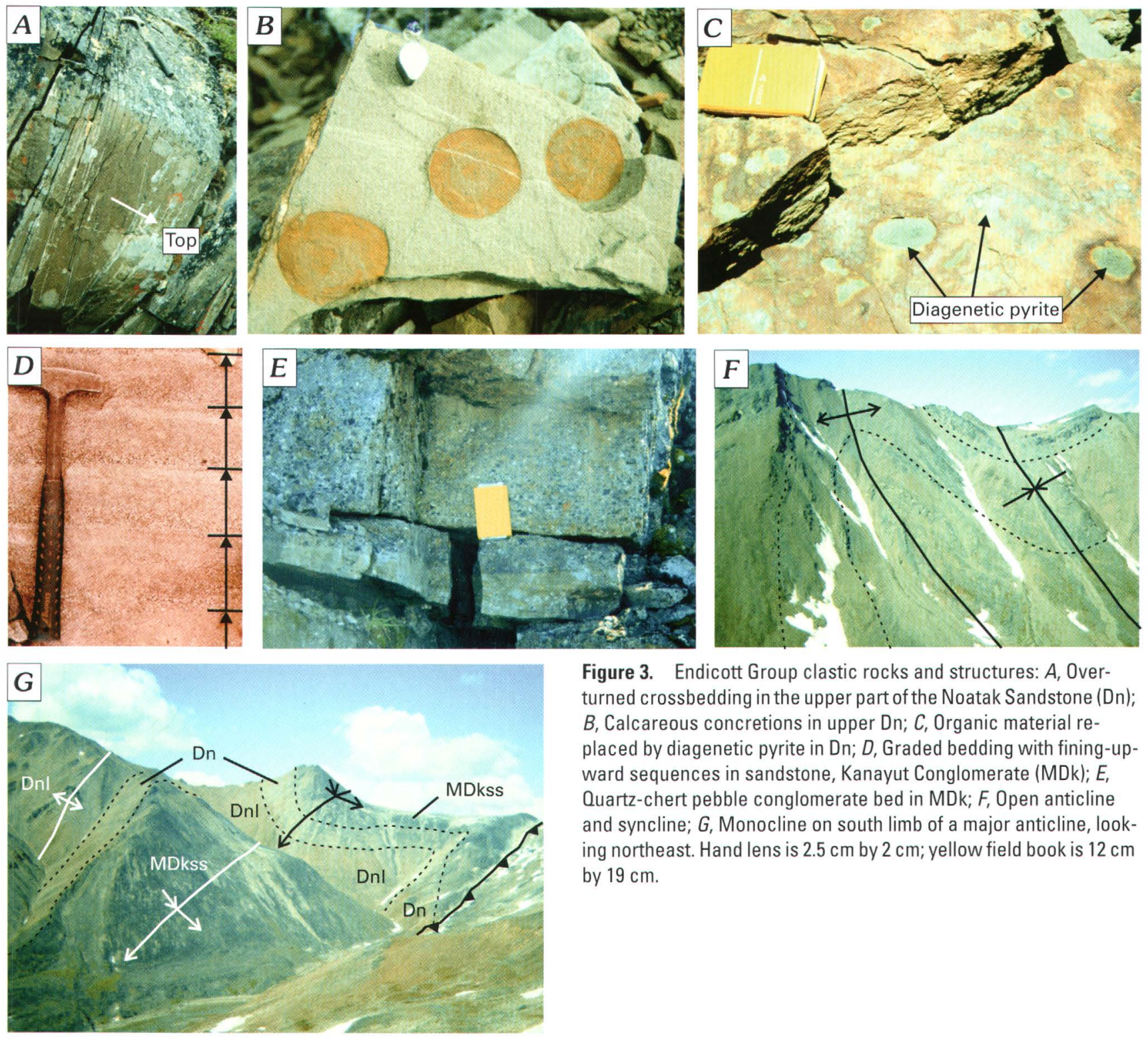

Figure 3. Endicott Group clastic rocks and structures: $A$, Overturned crossbedding in the upper part of the Noatak Sandstone (Dn); $B$, Calcareous concretions in upper $\mathrm{Dn} ; C$, Organic material replaced by diagenetic pyrite in $\mathrm{Dn} ; D$, Graded bedding with fining-upward sequences in sandstone, Kanayut Conglomerate (MDk); $E$, Quartz-chert pebble conglomerate bed in MDk; $F$, Open anticline and syncline; $G$, Monocline on south limb of a major anticline, looking northeast. Hand lens is $2.5 \mathrm{~cm}$ by $2 \mathrm{~cm}$; yellow field book is $12 \mathrm{~cm}$ by $19 \mathrm{~cm}$. 
dip moderately to steeply to the south. A distinctive, west-plunging monocline occurs on the south flank of the large anticline in the central map area (fig. $3 G$ ). A structural cross section through the Kady area (fig. 4) shows the relatively incompetent Hunt Fork Shale overlain by the Noatak Sandstone and the sandstonedominated lower part of the Kanayut Conglomerate, which form a relatively competent layer. Incompetent shale-rich units in the upper Kanayut Conglomerate are structurally thickened and internally folded within the cores of anticlines. Continued compression produced thrust faults subparallel to fold axes and a conjugate set of reverse faults. Offset on thrust faults in the Kady area is on the scale of a few hundred meters or less.

The Mesozoic structural style varies significantly from east to west. For example, the major anticline north of the monocline is overturned on its eastern end (fig. 2). To the west, the anticline progressively changes into an open fold, an open fold with an Mshaped core, and on the western end one limb is overturned and faulted away. Two north-northwest-trending, high-angle faults in the southern half of the map area (fig. 2) separate areas with different structural styles.

A late, east-trending normal(?) fault is present in the northern half of the map area (fig. 2). This fault is parallel to Mesozoic thrust faults and could also be interpreted as a thrust fault with younger-over-older-style displacement. One north-northwest-trending fault in the northern map area offsets the easttrending fold and fault structures.

\section{Mineralization}

\section{Distribution and Field Description of Sulfide Mineralization}

The Kanayut Conglomerate is the main host for veinbreccia and disseminated sulfide mineralization at Kady, with lesser amounts occurring in the Noatak Sandstone. Sulfide minerals include sphalerite, galena, chalcopyrite, and minor pyrite. Most mineralization occurs within a subparallel set of vein-breccia zones that strike approximately N. $25^{\circ} \mathrm{W}$. and dip steeply west (fig. 5). Vein-breccia zone $\mathrm{U}$ is the widest (as wide as $37 \mathrm{~m}$ ) and highest grade mineralized zone (figs. $6 A$ and $6 B$ ).
Vein-breccia zones B-C-E-F-G and L-K-H are interpreted to be formerly continuous and subparallel systems (fig. 6C) - both of these vein-breccia zones terminate at the Mesozoic thrust faults that bound their north and south sides, and the apparent changes in strike, in part, reflect topographic variations. Vein-breccia zone B-C-E-F-G is offset approximately $12 \mathrm{~m}$ along a small normal fault near $\mathrm{C}$, and there is a thrust fault between $\mathrm{F}$ and $\mathrm{G}$, and $\mathrm{K}$ and $\mathrm{H}$. This structural evidence supports the interpretation that the vein-breccia zones were present before Mesozoic deformation. Vein-breccia zones U-V, M, P and possibly A (fig. 5) are also part of this subparallel set. Vein-breccia zones with approximately east-west orientations include $\mathrm{D}, \mathrm{AB}, \mathrm{AC}$, and a small breccia zone near E (fig. 5). Vein-breccia zone D is parallel to the fold axis of an anticline, and its orientation may suggest that minor sulfide remobilization accompanied Mesozoic deformation.

Although most vein-breccia zones have an overall linear strike orientation, one vein at $\mathrm{H}$ is slightly folded (fig. $6 D$ ). Other veins at $\mathrm{H}$ occur in thin-sheeted vein sets (fig. $6 E$ ), or pinch out and are cut by late faults with minor slip (fig. $6 F$ ). Most veins at Kady are wider in brittle sandstone units and pinch out or disappear entirely in less competent shale units. The vein-breccia zones cross bedding at steep angles and are roughly perpendicular to the axial traces of large-scale Mesozoic folds and thrust faults (fig. 5). The approximately N. $55^{\circ} \mathrm{E}$. trend is the extensional direction in the Mesozoic contractional event and could be interpreted as evidence for vein formation during or after Mesozoic deformation. The offset of Mesozoic fold axes along several of the western vein-breccia zones (figs. 2,5 ) could either be interpreted as evidence (1) for vein formation after Mesozoic deformation, or (2) that Carboniferous mineralized zones acted as planes of weakness during Mesozoic deformation. Sheeted quartz veins at $\mathrm{S}$ are cut off by a later, inferred, Mesozoic fault. Mineralization has been variably deformed depending upon the competency of the host rock, degree of wallrock silica flooding, and the location within a given structure. Quartz-sulfide mineralization is often cut by penetrative cleavage, joints, and (or) stylolites.

Gray sandstone within the Kanayut Conglomerate contains sparse disseminated sphalerite and galena (generally less than 0.1 percent $\mathrm{Zn}+\mathrm{Pb}$ ), both adjacent to and distal from the veinbreccia zones (fig. 5). Sulfide-bearing sandstone is recognized

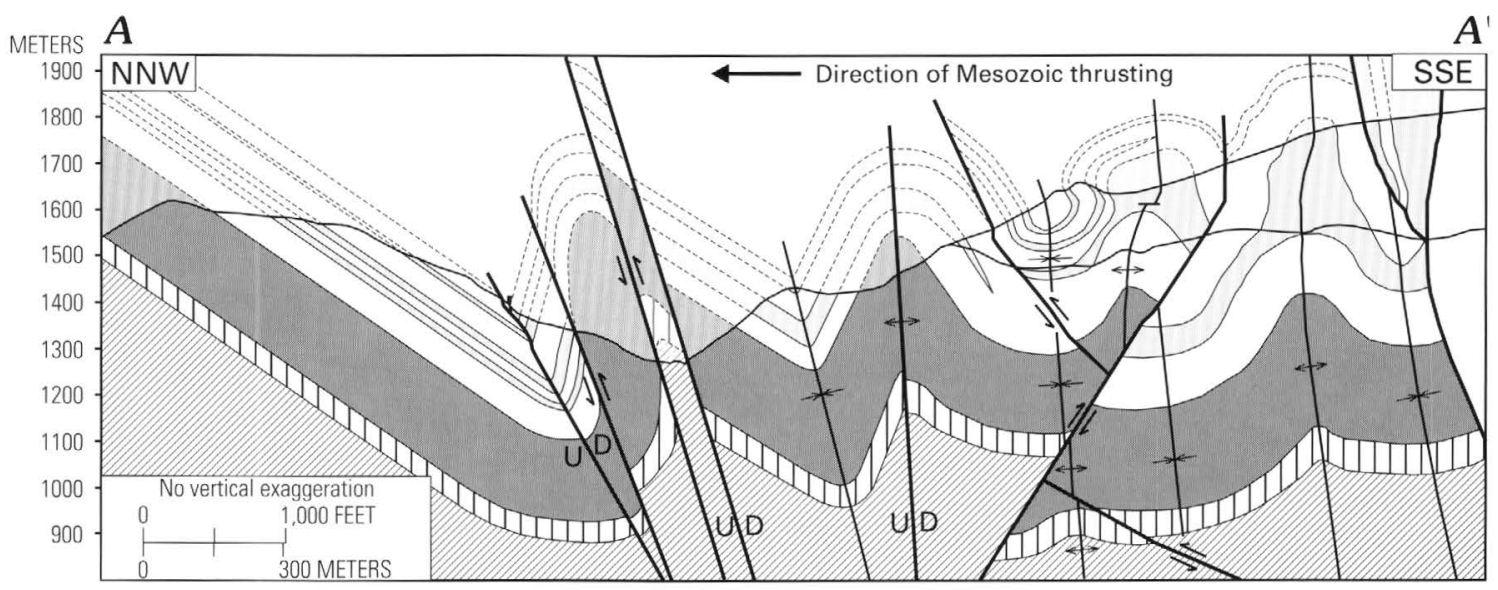

Figure 4. Structural cross section through the Kady area. 


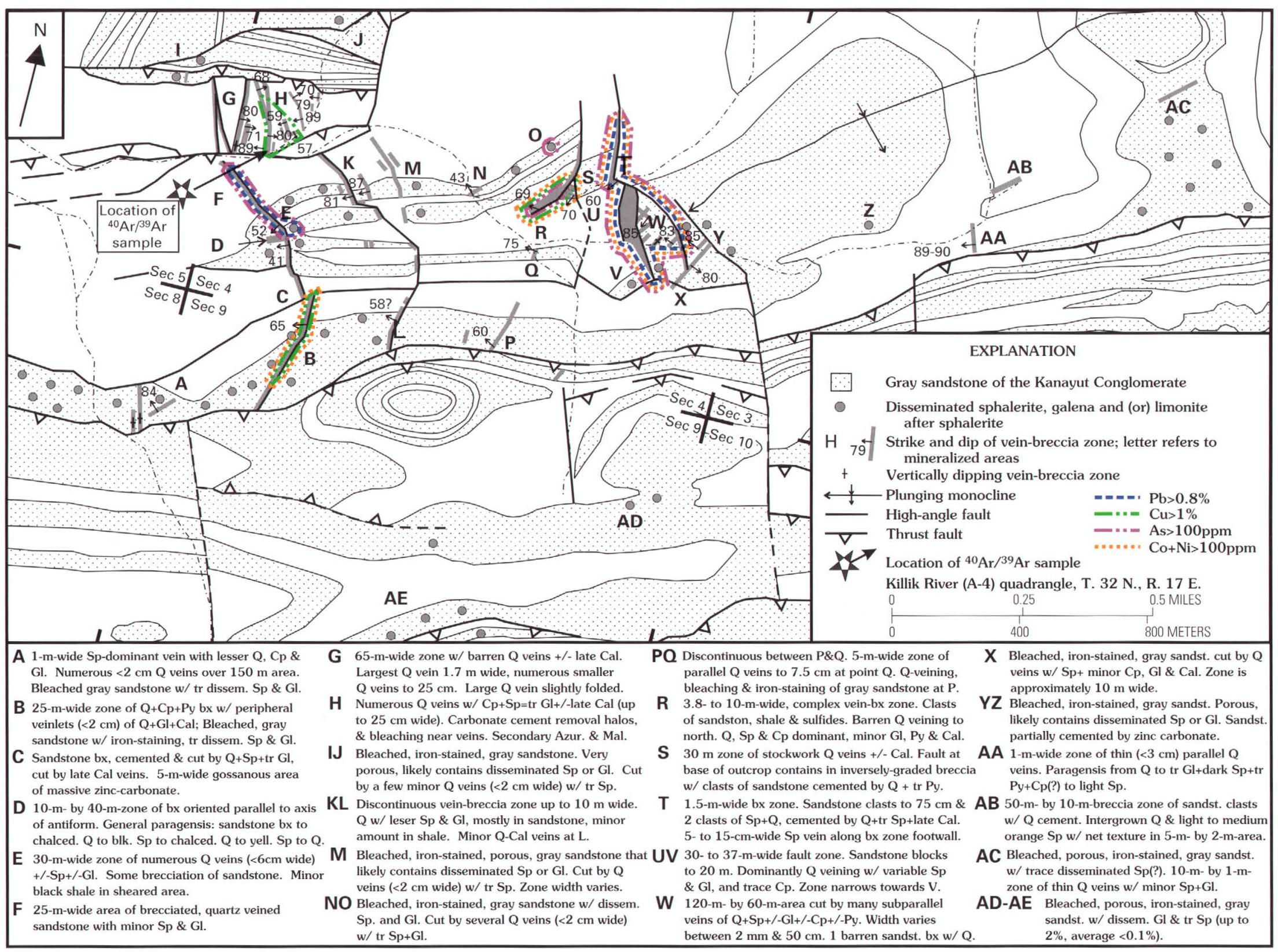

Figure 5. Map showing vein-breccia (gray lines) and disseminated (gray dots) sulfide mineralized areas. Gray sandstone within the Kanayut Conglomerate (stippled pattern) hosts most of the disseminated sulfides. Letters refer to individually described mineralized areas. Dips of mineralized vein-breccia zones are shown where measured. Map location shown in figure 2. Azur., azurite; Cal, calcite; $\mathrm{Cp}$, chalcopyrite; Gl, galena; Mal., malachite; Py, pyrite; 0 , quartz; Sp, sphalerite; amt., amount; blk., black; bx, breccia; chalced., 

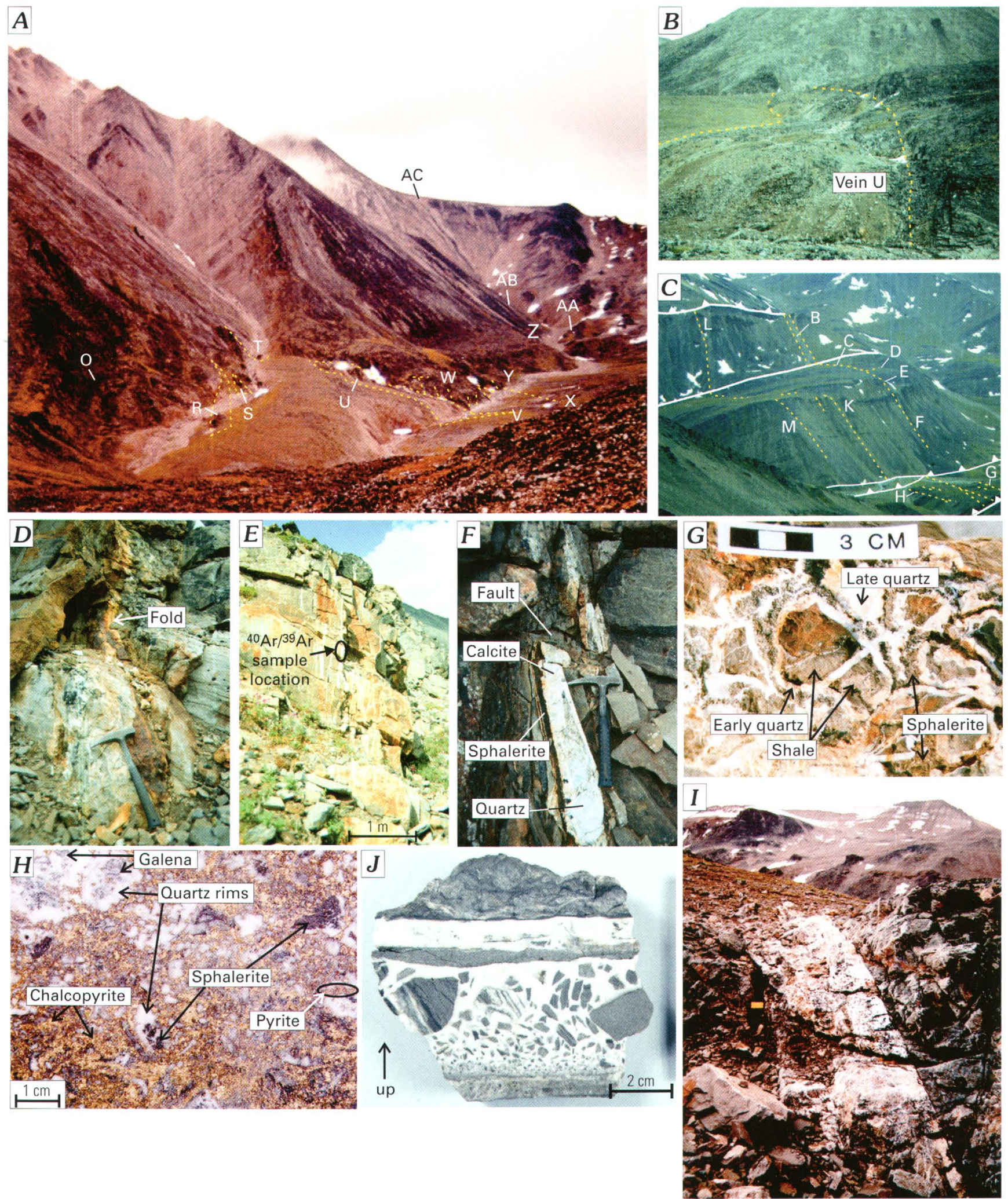

Figure 6. Vein-breccia zones and ore textures: $A_{t}$ View of vein-breccia zones looking east; $B$, Vein $U$ viewed from area $V$ (letters refer to individually described mineralized areas; see fig. 5); $C$, View of vein-breccia zones looking southwest; $D$, Folded quartz-sulfide vein in the Noatak Sandstone; $E$, Sheeted vein set at $\mathrm{H} ; F$, Pinched-out vein in shale bed in the Kanayut Conglomerate offset by late fault with minor slip, area $H$; $G$, Shale breccia clasts surrounded by milky quartz with open space between clasts filled with brown sphalerite and late quartz; $H$, Complex quartz-sulfide breccia, area R. Early galena, sphalerite, and pyrite fragments and (or) crystals are rimmed by milky quartz. Parage netically late, intergrown chalcopyrite and quartz fill leftover open space; I, Sharp-edged vein-breccia zone, vein T; yellow field book is 12 $\mathrm{cm}$ by $19 \mathrm{~cm} ; \mathrm{J}$, Inversely size graded breccia, area $\mathrm{S}$. 
in the field by its bleached, relatively light, gray color; iron staining (pale yellow to light orange red); highly reflective euhedral crystalline quartz faces inside pore spaces; and high porosity. Disseminated pyrite is sparsely distributed throughout the Kanayut Conglomerate and Noatak Sandstone, but its relationship (if any) to mineralization is unclear.

\section{Texture and Mineralogy of Individual Vein-Breccia Zones}

Individual vein-breccia zones vary in texture, width, and mineralogy (fig. 6). Textural variations include faulted host-rock blocks (as large as $20 \mathrm{~m}$ in vein $\mathrm{U}$ ) cut by thin veins, host-rock breccias cemented by sulfides and quartz (fig. $6 G$ ), symmetrical veins, veinlets, and complex sulfide-in-sulfide breccias (fig. $6 H$ ). Most breccia clasts are host-rock fragments, but clasts of veintype sphalerite in sandstone (as large as $25 \mathrm{~cm}$ in diameter) occur in vein T (fig. 6I). Host-rock breccias contain clasts that were torn from adjacent wallrocks and subsequently rotated and removed from their original locations. Vein $\mathrm{S}$ contains an unusual breccia with inversely size graded, rotated clasts, which is evidence for locally high fluid-flow rates (fig. $6 \mathrm{~J}$ ). A folded vein in area $\mathrm{W}$ has a tension gash that is symmetrically zoned with early brown sphalerite and late quartz. Sandstone along the margin of the vein is sheared, and there are peripheral barren quartz veins. The largest vein-breccia zones commonly have peripheral barren quartz veins and quartz-cemented host-rock breccias.

The relative proportions of the sulfides and their paragenetic sequences vary widely from vein to vein and within single vein-breccia zones. Locally, vein-breccia zones contain as much as 50 percent sulfides. If the entire length of the vein-breccia zones is considered (including barren quartz zones), the maximum sulfide content is less than 20 percent. Areas G, K, L, M, $\mathrm{N}$, and $\mathrm{Q}$ average less than 1 percent sulfides. Sphalerite is the dominant sulfide mineral at Kady, followed in abundance by galena. Chalcopyrite is generally minor, but in vein-breccia zone $\mathrm{B}$ it is abundant (as much as 10.9 percent $\mathrm{Cu}$ ) and constitutes as much as 98 percent of the sulfide present over a $2-\mathrm{m}$ by $20-\mathrm{m}$ area. The highest $\mathrm{Zn}$ and $\mathrm{Pb}$ grades occur in vein $\mathrm{U}$, and the highest concentrations of $\mathrm{Cu}$ occur in the $\mathrm{B}, \mathrm{H}$, and $\mathrm{R}$ veins. Pyrite occurs in significant quantities only in the $R$ and $B$ veins. Rare submicroscopic grains of pyrrhotite occur within sphalerite in vein $\mathrm{D}$, along with minor pyrite. Minor compositionally zoned pyrite and bravoite occur in vein U. Sulfosalts occur in trace amounts as microscopic blebs within galena.

Gangue minerals include abundant quartz, minor calcite, trace siderite and ankerite, and very rare chlorite. Vein quartz is usually crystalline and growth zoned but occasionally is dendritic, and there is an unusual area of chalcedonic banded quartz in vein D. Calcite, if present, always forms the paragenetically latest cement in the center of veins and breccias. Calcite is volumetrically most abundant in veins that cut carbonate-cemented sandstone, which suggests wallrock interaction buffered veinfluid chemistry $(\mathrm{pH})$. Calcite also occurs in sharp-edged veins that cut a sphalerite-cemented sandstone breccia in area C. Rare siderite and ankerite veins are paragenetically earlier than quartz-sulfide veins and only contain sulfides in area $\mathrm{H}$. Siderite, ankerite, and calcite also occur in veins and breccias not spatially associated with quartz veins or sulfide mineralization. Gangue minerals developed in wallrocks due to reactions with mineralizing fluids include siderite, ankerite, and recrystallized white mica.

Supergene weathering of a chalcopyrite-quartz vein at $\mathrm{H}$ has leached copper from chalcopyrite and precipitated malachite and minor azurite on top of calcite and quartz. Secondary azurite and malachite are also intergrown with zinc carbonate and limonite in a sandstone breccia near $\mathrm{H}$.

\section{Sphalerite Descriptions and Compositional Variations}

Sphalerite is the most common sulfide mineral at Kady, and it occurs throughout the paragenetic sequence of different veinbreccia zones. Most sphalerite is a dull to vitreous, mediumbrown color in outcrop, with minor local resinous yellow sphalerite. In doubly polished thin sections, sphalerite colors range from clear through shades of yellow, orange, reddish orange, and dark reddish orange, to an almost opaque black. The black sphalerite is actually light to medium yellow under high magnification, and the apparent black color is due to abundant microscopic inclusions of chalcopyrite. Pale-yellow sphalerite rarely contains small, grayish-brown, intensely growth zoned areas. Yellow and orange are the predominant colors in most sphalerite samples. Vein sphalerite is typically color-banded parallel to growth zones. Sphalerite in breccia-textured ore is intergrown with quartz and may be color-banded (either around sandstone clasts or as broken fragments of growth-zoned sphalerite veins) (fig. 7A), irregularly zoned (fig. 7B), sector zoned (fig. $7 C$ ), patchy, and (or) uniform in color.

Sphalerite exhibits a range in composition $\left(\mathrm{Zn}_{81.5-99.7}\right.$, $\left.\mathrm{Fe}_{0.1-17.9}, \mathrm{Cd}_{0.1-0.9}\right) \mathrm{S}$, but most sphalerite falls within the compositional range $\left(\mathrm{Zn}_{92.0-98.0}, \mathrm{Fe}_{1.5-8.0}, \mathrm{Cd}_{0.1-0.7}\right) \mathrm{S}$ (table 1). Most sphalerite did not contain Mn above the lower detection limit of 0.05 mole percent $\mathrm{MnS}$ at $2 \sigma$. Variation diagrams for mole percent $\mathrm{CdS}$ vs. FeS and mole percent FeS vs. sphalerite color (fig. 8) show the variety of sphalerite types and zoning. The color varies from clear to light yellow (0-2 mole percent $\mathrm{FeS}$ ) through dark reddish orange (14-18 mole percent $\mathrm{FeS}$ ) and is directly proportional to the iron content of the sphalerite.

Kady sphalerites fall into three different compositional groups (fig. 8). In group 1, sphalerite CdS values are highly variable, FeS values are uniformly low, and $\mathrm{CdS}$ does not vary with FeS. Sphalerite in vein $\mathrm{H}$ and late sphalerite in vein $\mathrm{U}$ occur in group 1. In group 2, sphalerite CdS values increase with FeS, and $\mathrm{FeS}$ values are less than 10 mole percent. The wide spread of values for vein R (fig. 8) may be due to redistribution of iron in sphalerite during extensive replacement by chalcopyrite. In group 3, sphalerite CdS values vary with FeS values but with a shallower slope than group-2 sphalerite. Sphalerites in group 3 also have higher FeS values. The three groups merge at low $\mathrm{CdS}$ and FeS values (fig. 8). The variety of sphalerite compositions may reflect varying chemical conditions in different parts of the Kady vein-breccia system. 


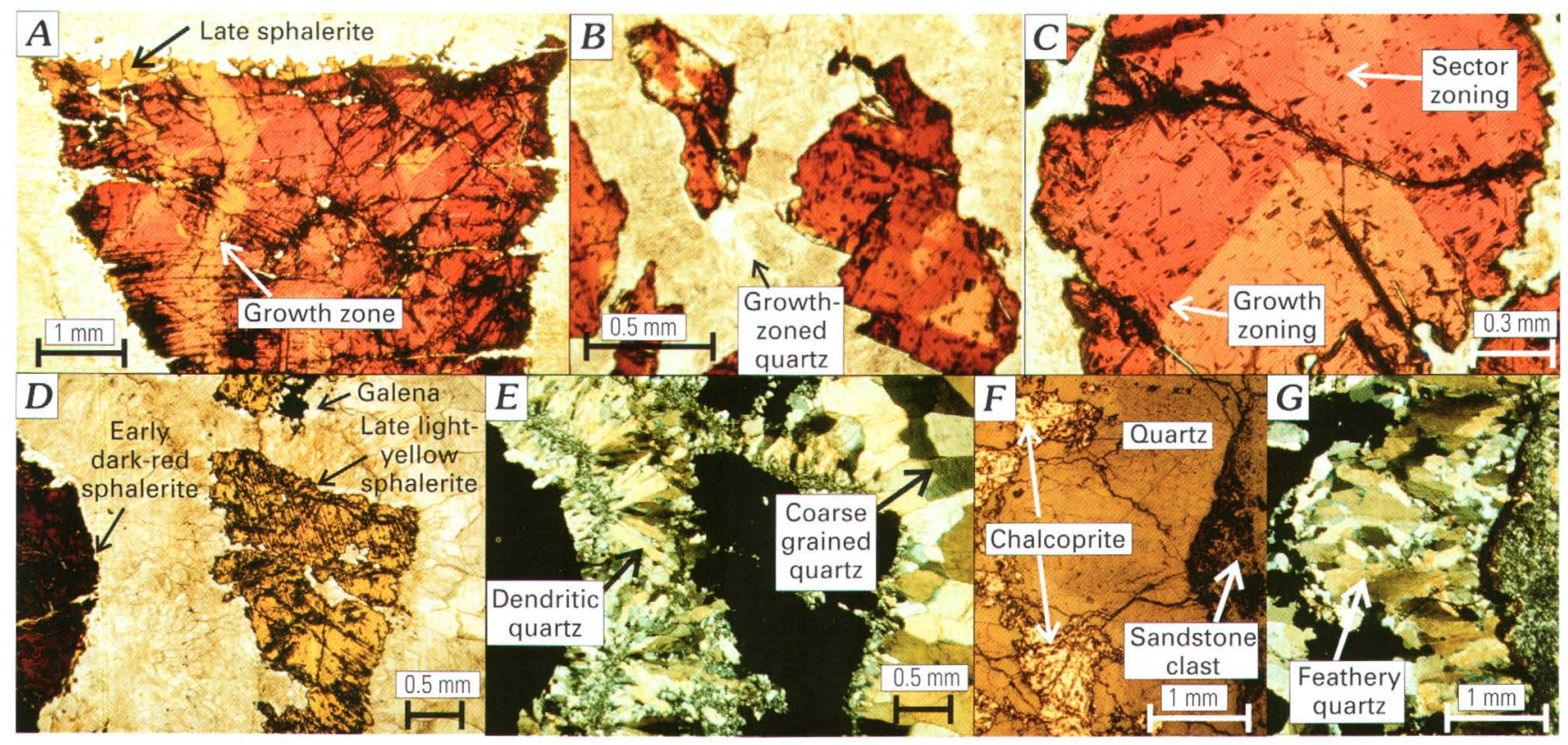

Figure 7. Quartz and sulfide textures in thin section: $A$, Vein $E$ (letters refer to individually described mineralized areas; see fig. 5); breccia fragment of growth-zoned vein-type sphalerite with a rim of late, low-iron, yellow sphalerite surrounded by quartz; $B$, Vein $E$; sphalerite with irregular color distribution surrounded by growth-zoned quartz crystals; $\mathcal{C}$, Vein $\mathrm{E}_{\text {; }}$ sphalerite crystal with both growth and sector zoning; $D$, Vein D; paragenetically early, dark-red sphalerite with trace pyrite, overgrown successively by early dendritic quartz, light-yellow sphalerite and minor galena, and paragenetically late coarse-grained quartz; $E$, Figure 7D in cross-polarized light showing early dendritic quartz and late coarse-grained quartz; $F$, Vein B; intergrown quartz and chalcopyrite adjacent to sandstone breccia clast; $G$, Figure $7 F$ in cross-polarized light showing feathery quartz adjacent to stylolitized contact with sandstone breccia clast.

In veins $\mathrm{D}$ and $\mathrm{U}$, sphalerite changes from early, relatively high iron sphalerite to late, low-iron sphalerite (figs. $7 D, 8$ ). This paragenetic change also occurs in sphalerite in vein AA. The paragenetically early, high-iron, dark reddish orange sphalerite in vein $\mathrm{D}$ contains isolated, rare, submicroscopic grains of pyrrhotite, and minor pyrite. Pyrite also occurs within quartz adjacent to the high-iron sphalerite but is not present within the paragenetically late, low-iron, clear to light-yellow sphalerite. This paragenetic evidence indicates that the sulfidation and oxidation state increased as sphalerite deposition progressed in vein $\mathrm{D}$. The early high-iron sphalerite is separated from the late low-iron sphalerite by dendritic quartz (fig. $7 E$ ), suggesting rapidly decreasing temperature conditions. Assuming sphalerite (maximum 17.9 mole percent $\mathrm{FeS}$ ), pyrite, and pyrrhotite last equilibrated between $150^{\circ} \mathrm{C}$ and $250^{\circ} \mathrm{C}$, a maximum pressure of about 1.1 to 1.3 kilobars is calculated for sulfide deposition at the Kady prospect (Toulmin and others, 1991).

\section{Major- and Trace-Element Composition of Mineralized Rocks}

Kady is predominantly a $\mathrm{Zn}-\mathrm{Pb}-\mathrm{Cu}-\mathrm{Ag}$ prospect, but variable amounts of $\mathrm{Ni}, \mathrm{Co}, \mathrm{Sb}, \mathrm{As}, \mathrm{Cd}, \mathrm{Fe}, \mathrm{Sn}$, and Au also characterize mineralized rock samples (Meyer and Kurtak, 1992).

Kady is geochemically similar to the other vein-breccia occurrences in the northern Brooks Range (Meyer and Kurtak, 1992), but Kady contains significantly more copper (as much as 10.9 percent) (fig. 9). Chalcopyrite is the only primary copper-bearing mineral observed in thin section (figs. $7 F$ and $7 G$ ). High $\mathrm{Zn}$ and $\mathrm{Cd}$ values reflect the high sphalerite content of most veins, and there is a direct correlation between the two elements. Co (as much as $230 \mathrm{ppm}$ ) and $\mathrm{Ni}$ (as much as $251 \mathrm{ppm}$ ) values reflect the presence of minor pyrite and bravoite. Minor $\mathrm{Co}$ and $\mathrm{Ni}$ also occur with iron sulfide in the Red Dog deposit and other vein-breccia occurrences in the northern Brooks Range (Meyer and Kurtak, 1992; Kelley and others, 1997). Sb-Ag $( \pm \mathrm{Pb})$ sulfosalts (identified with energy dispersive spectroscopy) are found within galena, and there is a direct correlation between $\mathrm{Ag}$ and $\mathrm{Sb}$ in most samples. The scatter may be due to stoichiometric variations and (or) the substitution of $\mathrm{Pb}$ into the sulfosalt structure. $\mathrm{Ba}$ is not common in most veins $(\leq 250 \mathrm{ppm})$ but sample 5604 (vein F) contains 875 ppm Ba (Meyer and Kurtak, 1992). Barite was not observed at Kady.

Rocks from vein-breccia occurrences in the northern Brooks Range show detectable levels of Te, Bi, Mo, and W in ICP-AES analyses (Meyer and Kurtak, 1992), elements that are not generally associated with sediment-hosted sulfide deposits (Goodfellow and others, 1993). These element concentrations correlated unusually well with $\mathrm{Zn}, \mathrm{Cu}$, and (or) $\mathrm{Pb}$ (e.g., $\mathrm{Zn}-\mathrm{W}$; $\mathrm{R}^{2}=0.999$ ), particularly in samples in which $\mathrm{Zn}, \mathrm{Pb}$, and $\mathrm{Cu}$ approached or exceeded their ICP-AES upper determination limits ( 2 percent $\mathrm{Zn}, 2$ percent $\mathrm{Cu}, 1$ percent $\mathrm{Pb}$ ). I interpret that interference in spectral lines caused readings that indicated false concentrations, and, therefore, the $\mathrm{Te}, \mathrm{Bi}, \mathrm{Mo}$, and $\mathrm{W}$ values are spurious. XRF analyses of several pulps from this data set verify the lack of $\mathrm{Bi}$ and $\mathrm{Mo}$, but interference problems were also encountered in the XRF analyses between $\mathrm{Pb}$ and $\mathrm{Bi}$ and $\mathrm{Mo}$ in a sample containing about 60 percent $\mathrm{Pb}$ (Werdon, 1998).

Relatively high levels of Sn, As, and Au were also reported in ICP analyses (Meyer and Kurtak, 1992), and in emission spectrographic data (Duttweiler, 1987), and Sn was reported in 


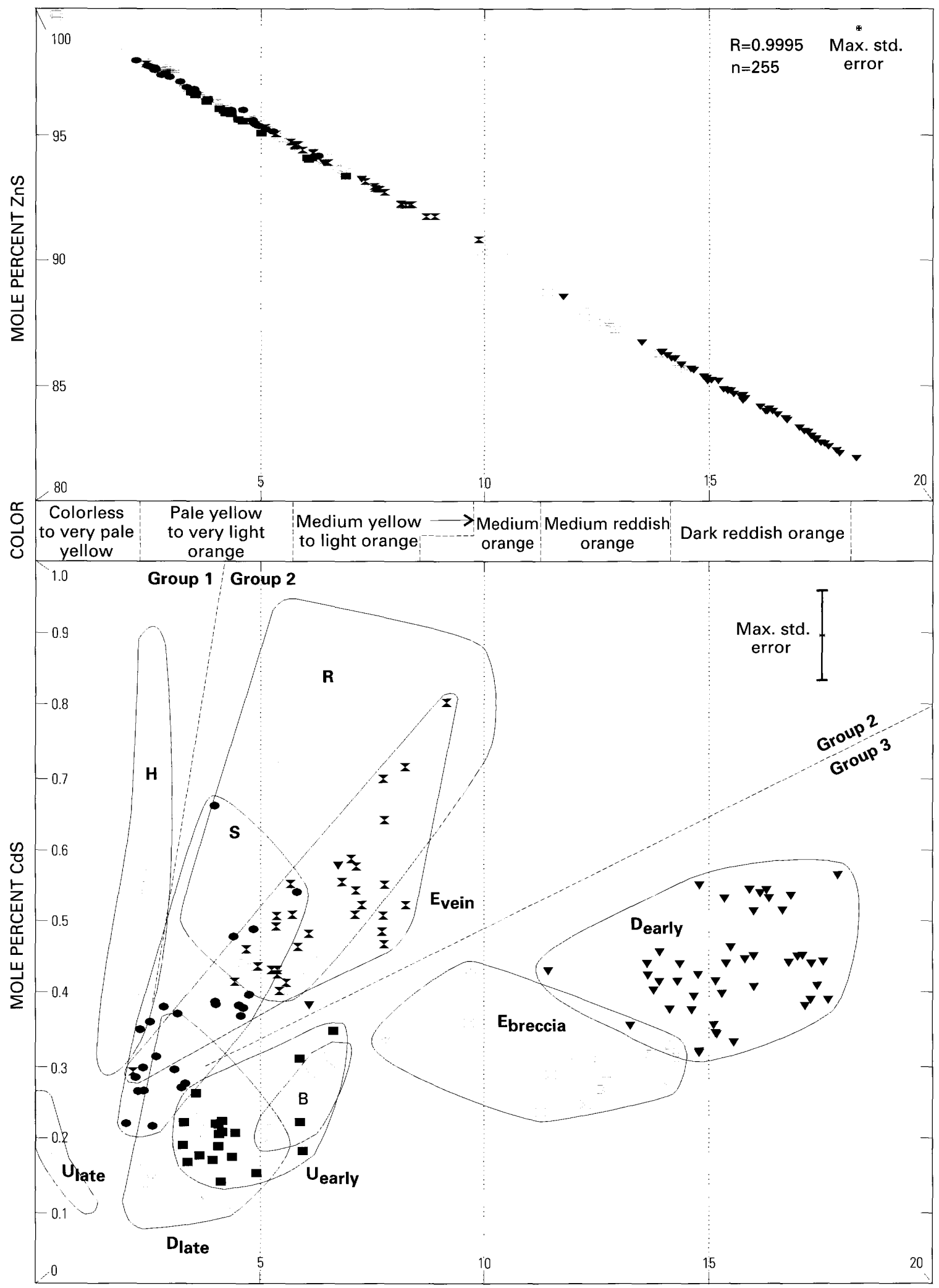

MOLE PERCENT FeS

Figure 8. Mole percent $\mathrm{FeS}, \mathrm{CdS}, \mathrm{ZnS}$ and color variations in sphalerite. Letters refer to individual mapped veins shown in figure 5. The sphalerite color correlates with mole percent FeS. The three sphalerite groups are discussed in the text. 
Table 1. Quantitative electron microprobe analyses of sphalerite.

\begin{tabular}{|c|c|c|c|}
\hline Vein & Sample description & $\begin{array}{c}\text { Sphalerite color(s) } \\
\text { (in 30- } \mu \mathrm{m} \text {-thick thin sections) }\end{array}$ & $\begin{array}{l}\text { Composition (mole percent) } \\
\text { (range } / \text { mean } / \text { median } / 1 \text { o) }\end{array}$ \\
\hline
\end{tabular}

S Brecciated, quartz-veined sandstone; angular, 1to 2-mm sphalerite fragments floating in a randomly oriented mosaic of clear, nongrowth zoned quartz crystals in a 0.5 - to 1.0 $\mathrm{cm}$-wide vein; minor chalcopyrite disease in sphalerite.

B Brecciated, quartz-veined sandstone; angular, 0.1to $1.5-\mathrm{mm}$ sphalerite fragments floating in a $2-$ $\mathrm{cm}$-wide, clear to milky quartz vein; minor chalcopyrite disease in sphalerite; trace chlorite(?) in center of vein.

R Complex sulfide breccia with sphalerite (black in hand specimen), galena, chalcopyrite, pyrite, and quartz; abundant chalcopyrite disease and veins in sphalerite; clasts include sphalerite, galena-quartz mixtures, and pyrite-quartz mixtures; clasts are rimmed by quartz, with leftover open space filled by intergrown chalcopyrite and quartz.

$\mathrm{H} \quad$ 5-cm-wide sphalerite (uniformly light brown in hand specimen) and quartz vein; sphalerite (early) cut and fragmented by irregular, symmetrically growth zoned milky quartz veins up to $0.5 \mathrm{~cm}$ wide; minor chalcopyrite disease

U Quartz-dominant, sulfide-bearing, 5-cm-wide vein cutting sandstone; paragenetically early quartz followed by co-precipitated quartz, chalcopyrite, galena, and compositionally zoned pyrite-bravoite, followed by quartz, then dark sphalerite rimmed with light sphalerite, then an unknown gray carbonate and finally, late calcite; sphalerite is cut by a vein of iron sulfide but it is not diseased by chalcopyrite.

D Complex quartz-sphalerite-sandstone breccia; breccia contains clasts of sandstone, sphalerite, and sphalerite clasts with quartz rims; quartzrimmed sandstone clasts (up to $4 \mathrm{~cm}$ ) entrained with sphalerite clasts (up to $3 \mathrm{~cm}$ ) in 10-m-wide vein-breccia zone; early reddishorange sphalerite clasts (containing and partially rimmed by pyrite cubes) surrounded by granular to dendritic to growth-zoned quartz (with trace pyrite in quartz near early sphalerite); discontinuously overgrown by clear to yellow sphalerite crystals (no pyrite), surrounded by minor chalcedonic quartz and late, open-space-filling, coarse-grained, growth-zoned quartz.
Irregular color zonation, colorless to pale yellow.

Poorly growth zoned, light to medium golden brown to yellow.

Unusually colored sphalerite; appears black, gray, or brown due to abundant chalcopyrite inclusions, but it is mostly light to medium yellow under high magnification.

Clear, very light orange, and pale yellow w/ irregular color zonation.

Growth zoned from early, light- and medium-orange sphalerite, to very light yellow, to late clear sphalerite.

Early sphalerite is medium to dark reddish orange w/ a couple of minor growth bands of medium yellow orange sphalerite.

Late sphalerite is clear to medium yellow.
ZnS 93.6 - 97.7/96.2/96.4/1.1

FeS $2.0-5.8 / 3.5 / 3.2 / 1.1$

CdS $0.2-0.7 / 0.4 / 0.4 / 0.1$

$\mathrm{MnS}$ not above lower detection limit. $\mathbf{n}=24$

ZnS $92.8-94.6 / 93.8 / 93.7 / 0.6$

FeS $5.2-6.8 / 6.0 / 6.1 / 0.6$

CdS $0.2-0.3 / 0.3 / 0.3 / 0.1$

MnS not above lower detection limit. $\mathbf{n}=10$

ZnS 89.3-96.0/94.0/94.4/1.6

FeS $3.5-9.9 / 5.5 / 5.1 / 1.5$

CdS $0.4-0.9 / 0.6 / 0.5 / 0.1$

$\mathrm{MnS}$ not above lower detection limit. $\mathbf{n}=36$

ZnS 96.5 - 98.1/97.1/97.1/0.3

FeS $1.6-2.6 / 2.3 / 2.4 / 0.2$

CdS $0.3-0.9 / 0.5 / 0.5 / 0.1$

MnS not above lower detection limit. $\mathbf{n}=25$

Early, orange sphalerite.

ZnS 93.0 - 96.5/95.4/95.7/1.0

FeS $3.3-6.7 / 4.4 / 4.1 / 1.0$

CdS $0.1-0.4 / 0.2 / 0.2 / 0.1$

MnS not above lower detection limit. $\mathbf{n}=20$

Late, clear sphalerite.

$\mathrm{ZnS} 98.8$ - 99.7/99.5/99.5/0.3

FeS $0.1-1.1 / 0.3 / 0.2 / 0.3$

CdS $0.1-0.3 / 0.2 / 0.2 / 0.0$

MnS not above lower detection limit. $\mathbf{n}=10$

Early, reddish-orange sphalerite.

ZnS 81.5 - 93.5/84.4/84.1/2.3

FeS $6.1-17.9 / 15.1 / 15.4 / 2.3$

CdS $0.3-0.6 / 0.4 / 0.4 / 0.1$

$\mathrm{MnS}$ not above lower detection limit. $\mathrm{n}=47$

Late, clear to yellow sphalerite.

ZnS $94.4-97.7 / 96.5 / 96.6 / 0.8$

FeS $2.2-5.4 / 3.3 / 3.2 / 0.8$

CdS $0.1-0.4 / 0.2 / 0.2 / 0.1$

$\mathrm{MnS}$ not above lower detection limit. $\mathbf{n}=38$ 
Table 1. Quantitative electron microprobe analyses of sphalerite-Continued.

\begin{tabular}{|c|c|c|c|}
\hline Vein & Sample description & 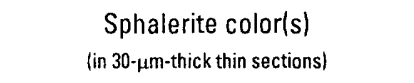 & $\begin{array}{l}\text { Composition (mole percent) } \\
\text { (range / mean /median / } 1 \sigma \text { ) }\end{array}$ \\
\hline $\mathrm{E}_{\text {breccia }}$ & $\begin{array}{l}\text { Angular, } 0.1 \text { - to } 1.5-\mathrm{mm} \text { fragments of sphalerite } \\
\text { and sandstone floating, with pyrite crystals, in } \\
\text { a } 3-\text { to } 4-\mathrm{cm} \text {-wide quartz vein; minor to } \\
\text { abundant chalcopyrite and iron sulfide disease } \\
\text { with small patches of chalcopyrite replacing } \\
\text { sphalerite. }\end{array}$ & $\begin{array}{l}\text { Medium orange red to light } \\
\text { orange, irregularly zoned. }\end{array}$ & $\begin{array}{l}\text { ZnS } 85.4-91.9 / 87.5 / 87.2 / 1.9 \\
\text { FeS } 7.8-14.3 / 12.2 / 12.5 / 1.9 \\
\text { CdS } 0.2-0.4 / 0.3 / 0.3 / 0.1 \\
\text { MnS up to } 0.1 \\
\mathbf{n}=15\end{array}$ \\
\hline $\mathrm{E}_{\text {vein }}$ & $\begin{array}{l}\text { Sphalerite-dominant vein with growth-zoned, } \\
\text { vein-type sphalerite fragments enclosed in a } \\
\text { matrix of finely crushed sphalerite intergrown } \\
\text { with quartz; minor chalcopyrite and iron } \\
\text { sulfide disease; some pyrite appears to replace } \\
\text { sphalerite; pyrite is euhedral in quartz adjacent } \\
\text { to sphalerite. }\end{array}$ & $\begin{array}{l}\text { Mostly medium yellow to light } \\
\text { orange; growth zoned; one } \\
\text { sphalerite vein fragment is } \\
\text { medium orange. }\end{array}$ & $\begin{array}{l}\text { ZnS } 90.0-97.5 / 93.1 / 93.0 / 1.6 \\
\text { FeS } 2.2-9.2 / 6.4 / 6.5 / 1.5 \\
\text { CdS } 0.3-0.8 / 0.5 / 0.5 / 0.1 \\
\text { MnS up to } 0.1 \\
\mathbf{n}=30\end{array}$ \\
\hline
\end{tabular}

XRF analyses (Werdon, 1998). Sn values occur within veins with high $\mathrm{Zn}$ concentrations, and there is a weak correlation between the elements. No Sn- or As-bearing minerals were observed in polished thin sections.

\section{Isotopic Work}

\section{Sulfur Isotopes}

Sulfur isotopic values (table 2) obtained for sulfides from vein-breccia zones $\mathrm{W}$ and $\mathrm{R}$ were used to estimate the temperature of sulfide deposition using the sulfur isotopic thermometer equations of Ohmoto and Rye (1979). Sulfur isotope geothermometry, based on the fractionation of sulfur isotopes between different sulfur-bearing compounds, assumes that equilibrium was obtained and preserved between two co-precipitated minerals. Sphalerite and galena in vein $\mathrm{W}$ are texturally intergrown and are interpreted to be co-precipitated. If isotopic equilibrium is assumed, the sulfide pair indicates a temperature of $209^{\circ} \pm 25^{\circ} \mathrm{C}$ during sulfide deposition. In vein-breccia zone R, sulfides occur in a complex breccia texture. Paragenetically early pyrite and sphalerite are overgrown by growth-zoned quartz. Paragenetically late galena, chalcopyrite, and quartz fill interstitial space left over between the pyrite-sphalerite-quartz clasts. If equilibrium at each stage is assumed, the early pyritesphalerite pair indicates a temperature of $251^{\circ} \pm 50^{\circ} \mathrm{C}$, and the late galena-chalcopyrite pair indicates a temperature of $162^{\circ} \pm 35^{\circ} \mathrm{C}$.

\section{Oxygen Isotopes}

Quartz was selected for oxygen isotopic analyses from both vein- and breccia-textured samples at Kady (table 3). Reliable oxygen-isotope compositions of ore-forming fluids are difficult to obtain from direct fluid-inclusion-extraction procedures because ore-forming fluids trapped in primary fluid inclusions can be contaminated by secondary fluid inclusions trapped during deformation. The oxygen-isotope composition of hydrothermal solutions can, however, be determined from quartz-water fractionation factors using fluid-inclusion homogenization temperatures and measured $\delta^{18} \mathrm{O}$ values of hydrothermal quartz. Fluid-inclusion analyses from primary, two-phase (water-vapor) fluid inclusions indicate vein quartz at Kady was deposited from

Table 2. Sulfur isotopic analyses of sulfide minerals.

[Vein locations are shown on fig. 5. Sulfur isotopic analyses for sphalerite, galena, chalcopyrite, and ore-stage pyrite are reported as per mil variations with respect to the Canyon Diablo iron meteorite. Samples were analyzed by Geochron Laboratories, Inc., Cambridge, Mass., and have analytical uncertainties of $\pm 0.2 \%$ at $2 \sigma]$

\begin{tabular}{cccc}
\hline Vein & Mineral & ${ }^{34} \mathrm{~S}(\%)$ & Sample description \\
\hline R & sphalerite & 11.4 & 4-m-wide complex vein-breccia zone with quartz $(70 \%)$, sphalerite $(16 \%)$, chalcopyrite \\
R & chalcopyrite & 10.9 & $(8 \%)$, galena $(1 \%)$, pyrite $(3 \%)$ and calcite $(2 \%)$. \\
R & galena & 7.8 & \\
R & ore-stage pyrite & 12.5 & \\
W & sphalerite & 12.7 & $0.37-\mathrm{m}$-wide vein of co-precipitated quartz (30\%) and light- to medium-brown sphalerite \\
W & galena & 9.6 & $(70 \%)$ with trace galena.
\end{tabular}


$\leq 6$ equivalent weight percent $\mathrm{NaCl}$ fluids at homogenization temperatures between $100^{\circ} \mathrm{C}$ and $145^{\circ} \mathrm{C}$ (not pressure corrected), and there is no evidence to suggest boiling (Werdon, 1998).

The $\delta^{18} \mathrm{O}$ data from quartz (sample U-2), and primary fluidinclusion homogenization temperatures in vein $U$ are used to calculate the oxygen-isotope composition of the hydrothermal fluid at Kady using the quartz-water fractionation factor equation of Matsuhisa and others (1979):

$$
\delta^{18} \mathrm{O}_{\mathrm{qtz}}-\delta^{18} \mathrm{O}_{\mathrm{f}}=\left(\frac{3.34 \times 10^{6}}{\mathrm{~T}^{2}}\right)-3.31
$$

where,

$\delta^{18} \mathrm{O}_{\mathrm{qtz}}$ is the $\delta^{18} \mathrm{O}$ data from quartz,

$\delta^{18} \mathrm{O}_{\mathrm{f}}$ is the $\delta^{18} \mathrm{O}$ composition of the hydrothermal fluid, and

$\mathrm{T}$ is the primary fluid-inclusion homogenization temperature in degrees Kelvin.

The fluid-inclusion measurements for vein $U$ were taken on a different sample than the $\delta^{18} \mathrm{O}$ measurements, but the quartz in both cases is spatially associated with chalcopyrite and galena. $\delta^{18} \mathrm{O}$ values of the hydrothermal quartz range from $13.6 \%$ o to $13.8 \%$ o (table 3 ), and primary fluid-inclusion homogenization temperatures range from $118^{\circ} \mathrm{C}$ to $143^{\circ} \mathrm{C}$ (Werdon, 1998). This corresponds to a range of calculated $\delta^{18} \mathrm{O}_{\mathrm{f}}$ values between $-4.9 \%$ and $-2.2 \%$ for the hydrothermal fluid at Kady.

Although no isotopic values for hydrogen $(\delta \mathrm{D})$ are available for the hydrothermal fluid at Kady, the calculated range of $\delta^{18} \mathrm{O}_{\mathrm{f}}$ values provides insight into the character and origin of the hydrothermal fluid. The source of the hydrothermal fluid at Kady can be modeled in two ways (fig. 10). The $\delta^{18} \mathrm{O}_{\mathrm{f}}$ values may suggest that the fluid was originally derived from a seawater and (or) a meteoric water source. The relative contribution of meteoric water and seawater cannot be determined, given the uncertainties in the ancient seawater field boundary (Sheppard, 1986). The other way to model the fluid is mixing between meteoric water and either a deep basinal brine or a metamorphic fluid.

\section{Host-Rock Diagenesis and Alteration}

\section{Clastic Components, Early Cements, and Diagenesis}

Clastic rocks of three Endicott-Group formations (Hunt Fork Shale, Noatak Sandstone, and Kanayut Conglomerate) distal to the vein-breccia zones (as far as $2 \mathrm{~km}$ away) were examined to determine which mineral assemblages characterized sedimentation, early cementation, and diagenesis. These features have a widespread distribution and show no spatial relationship to the vein-breccia zones.

Sandstones distal to the vein-breccia zones at Kady are predominantly (80-95 percent) composed of monocrystalline quartz (MCQ) and black chert fragments. The ratio of MCQ to chert varies from 50:50 to 95:5. Cherty argillite lithic fragments (1-10 percent) and white mica (1-10 percent) are common minor components. Plagioclase (1-4 percent), clay and (or) illite patches ( $0-13$ percent), chlorite ( $0-2$ percent), and accessory minerals (apatite, tourmaline, zircon, and rutile (anatase?) - less than or equal to 1 percent) are also present.
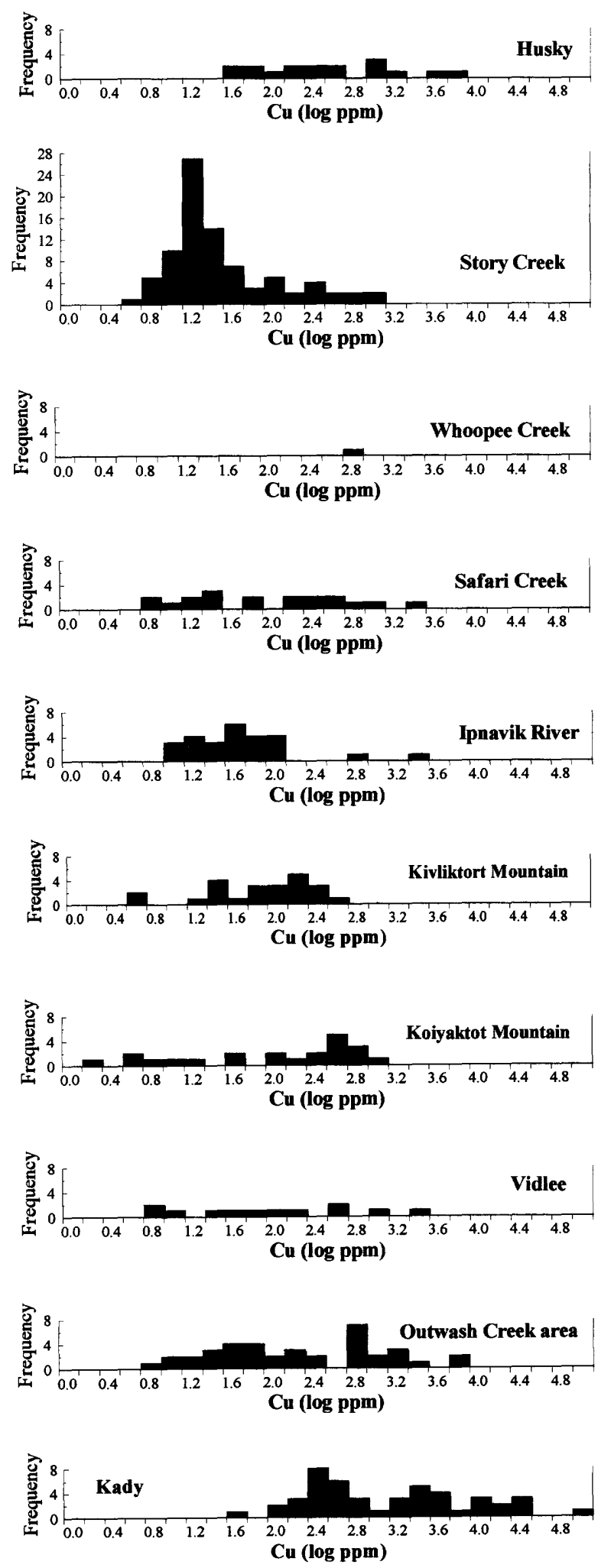

Figure 9. Histogram of copper analyses from mineralized hand samples from vein-breccia occurrences in the northern Brooks Range. Samples from Kady and the Outwash Creek area are more copper rich. Copper values are in log ppm units. ICP analyses are from Meyer and Kurtak (1992). 
Table 3. Oxygen isotopic analyses of hydrothermal quartz.

[Oxygen isotopic compositions obtained from hydrothermal quartz are reported as per mil (\%o) variations of ${ }^{18} \mathrm{O} /{ }^{16} \mathrm{O}$ with respect to standard mean ocean water (SMOW). Samples analyzed by Dr. K.L. Shelton, University of Missouri-Columbia. Analytical precision is generally better than $\pm 0.1 \%$ at $1 \sigma]$

\begin{tabular}{|c|c|c|}
\hline Vein & $\delta^{18} 0(\%)$ & Sample description \\
\hline$\overline{\mathrm{AB}}$ & 15.0 & $\begin{array}{l}\text { Breccia composed of light-gray, silicified siltstone fragments }(10 \%) \text { cemented by intergrown quartz }(45 \%) \text {, } \\
\text { medium reddish brown sphalerite }(45 \%) \text {, and trace chalcopyrite. }\end{array}$ \\
\hline $\mathrm{S}$ & 13.1 & $\begin{array}{l}\text { Vein quartz from } 8 \text {-cm-wide breccia-vein with sandstone clasts }(<3 \%) \text {, quartz }(45 \%) \text {, brown sphalerite }(50 \%) \text {, and } \\
\text { trace chalcopyrite }(<1 \%) \text { and pyrite }(<1 \%) \text {. }\end{array}$ \\
\hline $\mathrm{S}$ & 15.2 & Breccia quartz; same as above. \\
\hline $\mathbf{R}$ & 10.9 & Quartz vein with granular, fine-grained pyrite and minor chalcopyrite $(\sim 5 \%)$. \\
\hline A & 10.8 & 1-m-wide vein of medium- to dark-brown sphalerite $(94 \%)$ with lesser quartz $(5 \%)$ and chalcopyrite $(1 \%)$ \\
\hline A & 14.2 & $\begin{array}{l}\text { Light-gray, silicified siltstone fragments }(20 \%) \text { floating in mixed quartz }(60 \%) \text {, chalcopyrite }(15 \%) \text {, and medium- } \\
\text { brown sphalerite }(5 \%) \text {. }\end{array}$ \\
\hline U-1 & 15.4 & $\begin{array}{l}\text { Vein of quartz }(75 \%) \text {, medium- to dark-brown sphalerite }(24 \%) \text { and chalcopyrite }(1 \%) \text {; quartz cross-cuts } \\
\text { sphalerite subparallel to color banding. }\end{array}$ \\
\hline $\mathrm{U}-2$ & 13.6 & $\begin{array}{l}\text { Early quartz from a } 6.5-\mathrm{cm} \text {-wide symmetrical vein with early quartz }(3 \%)+\text { trace chalcopyrite }(<1 \%) \text {, growth- } \\
\text { zoned late quartz }(66 \%) \text {, and paragenetically late galena }(30 \%) \text {. }\end{array}$ \\
\hline $\mathrm{U}-2$ & 13.8 & Late quartz; same as above. \\
\hline
\end{tabular}

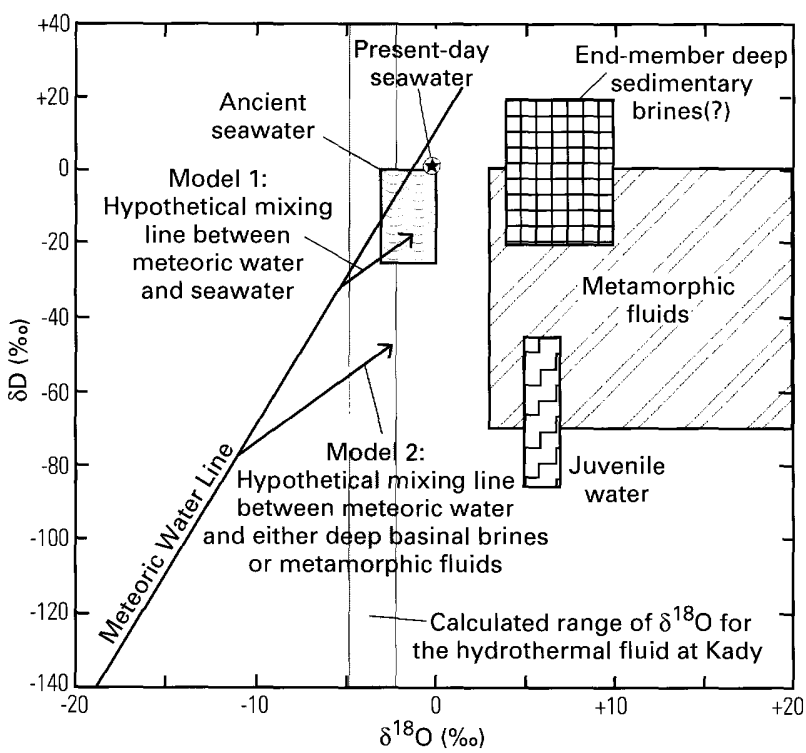

Figure 10. Calculated $\delta^{18} \mathrm{O}_{\text {fluid }}$ values for Kady. $\delta \mathrm{D}$ refers to the isotopic composition of hydrogen in water. Field boundaries from Ohmoto (1986), Sheppard (1986).

Euhedral to irregularly shaped plagioclase grains are completely albitized. Clastic grain-shaped clay and (or) very fine grained illite patches are possibly pseudomorphs after K-feldspar, although no detrital K-feldspar was identified in any stratigraphic unit at Kady. Scattered patches of chlorite are probably pseudomorphs after biotite, as one chlorite grain had a zircon inclusion with a metamict halo and rare partially chlorite altered biotite is present in a few sandstones. Some chlorite patches may be hornblende pseudomorphs as well because high-temperature fractions in ${ }^{40} \mathrm{Ar} /{ }^{39} \mathrm{Ar}$ sandstone spectra have high $\mathrm{Ca} / \mathrm{K}$ and $\mathrm{Cl} / \mathrm{K}$ ratios (Werdon and others, 1998). Large rutile (anatase?) grains are probably detrital, but widely distributed small grains are probably products of biotite destruction.

If the diagenetic fluids were K-feldspar- and mafic-mineraldestructive, they may have derived metals from structurally bound sites in $\mathrm{K}$-feldspar and mafic minerals by diagenetic processes. K-feldspar often contains structurally bound $\mathrm{Pb}^{+2}$ (which substitutes for $\mathrm{K}^{+1}$ ), and mafic minerals can contain elevated levels of $\mathrm{Cu}^{+2}$ and $\mathrm{Zn}^{+2}$. Therefore, sedimentary rocks of the Endicott Group may have provided a potential primary sedimentary source for base metals; however, mafic minerals are volumetrically minor, and K-feldspar may have been absent.

Organic plant material and fine-grained detrital carbonaceous fragments occur locally in the upper Noatak Sandstone, and more commonly in the Kanayut Conglomerate. Shales in the Kady area contain clay, quartz, chert, detrital white mica, limonite, and various carbonate minerals. Sandstone and shale in the Hunt Fork Shale and lower portion of the Noatak Sandstone are mineralogically similar to, but slightly less compositionally and texturally mature than, sandstone in the upper Noatak Sandstone and the Kanayut Conglomerate and exhibit similar diagenetic mineral-alteration assemblages.

Primary and early diagenetic cements identified petrographically and by energy dispersive spectroscopy (EDS) in the Endicott Group clastic rocks include: quartz, calcite, ankerite, siderite, an unidentified phosphate mineral, iron-manganese oxide/hydroxide, hematite, and organic material. The cement paragenesis of 117 individual samples is presented in the Appendix, and the overall cement paragenesis is summarized in figure 11. Quartz and ankerite are the most abundant early diagenetic cements.

Early diagenetic processes are very important in sandstone because the porosity can be significantly changed by early cementation or dissolution (development of secondary porosity) and because these processes influence later diagenetic and (or) hydrothermal processes. The distribution of carbonate in the Noatak Sandstone ranges from sandy limestone, to wellcemented calcareous sandstone, to concretionary and weakly carbonate cemented sandstone. The upper Noatak Sandstone contains small (2-6 mm), sparsely distributed, poikilitic ankerite crystals and minor early diagenetic quartz (EDQ) cement (fig. $12 A$ ). Early diagenetic ankerite often replaces chert grains inside poikilitic crystals. Gray sandstone in the Kanayut Conglomerate is weakly cemented by EDQ, as well as by disseminated carbonate (as much as 5 percent) in non-mineralized areas. 


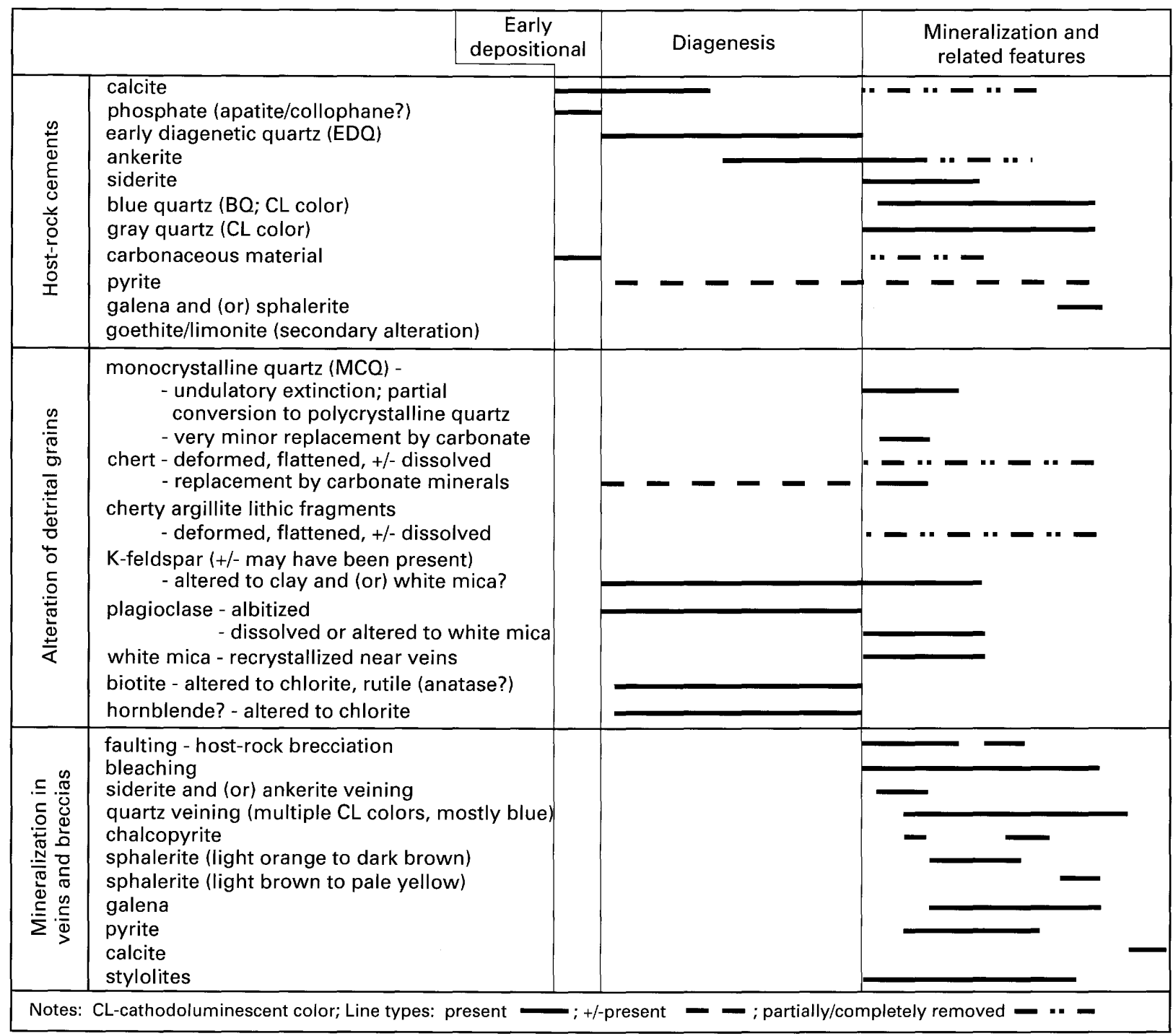

Figure 11. Summary of host-rock diagenesis and alteration, and ore paragenesis.

\section{Hydrothermal Cements, Veins, and Vein-Related Alteration}

Clastic rocks of the Endicott Group were examined to determine which mineral assemblages are related to the hydrothermal event. Interpreted alteration minerals are those that are spatially restricted to areas immediately adjacent to the veinbreccia zones (as much as $1 \mathrm{~m}$ away). Hydrothermal cements are present at varying distances from the vein-breccia zones, depending on the primary permeability of sandstone units.

Most sandstones in the Kanayut Conglomerate are partially to completely cemented by a combination of early diagenetic and hydrothermal quartz, which cannot be distinguished in transmitted light (fig. 12B). Cathodoluminescence is particularly useful for distinguishing early diagenetic quartz cement from later hydrothermal quartz cement. Unlike early diagenetic quartz, which is non-luminescent, hydrothermal quartz at Kady is typically light to medium neon blue (fig. 12C). The blue hydrothermal quartz is paragenetically later than early diagenetic quartz cement in the Kanayut Conglomerate and carbonate cement in the Noatak Sandstone.
The high permeability and porosity in both the upper, less well cemented part of the Noatak Sandstone and the sandstone of the Kanayut Conglomerate provided a site through which hydrothermal fluids could flow. Hydrothermal quartz, followed by disseminated galena and sphalerite, are paragenetically late cements in some gray sandstone units (fig. 5). Secondary goethite/limonite ( \pm anomalous $\mathrm{Zn}$ ) often occurs within pore spaces in the Kanayut Conglomerate, and zinc-carbonate cement occurs within the Noatak Sandstone in a small area near vein C.

In contrast to the relatively uniform color of hydrothermal cement, the cathodoluminescent color of hydrothermal quartz in veins is highly variable, including all shades of neon blue, gray, white, and pale brown (figs. 12C-12E). In larger, growth-zoned quartz veins, the cathodoluminescent colors alternate, particularly where there are alternating fluid-inclusion-rich and inclusion-poor bands. The cathodoluminescent colors in larger veins are relatively pale compared to very thin veins.

Extremely rare, paragenetically early siderite and ankerite veins hosted by the Noatak Sandstone are cut by later hydrothermal quartz veins (fig. 12C). Calcite occurs in veins that cut the Noatak Sandstone, and it occurs as the last mineral deposited in 


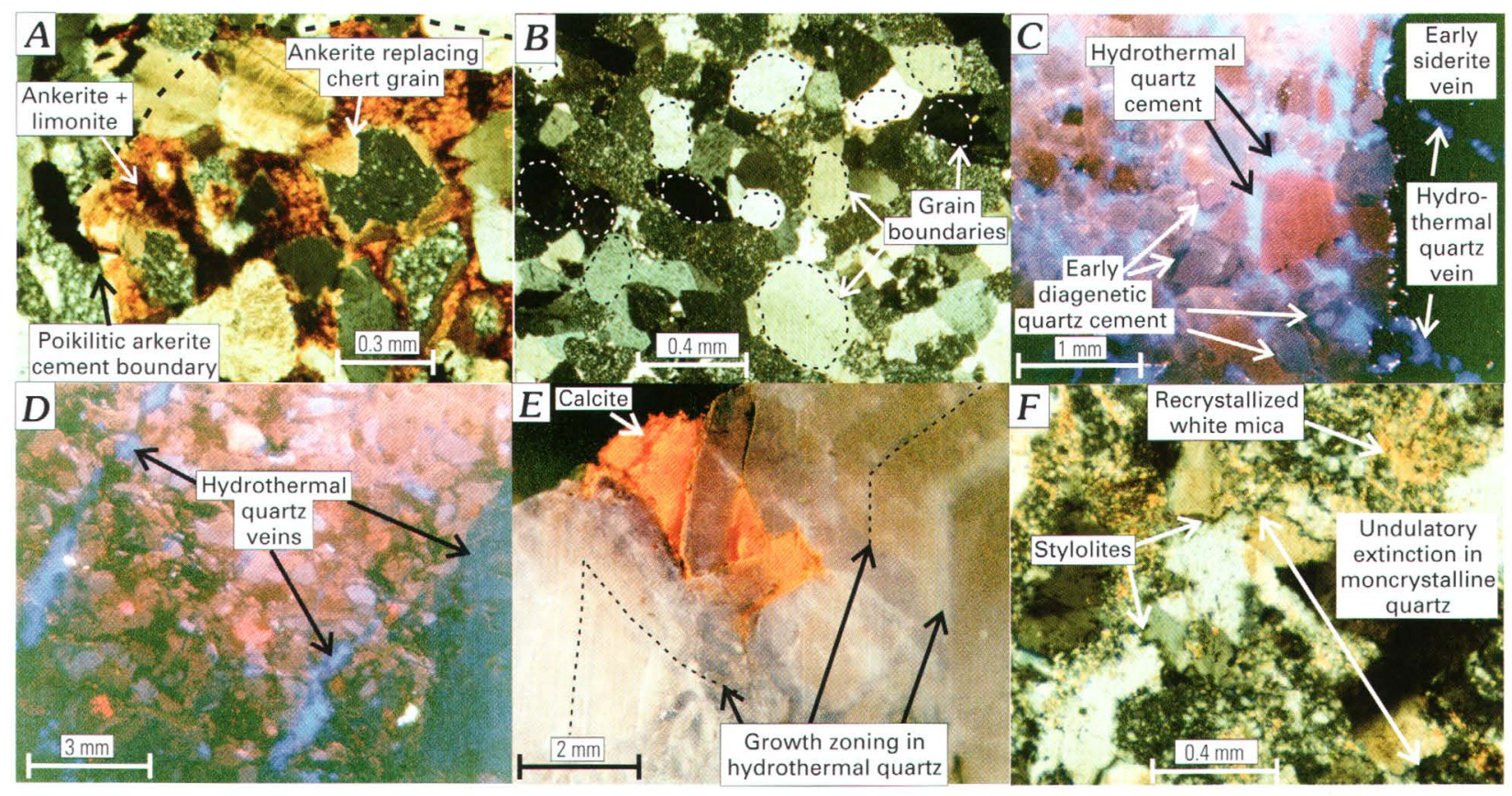

Figure 12. Petrographic and cathodoluminescent photos of host rocks and vein textures: $A$, Poikilitic ankerite cement in the Noatak Sandstone; chert clast partially replaced by ankerite; $B$, Texturally and compositionally mature, quartz-cemented sandstone in the Kanayut Conglomerate composed of monocrystalline quartz and chert grains; $C$, Sandstone cemented by sparse early-diagenetic quartz (non-luminescent) and late hydrothermal quartz (bright blue cathodoluminescent color). Late hydrothermal quartz veins cut an early siderite vein; $D$, Sandstone cut by a set of subparallel hydrothermal quartz veins; $E$, Faint cathodoluminescent color banding in growth-zoned hydrothermal quartz vein; F, Altered sandstone adjacent to vein $\mathrm{H}$ with structurally deformed clastic grains, stylolitization, and white mica (recrystallized and newly crystallized).

symmetrical veins and breccia-textured ore. The paragenetically late calcite in ore-related veins may have been locally derived from nearby carbonate-cemented units. There is an overall trend from relatively high iron carbonates (siderite and ankerite) in the early pre-sulfide stages to pure calcite in the post-sulfide stages of the hydrothermal system.

For alteration reactions to occur, the hydrothermal fluid must be at least slightly out of equilibrium with the wallrock sediments and the fluid must flow in volumetrically significant amounts. The clastic rocks at Kady are relatively siliceous and therefore alteration is not obvious in the field. Numerous subtle mineralogical, textural, and structural changes are observed in clastic rocks adjacent to the vein-breccia zones (fig. 11), and all of the features decrease in intensity with distance from the veinbreccia zones. The distance depends on the width of the veinbreccia zone and host-rock permeability but is generally limited to within $1 \mathrm{~m}$ of the vein-breccia zones.

The clastic rocks immediately adjacent to the vein-breccia zones are structurally deformed. In thin section, monocrystalline quartz grains and their quartz overgrowths exhibit undulatory extinction, and chert grains have been partially to completely flattened (fig. 12F). Thin barren quartz veins are commonly present immediately adjacent and subparallel to the vein-breccia zones (fig. 13).

Breccia clasts within the vein-breccia zones, adjacent clastic wallrocks, and disseminated $\mathrm{Pb}$ and (or) $\mathrm{Zn}$-sulfide-bearing gray sandstones are typically bleached relative to the fresh rocks. The color change from light gray to grayish white is primarily due to dissolution of chert grains and (or) recrystallization of black detrital chert grains to exclude carbon(?). In areas of intense silica dissolution, a pseudo-foliation is observed in hand sample. In thin section, these areas have numerous stylolites cutting chert and monocrystalline quartz grains, as well as stylolitized grain boundaries (fig. $12 F$ ). Carbonaceous material is not present in bleached, mineralized, gray sandstone; it was either not present (unlikely) or it was removed by the hydrothermal fluid. Alteration is less extensive (generally occurs less than $2 \mathrm{~cm}$ from vein margins) in the less permeable carbonatecemented sandstone and shale units. The carbonate-cemented sandstone and shale units are generally medium brown to maroon and are altered to lighter shades of these colors, or less commonly to light or medium forest green. The color change in these units is caused by the removal of iron carbonates, iron oxides, and (or) hydroxides.

To document chemical changes in carbonate minerals adjacent to mineralized vein-breccia zones, carbonate cements were semiquantitatively analyzed using EDS and cathodoluminescence. Carbonate cement in the Noatak Sandstone shows zoning (on a small scale) away from vein-breccia mineralization (figs. $13 A-13 E)$ in area $\mathrm{H}$. In area $\mathrm{H}, 2$ - to 6-mm-wide green, carbonate-removal alteration halos surround the sulfide-bearing quartz veins. The small carbonate-removal halo suggests that the ore fluids were weakly acidic. This is followed sequentially outward by ankerite, ankerite with elevated $\mathrm{Mn}$, calcite with trace $\mathrm{Fe}$ (iron oxide?), and finally a return to background ankerite about $0.5 \mathrm{~m}$ from the vein. The carbonate with the slightly elevated Mn content (fig. 13D) exhibits a much brighter orange color ( $\mathrm{Mn}$ is a cathodoluminescent activator element and $\mathrm{Fe}$ is a suppressor). Carbonate flooding of wallrocks is only present adjacent to veins that cut the carbonate-cemented Noatak Sandstone. 


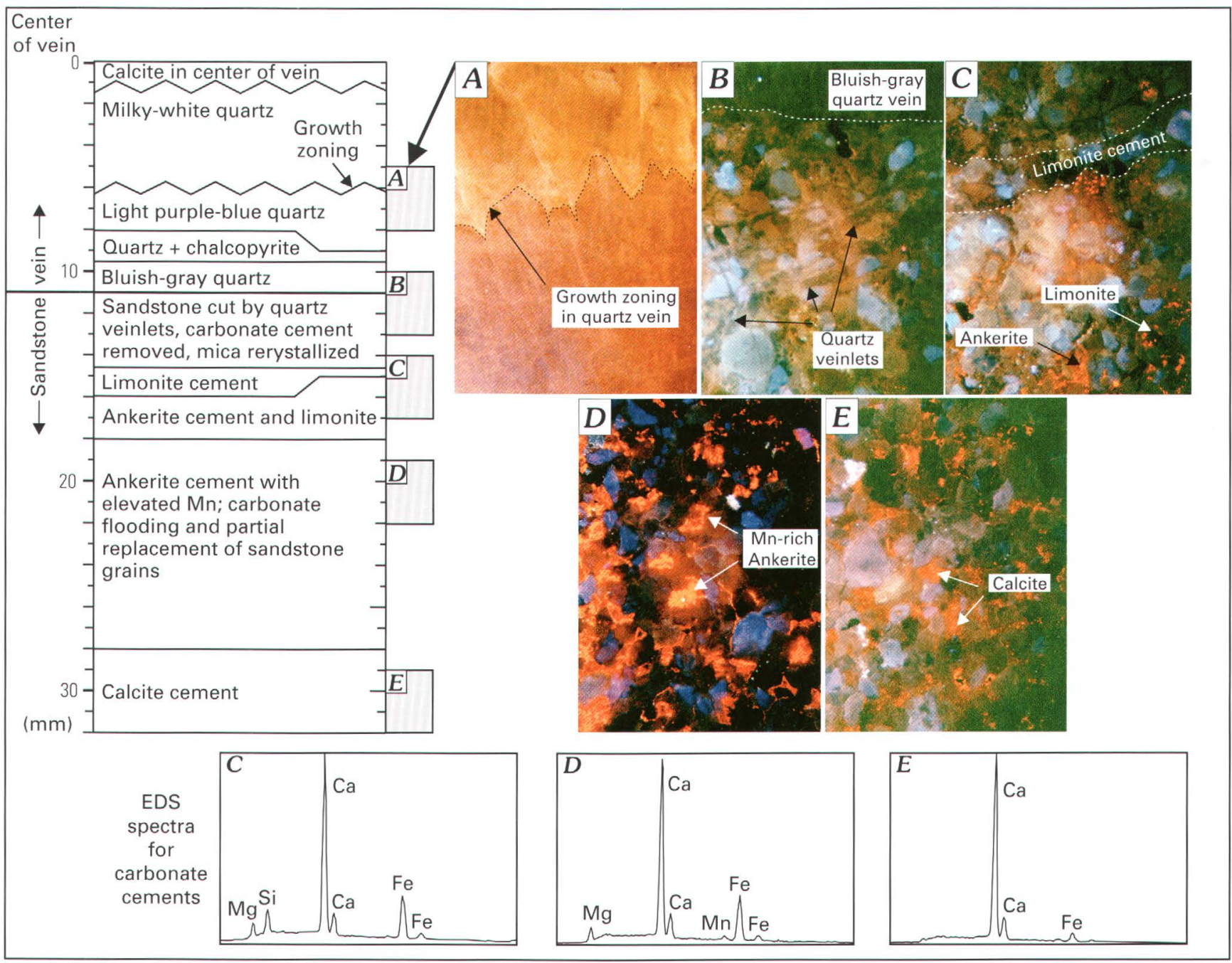

Figure 13. Alteration of carbonate cement in sandstone adjacent to vein $\mathrm{H}$ (see fig. 5 for location).

In these areas, ankerite replaces chert and, rarely, monocrystalline quartz grains. Compared to the Noatak Sandstone, carbonate cement within gray sandstone of the Kanayut Conglomerate is removed at greater distances (as much as 10 ? $\mathrm{m}$ ) from vein-breccia zones, presumably due to the higher primary permeability.

An important mineralogical change accompanying veinbreccia formation is the recrystallization of white mica. Detrital white mica grains are partially to completely pseudomorphed by fine-grained, recrystallized, white mica (fig. $12 F$ ), and patches of illite often become coarser grained adjacent to vein margins. Plagioclase is not present in the 2- to 6-mm-wide alteration halos surrounding veins in area $\mathrm{H}$, suggesting that it was either dissolved, or altered to white mica as well. Calculated vacuumderived ${ }^{40} \mathrm{Ar} /{ }^{39} \mathrm{Ar}$ closure temperatures for hydrothermal sericite are between $220^{\circ} \mathrm{C}$ and $240^{\circ} \mathrm{C}$ (McMaster, 1987), which implies an estimated minimum temperature of about $220^{\circ} \mathrm{C}$ for the hydrothermal fluids in the veins. Recrystallized white mica in sandstone within the sheeted vein-set in area $\mathrm{H}$ (fig. $6 E$ ) was dated by the ${ }^{40} \mathrm{Ar}{ }^{39} \mathrm{Ar}$ laser step-heating method (Werdon and others, 1998). It yielded Mississippian plateau ages of $324 \pm 2 \mathrm{Ma}$ and $325 \pm 2 \mathrm{Ma}$, and an isochron age of $324 \pm 3 \mathrm{Ma}$. The presence of non-recrystallized detrital white mica distal to the veinbreccia zones argues against the ${ }^{40} \mathrm{Ar} /{ }^{39} \mathrm{Ar}$ ages reflecting a regional heating event.

\section{Mineralization Model and Conclusions}

Because the formation of sediment-hosted base-metal deposits is often intimately linked to the development of the basin in which they formed, the timing of deposit formation with respect to basin evolution must be considered. The Kady vein-breccia prospect is interpreted as part of a region-wide $\mathrm{Zn}$ $\mathrm{Pb}-\mathrm{Ag}$ hydrothermal mineralizing event estimated to be between 337 and about $314 \mathrm{Ma}$ (Werdon and others, 1998). A Carboniferous age for the Kady prospect is supported by the Carboniferous ${ }^{40} \mathrm{Ar} /{ }^{39} \mathrm{Ar}$ ages of recrystallized white mica in wallrocks adjacent to the vein-breccia zones and by structural field evidence for a pre-Mesozoic age (offset by Mesozoic faults, minor folding). A period of regional extension, and volumetrically minor alkaline volcanic activity, occurred between 343 and $336 \mathrm{Ma}$ (Werdon and others, 1998). This presumably set up an elevated geothermal gradient that heated basinal fluids.

Potential metal sources at Kady include the Endicott Group clastics and (or) underlying basement rocks. Because basement rocks are structurally removed, it is not possible to evaluate this potential source. Sedimentary sources for base metals could include metals adsorbed on clays and Fe-Mn oxide cements, or metals diagenetically released from structurally bound mineral sites (e.g., biotite $\left(\mathrm{Zn}^{+2}, \mathrm{Cu}^{+2}\right)$ and 
K-feldspar $\left.\left(\mathrm{Pb}^{+2}\right)\right)$. All of these potential sources might have been available within the Endicott Group, but the exact source(s) is difficult to determine because metal contents of Endicott-Group units distal to and within mineralized areas have not been determined. The replacement of biotite by chlorite shows that mafic minerals were out of equilibrium with pore fluids in the Endicott Group, indicating that it has a favorable diagenetic history for being a metal source.

The mineralized zones contain brecciated and veined sandstone produced by minor tectonic movement and (or) hydrofracturing. The vertical offset of bedding is difficult to assess due to Mesozoic reactivation. Mississippian offset appears to have been greatest along the $U$ vein (perhaps $50 \mathrm{~m}$ ?). The presence of strata-bound zones of very low grade disseminated sulfide mineralization adjacent to the vein-breccia zones suggests that silica- and base-metal-bearing fluids flowed through poorly cemented sandstone beds. Hydrothermal cementation of the sediments in the Endicott Group, along with a high fluid pressure (as evidenced by large, rotated, displaced, and (or) size-graded breccia clasts), helped support and preserve the fault structures. The subparallel mineralized zones at Kady are modeled as steeply dipping listric(?) faults along which up-flowing basinal fluids traveled and sulfides were deposited (fig. 14).

Carboniferous units stratigraphically above the Kanayut Conglomerate have been structurally and (or) erosionally removed in the Kady area (except for small pieces of the Kayak Shale); therefore, it is not known what Mississippian facies were present above the vein-breccia zones, or if it was an area of emergence and non-deposition. The offset on Mesozoic thrust faults in the Kady area is relatively minor (less than a few hundred meters). Although no detailed structural maps are available between Kady and the mountain front, if the structural style in this area is similar to that in the Kady area, and if thrust-fault offsets are also relatively minor, the Pennsylvanian to Mississippian facies along the mountain front (fig. 1) could potentially be similar to the facies formerly overlying the Endicott Group in the Kady area. If the Kady deposit was overlain by basinal shales of the Kuna Formation, the low thermal conductivity and low permeability of these shaley capping sediments could reduce both conductive and convective heat loss from the basin (Goodfellow and others, 1993), thereby maintaining elevated temperatures within the Endicott Group reservoir until faulting occurred. This proposed basin model would be physically similar to the Sedex model of Lydon (1983).

During faulting, the pressure $(\mathrm{P})$ is interpreted to have changed from predominantly lithostatic to hydrostatic (fig. 14). Stratigraphic thickness estimates for units above the vein-breccia zones suggest that about $375 \mathrm{~m}$ of sediment may have been present over the top of the vein-breccia system. The depth of seawater covering the sediments is unknown. If the maximum temperature for the hydrothermal fluids was around $210^{\circ} \mathrm{C}$ to $250^{\circ} \mathrm{C}$, boiling would occur between $190 \mathrm{~m}$ and $410 \mathrm{~m}$ below sea level. In other words, if the water depth was less than $190 \mathrm{~m}$ to $410 \mathrm{~m}$, boiling would occur below the sea floor. Although the

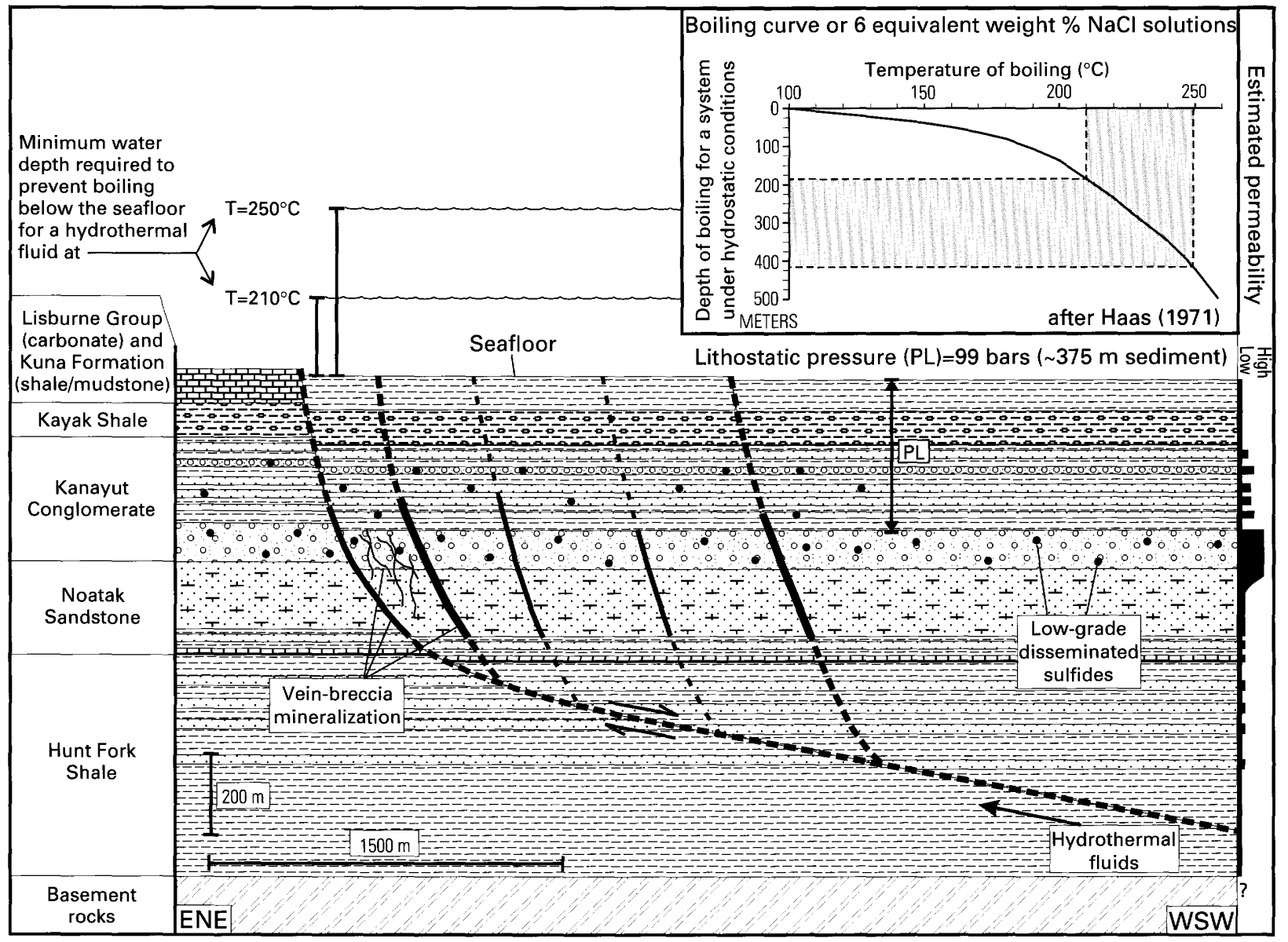

Figure 14. Schematic cross section and mineralization model for Kady. 
possibility of boiling in the upper levels of the vein-breccia system is within stratigraphic-thickness, salinity, and sulfur-isotopic-temperature constraints, there is no evidence to suggest boiling in fluid inclusions from hydrothermal-vein quartz at Kady (Werdon, 1998).

Textural, mineralogical, and fluid-inclusion data indicate that sulfides and quartz were deposited from slightly acidic, lowsalinity hydrothermal fluids under evolving chemical conditions (i.e., T, P, pH, fugacity of oxygen, fugacity of sulfur). A drop in temperature is suggested by the presence of dendritic quartz within some of the vein-breccia zones, the sulfur-pair temperature estimates in vein $\mathrm{R}$, and relatively low fluid-inclusion homogenization temperatures in late, vein-textured quartz. Most vein-breccia zones formed within the stability field for pyrite, but areas D and E evolved starting at the pyrite-pyrrhotite boundary. The decrease in sphalerite mole percent FeS values in many of the veins suggests that both the sulfidation and oxidation state of the fluid increased with time. The relatively high levels of $\mathrm{Cu}$ at Kady suggest that the fluids were relatively oxidized because copper is essentially immobile under reducing conditions at low temperatures. The removal of carbonaceous material within the mineralized area is consistent with a relatively oxidizing hydrothermal fluid. The $\mathrm{pH}$ increased in the late stages of the hydrothermal event due to buffering by wallrock carbonate cement.

The evidence and models discussed above suggest several reasons why a sedimentary exhalative deposit likely did not form above the Kady vein-breccia prospect. Although sulfur isotopic pairs and white mica ${ }^{40} \mathrm{Ar} /{ }^{39} \mathrm{Ar}$ closure temperatures indicate sulfides at Kady formed at a temperature of at least $220^{\circ} \mathrm{C}$ (and possibly as high as $250^{\circ} \mathrm{C}$ ), these relatively low temperatures cannot alone explain the lack of a Sedex deposit. Unlike the Red Dog and Drenchwater deposits, which are spatially associated with a few minor volcanic sills or dikes within and near mineralized areas, there are no volcanic sills or dikes within the Endicott Group in the Kady area. This could suggest that the Kady prospect had a shorter lived system of heat input to sustain hydrothermal fluid flow, but no data on how long the mineralizing systems were active is available.

The presence of sulfides within the Endicott Group suggests that metal-bearing solutions encountered changing physical and (or) chemical conditions that caused metals to drop out of solution before reaching the sea floor. Although there is no evidence for boiling within the vein-breccia system, the hydrostatic pressure may not have been great enough to keep the hydrothermal fluids from boiling in the subsurface above the level of the vein-breccia zones. If the water depth was great enough to prevent subsurface boiling, the low salinity values indicate that the ore fluid would be highly buoyant in seawater upon reaching the sea floor, and metals would be dispersed. The above information suggests that evaluation of Carboniferous facies, and water-depth estimates derived from them, are important to evaluating areas for their Sedex potential.

\section{Acknowledgments}

The Alaska Division of Geological and Geophysical Surveys and the U.S. Geological Survey provided financial support for this study. I wish to thank Rainer Newberry and Jeanine Schmidt for their numerous reviews and advice over the years on this project. Suggestions made by reviewers Gar Pessel, Ken Severin, Paul Layer, Jim Dover, and Karen Kelley also improved this paper. The help of Dennis Boyce in the field and discussions of Brooks Range geology with Gil Mull and Wes Wallace are also appreciated.

\section{References Cited}

Duttweiler, K.A., 1987, Use of factor analysis in locating base metal mineralization in the Killik River quadrangle, Alaska: U.S. Geological Survey Circular 998, p. 27-30.

Ellersieck, I., Tailleur, I.L., and Mull, C.G., 1990, Explanation to accompany reconnaissance geologic map of the Story Creek area, National Petroleum Reserve, Alaska: U.S. Geological Survey Open-File Report 90-533, $21 \mathrm{p}$.

Forrest, K., 1983, Geologic and isotopic studies of the Lik deposit and the surrounding mineral district, De Long Mountains, western Brooks Range, Alaska: University of Minnesota, unpub. Ph.D. dissertation, $161 \mathrm{p}$.

Gaccetta, J.D., and Church, S.E., 1989, Lead isotope data base for sulfide occurrences from Alaska: U.S. Geological Survey Open-File Report 89-688, $60 \mathrm{p}$.

Goodfellow, W.D., Lydon, J.W., and Turner, R.J.W., 1993, Geology and genesis of stratiform sediment-hosted (SEDEX) zinc-lead-silver sulfide deposits, in Kirkham, R.V., Sinclair, W.D., Thorpe, R.I., and Duke, J.M., eds., Mineral Deposit Modeling: Geological Association of Canada, Special Paper 40, p. 201-251.

Haas, J.L., 1971, The effect of salinity on the maximum thermal gradient of a hydrothermal system at hydrostatic pressure: Economic Geology, v. 66, p. 940-946.

Jansons, U., 1982, Zinc-lead occurrences in and near the National Petroleum Reserve in Alaska: U.S. Bureau of Mines Mineral Lands Assessment Report MLA 121-82, 55 p.

Jansons, U., and Parke, M.A., 1981, 1978 mineral investigations in the Misheguk Mountain and Howard Pass quadrangles, Alaska: U.S. Bureau of Mines Open-File Report 26-81, $195 \mathrm{p}$.

Kelley, K.D., Taylor, C.D., and Cieutat, B.A., 1997, Silver-lead-zinc mineral occurrences in the Howard Pass quadrangle, Brooks Range, Alaska, in Dumoulin, J., and Gray, J., eds., Geological Studies in Alaska by the U.S. Geological Survey, 1995: U.S. Geological Survey Professional Paper 1574, p. 101-110.

Lydon, J.W., 1983, Chemical parameters controlling the origin and deposition of sediment-hosted stratiform lead-zinc deposits, in Sangster, D.F., ed., Short Course in Sediment-Hosted Stratiform Lead-Zinc Deposits: Mineralogical Association of Canada, p. 175-250.

Matsuhisa, Y., Goldsmith, J.R., and Clayton, R.N., 1979, Oxygen isotopic fractionation in the system quartz-albite-anorthite-water: Geochimica et Cosmochimica Acta, v. 43, p. 1131-1140.

McMaster, D., 1987, A preliminary ${ }^{40} \mathrm{Ar} /{ }^{39} \mathrm{Ar}$ study of the thermal history and age of gold in the Red Lake greenstone belt: University of Toronto, unpub. M.S. thesis, $107 \mathrm{p}$.

Meyer, M.P., and Kurtak, J.M., 1992, Results of the 1991 U.S. Bureau of Mines Colville mining district study: U.S. Bureau of Mines Open-File Report 75-92, $101 \mathrm{p}$.

Moore, D.W., Young, L.E., Modene, J.S., and Plahuta, J.T., 1986, Geologic setting and genesis of the Red Dog zinc-lead-silver deposit, western Brooks Range, Alaska: Economic Geology, v. 81, p. 1696-1727. 
Moore, T.E., Wallace, W.K., Bird, K.J., Karl, S.M., Mull, C.G., and Dillon, J.T., 1994, Geology of northern Alaska, in Plafker, G., and Berg, H.C., eds., The Geology of Alaska: Boulder, Colo., Geology of North America, v. G1, Geological Society of America, p. 49-140.

Mull, C.G., Moore, T.E., Harris, E.E., Tailleur, I.L., 1994, Geologic map of the Killik River quadrangle: U.S. Geological Survey Open-File Report 94-679, 1 sheet, scale 1:125,000.

Mull, C.G., and Werdon, M.B., 1994, Generalized geologic map of the western Endicott Mountains, central Brooks Range, Alaska: Alaska Division of Geological and Geophysical Surveys Public Data File 94-55, 1 sheet, scale 1:250,000.

Ohmoto, H., 1986, Stable isotope geochemistry of ore deposits, in Valley, J.W., Taylor, H.P., Jr., and O'Neil, J.R., eds., Stable Isotopes in High Temperature Geological Processes: Reviews in Mineralogy, $v$. 16, p. 491-559.

Ohmoto, H., and Rye, R.0., 1979, Isotopes of sulfur and carbon, in Barnes, H.L., ed., Geochemistry of Hydrothermal Ore Deposits (2nd ed.): New York, John Wiley and Sons, p. 509-567.

Sheppard, S.M.F., 1986, Characterization and isotopic variations in natural waters, in Valley, J.W., Taylor, H.P., Jr., and O'Neil, J.R., eds.,
Stable Isotopes in High Temperature Geological Processes: Reviews in Mineralogy, v. 16, p. 165-183.

Toulmin, P., III, Barton, P.B., Jr., and Wiggins, L.B., 1991, Commentary on the sphalerite geobarometer: American Mineralogist, v. 76, p. 10381051.

Werdon, M.B., 1996, Drenchwater, Alaska: Zn-Pb-Ag mineralization in a mixed black shale-volcanic environment, in Coyner, A.R., and Fahey, P.L., eds., Geology and Ore Deposits of the American Cordillera: Geological Society of Nevada Symposium, Reno/Sparks, Nevada, April 1995, Proceedings, p. 13411354.

Werdon, M.B., 1998, Lead isotopic ratios, ICP and XRF analyses, and fluid inclusion data for the $\mathrm{Kady} \mathrm{Zn}-\mathrm{Pb}-\mathrm{Cu}-\mathrm{Ag}$ vein-breccia prospect, northern Brooks Range, Alaska: Alaska Division of Geological and Geophysical Surveys Public Data File 98-43, 8 p.

Werdon, M.B., Newberry, R.J., and Layer, P.W., 1998, ${ }^{40} \mathrm{Ar} /{ }^{39} \mathrm{Ar}$ laser step-heating data and spectra from sandstone and volcanic rocks in the northern Brooks Range, Alaska: Alaska Division of Geological and Geophysical Surveys Public Data File 98-33, 25 p.

Reviewers: Jeanine Schmidt, Rainer Newberry, Jim Dover, Karen Kelley, Gar Pessel, Ken Severin, and Paul Layer. 
Appendix 1. Summary of field, petrographic, energy dispersive spectroscopy (EDS), and cathodoluminescence data for clastic and lesser carbonate rocks and mineralization in the Kady area.

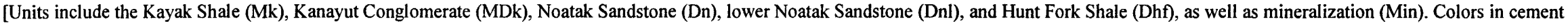

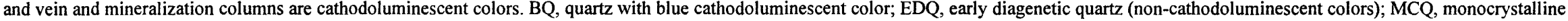
quartz]

\begin{tabular}{|c|c|c|c|c|}
\hline $\begin{array}{l}\text { Sample } \\
\text { number }\end{array}$ & Unit & Field description and petrographic observations & Cements (listed in paragenetic order) & Veins (minerals listed in paragenetic order) \\
\hline 1 & $\mathrm{Dn}$ & $\begin{array}{l}\text { Sandstone breccia: angular sandstone clasts }(\leq 3 \mathrm{~cm}) \text { floating in calcite. Light } \\
\text { tan-gray, fine grained, disseminated pyrite }(<1 \%) \text {, porous-leached. }\end{array}$ & EDQ, calcite. & Calcite. \\
\hline 2 & Dn & $\begin{array}{l}\text { Sandstone: gray-brown weathering, fine-medium grained, }<1 \% \text { disseminated } \\
\text { pyrite, porous-leached, grains of MCQ }(48 \%) \text {, chert }(49 \%) \text {, white mica, } \\
\text { lithics, chlorite (after biotite?) and fine-grained white mica/clay patches } \\
(3 \%) \text {. }\end{array}$ & $\begin{array}{l}\text { EDQ, ankerite (partially replacing } \\
\text { chert grains, } \pm \text { altered to goethite), } \\
\text { trace BQ. }\end{array}$ & $\mathrm{BQ}$. \\
\hline 3 & $\begin{array}{c}\text { Min- } \\
\text { Dn }\end{array}$ & $\begin{array}{l}\text { Sandstone: gray-brown weathering, fine-grained, porous-leached sandstone, } \\
\text { quartz vein is surrounded by carbonate flooding, grains of quartz, chert, } \\
\text { and }<3 \% \text { white mica, fine-grained white mica patches, Al- and Si-clay } \\
\text { grains, and lithics. }\end{array}$ & $\begin{array}{l}\text { EDQ, ankerite, minor siderite, } B Q ; \\
\text { ankerite extensively replaced } \\
\text { chert grains. }\end{array}$ & $\begin{array}{l}\text { Sandstone cut by BQ veins ( }+ \text { sphalerite } \\
+ \text { galena }+ \text { pyrite }+ \text { trace } \\
\text { chalcopyrite? } \pm \text { late calcite) that are } \\
\text { folded by foliation. One micritic } \\
\text { carbonate vein with late chlorite. }\end{array}$ \\
\hline 4 & MDk & $\begin{array}{l}\text { Sandstone: light gray-brown weathering, fine-grained sandstone with planar } \\
\text { beds, minor shale partings, Al- and Si-clay patches (after feldspar?). }\end{array}$ & EDQ, ankerite(?), siderite, BQ. & \\
\hline 5 & MDk & $\begin{array}{l}\text { Siltstone and shale: maroon, tan, and minor black, very fine grained, highly } \\
\text { foliated. }\end{array}$ & $\begin{array}{l}\text { Goethite after siderite, small calcite } \\
\text { patch. }\end{array}$ & \\
\hline 6 & MDk & Shale: maroon. & & \\
\hline 7 & MDk & Sandstone: fine grained, abundant carbonate cement. & $\begin{array}{l}\text { EDQ, siderite (replacing sandstone } \\
\text { grains), BQ (replacing siderite). }\end{array}$ & \\
\hline 8 & MDk & Sandstone: fine-coarse grained; trace rutile(?) grains. & $\begin{array}{l}\mathrm{EDQ} \text {, siderite, minor ankerite, } \mathrm{BQ} \text {, } \\
\text { rutile(?) (floating in } \mathrm{BQ} \text { ). }\end{array}$ & BQ spatially associated with siderite. \\
\hline 9 & MDk & Conglomerate: quartz and chert grains up to $2.5 \mathrm{~cm}$. & & \\
\hline 10 & MDk & Siltstone: reddish brown, very fine grained, contains tracks and burrows. & EDQ, siderite, $\mathrm{BQ}$ & BQ spatially associated with siderite. \\
\hline 11 & MDk & $\begin{array}{l}\text { Sandstone: light gray to white weathering, fine to coarse grained, minor } \\
\text { conglomerate pebbles. }\end{array}$ & $\mathrm{EDQ}$, minor ankerite, $\mathrm{BQ}$. & $\begin{array}{l}\text { Minor quartz }+ \text { carbonate }+ \text { chlorite } \\
\text { fractures and late ankerite veinlets. }\end{array}$ \\
\hline 12 & MDk & Sandstone: light green, fine grained, thin lens, minor disseminated pyrite. & $\mathrm{EDQ}$, ankerite, $\mathrm{BQ}$ & Late ankerite micro-veinlets. \\
\hline 13 & MDk & $\begin{array}{l}\text { Sandstone: light red weathering, light gray colored, fine grained, brecciated, } \\
\text { porous-leached sandstone with minor K-, Al-, and Si-clay(?) patches. }\end{array}$ & $\mathrm{EDQ}$, trace ankerite or siderite, $\mathrm{BQ}$. & $\begin{array}{l}\text { Mineralizing fluids flowed through } \\
\text { sandstone; fractures of quartz }+ \\
\text { epidote(???) + sulfides. }\end{array}$ \\
\hline 14 & MDk & $\begin{array}{l}\text { Sandstone: light red weathering, light gray colored, fine grained, porous- } \\
\text { leached. }\end{array}$ & $\mathrm{EDQ}$, trace $\mathrm{BQ}(?)$ & $\begin{array}{l}\text { Mineralizing fluids flowed through } \\
\text { sandstone. }\end{array}$ \\
\hline 15 & $\mathrm{MDk}$ & Sandstone: light red weathering, light gray colored, fine grained. & EDQ. & Thin veins of $B Q+$ sphalerite + galena. \\
\hline 16 & Dn & $\begin{array}{l}\text { Sandstone: brown weathering, fine grained, minor Al- and Si-clay grains, } \\
\text { porous-leached. }\end{array}$ & $\begin{array}{l}\text { EDQ, bright orange unknown } \\
\text { carbonate, } \mathrm{BQ} \text {, minor green } \\
\text { unknown cement. }\end{array}$ & \\
\hline 17 & MDk & $\begin{array}{l}\text { Sandstone: red-brown weathering, fine-medium grained, thin bedded, } \\
\text { porous-leached with grains of MCQ }(55 \%) \text {, chert }(30 \%) \text {, polycrystalline } \\
\text { quartz }(10 \%) \text {, and other }(5 \%) \text {. Brittle deformation. }\end{array}$ & $\begin{array}{l}\text { Abundant EDQ, ankerite, minor } \\
\text { BQ; some carbonate cement } \\
\text { replacing chert grains. }\end{array}$ & $\begin{array}{l}\text { Early ankerite veins cut by late } B Q \\
\text { veins. }\end{array}$ \\
\hline
\end{tabular}


Appendix 1. Summary of field, petrographic, energy dispersive spectroscopy (EDS), and cathodoluminescence data for clastic and lesser carbonate rocks and mineralization in the Kady area-Continued.

\begin{tabular}{|c|c|c|c|c|}
\hline $\begin{array}{l}\text { Sample } \\
\text { number }\end{array}$ & Unit & Field description and petrographic observations & Cements (listed in paragenetic order) & Veins (minerals listed in paragenetic order) \\
\hline 18 & MDk & $\begin{array}{l}\text { Sandstone: gray, fine grained, massive bedded, quartz and small black chert } \\
\text { grains, rutile, Al- and Si-clay(?) patches, minor disseminated pyrite. }\end{array}$ & EDQ. & $\begin{array}{l}\text { Mineralizing fluids flowed through } \\
\text { sandstone. }\end{array}$ \\
\hline 18.5 & MDk & $\begin{array}{l}\text { Siltstone: brown weathering, very fine grained, porous-leached sandstone } \\
\text { interbedded with shale. }\end{array}$ & Ankerite. & Pyrite + chalcopyrite + calcite veins. \\
\hline 19 & MDk & Siltstone: brown weathering, interbedded with shale. & Ankerite. & \\
\hline 20 & MDk & $\begin{array}{l}\text { Sandstone: brown, fine-medium grained, abundant carbonate cement, thin } \\
\text { bedded. }\end{array}$ & $\begin{array}{l}\text { Minor EDQ, ankerite (replacing } \\
\text { quartz and chert grains, } \pm \text { altered } \\
\text { to goethite). }\end{array}$ & $\begin{array}{l}\text { Ankerite replacing quartz and chert } \\
\text { grains, late ankerite micro-veinlets. }\end{array}$ \\
\hline 21 & MDk & $\begin{array}{l}\text { Sandstone: light gray, medium grained, massive to crossbedded, porous- } \\
\text { leached, entirely quartz cemented, slicks on fracture planes. }\end{array}$ & EDQ. & \\
\hline 22 & $\begin{array}{l}\text { Min- } \\
\text { MDk }\end{array}$ & $\begin{array}{l}\text { Sandstone: breccia of rounded sandstone fragments cemented by rock flour } \\
\text { and BQ, cut by late BQ veins. Sandstone is fine-medium grained, porous- } \\
\text { leached. }\end{array}$ & $\mathrm{EDQ}, \mathrm{BQ}$. & $\mathrm{BQ}+$ sphalerite + pyrite in fracture. \\
\hline 23 & $\begin{array}{l}\text { Min- } \\
\text { MDk }\end{array}$ & Sandstone: massive, cut by numerous sulfide-bearing quartz veins. & & \\
\hline 23.5 & Min & Mineralized breccia: chalcedonic quartz and sphalerite breccia. & & Chalcedonic quartz and sphalerite. \\
\hline 24 & Dn & $\begin{array}{l}\text { Sandstone: medium gray, fine grained, interbedded with green, chloritic, } \\
\text { foliated shale, area has lesser interbedded sandy carbonates and green, } \\
\text { maroon and black shale. Grains composed of quartz, plagioclase (4\%), } \\
\text { white mica (8-10\%), chlorite (after biotite?), chert (flattened, partially } \\
\text { dissolved). }\end{array}$ & EDQ(?), calcite (with trace Fe). & $\begin{array}{l}\text { Abundant carbonate and minor quartz } \\
\text { veining in the area. }\end{array}$ \\
\hline $25 \mathrm{a}$ & $\mathrm{MDk}$ & $\begin{array}{l}\text { Sandstone: light gray, fine grained, large-scale crossbeds, massive bedded, } \\
\text { very porous leached, slightly foliated, partial chert dissolution, grains of } \\
\text { MCQ, chert, lithics, plagioclase }(2-3 \%) \text {, white mica, trace zircon. }\end{array}$ & EDQ. & $\begin{array}{l}\text { Micro-veinlets of BQ parallel to } \\
\text { foliation adjacent to vein } B \text {. }\end{array}$ \\
\hline $25 b$ & Min & $\begin{array}{l}\text { Vein B: growth zoned quartz-galena-calcite veins cut sandstone } 25 \mathrm{a} \\
\text { peripheral to main quartz-chalcopyrite-pyrite zone. }\end{array}$ & & $\begin{array}{l}\text { Growth-zoned quartz (blue, tan, } \\
\text { yellow)+ galena with late calcite. }\end{array}$ \\
\hline 26 & Min & Mineralized vein-breccia zone: & & $\begin{array}{l}\text { Vein-breccia zone of quartz }+ \\
\text { chalcopyrite }+ \text { pyrite, secondary } \\
\text { azurite and malachite. }\end{array}$ \\
\hline 27 & MDk & Sandstone: fine-medium grained, large crossbed sets, porous-leached. & $\mathrm{EDQ}$, minor ankerite, $\mathrm{BQ}$. & Minor gray quartz veinlets. \\
\hline 28 & MDk & Sandstone: light gray, fine-medium grained, porous-leached. & $\mathrm{EDQ}$, trace $\mathrm{BQ}(?)$ & $\begin{array}{l}\text { Mineralizing fluids flowed through } \\
\text { rock. }\end{array}$ \\
\hline 29 & $?$ & $\begin{array}{l}\text { Sandstone and siltstone: brown to maroon, sandstone medium grained, } \\
\text { siltstone very fine grained, interbedded shale, minor carbonate lenses, } \\
\text { manganese-oxide coatings, rutile(?) grains. }\end{array}$ & $\begin{array}{l}\text { EDQ, ankerite ( } \pm \text { goethite altered), } \\
\text { siderite, }(\mathrm{BQ} \text { and } \mathrm{FeMnO}(\mathrm{OH}) \\
\text { replacing ankerite and siderite). }\end{array}$ & Abundant BQ + chalcopyrite veins. \\
\hline 30 & MDk & Sandstone: brown, fine grained, carbonate rich. & EDQ, abundant ankerite, BQ. & $\begin{array}{l}\text { Thin vein of gray quartz; BQ in diffuse } \\
\text { vein(?) }\end{array}$ \\
\hline 31 & $\begin{array}{l}\text { Min- } \\
\text { MDk }\end{array}$ & $\begin{array}{l}\text { Sandstone: light gray, fine-medium grained, bleached, light red iron } \\
\text { staining, porous-leached, reflective quartz surfaces, Al- and Si-clay } \\
\text { patches, galena cement. }\end{array}$ & $\begin{array}{l}\text { EDQ, abundant BQ + disseminated } \\
\text { galena, neon green unknown } \\
\text { cement. }\end{array}$ & $\begin{array}{l}\text { Mineralizing fluids passed through } \\
\text { rock, cut by gray and BQ veins. }\end{array}$ \\
\hline
\end{tabular}


Appendix 1. Summary of field, petrographic, energy dispersive spectroscopy (EDS), and cathodoluminescence data for clastic and lesser carbonate rocks and mineralization in the Kady area-Continued.

\begin{tabular}{|c|c|c|}
\hline $\begin{array}{l}\text { Sample } \\
\text { number }\end{array}$ & Unit & Field description and petrographic observations \\
\hline 32 & MDk & Siltstone: maroon and black, fine grained, interbedded with shale. \\
\hline 33 & MDk & Shale: greenish-brown to black paper shale, highly foliated. \\
\hline 34 & MDk & $\begin{array}{l}\text { Sandstone: grayish-green weathering, fine-medium grained, iron-stained, } \\
\text { porous-leached, crossbedded sandstone with fining-upward sets, minor } \\
\text { conglomerate lag beds to } 3 \mathrm{~cm} \text {, interbedded with gray shale; disseminated } \\
\text { pyrite, chlorite in sandstone. }\end{array}$ \\
\hline
\end{tabular}

MDk Sandstone: light gray weathering, medium gray color, medium grained, massive, jointed, porous-leached sandstone with patches and disseminated pyrite (partially altered to goethite).

36 Dn Sandstone: light greenish brown, fine grained, massive, \pm crossbedded, partially carbonate cemented, porous-leached.

37 Dn Sandstone: gray-green weathering, fine grained, porous-leached, minor pyrite patches, crossbed sets to $1 \mathrm{~m}$, sandstone breaks into thin planar pieces.

Dhf Sandstone: brown weathering, fine grained, micaceous, porous-leached, iron stained, interbedded with black shale.

39 Dhf Siltstone and shale: dark grayish green, interbedded with minor sandstone, fissile to thin bedded, fractured, iron stained.

40 Mk? Concretions: similar to those from the Kayak Shale near the Ivotuk airstrip, and elsewhere in the Killik River and Howard Pass quadrangles.

41 MDk Silty carbonate: dark reddish-brown weathering, with silver-blue weathering phosphatic fossils, minor lens, grains of quartz $(<30 \%)$.

MDk Sandstone: light gray, fine-medium grained, porous-leached, uniform grain size, minor slickenslides, cut by cleavage fractures.

43 Dn Sandstone: light gray-brown weathering, fine-medium grained, porousleached, thin bedded, poikilitic carbonate cement, disseminated pyrite altering to goethite.

MDk Sandstone: dark brown, fine grained, abundant goethite (after poikilitic carbonate cement), disseminated pyrite.

44b MDk Sandstone: dark brown, medium-coarse grained, abundant goethite (after carbonate) cement, disseminated pyrite.
Cements (listed in paragenetic order) Veins (minerals listed in paragenetic order)

Minor EDQ, abundant ankerite,

Early veins of $\mathrm{BQ}+$ siderite (vein rim)+ BQ.

ankerite (çenter); late gray quartz veinlets.

EDQ, goethite after carbonate, trace bright neon green unknown cement.

BQ-carbonate veinlets, may contain trace disseminated sphalerite + galena(?)

EDQ, zoned ankerite to siderite(?), pyrite, $\mathrm{BQ}$; minor carbonate replacement of sandstone grains.

$\mathrm{EDQ}$, minor ankerite ( \pm goethite altered).

EDQ, trace goethite after Fecarbonate.

$\mathrm{EDQ}$, ankerite ( \pm goethite altered).

Central cracks filled with siderite, calcite and pyrite.

Phosphatic fossils in mixed siderite, unknown green and yellow cements.

EDQ, abundant $\mathrm{BQ}$

Minor EDQ, ankerite (minor replacement of sandstone grains, \pm goethite altered), BQ (replacing goethite), bright green unknown cement.

Minor EDQ, goethite after poikilitic $\mathrm{Fe}$-carbonate, $\mathrm{BQ}$

EDQ, abundant goethite after siderite/ankerite (spatially associated with $\mathrm{BQ}$; replacing sandstone grains), BQ; unusual bright orange carbonate replacing(?) goethite and BQ.
BQ veins, \pm cut by micro-veinlets of ankerite.

Late $\mathrm{BQ}+$ chlorite + siderite veins, late ankerite in stylolites. Calcite + ankerite ( \pm minor goethite
altered) veins, and BQ veins. sandstone, outcrop cut by numerous quartz \pm carbonate veins.

Late stylolites filled with carbonate. 
Appendix 1. Summary of field, petrographic, energy dispersive spectroscopy (EDS), and cathodoluminescence data for clastic and lesser carbonate rocks and mineralization in the Kady area-Continued.

\begin{tabular}{|c|c|c|c|c|}
\hline $\begin{array}{l}\text { Sample } \\
\text { number }\end{array}$ & Unit & Field description and petrographic observations & Cements (listed in paragenetic order) & Veins (minerals listed in paragenetic order) \\
\hline 45 & MDk & $\begin{array}{l}\text { Coal and sandstone: light gray, fine-grained, planar to crossbedded, } \\
\text { carbonaceous sandstone interbedded with coal. }\end{array}$ & $\begin{array}{l}\mathrm{EDQ}+\text { coal or carbonaceous plant } \\
\text { material. }\end{array}$ & \\
\hline 46 & Dn & Sandstone: light brown, fine grained, slightly porous-leached. & $\mathrm{EDQ}$, ankerite, $\mathrm{BQ}$ & $\begin{array}{l}\text { Numerous zoned siderite }+ \text { ankerite }+ \\
\text { calcite veins and veinlets. }\end{array}$ \\
\hline 47 & Dn & $\begin{array}{l}\text { Sandstone: light brown, fine grained, crossbedded, minor mud-chip } \\
\text { conglomerates, porous-leached, disseminated pyrite, grains of MCQ } \\
(50 \%) \text {, chert }(42 \%) \text {, white mica }(<1 \%) \text {, cherty argillite lithics }(7 \%) \text {, small } \\
\mathrm{K}-, \mathrm{Al}-\text {, and } \mathrm{Si} \text {-clay or mica patches }(1 \%) \text {, trace epidote. Numerous } \\
\text { mineralized quartz + calcite veins with green, } 2-6 \mathrm{~mm} \text { carbonate-removal }\end{array}$ & $\begin{array}{l}\text { EDQ, ankerite ( } \pm \text { goethite altered), } \\
\text { pyrite; carbonate cement } \\
\text { removed adjacent to and } \\
\text { chemically zoned away from } \\
\text { quartz vein. }\end{array}$ & $\begin{array}{l}\text { Growth-zoned quartz vein with early } \\
\text { BQ and chalcopyrite to late white } \\
\text { quartz to late calcite in center; small } \\
\text { BQ veinlets along vein margin. }\end{array}$ \\
\hline
\end{tabular}

Fault Fault gouge: breccia composed of angular crystalline calcite fragments halos, chert more deformed and irregularly shaped within alteration halo, detrital white mica grains pseudomorphed by recrystallized white mica.

48b Min- Sandstone: light greenish brown, fine grained, abundant carbonate cement.

$\mathrm{Dn}$

Sandstone breccia: light greenish-brown, fine-grained, porous-leached sandstone with rutile grains.

Dn Sandstone: light greenish brown, fine grained, porous-leached; outcrop cut by veins.

51 MDk? Sandstone: light brown, massive to thin bedded.

52 MDk? Sandstone and siltstone: maroon and green, fine grained, interbedded.

53 Dn Sandstone: light brown, medium grained, massive; poikilitic carbonate lenses and concretions.

MDk- Conglomerate: gray/tan, carbonate cemented.

Dn?
MDk? Shale: dark grayish green, interbedded with sandstone.

Dn Sandstone: light brown, poikilitic carbonate cement.

MDk? Siltstone: maroon and green, fine grained, $\mathrm{MnO}$ tarnish, possibly burrowed, abundant zoned carbonate cement (unusual; most carbonate cement at Kady is not zoned).

\section{Al- and Si-clay, calcite, minor BQ.}

$\mathrm{EDQ}$, ankerite, minor $\mathrm{BQ}$; calcite(?) replacing sandstone grains.

$\mathrm{EDQ}$, calcite, very minor BQ.

EDQ, ankerite ( \pm goethite altered) $\mathrm{BQ}$.

EDQ, carbonate.

Minor EDQ, ankerite ( \pm minor goethite alteration), $\mathrm{BQ}$, trace pyrite; ankerite in concretion has replaced chert and MCQ grains. EDQ rims on sandstone grains are not present in center of concretion.

EDQ, carbonate.

EDQ, carbonate

Minor EDQ, distinctive zoned rhombohedral ankerite, calcite \& siderite.
Early ankerite veins cut by vein of quartz (margin) and calcite (center); chalcopyrite, pyrite \& galena associated with ankerite veins.

Quartz + chalcopyrite + trace galena + late calcite veins.

Veins of quartz pinch out/terminate into sphalerite and calcite. 
Appendix 1. Summary of field, petrographic, energy dispersive spectroscopy (EDS), and cathodoluminescence data for clastic and lesser carbonate rocks and mineralization in the Kady area-Continued.

\begin{tabular}{|c|c|c|}
\hline $\begin{array}{l}\text { Sample } \\
\text { number }\end{array}$ & Unit & Field description and petrographic observations \\
\hline 58 & MDk? & $\begin{array}{l}\text { Sandstone: brown, fine grained, very thin bedded, trough crossbedded, } \\
\text { abundant carbonate cement, brecciated. }\end{array}$ \\
\hline 59 & MDk & $\begin{array}{l}\text { Conglomerate and sandstone: light gray, medium to very coarse grained, } \\
\text { contains clasts of quartz, black chert and minor distinctive bright green } \\
\text { chert; cycles of conglomerate-massive sandstone-crossbedded sandstone. }\end{array}$ \\
\hline 60 & Dn & $\begin{array}{l}\text { Sandstone: brown, fine grained, carbonate cemented, poikilitic carbonate } \\
\text { concretions. }\end{array}$ \\
\hline 61 & Dnl & $\begin{array}{l}\text { Sandy carbonate: brown, fine-grained, bluish-silver and maroon, intra- } \\
\text { formational lithoclast (limestone) conglomerate with manganese tarnish } \\
\text { within sandy carbonate bed, carbonate concretions, small } 1-2 \mathrm{~cm} \\
\text { brachiopods. }\end{array}$ \\
\hline 62 & Dn & $\begin{array}{l}\text { Sandstone: brown, fine grained, poikilitic carbonate cement, black shale } \\
\text { partings/mud chips, crossbedded, minor plant debris. }\end{array}$ \\
\hline 63 & Dn & $\begin{array}{l}\text { Sandstone/siltstone: medium green, fine grained, very micaceous, mica } \\
\text { gives rock planar breakage habit, very porous leached grains. }\end{array}$ \\
\hline 64 & MDk? & Sandstone: fine-medium grained, interbedded with maroon shale. \\
\hline 65 & $\begin{array}{l}\text { MDk- } \\
\text { Dn? }\end{array}$ & $\begin{array}{l}\text { Sandstone: maroonish gray, bedding sets to } 0.7 \mathrm{~m} \text {, planar to sweeping } \\
\text { crossbeds, carbonate cemented. }\end{array}$ \\
\hline 66 & MDk & $\begin{array}{l}\text { Sandstone: maroon to yellow weathering, fine grained, massive to } \\
\text { crossbedded. }\end{array}$ \\
\hline $67 a$ & MDk & Sandstone: light gray weathering, massive. \\
\hline $67 \mathrm{~b}$ & MDk & $\begin{array}{l}\text { Siltstone: brownish-gray weathering, extremely fine-grained, quartz siltstone } \\
\text { being replaced by zoned, rhomb-shaped crystals of siderite. }\end{array}$ \\
\hline 68 & MDk & $\begin{array}{l}\text { Siltstone: light brownish-gray to maroon; disseminated pyrite. Siltstone } \\
\text { associated with maroon shales, siderite-cemented sandstones, minor coal, } \\
\text { iron-stones, manganese-staining, and Fe-concretions with pyritized plant } \\
\text { material in them. }\end{array}$ \\
\hline 69 & MDk & $\begin{array}{l}\text { Sandstone: maroon, fine grained, } \mathrm{K}-, \mathrm{Al}-\text {, and Si-clay or very fine-grained } \\
\text { white mica, slightly porous-leached. }\end{array}$ \\
\hline 70 & MDk & $\begin{array}{l}\text { Sandstone: grayish brown, coarse grained, mostly quartz and altered chert } \\
\text { grains. }\end{array}$ \\
\hline 71a & $\mathrm{MDk}$ & Concretion: sandy micritic carbonate with pyritized plant stem. \\
\hline $71 b$ & MDk & $\begin{array}{l}\text { Sandstone: light gray, fine-medium grained, light reddish-tan iron staining, } \\
\text { black lichen-covered outcrops, grains of rutile, unknown P- and Si- } \\
\text { bearing mineral (fluoresces), very porous leached. }\end{array}$ \\
\hline 72 & & Gossan C: gossanous Zn-carbonates. \\
\hline
\end{tabular}
Cements (listed in paragenetic order) Veins (minerals listed in paragenetic order)

EDQ, ankerite, siderite ( \pm goethite altered), $\mathrm{BQ}$.

EDQ, clay(?), minor goethite(?) after Fe-carbonate, BQ.

$\mathrm{EDQ}$, ankerite ( \pm goethite altered), $1 \%$ pyrite.

Calcite(?)/ankerite(?)

EDQ, abundant ankerite $( \pm$ minor goethite alteration), $\mathrm{BQ}$.

$E D Q$, trace BQ.

EDQ, unknown light orange carbonate cement, $\mathrm{BQ}$.

Ankerite.

EDQ, siderite ( \pm goethite altered).

Quartz, siderite.

Minor EDQ(?), ankerite, minor siderite.

EDQ, ankerite(?) ( \pm goethite altered), BQ.

EDQ(?), siderite ( \pm goethite altered); siderite replacing sandstone grains.

Siderite.

$\mathrm{EDQ}$, trace $\mathrm{BQ}$.
Sandstone brecciated, breccia cemented by calcite.

Outcrop is fractured/brecciated with quartz.

Calcite vein with trace ankerite along edge.

Early vein of botryoidal goethite (after Fe-carbonate?), cut by late $B Q$ veins.
Pyrite.

Mineralizing fluids passed through sandstone; veins of quartz + chalcopyrite.

Banded, secondary $\mathrm{Zn}(\mathrm{Cd}, \mathrm{Ca}$ $\pm \mathrm{Fe}) \mathrm{CO} 3$. 
Appendix 1. Summary of field, petrographic, energy dispersive spectroscopy (EDS), and cathodoluminescence data for clastic and lesser carbonate rocks and mineralization in the Kady area-Continued.

\begin{tabular}{|c|c|c|c|c|}
\hline $\begin{array}{l}\text { Sample } \\
\text { number }\end{array}$ & Unit & Field description and petrographic observations & Cements (listed in paragenetic order) & Veins (minerals listed in paragenetic order) \\
\hline 73 & $\begin{array}{l}\text { Min- } \\
\text { Dn }\end{array}$ & $\begin{array}{l}\text { Sandstone breccia and gossan C: sandstone exhibits at least two stages of } \\
\text { brecciation. }\end{array}$ & & $\begin{array}{l}\text { Sandstone breccia cemented by quartz }+ \\
\text { sphalerite }+ \text { minor galena, cut by } \\
\text { calcite veins. }\end{array}$ \\
\hline 74 & MDk & $\begin{array}{l}\text { Sandstone: brown, fine and coarse grained, iron stained, disseminated pyrite, } \\
\text { cut by en echelon, quartz-filled fractures. }\end{array}$ & $\begin{array}{l}\text { EDQ, ankerite(?) ( } \pm \text { trace goethite } \\
\text { alteration), pyrite }(1-2 \%) \text {, trace } \\
\text { BQ. }\end{array}$ & $\begin{array}{l}\text { Outcrop cut by thin quartz }+ \text { carbonate } \\
\text { veins. }\end{array}$ \\
\hline 75 & $\begin{array}{l}\text { Min- } \\
\text { MDk }\end{array}$ & Sandstone: light gray, fine grained, very porous leached, rutile. & $\begin{array}{l}\mathrm{EDQ} \text {, trace ankerite, trace siderite, } \\
\text { rutile(?) }\end{array}$ & $\begin{array}{l}\text { Siderite vein, cut by abundant, late, } \\
\text { sheeted micro-veinlets of gray quartz } \\
\text { and BQ with sulfide mineralization. }\end{array}$ \\
\hline 76 & MDk & $\begin{array}{l}\text { Graded sandstone breccia } \mathrm{S} \text { : fine grained, light gray, } \mathrm{Al} \text { - and Si-clay } \\
\text { patches, } 1 \% \text { rutile as grains/cement(?), porous-leached sandstone. Breccia } \\
\text { is inversely graded with clasts to } 2.5 \mathrm{~cm} \text {. }\end{array}$ & $\mathrm{EDQ}, \mathrm{BQ}$. & $\begin{array}{l}\text { Milky quartz cemented breccia }+ \text { trace } \\
\text { pyrite. }\end{array}$ \\
\hline 77 & MDk & $\begin{array}{l}\text { Sandstone: light grayish brown, medium grained, } 15-\text { to } 60-\mathrm{cm} \text {-thick planar } \\
\text { beds, disseminated pyrite altering to goethite, slightly porous-leached. }\end{array}$ & EDQ, pyrite, trace BQ. & \\
\hline 78 & $\begin{array}{l}\text { Min- } \\
\text { MDk }\end{array}$ & $\begin{array}{l}\text { Sandstone: light brownish-gray weathering, medium-grained, very porous } \\
\text { leached sandstone, cut by a large mineralized quartz-sphalerite dominant } \\
\text { vein-breccia zone and numerous smaller quartz veins. }\end{array}$ & $\mathrm{EDQ}$, trace $\mathrm{BQ}$. & $\begin{array}{l}\text { Gray quartz micro-veinlets and vein of } \\
\text { growth-zoned, grayish-BQ to white } \\
\text { quartz with sulfide mineralization, } \\
\text { cut by late fractures of BQ or } \\
\text { cerussite(?). }\end{array}$ \\
\hline 79 & Dn & Sandstone: light brown, fine grained. & $\begin{array}{l}\mathrm{EDQ} \text {, calcite, goethite after } \\
\text { siderite(?), BQ. }\end{array}$ & Outcrop cut by quartz veins. \\
\hline 80 & MDk & $\begin{array}{l}\text { Sandstone: light tan gray, fine grained, Al- and Si-clay patches, rutile, } \\
\text { porous-leached. }\end{array}$ & $\begin{array}{l}\text { EDQ, goethite after Fe-carbonate, } \\
\text { BQ. }\end{array}$ & Minor gray quartz veinlet. \\
\hline 81 & MDk & Sandstone: fine grained, trace disseminated pyrite, trough crossbedded. & $\begin{array}{l}\text { EDQ, ankerite ( } \pm \text { altered to } \\
\text { goethite). }\end{array}$ & $\mathrm{BQ}+$ ankerite(?) veins. \\
\hline 82 & MDk & $\begin{array}{l}\text { Shale: maroon, nearby green shale, occurs between MDk gray quartz } \\
\text { sandstone above and Dn brown carbonate-cemented sandstone below. }\end{array}$ & & \\
\hline $83 \mathrm{a}$ & MDk & $\begin{array}{l}\text { Sandstone: white and green mottled, fine grained, porous-leached, } 30-\mathrm{cm}- \\
\text { thick bed within maroon shale, chlorite slicks along bedding. }\end{array}$ & $\mathrm{EDQ}$, ankerite, siderite, trace $\mathrm{BQ}(?)$ & Gray-blue quartz vein. \\
\hline $83 b$ & MDk & $\begin{array}{l}\text { Sandstone: green and white mottled, fine grained, porous-leached, } 30-\mathrm{cm}- \\
\text { thick bed within maroon shale, chlorite slicks along bedding. }\end{array}$ & $\begin{array}{l}\mathrm{EDQ} \text {, minor ankerite, siderite, } \\
\text { minor chlorite(?) }\end{array}$ & Vein of BQ (rim) + calcite (center). \\
\hline 84 & MDk & $\begin{array}{l}\text { Sandstone: light gray, faint iron staining, medium grained, massive, thick } \\
\text { bedded, abundant white mica. }\end{array}$ & EDQ. & Minor quartz veining. \\
\hline 85 & MDk & $\begin{array}{l}\text { Sandstone: maroonish-gray weathering, fine-medium grained, } \pm \text { iron- } \\
\text { stained, trough(?) crossbedded, massive to thin bedded, porous-leached, } \\
\text { grains of MCQ }(50 \%) \text {, chert }(50 \%) \text {, rutile, white mica. }\end{array}$ & $\begin{array}{l}\text { EDQ, goethite after Fe-carbonate; } \\
\text { rims of chert grains partially } \\
\text { replaced by carbonate. }\end{array}$ & Gray quartz veins, fractured. \\
\hline $86 a$ & MDk & Sandstone: coarse grained. & $\begin{array}{l}\text { Phosphate mineral, goethite after } \\
\text { siderite(?) cement (notably no } \\
\text { EDQ). }\end{array}$ & \\
\hline $86 b$ & MDk & Sandstone: medium grained, porous-leached. & $\mathrm{EDQ}$, trace $\mathrm{BQ}(?)$ & Vein of siderite. \\
\hline
\end{tabular}



mineralization in the Kady area-Continued.

\begin{tabular}{|c|c|c|c|}
\hline $\begin{array}{l}\text { Sample } \\
\text { number }\end{array}$ & Unit & Field description and petrographic observations & Cements (listed in paragenetic order) \\
\hline 87 & MDk & $\begin{array}{l}\text { Sandstone: brown, fine grained, abundant, irregularly-zoned carbonate } \\
\text { cement, vugs of carbonate, angular sandstone grains, interbedded with } \\
\text { maroon, gray and green shale. }\end{array}$ & $\begin{array}{l}\text { Zoned calcite to siderite (notably } \\
\text { no EDQ). }\end{array}$ \\
\hline 88 & MDk & Sandstone: dark brown, medium-coarse grained, angular clastic grains. & $\begin{array}{l}\text { Sparse EDQ on a few grains, } \\
\text { abundant siderite. }\end{array}$ \\
\hline 89 & MDk & $\begin{array}{l}\text { Sandstone: light gray, fine grained, minor light red iron staining, very } \\
\text { porous leached, Al- and Si-clay patches. }\end{array}$ & $\begin{array}{l}\mathrm{EDQ}, \text { minor siderite }( \pm \text { minor } \\
\text { goethite alteration }), \mathrm{BQ}\end{array}$ \\
\hline 90 & MDk & $\begin{array}{l}\text { Sandstone: brown weathering, fine grained, thin bedded, very porous } \\
\text { leached, K-, Al-, and Si-clay or white mica (after feldspar?). }\end{array}$ & $\begin{array}{l}\text { EDQ, minor goethite after } \\
\text { siderite(?) }\end{array}$ \\
\hline 91 & MDk & Sandstone: light gray, fine grained, thin bedded. & $\begin{array}{l}\text { EDQ, minor siderite, possible } \\
\text { grayish BQ(?), minor neon, teal } \\
\text { green, unknown cement. }\end{array}$ \\
\hline 92 & MDk & $\begin{array}{l}\text { Sandstone: light gray, fine-medium grained, massive to crossbedded, } \\
\text { porous-leached; grains of MCQ, chert, lithics, rutile, apatite. }\end{array}$ & $\mathrm{EDQ}$, rutile(?), trace $\mathrm{BQ}$. \\
\hline 93 & $\begin{array}{l}\text { Min- } \\
\text { MDk }\end{array}$ & Sandstone: light gray, massive to crossbedded, porous-leached. & \\
\hline 94 & MDk & $\begin{array}{l}\text { Sandstone: light gray, fine grained, crossbedded, disseminated pyrite, plant } \\
\text { material, porous-leached, Al- and Si-clay and K-, Al-, Si-clay or white } \\
\text { mica. }\end{array}$ & $\begin{array}{l}\mathrm{EDQ} \text {, trace goethite after Fe- } \\
\text { carbonate, abundant } \mathrm{BQ} \text {. }\end{array}$ \\
\hline 95 & Dhf & $\begin{array}{l}\text { Siltstone and shale: dark greenish black, fine grained, finely interbedded, } \\
\text { porous-leached; chlorite, rutile(?) grains; mixed with fine-grained } \\
\text { sandstone. }\end{array}$ & $\begin{array}{l}\text { EDQ, goethite after Fe-carbonate, } \\
\text { pyrite. }\end{array}$ \\
\hline 96 & Dhf & $\begin{array}{l}\text { Sandstone: dark brownish gray, fine grained, crossbedded, black shale } \\
\text { partings, compact, very porous leached. }\end{array}$ & $\begin{array}{l}\mathrm{EDQ}, \text { ankerite ( } \pm \text { trace goethite? } \\
\text { alteration). }\end{array}$ \\
\hline 97 & Dn & $\begin{array}{l}\text { Sandstone: brown weathering, fine grained, trough crossbedded, abundant } \\
\text { disseminated pyrite patches, very porous leached, grains of MCQ, chert, } \\
\text { lithics, albite, chlorite (after biotite--one zircon grain with metamict } \\
\text { halo), white mica, Al- and Si-clay patches, chlorite, rutile, carbonaceous } \\
\text { plant fragments. }\end{array}$ & EDQ, siderite(?), trace BQ. \\
\hline 98 & MDk & $\begin{array}{l}\text { Sandstone: light brownish gray, medium grained, thin to trough crossbedded } \\
\text { (sets as thick as } 0.7 \mathrm{~m} \text { ), grains of } \mathrm{MCQ} \text {, chert, lithics, white mica. }\end{array}$ & $\begin{array}{l}\text { EDQ, ankerite ( } \pm \text { goethite } \\
\text { alteration); ankerite replaced } \\
\text { several chert grains. }\end{array}$ \\
\hline 99 & MDk & $\begin{array}{l}\text { Sandstone: light red iron stained, light gray colored, fine grained, porous- } \\
\text { leached, grains of MCQ, chert, white mica, lithics, plagioclase, trace } \\
\text { chlorite (after biotite), minor rutile, abundant K-, Al-, and Si-clay or } \\
\text { white mica (after feldspar?), plant fragments. }\end{array}$ & EDQ, rutile(?) \\
\hline 100 & $\begin{array}{l}\text { Min- } \\
\text { MDk }\end{array}$ & $\begin{array}{l}\text { Sandstone and shale: light gray sandstone and shale, light red iron staining. } \\
\text { sandstone is brecciated and cut by mineralized veins that tend to occur } \\
\text { along shale-rich layers. }\end{array}$ & \\
\hline
\end{tabular}

Mineralizing fluids flowed through sandstone; thin quartz vein.

Mineralizing fluids flowed through sandstone; $B Q$ veins, chlorite in one vein in thin section.

Numerous veins including a 1-m-wide sphalerite dominant vein with quartz, chalcopyrite and lesser galena. 
Appendix 1. Summary of field, petrographic, energy dispersive spectroscopy (EDS), and cathodoluminescence data for clastic and lesser carbonate rocks and mineralization in the Kady area-Continued.

\begin{tabular}{ccccc}
\hline $\begin{array}{c}\text { Sample } \\
\text { number }\end{array}$ & Unit & Field description and petrographic observations & Cements (listed in paragenetic order) & Veins (minerals listed in paragenetic order) \\
\hline 101 & MDk & $\begin{array}{c}\text { Sandstone: light gray, fine grained, very porous leached, minor rutile, } \\
\text { disseminated pyrite. }\end{array}$ & EDQ, pyrite. \\
102 & Dn & $\begin{array}{c}\text { Sandstone: light brownish gray, fine grained, thin crossbedded, very porous } \\
\text { leached, grains of quartz, chert, lithics, plagioclase, white mica, and }\end{array}$ & $\begin{array}{c}\text { EDQ, siderite ( } \pm \text { goethite } \\
\text { alteration), BQ. }\end{array}$ &
\end{tabular}

103 Dn Sandstone: light brown, fine grained, massive to crossbedded, very porous leached, grains of MCQ, chert, plagioclase, lithics, chlorite (after biotite) minor white mica, and trace apatite, rutile, minor Al- and Si-clay patches (after feldspar?).

104 Dn Sandstone: light brown weathering, fine grained, bleached, very porous leached, rutile.

105 MDk Sandstone: light gray, massive, crossbedded, trough crossbedded, plant material along bedding planes, very porous leached, rutile.

106 Min- Sandstone in fault: light brown weathering, medium grained, very porous

MDk leached; rutile grains.

107 Min- Sandstone in fault: brown weathering, iron stained, fine grained, slightly MDk porous-leached; chlorite(?) grains.

108 Min Vein:

109 Min- Sandstone: brown weathering, abundant disseminated pyrite, grains of MDk MCQ, chert, and brown lithics; cut by numerous mineralized veins.

110 Min- Sandstone and vein: growth-zoned quartz vein, chlorite; sandstone is

MDk bleached, brecciated, contains disseminated pyrite.

111 MDk Siltstone and shale: brown weathering, very fine grained, abundant white mica; 2.5 -cm-thick sets of crossbeds.

112 Dn? Sandstone: light brown, fine grained, abundant black shale rip-up clasts, ripple marks(?), small-scale crossbeds, porous-leached.

113 Min Vein:

114 MDk Sandstone: light gray, fine-medium grained, light yellow and red iron staining, massive trough crossbeds, porous-leached; rutile(?) grains.

115 Min Vein: vein in sandstone breccia.

116 Min- Sandstone: light gray weathering, light red iron staining, porous-leached, MDk massive, trough crossbedded, minor conglomerate beds.
Abundant EDQ, minor goethite after Fe-carbonate, pyrite, trace $\mathrm{BQ}$; carbonate partially replaced sandstone grains.

$E D Q$, thin goethite grain coatings.

$\mathrm{EDQ}, \mathrm{BQ}$.

EDQ, trace $B Q+$ rutile(?) (up to $1 \%)$.

EDQ, siderite, $\mathrm{BQ}$.

EDQ, pyrite cement, pyrite has partially replaced all grain types.

EDQ, calcite (with minor iron).

$\mathrm{EDQ}$, calcite, goethite after Fecarbonate, $\mathrm{BQ}$.

EDQ, possible late gray quartz(?)
Minor quartz veining.

Outcrop cut by quartz veins.

Quartz-veined joints.

Mineralizing fluids flowed through sandstone. Vein-breccia zones up to $30 \mathrm{~cm}$ wide, with quartz + sphalerite + galena.

$B Q$ veins.

Growth-zoned quartz (blue, grayishblue, and dull to bright yellow) with sulfides and neon blue cerussite.

Quartz + chalcopyrite + sphalerite + galena veins cut abundant disseminated pyrite.

Growth-zoned quartz: gray-blue (early) to $\tan$ (late).

Calcite vein.

Growth-zoned, gray-blue, tan, yellow quartz vein with chalcopyrite + sphalerite + galena.

Mineralizing fluids flowed through sandstone; many quartz veins (see sample 113).

Quartz vein with altered pyrite, chalcopyrite.

Mineralizing fluids flowed through sandstone. 
Appendix 1. Summary of field, petrographic, energy dispersive spectroscopy (EDS), and cathodoluminescence data for clastic and lesser carbonate rocks and mineralization in the Kady area-Continued.

\begin{tabular}{|c|c|c|c|c|}
\hline $\begin{array}{l}\text { Sample } \\
\text { number }\end{array}$ & Unit & Field description and petrographic observations & Cements (listed in paragenetic order) & Veins (minerals listed in paragenetic order) \\
\hline 117 & $\mathrm{Dn}$ & $\begin{array}{l}\text { Sandstone: gray-green, fine grained, trough crossbedded, disseminated } \\
\text { pyrite patches, porous-leached; grains of MCQ, chert, lithics, white mica, } \\
\text { abundant plant material. }\end{array}$ & $\begin{array}{l}\text { EDQ, } \mathrm{Zn} \text {-bearing goethite (after } \\
\text { poikilitic carbonate cement), } \\
\text { pyrite, } \mathrm{BQ} \text {, teal green unknown } \\
\text { cement. }\end{array}$ & $\begin{array}{l}\text { Mineralizing fluids flowed through } \\
\text { sandstone. }\end{array}$ \\
\hline
\end{tabular}




\title{
Chemical and Isotopic Data for Rocks and Ores from the Upper Triassic Greens Creek and Woewodski Island Volcanogenic Massive Sulfide Deposits, Southeastern Alaska
}

\author{
By Rainer J. Newberry and David A. Brew
}

\section{Abstract}

Comparison of chemical and isotopic data from samples of drill core and underground exposures at the volcanogenic massive sulfide (VMS) deposit at the Greens Creek mine on Admiralty Island and from samples of drill core at VMS prospects on Woewodski Island in the Duncan Canal area about $200 \mathrm{~km}$ to the south indicates that the ores and host rocks at both places have similar compositions. This evidence confirms that these two localities are within the regional VMS belt of Late Triassic age that has been inferred to extend from the Windy-Craggy deposit in British Columbia southeast to Zarembo Island, about $20 \mathrm{~km}$ southeast of Woewodski Island. Sparse but consistent fossil evidence indicates the belt's Late Triassic age. Although there are some felsic volcanic rocks near the Woewodski Island prospects, this VMS belt is distinguished by the overwhelming dominance of compositionally similar metabasalts. Specifically, the major- and trace-element chemical data indicate basaltic compositions for Greens Creek stratigraphic footwall rocks and sedimentary compositions for the stratigraphic hanging-wall rocks. The near absence of bimodal volcanism and the rareearth-element data indicate that the tectonic environment was not extensional, but was instead arc related. The corresponding data for the Woewodski Island rocks give similar results. Although the Woewodski Island metavolcanic rocks are not as deformed or altered as those at Greens Creek, we confidently assign them to the same volcanogenic massive sulfide belt on the basis of lithology, trace-element and rare-earth-element composition, and age.

\section{Introduction}

The deposit at the Greens Creek mine is the largest known of many volcanogenic massive sulfide (VMS) deposits in southeastern Alaska (fig. 1). It and the other Late Triassic deposits shown on figure 1 are part of a regional metallogenic belt that extends to the northwest into British Columbia, where it includes the Windy-Craggy deposit. This belt occurs in rocks assigned to the Alexander terrane by Berg and others (1978), but Brew and Ford (1994) and Brew (1996) interpret them instead to be equivalent to the Wrangellia terrane rocks exposed adjacent to the Alexander terrane. The Devonian(?) VMS deposits shown on figure 1 are in what Brew and Ford (1994) and Brew (1996) call the Behm Canal structural zone; their host rocks may actually be as old as Late Proterozoic and as young as late Paleozoic. The Late Proterozoic-early Paleozoic VMS deposits shown on figure 1 are all in the Alexander terrane.

Worldwide, VMS deposits of a given age, like the Late Triassic deposits that are the focus of this report, occur in clusters within larger belts. Thus, the presence of one major deposit signals the likelihood for others. Both the evaluation of undiscovered mineral resources and the strategies for exploration and development of Greens Creek-like deposits in southeastern Alaska depend on understanding the origin of the Greens Creek deposit.

Published models for the Greens Creek deposit include: (1) proximal VMS, ores related to felsic volcanics and phreatoclastic breccias (MacIntyre, 1986); (2) distal VMS, ores related to buildup in felsic tuff-like rocks (Dunbier and others, 1979); (3) sediment-hosted sulfide, with ores related to syn-sedimentary breccias and feeder veins (Dreschler and Dunbier, 1981); and (4) proximal VMS, with ores related to altered and deformed mafic volcanic rocks (Crafford, 1989). Given the degree of chemical and mineralogical alteration typically associated with the formation of VMS deposits (Date and others, 1983) and the highly sheared and foliated character of the rocks at Greens Creek, macroscopic and microscopic observations of color, texture, and mineralogy are insufficient to identify the rock protoliths.

The contrasting interpretations and uncertainties regarding the origin of the Greens Creek deposit prompted this study of the major- and minor-element compositions of the rocks. We also studied the composition of the rocks that enclose VMS 


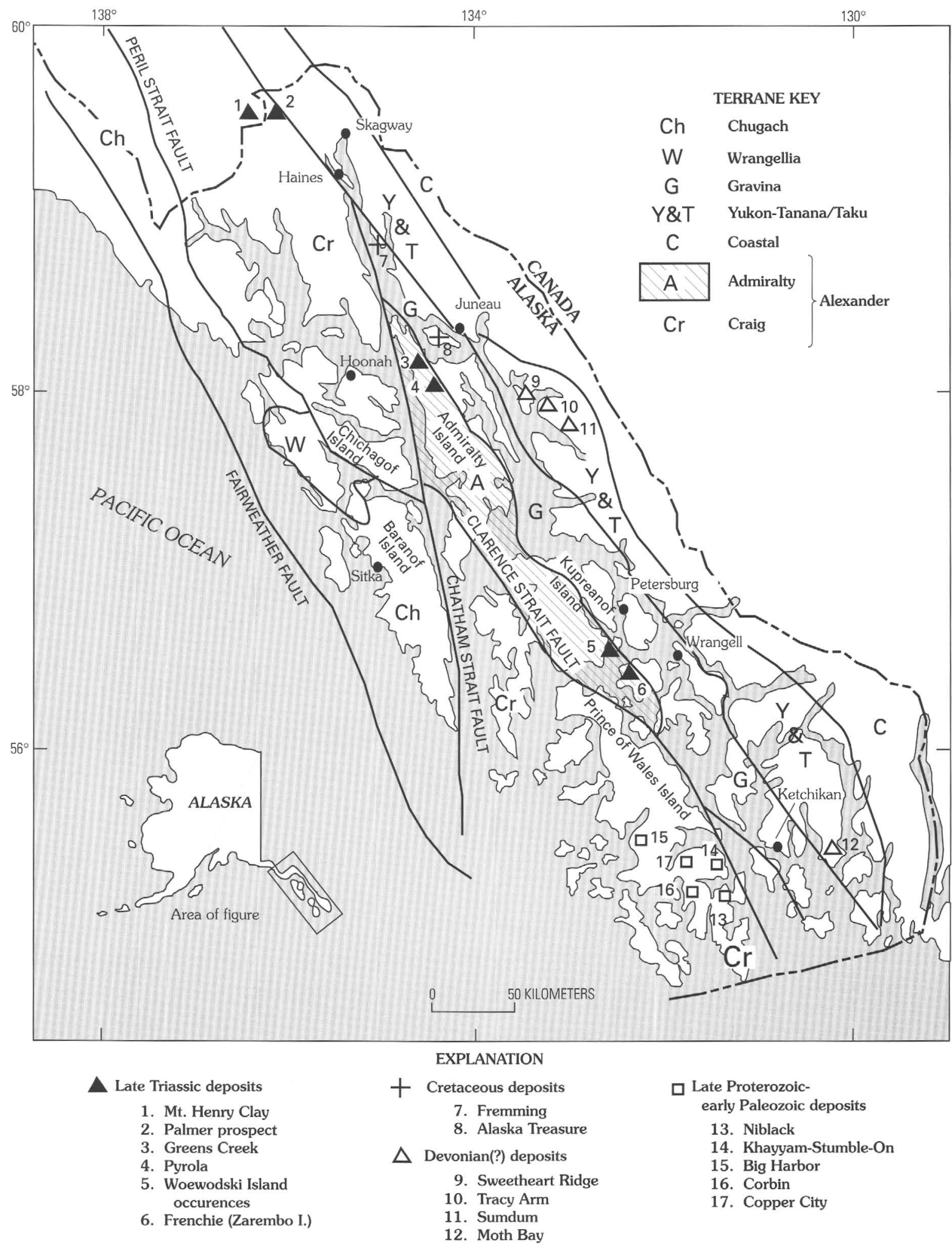

Figure 1. Location map showing major VMS (volcanogenic massive sulfide) deposits and prospects in southeastern Alaska and generalized lithotectonic terrane boundaries. Modified from and Newberry and others (1997).

prospects on Woewodski Island, in the Duncan Canal area (indicated by no. 5 on fig. 1); these prospects have been interpreted to be of the same Late Triassic age as the Greens Creek deposit (Berg, 1981; Berg and Grybeck, 1980). Finally, we studied the lead isotopes of ores from both Greens Creek and Woewodski
Island and also from several other VMS prospects in southeastern Alaska in order to further clarify the ages of the deposits.

A preliminary version of this report (Newberry and Brew, 1997) contains additional data and is referred to where we have truncated its data to present it in revised form in this article. 


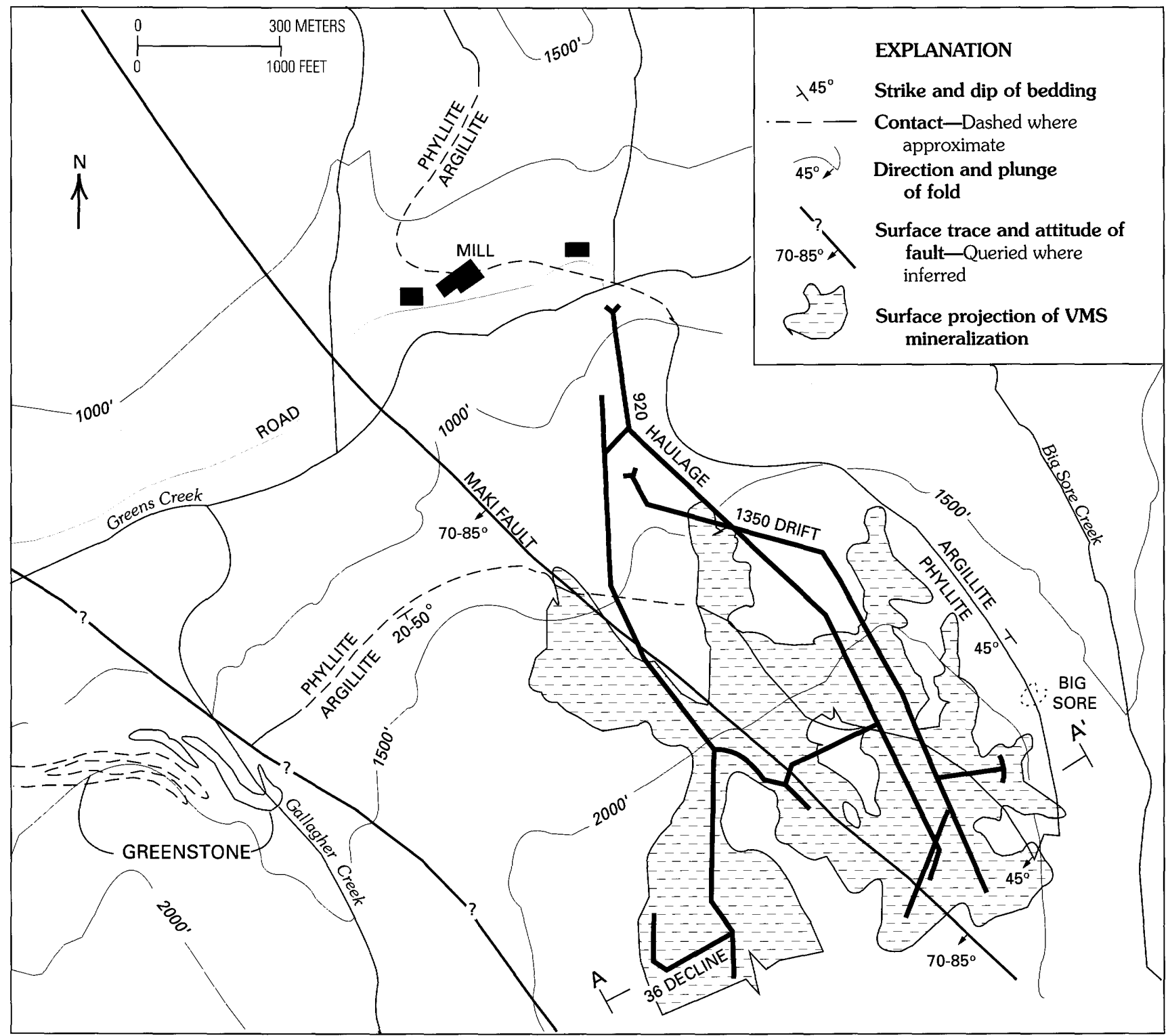

Figure 2. Generalized surface geology and surface projection of mineralized area at the Greens Creek deposit, southeastern Alaska. From Crafford (1989) and Newberry and others (1997).

\section{General Geology of the Greens Creek Deposit}

Massive sulfide at the Greens Creek deposit is discontinuously distributed along the contact between a structural hanging wall of thinly laminated quartz-mica-carbonate phyllite and a structural footwall of black graphitic meta-argillite (fig. 2). Halobia present in carbonate-rich black argillite clasts contained within pyritic massive sulfide ore indicate a Late Triassic maximum age for the deposit (N.J. Silberling, written commun., 1988, 1989, 1990; Crafford, 1989). Regional structural relationships and locally preserved graded bedding suggest that the stratigraphy at the mine is inverted and that the structural hanging-wall rocks originally underlay the VMS deposit. These stratigraphic footwall rocks are very fine grained ( $<0.2 \mathrm{~mm}$ typically), are strongly foliated, and have been described both as "mudstones," "tuffites," and "exhalites" with an upward-increasing felsic volcanic component (Dunbier and others, 1979) and as sedimentary rocks (Nokleberg and others, 1987). However, at least four folding events are documented at the mine (Crafford, 1989), and their cumulative effect has been to largely obliterate original stratigraphic footwall rock textures.

Given the inverted stratigraphic interpretation, the overall original stratigraphy of the Greens Creek mine is an upward progression from greater than $100 \mathrm{~m}$ of chloritic phyllite (chlorite $>$ carbonate $>$ quartz $>$ sericite), to 0 to $70 \mathrm{~m}$ of sericitic phyllite (sericite $>$ quartz $>$ carbonate, referred to later as "quartz-carbonate-mica phyllite"), to local siliceous pyritic phyllite, to 0 to $20 \mathrm{~m}$ of breccia, to massive sulfide, to (overlying) graphitic calc-argillite and limestone (Newberry and others, 1990). The thickness and silica content of sericitic phyllite increases dramatically in areas proximal to ore. The thicknesses of the breccia unit that is stratigraphically just below the ore horizon are greatest approximately 50 to $300 \mathrm{~m}$ laterally away from major sulfide thicknesses (Crafford, 1989). Carbonate-altered quartz-mariposite rocks are variably present near the ore horizon, but are distal to massive sulfide ore. Table 1 summarizes these stratigraphic and structural relations and 
Table 1. Generalized original stratigraphic section at the Greens Creek deposit, giving the single-letter abbreviations used in tables and figures.

\begin{tabular}{|c|c|c|c|c|}
\hline $\begin{array}{l}\text { Estimated } \\
\text { thickness }\end{array}$ & Major rock type(s) & Other rock type(s) & $\begin{array}{l}\text { Single- } \\
\text { letter } \\
\text { symbol }\end{array}$ & Notes \\
\hline \multirow[t]{2}{*}{$?$} & Graphitic calc-argillite & $\begin{array}{l}\text { Graphite-rich quartz-mica-carbonate rock } \\
\text { transitional to argillaceous limestone--..- }\end{array}$ & $\mathrm{h}$ & $\begin{array}{l}\text { Aka hanging-wall argillite, aka black argillite; aka black } \\
\text { [siliceous] argillite or calcareous argillite, aka siliceous } \\
\text { argillite; hard and siliceous within } 3 \text { to } 6 \mathrm{~m} \text { of ore. }\end{array}$ \\
\hline & Massive sulfide & 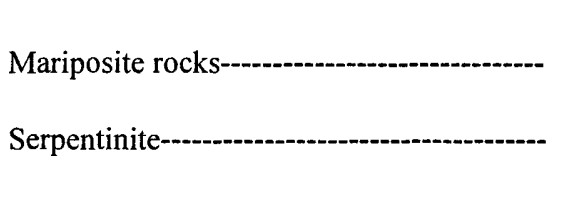 & $\mathrm{p}$ & $\begin{array}{l}\text { Carbonate-altered quartz-mariposite rocks variably present near } \\
\text { ore horizon--but distal to ore, they may be altered ultramafic } \\
\text { rocks (serpentinite); some black argillite may be interleaved } \\
\text { with ore; Halobia occur in carbonate-rich black argillite } \\
\text { clasts in pyritic massive sulfide ore. }\end{array}$ \\
\hline $0-20 \mathrm{~m}$ & 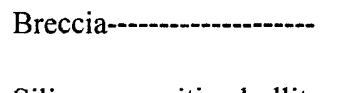 & 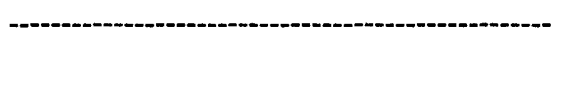 & $\mathrm{x}$ & $\begin{array}{l}\text { Aka siliceous breccia; thicknesses greatest } 50-300 \mathrm{~m} \text { away from } \\
\text { largest sulfide thicknesses. }\end{array}$ \\
\hline \multirow{2}{*}{$\begin{array}{l}\text { Locally } \\
\text { beneath ore }\end{array}$} & Siliceous pyritic phyllite & & & Occurs below most Cu-rich part of ore body. \\
\hline & ------------ & Chalcopyrite-quartz-pyrite-sericite rock---- & $\begin{array}{l}\mathrm{q} \\
\mathrm{q}\end{array}$ & \\
\hline $\begin{array}{l}\text { Locally } \\
\text { beneath ore }\end{array}$ & $\begin{array}{l}\text { Graphitic phyllite } \\
\text { (=visible graphite)------ }\end{array}$ & 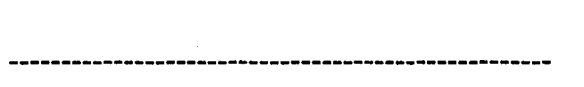 & $\mathrm{r}$ & Rare \\
\hline $0-70 \mathrm{~m}$ & $\begin{array}{l}\text { Sericitic phyllite-------- } \\
\text { (=sericite>quartz> } \\
\text { carbonate) }\end{array}$ & $\begin{array}{l}\text { Also contains ankerite, dolomite, calcite, } \\
\text { white mica, chlorite, Ti oxides; } \\
\text { mariposite, mariposite-bearing phyllite, } \\
\text { mariposite rock, mariposite phyllite, } \\
\text { mariposite-dolomite phyllite-------o--- } \\
\text { Quartz phyllite or pyrite-muscovite phyllite } \\
\text { or siliceous phyllite-- }\end{array}$ & $\mathrm{m}$ & $\begin{array}{l}\text { Aka quartz-carbonate-mica phyllite, aka "footwall phyllite," aka } \\
\text { muscovite phyllite, aka quartz phyllite, aka muscovite } \\
\text { phyllite, aka muscovite-dolomite phyllite; thickness and } \\
\text { silica content increases proximal to ore, carbonate content } \\
\text { may be }>10 \% \text {, more silicic and pyritic closer to ore; some } \\
\text { black argillite may be interleaved. }\end{array}$ \\
\hline$>100 \mathrm{~m}$ & $\begin{array}{l}\text { Chloritic phyllite }(= \\
\text { chlorite }>\text { carbonate }> \\
\text { quartz }>\text { sericite------- }\end{array}$ & 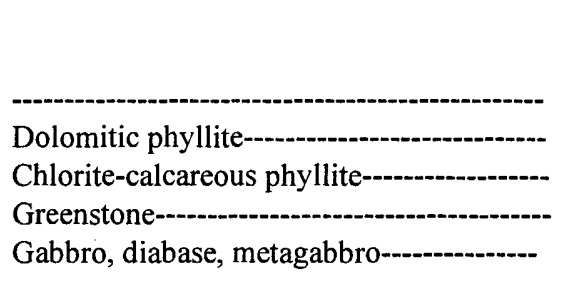 & $\begin{array}{l}\mathrm{c} \\
\mathrm{c} \\
\mathrm{c} \\
\mathrm{g} \\
\mathrm{g}\end{array}$ & \\
\hline Other & Felsic sill, keratophyre-- & 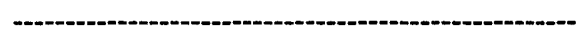 & k & Aka quartz-albite rock. \\
\hline
\end{tabular}


The structural hanging wall (i.e., stratigraphic footwall), quartz-carbonate-mica phyllite contains variable amounts of ankerite, dolomite, calcite, white mica, chlorite, mariposite, pyrite, and titanium oxides. These rocks were referred to as the "footwall phyllite" by Newberry and others (1997). The carbonate content of these phyllosilicate-rich rocks often exceeds 10 percent by volume, and the rocks typically become progressively more silicic and pyritic with increasing proximity to massive sulfide mineralization (Newberry and others, 1990). A local unit that is stratigraphically beneath the most $\mathrm{Cu}$-rich part of the orebody is a rock consisting of quartz, pyrite, sericite, and minor chalcopyrite referred to as "siliceous pyritic phyllite." An additional, and rare, rock type in the stratigraphic footwall package contains visible graphite and is referred to as "graphitic phyllite;" we interpret the organic carbon in this rock to indicate a sedimentary origin. Reflected light petrography indicates that most of the black color in the stratigraphic footwall rocks is, however, due instead to very fine grained sulfide minerals. The siliceous pyritic phyllite and the graphitic phyllite are below the footwall breccia mentioned previously.

Stratigraphic hanging-wall graphitic argillite, referred to variously as "black argillite," "black graphitic meta-argillite," or "graphitic calc-argillite," is graphite-rich quartz-mica-carbonate-graphite rock that is commonly transitional to argillaceous limestone (Newberry and others, 1990). Within about 3 to $6 \mathrm{~m}$ of massive sulfide, the black argillite is hard and siliceous. Black argillite is in places (structurally?) interleaved with massive sulfide and stratigraphic footwall phyllite.

\section{General Geology of Woewodski Island}

Rock exposures on Woewodski Island, $30 \mathrm{~km}$ south of Petersburg, Alaska (figs. 1, 3), are limited almost exclusively to beaches and stream cuts. Based on similarities to known fossiliferous Triassic rocks only a few kilometers to the northwest, most of the metavolcanic rocks on Woewodski Island have been interpreted to be of Late Triassic age (Berg, 1981; Berg and Grybeck, 1980; Brew, 1997a, 1997b) and some of indeterminate Mesozoic age. However, Cretaceous rocks of the Gravina assemblage are present just east of Woewodski Island, and it is not entirely clear where the contact between Triassic and Cretaceous rocks is located. Lithologies on Woewodski Island include black slate; greenstone of probable basaltic composition; basaltic tuff; limestone; and cherty, sulfide-bearing rocks. VMS mineralization has been identified at the Helen S. prospect, and prospects have been drilled near the center of the island (fig. 3). Due to the limited exposures, the ages and character of the ores and host rocks are not well constrained.

\section{Analytical Methods}

Seventy-one samples were selected for major- and minorelement analysis during detailed diamond drill core logging and underground and surface mapping in the Greens Creek deposit area. Ten samples from drill core of VMS prospects on central Woewodski Island were also analyzed. Outcrop samples were clean, fresh, 0.5 - to $2.0-\mathrm{kg}$, composite chip samples. Drill core was sampled by sawing complete $0.2-$ to $3.0-\mathrm{m}$ sections lengthwise, and the entire half of the core was ground for analysis. All samples were analyzed for major elements using standard fused-pellet X-ray fluorescence (XRF) techniques, for $\mathrm{FeO}$ by titration, and for minor and trace elements by energy-dispersive X-ray fluorescence in U.S. Geological Survey laboratories. Some of the same samples were checked by wavelength-dispersive XRF analyses at the University of Alaska-Fairbanks. All analytical methods are essentially those described by Arbogast (1996). CIPW norms were calculated from the major-element analyses using the "Petcal" program (R.D. Koch, written commun., 1980). In addition, 23 samples from Greens Creek were analyzed for rare earth and additional trace elements by standard neutron activation techniques (Arbogast, 1996). Galena-rich samples were analyzed (Richard Hurst, Chempet Research Corporation, Moorpark, Calif., written commun., 1995) for $\mathrm{Pb}$ isotopic ratios using standard extraction and analytical techniques, including correction for thermal fractionation (Cameron and others, 1969).

\section{Results}

Locations and brief descriptions of rocks from the Greens Creek mine area analyzed for this study are given in table 2 . This table also contains (in the column marked by an "*") the rock-type single-letter abbreviations that are employed as symbols on the compositional diagrams (figs. 4-13) and on the interpreted protolith diagrams, which are based on the major- and minor-element compositions. Table 1 explains these symbols and also links the samples to the stratigraphic package at Greens Creek. Major-oxide analyses (table 3 ) and minor-element analyses (tables 4 and 5) are given for selected samples from both the Greens Creek area and Woewodski Island. CIPW normative analyses are given in Newberry and Brew (1997, their table 3). Lead isotopic analyses for selected VMS deposits of southeastern Alaska are given in table 6.

\section{Interpretation}

\section{Compositional Characteristics and Protoliths of Rocks from the Greens Creek Deposit and Vicinity}

Major-element analyses of rocks from the Greens Creek mine area indicate broadly basaltic compositions (table 3 ) for the bulk of stratigraphic footwall rocks. However, extremely high LOI (loss on ignition = sum of $\mathrm{H}_{2} \mathrm{O}+\mathrm{CO}_{2}+\mathrm{S}$ ) values (Newberry and Brew, 1997, their table 2) and variably high normative corundum (Newberry and Brew, 1997, their table 3) indicate that these stratigraphic footwall rocks are either sedimentary or very strongly altered. Manganese oxide, which is commonly associated with hydrothermal alteration, is also present at high concentrations in some samples (table 3). Because $\mathrm{LOI}$ and $\mathrm{MnO}$ show a positive correlation (fig. $4 A$ ) for a variety of lithologies, it is likely that $\mathrm{MnO}, \mathrm{H}_{2} \mathrm{O}, \mathrm{CO}_{2}$, and $\mathrm{S}$ were introduced together, and the rocks have experienced hydrothermal alteration. Because the rocks have such high LOI's, it is best to compare oxide concentrations normalized on an anhydrous basis. 


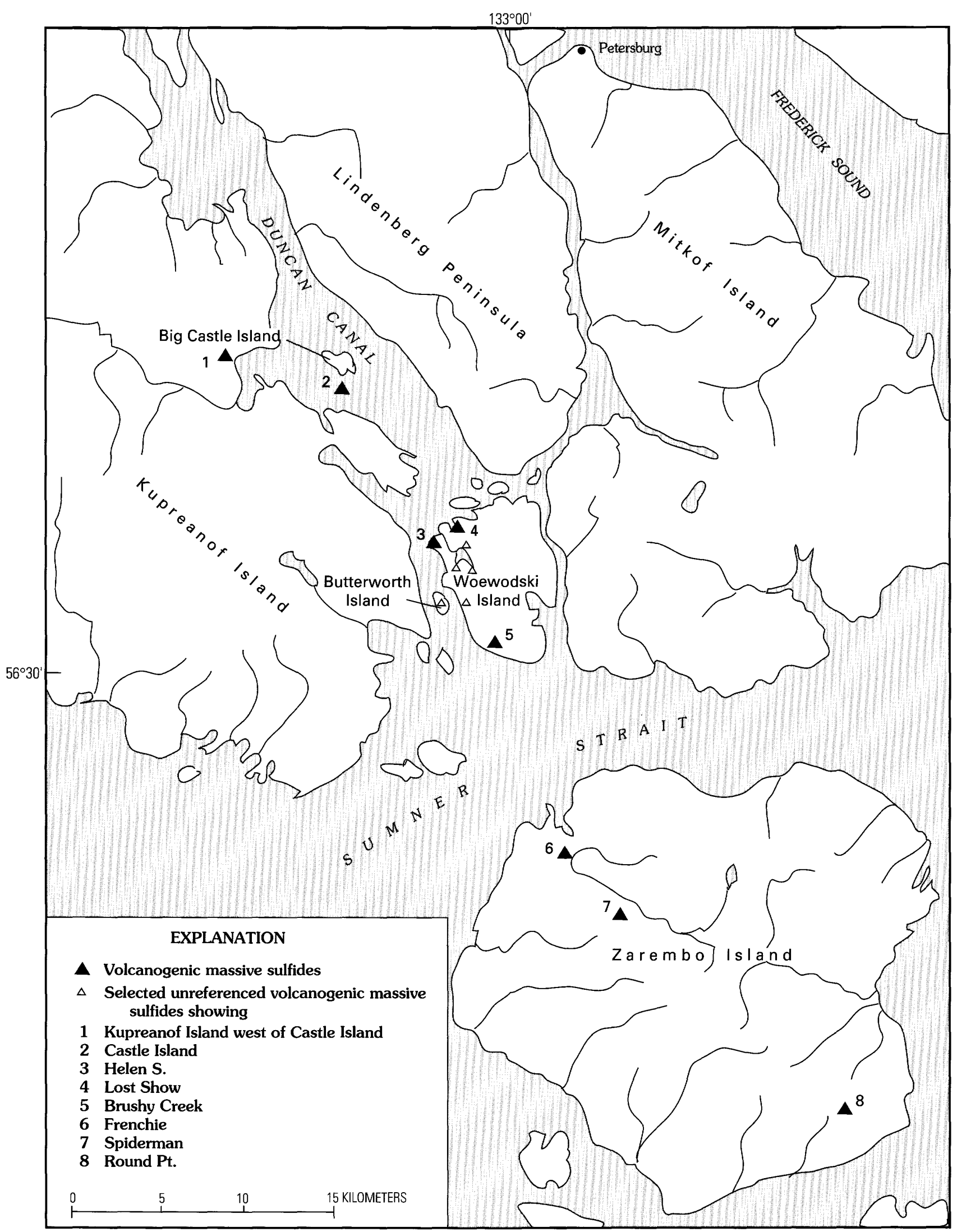

Figure 3. Locations of VMS (volcanogenic massive sulfide) showings in the Duncan Canal-Zarembo Island area, including Woewodski Island, southeastern Alaska. From Berg and Grybeck (1980) and Newberry and others (1997).

Of the various major oxides, $\mathrm{TiO}_{2}$ is among the most immobile during chemical alteration (Winchester and Floyd, 1977), and it is notable that the bulk of stratigraphic footwall rocks have $\mathrm{TiO}_{2}$ and also $\mathrm{SiO}_{2}$ contents compatible with mafic and ultramafic protoliths (BVSP, 1981) (fig. 4B). Obvious sedimentary rocks, i.e., the stratigraphic hanging-wall argillites ("h") and stratigraphic footwall graphitic phyllites ( "r"), have much higher $\mathrm{SiO}_{2}$ contents, but $\mathrm{TiO}_{2}$ concentrations are elevated relative to 

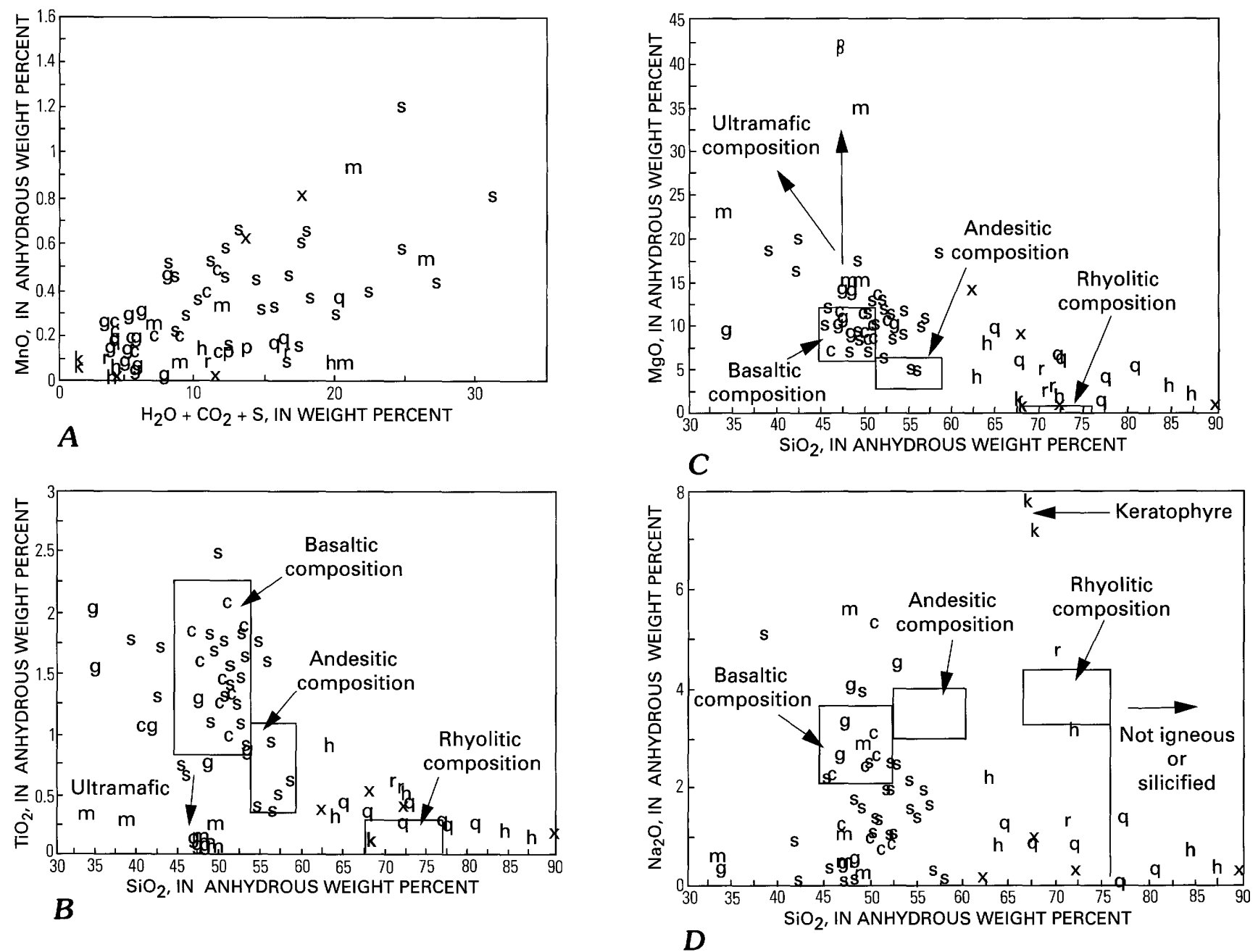

Figure 4. Major-element characteristics of rocks from the Greens Creek deposit and vicinity. Sample/rock type symbols plotted are as follows: Hanging-wall rocks: $h$, hanging-wall argillite; Footwall rocks: $r$, graphitic phyllite; $q$, quartzitic phyllite; $c$, chloritic phyllite/greenstone; $\mathrm{s}$, muscovite chlorite phyllite; $\mathrm{m}$, mariposite rock; $\mathrm{x}$, siliceous breccia; $\mathrm{g}$, gabbro; $\mathrm{p}$, serpentine. Other: $\mathrm{k}$, felsic sill. $A$, Anhydrous weight percent $\mathrm{MnO}$ plotted vs. sum of $\mathrm{H}_{2} \mathrm{O}+\mathrm{CO}_{2}+\mathrm{S}$, showing correlation between the two. $B$, Anhydrous weight percent $\mathrm{TiO}_{2}$ plotted vs. $\mathrm{SiO}_{2}$, showing that most stratigraphic footwall rocks have compositions consistent with mafic and ultramafic igneous rocks. $C_{r}$ Anhydrous weight percent $\mathrm{MgO}$ plotted vs. $\mathrm{SiO}_{2}$, showing that stratigraphic footwall rocks have compositions consistent with mafic and ultramafic igneous rocks. $D$, Anhydrous weight percent $\mathrm{Na}_{2} \mathrm{O}$ plotted vs. $\mathrm{SiO}_{2}$, showing major depletion in $\mathrm{Na}$ for most of the stratigraphic footwall rocks relative to normal igenous rocks and extreme enrichment in the sample of keratophyre ("k"). Compositional fields shown are from Streckeisen and LeMaitre (1979).

rhyolite (fig. 4B). Only a few of the samples have $\mathrm{SiO}_{2}$ and $\mathrm{TiO}_{2}$ contents appropriate for felsic igneous rocks (fig. $4 B$ ). The relatively close correspondence of the $\mathrm{SiO}_{2}$ and $\mathrm{TiO}_{2}$ is interesting and difficult to explain because $\mathrm{SiO}_{2}$ is generally judged to be mobile, and $\mathrm{TiO}_{2}$ immobile, during chemical alteration.

$\mathrm{MgO}$ contents of the greenstones and chloritic phyllites ("c"), serpentinites ("p"), gabbros ("g"), and mariposite-bearing phyllites ("m") are similarly appropriate for mafic and ultramafic rocks (fig. 4C). Many of the muscovite phyllites ("s") also show $\mathrm{SiO}_{2}, \mathrm{TiO}_{2}$, and $\mathrm{MgO}$ contents appropriate for basaltic rocks (figs. $4 B, 4 C$ ). $\mathrm{Na}_{2} \mathrm{O}$ concentrations, however, are extremely erratic (fig. $4 D$ ) and reflect the extreme mobility of $\mathrm{Na}^{+}$during hydrothermal alteration. Most of the samples in the data set are strongly to somewhat depleted in $\mathrm{Na}_{2} \mathrm{O}$ relative to unaltered igneous and sedimentary rocks (Taylor and McLennan, 1985) (fig. 4C); two samples from a felsic sill(?) from the mine area ("k") have extremely high $\mathrm{Na}_{2} \mathrm{O}$ and keratophyric compositions. Similarly, most of the samples have less than 3.0 percent $\mathrm{K}_{2} \mathrm{O}$ and, despite the apparent abundance of fine-grained white mica in the muscovite phyllites, are significantly depleted in $\mathrm{K}_{2} \mathrm{O}$ relative to felsic igneous rocks (table 3). Depletion in alkalis, especially $\mathrm{Na}_{2} \mathrm{O}$, is characteristic of alteration developed under some, dominantly felsic-rock-hosted, VMS deposits (e.g., Date and Watanabe, 1979; Date and others, 1983; Hashiguchi and others, 1983).

The CIPW Norms for the samples (Newberry and Brew, 1997, their table 3) show variably high corundum and quartz (Newberry and Brew, 1997, their fig. 6a). Some greenstones and chloritic phyllites have near-zero values for normative corundum and quartz; other footwall phyllites and metagabbros ("c," "g") have variably high corundum and low normative quartz, whereas the sedimentary rocks ("h," "r") have high normative quartz and variable corundum (Newberry and Brew, 1997, their fig. 6a). High normative corundum values in altered igneous 
Table 2. Locations and descriptions of Greens Creek (gc) and Woewodski Island (RN) samples.

[Abbreviations in "*" column explained in table 1. Abbreviations are used as symbols on some figures, and sample numbers are used in REE (rare-earth-element) plots. deg, degrees; min, minutes; sec, seconds; meta-hbl, meta-hornblende; musc, muscovite; grnst, greenstone; phyl, phyllite]

\begin{tabular}{|c|c|c|c|c|c|c|c|c|c|c|}
\hline \multirow{2}{*}{$\begin{array}{l}\text { Sample } \\
\text { no. }\end{array}$} & \multirow[t]{2}{*}{ Description } & \multirow[t]{2}{*}{ * } & \multirow[t]{2}{*}{ Location } & \multicolumn{3}{|c|}{ Latitude (W.) } & \multicolumn{3}{|c|}{ Longitude (N.) } & \multirow[t]{2}{*}{ Most likely protolith } \\
\hline & & & & deg & $\min$ & $\overline{\mathrm{sec}}$ & deg & $\min$ & sec & \\
\hline $\mathrm{gc} 78 \mathrm{~b}$ & siliceous breccia & $\mathrm{x}$ & 920 Adit, 1st $\mathrm{x}$-cut & 58 & 4 & 55 & 134 & 38 & 0 & silicified breccia \\
\hline $\mathrm{gc} 80 \mathrm{~d}$ & $\begin{array}{l}\text { pyrite dolomite } \\
\text { muscovite quartz } \\
\text { phyllite }\end{array}$ & $q$ & $\begin{array}{l}1350 \text { Adit: } x \text {-cut to } \\
\text { ore }\end{array}$ & 58 & 4 & 47 & 134 & 37 & 47 & altered sediment? \\
\hline $\mathrm{gc} 80 \mathrm{i}$ & $\begin{array}{l}\text { pyrite dolomite } \\
\text { muscovite quartz } \\
\text { phyllite }\end{array}$ & $q$ & $\begin{array}{l}1350 \text { Adit: } x \text {-cut to } \\
\text { ore }\end{array}$ & 58 & 4 & 47 & 134 & 37 & 47 & altered rock \\
\hline gc80k & $\begin{array}{l}\text { pyritic quartz muscovite } \\
\text { phyllite }\end{array}$ & $\mathbf{s}$ & $\begin{array}{l}1350 \text { Adit: } x \text {-cut to } \\
\text { ore }\end{array}$ & 58 & 4 & 47 & 134 & 37 & 47 & altered basalt \\
\hline $\mathrm{gc} 80 \mathrm{~m}$ & $\begin{array}{l}\text { pyrite dolomite } \\
\text { muscovite quartz } \\
\text { phyllite }\end{array}$ & $q$ & $\begin{array}{l}1350 \text { Adit: } x \text {-cut to } \\
\text { ore }\end{array}$ & 58 & 4 & 47 & 134 & 37 & 47 & altered basalt? \\
\hline $\mathrm{gc} 80 \mathrm{pq}$ & $\begin{array}{l}\text { pyritic quartz muscovite } \\
\text { phyllite }\end{array}$ & s & $\begin{array}{l}1350 \text { Adit: } x \text {-cut to } \\
\text { ore }\end{array}$ & 58 & 4 & 47 & 134 & 37 & 47 & altered basalt \\
\hline gc81a & graphitic phyllite & $\mathrm{r}$ & 1350 Adit & 58 & 4 & 47 & 134 & 37 & 47 & C-rich mudstone \\
\hline $\operatorname{gc} 82 a$ & $\begin{array}{l}\text { chlorite dolomite } \\
\text { phyllite }\end{array}$ & c & Greens Creek Road & 58 & 4 & 54 & 134 & 38 & 37 & basalt \\
\hline gc82c & $\begin{array}{l}\text { dolomite muscovite } \\
\text { quartz phyllite }\end{array}$ & $\mathrm{q}$ & Greens Creek Road & 58 & 4 & 54 & 134 & 38 & 37 & altered sediment(?) \\
\hline $\mathrm{gc} 83 \mathrm{~b}$ & $\begin{array}{l}\text { dark-gray quartz } \\
\text { phyllite }\end{array}$ & $q$ & Greens Creek Road & 58 & 4 & 49 & 134 & 38 & 50 & silicified sediment \\
\hline $\mathrm{gc} 83 \mathrm{c}$ & $\begin{array}{l}\text { typical muscovite } \\
\text { phyllite }\end{array}$ & $\mathrm{s}$ & Greens Creek Road & 58 & 4 & 49 & 134 & 38 & 50 & altered basalt \\
\hline gc84b & metagabbro & g & Greens Creek Road & 58 & 4 & 44 & 134 & 39 & 32 & gabbro \\
\hline $\mathrm{gc} 84 \mathrm{c}$ & mariposite rock & $\mathrm{m}$ & Greens Creek Road & 58 & 4 & 44 & 134 & 39 & 32 & altered mafic rock \\
\hline gc85b & greenstone & $\mathrm{c}$ & Greens Creek Road & 58 & 4 & 35 & 134 & 41 & 50 & basalt \\
\hline gc89a & black argillite & $\mathrm{h}$ & Greens Creek Road & 58 & 5 & 13 & 134 & 43 & 40 & carbonaceous mudstone \\
\hline gc90a & black siliceous argillite & $\mathrm{h}$ & Greens Creek Road & 58 & 5 & 33 & 134 & 44 & 0 & siliceous mudstone \\
\hline gc93a & siliceous breccia & $\mathrm{x}$ & Greens Creek Road & 58 & 5 & 54 & 134 & 44 & 21 & conglomerate(?) \\
\hline gc94a & $\begin{array}{l}\text { mariposite muscovite } \\
\text { dolomite phyllite }\end{array}$ & $\mathrm{m}$ & Greens Creek Road & 58 & 5 & 58 & 134 & 44 & 24 & altered ultramafic rock \\
\hline gc94b & $\begin{array}{l}\text { mariposite muscovite } \\
\text { dolomite phyllite }\end{array}$ & $\mathrm{m}$ & Greens Creek Road & 58 & 5 & 58 & 134 & 44 & 24 & altered mafic rock \\
\hline gc95a & graphitic phyllite & $\mathrm{r}$ & Greens Creek Road & 58 & 6 & 5 & 134 & 44 & 22 & C-rich mudstone \\
\hline gcl01a & metagabbro & $\mathrm{g}$ & Mariposite Ridge & 58 & 6 & 12 & 134 & 40 & 40 & gabbro \\
\hline $\mathrm{gcl} 102 \mathrm{a}$ & metagabbro & g & Mariposite Ridge & 58 & 6 & 16 & 134 & 40 & 36 & gabbro \\
\hline gc103a & metagabbro & $\mathrm{g}$ & Mariposite Ridge & 58 & 6 & 28 & 134 & 41 & 9 & gabbro \\
\hline gc121b & gray muscovite phyllite & s & 920 Adit "serp" & 58 & 4 & 55 & 134 & 37 & 55 & altered basalt \\
\hline gc122a & greenstone & c & GC 511 90-91' & 58 & 4 & 30 & 134 & 38 & 0 & basalt \\
\hline gc122b & quartz albite rock & $\mathrm{k}$ & GC 511 129.5' & 58 & 4 & 30 & 134 & 38 & 0 & keratophyre \\
\hline $\mathrm{gcl} 22 \mathrm{~d}$ & quartz albite rock & $\mathrm{k}$ & GC $511169.5^{\prime}$ & 58 & 4 & 30 & 134 & 38 & 0 & keratophyre \\
\hline $\mathrm{gc122e}$ & meta-diabase & $\mathrm{g}$ & GC $511203^{\prime}$ & 58 & 4 & 30 & 134 & 38 & 0 & altered diabase \\
\hline $\mathrm{gc122f}$ & greenstone & c & GC $511273^{\prime}$ & 58 & 4 & 30 & 134 & 38 & 0 & basalt \\
\hline $\mathrm{gc} 284 \mathrm{~b}$ & calcareous argillite & h & 920 Adit & 58 & 4 & 55 & 134 & 38 & 0 & calcareous mudstone \\
\hline $\mathrm{gc} 284 \mathrm{c}$ & $\begin{array}{l}\tan \text { muscovite dolomite } \\
\text { phyllite }\end{array}$ & s & 920 Adit & 58 & 4 & 55 & 134 & 38 & 0 & altered basalt \\
\hline $\operatorname{gc} 284 d$ & $\begin{array}{l}\text { tan muscovite dolomite } \\
\text { phyllite }\end{array}$ & s & 920 Adit & 58 & 4 & 55 & 134 & 38 & 0 & altered basalt \\
\hline $\operatorname{gc} 284 \mathrm{e}$ & $\begin{array}{l}\text { chlorite calcareous } \\
\text { phyllite }\end{array}$ & c & 920 Adit & 58 & 4 & 55 & 134 & 38 & 0 & basalt \\
\hline $\operatorname{gc} 284 f$ & siliceous argillite & $\mathrm{h}$ & 920 Adit & 58 & 4 & 55 & 134 & 38 & 0 & siliceous shale \\
\hline
\end{tabular}

rocks result from removal of $\mathrm{Na}_{2} \mathrm{O}, \mathrm{K}_{2} \mathrm{O}$, and $\mathrm{CaO}$ as $\mathrm{Al}_{2} \mathrm{O}_{3}$ is essentially immobile under most circumstances (Fyfe and others, 1978); the classification of such rocks based on the abundance of alkali elements is consequently misleading. The normative compositions, however, are consistent with maficultramafic protoliths for the less-altered greenstones, chloritic 
Table 2. Locations and descriptions of Greens Creek (gc) and Woewodski Island (RN) samples-Continued.

\begin{tabular}{|c|c|c|c|c|c|c|c|c|c|c|}
\hline \multirow{2}{*}{$\begin{array}{l}\text { Sample } \\
\text { no. }\end{array}$} & \multirow[t]{2}{*}{ Description } & \multirow[t]{2}{*}{$*$} & \multirow[t]{2}{*}{ Location } & \multicolumn{3}{|c|}{ Latitude (W.) } & \multicolumn{3}{|c|}{ Longitude (N.) } & \multirow[t]{2}{*}{ Most likely protolith } \\
\hline & & & & deg & $\min$ & sec & $\operatorname{deg}$ & $\min$ & $\sec$ & \\
\hline gc285a & mariposite phyllite & $\mathrm{m}$ & 1350 Adit & 58 & 4 & 47 & 134 & 37 & 47 & altered mafic rock \\
\hline $\mathrm{gc} 285 \mathrm{~b}$ & siliceous argillite & $\mathrm{h}$ & 1350 Adit & 58 & 4 & 47 & 134 & 37 & 47 & siliceous shale \\
\hline $\mathrm{gc} 285 \mathrm{~g}$ & mariposite phyllite & $\mathrm{m}$ & 1350 Adit & 58 & 4 & 47 & 134 & 37 & 47 & altered mafic rock \\
\hline $\operatorname{gc} 286 \mathrm{a}$ & meta-hbl gabbro & $\mathrm{g}$ & Mariposite Ridge & 58 & 6 & 10 & 134 & 39 & 40 & hornblende gabbro \\
\hline $\operatorname{gc} 286 b$ & greenstone & $\mathrm{c}$ & Mariposite Ridge & 58 & 6 & 10 & 134 & 39 & 40 & basalt \\
\hline $\mathrm{gc} 286 \mathrm{c}$ & metagabbro & $\mathrm{g}$ & Mariposite Ridge & 58 & 6 & 10 & 134 & 39 & 40 & gabbro \\
\hline $\operatorname{gc} 286 \mathrm{~d}$ & greenstone & $\mathrm{c}$ & Mariposite Ridge & 58 & 6 & 10 & 134 & 39 & 40 & basalt \\
\hline $\mathrm{gc} 286 \mathrm{e}$ & serpentinite & $\mathrm{p}$ & Mariposite Ridge & 58 & 6 & 10 & 134 & 39 & 40 & ultramafic rock \\
\hline $\mathrm{gc} 286 \mathrm{f}$ & greenstone & $\mathrm{c}$ & Mariposite Ridge & 58 & 6 & 10 & 134 & 39 & 40 & basalt \\
\hline $\mathrm{gc} 287 \mathrm{a}$ & $\begin{array}{l}\text { mariposite dolomite } \\
\text { phyllite }\end{array}$ & $\mathrm{m}$ & MPR-1 258-261' & 58 & 6 & 3 & 134 & 39 & 21 & altered mafic rock \\
\hline $\operatorname{gc} 287 \mathrm{~b}$ & $\begin{array}{l}\text { greenish-gray } \\
\text { muscovite phyllite }\end{array}$ & s & MPR-1 40-41' & 58 & 6 & 3 & 134 & 39 & 21 & altered basalt \\
\hline $\operatorname{gc} 287 \mathrm{c}$ & $\begin{array}{l}\text { tan muscovite dolomite } \\
\text { phyllite }\end{array}$ & $\mathrm{s}$ & MPR-1 166-168' & 58 & 6 & 3 & 134 & 39 & 21 & altered basalt \\
\hline $\mathrm{gc} 288 \mathrm{a}$ & $\begin{array}{l}\text { gray-tan muscovite } \\
\text { dolomite phyllite }\end{array}$ & $\mathrm{s}$ & PS 25 198-199' & 58 & 4 & 42 & 134 & 37 & 13 & altered basalt \\
\hline $\mathrm{gc} 288 \mathrm{~b}$ & $\begin{array}{l}\text { gray-tan muscovite } \\
\text { dolomite phyllite }\end{array}$ & $\mathrm{s}$ & PS 25 223-224' & 58 & 4 & 42 & 134 & 37 & 13 & altered basalt \\
\hline $\operatorname{gc} 288 \mathrm{c}$ & gray musc phyllite & $s$ & PS-25 46-47' & 58 & 4 & 42 & 134 & 37 & 13 & altered basalt \\
\hline gc288d & gray musc phyllite & s & PS 25 129-130' & 58 & 4 & 42 & 134 & 37 & 13 & altered basalt \\
\hline $\mathrm{gc} 288 \mathrm{e}$ & gray-tan phyllite & s & PS 25 274-275' & 58 & 4 & 42 & 134 & 37 & 13 & altered basalt \\
\hline $\mathrm{gc} 288 \mathrm{f}$ & pyrite muscovite phyl & $q$ & PS 25 372-374' & 58 & 4 & 42 & 134 & 37 & 13 & silicified rock \\
\hline $\operatorname{gc} 289 a$ & $\tan$ muscovite phyllite & $s$ & PS-5 20-25' & 58 & 4 & 42 & 134 & 37 & 13 & altered basalt \\
\hline $\mathrm{gc} 289 \mathrm{~b}$ & muscovite phyllite & $s$ & PS-5 135-139' & 58 & 4 & 42 & 134 & 37 & 13 & altered basalt \\
\hline $\operatorname{gc} 289 \mathrm{c}$ & gray-tan phyllite & s & PS-5 295-300' & 58 & 4 & 42 & 134 & 37 & 13 & altered basalt \\
\hline $\operatorname{gc} 289 \mathrm{~d}$ & graphitic phyllite & $\mathrm{r}$ & PS-5 80-81' & 58 & 4 & 42 & 134 & 37 & 13 & C-rich sediment \\
\hline $\operatorname{gc} 289 \mathrm{e}$ & gray-tan phyllite & s & PS-5 85-86' & 58 & 4 & 42 & 134 & 37 & 13 & altered basalt \\
\hline gc289f & gray-tan phyllite & s & PS-5 558-560' & 58 & 4 & 42 & 134 & 37 & 13 & altered basalt \\
\hline $\mathrm{gc} 289 \mathrm{~g}$ & breccia & $\mathrm{x}$ & PS-5 440-442' & 58 & 4 & 42 & 134 & 37 & 13 & silicified breccia \\
\hline $\mathrm{gc} 289 \mathrm{~h}$ & $\begin{array}{l}\text { greenish-gray } \\
\text { muscovite phyllite }\end{array}$ & $\mathrm{s}$ & PS-5 631-635' & 58 & 4 & 42 & 134 & 37 & 13 & altered basalt \\
\hline $\operatorname{gc} 289 \mathrm{i}$ & breccia & $\mathrm{x}$ & PS-5 366-369' & 58 & 4 & 42 & 134 & 37 & 13 & silicified breccia \\
\hline gc289j & gray siliceous phyllite & $q$ & PS-5 696-698' & 58 & 4 & 42 & 134 & 37 & 13 & silicified rock \\
\hline gc290a & gray muscovite phyllite & s & GC-139 955-957' & 58 & 4 & 27 & 134 & 37 & 2 & altered basalt \\
\hline gc291a & serpentinite & $\mathrm{p}$ & PS-83 978-980' & 58 & 4 & 23 & 134 & 38 & 51 & ultramafic rock \\
\hline gc293a & greenish-tan phyllite & $\mathrm{s}$ & GC-11 32-34' & 58 & 4 & 48 & 134 & 37 & 23 & altered basalt \\
\hline gc293b & chlorite phyllite & $\mathrm{c}$ & GC-11 89-90' & 58 & 4 & 48 & 134 & 37 & 23 & basalt \\
\hline gc293d & grayish-tan phyllite & s & GC-11 240-242' & 58 & 4 & 48 & 134 & 37 & 23 & altered basalt \\
\hline gc294a & greenish-tan phyllite & $s$ & GC-67 8-10' & 58 & 4 & 36 & 134 & 37 & 9 & altered basalt \\
\hline gc294b & gray musc phyllite & s & GC-67 78-80' & 58 & 4 & 36 & 134 & 37 & 9 & altered basalt \\
\hline gc294c & tan-gray phyllite & $\mathrm{s}$ & GC-67 101-102' & 58 & 4 & 36 & 134 & 37 & 9 & altered basalt \\
\hline $\operatorname{gc} 294 d$ & dark-gray phyllite & $\mathbf{S}$ & GC-67 156-157' & 58 & 4 & 36 & 134 & 37 & 9 & altered basalt \\
\hline RN116a & massive greenstone & $\mathrm{g}$ & - & 56 & 34 & 32 & 133 & 3 & 19 & - \\
\hline RN116d & pyritic greenschist & $\mathrm{a}$ & - & 56 & 34 & 32 & 133 & 3 & 19 & - \\
\hline RN116g & tuffaceous greenschist & $t$ & - & 56 & 34 & 32 & 133 & 3 & 19 & - \\
\hline RN118a & massive greenstone & $\mathrm{g}$ & - & 56 & 34 & 25 & 133 & 3 & 10 & - \\
\hline RN118b & masssive greenstone & $\mathrm{g}$ & - & 56 & 34 & 25 & 133 & 3 & 10 & - \\
\hline RN118d & cherty ironstone & - & - & 56 & 34 & 25 & 133 & 3 & 10 & - \\
\hline RN119c & tuffaceous greenschist & $\mathrm{t}$ & - & 56 & 34 & 27 & 133 & 3 & 0 & - \\
\hline RN120a & diabase(?) & $\mathrm{g}$ & - & 56 & 34 & 23 & 133 & 2 & 55 & - \\
\hline RN121a & amygdaloidal grnst & $\mathrm{g}$ & - & 56 & 34 & 25 & 133 & 3 & 30 & - \\
\hline RN123a & tuffaceous greenschist & $\mathrm{t}$ & - & 56 & 34 & 19 & 133 & 3 & 19 & - \\
\hline
\end{tabular}

phyllites, and gabbros; with non-igneous protoliths for the sedimentary rocks; and with a variety of apparent protoliths for the muscovite phyllites (Newberry and Brew, 1997, their fig. 6b).
Major oxide compositions are plotted in terms of the three components: alkalies $\mathrm{Na}_{2} \mathrm{O}+\mathrm{K}_{2} \mathrm{O}(\mathrm{A})$, calcium oxide $\mathrm{CaO}(\mathrm{C})$, and magnesium oxide $\mathrm{MgO}$ (F) (fig. 5). These plots help clarify 
Table 3. Major-oxide compositions for selected Greens Creek (gc) and Woewodski Island (RN) samples.

[Abbreviations in column "*" are explained in table 1.Abbreviations are used as symbols on some figures, and sample numbers are used in REE (rare-earth-element) plots. Almost all samples with LOI

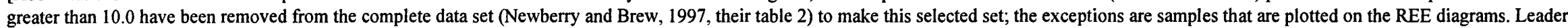
(-) indicates not analyzed. LOI, loss on ignition to $925^{\circ} \mathrm{C}$; Tot, total of anhydrous oxide components; Sum, oxide total $+\mathrm{S}+\mathrm{CO}_{2}+\mathrm{H}_{2} \mathrm{O}$ ]

\begin{tabular}{|c|c|c|c|c|c|c|c|c|c|c|c|c|c|c|c|c|c|c|}
\hline $\begin{array}{c}\text { Sample } \\
\text { no. }\end{array}$ & * & $\mathrm{SiO}_{2}$ & $\mathrm{Al}_{2} \mathrm{O}_{3}$ & $\mathrm{Fe}_{2} \mathrm{O}_{3}$ & $\mathrm{FeO}$ & $\mathrm{MgO}$ & $\mathrm{CaO}$ & $\mathrm{Na}_{2} \mathrm{O}$ & $\mathrm{K}_{2} \mathrm{O}$ & $\mathrm{TiO}_{2}$ & $\mathrm{P}_{2} \mathrm{O}_{5}$ & $\mathrm{MnO}$ & LOI & Tot & $S$ & $\mathrm{H}_{2} \mathrm{O}$ & $\mathrm{CO}_{2}$ & Sum \\
\hline gc78b & $\mathbf{x}$ & 85.7 & 4.12 & 2.70 & 0.16 & 0.60 & 0.80 & 0.20 & 1.00 & 0.22 & 0.08 & 0.02 & 2.49 & 95.6 & 2.76 & 0.63 & 1.76 & 100.8 \\
\hline $\mathrm{gc} 80 \mathrm{~d}$ & $q$ & 57.1 & 8.74 & 3.60 & 4.76 & 4.90 & 2.32 & 0.65 & 1.64 & 0.32 & 0.16 & 0.17 & 10.60 & 84.4 & 4.7 & 1.03 & 10.6 & 100.7 \\
\hline $\mathrm{gc} 80 \mathrm{i}$ & $q$ & 60.4 & 6.41 & 0.10 & 9.10 & 5.40 & 0.46 & 0.65 & 0.74 & 0.24 & 0.08 & 0.19 & 12.17 & 83.8 & 5.68 & 1.08 & 9.74 & 100.3 \\
\hline gc80k & $\mathrm{s}$ & 37.8 & 7.73 & 0.20 & 13.65 & 9.40 & 1.08 & 1.40 & 0.18 & 0.92 & 0.16 & 0.44 & 19.91 & 73.0 & 10.2 & 1.2 & 16 & 100.4 \\
\hline $\mathrm{gc} 80 \mathrm{~m}$ & $q$ & 51.7 & 8.04 & 0.40 & 9.33 & 7.60 & 0.26 & 0.95 & 0.72 & 0.36 & 0.14 & 0.37 & 15.23 & 79.9 & 7.44 & 1.47 & 11.4 & 100.2 \\
\hline $\mathrm{gc} 80 \mathrm{pq}$ & $\mathrm{s}$ & 22.0 & 11.1 & 0.22 & 12.72 & 11.30 & 7.50 & 0.65 & 1.48 & 0.90 & 0.16 & 0.81 & 16.39 & 68.8 & 10.6 & 2.2 & 18.9 & 100.5 \\
\hline gc81a & $r$ & 63.6 & 12.8 & 0.60 & 4.11 & 2.50 & 1.24 & 1.10 & 2.36 & 0.50 & 0.20 & 0.08 & 7.05 & 89.1 & 4.26 & 1.79 & 5.25 & 100.4 \\
\hline $\mathrm{gc} 82 \mathrm{a}$ & $\mathrm{c}$ & 47.4 & 14.0 & 3.20 & 7.39 & 10.90 & 8.08 & 2.30 & 0.92 & 1.22 & 0.12 & 0.26 & 3.14 & 95.8 & 0.01 & 3.64 & 0.08 & 99.5 \\
\hline $\mathrm{gc} 82 \mathrm{c}$ & $q$ & 69.3 & 10.7 & 1.30 & 5.26 & 5.80 & 0.22 & 0.70 & 1.74 & 0.44 & 0.16 & 0.18 & 3.55 & 95.8 & 0.32 & 3.68 & 0.1 & 99.9 \\
\hline $\mathrm{gc} 83 \mathrm{~b}$ & $q$ & 76.3 & 5.60 & 0.80 & 4.00 & 4.90 & 1.50 & 0.20 & 0.44 & 0.28 & 0.32 & 0.19 & 3.50 & 94.5 & 1.35 & 2.6 & 1.91 & 100.4 \\
\hline $\mathrm{gc} 83 \mathrm{c}$ & $\mathrm{s}$ & 45.6 & 13.8 & 2.80 & 11.70 & 11.50 & 0.76 & 1.24 & 0.24 & 1.40 & 0.44 & 0.36 & 7.32 & 89.8 & 3.76 & 6.45 & 0.26 & 100.3 \\
\hline $\mathrm{gc} 84 \mathrm{~b}$ & $\mathrm{~g}$ & 51.2 & 13.4 & 1.50 & 7.55 & 9.80 & 7.40 & 4.30 & 0.12 & 0.86 & 0.12 & 0.26 & 2.47 & 96.5 & 0.01 & 3.35 & 0.01 & 99.9 \\
\hline $\mathrm{gc} 84 \mathrm{c}$ & $\mathrm{m}$ & 44.8 & 19.6 & 0.86 & 7.23 & 13.90 & 0.46 & 5.20 & 0.70 & 0.10 & 0.04 & 0.25 & 6.24 & 93.1 & 0.32 & 5.91 & 0.21 & 99.6 \\
\hline $\mathrm{gc} 85 \mathrm{~b}$ & $\mathrm{c}$ & 47.8 & 17.4 & 2.40 & 8.06 & 8.00 & 3.30 & 5.00 & 0.84 & 0.94 & 0.44 & 0.13 & 4.00 & 94.3 & 0.01 & 4.36 & 0.51 & 99.2 \\
\hline $\mathrm{gc} 89 \mathrm{a}$ & $\mathrm{h}$ & 56.2 & 14.8 & 1.90 & 4.40 & 3.40 & 2.54 & 1.90 & 3.04 & 0.82 & 0.34 & 0.14 & 5.50 & 89.5 & 2.2 & 2.9 & 5.83 & 100.4 \\
\hline $\mathrm{gc} 90 \mathrm{a}$ & $\mathrm{h}$ & 82.9 & 6.08 & 0.25 & 2.11 & 2.60 & 0.48 & 0.55 & 1.14 & 0.22 & 0.06 & 1.80 & 1.65 & 98.2 & 0.01 & 1.7 & 0.65 & 100.6 \\
\hline gc93a & $x$ & 63.9 & 8.91 & 0.06 & 3.15 & 0.55 & 5.24 & 0.20 & 2.36 & 0.39 & 3.76 & 0.03 & 6.25 & 88.6 & 5.35 & 1.25 & 4.9 & 100.1 \\
\hline gc94b & $\mathrm{m}$ & 44.8 & 20.3 & 0.26 & 6.00 & 13.60 & 1.88 & 2.60 & 1.40 & 0.24 & 0.02 & 0.08 & 7.67 & 91.2 & 0.01 & 6.96 & 1.86 & 100.0 \\
\hline gc95a & $r$ & 68.0 & 14.1 & 1.50 & 3.73 & 2.10 & 0.44 & 4.50 & 1.08 & 0.60 & 0.30 & 0.10 & 2.49 & 96.5 & 0.17 & 1.78 & 2.4 & 100.8 \\
\hline gc101a & $\mathrm{g}$ & 45.4 & 21.6 & 1.02 & 2.21 & 8.60 & 11.5 & 3.80 & 0.03 & 0.08 & 0.01 & 0.05 & 5.46 & 94.3 & 0.01 & 5.18 & 0.2 & 99.7 \\
\hline $\mathrm{gc} 102 \mathrm{a}$ & $\mathrm{g}$ & 44.5 & 21.1 & 0.50 & 3.72 & 10.10 & 10.3 & 3.10 & 0.60 & 0.10 & 0.01 & 0.07 & 5.68 & 94.1 & 0.01 & 5.51 & 0.17 & 99.8 \\
\hline gc103a & $\mathrm{g}$ & 44.5 & 19.4 & 0.70 & 3.03 & 9.70 & 14.9 & 2.50 & 0.03 & 0.14 & 0.01 & 0.09 & 5.12 & 95.0 & 0.01 & 4.71 & 0.14 & 99.9 \\
\hline $\mathrm{gc} 121 \mathrm{~b}$ & $\mathrm{~s}$ & 53.2 & 11.0 & 1.55 & 7.62 & 16.6 & 0.56 & 0.10 & 0.01 & 0.56 & 0.03 & 0.22 & 7.35 & 91.5 & - & 6.76 & 0.94 & 99.2 \\
\hline $\mathrm{gc} 122 \mathrm{a}$ & c & 48.9 & 10.1 & 1.92 & 6.98 & 12.9 & 9.7 & 0.69 & 2.86 & 1.28 & 0.25 & 0.19 & 2.91 & 95.8 & - & 3.25 & 0.84 & 99.9 \\
\hline $\operatorname{gc} 122 b$ & $\mathrm{k}$ & 66.3 & 17.7 & 0.53 & 2.07 & 1.02 & 1.1 & 7.57 & 1.68 & 0.13 & 0.03 & 0.09 & 1.57 & 98.2 & - & 0.89 & 0.74 & 99.9 \\
\hline $\mathrm{gcl} 122 \mathrm{~d}$ & $\mathrm{k}$ & 67 & 17.9 & 0.84 & 1.34 & 0.54 & 1.7 & 7.00 & 1.81 & 0.13 & 0.02 & 0.06 & 1.16 & 98.3 & - & 0.71 & 0.6 & 99.7 \\
\hline $\operatorname{gc} 122 \mathrm{e}$ & $\mathrm{g}$ & 44.4 & 8.59 & 1.95 & 7.02 & 13.5 & 16.4 & 0.43 & 0.44 & 1.24 & 0.06 & 0.19 & 4.75 & 94.2 & - & 2.84 & 3.05 & 100.1 \\
\hline $\operatorname{gc} 122 \mathrm{f}$ & c & 43.5 & 10.6 & 2.08 & 8.49 & 10.7 & 12.9 & 1.14 & 1.58 & 1.49 & 0.2 & 0.2 & 5.98 & 92.9 & - & 3.06 & 3.34 & 99.3 \\
\hline gc $284 f$ & $\mathrm{~h}$ & 69.1 & 14.0 & 1.95 & 2.65 & 1.43 & 0.47 & 2.92 & 2.41 & 0.50 & 0.16 & 0.06 & $4.20^{1}$ & 99.85 & - & - & - & - \\
\hline gc $285 b$ & $\mathrm{~h}$ & 83.6 & 3.65 & 1.90 & 1.33 & 1.47 & 3.08 & 0.22 & 0.52 & 0.17 & 0.03 & 0.01 & $4.25^{1}$ & 100.2 & - & - & - & - \\
\hline gc286a & $\mathrm{g}$ & 32.4 & 12.4 & 2.20 & 20.0 & 8.89 & 13.10 & 0.37 & 0.18 & 1.92 & 2.12 & 0.29 & $4.41^{1}$ & 99.18 & - & - & - & - \\
\hline $\mathrm{gc} 286 \mathrm{~b}$ & c & 45.3 & 15.3 & 2.10 & 8.75 & 8.41 & 6.12 & 0.89 & 2.54 & 1.33 & 0.19 & 0.20 & $8.63^{1}$ & 99.76 & - & - & - & - \\
\hline gc $286 \mathrm{c}$ & $\mathrm{g}$ & 45.8 & 5.97 & 2.60 & 4.81 & 13.30 & 19.80 & 0.53 & 0.97 & 0.72 & 0.16 & 0.14 & $5.19^{1}$ & 99.99 & - & - & - & - \\
\hline $\operatorname{gc} 286 \mathrm{~d}$ & c & 48.2 & 14.5 & 3.40 & 10.8 & 7.97 & 5.52 & 2.90 & 0.01 & 1.99 & 0.21 & 0.22 & $3.91^{1}$ & 99.63 & - & - & - & - \\
\hline gc $287 \mathrm{~b}$ & $s$ & 47.9 & 16.1 & 3.30 & 8.60 & 5.74 & 3.01 & 2.30 & 3.06 & 1.00 & 0.50 & 0.52 & $6.94^{1}$ & 98.97 & - & - & - & - \\
\hline $\mathrm{gc} 287 \mathrm{c}$ & s & 47.9 & 13.0 & 6.30 & 5.14 & 4.05 & 3.00 & 1.63 & 3.12 & 0.81 & 0.42 & 0.45 & $9.63^{1}$ & 95.45 & - & - & - & - \\
\hline $\mathrm{gc} 288 \mathrm{f}$ & $q$ & 70.9 & 7.09 & 0.10 & 9.30 & 1.07 & 1.19 & 0.01 & 2.12 & 0.29 & 0.12 & 0.03 & $6.22^{1}$ & 98.44 & - & - & - & - \\
\hline gc289f & $\mathrm{s}$ & 49.8 & 15.5 & 2.60 & 7.00 & 8.21 & 2.51 & 1.96 & 2.00 & 1.63 & 0.17 & 0.47 & $6.98^{1}$ & 98.83 & - & - & - & - \\
\hline gc289j & $q$ & 74.3 & 10.1 & 0.20 & 3.20 & 3.66 & 0.52 & 1.23 & 2.44 & 0.26 & 0.06 & 0.15 & $2.93^{1}$ & 99.05 & - & - & - & - \\
\hline $\operatorname{gc} 290 \mathrm{a}$ & $\mathrm{s}$ & 35.8 & 16.2 & 1.00 & 10.4 & 16.80 & 2.15 & 0.10 & 0.20 & 1.45 & 0.02 & 0.33 & $9.16^{1}$ & 93.61 & - & - & - & - \\
\hline
\end{tabular}


Table 3. Major-oxide compositions for selected Greens Creek (gc) and Woewodski Island (RN) samples-Continued.

\begin{tabular}{|c|c|c|c|c|c|c|c|c|c|c|c|c|c|c|c|c|c|c|}
\hline $\begin{array}{c}\text { Sample } \\
\text { no. }\end{array}$ & * & $\mathrm{SiO}_{2}$ & $\mathrm{Al}_{2} \mathrm{O}_{3}$ & $\mathrm{Fe}_{2} \mathrm{O}_{3}$ & $\mathrm{FeO}$ & $\mathrm{MgO}$ & $\mathrm{CaO}$ & $\mathrm{Na}_{2} \mathrm{O}$ & $\mathrm{K}_{2} \mathrm{O}$ & $\mathrm{TiO}_{2}$ & $\mathrm{P}_{2} \mathrm{O}_{5}$ & $\mathrm{MnO}$ & LOI & Tot & $S$ & $\mathrm{H}_{2} \mathrm{O}$ & $\mathrm{CO}_{2}$ & Sum \\
\hline gc293a & $\mathrm{s}$ & 47.8 & 13.1 & 1.30 & 12.4 & 10.40 & 2.22 & 0.92 & 1.03 & 1.68 & 0.33 & 0.46 & $6.76^{1}$ & 98.38 & - & - & - & - \\
\hline gc293b & c & 46.6 & 12.6 & 2.30 & 9.64 & 9.43 & 4.25 & 0.73 & 1.40 & 1.69 & 0.17 & 0.39 & $9.63^{1}$ & 98.83 & - & - & - & - \\
\hline gc294a & s & 46.2 & 14.8 & 0.60 & 15.1 & 9.20 & 0.37 & 1.21 & 1.50 & 1.28 & 0.16 & 0.29 & $8.54^{1}$ & 99.25 & - & - & - & - \\
\hline gc294b & $\mathbf{s}$ & 45.3 & 10.8 & 2.00 & 8.60 & 10.20 & 5.94 & 1.67 & 0.35 & 1.28 & 0.19 & 0.67 & $9.08^{1}$ & 96.08 & - & - & - & - \\
\hline gc294c & s & 44.1 & 12.3 & 2.50 & 9.30 & 10.00 & 5.00 & 2.16 & 0.25 & 1.57 & 0.19 & 0.59 & $8.45^{1}$ & 96.41 & - & - & - & - \\
\hline RN116a & $\mathrm{g}$ & 43.6 & 13.2 & $11.6^{2}$ & - & 6.1 & 8.95 & 2.32 & 1.83 & 1.46 & 0.14 & 0.22 & $7.9^{1}$ & - & - & - & - & 97.3 \\
\hline RN116d & $\mathrm{a}$ & 47.6 & 13.7 & $14.5^{2}$ & - & 2.23 & 2.64 & 0.15 & 2.15 & 1.91 & 0.17 & 0.69 & $12.9^{1}$ & - & - & - & - & 98.6 \\
\hline RN116g & $\mathrm{t}$ & 45.3 & 15.7 & $10.4^{2}$ & - & 3.32 & 5.35 & 0.15 & 1.22 & 1.93 & 0.17 & 0.23 & $15.4^{1}$ & - & - & - & - & 99.2 \\
\hline RN118a & $\mathrm{g}$ & 42.7 & 15.0 & $13.7^{2}$ & - & 6.85 & 10.4 & 1.66 & 0.57 & 1.85 & 0.16 & 0.21 & $6.3^{1}$ & - & - & - & - & 99.4 \\
\hline RN118b & $\mathrm{g}$ & 51.1 & 14.4 & $9.38^{2}$ & - & 7.18 & 7.54 & 4.04 & 0.64 & 0.49 & 0.09 & 0.16 & $4.8^{1}$ & - & - & - & - & 99.8 \\
\hline RN118d & $\begin{array}{l}0 \\
-\end{array}$ & 33.3 & 7.33 & $30.9^{2}$ & - & 1.49 & 1.39 & 0.1 & 0.01 & 0.89 & 0.06 & 5.26 & $19.2^{1}$ & - & - & - & - & 99.9 \\
\hline RN119c & $t$ & 45.4 & 16.9 & $14.8^{2}$ & - & 2.33 & 1.11 & 0.1 & 2.61 & 1.26 & 0.32 & 1.13 & $13.2^{1}$ & - & - & - & - & 99.2 \\
\hline RN120a & $\mathrm{g}$ & 47.4 & 14.5 & $11.6^{2}$ & - & 7.26 & 10.3 & 3.1 & 0.65 & 1.36 & 0.11 & 0.19 & $3.8^{1}$ & - & - & - & - & 100.3 \\
\hline RN121a & $\mathrm{g}$ & 44.9 & 14.3 & $10.7^{2}$ & - & 6.75 & 11.1 & 2.85 & 1.13 & 1.25 & 0.11 & 0.16 & $4.9^{1}$ & - & - & - & - & 98.1 \\
\hline RN123a & $\mathrm{t}$ & 47.7 & 14.3 & $13.2^{2}$ & - & 3.0 & 3.2 & 0.1 & 1.39 & 1.39 & 0.13 & 0.46 & $14.2^{1}$ & - & - & - & - & 99.1 \\
\hline
\end{tabular}

1 Total is sum of anhydrous oxide components + LOI.

2 Total iron as $\mathrm{Fe}_{2} \mathrm{O}_{3}$. 
Table 4. Trace-element concentrations (in parts per million) of selected samples from Greens Creek (gc) and Woewodski Island (RN) by wavelength-dispersive $\mathrm{X}$-ray fluorescence analysis.

[Abbreviations in column "** are explained in table 1. Abbreviations are used as symbols on some figures, and sample numbers are used in REE (rare-earthelement) plots. Leader ( - ) indicates not analyzed]

\begin{tabular}{|c|c|c|c|c|c|c|c|c|c|c|c|c|c|}
\hline Sample no. & * & $\mathrm{Nb}$ & $\mathrm{Rb}$ & $\mathrm{Sr}$ & $\mathrm{Zr}$ & Y & $\mathrm{Ni}$ & $\mathrm{Cr}$ & $\mathrm{Ba}$ & $\mathrm{Ce}$ & La & $\mathrm{Cu}$ & $\mathrm{Zn}$ \\
\hline $\mathrm{gc78b}$ & $\mathrm{x}$ & $<10$ & 12 & 14 & 34 & 10 & 25 & 48 & 5000 & 34 & 58 & 10 & 820 \\
\hline gc80d & $\mathrm{q}$ & $<10$ & 20 & 46 & 92 & 16 & 12 & 11 & 780 & 28 & 10 & 10 & 90 \\
\hline $\mathrm{gc} 80 \mathrm{i}$ & $\mathrm{q}$ & $<10$ & 5 & 24 & 44 & $<10$ & 20 & 14 & 560 & 10 & 10 & 10 & 100 \\
\hline gc80k & $\mathrm{s}$ & $<10$ & 5 & 74 & 58 & $<10$ & 46 & 91 & 680 & 29 & 10 & 10 & 320 \\
\hline $\mathrm{gc} 80 \mathrm{~m}$ & $q$ & $<10$ & 5 & 60 & 58 & 10 & 28 & 68 & 2550 & 46 & 30 & 10 & 120 \\
\hline gc80pq & s & $<10$ & 28 & 215 & 76 & 46 & 50 & 129 & 5000 & 34 & 82 & 68 & 1500 \\
\hline gc81a & $r$ & 14 & 34 & 76 & 122 & 24 & 10 & 12 & 3450 & 48 & 48 & 10 & 40 \\
\hline gc82a & c & $<10$ & 5 & 112 & 68 & 20 & 88 & 355 & 830 & 34 & 24 & 24 & 455 \\
\hline gc82c & $q$ & $<10$ & 20 & 5 & 68 & 12 & 15 & 24 & 1100 & 44 & 10 & 10 & 100 \\
\hline $\mathrm{gc83b}$ & $q$ & $<10$ & 5 & 36 & 38 & $<10$ & 14 & 29 & 435 & 34 & 10 & 10 & 66 \\
\hline gc83c & $\mathrm{s}$ & $<10$ & 5 & 16 & 82 & 18 & 46 & 94 & 355 & 30 & 10 & 10 & 196 \\
\hline $\mathrm{gc} 84 \mathrm{~b}$ & $\mathrm{~g}$ & 14 & 5 & 285 & 82 & 16 & 234 & 610 & 205 & 22 & 10 & 24 & 100 \\
\hline $\mathrm{gc} 84 \mathrm{c}$ & $\mathrm{m}$ & $<10$ & 5 & 5 & 16 & $<10$ & 380 & 345 & 840 & 24 & 24 & 130 & 1500 \\
\hline gc85b & c & 14 & 5 & 325 & 52 & 18 & 63 & 134 & 210 & 54 & 26 & 70 & 98 \\
\hline gc89a & $\mathrm{h}$ & 16 & 74 & 60 & 140 & 32 & 22 & 28 & 3350 & 48 & 44 & 82 & 315 \\
\hline gc90a & $\mathrm{h}$ & $<10$ & 14 & 22 & 48 & $<10$ & 30 & 14 & 1100 & 40 & 24 & 24 & 66 \\
\hline gc93a & $\mathrm{x}$ & 14 & 46 & 136 & 62 & 44 & 102 & 355 & 2850 & 50 & 36 & 26 & 96 \\
\hline gc94a & $\mathrm{m}$ & $<10$ & 5 & 78 & 10 & $<10$ & 2500 & 4150 & 48 & 26 & 22 & 188 & 36 \\
\hline gc94b & $\mathrm{m}$ & $<10$ & 20 & 36 & 14 & $<10$ & 300 & 920 & 1850 & 20 & 10 & 48 & 36 \\
\hline gc95a & $\mathrm{r}$ & 12 & 12 & 16 & 104 & 18 & 12 & 17 & 590 & 42 & 10 & 54 & 90 \\
\hline gc101a & $\mathrm{g}$ & $<10$ & 5 & 24 & 12 & $<10$ & 240 & 980 & 118 & 64 & 22 & 10 & 10 \\
\hline $\mathrm{gc102a}$ & $\mathrm{g}$ & $<10$ & 5 & 24 & 10 & $<10$ & 260 & 620 & 225 & 30 & 10 & 10 & 32 \\
\hline gc103a & $\mathrm{g}$ & $<10$ & 5 & 5 & $<10$ & $<10$ & 250 & 1600 & 68 & 24 & 10 & 10 & 24 \\
\hline gc121b & $\mathrm{s}$ & $<10$ & 5 & 42 & 60 & 10 & 410 & 750 & 34 & $<30$ & $<30$ & 5 & 690 \\
\hline gc122a & c & 10 & 68 & 142 & 50 & 18 & 100 & 830 & 1900 & $<30$ & $<30$ & 5 & 130 \\
\hline gc122b & $\mathrm{k}$ & 16 & 56 & 160 & 120 & 12 & $<10$ & 10 & 1700 & 40 & $<30$ & 34 & 56 \\
\hline $\mathrm{gcl} 122 \mathrm{~d}$ & $\mathrm{k}$ & 12 & 62 & 790 & 118 & 10 & $<105$ & 10 & 1400 & $<30$ & $<30$ & 26 & 52 \\
\hline gc122e & $\mathrm{g}$ & $<10$ & 12 & 560 & 68 & 16 & 130 & 500 & 400 & $<30$ & $<30$ & 88 & 70 \\
\hline gc122f & c & $<10$ & 42 & 540 & 66 & 19 & 72 & 330 & 1600 & $<30$ & $<30$ & 72 & 68 \\
\hline gc284b & $\mathrm{h}$ & 10 & 14 & 126 & 74 & 26 & 11 & 124 & - & - & - & - & - \\
\hline $\operatorname{gc} 284 \mathrm{c}$ & $s$ & $<10$ & 22 & 60 & 78 & 16 & 98 & 114 & - & - & - & - & - \\
\hline $\operatorname{gc} 284 d$ & $\mathrm{~s}$ & $<10$ & 20 & 86 & 82 & 20 & 72 & 104 & - & - & - & - & - \\
\hline $\operatorname{gc} 284 \mathrm{e}$ & c & 12 & 54 & 90 & 104 & 32 & 90 & 142 & - & - & - & - & - \\
\hline gc284f & $\mathrm{h}$ & 16 & 42 & 30 & 110 & 26 & 10 & 30 & - & - & - & - & - \\
\hline $\operatorname{gc} 285 \mathrm{a}$ & $\mathrm{m}$ & 16 & 34 & 166 & 62 & 12 & 600 & 1650 & - & - & - & - & - \\
\hline gc285b & $\mathrm{h}$ & 12 & 16 & 54 & 50 & 16 & 10 & 54 & - & - & - & - & - \\
\hline gc285g & $\mathrm{m}$ & 12 & 88 & 70 & 28 & 12 & 405 & 530 & - & - & - & - & - \\
\hline $\mathrm{gc} 286 \mathrm{a}$ & $\mathrm{g}$ & $<10$ & 5 & 620 & 46 & 10 & 60 & 92 & - & - & - & - & - \\
\hline gc286b & c & 18 & 56 & 72 & 88 & 30 & 92 & 365 & - & - & - & - & - \\
\hline $\operatorname{gc} 286 \mathrm{c}$ & g & 16 & 30 & 255 & 34 & 14 & 88 & 250 & - & - & - & - & - \\
\hline gc286d & c & 22 & 5 & 150 & 126 & 48 & 76 & 140 & - & - & - & - & - \\
\hline gc286e & $\mathrm{p}$ & 10 & 5 & 12 & 16 & $<10$ & 2150 & 2600 & - & - & - & - & - \\
\hline $\mathrm{gc} 286 \mathrm{f}$ & c & 20 & 20 & 122 & 98 & 24 & 325 & 980 & - & - & - & - & - \\
\hline gc287a & $\mathrm{m}$ & 14 & 38 & 130 & 24 & 10 & 530 & 1450 & - & - & - & - & - \\
\hline gc287b & $\mathrm{s}$ & 10 & 50 & 270 & 92 & 30 & 45 & 132 & - & - & - & - & - \\
\hline $\operatorname{gc} 287 \mathrm{c}$ & $\mathrm{s}$ & 18 & 54 & 265 & 86 & 28 & 50 & 246 & - & - & - & - & - \\
\hline gc288a & $\mathbf{s}$ & 16 & 42 & 26 & 98 & 32 & 50 & 108 & - & - & - & - & - \\
\hline $\operatorname{gc} 288 b$ & $\mathbf{s}$ & 16 & 38 & 18 & 40 & 18 & 106 & 330 & - & - & - & - & - \\
\hline gc288c & $\mathrm{s}$ & 18 & 34 & 34 & 142 & 34 & 44 & 134 & - & - & - & - & - \\
\hline $\mathrm{gc} 288 \mathrm{~d}$ & $\mathrm{~s}$ & 14 & 16 & 230 & 38 & 18 & 150 & 780 & - & - & - & - & - \\
\hline gc288e & $\mathbf{s}$ & 20 & 34 & 134 & 58 & 38 & 116 & 650 & - & - & - & - & - \\
\hline
\end{tabular}

the nature of the rock protoliths. The rocks with sedimentary protoliths (stratigraphic hanging-wall argillite, "h;" and stratigraphic footwall graphitic phyllite, "r") plot with argillaceous and carbonate rocks. Some of the rocks of apparent maficultramafic parentage ("g," "c") plot in fields appropriate to basaltic rocks (fig. 5); however, the majority do not. If the 
Table 4. Trace-element concentrations (in parts per million) of selected samples from Greens Creek (gc) and Woewodski Island (RN) by wavelength-dispersive $\mathrm{X}$-ray fluorescence analysis-Continued.

\begin{tabular}{|c|c|c|c|c|c|c|c|c|c|c|c|c|c|}
\hline Sample no. & * & $\mathrm{Nb}$ & $\mathrm{Rb}$ & $\mathrm{Sr}$ & $\mathrm{Zr}$ & $\mathrm{Y}$ & $\mathrm{Ni}$ & $\mathrm{Cr}$ & $\mathrm{Ba}$ & $\mathrm{Ce}$ & $\mathrm{La}$ & $\mathrm{Cu}$ & $\mathrm{Zn}$ \\
\hline gc288f & $q$ & 12 & 14 & 42 & 54 & 18 & 11 & 40 & - & - & - & - & - \\
\hline gc289a & $\mathrm{s}$ & 14 & 18 & 106 & 66 & 28 & 98 & 194 & - & - & - & - & - \\
\hline $\operatorname{gc} 289 \mathrm{~b}$ & $\mathrm{~s}$ & 14 & 28 & 186 & 82 & 22 & 50 & 66 & - & - & - & - & - \\
\hline gc289c & $\mathrm{s}$ & 16 & 16 & 128 & 96 & 66 & 38 & 150 & - & - & - & - & - \\
\hline $\operatorname{gc} 289 d$ & $r$ & 18 & 40 & 86 & 76 & 30 & 26 & 18 & - & - & - & - & - \\
\hline $\operatorname{gc} 289 \mathrm{e}$ & $\mathrm{s}$ & 15 & 48 & 132 & 52 & 42 & 164 & 850 & - & - & - & - & - \\
\hline gc289f & $\mathrm{s}$ & 20 & 34 & 46 & 104 & 32 & 54 & 205 & - & - & - & - & - \\
\hline $\mathrm{gc} 289 \mathrm{~g}$ & $\mathrm{x}$ & 10 & 5 & 190 & 44 & 34 & 146 & 340 & - & - & - & - & - \\
\hline gc289h & $\mathrm{s}$ & 18 & 18 & 148 & 100 & 32 & 56 & 205 & - & - & - & - & - \\
\hline gc289i & $\mathrm{x}$ & 14 & 18 & 160 & 58 & 32 & 46 & 176 & - & - & - & - & - \\
\hline gc289j & $q$ & $<10$ & 62 & 24 & 100 & $<10$ & 10 & 24 & - & - & - & - & - \\
\hline gc290a & $\mathrm{s}$ & 28 & 135 & 102 & 280 & 30 & 126 & 176 & - & - & - & - & - \\
\hline gc291a & $\mathrm{p}$ & $<10$ & 5 & 40 & 12 & $<10$ & 2100 & 1350 & - & - & - & - & - \\
\hline gc293a & $\mathrm{s}$ & 18 & 24 & 38 & 82 & 50 & 50 & 62 & - & - & - & - & - \\
\hline gc293b & c & 14 & 24 & 74 & 108 & 32 & 58 & 158 & - & - & - & - & - \\
\hline $\operatorname{gc} 293 d$ & $\mathrm{~s}$ & 12 & 40 & 24 & 80 & 32 & 32 & 64 & - & - & - & - & - \\
\hline gc294a & $\mathrm{s}$ & 18 & 24 & 20 & 84 & 38 & 64 & 320 & - & - & - & - & - \\
\hline gc294b & $\mathrm{s}$ & 14 & 5 & 92 & 90 & 34 & 38 & 98 & - & - & - & - & - \\
\hline gc294c & $\mathrm{s}$ & 20 & 5 & 96 & 106 & 42 & 46 & 146 & - & - & - & - & - \\
\hline $\operatorname{gc} 294 d$ & $\mathrm{~s}$ & 21 & 5 & 20 & 86 & 26 & 240 & 630 & - & - & - & - & - \\
\hline RN116a & $\mathrm{g}$ & 14 & 32 & 144 & 94 & 26 & - & - & - & - & - & - & - \\
\hline RN116d & $\mathrm{a}$ & 12 & 46 & 44 & 124 & 33 & - & - & - & - & - & - & - \\
\hline RN116g & $t$ & 17 & 24 & 73 & 114 & 28 & - & - & - & - & - & - & - \\
\hline RN118a & $\mathrm{g}$ & 12 & 12 & 225 & 114 & 28 & - & - & - & - & - & - & - \\
\hline RN118b & $\mathrm{g}$ & 8 & 15 & 184 & 52 & 18 & - & - & - & - & - & - & - \\
\hline RN118d & - & 12 & 8 & 15 & 53 & 18 & - & - & - & - & - & - & - \\
\hline RN119c & $t$ & 13 & 44 & 57 & 88 & 24 & - & - & - & - & - & - & - \\
\hline RN120a & $\mathrm{g}$ & 12 & 20 & 270 & 87 & 27 & - & - & - & - & - & - & - \\
\hline RN121a & $\mathrm{g}$ & 10 & 23 & 180 & 74 & 20 & - & - & - & - & - & - & - \\
\hline RN123a & $\mathrm{t}$ & 14 & 24 & 44 & 88 & 25 & - & - & - & - & - & - & - \\
\hline
\end{tabular}

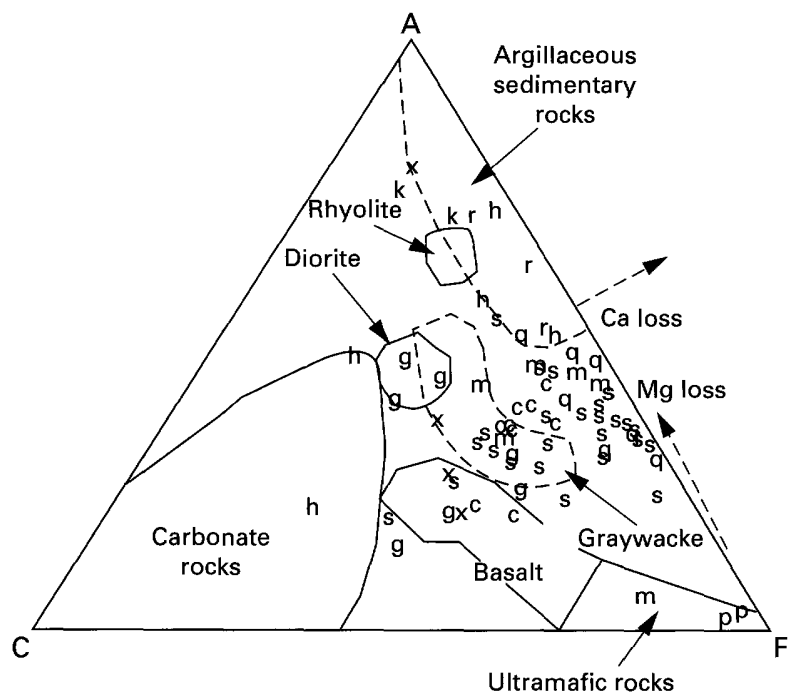

phyllites were originally unaltered sedimentary rocks, then they would have most likely been graywackes. However, less than a third of the analyses plot in the field of typical graywackes (fig. 5). One possible explanation is that the phyllites were derived from mafic rocks (including possibly mafic tuffs) that experienced various degrees of $\mathrm{Na}_{2} \mathrm{O}$ (fig. 4), and especially $\mathrm{CaO}$, loss during hydrothermal alteration.
Figure 5. Major-element compositions of rocks from the Greens Creek deposit and vicinity rocks on the ACF diagram (alkalies $\mathrm{Na}_{2} \mathrm{O}+\mathrm{K}_{2} \mathrm{O}(\mathrm{A})$, calcium oxide $\mathrm{CaO}$ (C), and magnesium oxide $\mathrm{MgO}(\mathrm{F})$; after Winkler, 1979), showing compositional relationships among rock types. Sample/ rock type symbols are as follows: Hanging-wall rocks: $h$, hanging-wall argillite; Footwall rocks: $r$, graphitic phyllite; $q$, pyritic muscovite quartz phyllite; c, chloritic phyllite and greenstones; $s$, muscovite phyllite; $m$, mariposite rock; $x$, siliceous breccia; $g$, gabbro; $p$, serpentine. Other: $k$, felsic sill. Note that stratigraphic hanging-wall rocks fall in well-defined fields of sedimentary rocks, whereas stratigraphic footwall rocks do not. Field boundaries from Winkler (1979).

Trace-element compositions most clearly suggest the mafic-ultramafic parentage for most of the Greens Creek stratigraphic footwall phyllites ("s," "c"). Notably, all these stratigraphic footwall rocks, (except the stratigraphic footwall graphitic ("r") and siliceous-pyritic ("q") phyllites) contain higher concentrations of $\mathrm{Cr}$ and $\mathrm{Ni}$ than do the rocks of sedimentary parentage (fig. 6). We interpret the low abundance of $\mathrm{Cr}$ and $\mathrm{Ni}$ in the graphitic phyllites (" $\mathrm{r}$ ") and in the local siliceous-pyritic phyllite ("q") unit as due to sedimentary parentage and extreme degree of hydrothermal leaching, respectively. Mariposite-bearing phyllites ("m"), serpentinites ("p"), and gabbroic-appearing ("g") rocks show concentrations appropriate to ultramafic rocks; chloritic phyllites and greenstones 
Table 5. Trace-element concentrations (in parts per million) for Greens Creek samples by neutron activation analysis.

[Abbreviations in column "*" are explained in table 1. Abbreviations are used as symbols on some figures, and sample numbers are used in REE (rare-earthelement) plots]

\begin{tabular}{cccccccccccccc}
\hline $\begin{array}{c}\text { Sample } \\
\text { no. }\end{array}$ & $*$ & $\mathrm{Ba}$ & $\mathrm{Sr}$ & $\mathrm{Co}$ & $\mathrm{Ni}$ & $\mathrm{Cr}$ & $\mathrm{Cs}$ & $\mathrm{Hf}$ & $\mathrm{Rb}$ & $\mathrm{Sb}$ & $\mathrm{Ta}$ & $\mathrm{Th}$ & $\mathrm{U}$ \\
\hline gc78b & $\mathrm{x}$ & 4570 & 43.1 & 7.37 & 27.9 & 48.6 & 0.93 & 0.84 & 26.2 & 15.2 & 0.158 & 1.03 & 0.97 \\
gc80d & $\mathrm{q}$ & 707 & 35 & 12.8 & 12.8 & 11.7 & 1.2 & 2.39 & 31.7 & 1.31 & 0.157 & 2.06 & 1.35 \\
gc80i & $\mathrm{q}$ & 515 & 49 & 18.5 & 23.7 & 15.6 & 0.78 & 1.09 & 15.4 & 1.8 & 0.126 & 1.14 & 0.58 \\
gc80k & $\mathrm{s}$ & 561 & 95.9 & 24.2 & 53.1 & 90.9 & 0.59 & 1.39 & 4.7 & 6.94 & 0.204 & 0.44 & 0.7 \\
gc80m & $\mathrm{q}$ & 2400 & 67.6 & 12.8 & 44.8 & 110 & 1.08 & 1.49 & 14.2 & 1.6 & 0.258 & 2.05 & 0.79 \\
gc80pq & $\mathrm{s}$ & 8760 & 171 & 21.4 & 63.1 & 111 & 1.9 & 1.66 & 33.5 & 7.34 & 0.272 & 1.13 & 1.13 \\
gc81a & $\mathrm{r}$ & 3530 & 68.4 & 10.2 & 6.42 & 14.5 & 2.18 & 3.36 & 44.5 & 2.01 & 0.239 & 2.69 & 1.63 \\
gc82a & $\mathrm{c}$ & 704 & 213 & 34 & 75.3 & 276 & 0.29 & 1.83 & 16.8 & 1.08 & 0.257 & 0.24 & 0.09 \\
gc82c & $\mathrm{q}$ & 1060 & 6 & 13.1 & 25.2 & 25.9 & 0.69 & 1.9 & 35.5 & 0.69 & 0.268 & 2.37 & 0.87 \\
gc83b & $\mathrm{q}$ & 404 & 59.2 & 9.91 & 26.7 & 29.1 & 0.27 & 0.97 & 9.08 & 0.56 & 0.146 & 1.32 & 1.76 \\
gc83c & $\mathrm{s}$ & 295 & 7 & 34.5 & 40.7 & 94.1 & 0.18 & 2.35 & 7.29 & 1.22 & 0.338 & 0.85 & 0.74 \\
gc84b & $\mathrm{g}$ & 155 & 346 & 38.6 & 241 & 550 & 0.07 & 2.04 & 6.57 & 2.89 & 0.417 & 1.04 & 0.43 \\
gc84c & $\mathrm{m}$ & 794 & 3 & 37.8 & 379 & 272 & 0.45 & 0.22 & 15.2 & 1.16 & 0.072 & 0.21 & 0.08 \\
gc85b & $\mathrm{c}$ & 176 & 330 & 24 & 61.5 & 109 & 0.26 & 3.21 & 14.5 & 0.49 & 0.727 & 3.41 & 1.48 \\
gc89a & $\mathrm{h}$ & 3230 & 75 & 8.1 & 23.5 & 57.5 & 4.41 & 3.14 & 79.8 & 1.3 & 0.613 & 4.33 & 4.29 \\
gc90a & $\mathrm{h}$ & 1020 & 22 & 12.4 & 37 & 14.5 & 1.21 & 1.39 & 24.4 & 0.62 & 0.255 & 1.96 & 0.77 \\
gc93a & $\mathrm{x}$ & 2490 & 169 & 15.9 & 134 & 235 & 3.72 & 1.62 & 49.8 & 21.6 & 0.307 & 2.49 & 4.73 \\
gc94a & $\mathrm{m}$ & 5.9 & 5 & 145 & 3550 & 2790 & 0.08 & 0.06 & 1.92 & 1.36 & 0.01 & 0.44 & 0.09 \\
gc9gc4b & $\mathrm{m}$ & 1910 & 57 & 41 & 306 & 796 & 2.4 & 0.24 & 34.8 & 0.52 & 0.009 & 0.11 & 0.16 \\
gc95a & $\mathrm{r}$ & 492 & 10 & 14 & 14.4 & 16.5 & 1.18 & 3.04 & 19.7 & 0.48 & 0.369 & 2.69 & 1.21 \\
gc101a & $\mathrm{g}$ & 58 & 8 & 25.4 & 186 & 1010 & 0.05 & 0.07 & 1.3 & 0.82 & 0.004 & 0.02 & 0.04 \\
gc102a & $\mathrm{g}$ & 165 & 24 & 36.7 & 252 & 767 & 0.24 & 0.13 & 12 & 0.37 & 0.014 & 0.04 & 0.12 \\
$103 \mathrm{a}$ & $\mathrm{g}$ & 35.2 & 4 & 29.9 & 229 & 1760 & 0.1 & 0.18 & 2.02 & 1.29 & 0.005 & 0.05 & 0.02
\end{tabular}

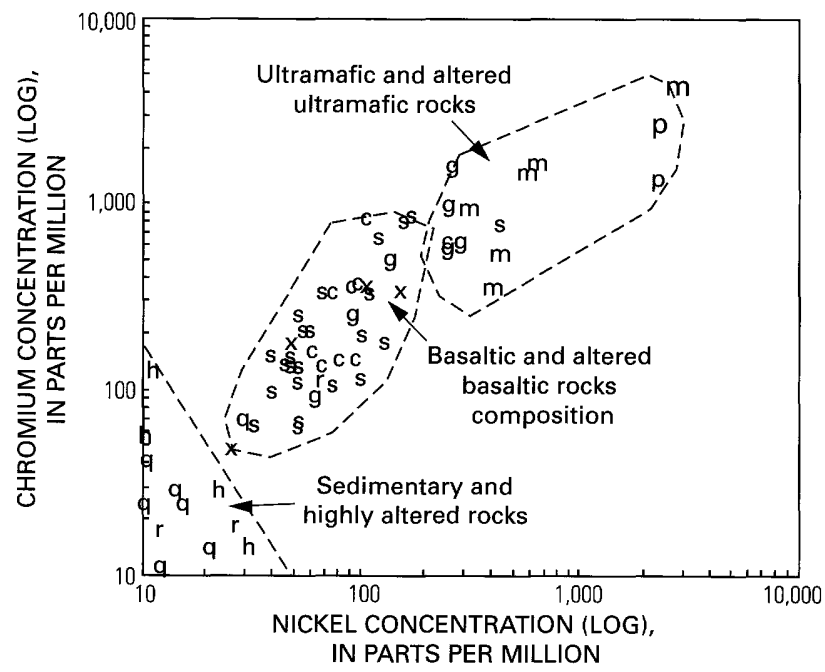

Figure 6. Ni versus $\mathrm{Cr}$ for rocks of the Greens Creek deposit and vicinity. Analyses by wavelength-dispersive $\mathrm{X}$-ray fluorescence method. Sample/rock type symbols plotted are as follows: Hanging-wall rocks: $h$, hanging-wall calcareous argillite; footwall rocks: $r$, graphitic phyllite; q, quartzitic phyllite; c, chloritic phyllite/ greenstone; $s$, muscovite chlorite phyllite; $m$, mariposite rock; $x$, siliceous breccia; $g$, gabbro; $p$, serpentine. Other: $k$, felsic sill. Field boundaries are based on data in BVSP (1981).

("c"), and muscovite phyllites ("s," i.e., non-graphitic stratigraphic footwall rocks) have $\mathrm{Cr}$-Ni ratios appropriate to basaltic rocks (fig. 6). The positive correlation of $\mathrm{Ni}$ and $\mathrm{Cr}$ concentrations for the bulk of stratigraphic footwall rocks contrasts sharply with the lack of a correlation between $\mathrm{Ni}$ and $\mathrm{Cr}$ in the metasedimentary rocks; we interpret both the absolute concentrations and element correlations to indicate that the bulk of footwall rocks were mafic volcanic rocks and not sediments derived from such rocks.

Because standard igenous rock chemical classification schemes are based on the mobile elements $\mathrm{Na}_{2} \mathrm{O}, \mathrm{K}_{2} \mathrm{O}$, and $\mathrm{CaO}$, and because these elements are tremendously mobile in VMS environments (Date and others, 1983), chemical classification is more reliably based on "immobile" elements (Winchester and Floyd, 1977). These elements, which include Ti, Zr, $\mathrm{Y}, \mathrm{Nb}$, and the rare earth elements, are much less susceptible to chemical transport during hydrothermal alteration than most major elements. Furthermore, we consider elemental ratios more reliable than elemental concentrations in most cases, because immobile-element ratios remain constant despite loss of mobile components or addition of new components (e.g., water, carbonate, sulfide). $\mathrm{A} \mathrm{SiO}_{2}$ vs. $\mathrm{Zr} / \mathrm{TiO}_{2}$ diagram (fig. 7A) shows that, with the exception of graphitic (" $r$ ") and siliceous ("q") phyllites, all the stratigraphic footwall rocks at Greens Creek have compositions consistent with basalt and andesite. The bulk of the footwall phyllites plot in a field defined as subalkalic basalt (fig. 7A).

Concentration of $\mathrm{SiO}_{2}$ is used in most igneous-rock classification schemes, but silica is clearly a mobile constituent in VMS deposits (Date and others, 1983) and at Greens Creek in particular. Abundant quartz veining in stratigraphic footwall rocks indicates that silica concentrations, which are in the range for andesite to rhyolite (fig. 7A) may be of secondary origin. 
Table 5. Trace-element concentrations (in parts per million) for Greens Creek samples by neutron activation analysis-Continued.

\begin{tabular}{|c|c|c|c|c|c|c|c|c|c|c|c|c|c|}
\hline $\begin{array}{c}\text { Sample } \\
\text { no. }\end{array}$ & $\mathrm{Zn}$ & $\mathrm{Zr}$ & Sc & La & $\mathrm{Ce}$ & $\mathrm{Nd}$ & $\mathrm{Sm}$ & Eu & Gd & Tb & $\mathrm{Tm}$ & $\mathrm{Yb}$ & Lu \\
\hline gc78b & 782 & 24.3 & 8.01 & 7.21 & 14.8 & 8.65 & 1.95 & 0.73 & 1.77 & 0.26 & 0.17 & 1.08 & 0.15 \\
\hline $\mathrm{gc} 80 \mathrm{~d}$ & 89.3 & 85.2 & 11.6 & 9.44 & 21.1 & 12 & 2.98 & 0.62 & 3.17 & 0.52 & 0.35 & 2.25 & 0.35 \\
\hline gc80i & 88.8 & 36.2 & 9.77 & 1.63 & 3.52 & 2.27 & 0.72 & 0.13 & 0.92 & 0.17 & 0.16 & 1.02 & 0.16 \\
\hline gc80k & 303 & 43 & 21.9 & 1.93 & 5.8 & 4 & 0.99 & 0.16 & 0.9 & 0.12 & 0.11 & 0.67 & 0.14 \\
\hline $\mathrm{gc} 80 \mathrm{~m}$ & 104 & 66 & 11.9 & 13 & 27.5 & 14.8 & 2.67 & 0.37 & 2.18 & 0.3 & 0.21 & 1.39 & 0.22 \\
\hline $\mathrm{gc} 80 \mathrm{pq}$ & 2260 & 92.4 & 26.7 & 8.22 & 17.4 & 9.79 & 2.83 & 0.79 & 3.63 & 0.63 & 0.41 & 2.63 & 0.38 \\
\hline gc81a & 39.1 & 136 & 14.6 & 13.6 & 30.3 & 17 & 4.19 & 0.85 & 4.7 & 0.72 & 0.48 & 3.03 & 0.46 \\
\hline gc82a & 303 & 100 & 40 & 6.18 & 14.6 & 9.42 & 3.22 & 1.19 & 4.32 & 0.77 & 0.48 & 3.04 & 0.43 \\
\hline $\mathrm{gc} 82 \mathrm{c}$ & 83.6 & 82.3 & 13.9 & 9.4 & 23.3 & 11.8 & 2.98 & 0.5 & 3.18 & 0.49 & 0.32 & 2.03 & 0.31 \\
\hline gc83b & 105 & 50.4 & 8.7 & 7.78 & 16.4 & 9.56 & 2.23 & 0.37 & 2.26 & 0.34 & 0.2 & 1.3 & 0.2 \\
\hline gc83c & 180 & 121 & 33.9 & 7.11 & 17.2 & 11.7 & 3.56 & 0.69 & 4.38 & 0.73 & 0.43 & 2.74 & 0.39 \\
\hline gc84b & 107 & 77.6 & 35.3 & 7.38 & 18.2 & 12.2 & 3.38 & 1.05 & 3.72 & 0.58 & 0.3 & 1.73 & 0.26 \\
\hline gc84c & 7350 & 17 & 6.92 & 1.51 & 3.44 & 1.81 & 0.47 & 0.21 & 0.49 & 0.07 & 0.05 & 0.23 & 0.04 \\
\hline gc85b & 71.7 & 125 & 26.4 & 19.4 & 44.2 & 20.8 & 4.46 & 1.2 & 3.87 & 0.61 & 0.37 & 2.34 & 0.36 \\
\hline $\mathrm{gc} 89 \mathrm{a}$ & 253 & 132 & 21.9 & 19.5 & 39.8 & 22.1 & 4.86 & 1.23 & 4.92 & 0.8 & 0.46 & 2.93 & 0.42 \\
\hline gc90a & 51.8 & 56.3 & 7.89 & 11.1 & 19.5 & 12.3 & 2.7 & 0.55 & 2.64 & 0.36 & 0.23 & 1.5 & 0.25 \\
\hline gc93a & 112 & 64.7 & 16.2 & 15.3 & 26.8 & 17.5 & 4.36 & 1.53 & 5.12 & 0.88 & 0.61 & 3.95 & 0.59 \\
\hline gc94a & 21.2 & 5.5 & 7.96 & 0.15 & 0.48 & 0.28 & 0.09 & 0.03 & 0.12 & 0.02 & 0.02 & 0.14 & 0.02 \\
\hline gc9gc4b & 45.2 & 8.3 & 42.1 & 0.74 & 1.4 & 0.96 & 0.35 & 0.1 & 0.52 & 0.1 & 0.07 & 0.43 & 0.07 \\
\hline gc95a & 71.8 & 122 & 13.2 & 9.93 & 22.3 & 12.7 & 3 & 0.79 & 3.19 & 0.56 & 0.4 & 2.71 & 0.41 \\
\hline gc101a & 14 & 8.6 & 17.7 & 0.29 & 0.76 & 0.61 & 0.21 & 0.18 & 0.29 & 0.06 & 0.03 & 0.19 & 0.03 \\
\hline $\mathrm{gc102a}$ & 31.4 & 4.8 & 12.5 & 0.58 & 1.4 & 0.98 & 0.32 & 0.23 & 0.41 & 0.07 & 0.04 & 0.26 & 0.04 \\
\hline $103 \mathrm{a}$ & 87.2 & 7 & 25 & 0.5 & 1.71 & 1.33 & 0.45 & 0.28 & 0.6 & 0.1 & 0.06 & 0.43 & 0.06 \\
\hline
\end{tabular}

Table 6. $\mathrm{Pb}$ isotopic data for galenas from Triassic and Cretaceous VMS prospects of southeastern Alaska.

[Lat, latitude; Long, longitude]

\begin{tabular}{lllllll}
\hline \multirow{2}{*}{ Name } & Host age & \multicolumn{2}{c}{ Location } & ${ }^{206} \mathrm{~Pb} /{ }^{204} \mathrm{~Pb}$ & ${ }^{207} \mathrm{~Pb} /{ }^{204} \mathrm{~Pb}$ & ${ }^{208} \mathrm{~Pb} /{ }^{204} \mathrm{~Pb}$ \\
\cline { 3 - 5 } & & Lat (N.) & Long $(\mathrm{W})$. & & & \\
\hline Greens Creek & Triassic & $58^{\circ} 4^{\prime}$ & $134^{\circ} 37^{\prime}$ & 18.635 & 15.5828 & 38.239 \\
Woewodski & Triassic & $56^{\circ} 34^{\prime}$ & $133^{\circ} 3^{\prime}$ & 18.672 & 15.559 & 37.954 \\
Fremming & Cretaceous & $58^{\circ} 52^{\prime}$ & $135^{\circ} 5^{\prime}$ & 18.404 & 15.466 & 37.708 \\
Alaska Treasure & Cretaceous & $58^{\circ} 15^{\prime}$ & $134^{\circ} 43^{\prime}$ & 18.425 & 15.497 & 37.924 \\
Alaska Treasure & Cretaceous & $58^{\circ} 15^{\prime}$ & $134^{\circ} 43^{\prime}$ & 18.412 & 15.475 & 37.692 \\
\hline
\end{tabular}

Classification based entirely on immobile element ratios (fig. $7 B$ ) indicates that, by such a scheme, none of the stratigraphic footwall rocks have protoliths more felsic than andesite or trachyandesite. Unfortunately, several of the samples of footwall phyllite ("r," "q," "cc," "s") contained Y or Nb below lower detection limits (table 4), so they could not be plotted. However, all these same samples contained $\mathrm{Zr} / \mathrm{TiO}_{2}$ ratios below 0.03 (fig. $7 A$ ), indicating a possible dacite or rhyodacite, but not rhyolite, parentage. Because most of the samples have immobile element ratios compatible with basalt, it suggests that their protoliths were basaltic (e.g., the rocks we have continued to call by their field names "graphitic phyllite" (" $r$ ") and even stratigraphic hanging-wall "black argillite" (h)). This is clearly a difficult interpretation and one that has bothered us throughout this study. Either these rocks had unusual sedimentary parents or they have been chemically and mechanically modified to the point that their original protoliths are undecipherable. The quartzose phyllites ("q") are equally problematic - they have experienced such intense hydrothermal alteration that it is unclear whether they represent silicified mafic rocks or silicified sediments-but, in any case, they definitely lack the immobiletrace-element characteristics of felsic volcanic rocks (fig. 7).

Immobile trace elements are also employed in characterization of the tectonic environment of basaltic rocks. The $\mathrm{Zr}$-Ti-Y diagram (fig. 8) of Pearce and Cann (1973) yields ambiguous results with these rocks: the least altered mafic rocks (greenstone and chloritic phyllite) plot in a field ("B") compatible with calc-alkalic arc, tholeiitic arc, or mid-ocean-ridge basalt (MORB). Notably, the majority of the least altered rocks plot outside of the field of "within plate" (extensional setting) basalt. A non-extensional setting is also compatible with the absence of felsic volcanic rocks in the stratigraphic sequence. Compositionally bimodal felsic plus mafic suites are characteristic of extensional settings, as in the Ambler VMS district of the central 


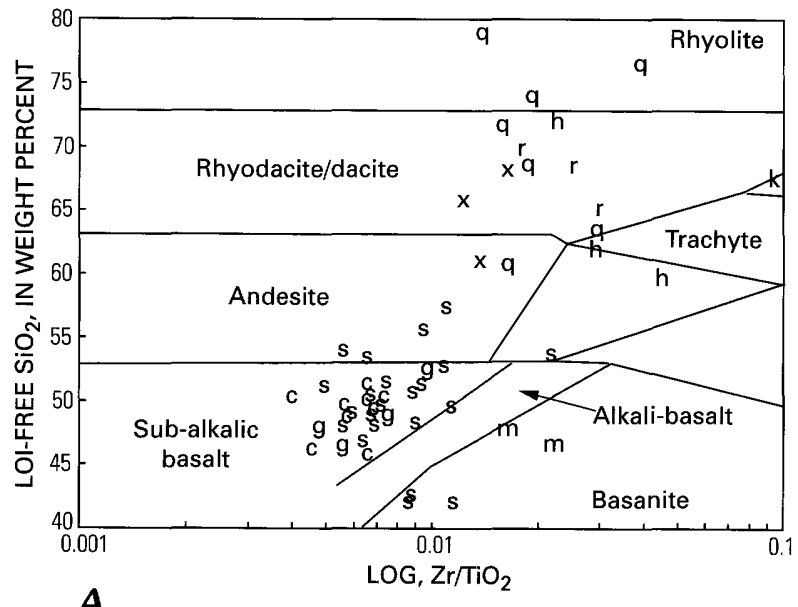

A

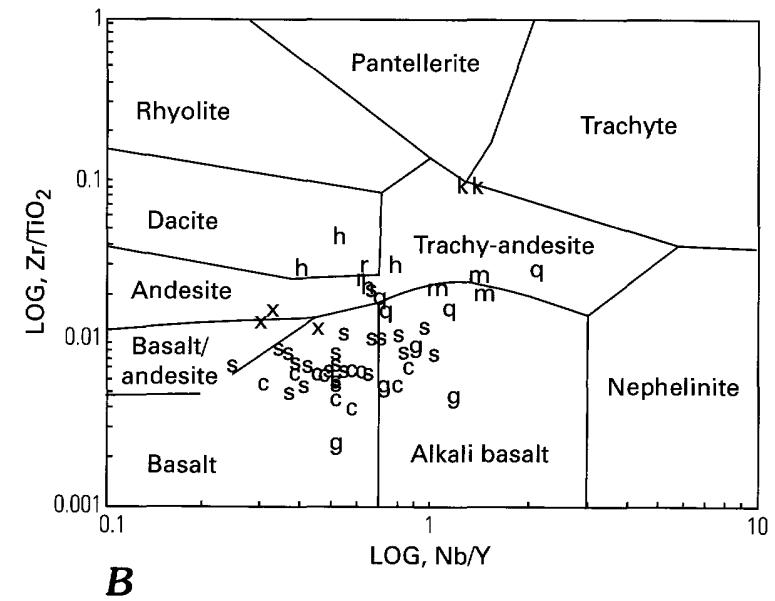

Figure 7. Trace-element characteristics of rocks from the Greens Creek deposit and vicinity. Analyses by wavelength-dispersive $X$-ray fluorescence method. Sample/rock type symbols plotted are as follows: Hanging-wall rocks: $h$, hanging-wall argillite; Footwall rocks: $r$, graphitic phyllite; q, quartzitic phyllite; c, chloritic phyllite/greenstone; s, muscovite-chlorite phyllite; m, mariposite rock; $x$, siliceous breccia; $g$, gabbro. Other: $k$, felsic sill. $A, \mathrm{SiO}_{2}$ plotted (calculated on an LOl-free basis) vs. $\mathrm{Zr} / \mathrm{TiO}_{2}$ diagram of Winchester and Floyd (1977). $\mathrm{B}, \mathrm{Zr} / \mathrm{TiO}_{2}$ plotted vs. $\mathrm{Nb} / \mathrm{Y}$ on the diagram of Winchester and Floyd (1977).

Brooks Range and the Bonnifield VMS district of the central Alaska Range (Gilbert and Bundtzen, 1979; Schmidt, 1988; Newberry and others, 1997). Sericitic phyllites ("s") have compositions that plot in and around the least altered mafic rocks ("c"), which lie in the MORB field (fig. 8). Such an association is consistent with a greater degree of hydrothermal alteration of the sericitic phyllites (e.g., fig. 4) and the fact that even the most immobile elements exhibit some mobility during extreme hydrothermal alteration (Winchester and Floyd, 1977).

Rare earth element (REE) concentrations are also employed to assess basalt tectonic environments. Existing REE data (Davis and Plafker, 1980; MacIntyre, 1986; McClelland and others, 1991; Gehrels and Barker, 1993) for well-characterized mafic metavolcanic rocks of northern southeastern Alaska show that units of different ages have contrasting patterns (fig. 9). In particular, the known Triassic basalts are different from MORB in that they lack light-REE depletion (fig. 9) but are different from

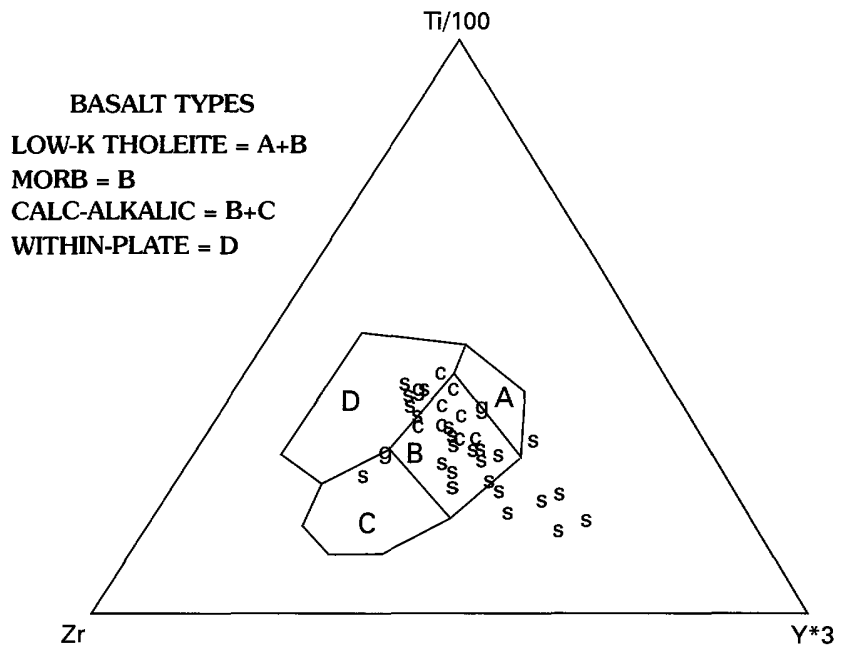

Figure 8. Basalt tectonic environment diagram of Pearce and Cann (1973) with data from those Greens Creek samples that exhibit $\mathrm{Ni}$-Cr compositional evidence (fig. 6) for a basaltic protolith. Sample/rock type symbols plotted are as follows: Footwall rocks: $c$, chloritic phyllite/greenstone; $s$, muscovite chlorite phyllite; $m$, mariposite rock; g, gabbro. MORB, mid-ocean-ridge basalt. Increased scatter of muscovite-chlorite phyllite data is most likely due to slight trace element mobility during hydrothermal alteration.

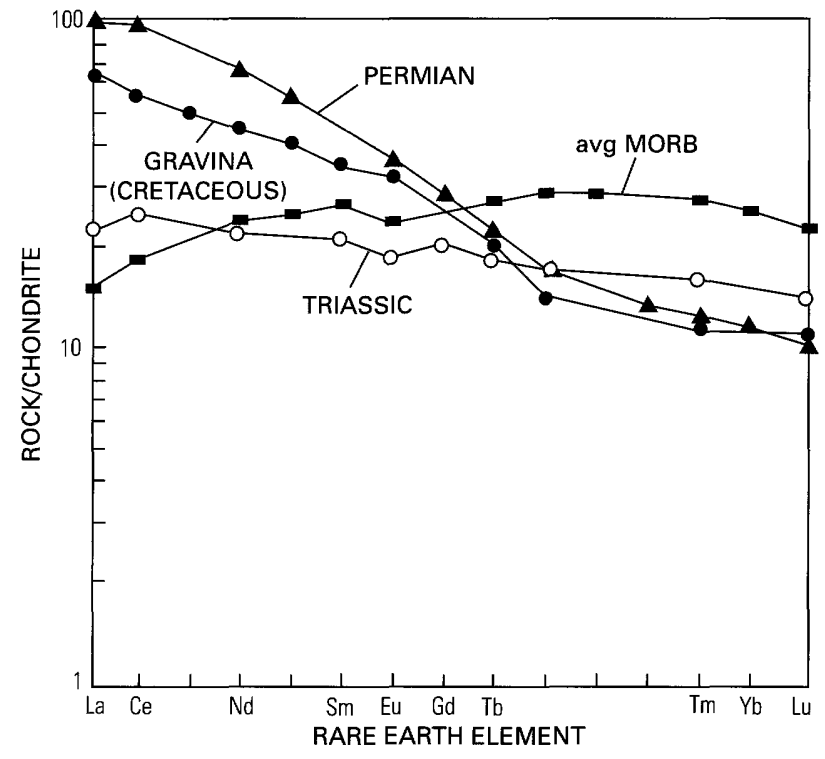

Figure 9. Chondrite-normalized average REE plots for basaltic rocks of northern southeastern Alaska compared to typical midocean-ridge basalt (MORB). Data for Alaskan rocks from Davis and Plafker (1980), Maclntyre (1986), McClelland and others (1991), and Gehrels and Barker (1993). Average MORB from BVSP (1981).

older and younger basalts in generally lacking light-REE enrichment. Within-plate (extensional) basalts invariably display light-REE enrichment, and arc-related basalts commonly exhibit relatively flat patterns (Henderson, 1984), like those exhibited by the Triassic basalts of southeastern Alaska. A.B. Ford and D.A. Brew (written commun., 1998) suggest that most of the compositional features of the Triassic basalts, including REE concentrations, are like those of flood basalts. 


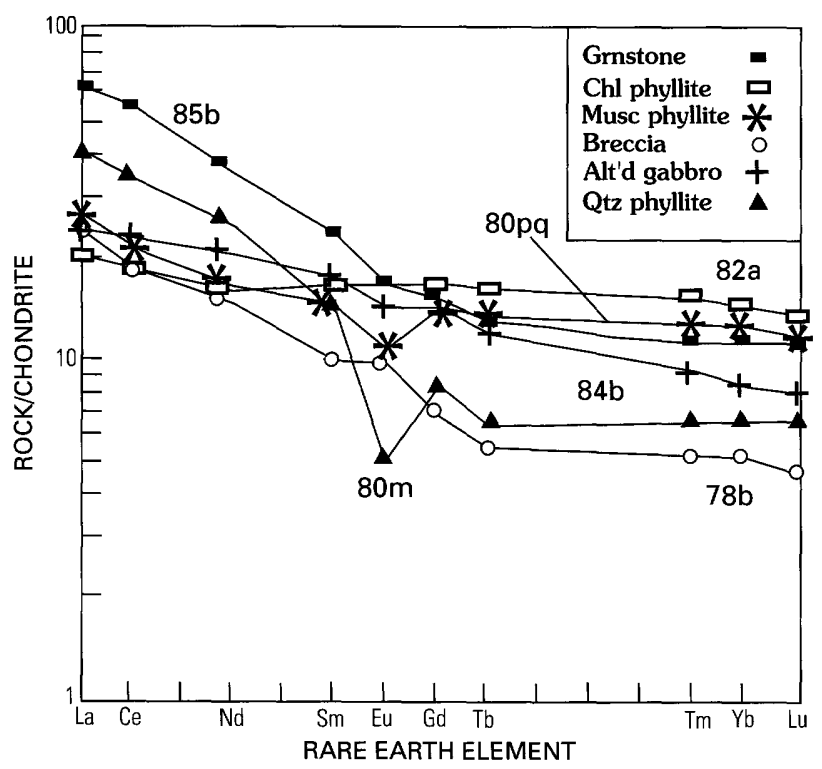

A

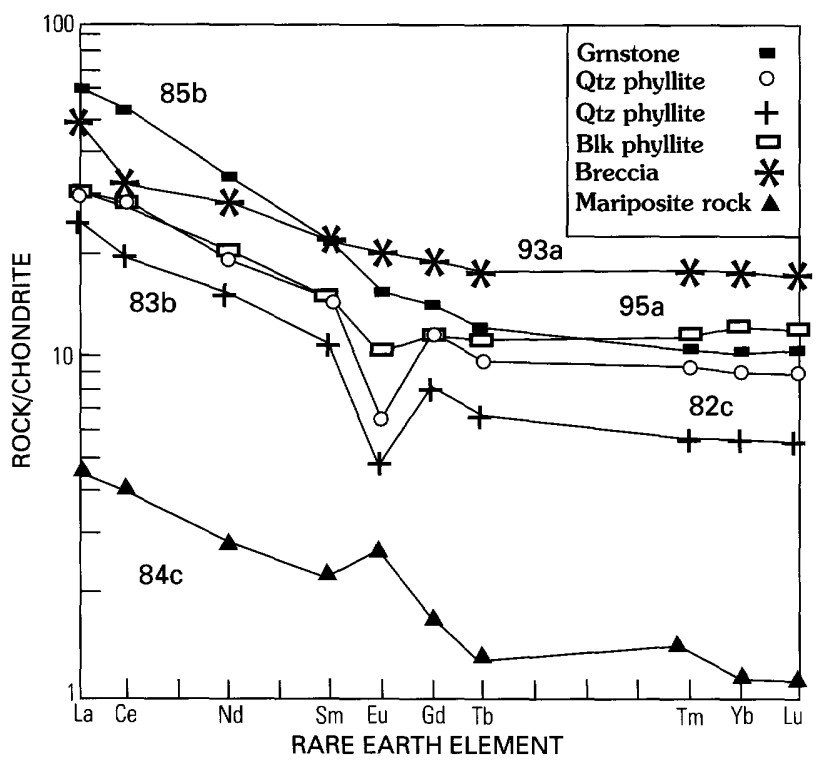

B

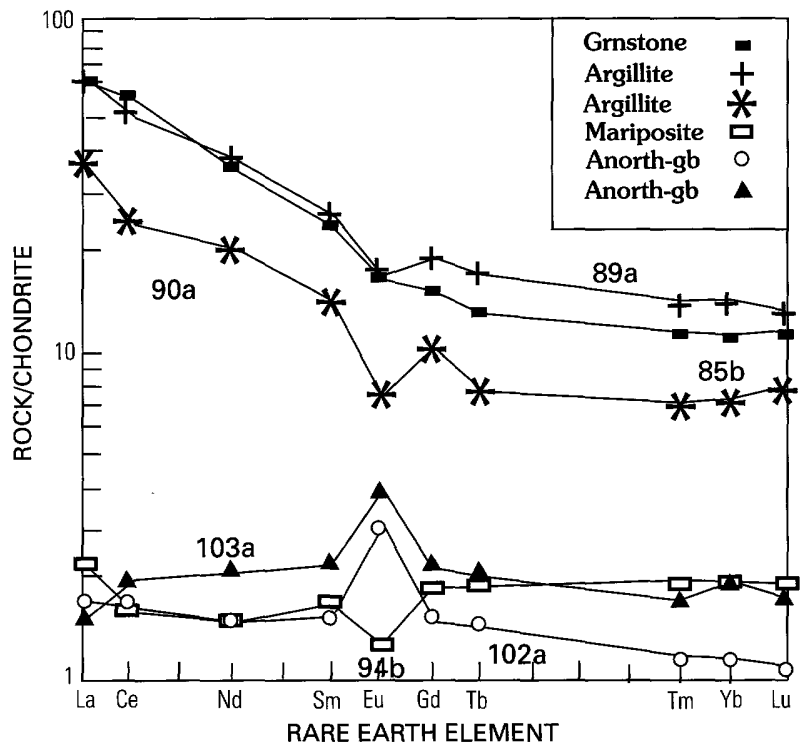

C

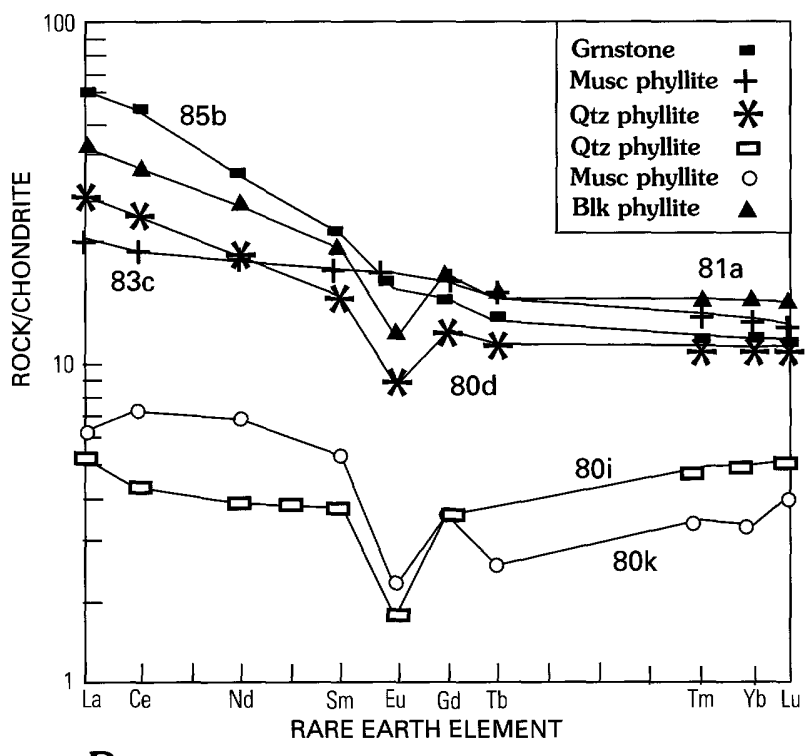

D

Figure 10. $A, B, C$, and $D$. Chondrite-normalized REE plots for rocks from the Greens Creek deposit and vicinity. Numbers (93a, for example) are abbreviated from the complete sample numbers shown in table 5. Line abbreviations: grnstone, greenstone; chl phyllite, chlorite phyllite; musc phyllite. muscovite phyllite; alt'd gabbro, altered gabbro; qtz phyllite, quartz phyllite; blk phyllite, black phyllite; anorth-gb, anorthitic gabbro. Sample $85 \mathrm{~b}$ is plotted on all four diagrams as a reference line.

The pattern of REE concentration data in rocks from the Greens-Creek-area rocks most closely resembles that of typical southeastern Alaska Triassic basalts (fig. 10), even though the range of concentrations differs. Most of the rocks identified by other trace and major elements as having mafic protoliths ("c"), such as sample 85b, exhibit relatively flat to slightly light REE-enriched patterns (fig. 10A). Rocks identified as having gabbroic parentage ("g") and mariposite-rich phyllites (" $m$ ", altered ultramafic rocks?) also exhibit flat patterns but with low REE abundances or positive Eu anomalies (figs. $10 \mathrm{~B}, 10 \mathrm{C}$ ) that are characteristic of mafic-ultramafic cumulate rocks (Henderson, 1984). The two breccia samples analyzed for REE's exhibit flat patterns similar to Triassic basalt (figs. 10A, 10B), suggesting that these two samples are also of basaltic parentage. Finally, all the other rocks analyzed-including graphitic (sedimentary protolith) rocks from both hanging wall and footwall-have patterns almost identical to the mafic or ultramafic protolith rocks, except that they display distinctly low relative Eu concentrations (fig. 10). In igneous rocks, Eu is present largely in feldspar, whereas the other (smaller) REE's are present largely in minor minerals (e.g., apatite). Feldspars are destroyed-and their Eu released-during both extreme hydrothermal alteration and normal weathering. Consequently, an interpretation of the REE patterns compatible with other trace- and major-element data is that the rocks surrounding the Greens Creek deposit have mafic/ultramafic protoliths. Further, the graphitic rocks are metasedimentary rocks derived from weathering of mafic 


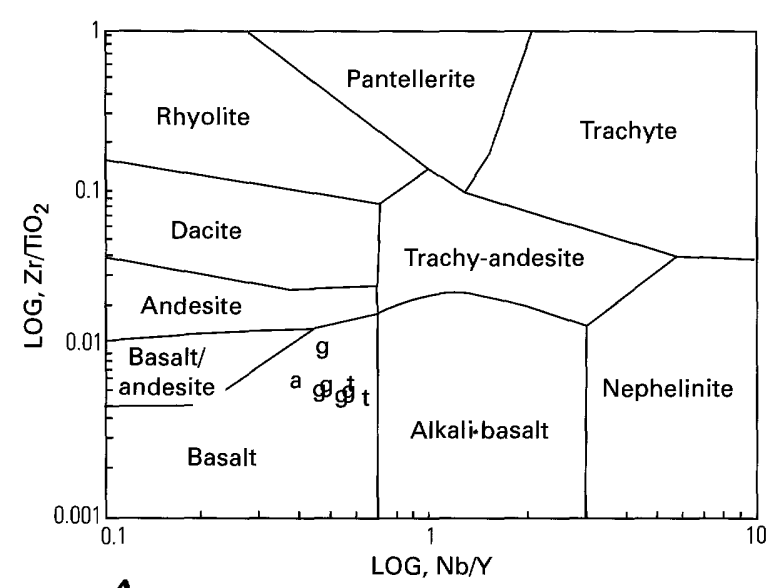

A

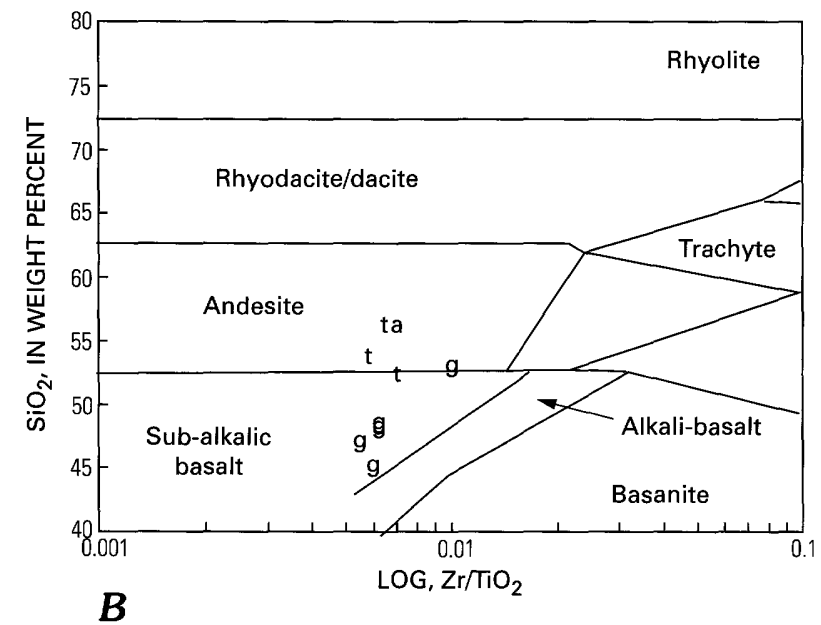

Figure 11. Classification of metavolcanic rocks on Woewodski Island using discrimination diagrams of Winchester and Floyd (1977). Symbols are as follows: $g$, greenstone; $a$, argillite; $t$, tuffaceous greenschist. $\mathrm{SiO}_{2}$ is plotted on a LOI-free basis. $A$, Classification based on immobile-element ratios; $B$, Classification based on silica and $\mathrm{Zr} / \mathrm{TiO}_{2}$. rocks, and silica-pyrite-rich rocks are extremely altered mafic or sedimentary rocks. Most notably, all the rocks sampled lack the heavy REE enrichment characteristic of felsic igneous rocks.

\section{Woewodski Island Metavolcanic Rocks- Character and Contrast with other Triassic Rocks, Including Those of the Greens Creek Deposit}

Metavolcanic rocks sampled from the vicinity of VMS prospects on Woewodski island were identified in the field as massive greenstone/diabase and as andesitic(?) tuff (table 2). Major-element compositions (Newberry and Brew, 1997, their fig. 15) are compatible with basaltic protoliths for the greenstones and indicate that the tuffaceous rocks are either not andesitic or are hydrothermally altered (table 3 ). In particular, the tuffaceous rocks exhibit very high LOI's (Newberry and Brew, 1997, their fig. 15a) and very low $\mathrm{Na}_{2} \mathrm{O}$ (Newberry and Brew, 1997, their fig. 15c). These rocks therefore may be very altered; nevertheless, the $\mathrm{SiO}_{2}$ and $\mathrm{MgO}$ contents are compatible with andesitic protoliths, but the $\mathrm{TiO}_{2}$ contents are more typical of basaltic rocks (Newberry and Brew, 1997; their fig. 15).

Despite significant major-element compositional differences, immobile element ratios (fig. 11A) for the massive and tuffaceous rocks are essentially identical and indicate basaltic protoliths. Classification based on silica and $\mathrm{Zr} / \mathrm{TiO}_{2}$ suggests that the tuffaceous rocks are andesitic (fig. 11B). These apparently contradictory classifications can be reconciled if the tuffaceous rocks are recognized as strongly altered basaltic tuffs, with addition of silica, losses in $\mathrm{MgO}$ and $\mathrm{Na}_{2} \mathrm{O}$, and large LOI.

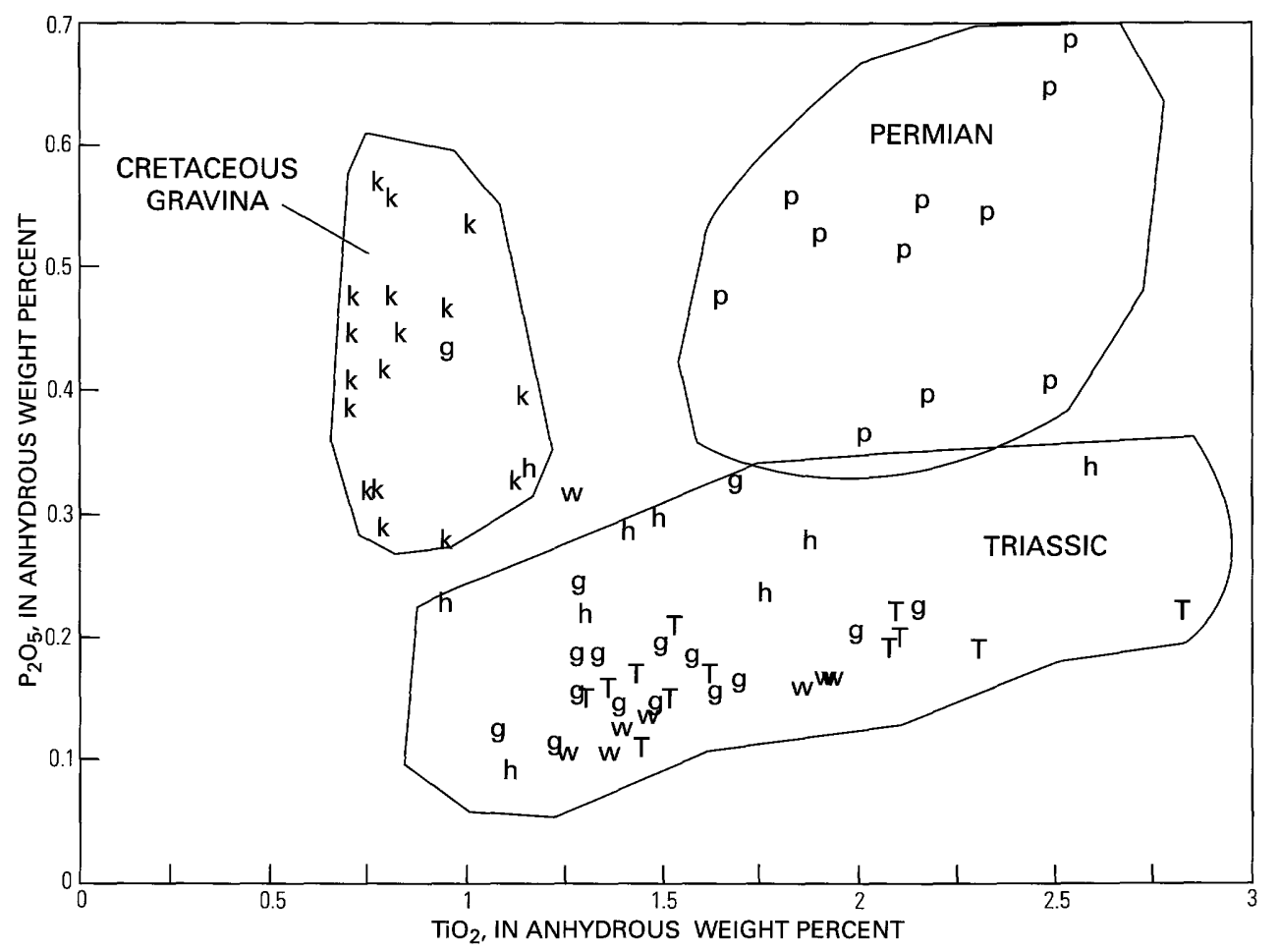

Figure 12. Anhydrous weight percent $\mathrm{P}_{2} \mathrm{O}_{5}$ plotted vs. $\mathrm{TiO}_{2}$ for metavolcanic rocks of northern southeastern Alaska, showing discrete compositional ranges for rocks of different ages. Symbols are as follows: T, Wrangellia Triassic; W, Woewodski Island; G, Greens Creek; H, Mt. Henry Clay; P, Permian; K, Gravina belt. Metavolcanic rocks from Woewodski Island (W) clearly fall in the range defined by Triassic basalts. Modified from Newberry and others (1995) with data from Ford and Brew (1993) and from this study. 


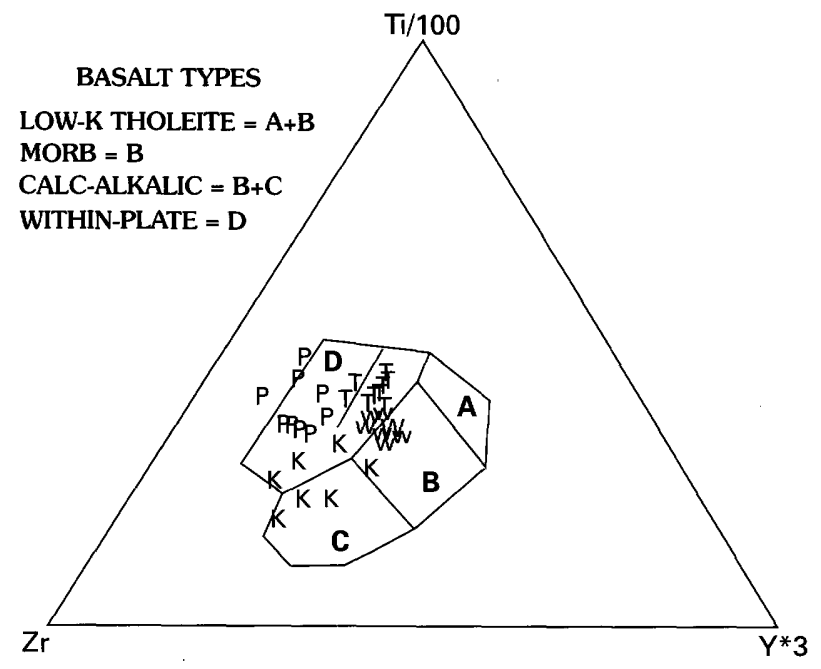

Figure 13. Basalt tectonic environment diagram of Pearce and Cann (1973) compared to data from Woewodski Island metavolcanic rocks and other metavolcanic rocks of northern southeastern Alaska. Symbols are as follows: T, Wrangeilia Triassic; W, Woewodski Island; P, Permian; K, Gravina belt. Data from Davis and Plafker (1980), McClelland and others (1991), Gehrels and Barker (1993), and from this study.
Concentrations of the relatively immobile oxides $\mathrm{P}_{2} \mathrm{O}_{5}$ and $\mathrm{TiO}_{2}$ suggest that the Woewodski Island metavolcanic rocks are basaltic and similar to those of known rocks of Triassic age (fig. 12). Newberry and others (1995) showed that metavolcanic rocks in northern southeastern Alaska could be distinguished based on concentrations of these elements. Although Woewodski Island lies near the contact between Alexander terrane and the Gravina belt (fig. 1), and although the Woewodski metavolcanic rocks are not as deformed or altered as those at Greens Creek, we confidently group them together on the basis of their chemistry, general lithologic similarity, and age.

Trace-element data also suggest similarities between the rocks of the Greens Creek area and those on Woewodski Island (fig. 13). Permian metabasalts and volcanic rocks of the dominantly Cretaceous Gravina belt plot as "within-plate" and calc-alkalic, respectively, whereas those of Woewodski Island plot in field "B," as do the least altered metabasaltic rocks from Greens Creek (fig. 8). However, other Triassic basalts from the Juneau-Haines area (Ford and Brew, 1993) contain relatively lower Y concentrations and do not plot with Woewodski Island data. It is unclear whether this apparent difference is statistically or geologically significant. The JuneauHaines-area basalts are from what is clearly Wrangellia terrane (Ford and Brew, 1993) that has oceanic or transitional

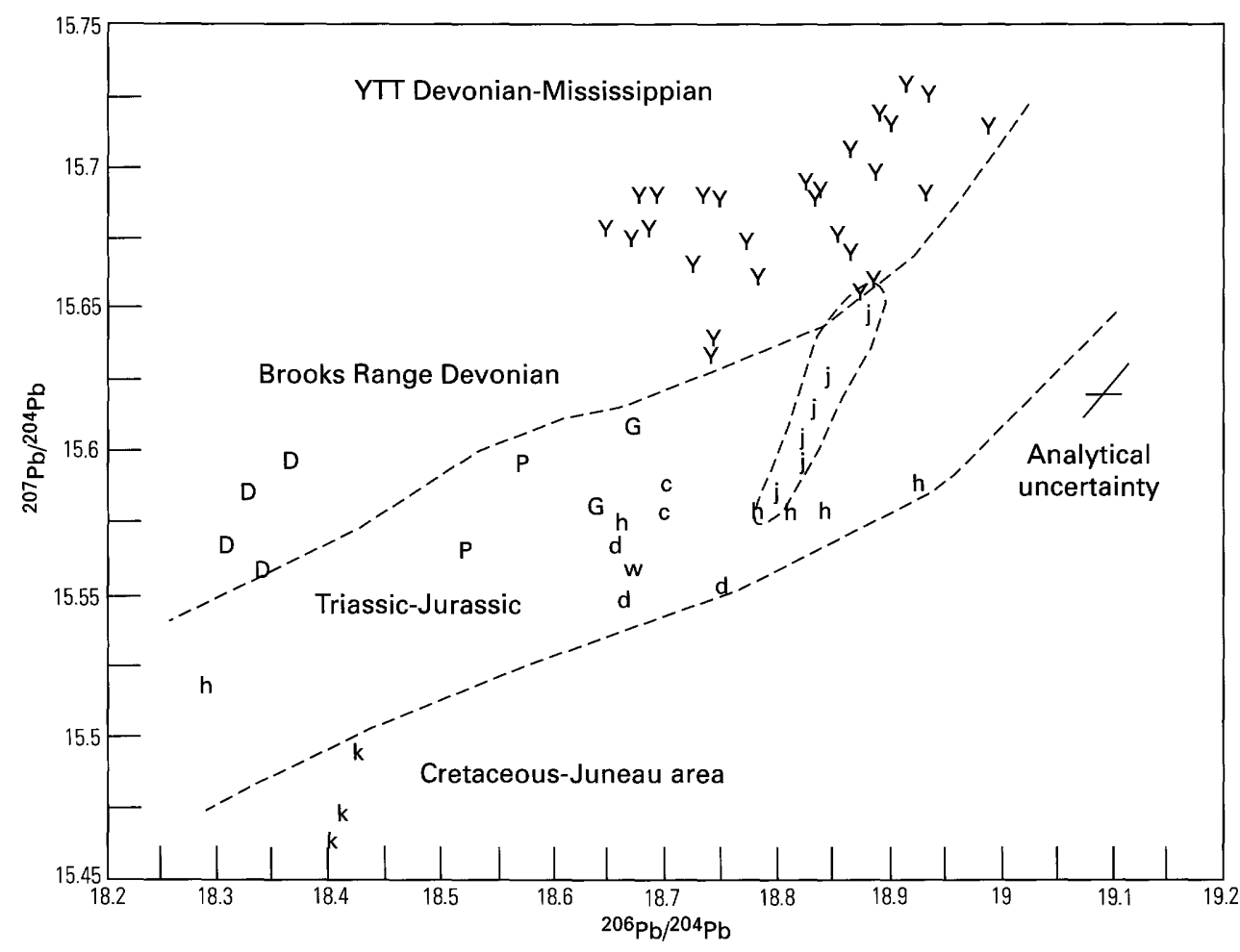

Figure 14. Common $\mathrm{Pb}$ isotopic data for galena from selected VMS deposits and prospects of Alaska and adjacent parts of Canada. Dashed-line age boundaries and dotted-line Eskay Creek sample group limit from Newberry and others (1997). Symbols are as follows: Y, Yukon-Tanana terrane (YTT); D, Brooks Range; H, Mt. Henry Clay; P, Pyrola; G, Greens Creek; C, Windy-Craggy; D, Duncan Canal; W, Woewodski Island; J, Eskay Creek; K, Juneau area. Data from Godwin and others (1988), Gaccetta and Church (1989), S.A. Church (written commun., 1996), Childe (1997), and from this study. 
crust as substrate to the Triassic basalts, whereas the volcanic rocks on Woewodski and at Greens Creek are from what Ford and Brew (1993) have tentatively interpreted to be Wrangelliaterrane-equivalent rocks that were erupted through a significant thickness of Alexander terrane crust.

\section{Age of VMS Deposits in the Greens Creek- Zarembo Island Belt}

Berg and Grybeck (1980) and Berg (1981) inferred that the VMS occurrences on Woewodski and Zarembo Islands were Late Triassic, based on Late Triassic fossils associated with VMS prospects nearby on Kupreanof Island. However, given the absence of local radiometric or fossil evidence and the presence of VMS prospects of other ages in southeastern Alaska (fig.1), this inference remains untested.

Our compositional data for metavolcanic rocks of Woewodski Island show them to be indistinguishable from Triassic basaltic rocks of the Greens Creek mine area. In addition, $\mathrm{Pb}$ isotopic data (table 6; fig. 14) for galenas from VMS ores indicates that the Woewodski Island VMS is most likely of Greens Creek age. Newberry and others (1997) have shown that Alaskan VMS deposits exhibit common $\mathrm{Pb}$ isotopic ratios that vary with age of the deposit. Our data (table 6) indicate that Woewodski Island and Greens Creek possess nearly identical common $\mathrm{Pb}$ isotopic ratios, both of which fall into the range of data for TriassicJurassic deposits of southeastern Alaska and nearby Canada. Additionally, prospects in Cretaceous Gravina group rocks of the Juneau area exhibit common $\mathrm{Pb}$ isotopic ratios that are significantly different from those of the Triassic-Jurassic deposits. Thus, it is almost certain that the VMS prospects on Woewodski Island and elsewhere in the Duncan Canal area are of the same age and origin as the Greens Creek deposit.

\section{Conclusions}

Major- and trace-element data for rocks at Greens Creek and on Woewodski Island indicate the stratigraphic footwall rocks have primarily basaltic and altered basaltic protoliths. There is no evidence for significant associated felsic volcanism at either area. Immobile-trace-element and rare-earth-element data suggest that the basaltic rocks are of immature arc affinity and do not represent an extensional environment. At Greens Creek, most of the phyllitic rocks that were stratigraphically beneath the ore are not of sedimentary parentage, and the geologic environment was not likely to have been a spreading center. Major- and minor-element and $\mathrm{Pb}$ isotopic similarities between rocks at Woewodski Island and at Greens Creek confirm previous suggestions that they are part of the same geologic/metallogenic belt.

\section{References Cited}

Arbogast, B.F., ed., 1996, Analytical methods manual for the Mineral Resources Program-U.S. Geological Survey: U.S. Geological Survey Open-File Report 96-525, 248 p.
Berg, H.C., 1981, Upper Triassic volcanogenic massive-sulfide metallogenic province identified in southeastern Alaska, in Albert, N.R.D., and Hudson, T., eds., The United States Geological Survey in Alaska: Accomplishments During 1979: U.S. Geological Survey Circular 823B, p. B104 - B108.

Berg, H.C., and Grybeck, D., 1980, Upper Triassic volcanogenic Zn-Pb$\mathrm{Ag}(-\mathrm{Cu}-\mathrm{Au})$-barite mineral deposits near Petersburg, Alaska: U.S. Geological Survey Open-File Report 80-527, 11 p.

Berg, H.C., Jones, D.L., and Coney, P.J., 1978, Pre-Cenozoic tectonostratigraphic terranes of southeastern Alaska and adjacent areas: U.S. Geological Survey Open-File Report 78-1085, scale 1:1,000,000, 2 sheets.

Brew, D.A., 1996, Geologic map of the Craig, Dixon Entrance, and parts of the Ketchikan and Prince Rupert quadrangles, southeastern Alaska: U.S. Geological Survey Miscellaneous Field Investigations Series Map MF-2319, 2 sheets, scale: 1:250,000, 53 p. pamphlet.

Brew, D.A., 1997a, Reconnaissance geologic map of the Petersburg C-3 quadrangle, southeastern Alaska: U.S. Geological Survey Open-File Report 97-156-I, scale 1:63,360, one sheet, 18 p. pamphlet.

Brew, D.A., 1997b, Reconnaissance geologic map of the Petersburg C-4 quadrangle, southeastern Alaska: U.S. Geological Survey Open-File Report 97-156-J, scale 1:63,360, one sheet, 21 p. pamphlet.

Brew, D.A., and Ford, A.B., 1994, The Coast Mountains plutonic-metamorphic complex between Skagway, Alaska, and Fraser, British Columbia-Geologic sketch and road log: U.S. Geological Survey Open-File Report 94-268, 25 p.

BVSP [Basaltic Volcanism Study Project], 1981, Basaltic volcanism on the terrestrial planets: New York, Pergamon Press, $1286 \mathrm{p}$.

Cameron, A.E., Smith, D.H., and Walker, R.L., 1969, Mass spectrometric analysis of nanogram quantities of lead: Analytical Chemistry, v. 41, p. 525-526.

Childe, F.C., 1997, Timing and tectonic setting of volcanogenic massive sulfide deposits in British Columbia: Constraints from U-Pb geochronology, radiogenic isotopes, and geochemistry: Vancouver, University of British Columbia, unpub. Ph.D. thesis, $270 \mathrm{p}$.

Crafford, T.C., 1989, The Greens Creek Ag-Au-Pb-Zn massive sulfide deposit, Admiralty Island, southeast Alaska [abs.]: Juneau, Alaska Miners Association Conference, April, 1989, p. 27-29.

Date, J., and Watanabe, Y., 1979, Alteration around Fukazawa Kuroko deposits, Akita Prefecture, with special reference to zonal distribution of alteration in hanging-wall rocks: Mining Geology, v. 29, p. 351-361. [In Japanese with abstract in English.]

Date, J., Watanabe, Y., and Saeki, Y., 1983, Zonal alteration around the Fukazawa Kuroko deposits, Akita Prefecture, northern Japan: Economic Geology Monograph 5, p. 365-386.

Davis, A., and Plafker, G., 1980, Comparative geochemistry and petrology of Triassic basaltic rocks from the Taku terrane on the Chilkat Peninsula and Wrangellia: Canadian Journal of Earth Sciences, v. 22, p. 183-194.

Dreschsler, J.S., and Dunbier, J., 1981, The Greens Creek ore deposit, Admiralty Island, Alaska [abs.]: Canadian Mining and Metallurgical Bulletin, v. 74, no. 833, p. 57.

Dunbier, J., Snow, G.G., and Butler, T.A., 1979, The Greens Creek project, Admiralty Island, Alaska [abs.]: Alaska Geological Society Symposium, Program and Abstracts, p. 40.

Ford, A.B., and Brew, D.A., 1993, Geochemical character of upper Paleozoic and Triassic greenstone and related metavolcanic rocks of the Wrangellia terrane in northern southeastern Alaska, inDusel-Bacon, C., and Till, A.B., eds., Geologic Studies in Alaska by the U.S. Geological Survey, 1992: U.S. Geological Survey Bulletin 2068, p. 197-217. 
Fyfe, W.S., Price, N.J., and Thompson, A.B., 1978, Fluids in the Earth's crust: Amsterdam, Elsevier, $247 \mathrm{p}$.

Gaccetta, J.D., and Church, S.E, 1989, Lead isotope data base for sulfide occurrences from Alaska: U.S. Geological Survey Open-File Report $89-688,60 \mathrm{p}$.

Gehrels, G.E., and Barker, F., 1993, Reconnaissance geochemistry of Permian and Triassic basalts of Taku and Wrangellia terranes, southeastern Alaska, in Dusel-Bacon, C., and Till, A.B., eds., Geologic Studies in Alaska by the U.S. Geological Survey, 1992: U.S. Geological Survey Bulletin 2068, p. 218-227.

Gilbert, W.G., and Bundtzen, T.K., 1979, Mid-Paleozoic tectonics, volcanism, and mineralization in the north-central Alaska Range: Journal of the Alaska Geological Society, v.1, p. F1-F22.

Godwin, C.I., Gabites, J.E., and Andrew, A., 1988, Leadtable: A galena lead isotope database for the Canadian Cordillera, with a guide to its use by explorationists: British Colimbia Ministry of Energy, Mines, and Petroleum Resources, Mineral Resources Division, Geological Survey Branch, Paper 1988-4, 188 p.

Hashiguchi, H., Yamada, R., and Inoue, T., 1983, Practical application of low $\mathrm{Na}_{2} \mathrm{O}$ anomalies in footwall acid lava for delimiting promising areas around the Kosaka and Fukazawa Kuroko deposits, Akita Prefecture, Japan: Economic Geology Monograph 5, p. 387-394.

Henderson, P., ed., 1984, Rare earth element geochemistry: Amsterdam, Elsevier, $510 \mathrm{p}$.

Macintyre, D.G., 1986, The geochemistry of basalts hosting massive sulphide deposits, Alexander terrane northwest British Columbia: British Columbia Ministry of Energy, Mines, and Petroleum Resources, Geological Fieldwork 1985, Paper 1986-1, p. 197-210.

McClelland, W.C., Gehrels, G.E., Samson, S.D., and Patchett, P.J., 1991, Protolith relations of the Gravina belt and Yukon-Tanana terrane in central southeastern Alaska: Journal of Geology, v. 100, p. 107-123.

Newberry, R.J., and Brew, D.A., 1997, The Upper Triassic Greens Creek VMS (volcanogenic massive sulfide) deposit and Woewodski Island VMS prospects, southeastern Alaska: Chemical and isotopic data for rocks and ores demonstrate similarity of these deposits and their host rocks: U.S. Geological Survey Open-File Report 97 $539,49 \mathrm{p}$.

Newberry, R.J., Brew, D.A., and Crafford, T.C., 1990, Genesis of the Greens Creek volcanogenic massive sulfide deposit, S.E. Alaska: A geochemical study: Geological Association of Canada, Program with Abstracts, v. 15, p. A96.

Newberry, R.J., Crafford, T.C., Newkirk, S.R., Young, L.E., Nelson, S.W., and Duke, N.A., 1997, Volcanogenic massive sulfide deposits of Alaska, in Goldfarb, R.J., and Miller, L.D., eds., Mineral Deposits of Alaska: Economic Geology Monograph 9, p. 120-150.

Newberry, R.J., McCoy, D.T., and Brew, D.A., 1995, Pluton-hosted gold ores in Alaska: Igneous vs. metamorphic origins: Resource Geology Special Issue 18, p. 57-100.

Nokleberg, W.J., Bundtzen, T.K., Berg, H.C., Brew, D.A., Grybeck, D., Robinson, M.S., Smith, T.E., and Yeend, W., 1987, Significant metalliferous lode deposits and placer districts of Alaska: U.S. Geological Survey Bulletin 1786, $104 \mathrm{p}$.

Pearce, J.A, and Cann, J.R., 1973, Tectonic setting of volcanic rocks determined using trace element analyses: Earth and Planetary Science Letters, v. 19, p. 290-300.

Schmidt, J.M., 1988, Mineral and whole rock compositions of seawaterdominated hydrothermal alteration at the Arctic VMS prospect, Alaska: Economic Geology, v. 83, p. 822-842.

Streckeisen, A.L., and LeMaitre, R.W., 1979, A chemical approximation to the modal QAPF classification of the igneous rocks: Neues Jahrbuch für Mineralogie Abhandlung, v. 136, p. 169-206.

Taylor, R.S., and McLennan, S.M., 1985, The continental crust: Its composition and evolution: Oxford, Blackwell, $321 \mathrm{p}$.

Winchester, J.A., and Floyd, P.A., 1977, Geochemical discrimination of different magma series and their differentiation products using immobile elements: Chemical Geology, v. 20, p. 325-343.

Winkler, H.G.F., 1979, Petrogenesis of metamorphic rocks (5th ed.): New York, Springer-Verlag, $348 \mathrm{p}$.

Reviewers: D.A. Singer, J.M. Schmidt. 


\title{
Core Lithofacies Analysis and Fluvio-Tidal Environments in the AK 94 CBM-1 Well, Near Wasilla, Alaska
}

\author{
By Romeo M. Flores, Mark D. Myers, Gary D. Stricker, and Julie A. Houle
}

\section{Abstract}

The AK 94 CBM-1 well penetrated and cored, from 108 to $378 \mathrm{~m}$, the Tyonek Formation (Miocene-Pliocene) near Wasilla, Alaska. Based on core and well-log descriptions, the Tyonek Formation consists of two major lithofacies types: fluvial dominated and tidallike. The upper (97 m thick) and lower (107 m thick) parts of the core consist of fluvial-dominated lithofacies occasionally interbedded with thin intervals of tidallike lithofacies, whereas the middle part (67 $\mathrm{m}$ thick) of the core predominantly contains tidallike lithofacies. Coal beds are mainly associated with the fluvial-dominated lithofacies where they were deposited in freshwater mires developed mainly on abandoned fluvial channel belts and subordinately on distal flood plains. In contrast, only thin coal lenses are present within the tidallike lithofacies, where they are associated with paleosols, well-drained mires, and freshwater lakes.

Sedimentary and biogenic structures suggest that the tidallike lithofacies formed in intertidal to subtidal environments. The most compelling evidence for this interpretation is the common occurrence of lenticular and flaserlike beds; foresets with reactivation surfaces; and rhythmic, mud-draped, bipolar ripple laminae. In addition, associated trace fossils of possibly Thalassinoides, Chondrites, Teichicnus, Gyrolithes, Planolites, and Paleophycus, as well as synaeresis cracks, support a tidal influence. These sedimentary structures are interbedded with heavily bioturbated units, suggesting subaqueous deposition. Alternatively, the only subaqueous fluvial setting that could produce most of these sedimentary structures is in a freshwater lacustrine setting with ebb and flow processes. Some of these tidallike deposits contain the freshwater lacustrine alga Pediastrum reported by Nichols (1998).

The alternating fluvial-dominated and tidallike lithofacies are similar to lithofacies found in the Tyonek Formation in the Chuitna drainage basin by Flores and others $(1994,1997)$. The discovery of these Tyonek tidallike lithofacies in the Chuitna River drainage basin and near Wasilla in the Upper Cook Inlet suggests that brackish water processes may have deposited part of the Tertiary coal-bearing deposits. Previous interpretations have limited these rocks to a nonmarine origin. If our interpretation of these rocks is correct, it requires their reevaluation and suggests that the generally accepted paleogeographic reconstructions of the Tertiary Cook Inlet Basin may need revision with respect to the location and distribution of marine-influenced sedimentation. However, if these rocks were deposited in freshwater lakes, the depositional processes that created these tidallike sedimentary structures need to be better documented.

\section{Introduction}

The Cook Inlet Basin (fig. 1) contains the second largest (the largest is the North Slope) amount of hypothetical coal resources in Alaska (Stricker, 1991). These coal resources are contained in the Oligocene to Pliocene Kenai Group (fig. 2) (Calderwood and Fackler, 1972), which is as much as 7,617 m thick in the offshore Cook Inlet Basin. The hypothetical coal resources of the basin exceed 1.5 trillion short tons of which 500 billion short tons are found onshore (Stricker, 1991; Smith, 1995).

The coal-bearing Kenai Group includes, from bottom to top, the Hemlock Conglomerate, and the Tyonek, Beluga, and Sterling Formations (fig. 2). These formations consist of interbedded conglomerate, sandstone, siltstone, mudstone, coal, and carbonaceous shale. Coals are mainly found in the Tyonek, Beluga, and Sterling Formations; however, the thickest coals are found in the Tyonek and Beluga.

The depositional environment of the Kenai Group has generally been interpreted as a nonmarine continental setting (Hite, 1976; Hayes and others, 1976). More specifically, the Beluga and Sterling Formations were interpreted as deposits of braided and meandering streams. Flores and Stricker (1993), and Flores and others $(1994,1997)$ reinterpreted the Beluga and Sterling Formations to be mixed anastomosed, meandering, and braided streams. In this setting, the thick and thin coal beds accumulated in raised mires and low-lying swamps, respectively. The Tyonek Formation has generally been interpreted as braided-stream deposits (McGee, 1972; Adkison and others, 1975; Dickinson and Campbell, 1978; Dickinson and others, 1995). However, tidallike deposits of the Tyonek in the Chuitna River drainage basin (fig. 1) were reported by Flores and others $(1994,1997)$.

The purpose of this paper is to report additional evidence for tidallike deposits in the Tyonek Formation near Wasilla (fig. 


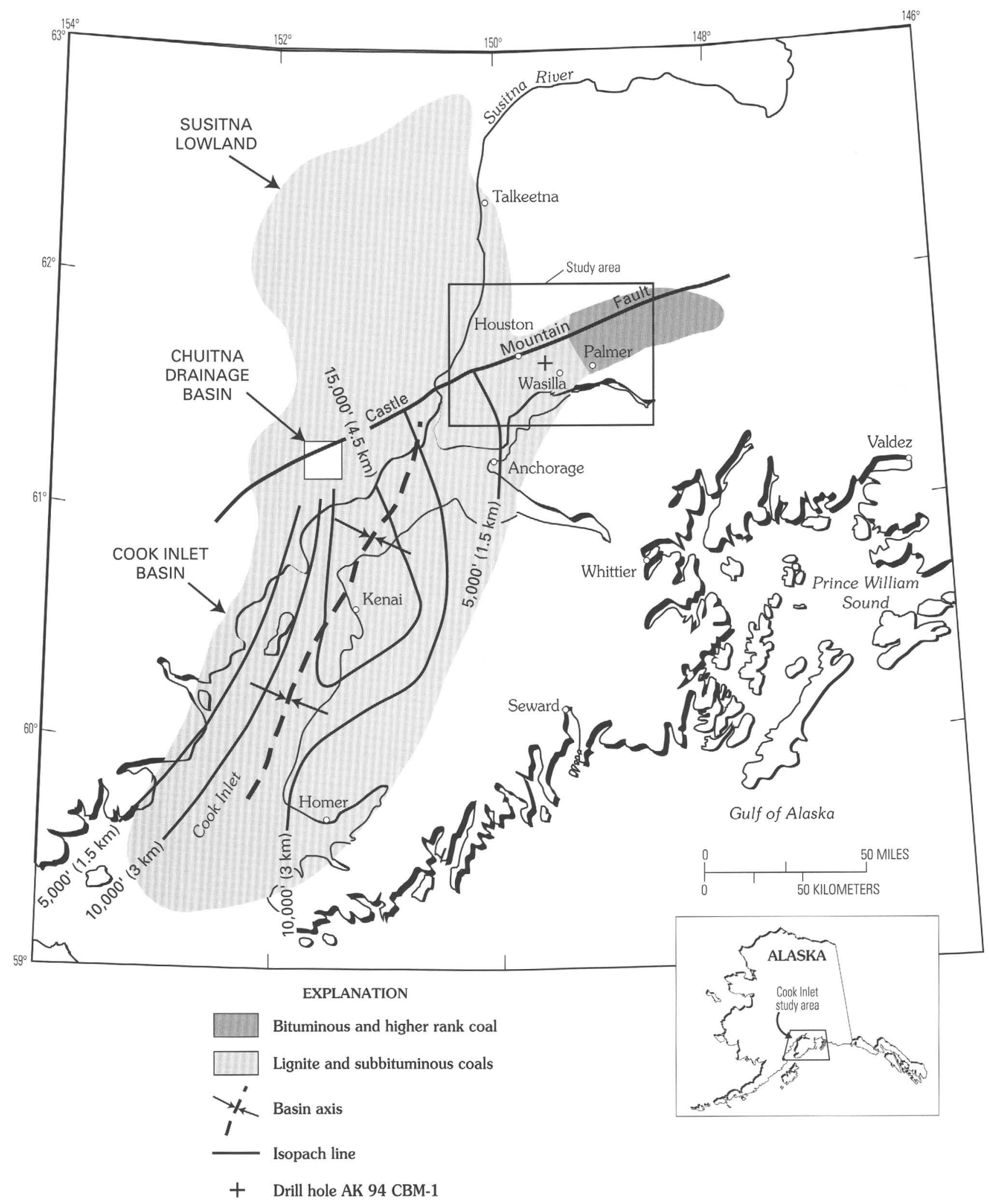

Figure 1. Offshore and onshore Cook Inlet Basin in south-central Alaska. Modified from Smith (1995).

1), which is about $161 \mathrm{~km}$ northeast of the Chuitna River drainage basin. These deposits were described from cores of the AK 94 CBM-1 well that was drilled for a coal-bed methane prospect by the Alaska Department of Natural Resources (Smith, 1995). This paper includes detailed lithofacies analysis, sedimentology, and interpretation of the depositional environments, which were not previously performed by Smith (1995).

\section{Geologic Setting}

The AK 94 CBM-1 well was drilled in 1994 at a location about $4.2 \mathrm{~km}$ northwest of Wasilla on the northeast margin of the Cook Inlet Basin (fig. 3). The northeast margin of the basin forms a northeast-trending trough bounded by the Castle 


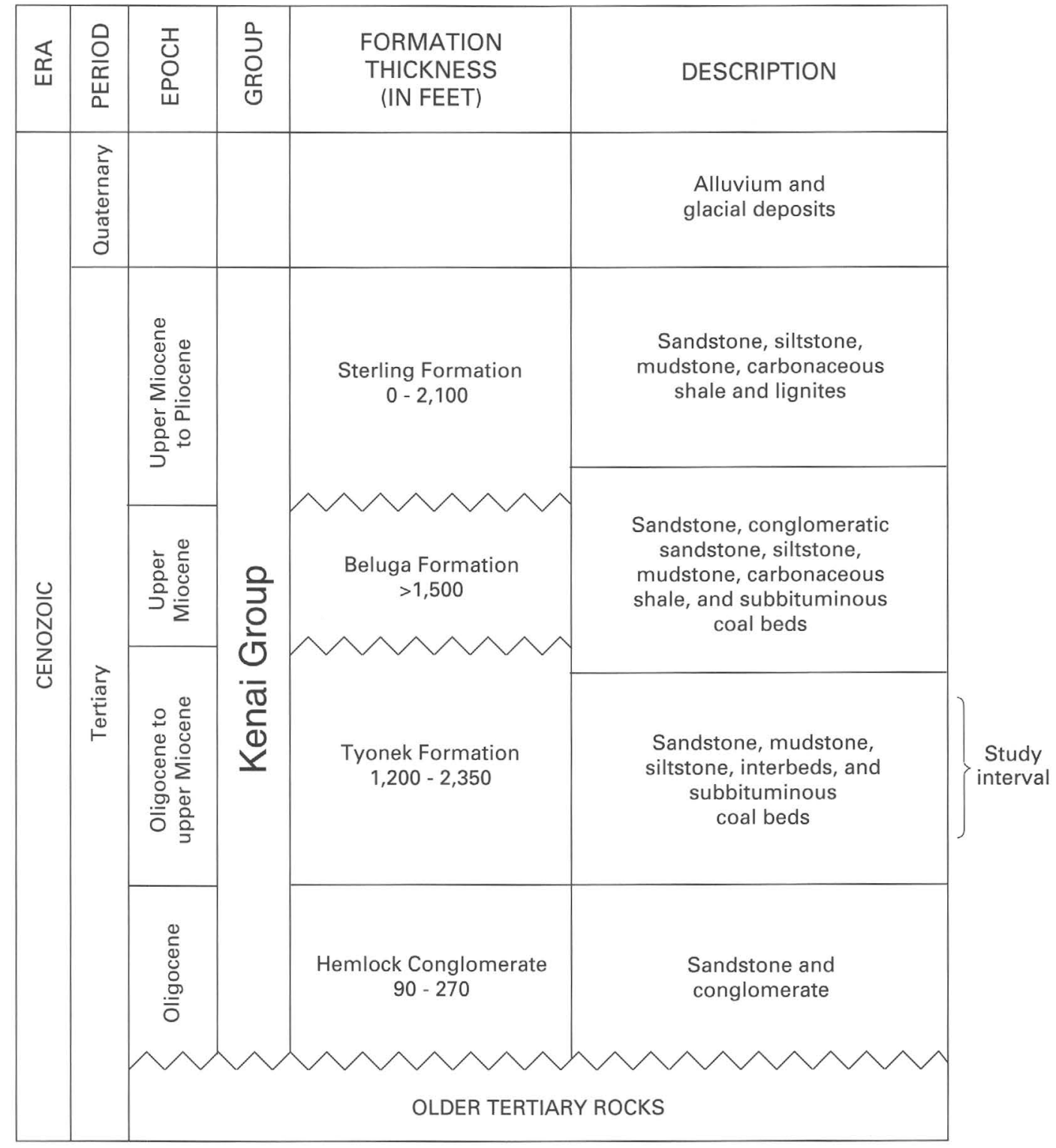

Figure 2. A general stratigraphic column showing the coal-bearing formations of the Kenai Group and the study interval in the Tyonek Formation in the upper Cook Inlet.

Mountain fault and Talkeetna Mountains on the north and the Chugach Mountains on the south (fig. 3). The western part of the trough, which is $40.2 \mathrm{~km}$ wide and $121 \mathrm{~km}$ long, contains the Tyonek Formation; in this area, it is more than $1,219 \mathrm{~m}$ thick and contains a cumulative thickness of greater than $30.5 \mathrm{~m}$ of coal beds at depths from 152 to $1,828 \mathrm{~m}$ (Smith, 1995). Smith (1995) reported that these coals are high-volatile bituminous with vitrinite reflectance $\left(R_{\mathrm{o}}\right)$ values ranging from 0.6 to 1.2 percent (fig. 3).

Johnsson and others (1993) reported that coals in the Kenai Group in the northern Cook Inlet Basin range from 0.2 to 0.9 percent $R_{0}$ in depths from 30.5 to $2,742 \mathrm{~m}$. Post-depositional uplift of the northern margin of the basin exposed older rocks (Paleocene to Oligocene) with high thermal maturity. For example, Paleocene to Eocene rocks, which contain high-volatile bituminous and anthracite coals, are exposed in the Matanuska Valley (Anonymous, 1990). Coal mines in this area have emitted methane gas, which caused outbursts and explosions in 1937 and 1957 (Barnes and Payne, 1956; Smith, 1995).
The high-thermal-maturity Tertiary coals, particularly in the northern margin of the Cook Inlet Basin, have been targeted for coal-bed methane exploration. Smith and Clough (1993) identified the Wasilla area as a prospect because of its proximity to infrastructure, including gas pipelines. This led to the drilling of the AK 94 CBM-1 well by the Department of Natural Resources, State of Alaska, to test for the presence of coal-bed methane in the Tyonek Formation (Smith, 1995). This test well will serve as an important guide to future coal-bed gas measurements and resource estimates of Tertiary coal-bearing rocks in the Cook Inlet Basin.

\section{Previous Work Performed on the AK 94 CBM-1 Well}

The AK 94 CBM-1 well was drilled to a total depth of $379 \mathrm{~m}$, and a continuous core of the Tyonek Formation was 


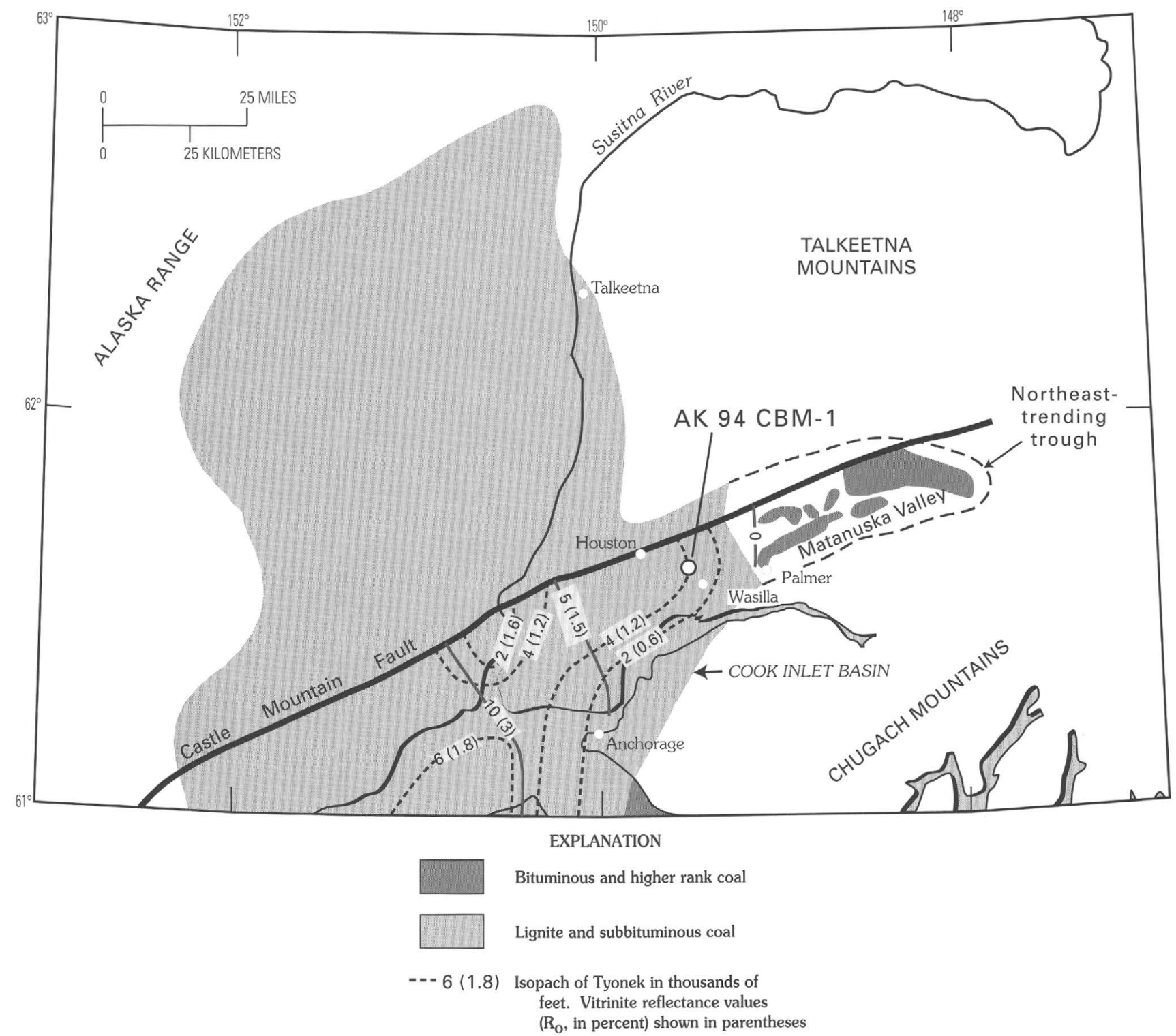

Figure 3. Upper Cook Inlet Basin showing location of the AK 94 CBM-1 well; coal rank in the Matanuska Valley; and coal rank, $\mathrm{R}_{0}$, and thickness of the Tyonek Formation. Modified from Smith (1995).

recovered from 108 to $379 \mathrm{~m}$ (Smith, 1995). The depths from 0 to $108 \mathrm{~m}$ were cased; however, rock samples indicate that the interval is composed of Quaternary glacial conglomerate. A suite of geophysical logs (e.g., gamma ray, density, spontaneous potential, and resistivity) was also run to evaluate the Tyonek rocks. The drilling project was mainly designed by Smith (1995) to collect samples from Tyonek coal beds that were greater than $0.6 \mathrm{~m}$ thick for proximate and geochemical analyses, and gas desorption. Thus, only coal beds that are less than $0.3 \mathrm{~m}$ thick were left in the core for detailed description. Smith (1995) noted that the coal beds are thick (as thick as 2.9 $\mathrm{m})$ at depth and their cumulative thickness is about $12.5 \mathrm{~m}$.

Out of the total of 18 coal beds drilled in the Tyonek Formation, 13 beds were sampled for gas content. Proximate analysis of these coals indicates an as-received moisture content of 4.8 to 9.02 percent, volatile matter of 5.57 to 22.85 percent, fixed carbon of 23.03 to 48.71 percent, and ash yield from 5.57 to 44.74 percent. In general, moisture and fixed carbon increased with depth. Gas content ranges from $63 \mathrm{ft}^{3} /$ short ton at standard temperature and pressure (STP) for coal beds at a shallow depth

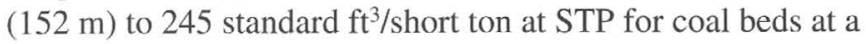
great depth $(366 \mathrm{~m})$. Carbon isotopes of the coal-bed gases range from -49.3 to $-43.3 \%$ (per mil) $\delta^{13} \mathrm{C}$, with slightly heavier isotope values at depth, indicating both a thermogenic and biogenic origin of the coal-bed gas. Composition of this gas is 90 percent methane with minor amounts of $\mathrm{CO}_{2}$ and $\mathrm{N}_{2}$. Vitrinite reflectance values range from 0.47 to 0.58 percent and generally increases with depth (Smith, 1995).

\section{Lithostratigraphy of the AK 94 CBM-1 Core}

Smith (1995) described the general lithology of the AK 94 CBM-1 core, from 379 to $108 \mathrm{~m}$ depth, as consisting of interbedded coal, mudstone, siltstone, sandstone, and conglomerate. Our detailed description of this 271-m-thick interval of 7.6-cmdiameter core included thickness, lithology, color, grain size, 
sorting, mineral composition, nature of contact, sedimentary structures, trace fossil content, and biological constituents (figs. $4 A-4 C)$.

In order to summarize the lithofacies association of the core, the Tyonek interval is divided into lower, middle, and upper parts based on the grain size, abundance, and thickness of diagnostic lithologic types. The lower part (fig. $4 A$ ), from 379 to $272 \mathrm{~m}$, is $107 \mathrm{~m}$ thick and consists of abundant conglomeratic sandstones and thick $(0.3-2.9 \mathrm{~m})$ coals and carbonaceous shales. The middle part (fig. $4 B$ ), from 272 to $205 \mathrm{~m}$, is $67 \mathrm{~m}$ thick and is composed of abundant mudstone, siltstone, fine-grained sandstone, and sparse, thin $(8.9 \mathrm{~cm}$ to $0.3 \mathrm{~m}) \mathrm{coal}$ and carbonaceous shale. The upper part (fig. 4C), from 205 to $108 \mathrm{~m}$, is $97 \mathrm{~m}$ thick and comprises abundant medium- to coarse-grained sandstones and thin to thick (1-3.5 ft or $0.3-1 \mathrm{~m})$ coal and carbonaceous shale.

The gamma-ray curve measures the natural radioactivity of rock types caused by absorption of thorium by clay minerals, potassium content of clay minerals (mainly illite), and uranium fixed by associated organic matter (Doveton, 1994). In general, low levels of radioactivity are observed in sandstone and siltstone, which is attributed to the amount of clay minerals, potassium feldspar, mica, and heavy minerals. Coal displays much lower gamma radiation compared to these rock types and is readily identifiable by the gamma-ray log curve. Density and resistivity curves were also used to identify the coal beds. Responses of both gamma-ray and spontaneous-potential logs are indirectly related to sediment grain size and may be used as a vertical grain-size profile (Galloway, 1968; Selley, 1978).

A comparison of the gamma-ray and spontaneous-potential logs of the conglomerates and sandstones have significantly different patterns (see fig. 4A). The gamma-ray log curve displays serrated cylindrical and bell shapes (rightward excursions). However, the spontaneous-potential log curve shows smooth cylindrical to combined smooth and serrated bell shapes (leftward excursions). The better definition of the spontaneous potential is probably due to changes in grain size of the sandstone. The cylindrical-shaped curve suggests uniform vertical grain size of the sandstone and the bell-shaped curve indicates fining upward.

\section{Lower Part of the Core}

The lower part (379-272 m) of the Tyonek interval (see fig. $4 A$ ) is dominated by medium- to coarse-grained sandstone and conglomerate, which make up as much as 60 percent of the total rock volume. The sandstones occur as fining-upward beds that are from 0.6 to $4.6 \mathrm{~m}$ thick and display erosional bases (fig. 5). The sandstones are light gray and show a "salt-and-pepper" texture (Krynine, 1950); light minerals consist mainly of quartz. The dark color is due to the presence of chert and other rock fragments (e.g., metamorphic and volcanic). Sedimentary structures consist of trough crossbeds that are $7.6 \mathrm{~cm}$ to $0.3 \mathrm{~m}$ in height (fig. 5). These structures are interbedded by a few planar crossbeds ( $0.3 \mathrm{~m}$ in height) with steep foresets (fig. 5). Asymmetrical and climbing ripple laminations $(7.6-\mathrm{cm}-$ to $0.6-\mathrm{m}-$ thick units) cap the trough and planar crossbedded units (fig. 6).

The conglomerates, which make up less than 5 percent of the total rock volume, have erosional bases and occur as 7.6-cm- to $0.6-\mathrm{m}$-thick beds (figs. $4 A$ and 5). They are gray and composed of rounded to subrounded pebbles and cobbles of quartz, black chert, and volcanic rock fragments. They are commonly framework supported, and a few are matrix supported. The framework-supported conglomerates exhibit clast imbrication.

Forty percent of the lower part of the Tyonek interval (fig. $4 A$ ) consists of interbedded, very fine to fine-grained sandstone, siltstone, mudstone, coal, and carbonaceous shale (fig. 7). Sandstones have sharp basal contacts, are gray in color, and vary from 0.3 to $1.5 \mathrm{~m}$ thick. They are mainly ripple laminated, lenticular and flaserlike bedded (with bipolar ripple laminae), and are vertically burrowed by nondescript trace fossils. Siltstones are gray, a few centimeters to $0.4 \mathrm{~m}$ thick, ripple laminated, burrowed, and are commonly interbedded with the sandstones. Mudstones are dark gray to black, depending on the amount of macerated plant content, and vary from 0.3 to $8.2 \mathrm{~m}$ thick. They are massive to crudely laminated, vertically and horizontally burrowed, and contain whole shells of bivalve mollusks or pelecypods. These bivalves are articulated, $3.8 \mathrm{~cm}$ wide and $6.3 \mathrm{~cm}$ long, and resemble a unionid clam in shape. Root marks, defined by vertical branchlike tubules lined by carbonaceous matter, are common in the sandstones, siltstones, and mudstones. Coal beds vary from 15.2 to $2.9 \mathrm{~m}$ in thickness (fig. $4 A$ ). They contains vitrinite bands indicating woody composition. They are commonly interbedded with carbonaceous shales, which vary from a few centimeters to $0.3 \mathrm{~m}$ thick. Carbonaceous shales are fissile and contain a mixture of mud and macerated plant fragments. Root marks are a common bioturbation feature in the carbonaceous shale.

\section{Middle Part of the Core}

The middle part (272-205 m) of the Tyonek interval (fig. $4 B$ ) is dominated by abundant fine-grained sandstones (fig. 8), which make up as much as 55 percent of the total rock volume. Sandstones display sharp to erosional bases and uniform grain size from bottom to top; they are from 0.3 to $7.9 \mathrm{~m}$ thick and are light gray and show a "salt-and-pepper" texture. Sedimentary structures consist mainly of foresets that range from 23 to $25 \mathrm{~cm}$ in height and are separated by clay-draped reactivation surfaces. Trough crossbeds, $8-15 \mathrm{~cm}$ in height, are common, but planar crossbeds, as much as $15 \mathrm{~cm}$ in height, and convolutions are rare. Lenticular bedding $(0.25-0.64 \mathrm{~cm}$ thick) and flaserlike ripple laminations are very common (fig. $8 A$ ). Ripple sets in the flaserlike laminations are bipolar and draped by burrowed mudstones (fig. $8 B$ ). The sandstones are heavily bioturbated, and the accompanying sedimentary structures have been partly to completely destroyed. Vertical burrows in the form of escape and branching structures are very common. Escape structures are $1.3 \mathrm{~cm}$ high and $2.5 \mathrm{~cm}$ wide, and the branching vertical burrows (e.g., Thalassinoides-like) are 5-8 cm wide and $1.5 \mathrm{~m}$ long (fig. 9). The branching burrows were open and passively infilled by overlying sand (fig. 9). Plant bioturbation in the form of root marks is rare.

The gamma-ray and spontaneous-potential logs of the sandstones have significantly different patterns (fig. 4B). The gammaray $\log$ curve displays mainly a serrated bell shape (rightward excursions). However, the spontaneous-potential log curve shows combined smooth and serrated bell shapes and funnel 


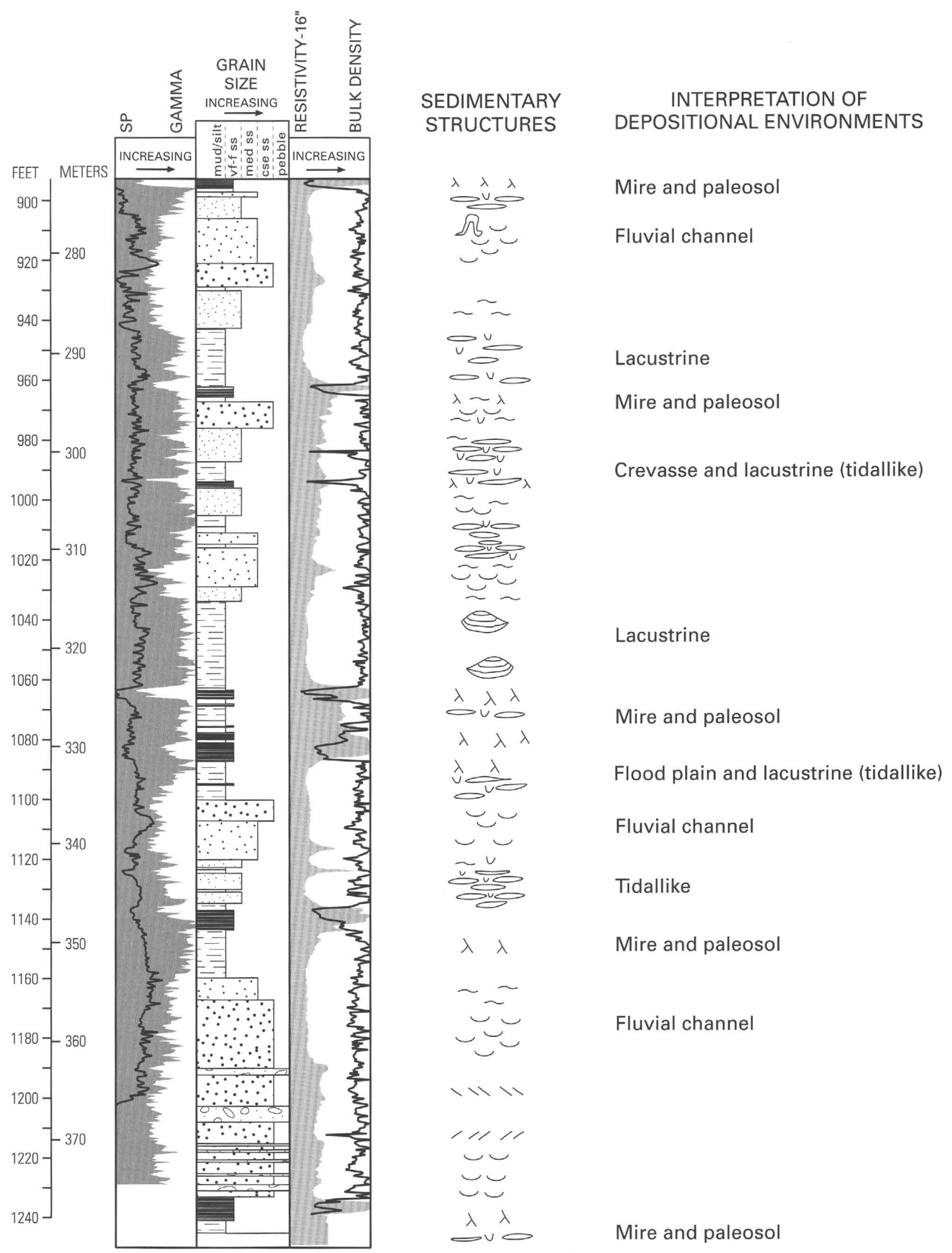

A

Figure 4A. Lithostratigraphy, geophysical logs, and lithofacies variation of the lower part (379-272 m) of the AK 94 CBM-1 core. 


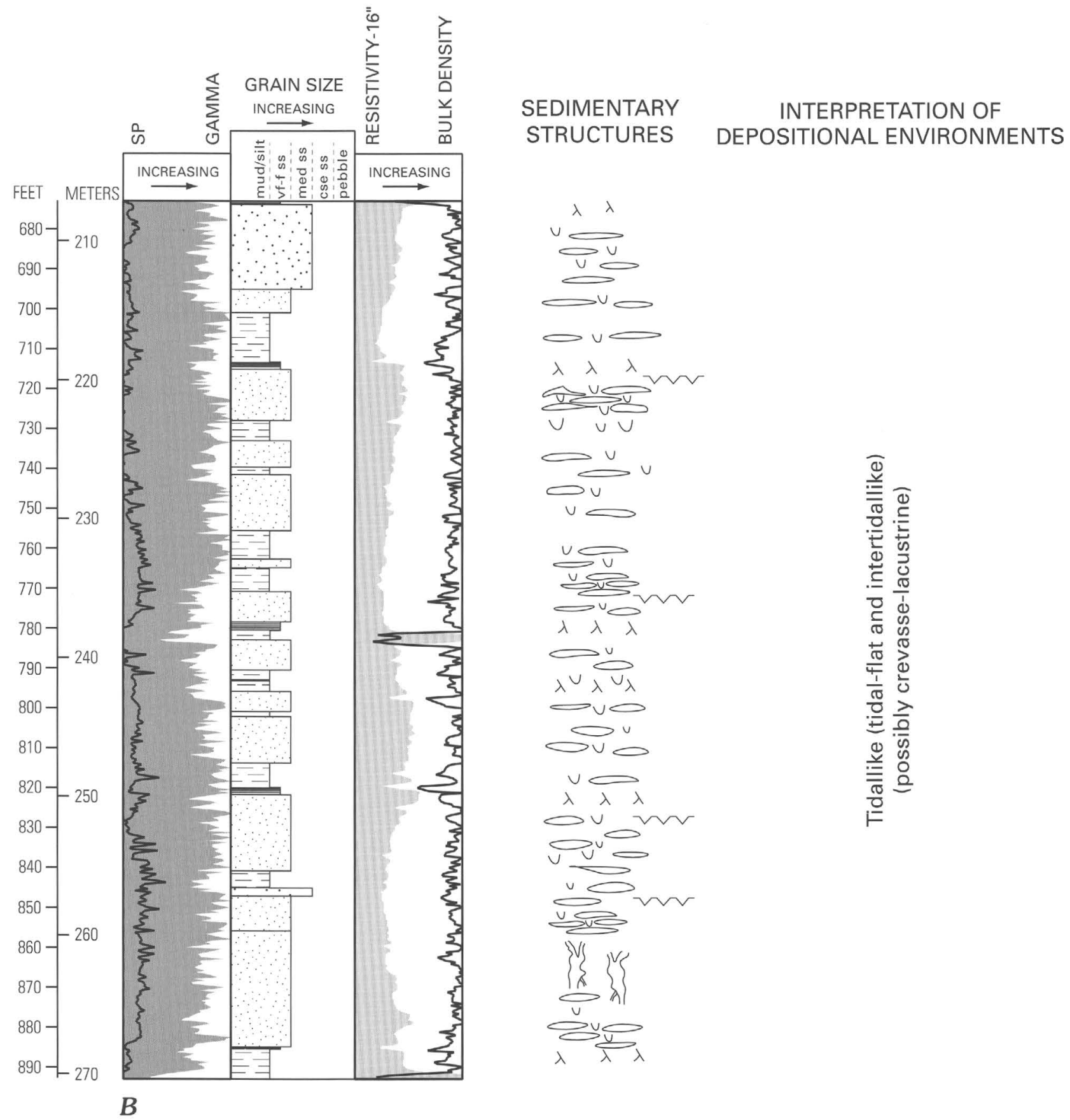

Figure 4B. Lithostratigraphy, geophysical logs, and lithofacies variation of the middle part (272-205 m) of the AK 94 CBM-1 core.

shapes (leftward excursions). The spontaneous-potential log curve is probably due to fining-upward grain size of the sandstone. The funnel shape curve of the spontaneous potential curve suggests coarsening-upward vertical grain size of the sandstone.

Forty-five percent of the total rock volume of the middle part of the Tyonek interval (fig. $4 B$ ) is composed of siltstone, mudstone, carbonaceous mudstone, and coal (fig. 10). The siltstones and mudstones are light to dark gray and are commonly intercalated, making up units as much as $2.7 \mathrm{~m}$ thick. These units are commonly lenticular and (or) flaser bedded with lenses of siltstones draped by mudstones. Wispy lenses or "starved ripples" in siltstones are also common. Siltstone lenses are as much as $0.6 \mathrm{~cm}$ thick and are vertically burrowed by trace fossils- the burrows include small oval burrows of possibly Chondrites and Teichicnus (Pemberton and Wightman, 1992; Beynon and Pemberton, 1992) at 264.3-264.4 m. Possible trace fossils of Planolites and Paleophycus (Pemberton and Wightman, 1992; Beynon and Pemberton, 1992) are found at $264.5 \mathrm{~m}$. A Thalassinoideslike (Pemberton and Wightman, 1992; Beynon and Pemberton, 1992) trace fossil (fig. 9) is found at $263.4-262.9 \mathrm{~m}$. The mudstones are heavily bioturbated vertically by wormlike or spiroform burrows (possibly Gyrolithes) that are $1 \mathrm{~mm}$ in diameter and $1.3 \mathrm{~cm}$ long. These burrowed horizons are commonly associated with mud and synaeresis cracks. Discrete units of black, 


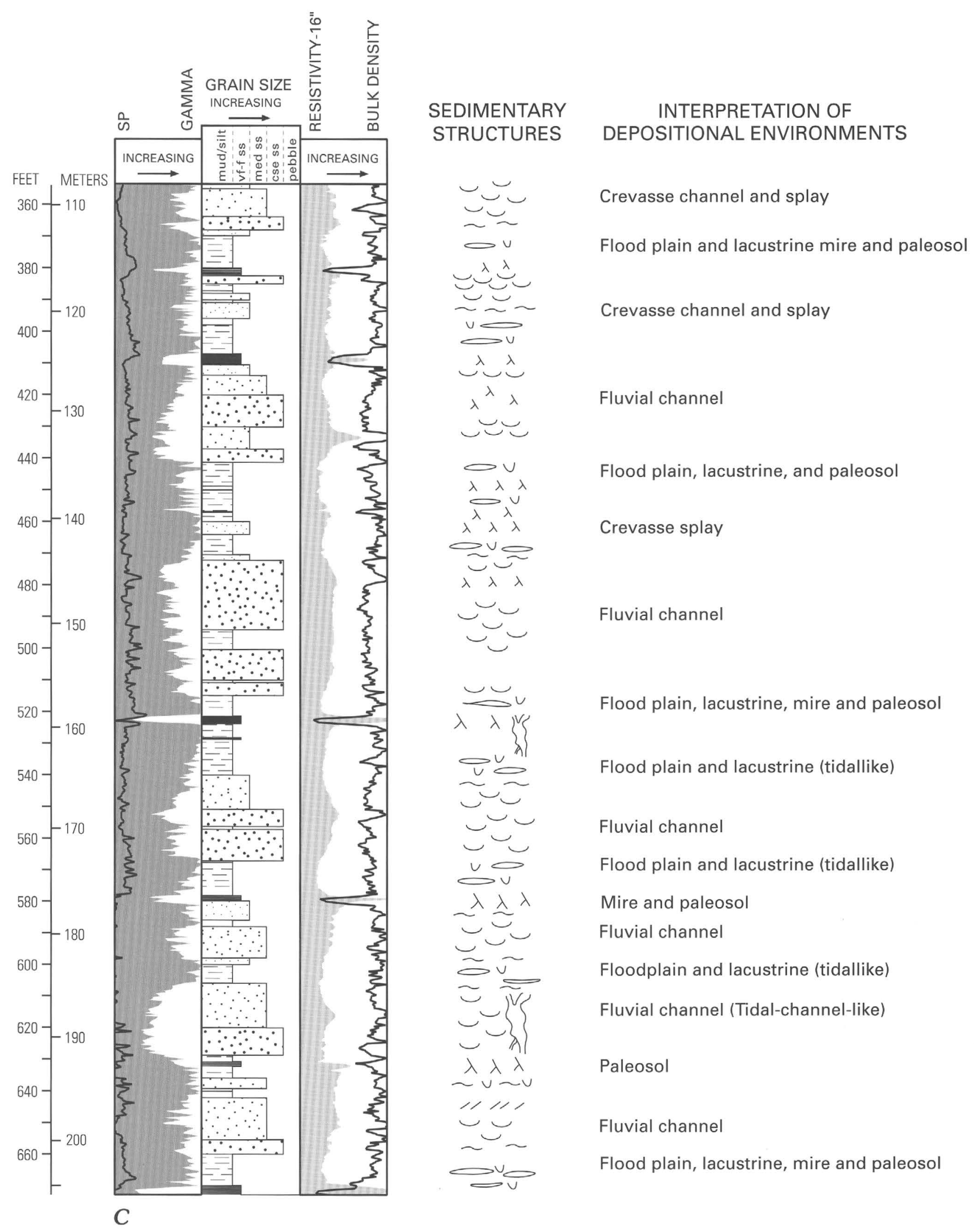

Figure 4C. Lithostratigraphy, geophysical logs, and lithofacies variation of the upper part (205-108 m) of the AK 94 CBM-1 core.

carbonaceous mudstones are as much as $0.6 \mathrm{~m}$ thick and are bioturbated by plant rootlets associated with yellow mottles.
These mudstone units are interbedded with thin, vitrain-rich, coaly layers that are as much as $8.2 \mathrm{~cm}$ thick. 


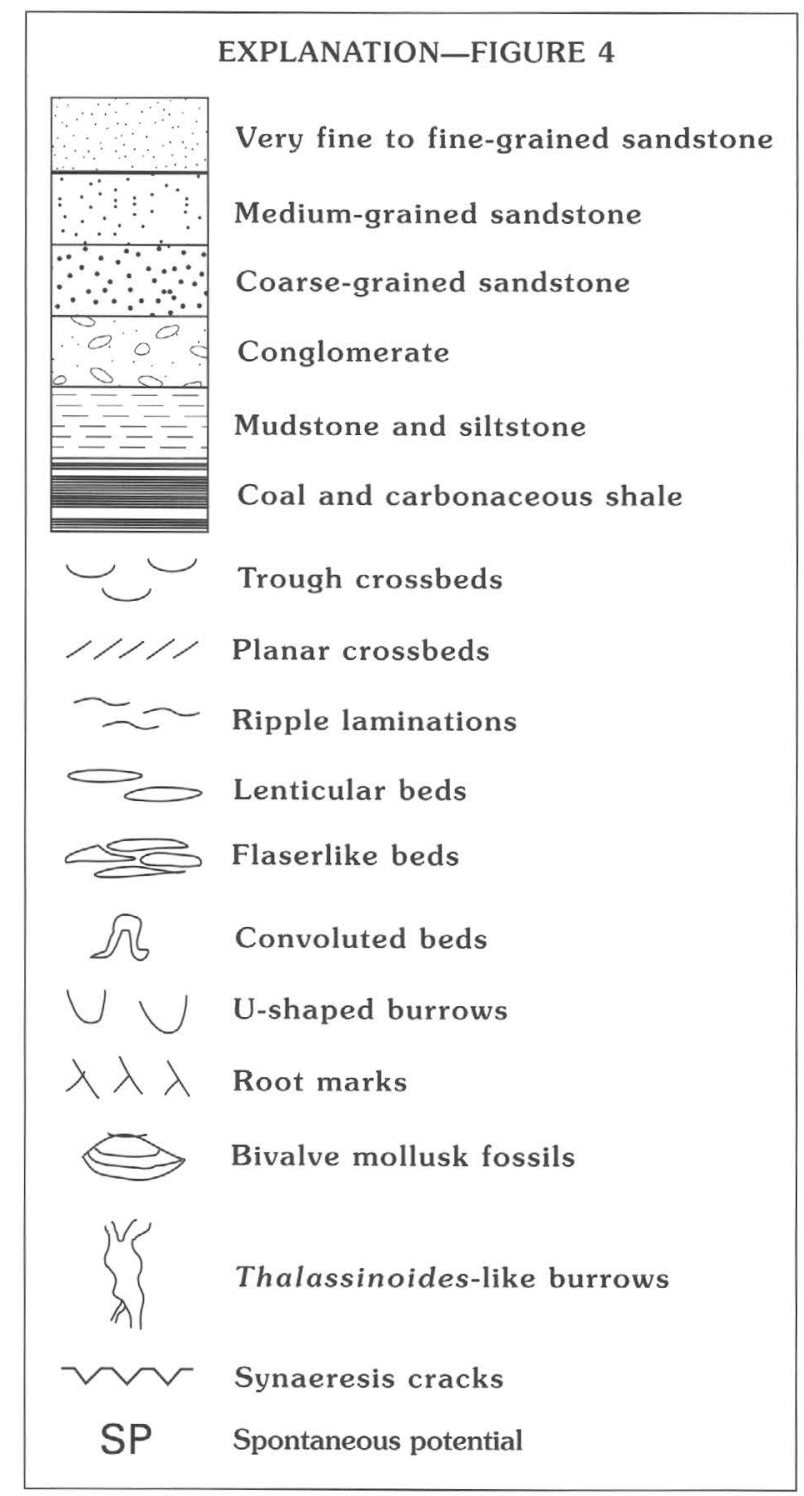

\section{Upper Part of the Core}

The upper part (205-108 m) of the Tyonek interval (fig. $4 C$ ) is dominated by fine- to coarse-grained sandstones, which make up about 55 percent of the total rock volume, and commonly by thin coal beds. The sandstones occur as fining-upward beds that are from 4.6 to $9.1 \mathrm{~m}$ thick and display sharp to erosional bases (fig. 11). The sandstones are light gray and show a "salt-and-pepper" texture. Sedimentary structures commonly consist of trough crossbeds that are as much as $0.3 \mathrm{~m}$ in height (fig. 11). Massive beds as much as $1.8 \mathrm{~m}$ thick are commonly interbedded with the trough crossbeds. Planar crossbeds (as much as $1.5 \mathrm{~cm}$ in height) and slump structures are sparse. Asymmetrical and climbing ripple laminations in units as much as $0.6 \mathrm{~m}$ thick cap the fining-upward sandstones. Bioturbation by trace fossils and plants is common. Trace-fossil burrows are vertical (fig. 11) and branchlike or Thalassinoides-like and are as much as $10 \mathrm{~cm}$ in diameter and $0.5 \mathrm{~m}$ long. Carbonaceous plant root marks are common in the upper part of the finingupward sandstones. The root marks are both younger and older than the vertical burrows.
The gamma-ray log curve (fig. 4C) displays serrated-bell and cylindrical shapes (rightward excursions). The spontaneouspotential log curve shows combined smooth- and serrated-bell and cylindrical shapes and funnel shape (leftward excursions). The spontaneous-potential log curve is probably due to uniform vertical and fining-upward grain size of the sandstones. These vertical grain-size variations of the sandstones are reflected by the gamma-ray log curves. The funnel shape curve of the spontaneous potential curve suggests coarsening-upward vertical grain size of the sandstones.

Forty-five percent of the upper part of the Tyonek interval (fig. $4 C$ ) consists of siltstone, mudstone, coal and carbonaceous shale (fig 12). Siltstones are light gray, a few centimeters to 1.4 $\mathrm{m}$ thick, contain very fine grained sandstone lenses (lenticular bed), and are rippled, rooted, and burrowed (fig. 11). Mudstones are gray to black, depending on the amount of carbonaceous matter content, and vary from 0.3 to $2.7 \mathrm{~m}$ thick (fig 12 ). They are massive to crudely laminated, rippled (lenticular beds and "starved ripples"), rooted, and vertically burrowed. Vertical burrows in the mudstones are 0.6 to $2.5 \mathrm{~cm}$ in diameter, more than 8 $\mathrm{cm}$ long, and sand-infilled. Carbonaceous root marks penetrate these branchlike vertical burrows. Coal and carbonaceous shales are interbedded, forming units as thick as $0.9 \mathrm{~m}$. Coals are brightly banded, indicating woody composition. Carbonaceous shales are commonly rooted (fig. 12).

\section{LOWER PART OF THE CORE}

\section{Lithofacies Associations and Sequences}

Lithofacies associations of the Tyonek Formation may be recognized as a depositionally related group of lithofacies that occur together. Lithofacies sequences of the Tyonek Formation are recognized as a series of lithofacies that pass vertically from one lithofacies to another.

Three thick, fining-upward sandstone units occur as lithofacies sequences in the lower part of the Tyonek interval (fig. 4A), from bottom to top, 376-353 m, 344-334 m, and 287-273 m. These 12- to 23-m-thick sandstones consist of stacked, multiple scoured units, each of which fines upward. The fining-upward sandstones at 376-353 m are associated with erosionally based conglomerates that are either singular or stacked units. This lithofacies association is mainly found at $374.6-362.7 \mathrm{~m}$, where the conglomerates occur. In addition, pebble floats are associated with erosionally based sandstones in the middle part of the succession. The fining-upward sandstones at $287-272 \mathrm{~m}$ are associated with some conglomerates that mark erosional bases below the sandstones.

The fining-upward sandstone lithofacies sequences are interbedded, from bottom to top, with lithofacies associations consisting of coal, carbonaceous shale, sandstone, siltstone, and mudstone at 379-376 m, 353-344 m, 334-287 m, and 273-272 $\mathrm{m}$. The lithofacies association at $379-376 \mathrm{~m}$ consists mainly of coal and carbonaceous shale underlain by mudstone and siltstone, which are rooted, burrowed, lenticular bedded, and rippled. The lithofacies association at 353-344 m includes, from bottom to top, a rooted mudstone capped by vitrinite lenses, coal 

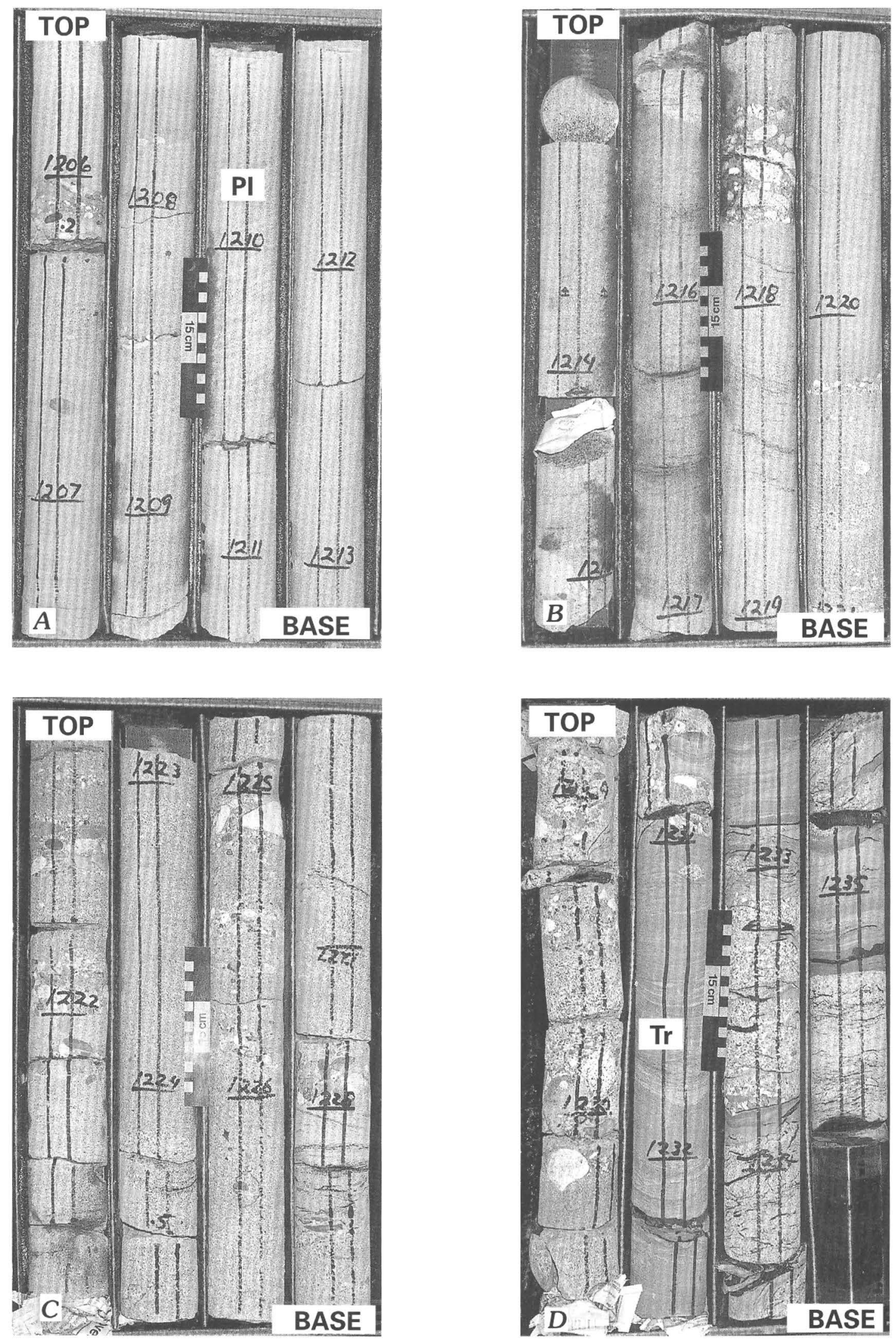

Figure 5. Stacked, erosionally based, fining-upward sandstones interbedded with conglomerates from 376.4 to $367.4 \mathrm{~m}$ in the lower part of the core (photos $A$, from 376.3 to $369.9 \mathrm{~m} ; B$, from 369.9 to $372.2 \mathrm{~m}$; $C$, from 372.2 to 374.6 $\mathrm{m}$; and $D$, from 374.6 to $376.8 \mathrm{~m}$ ). Trough ( $\mathrm{Tr}$ ) and planar (PI) crossbeds in the fining-upward sandstones from 375.6$375.0 \mathrm{~m}$ and 371.8 to $367.7 \mathrm{~m}$, respectively. Numbers on core show depth in feet. To convert from feet to meters multiply by 0.304 (note centimeter scale on photo). 


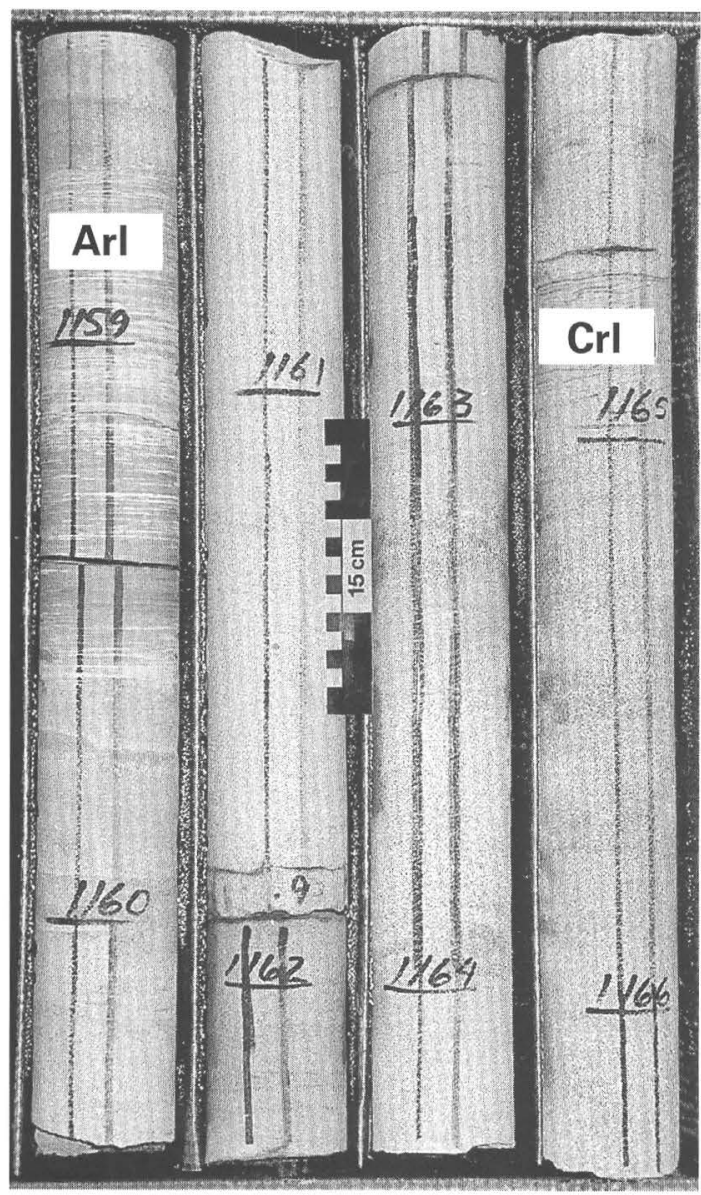

Figure 6. Climbing ripple laminations ( $\mathrm{Crl}$ ) interbedded with asymmetrical ripple laminations (Arl) that cap the stacked, fining-upward sandstones from 355.2 to $353.1 \mathrm{~m}$ in the lower part of the core. Numbers on core show depth in feet. To convert from feet to meters multiply by 0.304 (note centimeter scale on photo).

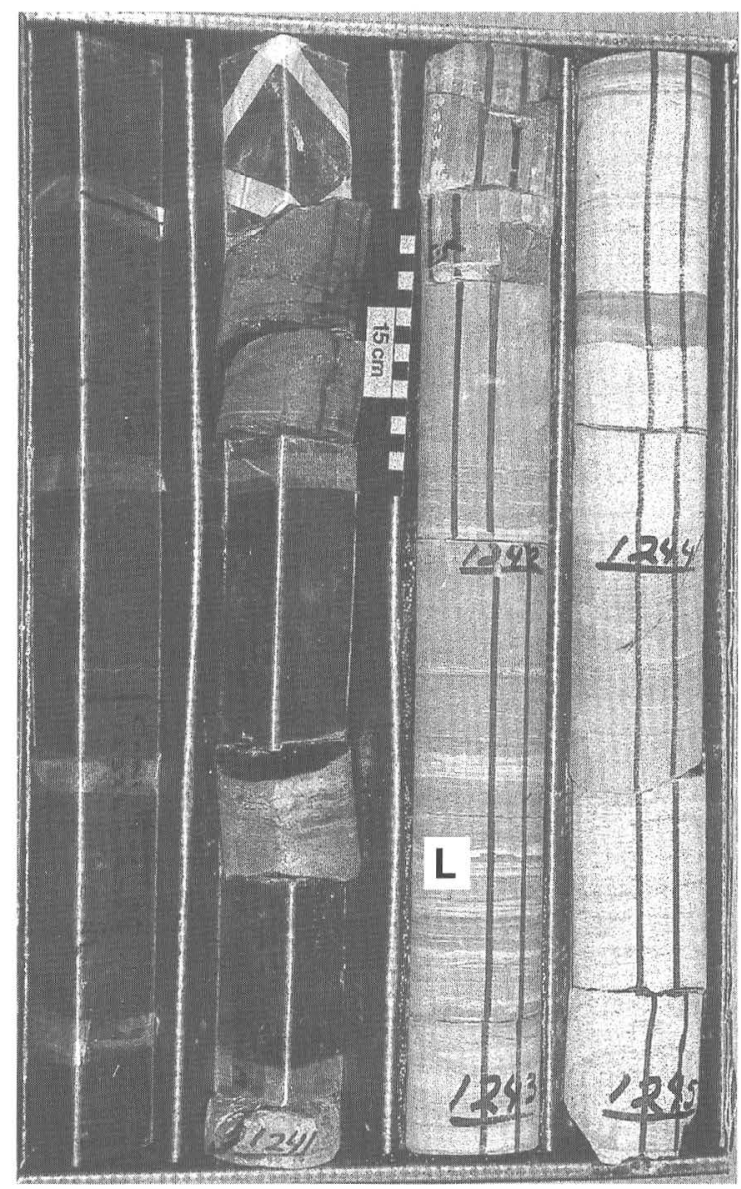

Figure 7. Interbedded coal, carbonaceous mudstone, siltstone, and sandstone from 379.3 to $377.1 \mathrm{~m}$ in the lower part of the core. A part of the coal bed is missing and was sampled for analysis (378-377). L, lenticular bedding. Numbers on core show depth in feet. To convert from feet to meters multiply by 0.304 (note centimeter scale on photo). and carbonaceous shale, and interbedded burrowed mudstone; tonstein; fining-upward, rippled-burrowed sandstone; and siltstone. The lithofacies association at 334-287 m consists of coal and rooted carbonaceous shale interbedded with lenticular-bedded, mud-draped (flaserlike) sandstone, siltstone, and mudstone in the lower part. A massive mudstone containing bivalve mollusks interbedded with a multi-erosional, 7.3-m-thick, finingupward sandstone occurs in the middle part. A lithofacies association of coal and carbonaceous shale interbedded with mudstone and stacked, multi-erosional, 1.5- to 3.3-m-thick, finingupward sandstone occurs in the upper part. The lithofacies association at 273-272 m consists mainly of coal and carbonaceous shale. A mudstone at $260.4 \mathrm{~m}$ (below a coal) analyzed by Nichols (1998) for palynomorphs yielded the freshwater alga Pediastrum and common conifer pollen.

\section{Interpretation}

The thick, fining-upward sandstone sequences are interpreted as deposits of major fluvial channels. The stacked, multiple scoured units represent deposits of shallow (thin) to deep (thick) subchannels. The thin, erosionally based conglomerates are interpreted as channel-floor and gravel-bar deposits similar to braided streams (Webb, 1994). Pebbles in the sandstone reflect debris-flow deposits. The lithofacies association of sandstone and conglomerate suggests that the major fluvial channels were braided, consisting of subchannels that were aerially separated by gravel bars (Best and Bristow, 1993). The fining-upward sandstones floored by conglomerates indicate channel-fill during waxing to waning channel flows (Allen, 1965).

The coal and carbonaceous shale lithofacies associations are interpreted as mire deposits in freshwater environments as indicated by the alga Pediastrum. The massive mudstones containing bivalve mollusks or pelecypods suggest deposition in a subaqueous, probably freshwater, lacustrine environment. The lithofacies association of mudstone with lenticular-bedded, mud-draped sandstone, siltstone, and mudstone reflects deposition either in a wave-influenced flood plain crevasse and lacustrine environment, or tidallike environment. The thin, finingupward sandstone lithofacies sequence is interpreted as deposits 

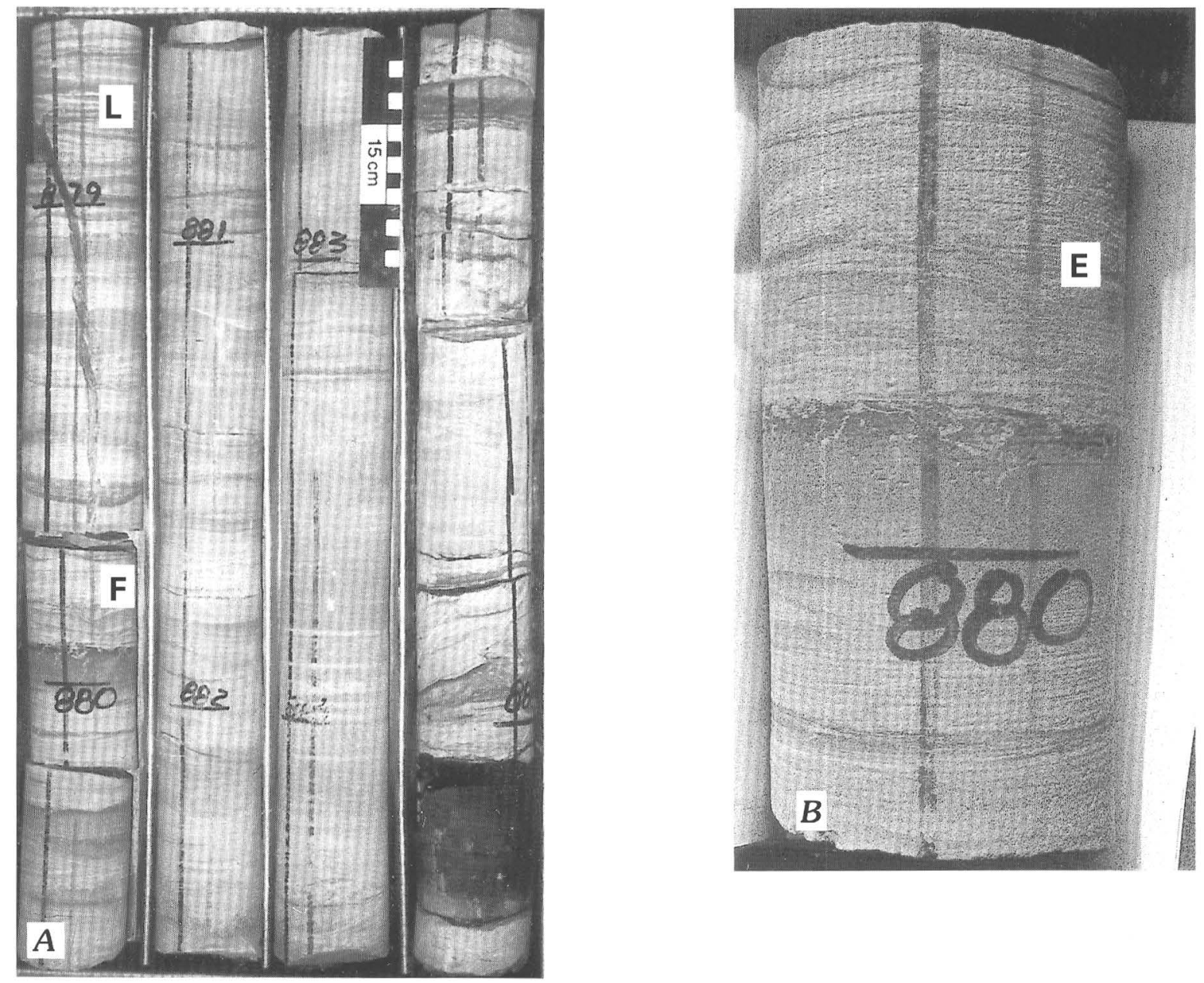

Figure 8. $\quad A$, Siltstones, mudstones, and silty sandstones containing lenticular ( $L$ ) and flaserlike $(\mathrm{F})$ beds underlain by a thin coal and carbonaceous shale lithofacies from 270.1 to $267.9 \mathrm{~m}$. B. Flaserlike beds with bipolar ripple sets draped by burrowed mudstone lenses and a mudstone exhibiting synaeresis or shrinkage cracks (SC). E, escape structure. Numbers on core show depth in feet. To convert from feet to meters multiply by 0.304 (note centimeter scale on photo).

of minor, sinuous or meandering fluvial channels that drained the mires, flood plains, and lacustrine environments (Miall, 1996). They also served as minor drainages that were laterally contemporaneous to the major braided fluvial channels in the alluvial plain.

\section{MIDDLE PART OF THE CORE}

\section{Lithofacies Associations and Sequences}

The lithofacies association in the middle part of the core consists mainly of erosionally based, thick, heavily bioturbated, fine-grained sandstones at 272-242 m (fig. 4B). The fine-grained sandstones are mainly lenticular, wispy, and flaserlike bedded and contain subordinate crossbed foresets with clay-draped reactivation surfaces and opposed foresets in ripple laminae. These sedimentary structures are partly destroyed by "tidallike" burrows and U-shaped escape burrows. The fine-grained sandstones are associated with minor siltstones and mudstones that are commonly lenticular and flaserlike bedded. Lithofacies association of minor amounts of rooted carbonaceous mudstones with the siltstones and mudstones are at 250-249 m and 243-242.8 m.
The lithofacies association from 242-205 $\mathrm{m}$ of the middle part of the core is mainly composed of sharp-based, thin, heavily bioturbated, fine-grained sandstones. These are commonly lenticular and flaserlike bedded with the mud-drapes being burrowed. These sedimentary structures are locally rhythmic and are commonly destroyed by vertical burrows and root marks. Root marks are in the form of yellow mottles. The finegrained sandstones are commonly associated with thin to thick siltstones and mudstones. The siltstones and mudstones in this lithofacies association are characterized by lenticular, wispy, and flaserlike beds that are almost destroyed by vertical burrows and root marks. The root marks and mottled structures are associated with thin coal and carbonaceous mudstones. A rooted carbonaceous mudstone analyzed for palynomorphs by Nichols (1998) yield abundant pine and hemlock pollen.

\section{Interpretation}

The lithofacies association in the middle part of the core is very similar to that described as tidallike deposits by Flores and others (1997) in core from the Chuitna drainage. The thin to thick, erosional- to sharp-based, fine-grained sandstone 


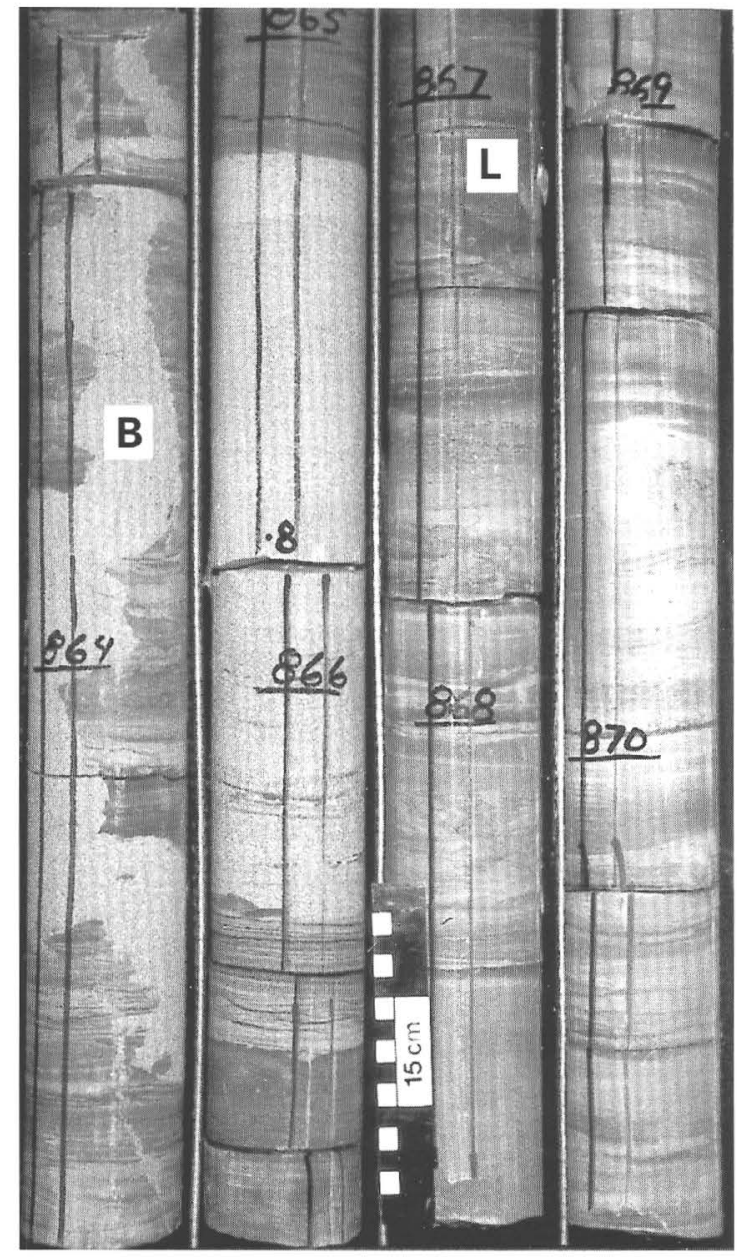

Figure 9. Siltstones, mudstones and silty sandstones displaying lenticular beds (L) and a Thalassinoides-like burrow (B) from 265.4 to $262.9 \mathrm{~m}$. Numbers on core show depth in feet. To convert from feet to meters multiply by 0.304 (note centimeter scale on photo).

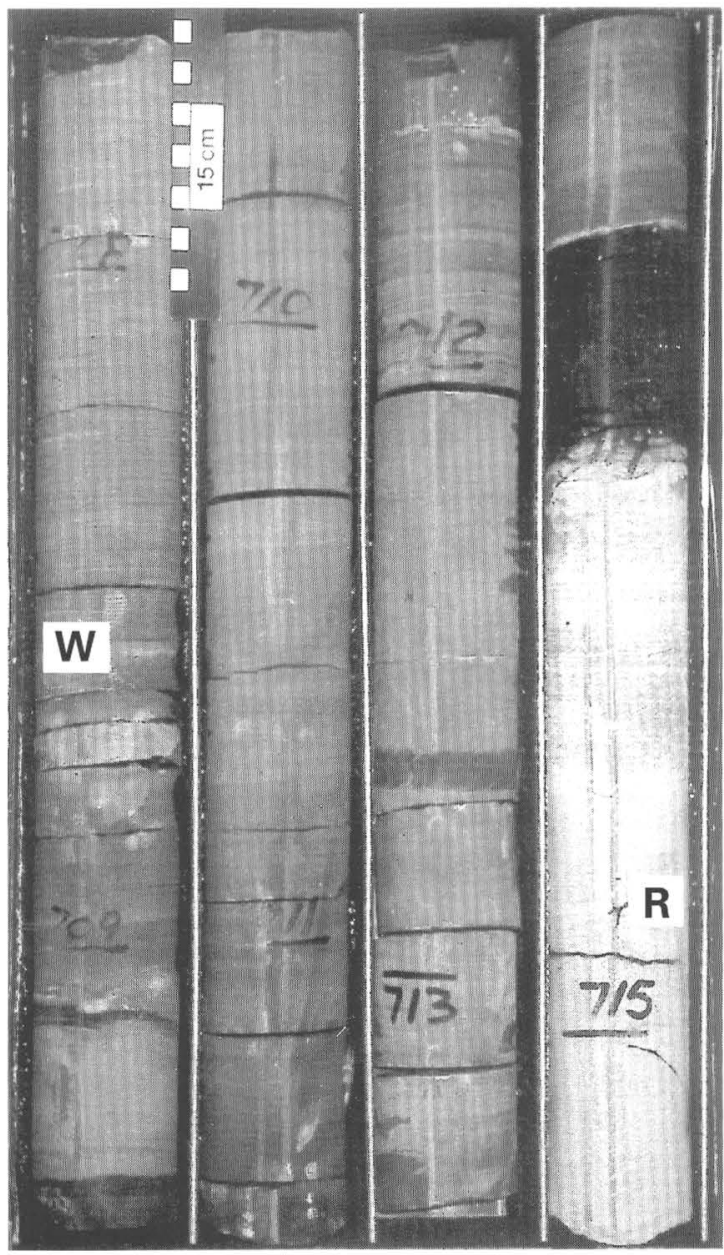

Figure 10. Mudstones, siltstones, carbonaceous shales, and coals from 217.8 to $215.7 \mathrm{~m}$ in the middle part of core. $\mathrm{W}$, wispy structure. $\mathrm{R}$, root mark. Numbers on core show depth in feet. To convert from feet to meters multiply by 0.304 (note centimeter scale on photo). lithofacies are interpreted by Flores and others (1997) as deposits in tidal-channel to tidal-flat-like environments similar to sediments described by Evans (1975), Visser (1980), Reinson (1989), Nio and Yang (1991), Flores and Johnson (1995), and Flores and Sykes (1996). The Thalassinoides-like burrows suggest that these lithofacies associations were influenced by brackish or marine water. However, MacKenzie (1975) suggested that the same burrows may have been generated by some species of clams. If this is correct, we suggest that these clams may be similar to the unionid bivalve mollusks found in the mudstones in the lower part of the core, interpreted to be deposited in a large flood-plain freshwater lake. The tidallike deposits may have been deposited in crevasse splays and reworked by burrowing animals as well as ebb-and-flow processes. The presence of burrowed lenticular and flaserlike beds in the sandstone and siltstone, and in the wispy beds, indicates intertidal-subtidal-like environments (Reineck and Wunderlich 1968). The opposed bipolar ripple laminae and development of reactivation surfaces probably reflects successive tidallike ebb and flow. The possible trace fossils Thalassinoides, Chondrites, Teichicnus, Gyrolithes, Planolites, and Paleophycus reflect brackish-marine influence and may support tidallike deposition of the associated rocks
(Pemberton and Wightman, 1992; Beynon and Pemberton, 1992). Furthermore, the presence of synaeresis or shrinkage cracks support the idea of large fluctuations in salinity (Burst, 1965). The rooted and mottled mudstone lithofacies are interpreted as paleosols or millisols (Retallack, 1988). The coal and carbonaceous mudstone lithofacies associated with the paleosols suggest deposition either in low-lying or well-drained mires. Retallack (1988) recognized this coal and carbonaceous mudstone lithofacies as a form of paleosol or histosol.

\section{Upper Part of the Core}

\section{Lithofacies Associations and Sequences}

The upper part of the core from $205-108 \mathrm{~m}$ is dominated by lithofacies sequences of erosional-based, fining-upward (medium to fine) sandstone (fig. $4 C$ ). The sandstone commonly displays branching Thalassinoides-like vertical burrows. The thick, fining-upward sandstones are found at 201-125 m. At these depths, the sandstones are commonly associated with 

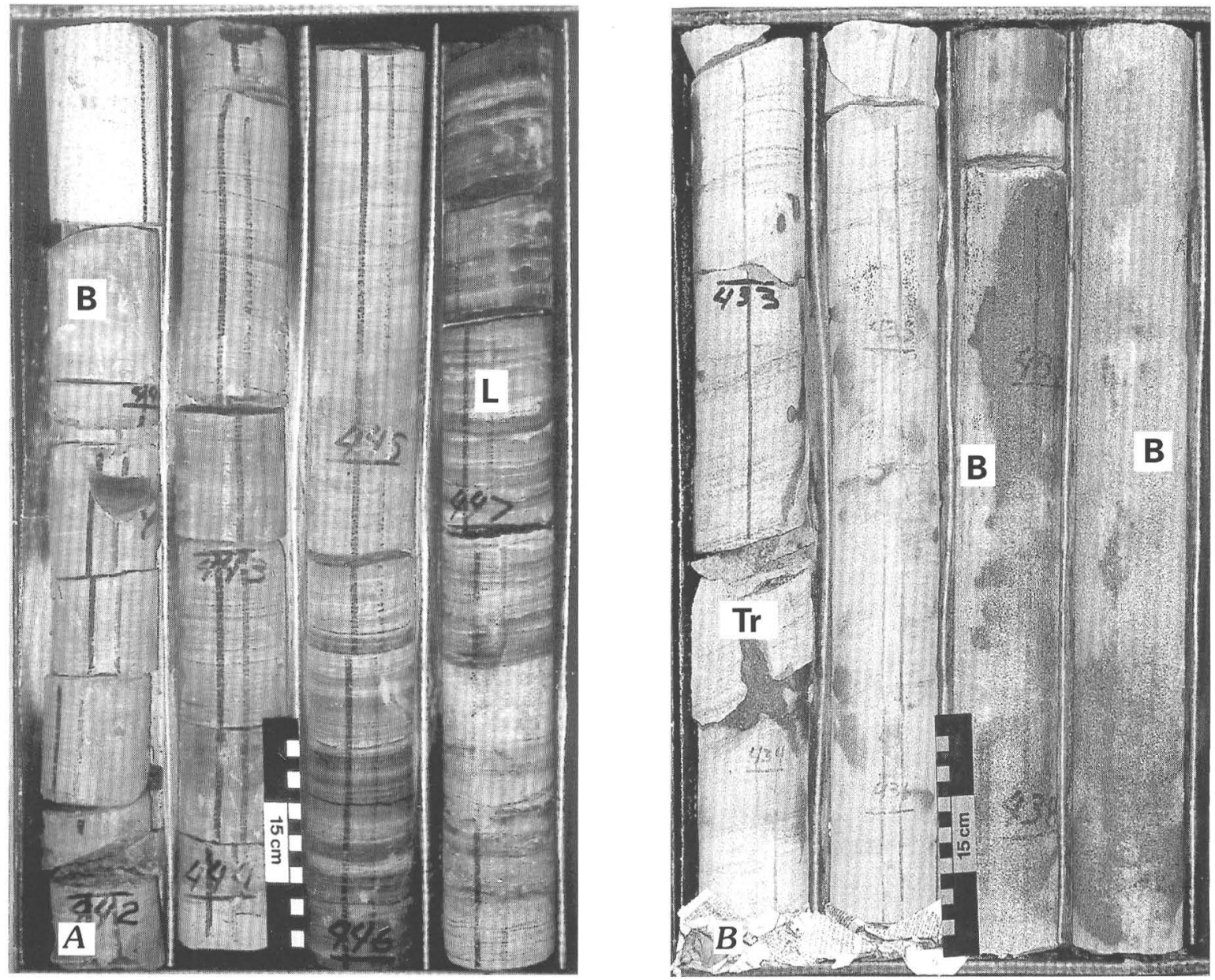

Figure 11. $A$, Burrowed mudstones and siltstones from $149.6-134.5 \mathrm{~m}$, and $B$, sandstones containing trough crossbeds (Tr) and burrows (B) from 134.5-132.1 m. Numbers on core show depth in feet. To convert from feet to meters multiply by 0.304 (note centimeter scale on photo).

coarsening-upward lithofacies sequences of rippled ("starved") mudstones and burrowed, lenticular, flaser-bedded siltstones, and sandstones. In addition, thick coals and carbonaceous shales are interbedded with these lithofacies associations. Mudstones at $174.6 \mathrm{~m}$ and $202 \mathrm{~m}$ (above coals), analyzed by Nichols (1998) for palynomorphs, yielded the freshwater alga Pediastrum and common conifer pollen. Thin fining-upward sandstone lithofacies sequences are found at $120-117 \mathrm{~m}$. Thin coal and rooted carbonaceous shales and mudstones cap the fining-upward sandstone lithofacies sequences. These lithofacies sequences are underlain by coarsening-upward lithofacies sequences of burrowed mudstones, and rippled siltstones and sandstones.

\section{Interpretation}

The thin to thick, fining-upward sandstone lithofacies sequences are interpreted as fluvial channels. Because these channels exhibit Thalassinoides-like burrows, it suggests reworking of the fluvial sediments by either crustaceanlike organisms typical of tidal-flat-like settings (Reineck, 1967) or some species of pelecypods (MacKenzie, 1975). The tidal influence may be indicated by the lenticular and flaser-bedded units. The coarsening-upward lithofacies sequences of mudstones, siltstones, and sandstones represent fluvial aggradational complexes (e.g., crevasse channels and splays) in subaqueous floodplain or freshwater lacustrine environments. Thus, the fluvial channels overlying these coarsening-upward lithofacies sequences may represent crevasse channels that were reoccupied by flow-through systems. The coal and carbonaceous shales and rooted mudstones are interpreted as mire and paleosol deposits on abandoned deposits of these flow-through channel systems. These mires were inundated by freshwater lakes, as indicated by the presence of the freshwater alga Pediastrum.

\section{Summary and Conclusions}

The Tyonek interval in the AK 94 CBM-1 well contains interbedded fluvial-dominated and tidallike lithofacies. The fluvial-dominated lithofacies are found in the lower and upper parts of the core. The tidallike lithofacies are found in the middle part of the core; however, tidallike lithofacies are associated with the fluvial-dominated lithofacies. These alternating fluvialdominated and tidallike lithofacies are similar to lithofacies found in the Tyonek Formation in the Chuitna drainage basin described by Flores and others $(1994,1997)$. 


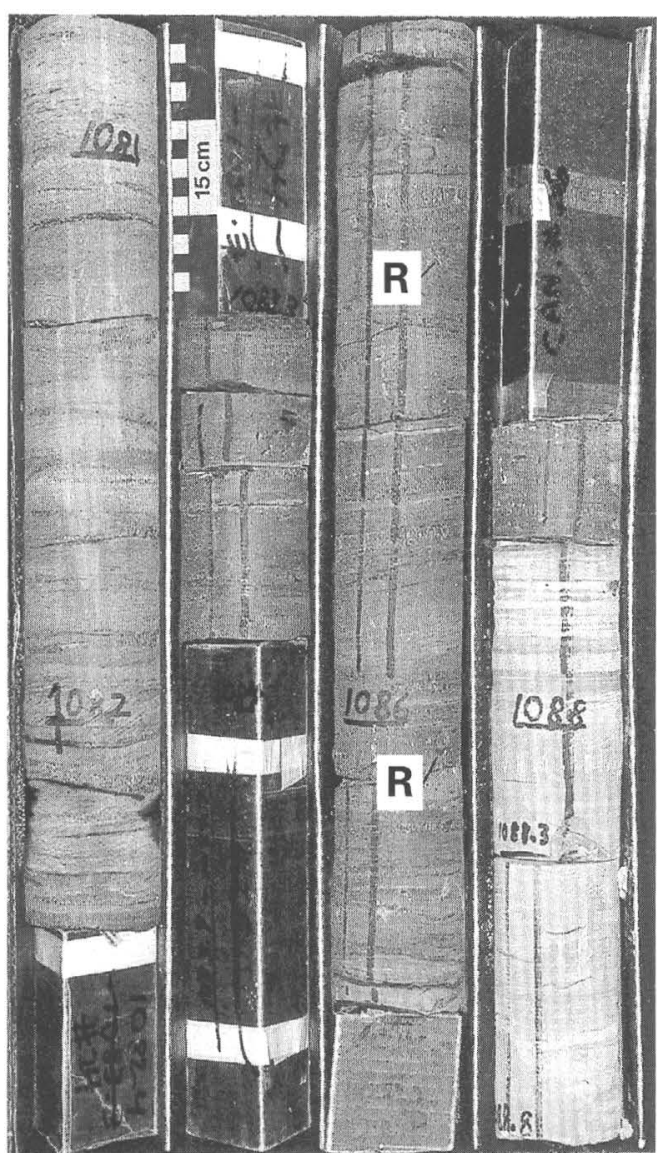

Figure 12. Siltstones and mudstones interbedded with carbonaceous shales and coals (removed for analysis) from 329.4 to $331.9 \mathrm{~m}$. Mudstones are mainly rooted ( $R$ ). Numbers on core show depth in feet. To convert from feet to meters multiply by 0.304 (note centimeter scale on photo).

The coal beds are mainly associated with the fluvial-dominated lithofacies. Here, the coal beds formed in freshwater mires developed mainly on abandoned fluvial channel belts and, subordinately, on distal flood plains. Paleosols and well-drained mires, forming only thin coal lenses, are associated with the tidallike lithofacies. The tidallike lithofacies are interpreted to form in intertidal-subtidal-like environments. Perhaps the most compelling characteristics of these tidallike lithofacies are the lenticular and flaserlike beds; foresets with reactivation surfaces; and rhythmic, mud-draped, bipolar ripple laminae. Associated trace fossils (Thalassinoides-like, Chondrites, Teichicnus, Gyrolithes, Planolites, and Paleophycus) and synaeresis cracks with these lithofacies support a brackish-marine influence (Burst, 1965; Pemberton and Wightman, 1992; Beynon and Pemberton, 1992). Subaqueous deposition is suggested by sedimentary structures that are laced with heavy bioturbation. The only subaqueous fluvial setting that is equivalent to a tidallike setting in which most of these sedimentary structures may be formed is possibly in a freshwater lacustrine setting with ebb-and-flow processes, possibly driven by wind storms.

The discovery of these Tyonek tidallike lithofacies near Wasilla and in the Chuitna River drainage basin (Flores and others, 1994, 1997) in the upper Cook Inlet suggests that the processes that formed them are more prevalent and widespread than originally thought. Another interpretation could be that tidallike processes in freshwater lakes in fluvial-dominated environments, particularly in the Cook Inlet Basin, have been overlooked. It is an enigma that these lithofacies have not been found associated with fluvial-dominated lithofacies of the Kenai Group during previous studies. The generally accepted paleogeographic reconstructions of the Tertiary Cook Inlet Basin may need to be revised with respect to the paleogeography and distribution of brackish-marine-influenced sedimentation. Thus, based on this study and previous work by Flores and others (1994, 1997), the estuarine and marine environments that existed during deposition of the Tyonek are probably found in the southern and central part of the Cook Inlet Basin.

\section{Acknowledgments}

This investigation is a cooperative work between the Alaska Department of Natural Resources, Oil and Gas Division and the U.S. Geological Survey. Lisa R. Bader and Allan M. Ochs of the U.S. Geological Survey assisted in drafting figures, and their work is appreciated. Assistance by Dr. John W. Reeder, Curator of the Alaska Geologic Materials Center, is appreciated for permission to describe the cores in their facilities. Appreciation is extended to Bo Henk of the University of Alberta and on sabbatical from ARCO, Anchorage, for identifying the trace fossils of the cores. Dr. Robert Blodgett of the University Oregon identified the unionid bivalve fossil.

\section{References Cited}

Adkison, W.L., Kelley, J.S., and Newman, K.R., 1975, Lithology and palynology of Tertiary rocks exposed near Capps Glacier and along Chuitna River, Tyonek quadrangle, southern Alaska: U.S. Geological Survey Open-File Report 75-21, 58 p., 1 plate.

Allen, J.R.L., 1965, Fining upward cycles in alluvial successions: Liverpool and Manchester Geological Journal, v. 4, p. 229-246.

Anonymous, 1990, Alaska's high-rank coals: Department of Natural Resources, Division of Geological and Geophysical Surveys, Information Circular 33, $36 \mathrm{p}$.

Barnes, F.F., and Payne, T.G., 1956, The Wishbone Hill district, Matanuska coal field, Alaska: U.S. Geological Survey Bulletin 1016, 88 p.

Best, J.L., and Bristow, C.S., 1993, Braided rivers: Geological Society Special Publication, $367 \mathrm{p}$.

Beynon, B.M., and Pemberton, S.G., 1992, Ichnological signature of a brackish water deposits: An example from the Lower Cretaceous Grand Rapids Formation, Cold Lake Oil Sands area, Alberta, in Pemberton, S.G., ed., Applications of Ichnology to Petroleum Exploration, A Core Workshop. SEPM (Society for Sedimentary Geology), p. 199221.

Burst, J.F., 1965, Subaqueously formed shrinkage cracks in clay: Journal of Sedimentary Petrology, v. 35, p. 348-353.

Calderwood, K.W., and Fackler, W.C., 1972, Proposed stratigraphic nomenclature for Kenai Group, Cook Inlet basin, Alaska: American Association of Petroleum Geologists Bulletin, v. 56, p. 739-754.

Dickinson, K.A., and Campbell, J.A., 1978, Sedimentary facies in Tertiary rocks in the Tyonek quadrangle, in Geological Survey Research 1978: U.S. Geological Survey Professional Paper 1100, p. 84. 
Dickinson, K.A., Campbell, J.A., and Dula, W.F., Jr.,1995, Geology, geochemistry, and uranium favorability of Tertiary continental sedimentary rocks in the northwestern part of the Cook Inlet area, Alaska: U.S. Geological Survey Bulletin 2098, p. B1-B37.

Doveton, J.H., 1994, Geologic log interpretation: Society of Economic Paleontologists and Mineralogists Short Course Notes No. 29, 169 p.

Evans, G., 1975, Intertidal flat deposits of the Wash, western margin of the North Sea, in Ginsburg, R.N., ed., Tidal Deposits-A Casebook of Recent Examples and Fossil Counterparts: New York, Springer-Verlag, p. 13-20.

Flores, R.M., and Johnson, S.Y., 1995, Sedimentology and lithofacies of the Eocene Skookumchuck Formation in the Centralia coal mine, southwest Washington, in Fritsche, A.E., ed., Cenozoic Paleogeography of the Western United States-II: Pacific Section, SEPM (Society for Sedimentary Geology), Book 75, p. 274-290.

Flores, R.M., and Stricker, G.D., 1993, Responses of coal splitting and associated drainage pattern to syntectonism in the Paleocene and Eocene Chickaloon Formation, Matanuska coalfield, Alaska, in Rao, P.D., and Walsh E., eds., Focus on Alaska's Coal 1993: Mineral Industry Research Laboratory, University of Alaska, p. 190-226.

Flores, R.M., Stricker, G.D., and Roberts, S.B., 1994, Miocene coal-bearing strata of the Tyonek Formation: Braided-stream deposits in the Chuit Creek-Chuitna River drainage basin, southern Alaska, in Till, A.B., and Moore, T.E., eds., Geologic Studies in Alaska by the U.S. Geological Survey, 1993: U.S. Geological Survey Bulletin 2107, p. 95114.

Flores, R.M., Stricker, G.D., and Stiles, R.B., 1997, Tidal influence on deposition and quality of coals in the Miocene Tyonek Formation, Beluga coal field, upper Cook Inlet, Alaska, in Dumoulin, J.A., and Gray, J.E., eds., Geologic Studies in Alaska by the U.S. Geological Survey, 1995: U.S. Geological Survey Professional Paper 1574, p. 137-156.

Flores, R.M., and Sykes, R., 1996, Depositional controls on coal distribution and quality in the Eocene Brunner coal measures, Buller coalfield, South Island, New Zealand: International Journal of Coal Geology, v. 29, p. 291-336.

Galloway, W.E., 1968, Depositional systems of the lower Wilcox Group, north-central Gulf Coast Basin: Gulf Coast Association of Geological Society Transaction, v. 18, p. 275-289.

Hayes, J.B., Harms, J.C., and Wilson, T.W., 1976, Contrasts between braided and meandering stream deposits, Beluga and Sterling Formations (Tertiary), Cook Inlet, Alaska, in Miller, T.P., ed., Recent and Ancient Sedimentary Environments in Alaska: Anchorage, Alaska Geological Society Symposium Proceedings, p. J1-J27.

Hite, D.M., 1976, Some sedimentary aspects of the Kenai Group, Cook Inlet, Alaska, in Miller, T.P., ed., Recent and Ancient Sedimentary Environments in Alaska: Alaska Geological Society Symposium Proceedings: Anchorage, Alaska, p. 11-122.

Johnsson, M.J., Howell, D.G., and Bird, K.J., 1993, Thermal maturity patterns in Alaska: Implications for tectonic evolution and hydrocarbon potential: American Association of Petroleum Geologists Bulletin, v. 77, p. 1874-1903.

Krynine, P.D., 1950, Petrology, stratigraphy and origin of the Triassic sedimentary rocks of Connecticut: Connecticut Geology and Natural History Survey, 239 p.
MacKenzie, D.B., 1975, Tidal sand flat deposits in Lower Cretaceous Dakota Group near Denver, Colorado, in Ginsburg, R.N., ed., Tidal Deposits-A Casebook of Recent Examples and Fossil Counterparts: New York, Springer-Verlag, p. 117-125.

McGee, D.C., 1972, Coal reserves, Beluga and Chuitna Rivers and Capps Glacier areas Alaska: Alaska Geological and Geophysical Surveys, 5 p., 3 plates.

Miall, A.D., 1996, The geology of fluvial deposits: sedimentary facies, basin analysis, and petroleum geology: New York, Springer-Verlag, $582 \mathrm{p}$.

Nichols, D.J., 1998, Report on samples from Tyonek Formation facies possibly deposited under marine influence: U.S. Geological Survey Administrative Report, $2 \mathrm{p}$.

Nio, S.D., and Yang, C., 1991, Diagnostic attributes of clastic tidal deposits-A review, in Smith, D.G., Reinson, G.E., Zaitlin, B.A., and Rahmani, R.A., eds., Clastic Tidal Sedimentology: Canadian Society of Petroleum Geology Memoir 16, p. 3-28.

Pemberton, S.G., and Wightman, D.M., 1992, Ichnological characteristics of brackish waster deposits, in Pemberton, S.G., ed., Applications of Ichnology to Petroleum Exploration, A Core Workshop: SEPM (Society for Sedimentary Geology), p. 141-167.

Reineck, H.E., 1967, Layered sediments of tidal flats, beaches, and shelf bottoms of the North Sea, in Lauff. G.H., ed., Estuaries: American Association of Advancement of Sciences, p. 191-206.

Reineck, H.E., and Wunderlich, F., 1968, Classification and origin of flaser and lenticular bedding: Sedimentology, v. 11, p. 99-104.

Reinson, G.E., 1989, Tide-influenced channel deposits in the Lower Cretaceous Glauconitic Member, southern Alberta, in Reinson, G.E., ed., Modern and Ancient Examples of Clastic Tidal Deposits-A Core and Peel Workshop: Calgary, Canadian Society of Petroleum Geologists, Second International Research Symposium on Clastic Tidal Deposits, Calgary, p. 98-104.

Retallack, G.J., 1988, Field recognition of paleosols, in Reinhardt, J., and Sigleo, W.R., eds., Paleosols and Weathering Through Geologic Time: Principles and Applications: Geological Society of America Special Paper 216, p. 1-20.

Selley, R.C., 1978, Ancient sedimentary environments: Ithaca, New York, Cornell University Press, $287 \mathrm{p}$.

Smith, T.N., 1995, Coalbed methane potential for Alaska and drilling results for the Upper Cook Inlet Basin: Intergas ‘95, May 15-19, 1995, Tuscaloosa, Alabama, University of Alabama, p. 1-21.

Smith, T.N., and Clough, J.G., 1993, Coalbed methane potential for Alaska: 1993 American Association of Petroleum Geologists Annual Convention Program Abstracts, p. 184.

Stricker, G.D., 1991, Economic Alaskan coal deposits, in Gluskoter, H.J., Rice D.D., and Taylor, R.B., eds., The Geology of North America: Economic Geology, U.S., v. P-2, p. 591-602.

Visser, M.J., 1980, Neap-spring cycles reflected in Holocene subtidal large-scale bedform deposits: A preliminary note: Geology, v. 8, p. 543-546.

Webb, E.K., 1994, Simulating the three-dimensional distribution of sediment units in braided stream deposits: Journal of Sedimentary Research, B64, p. 219-231. 


\title{
Lower Paleozoic Deep-Water Facies of the Medfra Area, Central Alaska ${ }^{1}$
}

\author{
By Julie A. Dumoulin, Dwight C. Bradley, Anita G. Harris, and John E. Repetski
}

\section{Abstract}

Deep-water facies, chiefly hemipelagic deposits and turbidites, of Cambrian through Devonian age are widely exposed in the Medfra and Mt. McKinley quadrangles. These strata include the upper part of the Telsitna Formation (MiddleUpper Ordovician) and the Paradise Fork Formation (Lower Silurian-Lower Devonian) in the Nixon Fork terrane, the East Fork Hills Formation (Upper Cambrian-Lower Devonian) in the East Fork subterrane of the Minchumina terrane, and the chert and argillite unit (Ordovician) and the argillite and quartzite unit (Silurian-Devonian? and possibly older) in the Telida subterrane of the Minchumina terrane.

In the western part of the study area (Medfra quadrangle), both hemipelagic deposits and turbidites are largely calcareous and were derived from the Nixon Fork carbonate platform. Eastern exposures (Mt. McKinley quadrangle; eastern part of the Telida subterrane) contain much less carbonate; hemipelagic strata are mostly chert, and turbidites contain abundant rounded quartz and lesser plagioclase and potassium feldspar. Deepwater facies in the Medfra quadrangle correlate well with rocks of the Dillinger terrane exposed to the south (McGrath quadrangle), but coeval strata in the Mt. McKinley quadrangle are compositionally similar to rocks to the northeast (Livengood quadrangle). Petrographic data thus suggest that the Telida subterrane as presently defined is an artificial construct made up of two distinct sequences of disparate provenance.

Restoration of 90 and $150 \mathrm{~km}$ of dextral strike-slip on the Iditarod and Farewell faults, respectively, aligns the deep-water strata of the Minchumina and Dillinger terranes in a position east of the Nixon Fork carbonate platform. This restoration supports the interpretation that lower Paleozoic rocks in the Nixon Fork and Dillinger terranes, and in the western part of the Minchumina terrane (East Fork subterrane and western part of the Telida subterrane), formed along a single continental margin.

\footnotetext{
1 During field work in 1998, we discovered a previously unreported belt of SilurianDevonian deep-water facies in the northeastern corner of the Medfra quadrangle. These rocks are described in Dumoulin, J.A., Bradley, D.C., and Harris, A.G., in press, Paleozoic strata of the Dyckman Mountain area, northeastern Medfra quadrangle, Alaska, in Kelley, K.D., and Gough, L.P., eds., Geologic Studies in Alaska by the U.S. Geological Survey, 1998: U.S. Geological Survey Professional Paper 1615.
}

Rocks in the eastern part of the Telida subterrane are compositionally distinct from those to the west and may have had a different origin and history.

\section{Introduction}

Lower Paleozoic rocks deposited in deep-water, off-platform settings occur widely throughout central Alaska. In this paper we describe the lithofacies, biostratigraphy, depositional environments, and regional correlation of Upper Cambrian through Lower Devonian deep-water strata exposed in parts of the Medfra and Mt. McKinley 1:250,000 quadrangles (figs. 1, 2 ). These rocks have been variously correlated but have received little detailed study. They were assigned to the Nixon Fork and Minchumina terranes by Patton and others (1994) but were included in the White Mountain sequence of the Farewell terrane by Decker and others (1994).

The terminology used for lower Paleozoic rocks in central Alaska is confusing and contentious. Deep-water strata in the McGrath and Lime Hills quadrangles south of the Medfra area, considered part of the White Mountain sequence by Decker and others (1994), have also been called the Dillinger terrane (Jones and others, 1981; Silberling and others, 1994) or sequence (Gilbert and Bundtzen, 1984). Coeval platform facies in this area, called Nixon Fork terrane or sequence by other authors (Silberling and others, 1994; Gilbert and Bundtzen, 1984), are included by Decker and others (1994) in their White Mountain sequence. We agree with Decker and others (1994) that lower Paleozoic strata in their Farewell terrane probably formed along a single continental margin but find their terminology for subdivisions of the Farewell awkward and inadequate. In this paper we follow the terrane terminology of Silberling and others (1994) except where noted and refer to lower Paleozoic deep-water facies in the McGrath and Lime Hills quadrangles as the "Dillinger terrane" (fig. 1). We use terrane, however, in the older sense of the word to indicate a belt of related rocks and not as redefined (e.g., Jones and others, 1981) to require fault boundaries and imply "exotic" origins for these belts.

Our lithologic and biostratigraphic data indicate that lower Paleozoic deep-water facies of the Medfra quadrangle correlate relatively well with deep-water facies of the Dillinger terrane 


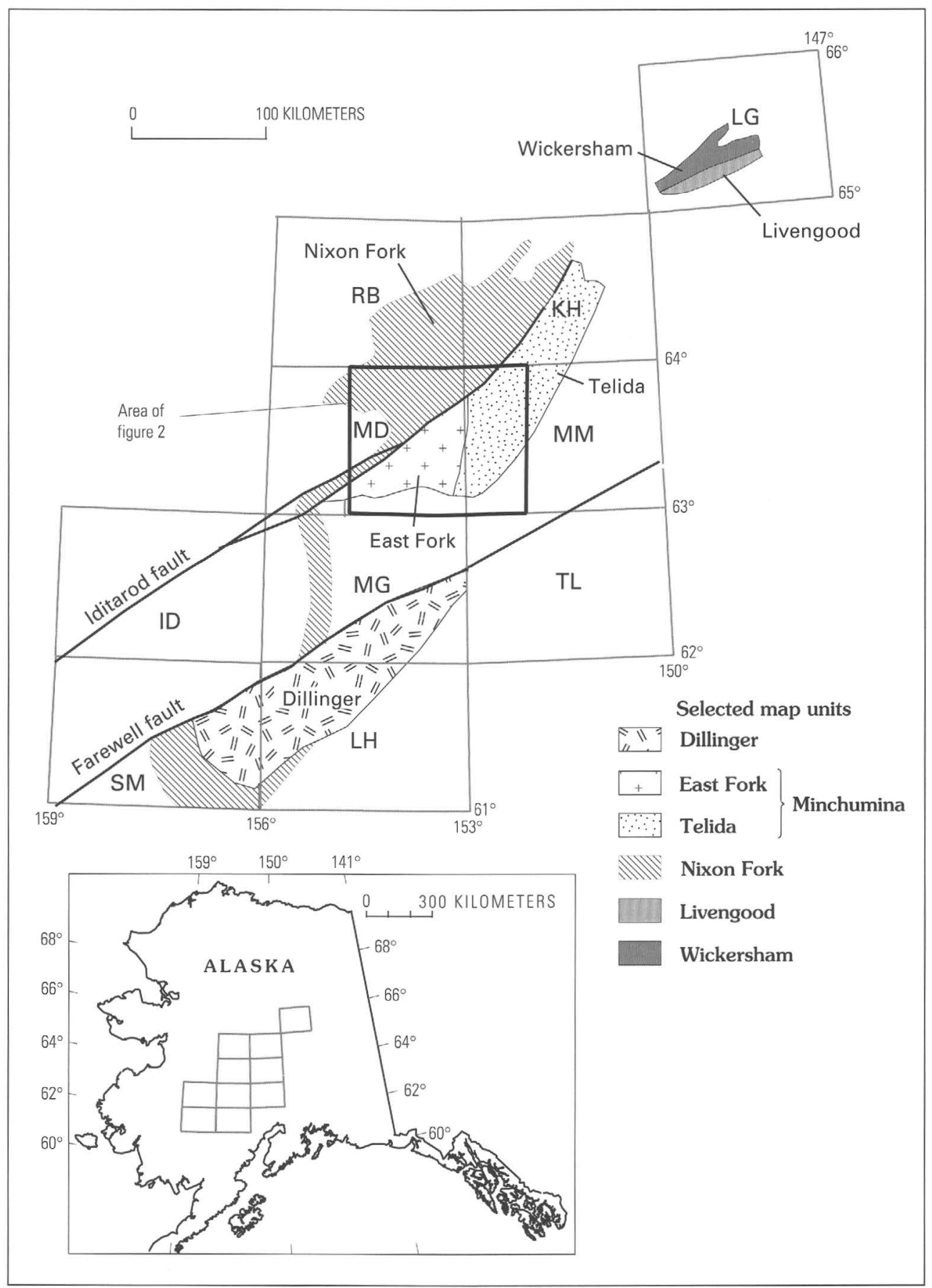

Figure 1. Location of quadrangles and selected tectonostratigraphic terranes and subterranes mentioned in text; East Fork and Telida are subterranes of Minchumina terrane. Dillinger and Nixon Fork south of lat $63^{\circ} \mathrm{N}$. modified from Decker and others (1994) and Silberling and others (1994); East Fork, Nixon Fork north of lat $63^{\circ} \mathrm{N}$., and Telida from Patton and others (1994); Livengood and Wickersham from Silberling and others (1994). The Farewell terrane of Decker and others (1994) includes the Nixon Fork, Minchumina, and Dillinger terranes shown here. Quadrangles: ID, Iditarod; KH, Kantishna River; LG, Livengood, LH, Lime Hills; MD, Medfra; MG, McGrath; MM, Mt. McKinley; RB, Ruby; SM, Sleetmute; TL, Talkeetna.

exposed to the south (McGrath quadrangle). Coeval deep-water strata in the Mt. McKinley quadrangle have stronger lithologic similarities to rocks of the Wickersham and Livengood terranes to the northeast (Livengood quadrangle).

\section{Previous Work and Methods}

Lower Paleozoic deep-water strata in the Medfra quadrangle were briefly described by Patton and others (1980) and Dutro 


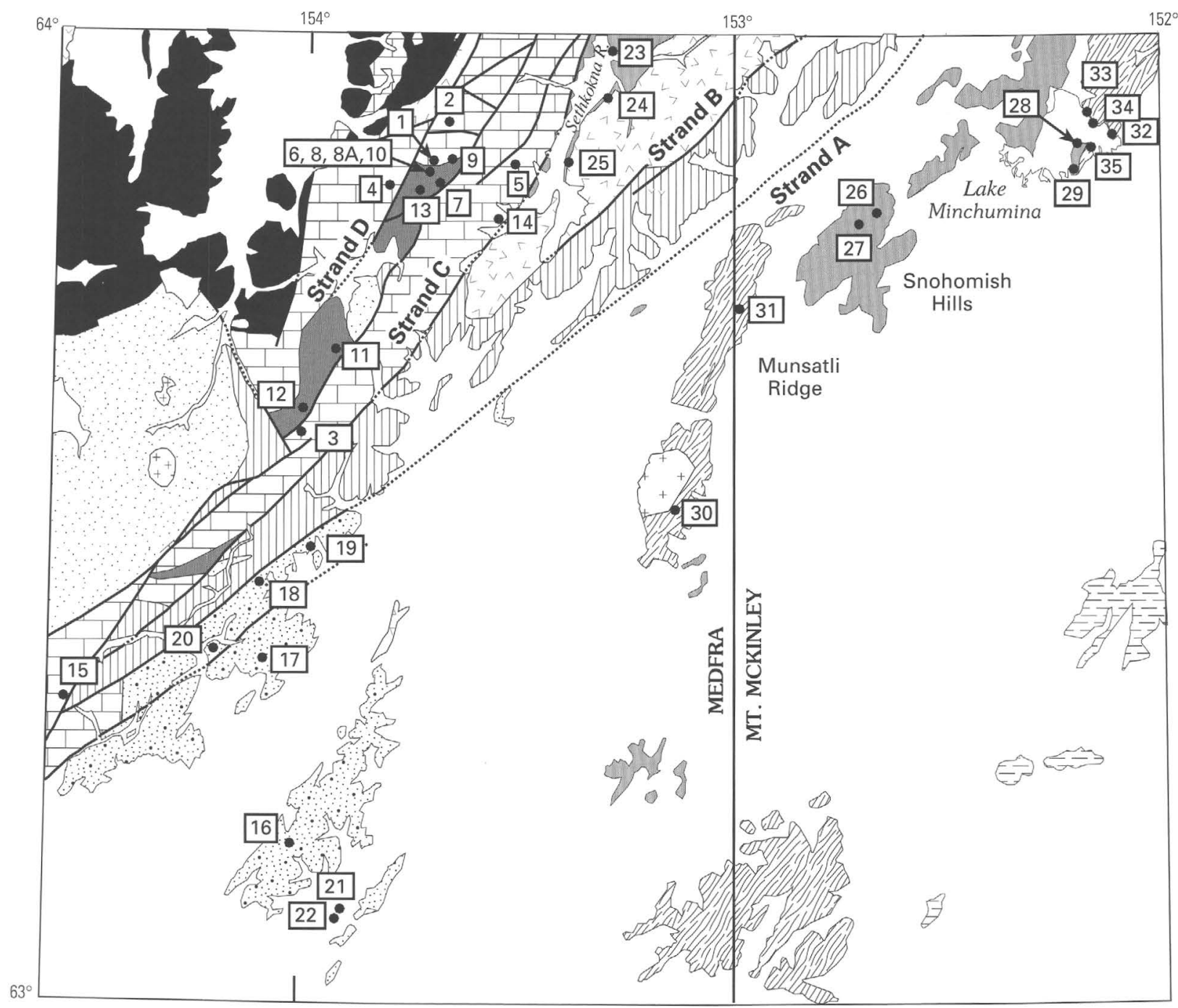

\section{EXPLANATION}

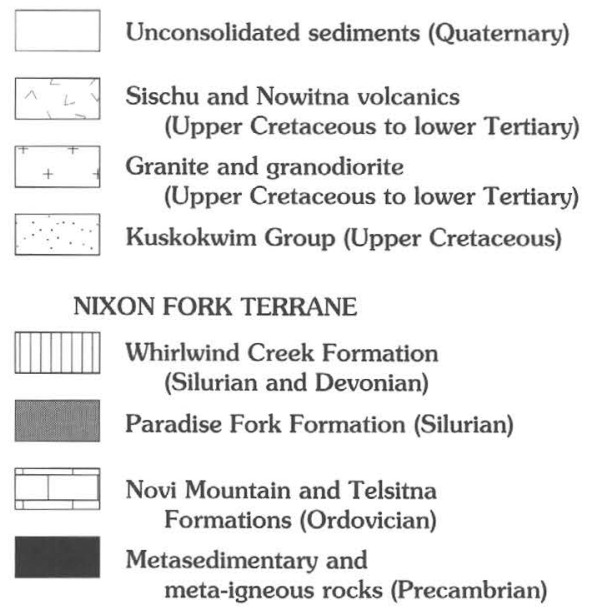

MINCHUMINA TERRANE, EAST FORK SUBTERRANE

East Fork Hills Formation (Cambrian to Devonian)

MINCHUMINA TERRANE, TELIDA SUBTERRANE

Chert and argillite unit (Ordovician)

QIIIT Argillite and quartzite unit

(Proterozoic to Devonian)

\section{YUKON-TANANA TERRANE}

Metamorphic rocks, undivided (Proterozoic to Devonian)

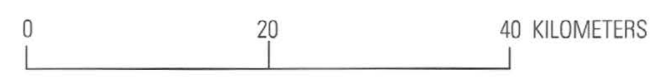

Figure 2. Location of lithologic and fossil collections and structural data from study area in Medfra (MD) and Mt. McKinley (MM) quadrangles. Geologic mapping from Patton and others (1980) (MD) and Wilson and others (1998) (MM). Letters A, B, C, and D refer to strands of the Iditarod fault system mentioned in text. Outcrops between Iditarod fault strands B and C shown here as chert and argillite unit were mapped as Pzc by Patton and others (1980). Novi Mountain and Telsitna Formations as shown include outcrops of Whirlwind Creek Formation too small to show at scale of map. Newly recognized deep-water facies mentioned in footnote on first page of this article are located between localities 25 and 31 in belt shown as Whirlwind Creek Formation. 
and Patton (1982); sparse lithologic and fossil data from correlative rocks in the Mt. McKinley and Kantishna River quadrangles were reported by Chapman and others (1975), Chapman and Yeend (1981), Chapman and others (1981), and Patton and others (1994).

We examined lower Paleozoic rocks in the Medfra area at 36 localities. Microlithofacies were established through field observations and study of about 80 thin sections. Conodont age and biofacies determinations are based on 14 new collections and 13 older, unpublished collections reexamined for this paper (table 1). Interpretations of depositional environments follow models in Wilson (1975), Cook and others (1983), and Scholle and others (1983).

\section{Lithofacies, Age, and Depositional Environment}

Deep-water strata described here belong to three formations (Dutro and Patton, 1982) and several unnamed map units (Patton and others, 1980; Chapman and Yeend, 1981) that have been grouped into two terranes (Patton and others, 1994). We follow below the terrane terminology of Patton and others (1994) unless otherwise noted.

\section{Nixon Fork Terrane}

Precambrian through Mesozoic strata of the Nixon Fork terrane form a southwest-trending belt in the eastern and central Medfra quadrangle (fig. 1). Some 5,500 m of Ordovician through Devonian rocks in this terrane comprise a chiefly platform carbonate succession interrupted by an interval of deeper water facies. Four formations are recognized (Dutro and Patton, 1982); deep-water strata occur in the uppermost part of the Telsitna Formation (Ordovician) and throughout the Paradise Fork Formation (Silurian and Lower Devonian). The Novi Mountain Formation (Lower Ordovician) and the Whirlwind Creek Formation (Upper Silurian and Devonian) consist exclusively of shallow-water facies. In this report, we focus on deep-water facies within the lower Paleozoic succession. The Nixon Fork terrane is cut by a series of northeast-striking faults (Patton and others, 1980); details of stratigraphy, lithofacies, and thermal history differ across these faults, as will be detailed below.

\section{Telsitna Formation}

The Telsitna Formation consists of about 2,000 $\mathrm{m}$ of limestone and lesser dolostone (Dutro and Patton, 1982) deposited largely in supratidal to shallow subtidal settings (Measures and others, 1992). In a section just south of the type section (fig. 2, loc. 1), the uppermost part of the Telsitna is light-gray-weathering, dark-brownish-gray micrite, partly dolomitized, in 10- to 30-cm-thick beds. Stringers and nodules of tan, gray, or black chert, generally a few centimeters thick, parallel bedding. Bioherms a few meters across, made up of corals and (or) stromatoporoids, are locally abundant. These beds contain conodonts of probable Late Ordovician age deposited in a warm, shallowwater setting (table 1, loc. 1; fig. 3A). Lithologically similar strata comprise the upper part of the type section, $4.5 \mathrm{~km}$ to the north (fig. 2, loc. 2). Conodonts near the top of this section are early Late Ordovician (middle Edenian-early Maysvillian) and indicate a tropical, shallow-water environment (table 1, loc. 2). Corals and brachiopods in the uppermost beds in this section are also of Maysvillian age (Dutro and Patton, 1982).

Distinctly different facies, however, characterize the uppermost Telsitna Formation in an elongate fault block southeast of the type section (fig. 2, loc. 3). In this area, several hundred meters of dark-gray-weathering, grayish-black micrite, in platy to irregular beds a few millimeters to $20 \mathrm{~cm}$ thick, forms the top of the Telsitna. Subordinate silt- to sand-sized clasts are disseminated throughout this micrite and are locally concentrated into graded layers a few millimeters thick. Clasts include peloids, calcitized radiolarians, and calcareous and siliceous sponge spicules (some radiolarians and spicules are pyritized) (figs. 3B, $3 C$ ). A few samples contain fragments of pelmatozoans, brachiopods, ostracodes, trilobites, and possible algae. These beds produced three conodont collections; the most diagnostic samples are very latest Ordovician (table 1, loc. 3; figs. 4M-4AA). All collections are distal winnows deposited in a deep-shelf to basinal setting.

Conodonts indicative of a deep-water depositional environment were also recovered from the upper part of the Telsitna Formation in fault blocks west and east of the type area (table 1, locs. 4,5$)$. Strata at both localities are fine-grained, brownishgray, locally bioclastic limestone. The western collection (loc. 4) is late Middle Ordovician; the eastern faunule (loc. 5) is correlative or slightly younger.

Lithologic and paleontologic data thus demonstrate that different sections of the uppermost Telsitna Formation are not precisely coeval and formed in distinctly different depositional environments. Upper Ordovician strata in and south of the type section (table 1, locs. 1 and 2) accumulated in a shallow-water, inner-shelf or platform setting. The slightly younger section at locality 3 consists of hemipelagic sediment derived from a carbonate platform but deposited in a slope or basin environment. Sections at localities 4 and 5 appear intermediate between these two extremes and probably formed in an outer-shelf or outerplatform setting.

Telsitna Formation conodonts from locality 3 have notably higher color alteration indices (CAI's) than do those from the type area (4.5 vs. 2 and 3.5). Similarly sharp contrasts in conodont CAI's from correlative strata occur in other parts of the Nixon Fork terrane (Savage and others, 1995; A.G. Harris and J.E. Repetski, unpub. data, 1997). CAI contrasts - along with the differences in lithofacies, age, and depositional environment documented above - suggest that discrete fault blocks in the Medfra quadrangle preserve fragments of the Nixon Fork platform with divergent depositional and thermal histories. These differences are not confined to Upper Ordovician rocks, as will be seen below.

\section{Paradise Fork Formation}

The Paradise Fork Formation is a sequence of dark-gray, thin-bedded limestone and black shale that forms a southwesttrending, 40-km-long synform through the northeastern part of 
Table 1. Conodont data for localities shown on figure 2.

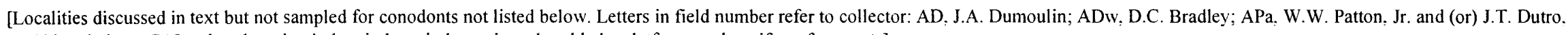
Jr. Abbreviations: CAI, color alteration index; indets., indeterminate bar, blade, platform, and coniform fragments]

\begin{tabular}{|c|c|c|c|c|c|}
\hline $\begin{array}{l}\text { Locality no., } \\
\text { (terrane or } \\
\text { subterrane; } \\
\text { unit) }\end{array}$ & $\begin{array}{l}\text { Quadrangle } \\
\text { latitude/ } \\
\text { longitude }\end{array}$ & $\begin{array}{l}\text { Conodont fauna and CAI } \\
\text { [field no.; USGS collection no.] }\end{array}$ & Age & Biofacies & Remarks \\
\hline $\begin{array}{c}1 \text { (Nixon } \\
\text { Fork; } \\
\text { Telsitna Fm.) }\end{array}$ & $\begin{array}{l}\text { Medfra D-2 } \\
63^{\circ} 51.90^{\prime} \\
153^{\circ} 43.18^{\prime}\end{array}$ & $\begin{array}{l}3 \text { belodinids of probable Late Ordovician } \\
\text { morphotype } \\
7 \text { Panderodus gracilis (Branson \& Mehl) } \\
1 \text { unassigned coniform } \\
\text { CAI }=2 \\
{[97 \mathrm{AD} 14 \mathrm{~B} ; 11504-\mathrm{CO}]}\end{array}$ & $\begin{array}{l}\text { Middle-Late Ordovician, } \\
\text { probably Late Ordovician on } \\
\text { the basis of the belodinid } \\
\text { morphotype. }\end{array}$ & $\begin{array}{l}\text { Indeterminate (too few } \\
\text { conodonts); probably, warm, } \\
\text { shallow-water depositional } \\
\text { environment. }\end{array}$ & $\begin{array}{l}\text { Medium-gray-weathering, light- } \\
\text { gray, fine-grained dolostone } \\
\text { containing abundant } \\
\text { colonial corals. Sample } \\
\text { weight } 8.8 \mathrm{~kg} \text {. }\end{array}$ \\
\hline $\begin{array}{l}2 \text { (Nixon } \\
\text { Fork; } \\
\text { Telsitna Fm.) }\end{array}$ & $\begin{array}{l}\text { Medfra D-2 } \\
63^{\circ} 54.50^{\prime} \\
153^{\circ} 40.50^{\prime}\end{array}$ & $\begin{array}{l}1 \text { belodinid } \\
1 \text { Culumbodina occidentalis Sweet } \\
1 \text { Drepanoistodus sp. indet. } \\
1 \text { Panderodus sp. } \\
4 \text { indet. coniform fragments } \\
\text { CAI }=3.5 \\
{[79 A P a 99 a ; 11524-\mathrm{CO}]}\end{array}$ & $\begin{array}{l}\text { Age is middle Edenian-early } \\
\text { Maysvillian (early-middle } \\
\text { Late Ordovician), probably } \\
\text { early Maysvillian because } \\
\text { corals and brachiopods from } \\
\text { this interval indicate a } \\
\text { Maysvillian age. }\end{array}$ & $\begin{array}{l}\text { Indeterminate (too few } \\
\text { conodonts). Conodonts are } \\
\text { indicative of a tropical } \\
\text { shallow-water depositional } \\
\text { setting. C. occidentalis is the } \\
\text { most biostratigraphically } \\
\text { diagnostic element and a } \\
\text { component of the western } \\
\text { North American Midcontinent } \\
\text { province. }\end{array}$ & $\begin{array}{l}\text { Thick-bedded limestone at } \\
\sim 5,700 \mathrm{ft} \text {. above base of } \\
\text { type section. }\end{array}$ \\
\hline $\begin{array}{c}3 \text { (Nixon } \\
\text { Fork; } \\
\text { Telsitna Fm.) }\end{array}$ & $\begin{array}{l}\text { Medfra C-3 } \\
63^{\circ} 35.89^{\prime} \\
154^{\circ} 01.76^{\prime}\end{array}$ & $\begin{array}{l}1 \text { ozarkodinid P element (fig. } 4 \mathrm{~V} \text { ) } \\
36 \text { Panderodus sp. } \\
\text { Paroistodus? n. sp. A of Nowlan and } \\
\quad \text { others, } 1988 \\
28 \mathrm{M} \& 46 \mathrm{~S} \text { elements (figs. } 4 \mathrm{O}-\mathrm{S} \text { ) } \\
\text { Periodon grandis (Ethington)? } \\
\quad 1 \mathrm{~Pa}, 1 \mathrm{M} \& 2 \text { Sc elements (figs. } 4 \mathrm{~W}- \\
\quad \mathrm{Y} \text { ) } \\
4 \text { Pseudooneotodus mitratus } \\
\quad \text { (Moskalenko) (figs. } 4 \mathrm{M}, \mathrm{N}) \\
13 \text { indet. fragments } \\
6 \text { Strachanognathus parvus } \text { Rhodes (figs. } \\
\quad 4 \mathrm{~T}, \mathrm{U} \text { ) } \\
\text { CAI=4.5 } \\
\text { [97AD34A; 11510-CO) }\end{array}$ & $\begin{array}{l}\text { Age is late Middle-middle Late } \\
\text { Ordovician, probably Late } \\
\text { Ordovician on the basis of the } \\
\text { morphology of the } \\
\text { ozarkodinid P element. }\end{array}$ & $\begin{array}{l}\text { Paroistodid-panderodid biofacies: } \\
\text { faunule represents a distal } \\
\text { (deep shelf to basinal) } \\
\text { winnow. Cosmopolitan and } \\
\text { (or) cool-water faunule. }\end{array}$ & $\begin{array}{l}\text { Dark-gray-weathering, grayish- } \\
\text { black micrite in irregular } \\
20 \text {-cm beds; contains graded } \\
\text { laminae of peloids and } \\
\text { bioclasts (chiefly calcitized } \\
\text { radiolarians and calcareous } \\
\text { and pyritized sponge } \\
\text { spicules, lesser pelmatozoan } \\
\text { and brachiopod fragments). } \\
\text { Sample weight } 10.4 \mathrm{~kg} \text {. }\end{array}$ \\
\hline
\end{tabular}


Table 1. Conodont data for localities shown on figure 2-Continued.

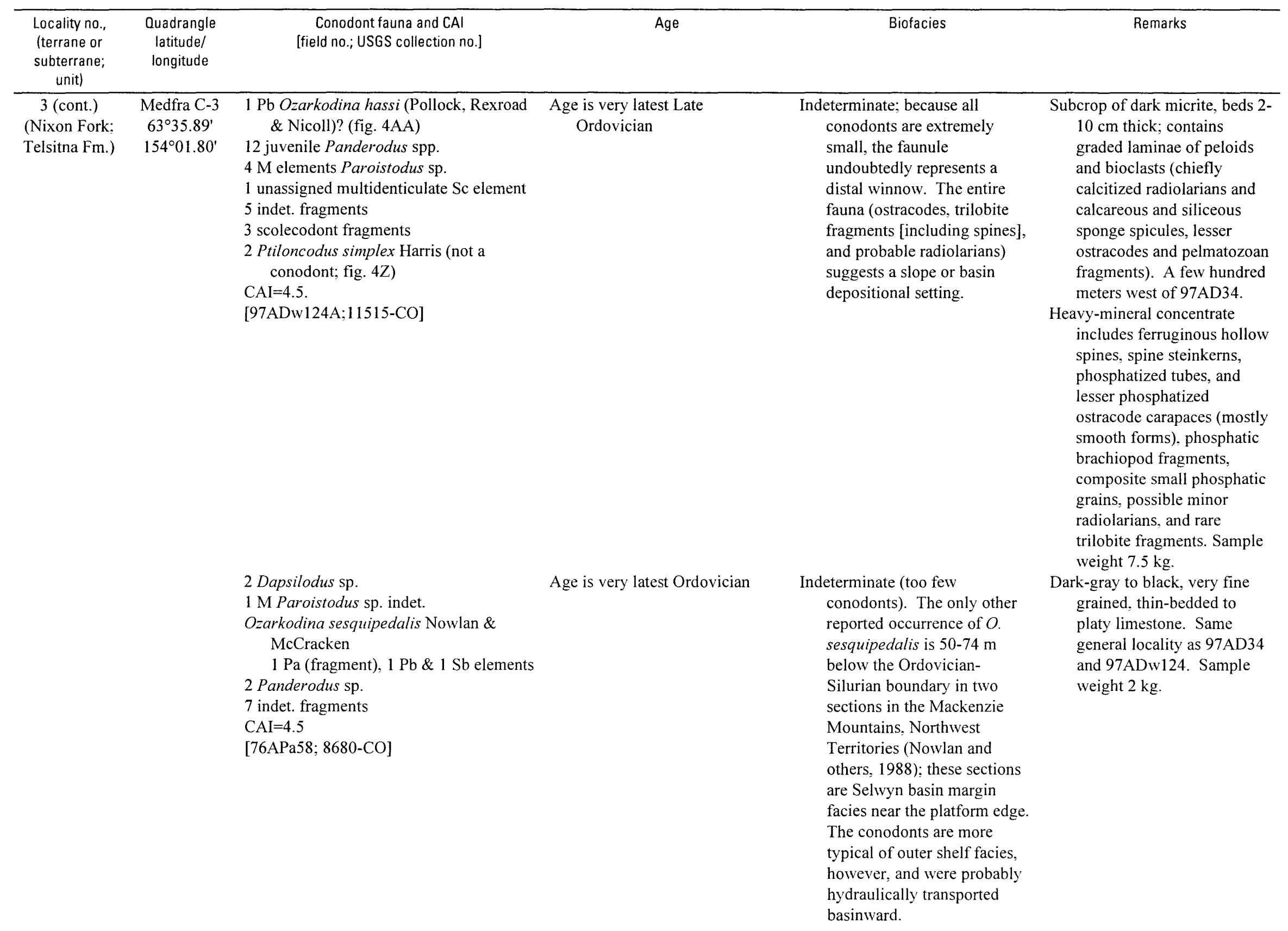


Table 1. Conodont data for localities shown on figure 2-Continued.

\begin{tabular}{|c|c|c|c|c|c|}
\hline $\begin{array}{l}\text { Locality no., } \\
\text { (terrane or } \\
\text { subterrane; } \\
\text { unit) }\end{array}$ & $\begin{array}{l}\text { Quadrangle } \\
\text { latitude/ } \\
\text { longitude }\end{array}$ & $\begin{array}{l}\text { Conodont fauna and CAI } \\
\text { [field no.; USGS collection no.] }\end{array}$ & Age & Biofacies & Remarks \\
\hline $\begin{array}{c}4 \text { (Nixon } \\
\text { Fork; } \\
\text { Telsitna Fm.) }\end{array}$ & $\begin{array}{c}\text { Medfra D-2 } \\
63^{\circ} 50.42^{\prime} \\
153^{\circ} 49.33^{\prime}\end{array}$ & $\begin{array}{l}1 \text { Belodina compressa (Branson \& Mehl) } \\
1 \text { juvenile Belodina } \mathrm{sp} \text {. } \\
2 \text { Paroistodus } \mathrm{sp} \text {. } \\
2 \text { Drepanoistodus } \text { sp. } \\
10 \text { Panderodus unicostatus (Branson \& } \\
\quad \text { Mehl) } \\
2 \text { Paroistodus } \mathrm{sp} \text {. } \\
\text { Periodon aculeatus } \text { Hadding } \\
\quad 3 \mathrm{~Pa}, 4 \mathrm{M}, 1 \mathrm{Sb} \& 4 \mathrm{Sc} \text { element } \\
\quad \text { fragments } \\
1 \text { Staufferella } \text { sp. } \\
1 \text { Strachanognathus parvus } \text { Rhodes } \\
6 \text { unassigned coniform fragments and } \\
\quad \text { juveniles } \\
\text { CAI }=3.5 \\
\text { [76APa34A; } 8660-\mathrm{CO}]\end{array}$ & $\begin{array}{l}\text { Age is late Middle Ordovician; } B \text {. } \\
\text { compressa Zone (late } \\
\text { Blackriveran, late early } \\
\text { Caradocian) }\end{array}$ & $\begin{array}{l}\text { Mixed biofacies but chiefly } \\
\text { pelagic realm cosmopolitan } \\
\text { (e.g., Periodon, Dapsilodus, } \\
\text { Strachanognathus)), } \\
\text { pandemic (Panderodus), and } \\
\text { rare tropical cosmopolitan } \\
\text { (Belodina) taxa. Rock } \\
\text { probably represents outer } \\
\text { shelf or off-shelf depositional } \\
\text { setting. }\end{array}$ & $\begin{array}{l}\text { Light-gray to brownish-gray, } \\
\text { thin-bedded, fine-grained } \\
\text { limestone. Sample weight } 2 \\
\text { kg. }\end{array}$ \\
\hline $\begin{array}{l}4 \text { (Nixon } \\
\text { Fork; } \\
\text { Whirlwind } \\
\text { Creek Fm.?) }\end{array}$ & $\begin{array}{l}\text { Medfra D-2 } \\
63^{\circ} 52.11^{\prime} \\
153^{\circ} 50.32^{\prime}\end{array}$ & $\begin{array}{l}\text { Ozarkodina confluens (Branson \& Mehl) } \\
\quad 6 \mathrm{~Pa}, 1 \mathrm{~Pb}, 1 \mathrm{M}, 1 \mathrm{Sa}, 1 \mathrm{Sb} \& 1 \mathrm{Sc} \\
\text { elements } \\
\text { digyrate apparatus (oulodid?) } \\
\quad 2 \mathrm{~Pb} \text { element fragments } \\
17 \text { indet. fragments } \\
\mathrm{CAI}=2.5-3 \\
\text { [97ADw143B; } 12599-\mathrm{SD}]\end{array}$ & $\begin{array}{l}\text { Ludlovian-Pridolian (Late } \\
\text { Silurian and probably not } \\
\text { early Ludlovian) }\end{array}$ & $\begin{array}{l}\text { Indeterminate (too few } \\
\text { conodonts); } O \text {. confluens as } \\
\text { well as the amphiporids } \\
\text { suggest at least partial } \\
\text { restriction. }\end{array}$ & $\begin{array}{l}\text { Amphipora-bearing dolostone } \\
\text { and dolomicrite rubble. } \\
\text { Patton and others (1980) } \\
\text { mapped these rocks as Od, } \\
\text { but the conodonts indicate } \\
\text { Whirlwind Creek } \\
\text { Formation. } 2 \mathrm{~km} \text { northwest } \\
\text { of, and apparently } \\
\text { overlying, 76APA34A. } \\
\text { Sample weight } 9.5 \mathrm{~kg} \text {. }\end{array}$ \\
\hline $\begin{array}{c}5 \text { (Nixon } \\
\text { Fork; } \\
\text { Telsitna Fm.) }\end{array}$ & $\begin{array}{c}\text { Medfra D-2 } \\
63^{\circ} 51.50^{\prime} \\
153^{\circ} 31.33^{\prime}\end{array}$ & $\begin{array}{l}8 \text { Belodina? repens Moskalenko s.f. } \\
5 \text { juvenile belodinids } \\
2 \text { M elements Drepanoistodus sp. } \\
13 \text { Panderodus } \text { sp. } \\
6 \text { Plectodina? tunguskaensis } \\
\quad \text { (Moskalenko) } \\
1 \text { unassigned } \mathrm{Pb} \text { element } \\
\mathrm{CAI}=3.5 \\
{[76 \mathrm{APa} 39 ; 8679-\mathrm{CO}]}\end{array}$ & $\begin{array}{l}\text { Age is late Middle-Late } \\
\text { Ordovician, possibly Late } \\
\text { Ordovician }\end{array}$ & $\begin{array}{l}\text { Indeterminate; likely a distal } \\
\text { winnow into deeper water } \\
\text { facies. The species } \\
\text { association is chiefly } \\
\text { Siberian-Alaskan with minor } \\
\text { pandemics. }\end{array}$ & $\begin{array}{l}\text { Massive-bedded, brownish- } \\
\text { gray, fine-grained to finely } \\
\text { crystalline limestone } \\
\text { containing gastropods, } \\
\text { trilobites, and cephalopods } \\
\text { of probable Ordovician age. } \\
\text { Sample weight } 2 \mathrm{~kg} \text {. }\end{array}$ \\
\hline
\end{tabular}


Table 1. Conodont data for localities shown on figure 2-Continued.

\begin{tabular}{|c|c|c|c|c|c|}
\hline $\begin{array}{l}\text { Locality no., } \\
\text { (terrane or } \\
\text { subterrane; } \\
\text { unit) }\end{array}$ & $\begin{array}{l}\text { Quadrangle } \\
\text { latitude/ } \\
\text { longitude }\end{array}$ & $\begin{array}{c}\text { Conodont fauna and CAI } \\
\text { [field no.; USGS collection no.] }\end{array}$ & Age & Biofacies & Remarks \\
\hline $\begin{array}{c}6 \text { (Nixon } \\
\text { Fork; } \\
\text { Paradise Fork } \\
\text { Fm.) }\end{array}$ & $\begin{array}{c}\text { Medfra D-2 } \\
63^{\circ} 51.80^{\prime} \\
153^{\circ} 43.08^{\prime}\end{array}$ & $\begin{array}{l}120 \text { Panderodus unicostatus (Branson \& } \\
\quad \text { Mehl) } \\
\text { Ozarkodina excavata (Branson \& Mehl) } \\
\quad 1 \mathrm{~Pa}, 1 \mathrm{~Pb} \& 2 \mathrm{Sc} \text { elements } \\
\text { Pterospathodus procerus (Walliser) (figs. } \\
\quad 4 \mathrm{NN}-\mathrm{PP} \text { ) } \\
\quad 7 \mathrm{~Pa} \& 2 \mathrm{~Pb} \text { elements } \\
5 \text { indet. fragments } \\
\mathrm{CAI}=2.5 \\
{[97 \mathrm{ADw} 103 ; 12598-\mathrm{SD}]}\end{array}$ & $\begin{array}{l}\text { Pt. celloni Zone into } K \text {. } \\
\text { ranuliformis Zone (= late } \\
\text { Llandoverian-very early } \\
\text { Wenlockian; = middle Early } \\
\text { Silurian) }\end{array}$ & $\begin{array}{l}\text { Panderodid biofacies: postmortem } \\
\text { transport from or within a mid } \\
\text { shelf or slope depositional } \\
\text { setting. }\end{array}$ & $\begin{array}{l}\text { Dark-gray, finely laminated, } \\
\text { fissile micrite. Overlies } \\
\text { 97AD14 (loc. 1) and } \\
\text { underlies 97AD13C (loc. 8); } \\
\text { near base of formation. } \\
\text { Same general locality as } \\
\text { 77APa1 } 12 \text { (table 2). } \\
\text { Heavy-mineral concentrate } \\
\text { includes pyritized sponge } \\
\text { spicules. Sample weight } 7.9 \\
\text { kg. }\end{array}$ \\
\hline $\begin{array}{c}7 \text { (Nixon } \\
\text { Fork; } \\
\text { Paradise Fork } \\
\text { Fm.) }\end{array}$ & $\begin{array}{c}\text { Medfra D-2 } \\
63^{\circ} 50.92^{\prime} \\
153^{\circ} 41.33^{\prime}\end{array}$ & $\begin{array}{l}1 \text { incomplete, robust, hyaline, coniform- } \\
\text { like Sc element of Middle Ordovician- } \\
\text { Silurian morphotype } \\
\text { CAI=2 or } 3 \\
\text { [97AD8B] }\end{array}$ & Middle Ordovician-Silurian & $\begin{array}{l}\text { Indeterminate (too few } \\
\text { conodonts). }\end{array}$ & $\begin{array}{l}\text { Light-gray-weathering, grayish- } \\
\text { black, fine-grained } \\
\text { limestone in beds } 2-5 \mathrm{~cm} \\
\text { thick, with graded laminae } \\
\text { of silt- to sand-sized micritic } \\
\text { clasts. Upper part of unit. } \\
\text { Heavy-mineral concentrate } \\
\text { includes minor schistose } \\
\text { (chloritic-muscovitic) } \\
\text { lithoclasts. Sample weight } \\
9.7 \mathrm{~kg} \text {. }\end{array}$ \\
\hline $\begin{array}{c}8 \text { (Nixon } \\
\text { Fork; } \\
\text { Paradise Fork } \\
\text { Fm.) }\end{array}$ & $\begin{array}{c}\text { Medfra D-2 } \\
63^{\circ} 51.67^{\prime} \\
153^{\circ} 43.08^{\prime}\end{array}$ & $\begin{array}{l}\text { Kockelella patula Walliser } \\
\quad 1 \mathrm{~Pa}, 1 \mathrm{~Pb} \& 1 \mathrm{Sb} \text { elements (figs. } \\
\quad 4 \mathrm{QQ}-\mathrm{SS} \text { ) } \\
2 \text { unassigned Sc ( } 2 \text { morphotypes) } \\
\text { elements } \\
4 \text { indet. fragments } \\
\text { CAI=2 } \\
\text { [97AD13; 12594-SD] }\end{array}$ & $\begin{array}{l}\text { K. patula Zone ( }=\text { K. ranuliformis } \\
+K \text {. amsdeni Zones of } \\
\text { Barrick and Klapper, 1976). } \\
\text { K. patula has been found with } \\
\text { Cyrtograptus rigidus Zone } \\
\text { graptolites in the Carnic Alps } \\
\text { (Jaeger, 1975). Kleffner } \\
\text { (1995), on the basis of } \\
\text { graphic correlation, indicates } \\
\text { the range of } K \text {. patula is } \\
\text { within the } C \text {. lundgreni Zone } \\
\text { which, according to him, } \\
\text { occupies most of the middle } \\
\text { Wenlockian. }\end{array}$ & $\begin{array}{l}\text { Indeterminate (too few } \\
\text { conodonts); the kockelellids } \\
\text { were derived from a shelf or } \\
\text { platform depositional } \\
\text { environment. K. patula is } \\
\text { common in Europe but rare in } \\
\text { North America. }\end{array}$ & $\begin{array}{l}\text { Medium-gray-weathering, } \\
\text { grayish-black, finely } \\
\text { laminated micrite with } \\
\text { minor calcitized } \\
\text { radiolarians. Several } \\
\text { hundred meters } \\
\text { stratigraphically above } \\
\text { 97ADw103 (loc. } 6 \text { ). } \\
\text { Heavy-mineral concentrate } \\
\text { includes minor schistose } \\
\text { (chloritic-muscovitic) } \\
\text { lithoclasts. Sample weight } \\
9.7 \mathrm{~kg} \text {. }\end{array}$ \\
\hline
\end{tabular}


Table 1. Conodont data for localities shown on figure 2-Continued.

\begin{tabular}{|c|c|c|c|c|c|}
\hline $\begin{array}{l}\text { Locality no., } \\
\text { (terrane or } \\
\text { subterrane; } \\
\text { unit) }\end{array}$ & $\begin{array}{l}\text { Quadrangle } \\
\text { latitude/ } \\
\text { longitude }\end{array}$ & $\begin{array}{l}\text { Conodont fauna and CAI } \\
\text { [field no.; USGS collection no.] }\end{array}$ & Age & Biofacies & Remarks \\
\hline $\begin{array}{l}\text { 8A (Nixon } \\
\text { Fork; } \\
\text { Paradise Fork } \\
\text { Fm.) }\end{array}$ & $\begin{array}{c}\text { Medfra D-2 } \\
63^{\circ} 51.50^{\prime} \\
153^{\circ} 42.50^{\prime}\end{array}$ & $\begin{array}{l}7 \text { juvenile Dapsilodus } \text { sp. indet. elements } \\
1 \text { juvenile Sb? element } \text { Oulodus? sp. } \\
\text { indet. } \\
1 \text { Pa element fragment Ozarkodina } \\
\text { excavata (Branson \& Mehl)? } \\
1 \text { Panderodus unicostatus (Branson \& } \\
\text { Mehl) element } \\
1 \text { juvenile Pa element Polygnathus sp. } \\
\text { indet. of late Emsian or younger } \\
\text { Devonian morphotype } \\
1 \text { Pseudooneotodus bicornis Drygant } \\
\text { element } \\
\text { CAI=2-2.5 } \\
\text { [98ADw206; 12616-SD] }\end{array}$ & $\begin{array}{l}\text { Conodonts in this sample } \\
\text { represent two ages: late } \\
\text { Llandoverian to at least latest } \\
\text { Ludlovian (Ps. bicornis) and } \\
\text { late Emsian or even younger } \\
\text { Devonian (juvenile } \\
\text { polygnathid). Ps. bicornis has } \\
\text { recently been shown to range } \\
\text { at least into the } O z \text {. } \\
\text { remscheidensis Zone in } \\
\text { Sardinia (Corradini and } \\
\text { others, 1998); until the } \\
\text { Sardininian report the species } \\
\text { had not been reported above } \\
\text { the Wenlockian (Kleffner, } \\
\text { 1995). About } 20 \% \text { of the } \\
\text { slabs in 98ADw206 contained } \\
\text { the graptolite Monograptus } \\
\text { uniformis of early } \\
\text { Lochkovian age (table 2). } \\
\text { We interpret this sample as a } \\
\text { mix of Llandoverian- } \\
\text { Ludlovian, early Lochkovian, } \\
\text { and late Emsian (or younger } \\
\text { Devonian) weakly laminated } \\
\text { limestones. }\end{array}$ & $\begin{array}{l}\text { Indeterminate (too few } \\
\text { conodonts); those that are } \\
\text { present are mainly extremely } \\
\text { small coniform elements that } \\
\text { indicate a distal winnow. The } \\
\text { conodonts and graptolites } \\
\text { from this collection indicate } \\
\text { mixed ages, making the } \\
\text { sample ineligible for biofacies } \\
\text { analysis. }\end{array}$ & $\begin{array}{l}\text { Dark gray, weakly laminated } \\
\text { calcisiltstone with rare } \\
\text { micrite clasts and small } \\
\text { fossil fragments. Sample } \\
\text { weight } 8.1 \text { kg. } \\
\text { Heavy-mineral concentrate } \\
\text { includes minor } \\
\text { metacarbonate rock } \\
\text { fragments (talc-actinolite- } \\
\text { tremolite) and rare } \\
\text { phosphatic brachiopod } \\
\text { fragments. }\end{array}$ \\
\hline $\begin{array}{l}11 \text { (Nixon } \\
\text { Fork; } \\
\text { Paradise Fork } \\
\text { Fm.) }\end{array}$ & $\begin{array}{l}\text { Medfra C-2 } \\
63^{\circ} 40.33^{\prime} \\
153^{\circ} 57.52^{\prime}\end{array}$ & $\begin{array}{l}\text { Kockelella sp. indet. or another Silurian } \\
\quad \text { digyrate apparatus } \\
\quad 3 \mathrm{~Pb}, 3 \mathrm{M} \& 2 \mathrm{Sb} \text { elements } \\
\text { CAI }=2.5-3 \\
{[77 \mathrm{APa} 130 ; 9145-\mathrm{CO}]}\end{array}$ & $\begin{array}{l}\text { Silurian, late Llandoverian into } \\
\text { late Ludlovian }\end{array}$ & $\begin{array}{l}\text { Indeterminate (too few } \\
\text { conodonts). Normal-marine } \\
\text { depositional setting. }\end{array}$ & $\begin{array}{l}\text { Brownish-gray limestone. } \\
\text { Sample weight } 2.5 \mathrm{~kg} .\end{array}$ \\
\hline $\begin{array}{l}12 \text { (Nixon } \\
\text { Fork; } \\
\text { Paradise Fork } \\
\text { Fm.) }\end{array}$ & $\begin{array}{l}\text { Medfra C-3 } \\
63^{\circ} 37^{\prime} \\
154^{\circ} 00.75^{\prime}\end{array}$ & $\begin{array}{l}3 \text { Panderodus } \mathrm{sp} \text {. } \\
10 \text { indet. coniform fragments } \\
\mathrm{CAI}=4.5 \\
{[77 \mathrm{APa} 134 ; 9146-\mathrm{CO}]}\end{array}$ & $\begin{array}{l}\text { Middle Ordovician-Silurian } \\
\text { (other coniform elements are } \\
\text { not Devonian morphotypes) }\end{array}$ & $\begin{array}{l}\text { Indeterminate (too few } \\
\text { conodonts). }\end{array}$ & $\begin{array}{l}\text { Light-brownish-gray limestone. } \\
\text { Sample weight } 2.7 \mathrm{~kg} \text {. }\end{array}$ \\
\hline
\end{tabular}


Table 1. Conodont data for localities shown on figure 2-Continued.

\begin{tabular}{|c|c|c|c|c|c|}
\hline $\begin{array}{l}\text { Locality no., } \\
\text { (terrane or } \\
\text { subterrane; } \\
\text { unit) }\end{array}$ & $\begin{array}{c}\text { Quadrangle } \\
\text { latitude/ } \\
\text { longitude }\end{array}$ & $\begin{array}{l}\text { Conodont fauna and CAI } \\
\text { [field no.; USGS collection no.] }\end{array}$ & Age & Biofacies & Remarks \\
\hline \multirow[t]{2}{*}{$\begin{array}{l}13 \text { (Nixon } \\
\text { Fork; } \\
\text { Whirlwind } \\
\text { Creek Fm.) }\end{array}$} & $\begin{array}{c}\text { Medfra D-2 } \\
63^{\circ} 50.23^{\prime} \\
153^{\circ} 44.47^{\prime}\end{array}$ & $\begin{array}{l}\text { BARREN } \\
\text { [97AD12] }\end{array}$ & & & $\begin{array}{l}\text { Thick-bedded to massive, light- } \\
\text { gray, vuggy dolostone, } \\
\text { locally cherty, with molds } \\
\text { of brachiopods(?), underlies } \\
\text { Amphipora-bearing } \\
\text { dolostone. Mapped by } \\
\text { Patton and others (1980) as } \\
\text { Paradise Fork Formation } \\
\text { [Sls] but represents shallow- } \\
\text { water facies. Lies just } \\
\text { above Sls. Sample weight } \\
9.1 \text { kg. }\end{array}$ \\
\hline & $\begin{array}{c}\text { Medfra D-2 } \\
63^{\circ} 50.08^{\prime} \\
153^{\circ} 45.30^{\prime}\end{array}$ & $\begin{array}{l}1 \text { Dapsilodus sp. element } \\
2 \text { Sb elements of "Ligonodina" confluens } \\
\quad \text { confluens of Jeppsson (1972) } \\
\text { Ozarkodina confluens (Branson \& Mehl) } \\
\quad \text { (at least } 3 \text { morphotypes) } \\
\quad 19 \mathrm{~Pa}, 7 \mathrm{~Pb}, \& 2 \text { Sb elements } \\
\text { Ozarkodina excavata (Branson \& Mehl) } \\
\quad 1 \text { Sa and } 1 \text { Sb elements } \\
4 \text { Panderodus unicostatus (Branson \& } \\
\text { Mehl) elements } \\
\text { CAI=2.5 } \\
\text { [98ADw211; 12617-SD] }\end{array}$ & $\begin{array}{l}\text { Late Silurian; within } A \text {. } \\
\text { ploeckensis Zone to very high } \\
\text { in the } O z \text {. remscheidensis } \\
\text { Zone (middle Gorstian } \\
\text { [=within early Ludlovian] to } \\
\text { within the late Pridolian) }\end{array}$ & $\begin{array}{l}\text { Ozarkodinid biofacies; these } \\
\text { conodonts were deposited in } \\
\text { the shallow part of the } \\
\text { ozarkodinid biofacies, as } O z \text {. } \\
\text { confluens is chiefly a shallow- } \\
\text { water species. }\end{array}$ & $\begin{array}{l}\text { Thick-bedded, finely crystalline } \\
\text { dolostone; } 1 \mathrm{~km} \text { west of, } \\
\text { and at a stratigraphic level } \\
\text { close to, } 97 \mathrm{AD} 12 . \text { Sample } \\
\text { weight } 10.3 \mathrm{~kg} \text {. }\end{array}$ \\
\hline $\begin{array}{l}14 \text { (Nixon } \\
\text { Fork; } \\
\text { Whirlwind } \\
\text { Creek Fm.) }\end{array}$ & $\begin{array}{c}\text { Medfra D-2 } \\
63^{\circ} 48.80^{\prime} \\
153^{\circ} 33.27^{\prime}\end{array}$ & $\begin{array}{l}1 \mathrm{~Pb} \text { element fragment Oulodus sp. indet. } \\
\text { Ozarkodina remscheidensis (Ziegler) } \\
8 \mathrm{~Pa} \text { elements (mostly incomplete) } \\
\text { Ozarkodina confluens (Branson \& Mehl) } \\
\text { or Pandorinellina optima } \\
\text { (Moskalenko) } \\
7 \mathrm{~Pa} \text { elements (mostly incomplete) } \\
\text { Ozarkodina spp. indet. } \\
2 \mathrm{~Pa}, 3 \mathrm{~Pb} \text { ( } 2 \text { morphotypes), } 4 \mathrm{M}, 2 \\
\mathrm{Sa}, 10 \mathrm{Sb} \& 5 \mathrm{Sc} \text { elements } \\
99 \text { chiefly incomplete Panderodus spp. } \\
\text { elements } \\
174 \text { indet. fragments } \\
\mathrm{CAI}=4\end{array}$ & $\begin{array}{l}\text { Age is late Ludlovian-Pridolian } \\
\text { (Late but not earliest Late } \\
\text { Silurian) or late Lochkovian } \\
\text { (late early Early Devonian); } \\
\text { probably the Silurian range } \\
\text { because of the variety of } O \text {. } \\
\text { remscheidensis morphotypes } \\
\text { and because the } 7 \text { Pa elements } \\
\text { attributed to either } O \text {. } \\
\text { confluens (a Late Silurian } \\
\text { species) or Pa. optima (a late } \\
\text { Lochkovian species) are more } \\
\text { like } O \text {. confluens. }\end{array}$ & $\begin{array}{l}\text { Panderodid biofacies; the } \\
\text { panderodids and the type of } \\
\text { ozarkodinids indicate a } \\
\text { shallow-water depositional } \\
\text { setting. The abundant but } \\
\text { chiefly broken conodonts } \\
\text { indicate a high-energy setting. }\end{array}$ & $\begin{array}{l}\text { Skeletal packstone in } 3-20 \mathrm{~cm} \\
\text { beds; about 2-3 m of section } \\
\text { here. Mapped by Patton } \\
\text { and others (1980) as } \\
\text { Telsitna Ridge Formation } \\
\text { [Od], but the conodonts } \\
\text { suggest this sample is from } \\
\text { the Whirlwind Creek } \\
\text { Formation. Strata appear to } \\
\text { overlie Od. Sample weight } \\
9.9 \mathrm{~kg} \text {. }\end{array}$ \\
\hline
\end{tabular}


Table 1. Conodont data for localities shown on figure 2-Continued.

\begin{tabular}{|c|c|c|c|c|c|}
\hline $\begin{array}{l}\text { Locality no., } \\
\text { (terrane or } \\
\text { subterrane; } \\
\text { unit) }\end{array}$ & $\begin{array}{l}\text { Quadrangle } \\
\text { latitude/ } \\
\text { longitude }\end{array}$ & $\begin{array}{l}\text { Conodont fauna and CAI } \\
\text { [field no.; USGS collection no.] }\end{array}$ & Age & Biofacies & Remarks \\
\hline $\begin{array}{l}15 \text { (Nixon } \\
\text { Fork; } \\
\text { Whirlwind } \\
\text { Creek Fm.) }\end{array}$ & $\begin{array}{c}\text { Medfra B-4 } \\
63^{\circ} 18.67^{\prime} \\
154^{\circ} 34.17^{\prime}\end{array}$ & $\begin{array}{l}\text { Oulodus } \mathrm{n} \text {. sp. } \\
2 \mathrm{~Pa}, 1 \mathrm{~Pb}, 4 \mathrm{Sa}, 2 \mathrm{Sb} \& 2 \mathrm{Sc} \text { elements } \\
\text { (distinctive oulodid with outer lower } \\
\text { margin of cusp extended as prong on } \\
\text { the } \mathrm{Pa}, \mathrm{Sa} \& \mathrm{Sb} \text { elements) } \\
\text { Ozarkodina confluens (Branson \& Mehl)? } \\
\quad 1 \mathrm{~Pa}, 2 \mathrm{~Pb}, 3 \mathrm{M}, 1 \mathrm{Sa} \& 6 \mathrm{Sc} \text { elements } \\
4 \text { indet. fragments } \\
\mathrm{CAI}=3.5 \\
\text { [79APa82B; } 11994-\mathrm{SD}]\end{array}$ & $\begin{array}{l}\text { Wenlockian-middle Pridolian } \\
\text { (late Early-Late, but not } \\
\text { latest, Silurian.) }\end{array}$ & $\begin{array}{l}\text { Oulodid-ozarkodinid biofacies: } \\
\text { shallow-water, relatively high } \\
\text { energy depositional setting. }\end{array}$ & $\begin{array}{l}\text { Limestone in thrust sheet at } \\
\text { Limestone Hill, mapped as } \\
\text { Telsitna Formation [Od] by } \\
\text { Patton and others (1980). } \\
\text { Sample weight } 6.0 \mathrm{~kg} \text {. }\end{array}$ \\
\hline \multirow[t]{2}{*}{$\begin{array}{l}16 \text { (East } \\
\text { Fork; East } \\
\text { Fork Hills } \\
\text { Fm.) }\end{array}$} & $\begin{array}{c}\text { Medfra A-3 } \\
63^{\circ} 09.25^{\prime} \\
154^{\circ} 02.29^{\prime}\end{array}$ & $\begin{array}{l}2 \text { Cordylodus proavus Müller } \\
1 \text { Hirsutodontus hirsutus } \text { Miller } \\
2 \text { indet. coniform elements } \\
\text { CAI=5 } \\
{[97 \mathrm{AD} 30 \mathrm{~B} ; 11508-\mathrm{CO}]}\end{array}$ & $\begin{array}{l}\text { Age is latest Late Cambrian ( } C o \text {. } \\
\text { proavus Zone through } \\
\text { succeeding } C o \text {. intermedius } \\
\text { Zone) }\end{array}$ & $\begin{array}{l}\text { Indeterminate (too few } \\
\text { conodonts). }\end{array}$ & $\begin{array}{l}\text { Subcrop of medium-gray- } \\
\text { weathering, medium-gray, } \\
\text { fine-grained limestone in } \\
\text { beds } 0.5 \text { to } 5 \mathrm{~cm} \text { thick with } \\
\text { grayish-orange dolomitic } \\
\text { layers, parallel- and cross- } \\
\text { laminated. Minor quartz } \\
\text { and plagioclase silt. Type } \\
\text { section of East Fork Hills } \\
\text { Formation. } \\
\text { Heavy-mineral concentrate } \\
\text { includes muscovite, } \\
\text { lithoclasts, and rare } \\
\text { phosphatic brachiopod } \\
\text { fragments. Sample weight } \\
7.5 \mathrm{~kg} \text {. }\end{array}$ \\
\hline & & $\begin{array}{l}3 \text { indet. coniform elements } \\
\mathrm{CAI}=4.5 \\
{[\mathrm{D} 8-26]}\end{array}$ & Age is latest Cambrian-Devonian & $\begin{array}{l}\text { Indeterminate (too few } \\
\text { conodonts). }\end{array}$ & $\begin{array}{l}\text { Schistose limestone. Same } \\
\text { general locality as } \\
\text { 97AD30B. Sample weight } \\
2.9 \mathrm{~kg} \text {. }\end{array}$ \\
\hline
\end{tabular}


Table 1. Conodont data for localities shown on figure 2-Continued.

\begin{tabular}{|c|c|c|}
\hline $\begin{array}{c}\text { Locality no., } \\
\text { (terrane or } \\
\text { subterrane; } \\
\text { unit) }\end{array}$ & $\begin{array}{c}\text { Quadrangle } \\
\text { latitude/ } \\
\text { longitude }\end{array}$ & $\begin{array}{l}\text { Conodont fauna and CAI } \\
\text { [field no.; USGS collection no.] }\end{array}$ \\
\hline $\begin{array}{l}17 \text { (East } \\
\text { Fork; East } \\
\text { Fork Hills } \\
\text { Fm.) }\end{array}$ & $\begin{array}{c}\text { Medfra B-3 } \\
63^{\circ} 21.10^{\prime} \\
154^{\circ} 06.44^{\prime}\end{array}$ & $\begin{array}{l}\text { Many conodonts are deformed. } \\
31 \text { Cordylodus proavus Müller (fig. 4D) } \\
9 \text { Eoconodontus notchpeakensis (Miller) } \\
\quad \text { (fig. 4E) } \\
22 \text { Hirsutodontus hirsutus Miller (figs. } \\
\quad \text { 4A-C) } \\
57 \text { Teridontus nakamurai (Nogami) (fig. } \\
\quad \text { 4F) } \\
109 \text { indet. and unassigned fragments } \\
\text { CAI=5-5.5 } \\
\text { [97AD32C; } 11509-\mathrm{CO}] \\
1 \text { Cordylodus proavus Müller } \\
1 \text { Teridontus nakamurai (Nogami) } \\
\text { CAI=about } 5 \\
\text { [P5-27; 8841-CO] }\end{array}$ \\
\hline $\begin{array}{c}18 \text { (East } \\
\text { Fork; East } \\
\text { Fork Hills } \\
\text { Fm.) }\end{array}$ & $\begin{array}{c}\text { Medfra B-3 } \\
63^{\circ} 25.99^{\prime} \\
154^{\circ} 07.40^{\prime}\end{array}$ & $\begin{array}{l}\text { Ansella } \mathrm{sp} \text {. indet. } \\
1 \mathrm{~Pa}, 1 \mathrm{M}, 1 \mathrm{Sb} \& 1 \mathrm{Sc} \text { elements } \\
2 \text { Belodina } \mathrm{sp} . \\
3 \text { Drepanoistodus } \mathrm{sp} . \\
\text { Erraticodon balticus } \text { Dzik } \\
1 \mathrm{~Pa}, 2 \mathrm{M}, 2 \mathrm{Sb} \& 1 \mathrm{Sc} \text { elements } \\
13 \text { Panderodus } \mathrm{sp} . \\
2 \text { Paroistodus? mutatus (Rhodes) } \\
\text { Periodon aculeatus } \mathrm{Hadding} \\
\quad 25 \mathrm{~Pa}, 8 \mathrm{~Pb}, 51 \mathrm{M}, 7 \mathrm{Sa}, 26 \mathrm{Sb} \& 27 \\
\text { Sc elements } \\
33 \text { Protopanderodus } \text { cf. } P \text {. varicostatus } \\
\quad \text { (Sweet \& Bergström) } \\
\text { Pygodus anserinus Lamont \& Lindström } \\
3 \mathrm{P} \& 20 \mathrm{~S} \text { elements } \\
1 \text { Spinodus ramosus } \text { (Hadding) } \\
57 \text { indet. fragments } \\
\text { CAI }=4.5 \\
\text { [97AD29C: } 11507-\mathrm{CO}]\end{array}$ \\
\hline
\end{tabular}

Age is latest Late Cambrian (Co.
proavus Zone through
succeeding Co. intermedius
Zone)

Zone)

\section{Age is latest Cambrian-very early Indeterminate (too few} Early Ordovician

\section{Pygodus anserinus Zone (middle} Middle Ordovician
Cordylodid biofacies: fauna

includes chiefly open-marine

pandemic forms (cordylodids,

teridontids, and

eoconodontids) and minor

tropical cosmopolites $(H$.

hirsutus). Outer shelf or

deeper depositional setting. pandemics but would not

likely occur in restricted shallow-water marine deposits.

Periodontid-protopanderodid biofacies: slope to basinal depositional setting.
Good outcrop of gray- and yellow-weathering, very fine grained limestone in $\mathrm{mm}$ to $8-\mathrm{cm}$ beds with parallel- and crosslaminations and climbing ripples. Silty laminae contain dolomite, quartz, plagioclase, white mica, and metamorphic lithic clasts. Sample weight $9.3 \mathrm{~kg}$.

Silty limestone. Same general locality as $97 \mathrm{AD} 32 \mathrm{C}$. Sample weight $2.8 \mathrm{~kg}$.

Medium-gray-weathering, darkgray, fine-grained limestone in 2- to $5-\mathrm{cm}$ beds that are delineated by orange silty laminae: interbedded with tan-weathering, dark-gray to black cherty argillite.

Limestone contains 5-15\% bioclasts, mostly radiolarians (some calcitized) and lesser pelmatozoan fragments.

Heavy-mineral concentrate includes phosphatized grains and bioclasts and phosphatic brachiopod fragments. Sample weight $9.7 \mathrm{~kg}$. 
Table 1. Conodont data for localities shown on figure 2-Continued.

\begin{tabular}{|c|c|c|c|c|c|}
\hline $\begin{array}{l}\text { Locality no., } \\
\text { (terrane or } \\
\text { subterrane; } \\
\text { unit) }\end{array}$ & $\begin{array}{l}\text { Quadrangle } \\
\text { latitude/ } \\
\text { longitude }\end{array}$ & $\begin{array}{l}\text { Conodont fauna and CAI } \\
\text { [field no.; USGS collection no.] }\end{array}$ & Age & Biofacies & Remarks \\
\hline \multirow[t]{2}{*}{$\begin{array}{l}19 \text { (East } \\
\text { Fork; East } \\
\text { Fork Hills } \\
\text { Fm.) }\end{array}$} & $\begin{array}{l}\text { Medfra B-3 } \\
63^{\circ} 28.28 \\
153^{\circ} 59.80^{\prime}\end{array}$ & $\begin{array}{l}1 \text { Panderodus sp. indet. } \\
\text { CAI=indet. } \\
\text { [97AD28A] }\end{array}$ & $\begin{array}{l}\text { Middle Ordovician-Middle } \\
\text { Devonian }\end{array}$ & $\begin{array}{l}\text { Indeterminate (too few } \\
\text { conodonts). }\end{array}$ & $\begin{array}{l}\text { Medium-gray-weathering, } \\
\text { medium-dark-gray, fine- } \\
\text { grained limestone in 1- to 2- } \\
\text { cm beds with yellow. } \\
\text { dolomitic, peloidal laminae. } \\
\text { Sample weight } 9.7 \mathrm{~kg} \text {. }\end{array}$ \\
\hline & & $\begin{array}{l}1 \text { juvenile belodinid } \\
1 \text { juvenile } \text { Panderodus sp. indet. } \\
\text { CAI }=6-6.5 \\
{[D 7-24 ; 8861-\mathrm{CO}]}\end{array}$ & $\begin{array}{l}\text { Age is early, but not earliest, } \\
\text { Middle and Late Ordovician }\end{array}$ & $\begin{array}{l}\text { Indeterminate (too few } \\
\text { conodonts); postmortem } \\
\text { winnow. }\end{array}$ & $\begin{array}{l}\text { Medium-dark-gray limestone. } \\
\text { Same general locality as } \\
\text { 97AD28A. Sample weight } \\
3.0 \mathrm{~kg} .\end{array}$ \\
\hline $\begin{array}{l}20 \text { (East } \\
\text { Fork; East } \\
\text { Fork Hills } \\
\text { Fm.) }\end{array}$ & $\begin{array}{l}\text { Medfra B-3 } \\
63^{\circ} 21^{\prime} \\
154^{\circ} 13^{\prime}\end{array}$ & $\begin{array}{l}3 \text { small coniform elements of an } \\
\quad \text { icriodid(?) or an Early Ordovician } \\
\text { coniform apparatus } \\
\text { CAI }=4.5-5 \\
\text { [78ADu24; } 9977-\mathrm{SD}]\end{array}$ & Ordovician-Devonian & $\begin{array}{l}\text { Indeterminate (too few } \\
\text { conodonts); distal winnow. }\end{array}$ & Sample weight $3.2 \mathrm{~kg}$ \\
\hline $\begin{array}{l}21 \text { (East } \\
\text { Fork; East } \\
\text { Fork Hills } \\
\quad \text { Fm.) }\end{array}$ & $\begin{array}{l}\text { Medfra A-2 } \\
63^{\circ} 05.77^{\prime} \\
153^{\circ} 53.83^{\prime}\end{array}$ & $\begin{array}{l}3 \text { coniform elements Late Ordovician- } \\
\text { earliest Devonian morphotype } \\
1 \mathrm{P} \text { element fragment of Silurian- } \\
\text { Devonian morphotype } \\
\text { CAI }=5.5 \\
{[76 \mathrm{APa} 112 ; 9731-\mathrm{SD}]}\end{array}$ & Silurian-early Early Devonian & $\begin{array}{l}\text { Indeterminate (too few } \\
\text { conodonts); normal-marine } \\
\text { depositional setting. }\end{array}$ & $\begin{array}{l}\text { Dark-gray, laminated, fine- } \\
\text { grained limestone. }\end{array}$ \\
\hline $\begin{array}{l}22 \text { (East } \\
\text { Fork; East } \\
\text { Fork Hills } \\
\quad \text { Fm.) }\end{array}$ & $\begin{array}{c}\text { Medfra A-2 } \\
63^{\circ} 05.13^{\prime} \\
153^{\circ} 54.45^{\prime}\end{array}$ & $\begin{array}{l}1 \mathrm{~Pa} \text { Eognathodus cf. E. sulcatus (Philip) } \\
1 \mathrm{~Pa} \text { Ozarkodina } \text { sp. indet. } \\
\text { CAI=5.5 } \\
{[78 \mathrm{ADu} 27 ; 9978-\mathrm{SD}]}\end{array}$ & Pragian (middle Early Devonian) & $\begin{array}{l}\text { Indeterminate (too few } \\
\text { conodonts); normal-marine } \\
\text { depositional setting. }\end{array}$ & Sample weight $2 \mathrm{~kg}$. \\
\hline
\end{tabular}


Table 1. Conodont data for localities shown on figure 2-Continued.

\begin{tabular}{|c|c|c|c|c|c|}
\hline $\begin{array}{l}\text { Locality no., } \\
\text { (terrane or } \\
\text { subterrane; } \\
\text { unit) }\end{array}$ & $\begin{array}{l}\text { Quadrangle } \\
\text { latitude/ } \\
\text { longitude }\end{array}$ & $\begin{array}{l}\text { Conodont fauna and CAI } \\
\text { [field no.; USGS collection no.] }\end{array}$ & Age & Biofacies & Remarks \\
\hline $\begin{array}{c}23 \text { (Telida; } \\
\text { chert and } \\
\text { argillite unit) }\end{array}$ & $\begin{array}{c}\text { Medfra D-1 } \\
63^{\circ} 59.17^{\prime} \\
153^{\circ} 17.67^{\prime}\end{array}$ & $\begin{array}{l}2 \text { Cordylodus intermedius Furnish (fig. } \\
4 \mathrm{G} \text { ) } \\
6 \text { Drepanoistodus cf. D. pervetus Nowlan } \\
\text { (fig. } 4 \mathrm{H} \text { ) } \\
18 \text { unassigned drepanodontiform elements } \\
18 \text { Laurentoscandodus triangularis } \\
\text { (Furnish) (fig. } 4 \mathrm{~J} \text { ) } \\
1 \text { New genus \& new species? } \\
4 \text { Oneotodus simplex Furnish } \\
3 \text { "Oneotodus" cf. "O." variabilis } \\
\text { Lindström } \\
13 \text { Rossodus manitouensis Repetski \& } \\
\text { Ethington (fig. } 4 \mathrm{I} \text { ) } \\
3 \text { Rossodus? sp. } \\
1 \text { unassigned scandodontiform element } \\
8 \text { Scolopodus sulcatus Furnish (fig. } 4 \mathrm{~K} \text { ) } \\
6 \text { Variabiloconus bassleri (Furnish) (fig. }\end{array}$ & $\begin{array}{l}\text { Age is early Early Ordovician } \\
\text { (Rossodus manitouensis } \\
\text { Zone; early Ibexian). } \\
\text { This collection is the same age } \\
\text { and contains many of the } \\
\text { same conodont species as the } \\
\text { oldest samples from the type } \\
\text { section of the Novi Mountain } \\
\text { Formation in the Medfra D-1 } \\
\text { quadrangle. }\end{array}$ & $\begin{array}{l}\text { Laurentoscandodid-rossodid } \\
\text { biofacies; tropical, normal- } \\
\text { marine shelf or platform } \\
\text { depositional setting. Species } \\
\text { association includes some } \\
\text { North American Midcontinent } \\
\text { elements, but tropical } \\
\text { cosmopolites and some } \\
\text { pandemics predominate. }\end{array}$ & $\begin{array}{l}\text { Distal, thin-bedded, silty } \\
\text { limestone turbidites with } \\
\text { local black carbonaceous } \\
\text { partings. Sample is from } \\
\text { 12-cm-thick bed of } \\
\text { medium-dark-gray, fine- } \\
\text { grained, dolomitic limestone } \\
\text { with parallel- and cross- } \\
\text { laminations and starved } \\
\text { ripples; laminae contain } \\
\text { peloids and lesser quartz } \\
\text { and plagioclase silt. Pzc } \\
\text { map unit of Patton and } \\
\text { others (1980). } \\
\text { Heavy-mineral concentrate } \\
\text { includes schistose grains. } \\
\text { Sample weight } 8.6 \mathrm{~kg} \text {. }\end{array}$ \\
\hline
\end{tabular}

7 unassigned coniform elements

$\mathrm{CAI}=4$

[97ADw116A; 11513-CO] 
Table 1. Conodont data for localities shown on figure 2-Continued.

\begin{tabular}{|c|c|c|c|c|c|}
\hline $\begin{array}{l}\text { Locality no., } \\
\text { (terrane or } \\
\text { subterrane; } \\
\text { unit) }\end{array}$ & $\begin{array}{l}\text { Quadrangle } \\
\text { latitude/ } \\
\text { longitude }\end{array}$ & $\begin{array}{l}\text { Conodont fauna and CAI } \\
\text { [field no.; USGS collection no.] }\end{array}$ & Age & Biofacies & Remarks \\
\hline $\begin{array}{l}33 \text { (Telida; } \\
\text { argillite and } \\
\text { quartzite } \\
\text { unit) }\end{array}$ & $\begin{array}{c}\text { Mt. McKinley } \\
\text { D-5 } \\
63^{\circ} 55.08^{\prime} \\
152^{\circ} 10^{\prime}\end{array}$ & $\begin{array}{l}\text { Aspelundia expansa Armstrong } \\
4 \mathrm{~Pb} \text { and } 1 \mathrm{M} \text { elements (figs. 4BB- } \\
\mathrm{DD} \text { ) } \\
\text { Aspelundia fluegeli (Walliser) } \\
3 \mathrm{~Pa}, 4 \mathrm{~Pb}, 1 \mathrm{Sb} 1 \& 1 \mathrm{Sb} 2 \text { elements } \\
\text { (figs. } 4 \mathrm{EE}-\mathrm{GG} \text { ) } \\
\text { Aspelundia spp. vicarious elements } \\
8 \mathrm{M}, 2 \mathrm{Sb} \& 9 \mathrm{Sc} \text { (some of the Sc } \\
\text { elements could belong in Ozarkodina) } \\
\text { (fig. } 4 \mathrm{HH} \text { ) } \\
1 \text { fragment Carniodus? sp. indet. } \\
\text { Dapsilodus obliquicostatus (Branson \& } \\
\text { Mehl) (figs. 4JJ-MM) } \\
4 \mathrm{M} \& 30 \mathrm{~S} \text { elements } \\
1 \text { Sb element Distomodus sp. indet. (fig. } \\
\text { 4II) } \\
1 \text { unassigned oistodontiform element of } \\
\text { Ordovician morphotype } \\
1 \text { Pa? element Ozarkodina? sp. indet. } \\
31 \text { Panderodus unicostatus (Branson \& } \\
\text { Mehl) } \\
2 \text { Walliserodus sp. indet. } \\
1 \text { unassigned Sb element } \\
1 \text { unassigned Sc element of deep-water } \\
\text { morphotype } \\
94 \text { indet. fragments } \\
\text { CAI=4-4.5 } \\
\text { [97AD54C; } 12597-S D \text { ] }\end{array}$ & $\begin{array}{l}\text { Age is early Early Silurian; } \\
\text { middle-late Llandoverian but } \\
\text { could be middle } \\
\text { Llandoverian. According to } \\
\text { Armstrong (1990), } \\
\text { Aspelundia expansa may not } \\
\text { extend above the middle } \\
\text { Llandoverian. }\end{array}$ & $\begin{array}{l}\text { Aspelundid-coniform biofacies. } \\
\text { Post-mortem transport from } \\
\text { or within this biofacies, which } \\
\text { represents outer shelf or slope } \\
\text { depositional setting according } \\
\text { to Armstrong (1990). }\end{array}$ & $\begin{array}{l}\text { Light-olive-gray-iveathering, } \\
\text { medium-dark-to dark-gray, } \\
\text { very fine grained dolomitic } \\
\text { micrite with silty laminae } \\
\text { rich in calcareous sponge } \\
\text { spicules, quartz, and lesser } \\
\text { plagioclase. From } 20 \text {-cm } \\
\text { chunk presumed fallen from } \\
\text { adjacent cliffs and } \\
\text { interbedded with argillite } \\
\text { and sandstone. } \\
\text { Heavy-mineral concentrate } \\
\text { includes phosphatized } \\
\text { bioclasts and rare } \\
\text { phosphatic brachiopod } \\
\text { fragments and phosphatized } \\
\text { radiolarians. Sample weight } \\
4.2 \text { kg. }\end{array}$ \\
\hline
\end{tabular}



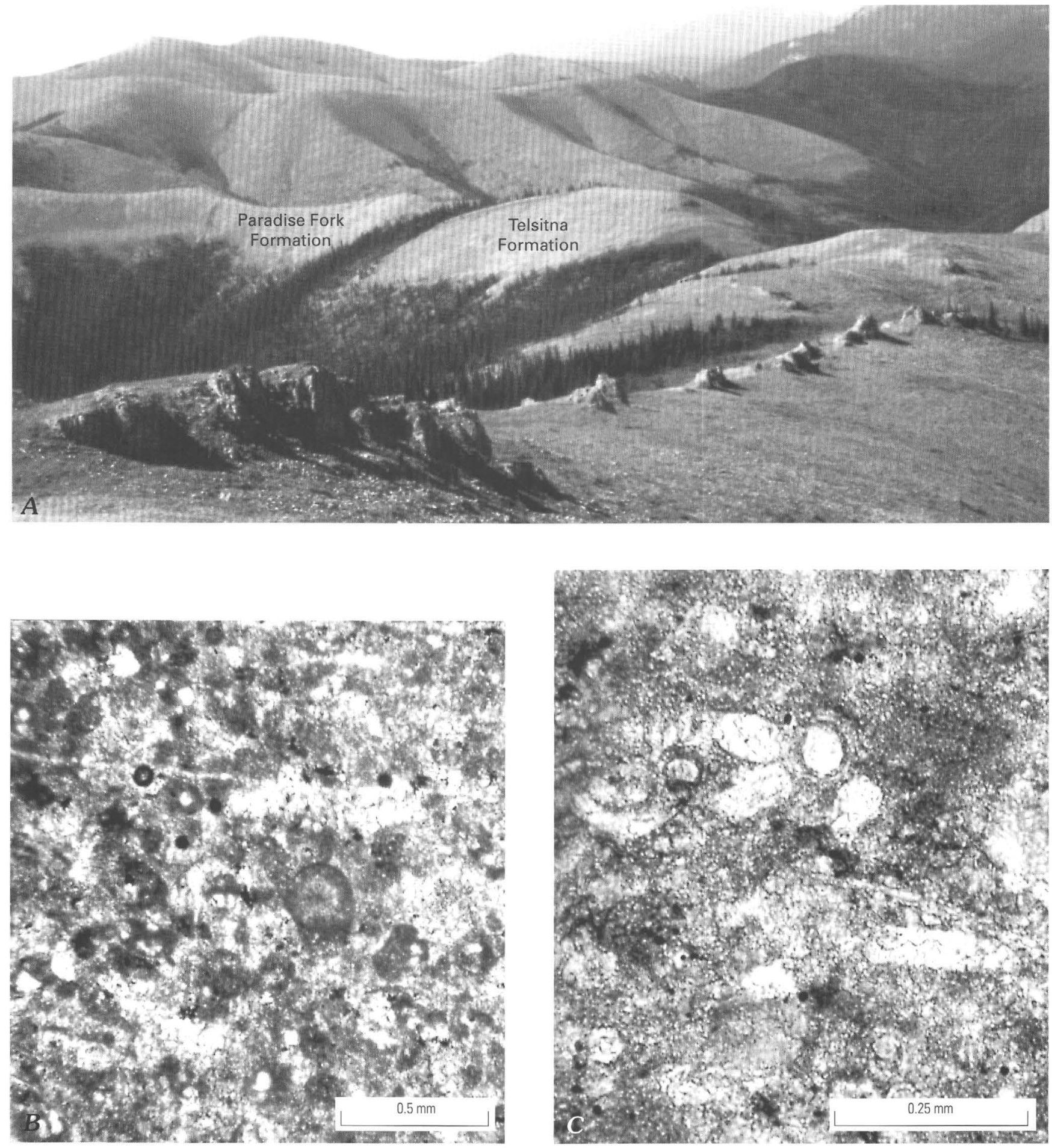

Figure 3. A, View to south of shallow-water facies (coralline dolostone) in the upper part of the Telsitna Formation (fig. 2, loc. 1) overlain by deep-water facies (shale and fissile to laminated limestone) in the lower part of the Paradise Fork Formation (fig. 2, locs. 6, 8); Nixon Fork terrane. $B, C$, Sedimentary features of the upper part of the Telsitna Formation (fig. 2, loc. 3). B, Lamina rich in peloids and calcitized radiolarians; station 97AD34. C, Calcareous sponge spicules in micrite; station 97ADw124.

the Medfra quadrangle (Patton and others, 1980). It is generally poorly exposed, but appears to be at least $1,000 \mathrm{~m}$ thick (Dutro and Patton, 1982). Dutro and Patton (1982) assigned a middle Early Silurian age to the Paradise Fork based on graptolites and ostracodes and suggested that the unit was deposited in a relatively deep water setting. Our studies confirm a deep-water depositional environmental for this unit but indicate that it is, in part, as young as Early Devonian.
We examined the Paradise Fork Formation at six partial sections in and near its type locality (fig. 2, locs. 6-10) and reexamined conodont collections from two additional sections (fig. 2 and table 1, locs. 11, 12). The lower part of the Paradise Fork (locs. 6, 8,9) is grayish-brown-weathering, dark-gray to black, fissile to laminated limestone and limy shale in 3- to 5-cm-thick beds. Limestones are micrite and calcareous siltstone with 5-20 percent calcitized radiolarians and rare calcareous and pyritized 
sponge spicules (figs. $5 B, 5 C$ ); fine dolomite rhombs and rare quartz silt form the laminae. The upper part of the formation (locs. 7,10 ) is chiefly light-gray-weathering, medium-gray to black limestone with tan-weathering dolomitic partings (fig. $5 \mathrm{~A}$ ). Beds are $1-15 \mathrm{~cm}$ thick (most $\leq 5 \mathrm{~cm}$ ), with well-developed parallel- and cross-laminae, and consist of graded alternations of fine- to medium-grained calcareous sandstone, siltstone, and micrite. Sandy layers contain rounded to irregular micrite clasts $(40-400 \mu \mathrm{m})$ in a sparry matrix (fig. $5 \mathrm{D})$; rare bioclasts in these strata include pelmatozoan debris (locally silicified), ostracodes, calcareous sponge spicules, and calcitized radiolarians. Minor metamorphic lithic clasts occur in coarser layers and laminae throughout the formation (table 1, locs. 7, 8, 8A). The uppermost part of the Paradise Fork, known only from a single rubble outcrop (loc. $8 \mathrm{~A}$ ), is dark-gray, weakly laminated calcisiltstone with rare micritic clasts and fossil fragments.

Conodont collections support a middle Early Silurian age for much of the Paradise Fork Formation, but the uppermost part of the unit is at least as young as Early Devonian. Fissile, very finely laminated limestone near the base of the unit, at about the same locality and horizon that produced late Llandoverian to early Wenlockian graptolites (table 2, loc. 6) (Dutro and Patton, 1982), yielded late Llandoverian to very early Wenlockian conodonts that indicate post-mortem transport from or within a midshelf or slope depositional setting (table 1, loc. 6; figs. $4 N N-$ $4 P P)$. Laminated limestone a few hundred meters higher in the section contains early to middle Wenlockian conodonts (table 1 , loc. 8; figs. $4 Q Q-4 S S$ ) redeposited from a shelf or platform environment. Dutro and Patton (1982) reported ostracodes of probable Wenlockian age from near the top of the Paradise Fork, and no fossils younger than Wenlockian have been found in outcrop. An isolated rubble outcrop, however, $0.5 \mathrm{~km}$ southeast of locality 8 (tables 1 and 2, loc $8 \mathrm{~A}$ ), yielded a mixed fauna that indicates a surprisingly young age for the uppermost part of the Paradise Fork. A single collection of apparently uniform lithology from this locality contained numerous specimens of the early Lochkovian graptolite Monograptus uniformis. Conodonts from the same collection are of two ages: late Llandoverian to at least latest Ludlovian and late Emsian or even younger Devonian. This collection most likely represents rubble derived from a relatively condensed section of Wenlockian to Emsian (or possibly even younger Devonian) age.

The Paradise Fork Formation originated as hemipelagic deposits and distal turbidites derived from a carbonate platform. Fine-grained "background" sediment (laminated radiolarianbearing micrite) predominates in the lower part of the unit. Turbidites (calcareous siltstone and sandstone) are most notable in the upper part of the section and increase upward in thickness and abundance. The uppermost section appears extremely condensed.

In the southern part of its type locality (fig. 2, loc. 13), the Paradise Fork Formation interfingers with shallow-water strata. These rocks are thick- to massive-bedded, light-gray, vuggy dolostone with locally abundant tubular stromatoporoids (Amphipora sp.). A sample from locality 13 contained conodonts of early Ludlovian to late Pridolian age; strata here are lithologically similar to, and probably correlative with, the lower part of the Whirlwind Creek Formation of Late Silurian-Early Devonian age (Dutro and Patton, 1982).
Fault blocks adjacent to those containing the Paradise Fork Formation display a different Silurian stratigraphy. Six kilometers west of the Paradise Fork type locality (fig. 2, loc. 4), Ordovician deep-water limestone at the top of the Telsitna Formation is overlain by Upper Silurian amphiporid dolostone; strata equivalent in age and lithology to the lower part of the Paradise Fork are missing. A similar sequence occurs $8 \mathrm{~km}$ east of the Paradise Fork type locality (fig. 2 and table 1, loc. 14), where shallow-water skeletal packstone of probable late Late Silurian age overlies deep-water Ordovician facies. Deep-water Silurian rocks are also absent from Nixon Fork sections throughout the southern half of the Medfra quadrangle (Patton and others, 1980). In this area, shallow-water facies of the Whirlwind Creek Formation directly overlie the Telsitna Formation (Dutro and Patton, 1982). The age of the lower part of the Whirlwind Creek is not well constrained. Dutro and Patton (1982) suggested that the basal strata are Ludlovian or younger, but a conodont collection that could be as old as Wenlockian was obtained from locality 15 (table 1).

Deep-water strata of the Paradise Fork Formation are thus quite limited in extent but not in age. They appear to have formed in a small, relatively long lived basin that developed within the Nixon Fork platform. Deep-water facies found in some sections of the upper Telsitna Formation suggest that basinal conditions were locally established by Late (possibly late Middle) Ordovician time. Shallow-water strata precisely coeval with the thick Lower Silurian part of the Paradise Fork Formation have not yet been identified in the Nixon Fork terrane. The condensed, upper part of the Paradise Fork interfingers with shallow-water facies of Late Silurian age.

\section{Minchumina Terrane}

The Minchumina terrane lies directly southeast of the Nixon Fork terrane in the Medfra and Mt. McKinley quadrangles and extends some $300 \mathrm{~km}$ into the Kantishna River quadrangle (fig. 1). Two subterranes are recognized (Patton and others, 1994). The East Fork subterrane is confined to the Medfra quadrangle and comprises the East Fork Hills Formation (Upper Cambrian through Lower Devonian; age revised herein). The more extensive Telida subterrane consists of sparse, discontinuous exposures of Precambrian(?) and Paleozoic rocks.

\section{East Fork Subterrane}

The East Fork Hills Formation is a sequence of laminated, locally dolomitic limestone that forms a northeast-trending belt at least $60 \mathrm{~km}$ long and $40 \mathrm{~km}$ wide in the southeastern part of the Medfra quadrangle (Patton and others, 1980) (fig. 1). The unit was named by Dutro and Patton (1982), who reported a thickness of several hundred meters, an age (based on sparse conodont collections) of Early Ordovician to Middle Devonian, and a deep-water depositional environment. Exposures of the East Fork Hills Formation are uniformly poor, and relations with other units are obscure. Shallow-water facies of the Nixon Fork 


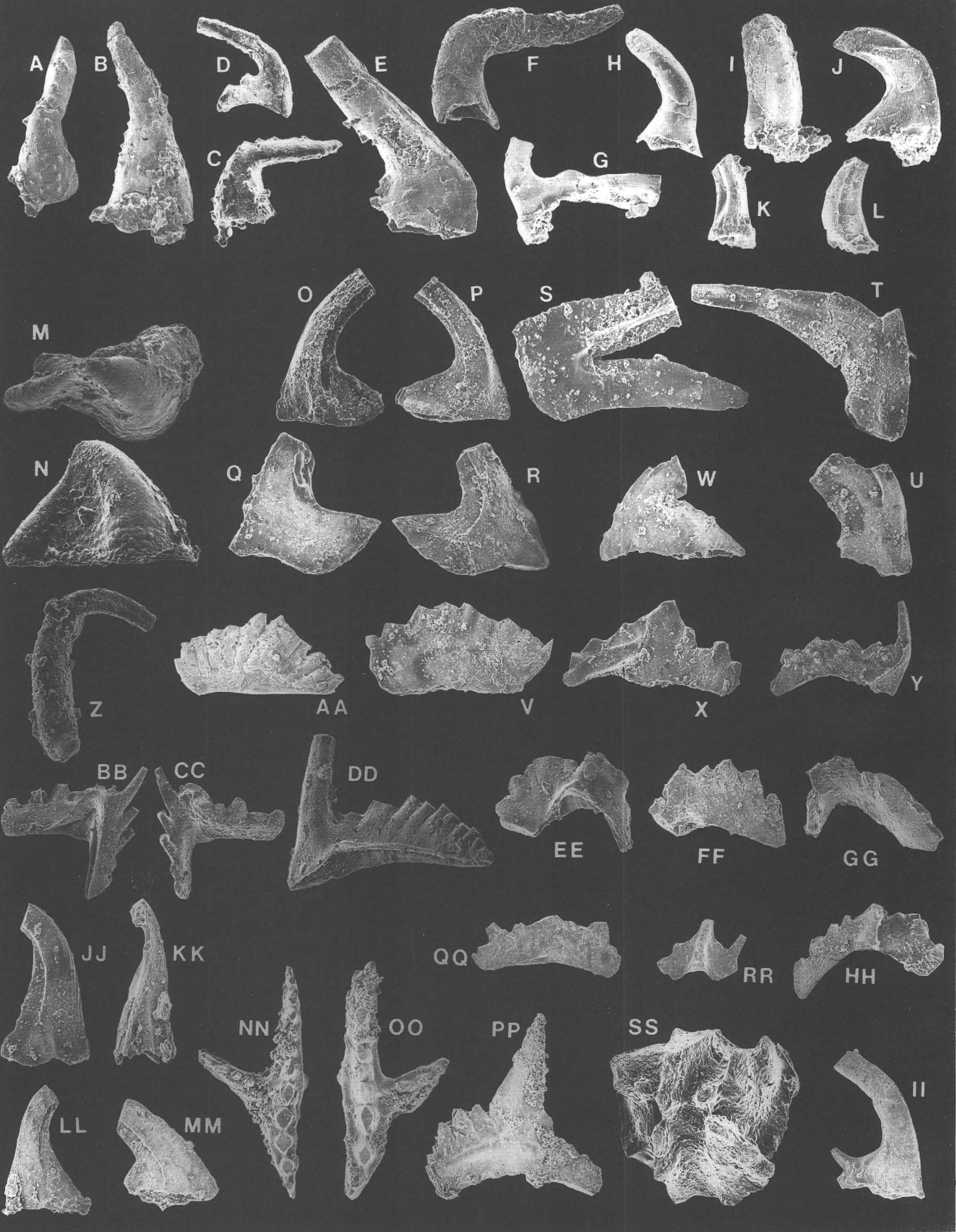


Figure 4. Early Paleozoic conodonts from deep-water facies in the Medfra area, central Alaska (scanning electron photomicrographs); figure $4 Z$ is not a conodont. Illustrated specimens are reposited in the U.S. National Museum, USNM, Washington, D.C. See table 1 for lithostratigraphic description, faunal analysis, and age assignment of collections and figure 2 for their geographic and geologic position.

A-F, very Late Cambrian, East Fork Hills Formation, USGS colln. 11509-CO (fig. 2, loc. 17), x100 except D x50.

A-C, Hirsutodontus hirsutus Miller, antero-lateral and two lateral views, USNM 497666-68.

D, Cordylodus proavus Müller, outer lateral view, USNM 497669.

E, Eoconodontus notchpeakensis (Miller), outer lateral view, USNM 497670.

F, Teridontus nakamurai (Nogami), outer lateral view, USNM 497671.

$\mathrm{G}-\mathrm{L}$, Rossodus manitouensis Zone, early Early Ordovician, argillite and quartzite unit, Telida subterrane, USGS colln. 11513-CO (fig. 2, loc. 23), x100.

G, Cordylodus intermedius Furnish, outer lateral view, USNM 497672.

H, Drepanoistodus cf. D. pervetus Nowlan, outer lateral view, USNM 497673.

I, Rossodus manitouensis Repetski and Ethington, antero-lateral view, USNM 497674.

$\mathrm{J}$, Laurentoscandodus triangularis (Furnish), outer lateral view, USNM 497675.

K, Scolopodus sulcatus Furnish, postero-lateral view, USNM 497676.

L, Variabiloconus bassleri (Furnish), inner lateral view, USNM 497677.

M-Y, late Middle-Late Ordovician, upper part of Telsitna Formation, USGS colln. 11510-CO (fig. 2, loc. 3), $\mathrm{x} 100$.

M, N, Pseudooneotodus mitratus (Moskalenko), upper and lateral views, USNM 497678.

0-S, Paroistodus? sp. A of Nowlan and others (1988), inner and outer lateral views of two $\mathrm{S}$ elements and inner lateral view of $\mathrm{M}$ element, USNM 497679-81.

T, U, Strachanognathus parvus Rhodes, outer and inner lateral views, USNM 497683-84.

V, Ozarkodinid P element, outer lateral view, USNM 497685.
W-Y, Periodon grandis (Ethington)?, M, Pa, and Sb elements, two inner and an outer lateral views, USNM 497686-88.

$Z, A A$, very latest Late Ordovician, upper part of Telsitna Formation, USGS colln. 11515-CO (fig. 2, loc. 3).

$Z$, Ptiloncodus simplex Harris, lateral view, $\mathrm{x} 125$, USNM 497689. This phosphatic microfossil is known chiefly as discrete elements but does occur in clusters (Tipnis, 1979); its taxonomic affinities remain uncertain. It is rare to common in late Early to Late Ordovician conodont collections in North America.

AA, Ozarkodina hassi (Pollock, Rexroad, and Nicoll)?, $\mathrm{Pb}$ element, inner lateral view, $\mathrm{x} 75$, USNM 497690 .

BB-MM, middle and late Llandoverian, argillite and quartzite unit, Telida subterrane, USGS colln. 12597-SD

(fig. 2, loc. 33), x75 except DD x65.

$\mathrm{BB}-\mathrm{DD}$, Aspelundia expansa Armstrong, $\mathrm{Pb}$ and $\mathrm{M}$ elements, inner and outer lateral views of $\mathrm{Pb}$ and inner lateral view of M, USNM 497691-92.

$\mathrm{EE}-\mathrm{GG}$, Aspelundia fleugli (Walliser), $\mathrm{Pb}, \mathrm{Pa}$, and $\mathrm{Pb}$ elements, inner, inner, and outer lateral views, USNM 497693-95.

$\mathrm{HH}$, Aspelundia sp. indet. vicarious Sb element, inner lateral view, USNM 497696.

II, Distomodus sp. indet., Sb element, inner lateral view, USNM 497697.

JJ-MM, Dapsilodus obliquicostatus (Branson \& Mehl), three $S$ and $1 \mathrm{M}$ (fig. 4MM) elements, two inner and two outer lateral views, USNM 497698700.

NN-PP, Pterospathodus celloni Zone into K. ranuliformis Zone, late Llandoverian and very early Wenlockian, lower part of Paradise Fork Formation, USGS colln. 12598-SD (fig. 2, loc. 6), Pt. procerus (Walliser), $\mathrm{Pa}$ and $\mathrm{Pb}$ elements, upper and inner lateral views, x75, USNM 497701-03.

QQ-SS, Kockelella patula Zone, lower part of Paradise Fork Formation, USGS colln. 12594-SD (fig. 2, loc. 8), K. patula Walliser, $\mathrm{Pb}, \mathrm{Sb}$, and $\mathrm{Pa}$ elements, inner lateral and upper views, x50, USNM 49770406. terrane occur in fault contact to the northwest and deeper water facies of the Telida subterrane are exposed to the east and northeast (Patton and others, 1980) (fig. 2).

Our studies revise the age of the East Fork Hills Formation to Late Cambrian through Early Devonian and indicate that at least three subunits can be distinguished based on lithofacies and conodont faunas. The first subunit makes up the central part of the outcrop belt (fig. 2, locs. 16, 17) and includes the type locality (loc. 16) along the crest of the East Fork Hills. Thinbedded $(0.5$ to $8 \mathrm{~cm})$, fine-grained, medium-gray limestone and grayish-orange silty limestone comprise this subunit; beds contain parallel-laminae and small-scale cross-laminae, climbing ripples, and possible flute casts. Limestone layers are chiefly slightly recrystallized micrite. Silty layers are finely crystalline (40-60 $\mu \mathrm{m}$ ) dolomite with 1-15 percent detrital grains (mostly quartz, plagioclase feldspar, white mica, and metamorphic lithic clasts). Collections from this subunit produced phosphatic brachiopod fragments and latest Late Cambrian conodonts indicative of an outer-shelf or deeper depositional setting (table 1, locs. 16, 17; figs. $4 A-4 F$ ). 

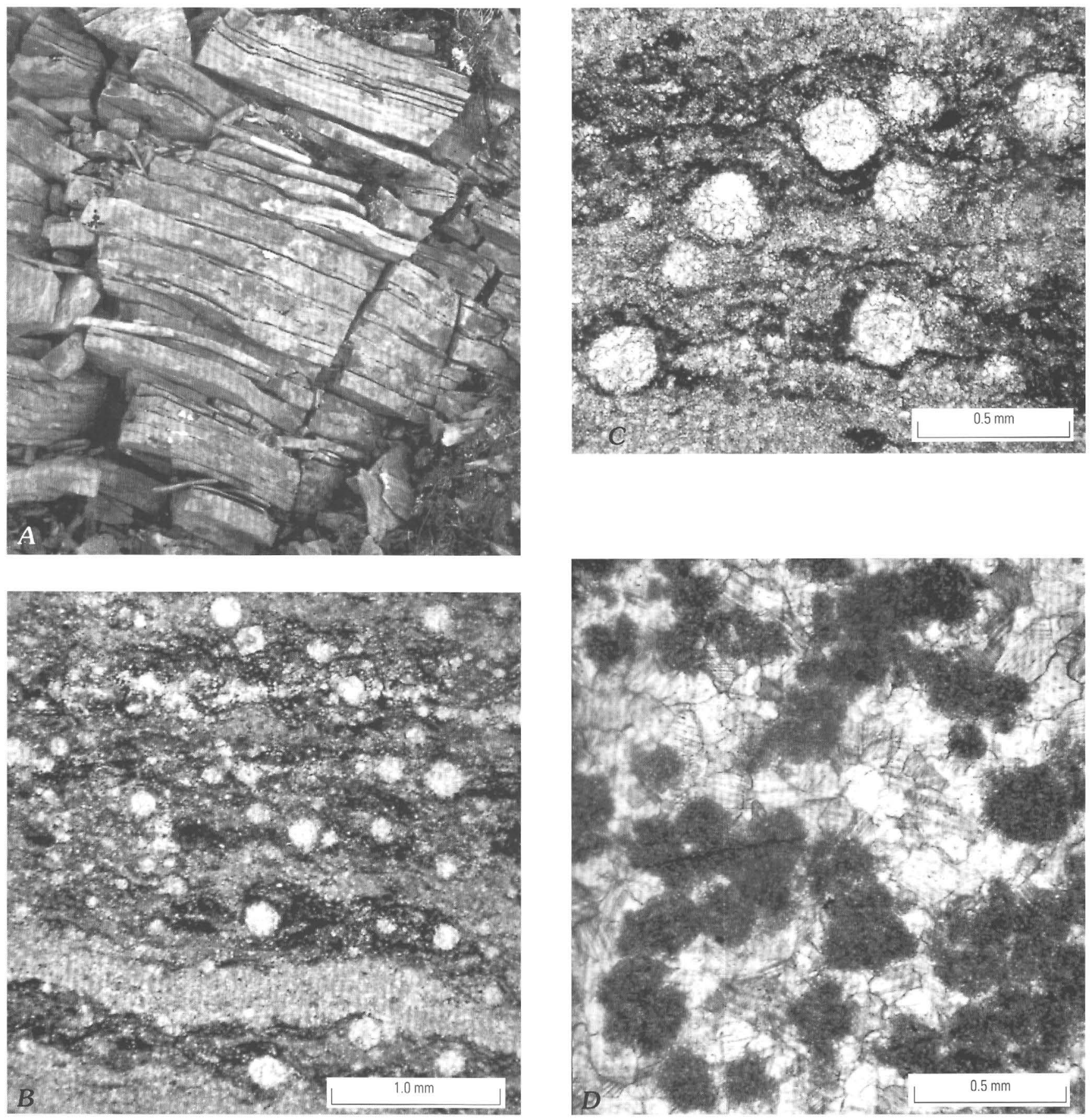

Figure 5. Sedimentary features of the Paradise Fork Formation, Nixon Fork terrane. $A$, Thin-bedded, fine-grained, distal carbonate turbidites; upper part of unit (fig. 2, loc. 7). B, C. Photomicrographs of calcitized radiolarians in micrite matrix; lower part of unit (fig. 2, loc. 8). D, Photomicrograph of turbidites shown in $A$, made up of sand- to silt-sized micrite clasts in sparry calcite matrix.

The second subunit forms a fault-bounded block along the northwestern edge of the outcrop belt (fig. 2, locs. 18, 19, 20). It consists chiefly of limestone with subordinate dark chert and siliceous siltstone; basalt rubble occurs at locality 18 . Limestone is slightly recrystallized micrite with thin concentrations of peloids and (or) bioclasts, including locally abundant calcitized radiolarians and rare pelmatozoan and brachiopod fragments. Some samples contain thin laminae of chert or dolomite; noncarbonate detritus ( $<5$ percent of most samples) is largely white mica and clasts of silty argillite. Where biostratigraphically diagnostic, conodonts from this subunit are of middle Middle Ordovician age and indicate a slope to basinal setting (table 1, loc. 18).
A distinctive conodont fauna delineates a third subunit of the East Fork Hills Formation in the southeastern part of the outcrop belt. Dark-gray, fine-grained, laminated limestone at two localities contains the youngest conodonts definitively identified from this formation (fig. 2 and table 1, locs. 21, 22). One collection could be as old as Silurian but is no younger than early Early Devonian (Lochkovian); the other is of middle Early Devonian (Pragian) age. Conodonts from both samples denote a normal-marine depositional setting.

Lithofacies, sedimentary structures, and conodont biofacies suggest an outer-shelf or deeper depositional setting for all three subunits of the East Fork Hills Formation. Laminated limestones 
Table 2. Other fossil data for localities shown on figure 2.

[Letters in field number refer to collector: CH, R. Chapman and M. Churkin; Dw, D.C. Bradley; Pa, W.W. Patton, Jr.; Rb and Wr, F.R. Weber]

\begin{tabular}{|c|c|c|c|c|}
\hline $\begin{array}{c}\text { Locality no. } \\
\text { (terrane or } \\
\text { subterrane; unit) }\end{array}$ & $\begin{array}{l}\text { Quadrangle } \\
\text { latitude/ } \\
\text { longitude }\end{array}$ & $\begin{array}{c}\text { Fauna } \\
\text { [field no.; USGS collection no.] }\end{array}$ & Age & Source \\
\hline $\begin{array}{c}6 \text { (Nixon Fork; } \\
\text { Paradise Fork Fm.) }\end{array}$ & $\begin{array}{c}\text { Medfra D-2 } \\
63^{\circ} 51.80^{\prime} \\
153^{\circ} 43.08^{\prime}\end{array}$ & $\begin{array}{l}\text { Graptolites: Monograptus cf. } \\
\text { M. parapriodon Boucek, } \\
\text { Paraplectograptus aff. } P \text {. } \\
\text { eiseli (Manck) } \\
\text { [77APa1 12B] }\end{array}$ & $\begin{array}{l}\text { Early Silurian (late } \\
\text { Llandoverian-early } \\
\text { Wenlock; probably late } \\
\text { Llandoverian, } \\
\text { Monoclimacis crenulata } \\
\text { Zone }\end{array}$ & $\begin{array}{l}\text { Written report from C. Carter } \\
\text { to W.W. Patton, Jr., } 1978\end{array}$ \\
\hline $\begin{array}{l}\text { 8A (Nixon Fork; } \\
\text { Paradise Fork Fm.) }\end{array}$ & $\begin{array}{c}\text { Medfra D-2 } \\
63^{\circ} 51.50^{\prime} \\
153^{\circ} 42.50^{\prime}\end{array}$ & $\begin{array}{l}\text { Graptolites: Monograptus } \\
\text { uniformis Pribyl } \\
\text { [98ADw206] }\end{array}$ & $\begin{array}{l}\text { Age is early Early Devonian } \\
\text { (Lochkovian; Monograptus } \\
\text { uniformis Zone) }\end{array}$ & $\begin{array}{l}\text { Written report from S. } \\
\text { Finney, California State } \\
\text { University (Long Beach), } \\
\text { to D. Bradley, } 1998\end{array}$ \\
\hline $\begin{array}{c}28 \text { (Telida; } \\
\text { chert and argillite } \\
\text { unit) }\end{array}$ & $\begin{array}{l}\text { Mt. McKinley D-5 } \\
63^{\circ} 53.17^{\prime} \\
152^{\circ} 11^{\prime}\end{array}$ & $\begin{array}{l}\text { Graptolites: poorly preserved } \\
\text { Orthograptus(?) cf. } O . \\
\text { quadrimucronatus (Hall) } \\
\text { [79CH89] }\end{array}$ & $\begin{array}{l}\text { Middle Ordovician (approx. } C \text {. } \\
\text { tubuliferous Zone) }\end{array}$ & $\begin{array}{l}\text { Written report from C. Carter } \\
\text { to R. Chapman, } 1979\end{array}$ \\
\hline $\begin{array}{c}29 \text { (Telida; } \\
\text { chert and argillite } \\
\text { unit) }\end{array}$ & $\begin{array}{l}\text { Mt. McKinley D-5 } \\
63^{\circ} 51.5^{\prime} \\
152^{\circ} 12^{\prime}\end{array}$ & $\begin{array}{l}\text { Graptolites: Orthoretiolites } \\
\text { hami Whittington and } \\
\text { Orthograptus sp. } \\
\text { [79CH88] }\end{array}$ & $\begin{array}{l}\text { Middle Ordovician (approx. } C \text {. } \\
\text { tubuliferous Zone) }\end{array}$ & $\begin{array}{l}\text { Written report from C. Carter } \\
\text { to R. Chapman, } 1979\end{array}$ \\
\hline $\begin{array}{l}35 \text { (Telida; } \\
\text { argillite and } \\
\text { quartzite unit) }\end{array}$ & $\begin{array}{c}\text { Mt. McKinley D-5 } \\
63^{\circ} 53^{\prime} \\
152^{\circ} 9.5^{\prime}\end{array}$ & $\begin{array}{l}\text { Corals: Favosites(?) } \\
\text { [59ARb86] (in place) and } \\
\text { Xystriphyllum sp. } \\
\text { [78AWr8] (float-may not } \\
\text { be from argillite and } \\
\text { quartzite unit) }\end{array}$ & $\begin{array}{l}\text { Silurian or Devonian; } \\
\text { Xystriphyllum sp. is } \\
\text { restricted to latest Silurian } \\
\text { (Pridolian)-early Middle } \\
\text { Devonian }\end{array}$ & $\begin{array}{l}\text { Written report from W.A. } \\
\text { Oliver, Jr., to F.R. Weber, } \\
\text { 1997. Favosites(?) was } \\
\text { originally identified } \\
\text { (Oliver and others, 1975; } \\
\text { Chapman and others, } \\
\text { 1981) as Saffordophyllum } \\
\text { sp. of Middle-Late } \\
\text { Ordovician age }\end{array}$ \\
\hline
\end{tabular}

formed as hemipelagic deposits and distal turbidites derived from a carbonate platform. "Common" penecontemporaneous slump structures reported by Patton and others (1980) imply a slope environment for at least some parts of the unit. Radiolarians and periodontid and protopanderodid conodonts in the second subunit indicate that it may have accumulated in somewhat deeper water conditions than did the rest of the East Fork Hills Formation.

\section{Telida Subterrane}

Exposures in the Telida subterrane are scarce, discontinuous, and have yielded few fossils, so the detailed stratigraphy of these rocks remains uncertain. Patton and others (1994) recognized four units, in ascending stratigraphic order: (1)
pre-Ordovician limestone and phyllite; (2) pre-Ordovician(?) and Ordovician argillite and quartzite; (3) Ordovician (and younger?) chert and argillite; and (4) Middle to Upper Devonian limestone. We report here new lithologic and paleontologic data from the argillite and quartzite unit and the chert and argillite unit in the Medfra and Mt. McKinley quadrangles. Our studies suggest that the chert and argillite unit is older than at least some parts of the argillite and quartzite unit.

\section{Chert and Argillite Unit}

The chert and argillite unit includes map units Pzc (lower Paleozoic chert and phyllite of Patton and others, 1980) in the Medfra quadrangle, DOc (Ordovician through Devonian chert) 
in the northwestern part of the Mt. McKinley quadrangle (Chapman and Yeend, 1981), and Oc (Ordovician chert and slate unit) in the western Kantishna River quadrangle (Chapman and others, 1975). We examined this unit at six localities in the study area (fig. 2, locs. 23-28).

The westernmost outcrops of the chert and argillite unit occur in a fault block bounded by fragments of the Nixon Fork terrane (figs. 1, 2; locs. 23-25). Discontinuous exposures along the Sethkokna River consist of fine-grained limestone, silty dolostone, argillite, and chert; all lithologies are thin bedded $(<0.5-12 \mathrm{~cm}$, most $2-5 \mathrm{~cm})$ with black carbonaceous partings. Carbonate rocks are medium to dark gray, weather olive gray to dark yellowish brown, and are locally graded, with well-developed parallel- and cross-laminae, starved ripples, and load casts (figs. 6A, 6B). Samples consist of slightly recrystallized calcite and (or) dolomicrite, locally abundant peloids and calcareous sponge spicules, and $<5-50$ percent detrital quartz, plagioclase, and metamorphic lithoclasts (fig. $6 \mathrm{C}$ ). Chert is dark gray to brown to black and contains siliceous sponge spicules and radiolarian ghosts (fig. $6 D$ ).

Exposures of the chert and argillite unit in the Mt. McKinley quadrangle are similar to those in the Medfra quadrangle but lack a carbonate component (fig. 2, locs. 26-28). Rubble in the Snohomish Hills is laminated, very light gray to black, spiculitic, radiolarian chert and lesser tan argillite; outcrops at Lake Minchumina are light olive gray silty argillite.

The chert and argillite unit yields fossils of Early and Middle Ordovician age (table 1, loc. 23; table 2, locs. 28, 29). Limestone in the Medfra quadrangle (loc. 23) produced abundant conodonts of early Early Ordovician age (Rossodus manitouensis Zone; figs. $4 G-4 L$ ); this fauna is the same age as the oldest part of the Novi Mountain Formation in the Nixon Fork terrane and contains many of the same species found in Novi Mountain samples. Argillite contains late Middle Ordovician (Caradocian) graptolites at Lake Minchumina (fig. 2 and table 2, locs. 28, 29) and a less diagnostic fauna of Middle(?) Ordovician age in the southwestern part of the Kantishna River quadrangle, $50 \mathrm{~km}$ to the north (Chapman and others, 1981).

Lithofacies, sedimentary structures, and biofacies indicate an off-platform setting for the chert and argillite unit. Calcareous beds are distal turbidites, derived at least in part from the Nixon Fork terrane. Argillite and chert formed as hemipelagic deposits.

\section{Argillite and Quartzite Unit}

We examined the argillite and quartzite unit at five localities (fig. 2, locs. 30-34). These rocks are mapped as Pzp€q (Precambrian or lower Paleozoic quartzite, grit, and argillite) in the Medfra quadrangle (Patton and others, 1980), DOs (Ordovician to Devonian shaly rocks) in the northwestern part of the Mt. McKinley quadrangle (Chapman and Yeend, 1981), and €qs (Cambrian quartzite, metasiltstone, slate, and grit) in the western Kantishna River quadrangle (Chapman and others, 1975).

Along Munsatli Ridge (fig. 2, locs. 30, 31) and on the east side of Lake Minchumina (fig. 2, loc. 32), the argillite and quartzite unit consists chiefly of sandstone, pebbly sandstone, and fine-grained conglomerate in slabby beds $3-10 \mathrm{~cm}$ thick. Fresh surfaces are parallel-laminated and range from very pale orange to olive or blue-gray. Some sandstones are graded, and some contain thin argillite interbeds. Sandstone samples are very poorly sorted; most are medium grained but contain notable (510 percent) outsized clasts, $4-10 \mathrm{~mm}$ in diameter. Clasts are angular to rounded and are chiefly monocrystalline quartz $(\mathrm{Qm})$ with undulous extinction $(\mathrm{Qm}=60-95$ percent, generally $>80$ percent). Other framework grains include feldspar (both plagioclase and microcline, based on twinning), polycrystalline quartz, metasedimentary lithic clasts, and rare tourmaline. Matrix, mostly finely intergrown quartz and phyllosilicates, makes up $<5$ to $>30$ percent of the samples examined. Grain boundaries are generally recrystallized, and some samples are semischists.

More heterogeneous strata also included in the argillite and quartzite unit crop out along the northeastern shore of Lake Minchumina (fig. 2, locs. 33, 34). These rocks consist of strongly cleaved, silty to cherty argillite, phyllite, and chert, intercalated with intervals of sandstone and carbonate that are $20 \mathrm{~cm}$ to $1.5 \mathrm{~m}$ thick (fig. $7 A$ ). Finer grained rocks range from reddish brown to silvery gray to black; siliceous sponge spicules and radiolarian ghosts are locally abundant in some cherty layers. Sandstones weather white to orange to brownish gray and are medium to dark gray, very fine to medium grained, poorly sorted, and locally cross-laminated and graded. Some samples are mostly rounded to angular monocrystalline quartz with lesser plagioclase and microcline feldspar and are virtually identical to strata described above from Munsatli Ridge. Others contain a similar suite of quartz and subordinate feldspar as well as 10 to 70 percent carbonate, chiefly dolomite, that occurs as clasts and matrix (figs. $7 B-7 D$ ). Clasts are finely crystalline dolomite mosaics and lesser pelmatozoan fragments. Rare carbonate layers are mostly finely crystalline $(5-40 \mu \mathrm{m})$ dolomite with abundant calcareous sponge spicules, lesser calcitized radiolarians, and minor detrital quartz and feldspar (fig. 7E).

No fossils have been obtained from the argillite and quartzite unit along Munsatli Ridge, but several collections constrain the age of the rocks at Lake Minchumina. Finegrained dolostone from locality 33 (fig. 2; table 1) contains abundant conodonts of Early Silurian (middle-late Llandoverian, possibly middle Llandoverian) age (figs. $4 B B-4 M M$ ); the heavy-mineral concentrate from this sample contains rare phosphatic brachiopod fragments and phosphatized radiolarians. The conodont faunule represents postmortem transport from or within the aspelundid-coniform biofacies, which indicates an outer-shelf or slope depositional setting.

Several collections of corals from locality 35 (fig. 2; table 2, loc. 35) are of Silurian or Devonian age (W.A. Oliver, Jr., 1997; written commun. to F. Weber). The most diagnostic form is a ptenophylid coral—Xystriphyllum sp., of latest Silurian (Pridolian) to early Middle Devonian age-found in a beach cobble that may not have come from the argillite and quartzite unit (Chapman and others, 1981). A second coral was collected from outcrop. This specimen was originally identified as Saffordophyllum sp. of Middle to Late Ordovician age (Oliver and others, 1975; Chapman and others, 1981) but is now identified as Favosites? sp. of Silurian or Devonian age (W.A. Oliver, Jr., 1997; written commun. to F. Weber). Two-hole crinoid columnals, indicative of a late Early-early Middle Devonian (EmisianEifelian) age, are also reported from beach float found near locality 35 (R.B. Blodgett, Oregon State University, 1997, written commun. to F. Weber). 

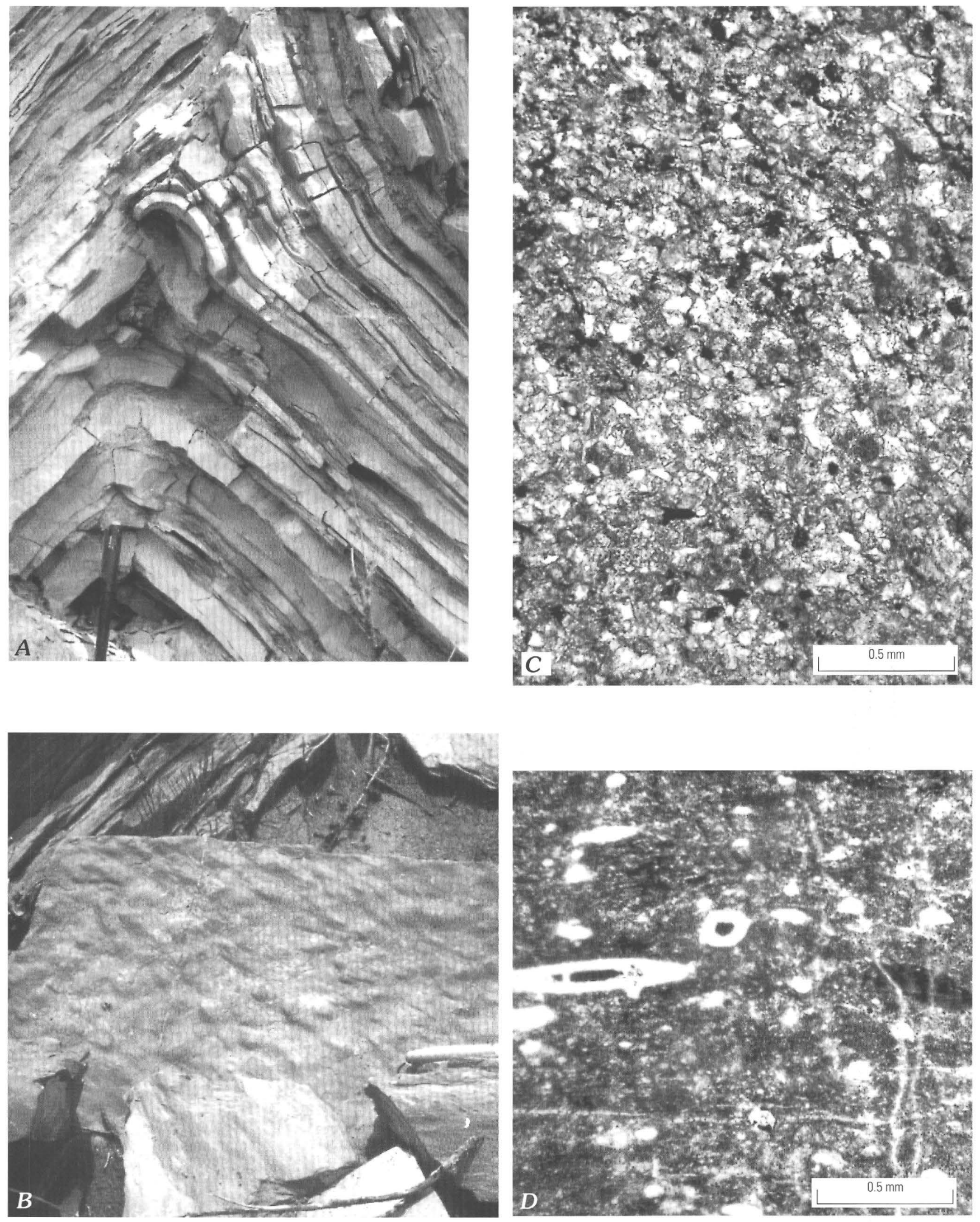

Figure 6. Sedimentary features of the chert and argillite unit, Telida subterrane, Minchumina terrane. $A-C$, Outcrop views and photomicrograph $(C)$ of distal, thin-bedded, silty limestone turbidites (fig. 2, loc. 23). Note black carbonaceous partings in $A$, load casts on bed bottom in $B$, and parallel laminae rich in peloids (dark grains) and quartz and lesser plagioclase silt (light grains) in $C$. $D$, Photomicrograph of chert with abundant siliceous sponge spicules; some spicule centers are pyritized (fig. 2, loc. 24). 

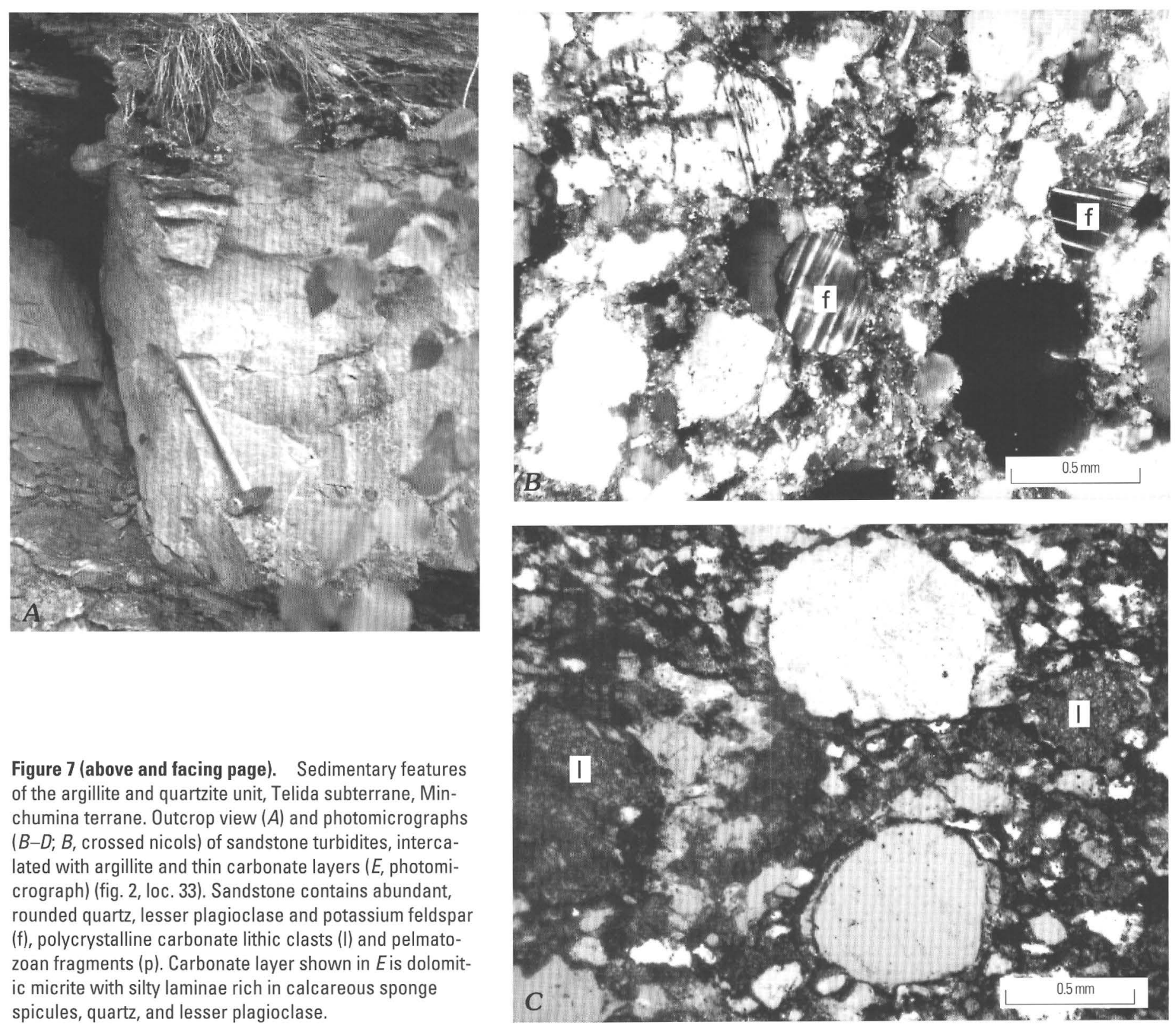

Figure 7 (above and facing page). Sedimentary features of the argillite and quartzite unit, Telida subterrane, Minchumina terrane. Outcrop view $(A)$ and photomicrographs $(B-D ; B$, crossed nicols) of sandstone turbidites, intercalated with argillite and thin carbonate layers ( $E$, photomicrograph) (fig. 2, loc. 33). Sandstone contains abundant, rounded quartz, lesser plagioclase and potassium feldspar (f), polycrystalline carbonate lithic clasts (I) and pelmatozoan fragments (p). Carbonate layer shown in $E$ is dolomitic micrite with silty laminae rich in calcareous sponge spicules, quartz, and lesser plagioclase.

area between locs. 28, 29, and 35 on fig. 2) to the argillite and quartzite unit (Chapman and others, 1981; Patton and others, 1994). If graptolite-bearing strata on the north and south sides of Yutokh Hill are included in the chert and argillite unit, however, a different stratigraphy results (F. Weber, written commun., 1997). In this interpretation, the argillite and quartzite unit, exposed at the top and on the east side of Yutokh Hill, unconformably overlies the chert and argillite unit (the contact could also be a low-angle thrust fault). Although structure in this area is complex (see further discussion below) our new fossil data, and the reinterpreted age of the in situ coral from Yutokh Hill, suggest that the chert and argillite unit at Lake Minchumina is Ordovician and the argillite and quartzite unit is Silurian and perhaps, in part, Devonian. It is possible, however, that the argillite and quartzite unit includes strata of several ages. Previous workers (Patton and others, 1980; Chapman and others, 1975) correlated this unit with Cambrian-Upper Proterozoic rocks, such as the Wickersham unit in the Livengood quadrangle, on 

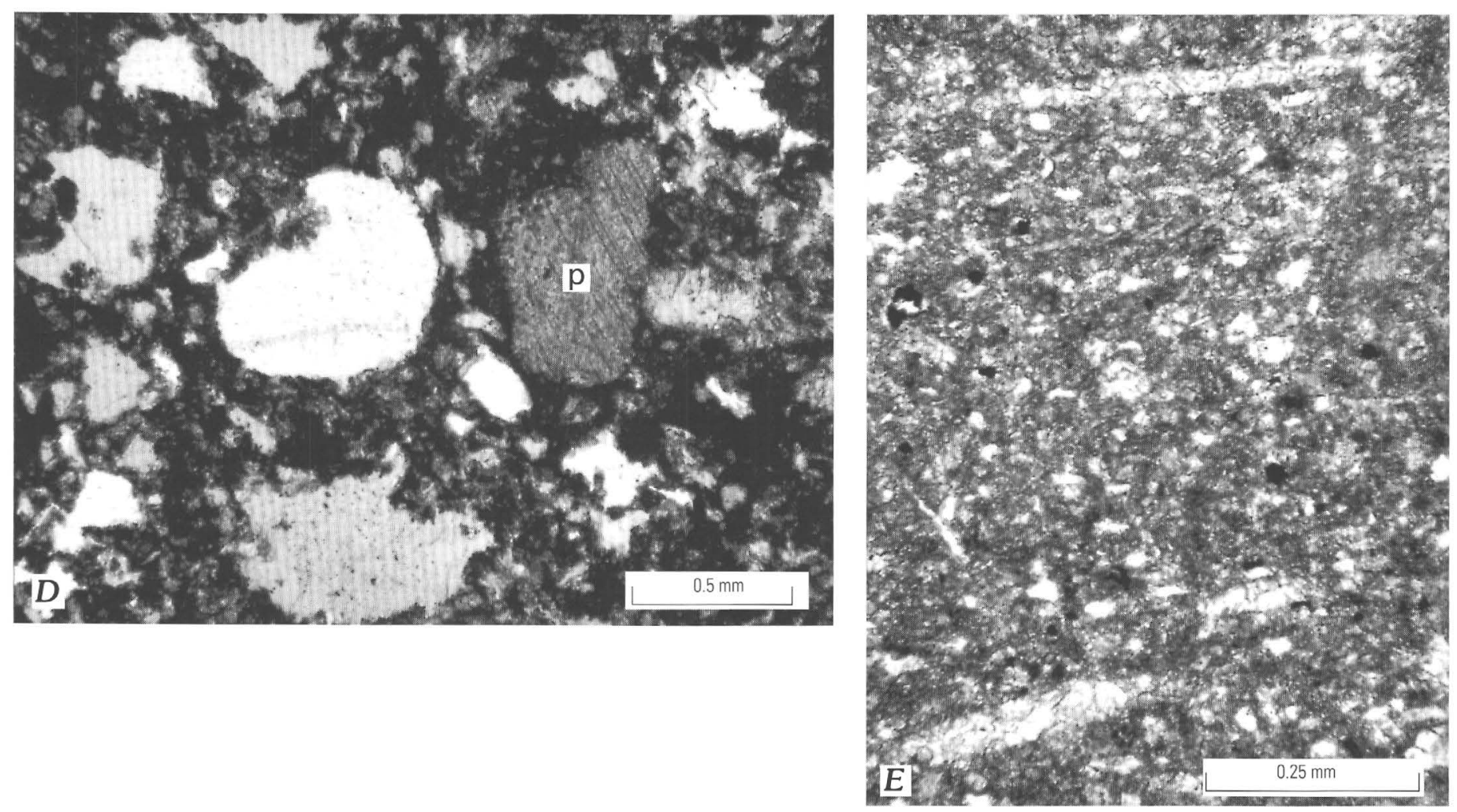

lithologic grounds-grits in the Wickersham are bimodal quartzite with locally abundant potassium and plagioclase feldspar (Weber and others, 1992). The compositional ties that we noted in our study area between calcareous and noncalcareous turbidites in the argillite and quartzite unit need not signify stratigraphic equivalence but could instead indicate erosion and reworking of older material into younger turbidites. Thus, undated, noncalcareous parts of the argillite and quartzite unit (for example, rocks at Munsatli Ridge) could be Cambrian or older and could have provided a source for the coarse quartz and feldspar found in the Silurian turbidites at Lake Minchumina.

\section{Correlation}

Lithologic and paleontologic data detailed above constrain correlations between the Nixon Fork and Minchumina terranes and provide a basis for regional comparison of these strata with coeval deep-water facies exposed to the south (Dillinger terrane). In this section, we compare Upper Cambrian to Lower Devonian deep-water sequences in the Nixon Fork, Minchumina, and Dillinger terranes and consider depositional and tectonic factors affecting their correlation.

\section{Stratigraphic Constraints and Implications}

The lower Paleozoic stratigraphies of the Nixon Fork, Minchumina, and Dillinger terranes are compared in figure 8. Gaps remain in these stratigraphies (particularly for the Minchumina terrane), but the available data outline several interesting patterns. In the discussion that follows, "Nixon Fork terrane" refers only to rocks in the Medfra quadrangle and not to correlative strata in the McGrath and Lime Hills quadrangles to the south that some workers have included in this terrane.

\section{Nixon Fork Terrane}

Deep-water facies in the Nixon Fork terrane accumulated primarily at times when deposition is not recorded in adjacent deep-water sequences. In the Nixon Fork, deep-water sediments formed chiefly during the Late Ordovician, Early Silurian, and Early Devonian, but strata of Late Ordovician age and some intervals in the Early Silurian have not been identified in the Minchumina terrane or in the paleontologically well constrained Dillinger terrane. If these terranes indeed represent parts of a single continental margin, depositional patterns suggest that the shelf edge along this margin stepped back (retreated landward) in Late Ordovician and Early Silurian time. During this period, turbidites and hemipelagic material were trapped near the platform in sequences such as the Paradise Fork Formation and did not reach more distal sequences such as the Dillinger that formed farther from the continental margin.

\section{East Fork Subterrane of the Minchumina Terrane}

Well-dated intervals in the East Fork subterrane correlate in part with platform facies of the Nixon Fork terrane, do not match dated intervals in the Telida subterrane, and correlate well with the Dillinger terrane. The general correlation between Nixon Fork and East Fork rocks supports the suggestion that deepwater facies in the East Fork were derived from the Nixon Fork platform. Strata as old as the Late Cambrian part of the East Fork, however, have not been identified in the Nixon Fork terrane in the Medfra quadrangle. The base of the Novi Mountain Formation, the oldest dated unit in the Nixon Fork terrane in this area, is paleontologically well constrained at its type section as early Early Ordovician (R. manitouensis Zone) (A.G. Harris and J.E. Repetski, unpub. data, 1997). Not all sections of the Novi 
Mountain have been dated, however, and it is possible that, outside its type area, the unit is as old as Late Cambrian. Alternatively, Cambrian strata in the East Fork may have accumulated before the Nixon Fork platform was established and may have derived from a more distant source. Upper Cambrian beds in the East Fork correlate well, biostratigraphically and lithologically, with the Lyman Hills Formation (Bundtzen and others, 1994) in the Dillinger terrane and could have had the same provenance.

Middle Ordovician and Lower Devonian strata in the East Fork subterrane correlate well with, and could have been derived from, the Nixon Fork platform. Ordovician and Devonian East Fork rocks also correlate with parts of the Post River Formation and the Barren Ridge Limestone, respectively, in the Dillinger terrane (fig. 8). However, the Dillinger terrane is characterized by a thick sandstone turbidite unit of Silurian age, the Terra Cotta Mountains Sandstone (Churkin and Carter, 1996). No lithologic or biostratigraphic match for this unit has been found in the East Fork subterrane.

Our biostratigraphic data indicate that strata in the East Fork subterrane accumulated during Late Cambrian, Middle Ordovician, and Early Devonian time. These data could be an artifact of the poor exposures characteristic of this subterrane. It is also possible, however, that carbonate turbidites and associated hemipelagites were generated chiefly at certain times during the long history of the Nixon Fork platform, and the stratigraphy of the East Fork subterrane reflects this discontinuous generation. Episodic transfer of sediment from shelf to basin could be due to tectonic events, eustatic fluctuations, and (or) autocyclic changes in platform sedimentation, among other factors.

\section{Telida Subterrane of the Minchumina Terrane}

Lower Ordovician strata in the Telida subterrane have the strongest ties to the Nixon Fork platform of all deep-water rocks in the study area. Conodonts from calcareous turbidites at locality 23 (fig. 2) match those from the lower part of the Novi Mountain Formation species for species (table 1) (A.G. Harris and J.E. Repetski, unpub. data, 1997). These Telida strata also correlate well, biostratigraphically and lithologically, with the lower siltstone member of the Post River Formation in the Dillinger terrane (Churkin and Carter, 1996). Middle Ordovician rocks in the Telida subterrane correlate with broadly dated parts of the Nixon Fork platform and with the middle part of the Graptolite Canyon Member of the Post River Formation. Telida sandstones at Lake Minchumina correlate with, or are just slightly older than, the lower part of the Paradise Fork Formation in the Nixon Fork terrane and correlate well with the upper part of the Post River Formation. Lake Minchumina sandstones are at least in part older than, and differ in composition from, the Terra Cotta Mountains Sandstone in the Dillinger terrane, as will be discussed below.

\section{Compositional Trends}

Several notable patterns in the composition of Medfra-area deep-water facies can be discerned. These patterns reflect variations in the proportion of carbonate to noncarbonate detritus, and in the types of noncarbonate detritus, that are found in deepwater strata of the Nixon Fork and Minchumina terranes.

\section{Carbonate Input}

Turbidites and hemipelagic strata show similar carbonateto-noncarbonate ratios in the study area. In the Nixon Fork terrane, the East Fork subterrane, and the westernmost exposures of the Telida subterrane, turbidites consist chiefly of carbonate material and were derived principally from a shallowwater carbonate platform, presumably the Nixon Fork platform. Turbidites in the eastern exposures of the Telida subterrane are much less calcareous and include detritus - such as coarse, rounded quartz grains and microcline-not found in turbidites in the other sequences. Finer grained strata show similar trends. In the Nixon Fork terrane, the East Fork subterrane, and the western Telida subterrane, hemipelagic deposits are chiefly micrite and shale, but in the eastern part of the Telida subterrane these deposits are much less terrigenous and consist predominantly of chert. The chert and argillite unit of the Telida subterrane is lithologically similar to, but somewhat older than, the Livengood Dome chert (Upper Ordovician; Chapman and others, 1980) in the Livengood quadrangle to the northeast (Livengood terrane of Silberling and others, 1994) (fig. 1).

Compositional data thus suggest that strata in the eastern Telida subterrane accumulated farthest from the continental margins, experienced relatively little input from carbonate platforms adjacent to these margins, and received some noncarbonate detritus that did not reach the other deep-water sequences in the study area.

\section{Noncarbonate Components}

Noncarbonate detritus occurs sparsely in most parts of the Nixon Fork terrane and the East Fork subterrane but is a significant component in turbidites in the western part of the Telida subterrane. Plagioclase feldspar and metamorphic lithic clasts made up of quartz, chlorite, and (or) white mica are notable minor constituents of Upper Cambrian strata in the East Fork subterrane, Lower Ordovician turbidites in the western Telida subterrane, and Lower Ordovician rocks in the Nixon Fork platform succession; rare metamorphic clasts were also noted in the Silurian-Lower Devonian Paradise Fork Formation. The clasts, and perhaps the feldspar, may have been derived from metamorphosed basement rocks that underlie the Nixon Fork platform succession; these rocks are of early Paleozoic or Precambrian age and include quartz-chlorite-muscovite schist and quartz-plagioclase porphyry (map units Pzp $€_{p}$ and Pzp $€_{v}$ of Patton and others, 1980).

Noncarbonate detritus is a significant component in turbidites of the argillite and quartzite unit in the Telida subterrane. Correlation of these turbidites with the Terra Cotta Mountains Sandstone in the Dillinger terrane has been suggested ( $T$. Bundtzen, Pacific Rim Inc., oral commun., 1997) and is broadly supported by age data from the exposures at Lake Minchumina, although the Minchumina strata are slightly older than the base of the Terra Cotta in its type area (fig. 8) (Churkin and Carter, 1996). Published petrographic descriptions of the Terra Cotta, however, suggest significant compositional differences between the two units. Slate clasts, polycrystalline quartz, and white mica are important constituents of the Terra 


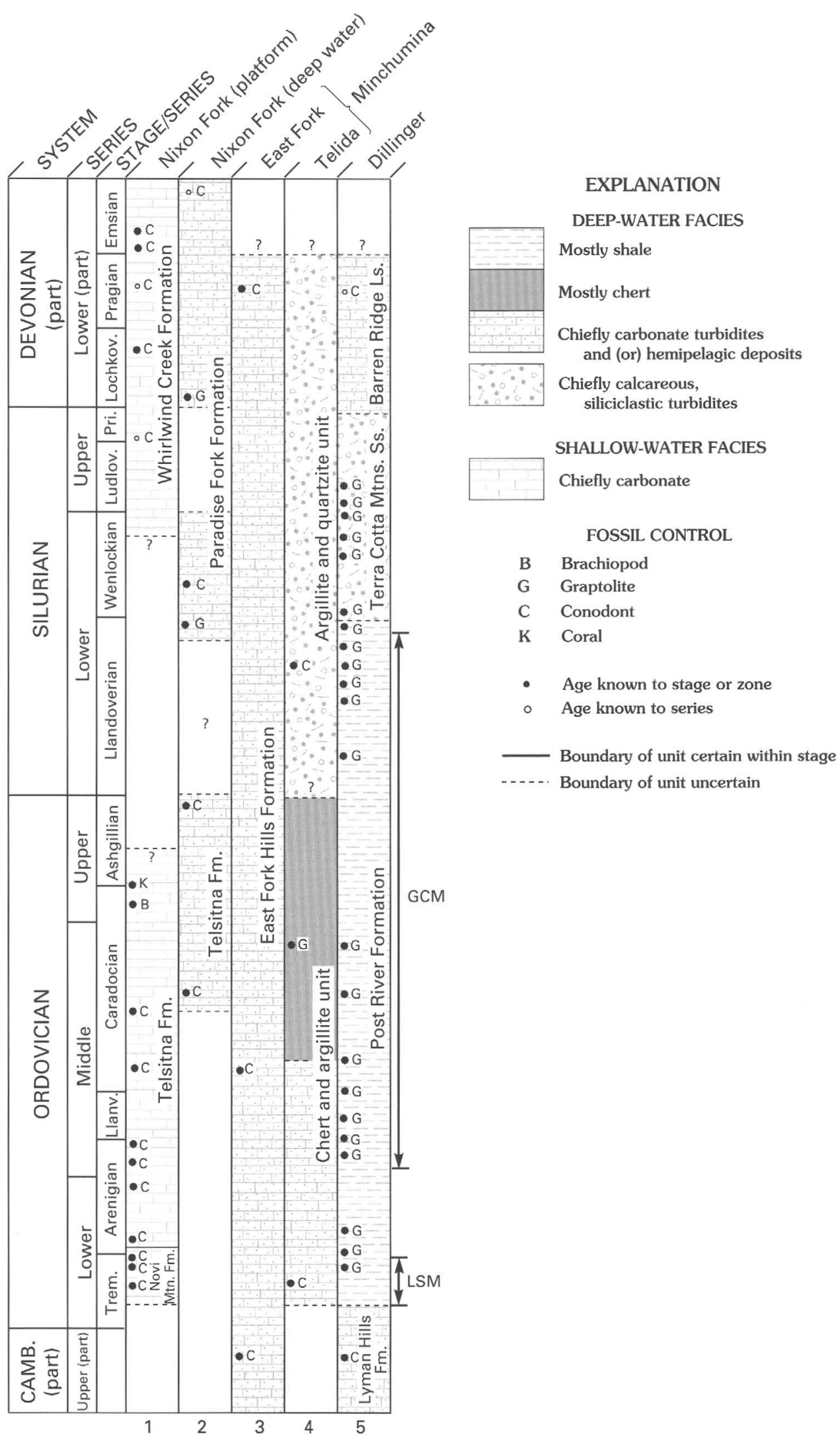

Figure 8. Correlation, lithologies, fossil control, and depositional environments of uppermost Cambrian to Lower Devonian rocks in selected areas of central Alaska. Only fossil groups that most narrowly restrict age of collection or unit are listed. Age of argillite and quartzite unit (column 4) is poorly constrained. Parts of this unit could be older than chert and argillite unit but youngest beds could be as young as Devonian; see text for discussion. Arrows adjacent to column 5 indicate lower siltstone member (LSM) and Graptolite Canyon Member (GCM) of the Post River Formation. Data sources as follows: column 1, Dutro and Patton (1982), A.G. Harris and J.E. Repetski, unpub. data; columns 2-4, this paper; column 5, Bundtzen and others (1994), Churkin and Carter (1996). The former Llandeilian Series is now considered a stage of the Llanvirnian Series (Fortey and others, 1995). 
Cotta (Bundtzen and others, 1994; Churkin and Carter, 1996) but are rare or absent in Telida turbidites, whereas the potassium feldspar and rounded, bimodal, monocrystalline quartz that are so notable in the Telida turbidites are not reported from the Terra Cotta.

As noted above, parts of the argillite and quartzite unit in the Telida subterrane could be of Cambrian-Precambrian age and equivalent to the Wickersham unit (and related rocks) in the Livengood area to the north (Wickersham terrane of Silberling and others, 1994) (fig. 1). Erosion of these older Telida rocks could then have provided quartz and feldspar to the Silurian turbidites at Lake Minchumina. If all parts of the argillite and quartzite unit are of Silurian and younger age, grits such as the Wickersham still seem to be a likely source for the quartz and feldspar found in Telida subterrane turbidites. Whatever the age of the turbidites in the argillite and quartzite unit, their composition implies ties to rocks currently exposed to the northeast (Livengood quadrangle) rather than to the south (McGrath quadrangle). Petrographic data thus indicate that the Telida subterrane contains at least two distinct sequences, each derived from a different source, and these sequences need not have had a shared Paleozoic history.

\section{Structure}

Paleozoic rocks of the study area have been affected by two principal deformations: an earlier folding about northeast axes, followed by dextral strike-slip on the Iditarod fault system. The focus will be here on aspects of the structure that bear on the palinspastic relations among the strata of interest.

The Iditarod fault is one of the most significant strike-slip faults in Alaska (Grantz, 1966). In the Iditarod quadrangle, the fault cuts a Late Cretaceous to early Tertiary volcano-plutonic complex and separates its two halves-the Iditarod Volcanics and the Beaver Mountains volcanic field—by 88 to $94 \mathrm{~km}$ in a dextral sense (Miller and Bundtzen, 1988). Similarly, in the McGrath and Medfra quadrangles, the eastern contact between Cretaceous siliciclastic strata of the Kuskokwim Group and Paleozoic carbonate rocks of the Nixon Fork terrane shows a dextral map separation of about $90 \mathrm{~km}$. Although the Iditarod fault has only one strand of any significance in the Iditarod quadrangle (Miller and Bundtzen, 1988), several splays are mapped in the Medfra quadrangle (Patton and others, 1980). Presumably, dextral displacement across all of the splays sums to at least $\sim 90$ $\mathrm{km}$ - or even more if some dextral strike-slip preceded deposition of the Kuskokwim Group. There are no piercing points on any of these splays, so map separation cannot be measured; they are presumed to be dextral strike-slip faults because of their continuity and subparallelism with the Iditarod fault in the Iditarod quadrangle.

In the area of figure 2, the Iditarod fault has four main strands, labeled A through D (fig. 9), and several minor ones. Strand A juxtaposes deep-water facies of the Minchumina terrane against shallow-marine carbonate rocks of the Upper Silurian-Devonian Whirlwind Creek Formation. Strand A is presumably the main strand of the Iditarod fault system in this area; horizontal displacement is likely to be several tens of kilometers. Strand B juxtaposes the Whirlwind Creek Formation against the
Upper Cretaceous to lower Tertiary Sischu volcanics. There is no direct evidence for the amount of displacement but it is inferred to have a small component of northwest-side-down vertical displacement and perhaps a few to a few tens of kilometers of dextral horizontal displacement. Strand C juxtaposes deep-water facies of the chert and argillite unit, which depositionally underlie the Sischu volcanics, against coeval shallow-marine facies of the Nixon Fork terrane. As noted above, an Early Ordovician conodont fauna in the chert and argillite unit precisely matches the conodont fauna of the age-equivalent Novi Mountain Formation. Significant fault displacement, either strike-slip, thrusting, or both, is needed to explain the current close juxtaposition of environments that must once have been farther apart.

A second regional-scale dextral strike-slip fault, the Farewell, lies to the south of the present study area, but it does bear on the palinspastic relations between the Minchumina and Dillinger terranes. In the McGrath and Lime Hills quadrangles, a Late Silurian to Early Devonian algal barrier reef complex in the Nixon Fork terrane shows a dextral map separation of 145 to 153 km (Blodgett and Clough, 1985).

The Paleozoic rocks that were rearranged along these various strike-slip faults had already been folded. Fold axial traces trend roughly northeast-southwest in both the Nixon Fork and Minchumina terranes. Nixon Fork strata typically are deformed into open to tight folds with wavelengths as long as a few kilometers. On this basis, we suggest that each of the fault-bounded tracts containing Nixon Fork strata were probably not much wider prior to shortening than they are today-perhaps 10-20 percent wider. Shortening across the Minchumina terrane cannot be estimated with confidence but was undoubtedly far greater than in the Nixon Fork. Shoreline exposures at Lake Minchumina disclose the presence of inverted subhorizonal beds and nearly recumbent isoclinal folds (T.M. Kusky, Boston University, unpub. data, 1997) that require extreme amounts of shortening for at least some rocks in the belt. Large amounts of shortening are not suggested by the simple map pattern of the Minchumina terrane (fig. 2), but this is strictly an artifact of the mapping conditions: outcrops and helicopter landings are so sparse that entire mountains were assigned to a single unit on the basis of one or two rubble outcrops. It seems likely that the belt of northwest-directed thrust faults mapped in the Livengood quadrangle (Weber and others, 1992) continues along strike into the area of figure 2 and that the structure in the Minchumina terrane is as complex as in the southeastern part of the Livengood quadrangle.

Figure 9 shows the palinspastic distribution of our sample localities. On the Farewell fault, we restored $150 \mathrm{~km}$ of dextral map-separation (Blodgett and Clough, 1985). Where the Iditarod fault has a single strand (extreme western part of fig. 9, and beyond), we restored $90 \mathrm{~km}$ of dextral map separation (Miller and Bundtzen, 1988). For lack of any concrete evidence, we arbitrarily restored $30,20,20$, and $20 \mathrm{~km}$ of dextral map separation across strands A, B, C, and D, respectively. Regardless of the displacements assigned to the individual strands, the total on all four should sum to $90 \mathrm{~km}$; the relative positions of rocks northwest of strand D and southeast of strand A are the same regardless of the details. Figure 9 does not restore any shortening-only strike-slip. As noted above, shortening was relatively minor in the Nixon Fork terrane but probably quite severe in the 
Minchumina terrane. The distance across the Minchumina terrane between the Farewell fault and Iditarod fault systems was undoubtedly wider than at present.

In figure 9, deep-water strata between strands B and C remain isolated from the broader tract of the Minchumina terrane, and there seems no way to bring these tracts together given only $90 \mathrm{~km}$ of only dextral displacement on strands A through D combined. One possible explanation for this map pattern is that deeper water strata between strands B and C accumulated in an intrashelf basin in the Nixon Fork platform, as implied by the restoration in figure 9. A second possibility is that these deepwater facies record a northwestward Ordovician transgression across the Nixon Fork platform that was followed by southeastward progradation of this platform during Silurian-Devonian time, prior to any shuffling on strike-slip faults. A third possibility is that rocks between strands $\mathrm{B}$ and $\mathrm{C}$ represent a piece of the Minchumina terrane that was thrust into position from the southeast, prior to any shuffling on strike-slip faults, over shallow-water strata of the Nixon Fork platform that lie between strands A and B. A fourth possibility is that the shallowwater rocks between strands A and B belong to the Minchumina and not the Nixon Fork terrane; in this interpretation, fault strand C, not strand A, marks the boundary between the Nixon Fork and Minchumina terranes (Patton and others, 1984; Patton and others, 1994). These alternatives cannot be evaluated without more information.

\section{Biogeography}

The ultimate origin of lower Paleozoic rocks in central Alaska remains contentious. Some authors have suggested that the Nixon Fork, Minchumina, and Dillinger terranes represent

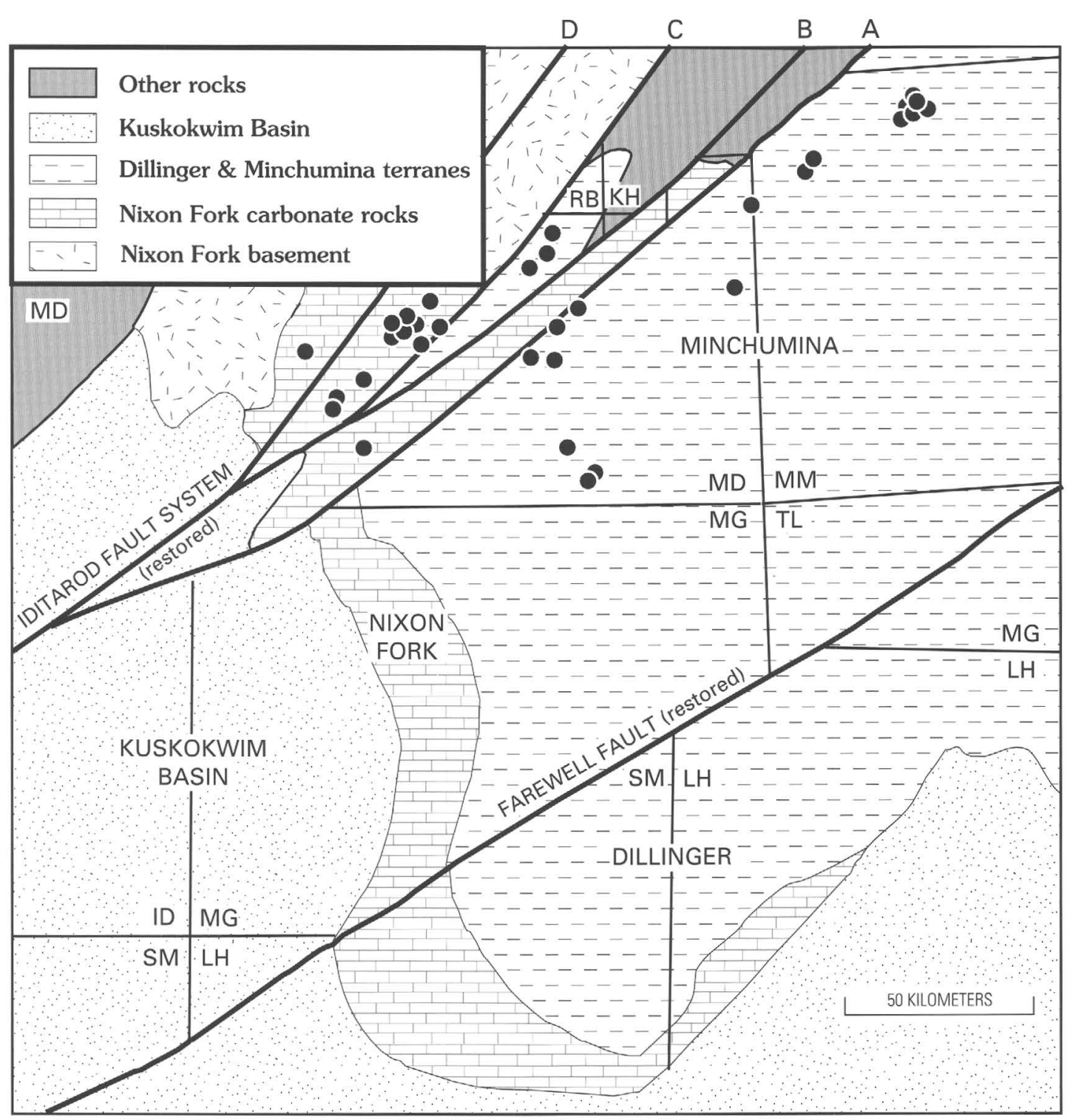

Figure 9. Palinspastic map of study area. Letters A, B, C, and D refer to strands of the Iditarod fault system mentioned in text. Restoration of 90 and $150 \mathrm{~km}$ of dextral strike-slip on the Iditarod and Farewell faults, respectively, aligns the deep-water Dillinger and Minchumina terranes in a position east of the Nixon Fork carbonate platform. Terrane affinity of lower Paleozoic rocks between fault strands $\mathrm{A}$ and $\mathrm{C}$ is uncertain; see text for dicussion. Black dots are locations plotted in figure 2. Fine lines are quadrangle boundaries; quadrangle abbreviations as in figure 1. 
displaced fragments of the North American continental margin (Decker and others, 1994), but, more recently, others have interpreted these terranes as a sequence rifted away from the Siberian craton (Blodgett and Brease, 1997).

Biogeographic affinities of lower Paleozoic faunas can constrain the paleogeographic position of central Alaska, but the faunas discussed above are not particularly useful for such analyses. Deep-water conodont faunas, and most conodont faunas of Silurian and Devonian age, are relatively cosmopolitan. With the exception of a faunule from the upper part of the Telsitna Formation in the Nixon Fork terrane (loc. 5, fig. 2; table 1), conodont collections from deep-water facies in the Medfra area consist of tropical cosmopolites, pandemics, and North American Midcontinent species. The collection at locality 5 , however, of late Middle-Late Ordovician age, consists chiefly of Siberian-Alaskan province (SAP) elements with minor pandemics.

SAP elements are common in some collections from shallow-water facies of the Nixon Fork terrane, particularly those of late Early-Middle Ordovician age (Dumoulin, Bradley, and others, 1998). They are also noteworthy in some Early, Middle, and Late Ordovician faunas from the western and central Brooks Range and Seward Peninsula of northern Alaska (Dumoulin and Harris, 1994). A full explication of the biogeography of lower Paleozoic rocks in central Alaska is beyond the scope of this paper, but it is worth noting here that Siberian-Alaskan province elements have not been identified from Paleozoic conodont faunas of east-central Alaska or northwestern Canada (Dumoulin, Harris, and de Freitas, 1998; A.G. Harris, unpub. data).

\section{Conclusions}

Deep-water facies of Cambrian through Devonian age crop out widely in the eastern Medfra and western Mt. McKinley quadrangles and have been included in several discrete terranes. Calcareous hemipelagic deposits and fine-grained carbonate turbidites comprise the upper part of the Telsitna Formation (Middle-Upper Ordovician) and the Paradise Fork Formation (Lower Silurian-Lower Devonian) in the Nixon Fork terrane, the East Fork Hills Formation (Upper Cambrian-Lower Devonian) in the East Fork subterrane of the Minchumina terrane, and western exposures of the chert and argillite unit (Ordovician) in the Telida subterrane of the Minchumina terrane. These strata were derived largely from the Nixon Fork carbonate platform and correlate well with parts of the Dillinger terrane exposed to the south. The chert and argillite unit (eastern outcrops) and the argillite and quartzite unit (Silurian-Devonian? and possibly older) in the Telida subterrane of the Minchumina terrane consist chiefly of siliceous hemipelagic deposits and quartzofeldspathic turbidites and may correlate with parts of the Wickersham and Livengood terranes to the northeast (Livengood quadrangle). The Telida subterrane as presently defined thus includes two roughly coeval sequences of disparate origin.

Deep-water strata of the Minchumina and Dillinger terranes restore to a position east of the Nixon Fork carbonate platform when 90 and $150 \mathrm{~km}$ of dextral strike-slip on the Iditarod and Farewell faults, respectively, are removed. This restoration and our petrographic and paleontologic data suggest that lower Paleozoic rocks in the Nixon Fork and Dillinger terranes, as well as those in the western part of the Minchumina terrane, formed along a single continental margin. Strata in the eastern part of the Minchumina terrane (eastern part of the Telida subterrane) differ in composition and provenance from those to the west and may have had a distinct geologic history.

\section{Acknowledgments}

Tom Bundtzen and Grant Abbott accompanied the field party and provided valuable insights about potentially correlative rocks in the Alaska Range and Yukon. Tim Kusky helped with structural studies at Lake Minchumina. Stan Finney identified a key graptolite collection. We thank Jack and Sherry Hayden of Denali West Lodge for logistical support.

\section{References Cited}

Armstrong, H.A., 1990, Conodonts from the Upper Ordovician-Lower Silurian carbonate platform of north Greenland: Gronlands Geologiske Undersogelse Bulletin 159, 151 p.

Barrick, J.E., and Klapper, G., 1976, Multielement Silurian (late Llandoverian-Wenlockian) conodonts of the Clarita Formation, Arbuckle Mountains, Oklahoma, and phylogeny of Kockelella: Geologica et Palaeontologica, v. 10, p. 59-100.

Blodgett, R.B., and Clough, J.G., 1985, The Nixon Fork Terrane-Part of an in situ peninsular extension of the Paleozoic North American continent [abs.]: Geological Society of America, Abstracts with Programs, v. 17, no. 6, p. 342.

Blodgett, R.B., and Brease, P.F., 1997, Emsian (late Early Devonian) brachiopods from Shellabarger Pass, Talkeetna C-6 quadrangle, Denali National Park, Alaska indicate Siberian origin for Farewell terrane [abs.]: Geological Society of America, Abstracts with Programs, v. 29 , no. 5 , p. 5.

Bundtzen, T.K., Laird, G.M., Blodgett, R.B., Clautice, K.H., and Harris, E.E., 1994, Geology of the Gagaryah River area, Lime Hills C-5 and C-6 quadrangles, southwest Alaska: Alaska Divison of Geological \& Geophysical Surveys Public Data File 94-40, 17 p., 1 plate, scale $1: 63,360$.

Chapman, R.M., Churkin, Michael, Jr., Carter, Claire, and Trexler, J.H., Jr., 1981, Ordovician graptolites and early Paleozoic radiolarians in the Lake Minchumina area date a regional shale and chert belt, in Albert, N.R.D., and Hudson, Travis, eds., The U.S. Geological Survey in Alaska: Accomplishments During 1979: U.S. Geological Survey Circular 823-B, p. B32-B34.

Chapman, R.M., Weber, F.R., Churkin, Michael, Jr., and Carter, Claire, 1980, The Livengood Dome Chert: A new Ordovician formation in central Alaska and its relevance to displacement on the Tintina fault, in Shorter Contributions to Stratigraphy and Structural Geology, 1979: U.S. Geological Survey Professional Paper 1126-F, p. F1-F13.

Chapman, R.M., and Yeend, Warren, 1981, Geologic reconnaissance of the east half of Kantishna River quadrangle and adjacent areas, in Albert, N.R.D., and Hudson, Travis, eds., The U.S. Geological Survey in Alaska: Accomplishments During 1979: U.S. Geological Survey Circular 823-B, p. B30-B32.

Chapman, R.M., Yeend, W.E., and Patton, W.W., Jr., 1975, Preliminary reconnaissance map of the western half of Kantishna River quadrangle, Alaska: U.S. Geological Survey Open-File Map 75-351, scale $1: 250,000$. 
Churkin, Michael, Jr., and Carter, Claire, 1996, Stratigraphy, structure, and graptolites of an Ordovician and Silurian sequence in the Terra Cotta Mountains, Alaska Range, Alaska: U.S. Geological Survey Professional Paper 1555, $84 \mathrm{p}$.

Cook, H.E., Hine, A.C., and Mullins, H.T., 1983, Platform margin and deep water carbonates: Society of Economic Paleontologists and Mineralogists Short Course Notes No. 12, 573 p.

Corradini, Carlo, Ferretti, Annalisa, Serpagli, Enrico, and Barca, Sebastiano, 1998, The Ludlow-Pridoli section Genna Ciuerciu, west of Silius: Giornale di Geologia, ser. 3, Special Issue, ECOS VII-Sardinia Guidebook, p. 112-118.

Decker, John, Bergman, S.C., Blodgett, R.B., Box, S.E., Bundtzen, T.K., Clough, J.G., Coonrad, W.L., Gilbert, W.G., Miller, M.L., Murphy, J.M., Robinson, M.S., and Wallace, W.K., 1994, Geology of southwestern Alaska, in Plafker, George, and Berg, H.C., eds., The Geology of Alaska: Boulder, Colo., Geological Society of America, The Geology of North America, v. G-1, p. 285-310.

Dumoulin, J.A., Bradley, D.C., Harris, A.G., and Repetski, J.E., 1998, Sedimentology, conodont biogeography, and subsidence history of the Nixon Fork terrane, Medfra quadrangle, Alaska [abs.]: Celle, Germany, III International Conference on Arctic Margins Abstracts, p. 49.

Dumoulin, J.A., and Harris, A.G., 1994, Depositional framework and regional correlation of pre-Carboniferous metacarbonate rocks of the Snowden Mountain area, central Brooks Range, northern Alaska: U.S. Geological Survey Professional Paper 1545, 74 p.

Dumoulin, J.A., Harris, A.G., and de Freitas, T.A., 1998, Facies patterns and conodont biogeography in Arctic Alaska and the Canadian Arctic Islands: Evidence against juxtaposition of these areas during early Paleozoic time [abs.]: Celle, Germany, III International Conference on Arctic Margins Abstracts, p. 50.

Dutro, J.T., Jr., and Patton, W.W., Jr., 1982, New Paleozoic formations in the northern Kuskokwim Mountains, west-central Alaska, in Stratigraphic Notes, 1980-1982: U.S. Geological Survey Bulletin 1529-H, p. $\mathrm{H} 13-\mathrm{H} 22$.

Fortey, R.A., Harper, D.A.T., Ingham, J.K., Owen, A.W., and Rushton, A.W.A., 1995, A revision of Ordovician series and stages from the historical type area: Geological Magazine, v. 132, no. 1, p. 15-30.

Gilbert, W.G., and Bundtzen, T.K., 1984, Stratigraphic relationship between Dillinger and Mystic terranes, western Alaska Range, Alaska [abs.]: Geological Society of America Abstracts with Programs, v. 16 , no. 5 , p. 286.

Grantz, Arthur, 1966, Strike-slip faults in Alaska: U.S. Geological Survey Open-File Report 267, $82 \mathrm{p}$.

Jaeger, Hermann, 1975, Die Graptolithenführung im Silur/Devon des CelIon-Profils (Karnische Alpen): Carinthia II, p. 111-126.

Jeppsson, Lennart, 1972, Some Silurian conodont apparatuses and possible conodont dimorphism: Geologica et Palaeontologica, no. 6, p. 51-69.

Jones, D.L., Silberling, N.J., Berg, H.C., and Plafker, George, 1981, Map showing tectonostratigraphic terranes in Alaska, columnar sections, and summary description of terranes: U.S. Geological Survey Open-File Report 81-792, 20 p., 2 sheets, scale 1:2,500,000.

Kleffner, M.A., 1995, A conodont- and graptolite-based Silurian chronostratigraphy, in Mann, K.O., and Lane, H.R., eds., Graphic
Correlation: SEPM Society for Sedimentary Geology Special Publication No. 53, p. 159-176.

Measures, E.A., Rohr, D.M., and Blodgett, R.B., 1992, Depositional environments and some aspects of the fauna of Middle Ordovician rocks of the Telsitna Formation, northern Kuskokwim Mountains, Alaska, in Bradley, D.C., and Dusel-Bacon, Cynthia, eds., Geologic Studies in Alaska by the U.S. Geological Survey, 1991: U.S. Geological Survey Bulletin 2041, p. 186-201.

Miller, M.L., and Bundtzen, T.K., 1988, Right-lateral offset solution for the Iditarod-Nixon Fork fault, western Alaska: U.S. Geological Survey Circular 1016, p. 99-103.

Nowlan, G.S., McCracken, A.D., and Chatterton, B.D.E., 1988, Conodonts from Ordovician-Silurian boundary strata, Whittaker Formation, Mackenzie Mountains, Northwest Territories: Geological Survey of Canada Bulletin 373, $99 \mathrm{p}$.

Oliver, W.A., Jr., Merriam, C.W., and Churkin, Michael, Jr., 1975, Ordovician, Silurian, and Devonian corals of Alaska: U.S. Geological Survey Professional Paper 823-B, p. 13-44.

Patton, W.W., Jr., Box, S.E., Moll-Stalcup, E.J., and Miller, T.P., 1994, Geology of west-central Alaska, in Plafker, George, and Berg, H.C., eds., The Geology of Alaska: Boulder, Colo., Geological Society of America, The Geology of North America, v. G-1, p. 241-269.

Patton, W.W., Jr., Moll, E.J., Dutro, J.T., Jr., Silberman, M.L., and Chapman, R.M., 1980, Preliminary geologic map of the Medfra quadrangle, Alaska: U.S. Geological Survey Open-File Report 80-811A, scale $1: 250,000$.

Patton, W.W., Jr., Moll, E.J., and King, H.D., 1984, The Alaskan Mineral Resources Assessment Program: Guide to information contained in the folio of geologic and mineral-resource maps of the Medfra quadrangle, Alaska: U.S. Geological Survey Circular 928, 11 p.

Savage, N.M., Rohr, D.M., and Blodgett, R.B., 1995, Late Silurian conodonts from the Medfra B-4 quadrangle, west-central Alaska [abs.]: Geological Society of America Abstracts with Programs, v. 27, no. 5, p. 76-77.

Scholle, P.A., Bebout, D.G., and Moore, C.H., 1983, Carbonate depositional environments: American Association of Petroleum Geologists Memoir 33, 708 p.

Silberling, N.J., Jones, D.L., Monger, J.W.H., Coney, P.J., Berg, H.C., and Plafker, George, 1994, Lithotectonic terrane map of Alaska and adjacent parts of Canada, in Plafker, George, and Berg, H.C., eds., The Geology of Alaska: Boulder, Colo., Geological Society of America, The Geology of North America, v. G-1, plate 3, 1 sheet, scale $1: 2,500,000$.

Tipnis, R.S., 1979, Fused clusters of Ptiloncodus simplex Harris: An Ordovician phosphatic microfossil, in Current Research, Part C: Geological Survey of Canada Paper 79-1C, p. 51-54.

Weber, F.R., Wheeler, K.L., Rinehart, C.D., Chapman, R.M., and Blodgett, R.B., 1992, Geologic map of the Livengood quadrangle, Alaska: U.S. Geological Survey Open-File Report 92-562, 20 p., scale 1:250,000.

Wilson, F.H., Dover, J.H., Bradley, D.C., Weber, F.R., Bundtzen, T.K., and Haeussler, P.J., 1998, Geologic map of central (Interior) Alaska: U.S. Geological Survey Open-File Report 98-133, 3 sheets, scale $1: 500,000,63 \mathrm{p}$.

Wilson, J.L., 1975, Carbonate facies in geologic history: New York, Springer-Verlag, $471 \mathrm{p}$. 



\title{
Alagogshak Volcano: A Pleistocene Andesite-Dacite Stratovolcano in Katmai National Park
}

\author{
ByWes Hildreth, Judy Fierstein, Marvin A. Lanphere, and David F. Siems
}

\section{Abstract}

Alagogshak volcano, a newly recognized volcanic-front stratovolcano on the Alaska Peninsula rangecrest, $15 \mathrm{~km}$ southwest of Katmai Pass, produced $10-18 \mathrm{~km}^{3}$ of andesite-dacite eruptive products during several episodes of activity in the middle and late Pleistocene. From a central vent marked by hydrothermal alteration and remnants of a cratered fragmental cone on the present-day drainage divide, glacially incised stacks of lava flows (57-66 percent $\mathrm{SiO}_{2}$ ) dip radially and extend 6-10 $\mathrm{km}$ in most directions. Lava flows that make up four ridge-capping outliers well west of the volcano may also have erupted there. The medium-K calcalkaline Alagogshak eruptive suite is compositionally varied, probably reflecting independent evolution of different magma batches supplied in several episodes spread intermittently over at least 600,000 years. In contrast, the exclusively Holocene andesite-dacite suite (59-64 percent $\mathrm{SiO}_{2}$ ) produced by the Mount Martin cone, which is centered $3 \mathrm{~km}$ northeast of Alagogshak, yields far more coherent compositional trends.

\section{Introduction}

While studying the volcanoes of the Katmai cluster (fig. 1), we recognized a glacially eroded vent complex of Pleistocene age on the Alaska Peninsula drainage divide, $3 \mathrm{~km}$ southwest of the active crater of Mount Martin. Mapping in 1996 and 1997 demonstrated this vent to be the source of most of the glaciated lava flows nearby and showed Mount Martin to be a small, much younger edifice, constructed entirely during the Holocene. This note describes the remnants of what we call the Alagogshak volcano, estimates its original extent and volume, and compares the compositions of its eruptive products with those of nearby Mount Martin.

\section{Glacially Eroded Ediface}

Surviving remnants of Alagogshak volcano are mapped in figure 2. The high point today is a rounded ice-capped knob at an elevation of about $6,020 \mathrm{ft}(1,835 \mathrm{~m})$ on the rangecrest $12.5 \mathrm{~km}$ northeast of Kejulik Pass, $15 \mathrm{~km}$ southwest of Katmai Pass, and $95 \mathrm{~km}$ southeast of the town of King Salmon (fig. 1). This summit forms the east rim of a glacially gutted vent complex, from which lavas flowed north toward Angle Creek, south into the forks of Kejulik River, and southeast into Alagogshak Creek.

The vent is an area of focused hydrothermal alteration about $800 \mathrm{~m}$ wide, now hollowed out as a cirque-amphitheater (fig. 3) occupied by a northwest-draining glacier. Preserved along its south rim (the present rangecrest) are outward-dipping agglutinates, beds of coarse scoria (57-58 percent $\mathrm{SiO}_{2}$ ), and poorly sorted layers of phreatomagmatic ejecta rich in dense glassy blocks. Preserved along the north rim of the cirque (fig. 3) are part of the fumarolically acid altered core, a remnant of the old crater rim draped by stratified ejecta that dip both inward and outward, and an effusive lava flow of black glassy dacite (63.7 percent $\mathrm{SiO}_{2}$ ) that dips $20^{\circ} \mathrm{NW}$. and is as thick as $140 \mathrm{~m}$.

Stacks of lava flows that dip radially away from the vent region are well preserved in four main sectors (fig. 2). In all sectors, the lavas were emplaced onto a ruggedly incised glacial terrain that had more than $1,200 \mathrm{~m}$ of local relief, cut entirely into subhorizontal siltstone and sandstone of the Jurassic Naknek Formation (Riehle and others, 1993). To the south, at the head of the east fork of Kejulik River, a set of glaciated benches that steps down from the summit to an elevation as low as $1,500 \mathrm{ft}$ $(450 \mathrm{~m})$ consists of a stack of at least seven andesitic lava flows (57-63 percent $\mathrm{SiO}_{2}$ ), which range from 10 to $30 \mathrm{~m}$ thick. At their present-day distal extremity, canyon-wall remnants of these flows range in elevation from $1,500 \mathrm{ft}$ to as high as $2,300 \mathrm{ft}(700$ $\mathrm{m}$ ), indicating that the stack was once at least $250 \mathrm{~m}$ thick there and suggesting that intracanyon lava flows formerly extended much farther downstream.

To the southwest, the Kejulik River cleaver is a stack of about 20 andesite-dacite lava flows (fig. 4) that dip gently away from the vent and range from 8 to $40 \mathrm{~m}$ thick and from 58 percent to 66 percent $\mathrm{SiO}_{2}$. The greatest thickness of the stack preserved is $950 \mathrm{ft}(300 \mathrm{~m})$. One of the lavas thickens southward as a formerly intracanyon flow as thick as $80 \mathrm{~m}$ that today forms a sinuous interfluve along the east wall of the main Kejulik River (fig. 2), where its lowest basal elevation is $1,300 \mathrm{ft}(400 \mathrm{~m})$.

To the northwest, two glaciated lava-flow stacks of different ages face each other across the outlet gorge that drains the 


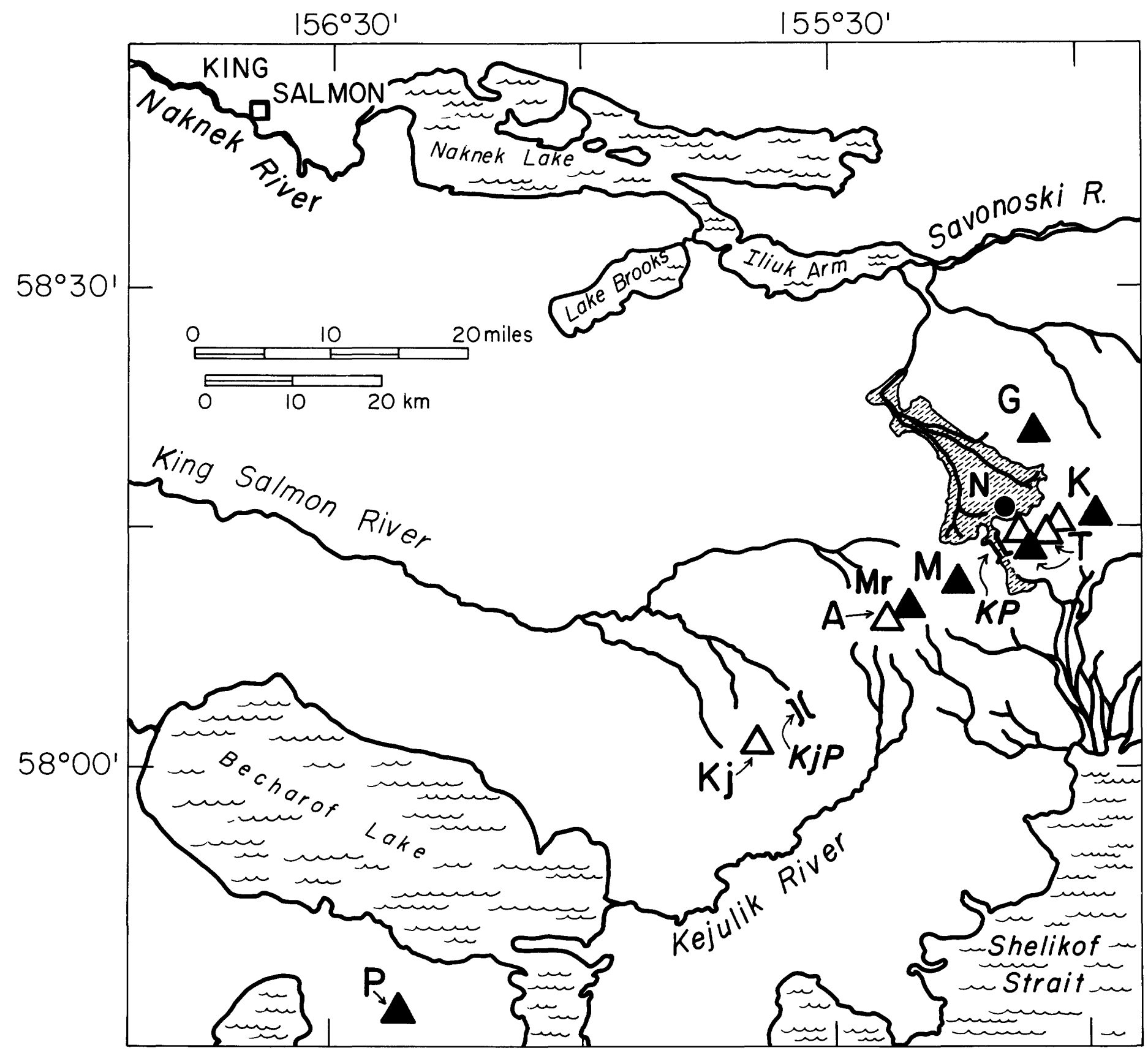

Figure 1. Map showing part of the stratovolcanic chain along the Alaska Peninsula, southeast of the town of King Salmon. Solid triangles indicate cones active during the Holocene; open triangles indicate Pleistocene cones long extinct. The Katmai volcanic cluster includes Alagogshak (A), Martin (Mr), Mageik (M), Griggs (G), Trident (T; three extinct cones and one recently active cone), and Mount Katmai (K). Farther southwest are Kejulik $(K j)$ and Peulik (P) volcanoes. Solid circle (N) indicates Novarupta, site of the great explosive eruption of 1912, when ignimbrite (shaded valley fill) was emplaced in the Valley of Ten Thousand Smokes (Hildreth, 1983). Low points along the volcanic axis, which here forms the Alaska Peninsula drainage divide, include Katmai Pass (KP) and Kejulik Pass (KjP).

vent-cirque glacier toward Angle Creek. Today, both stacks reach about $6 \mathrm{~km}$ from the vent, dipping $5^{\circ}-10^{\circ} \mathrm{NNW}$, but they originally extended many kilometers farther. The western (older) stack is a set of three or four andesite flows (60-63 percent $\mathrm{SiO}_{2}$ ), each 50 to $125 \mathrm{~m}$ thick, that widely exhibit basal colonnades and thick entablatures of slender glassy columns that suggest ice-contact emplacement. The eastern stack begins with three dacite lava flows (each 64 percent $\mathrm{SiO}_{2}$ ), one of which is $200 \mathrm{~m}$ thick, glassy, columnar, probably englacial, and descends to a basal elevation as low as $1,900 \mathrm{ft}(580 \mathrm{~m})$ about $90 \mathrm{~m}$ lower than the nearby older stack. The dacites are overlain proximally by three or four more andesite-dacite lava flows (58-64 percent $\mathrm{SiO}_{2}$ ) that are extensively covered by ice and till but include the thick crater-rim dacite illustrated in figure 3.

To the southeast, a single thick glassy andesite lava flow (61 percent $\mathrm{SiO}_{2}$ ) descended steeply from the rangecrest and spread into Alagogshak Creek, where it now forms a glacially scoured plateau. Its base is today as low as $1,500 \mathrm{ft}(450 \mathrm{~m})$, but because the flow thickens to more than $100 \mathrm{~m}$ at its distal extremity, it must have extended much farther downstream prior to glacial excavation. 


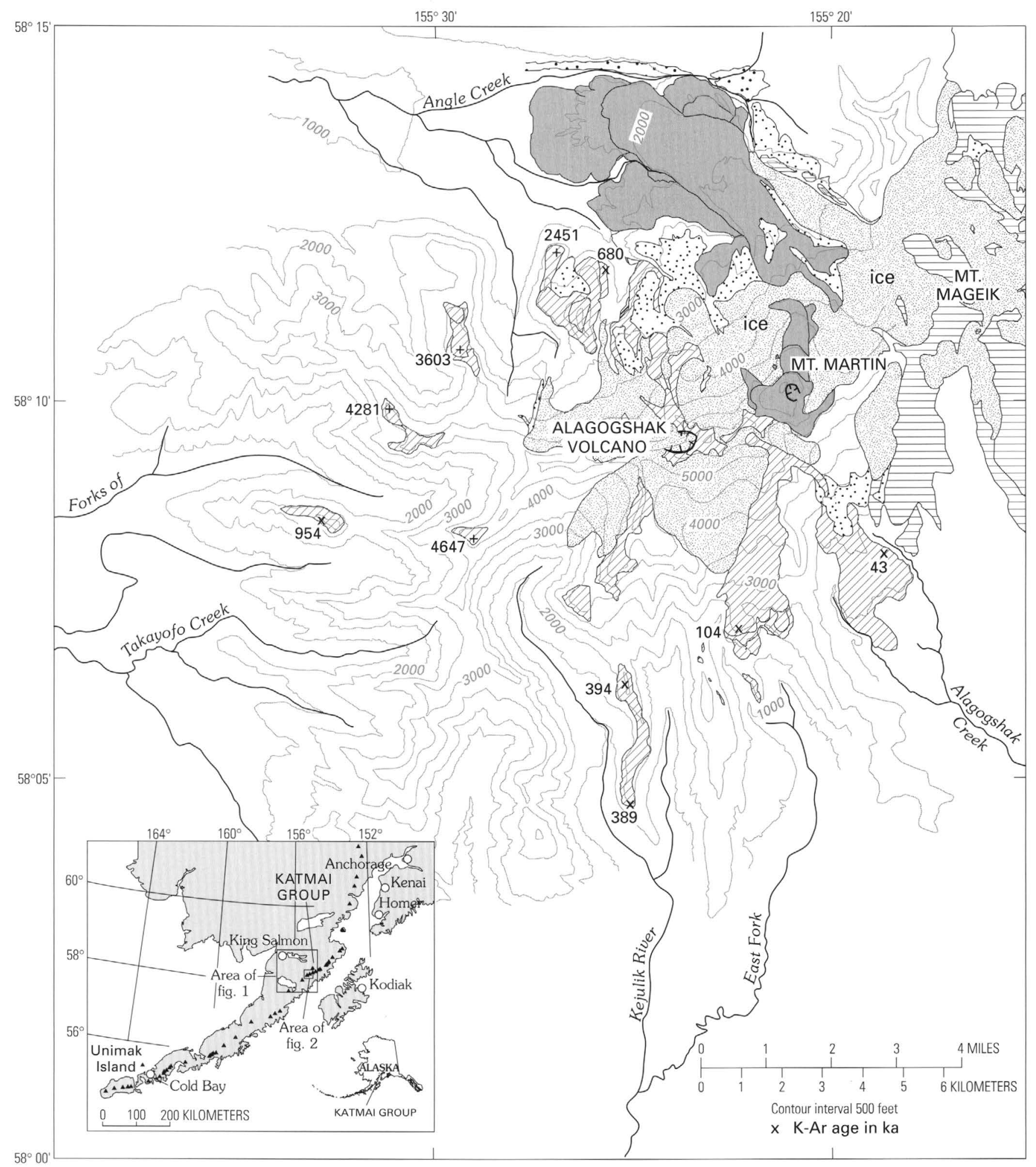

Figure 2. Distribution of Holocene eruptive products of Mount Martin (dark shading) and glacially eroded Pleistocene lavas of Alagogshak volcano (diagonal pattern). Their craters are indicated by hachures. Also shown is the western half of Mount Mageik (horizontal pattern) and various glacial deposits (stippled pattern). Pale shading is glacial ice, and uncolored regional basement is Jurassic Naknek Formation (plus scattered surficial deposits and a few Tertiary intrusive rocks, not indicated). Elevations (+) in feet (1 m=3.28 ft). Locations of samples dated by K-Ar method (table 1, this report; Shew and Lanphere, 1992) are indicated by "X" accompanied by age (in ka). Topographic base simplified from USGS 1:63,360 quadrangles Mt. Katmai $A-4$ and $A-5$.

\section{Western Outliers}

In addition to the four unequivocal outflow sectors just described, four craggy outliers of ridge-capping andesite 6-10 $\mathrm{km}$ west of the Alagogshak vent (fig. 2) may also have erupted there.
The northernmost outlier, at peak 3603 on the divide between tributaries of Angle Creek, consists of three, gently northwest dipping, andesite lava flows (57-61 percent $\mathrm{SiO}_{2}$ ), each as thick as $100 \mathrm{~m}$. Thick flow-breccia and glassy columnar zones suggest ice-contact emplacement. The southernmost (and 


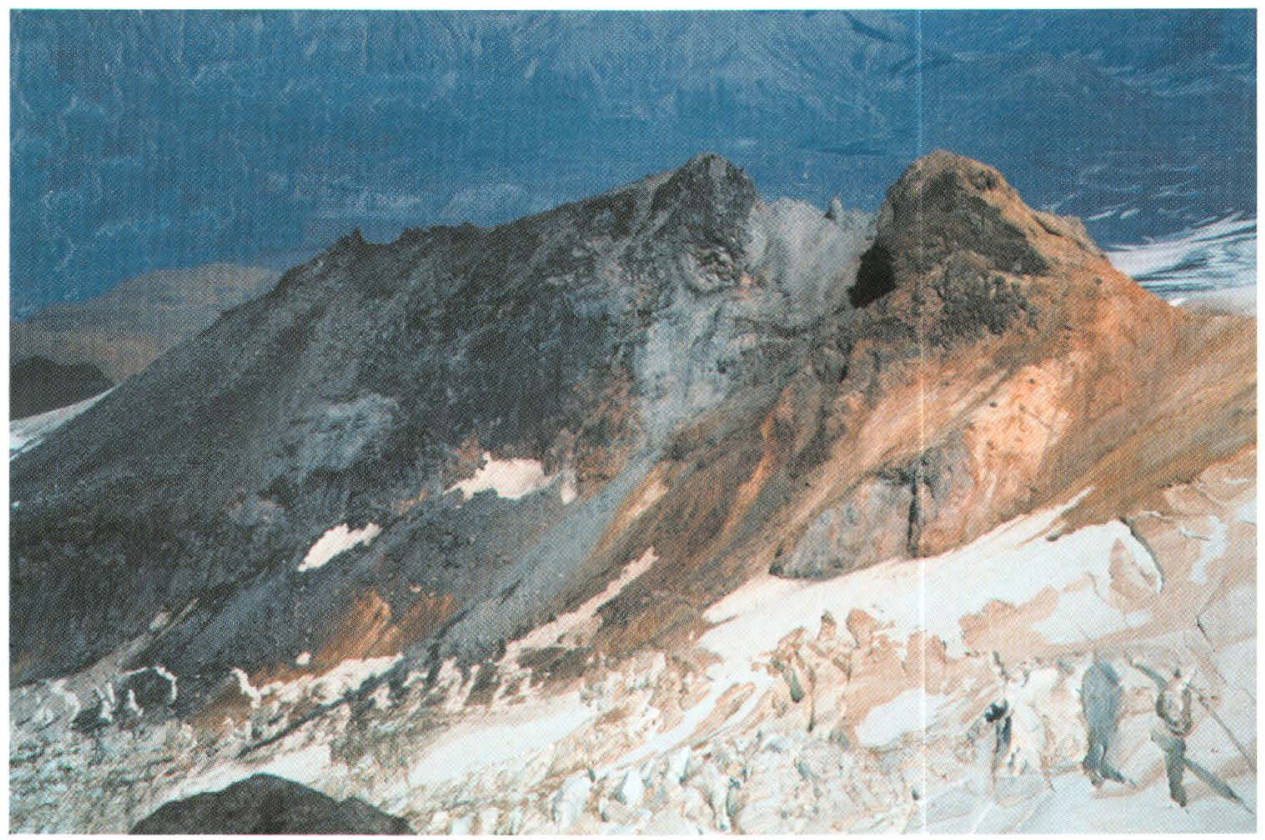

Figure 3. View northward from south-rim rangecrest across vent area of Alagogshak volcano, glacially gutted to produce an $800-\mathrm{m}$-wide cirque-amphitheater that opens to the northwest (left). Acid-altered crater-fill forms lower wall beyond ice. Black ridge is part of a 140-m-thick dacitic outflow lava (samples K-2089, 2089A) that dips $20^{\circ} \mathrm{NW}$. (left). Knob 5290 at upper right is a crater-rim remnant, across which stratified ejecta drape both inward and outward.

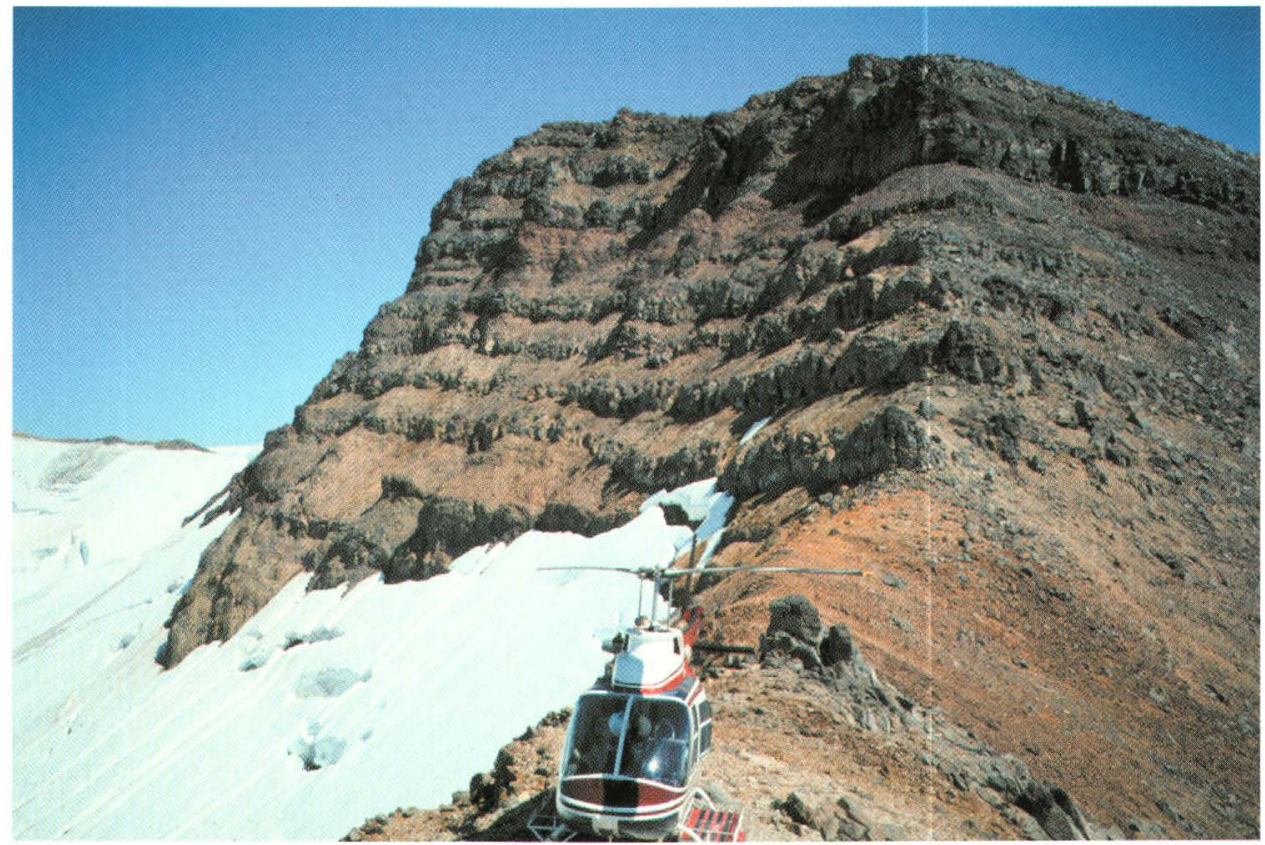

Figure 4. Stack of twelve andesite lava flows (exposed above the ice) along upper part of Kejulik cleaver, at elevation 4,900-5,150 ft, $3 \mathrm{~km}$ southwest of the Alagogshak vent. View toward the northeast. As many as eight more flows, stratigraphically lower in the stack, form basal part of the cleaver (behind the camera).

smallest) of the outliers, capping peak 4647 on the divide between Kejulik River and Takayofo Creek, is a single lava flow of silicic andesite (62 percent $\mathrm{SiO}_{2}$ ) as thick as $200 \mathrm{~m}$. Between them, on the Takayofo-Angle Creek divide, a third outlier consists of coarse flow-breccia and four lava flows, three of which are atypically mafic (52-53 percent $\mathrm{SiO}_{2}$ ) for Alagogshak and unusually rich in big clinopyroxene and plagioclase phenocrysts. Overlying these on the northwestern spur of the outlier (peak 4281), the fourth lava consists of a 100-m-thick flow of ordinary Alagogshak-type andesite ( 58 percent $\mathrm{SiO}_{2}$ ). Finally, the fourth 
and westernmost outlier, which caps the ridge dividing two tributaries of Takayofo Creek (fig. 2), consists of a single phenocryst-rich andesite lava flow (61-62 percent $\mathrm{SiO}_{2}$ ) as thick as $200 \mathrm{~m}$. Shew and Lanphere (1992) reported a K-Ar age of $954 \pm 109$ ka for plagioclase separated from this flow.

Basal contacts of the outliers dip gently away from the Alagogshak center, except for the westernmost outlier, which is subhorizontal. As each of the four remnants rests upon Jurassic sedimentary rocks along low-angle contacts that are now perched $300-600 \mathrm{~m}$ above the floors of adjacent glacial valleys, it is clear on physiographic grounds that all the outliers are relatively old. No other source vent or dike has been recognized, however, between Alagogshak volcano and the still older Kejulik stratocone, $21 \mathrm{~km}$ farther southwest (Riehle and others, 1993;

Shew and Lanphere, 1992). Our favored but inconclusive inference is that the lava flows of the outliers, at least most of them, represent eruptive phases of Alagogshak volcano older than the episodes that produced the andesites and dacites in the four more proximal sectors described above.

\section{Geochronology}

$\mathrm{K}$-Ar ages were measured for whole-rock samples of andesitic lava flows in three of the proximal sectors described (table 1), employing the multiple-collector mass spectrometer at the U.S. Geological Survey (USGS) in Menlo Park (Stacey and others, 1981) and following the methods described by Hildreth and Lanphere (1994). Plagioclase K-Ar ages were reported previously by Shew and Lanphere (1992) for lava flows in the fourth sector, as well as for the old, westernmost outlier.

In the older stack of andesite lavas in the northwest sector, the basal flow (resting on Jurassic strata) gives an age of $680 \pm 20$ $\mathrm{ka}$. To the south, the top flow of the stack of seven andesite lavas at the head of the east fork of Kejulik River yields an age of $104 \pm 10 \mathrm{ka}$. To the southeast, the thick plateau-forming andesite lava flow at the head of Alagogshak Creek gives an age of $43 \pm 8$ $\mathrm{ka}$. All three nonglassy whole-rock samples provided satisfactory yields of radiogenic argon (6-13 percent ${ }^{40} \mathrm{Ar}^{*}$; table 1 ), permitting high-precision age determinations.
In the southwest sector, plagioclase separated from two of three andesite-dacite lava flows that make up the sinuous remnant of an intracanyon stack along the main fork of Kejulik River provided accordant ages of $394 \pm 46 \mathrm{ka}$ and $389 \pm 71 \mathrm{ka}$ (Shew and Lanphere, 1992). Finally, Shew and Lanphere (1992) measured an age of $954 \pm 109$ ka for plagioclase from the crystalrich andesite that makes up the westernmost outlier (fig. 2).

The apparent longevity of more than 600 k.y. (or 900 k.y. if the westernmost outlier did indeed erupt from the Alagogshak center) is greater than that of many andesite-dacite stratovolcanoes, but it is not extraordinary. In the Cascade arc, for example, high-precision K-Ar measurements in our Menlo Park laboratory indicate that the Adams, Baker, Hood, Lassen, Mazama, and Rainier volcanic centers have erupted intermittently for comparably long periods of time. Owing in part to its modest eruptive volume, however, the longevity of volcanism at Alagogshak seems to require that long periods of repose separated its episodes of eruptive activity.

\section{Eruptive Volume}

Much of Alagogshak volcano has been erosively stripped. Rapid rates of erosion have been promoted by Pleistocene expansions of glacial ice that repeatedly covered most of the Alaska Peninsula (Riehle and Detterman, 1993). Areas still covered by products of the Alagogshak center thus sum to less than $20 \mathrm{~km}^{2}$, less even than the area $\left(33 \mathrm{~km}^{2}\right)$ covered by the postglacial products of Mount Martin (fig. 2). Distribution of the remnants shown in figure 2 clearly indicates, however, that the area originally covered by Alagogshak lavas was at least $60 \mathrm{~km}^{2}$ and, if the western outliers and distal intracanyon lava tongues are included, perhaps as much as $90 \mathrm{~km}^{2}$.

Volume reconstruction is somewhat less certain. Because the central vent of Alagogshak straddles a rangecrest arête of Jurassic sandstone that today extends (at 1,550-1,620 m elevation) for more than $4 \mathrm{~km}$ west of its $1,835-\mathrm{m}$ summit, it is obvious that a cone model is inappropriate for the volume calculation. From a modest vent cone perched on a basement high, lavas flowed steeply outward into cirques and valleys to the north, south, and west. Judging by the rim-draping fall and surge

Table 1. Whole-rock potassium-argon ages and analytical data.

[Analysts: potassium by D.F. Siems; argon by F.S. McFarland and J.Y. Saburomaru (Menlo Park, Calif.). Constants: $\left.\lambda_{\varepsilon}=0.581 \times 10^{-10} \mathrm{y}^{-1} ; \mathrm{lb}=4.962 \times 10^{-10} \mathrm{y}^{-1} ;{ }^{40} \mathrm{~K} / \mathrm{K}=1.167 \times 10^{-4} \mathrm{~mol} / \mathrm{mol}\right]$

\begin{tabular}{|c|c|c|c|c|c|c|}
\hline \multirow{2}{*}{$\begin{array}{l}\text { Sample } \\
\text { number }\end{array}$} & \multirow{2}{*}{$\begin{array}{l}\text { Location } \\
\text { (see fig. 2) }\end{array}$} & \multicolumn{2}{|c|}{ Weight percent } & \multicolumn{2}{|c|}{ Badiogenic ${ }^{40} \mathrm{Ar}$} & \multirow{2}{*}{$\begin{array}{c}\text { Calculated } \\
\text { age }\end{array}$} \\
\hline & & $\mathrm{SiO}_{2}$ & $\mathrm{~K}_{2} \mathrm{O}$ & $10^{-13} \mathrm{~mol} / \mathrm{g}$ & Percent & \\
\hline K-2049 & $\begin{array}{l}\text { Alagogshak Creek: } \\
\text { Plateau-forming } \\
\text { lava flow, } 2,300 \mathrm{ft}\end{array}$ & 61.1 & $1.644 \pm 0.008$ & 1.007 & 6.0 & $43 \pm 8 \mathrm{ka}$ \\
\hline $\mathrm{K}-2135$ & $\begin{array}{l}\text { Kejulik River, East } \\
\text { Fork: Top lava of } \\
\text { stack at } 2,500 \mathrm{ft} \mathrm{rim}\end{array}$ & 57.7 & $1.580 \pm 0.004$ & 2.369 & 9.0 & $104 \pm 10 \mathrm{ka}$ \\
\hline K-2074 & $\begin{array}{l}\text { Angle Creek, SE. } \\
\text { Fork: Basal lava } \\
\text { flow, W. wall of } \\
\text { gorge at } 2,300 \mathrm{ft}\end{array}$ & 60.0 & $1.419 \pm 0.001$ & 13.90 & 13.3 & $680 \pm 20 \mathrm{ka}$ \\
\hline
\end{tabular}


deposits preserved on remnants of the crater walls (fig. 3), the vent cone is unlikely to have been more than 100-200 m higher than the present summit. By comparison, the present-day active crater of Mount Martin is about $225 \mathrm{~m}$ deep and the crater of Mount Mageik only about $100 \mathrm{~m}$. Conservatively, then, if we assume original thicknesses of only $500 \mathrm{~m}$ for the perched vent region and an average of $250 \mathrm{~m}$ for the flanking stacks of outflow lavas, we estimate $10-13 \mathrm{~km}^{3}$ for the Alagogshak edificeor $13-18 \mathrm{~km}^{3}$ if all the western outliers are assigned to Alagogshak. This is much larger than our estimate for modern Mount Martin $\left(7 \mathrm{~km}^{3}\right)$ but far less voluminous than for nearby Mageik $\left(30 \mathrm{~km}^{3}\right)$ and Griggs $\left(20-25 \mathrm{~km}^{3}\right)$ volcanoes. No evidence has been found of voluminous tephra that might add appreciably to Alagogshak's eruptive volume, nor would the former existence of much glacially stripped fallout be expected on the basis of the generally low explosivity eruptive products preserved.

\section{Mount Martin}

Mount Martin is a small Holocene successor volcano that barely overlaps the northeastern edge of the glaciated Alagogshak edifice. Its inception postdates the last known activity at Alagogshak by about 30,000 years (table 1), and its fumarolically active crater lies $3 \mathrm{~km}$ northeast of the ravaged central vent of Alagogshak. Mount Martin consists of a small fragmental cone and, descending $10 \mathrm{~km}$ northwestward, a staircase of 10 overlapping coulees of blocky dacite, each 75-100 m thick. Although its summit exceeds $6,100 \mathrm{ft}(1,860 \mathrm{~m})$ in elevation, the 2-km-wide cone itself has local relief of only $500 \mathrm{~m}$, owing to its construction (like Alagogshak) upon a high ridge of Jurassic basement rocks. Of the total eruptive volume estimated for Mount Martin $\left(7 \mathrm{~km}^{3}\right)$, the small cone thus makes up less than 5 percent and the $31-\mathrm{km}^{2}$ lava-flow field about 95 percent (fig. 2). Scoriaceous and massive glassy (phreatomagmatically ejected) blocks of the cone are andesitic (58.9-61 percent $\mathrm{SiO}_{2}$ ), whereas the sequence of coulees is largely dacitic (62.5-64.2 percent $\mathrm{SiO}_{2}$; table 2). Despite its high ring of active glaciers (fig. 2), erosion of the cone and coulees is insignificant, indicating that Mount Martin is a Holocene volcano in its entirety. The glaciated lava flows adjacent to Martin's western flank are readily distinguishable, chemically and stratigraphically, as having erupted at Alagogshak volcano. The cone of Mount Martin is marked by a persistent steam plume derived from as many as 20 vigorous fumaroles that are precipitating sulfur in the talus northwest of a shallow acid lake on the floor of its 300 -m-wide crater.

\section{Composition of Eruptive Products}

Nearly all lava flows from Mount Martin and most of the exposed flows surviving at Alagogshak were sampled, as were glassy blocks of juvenile ejecta from the rims of both craters. Major-element determinations by X-ray fluorescence spectroscopy are given in table 2 and illustrated in figure 5. Essentially all samples from both volcanoes are plagioclase-rich two-pyroxene andesites and dacites, all of which also carry Fe-Ti-oxide microphenocrysts, but none of which contain amphibole, biotite,
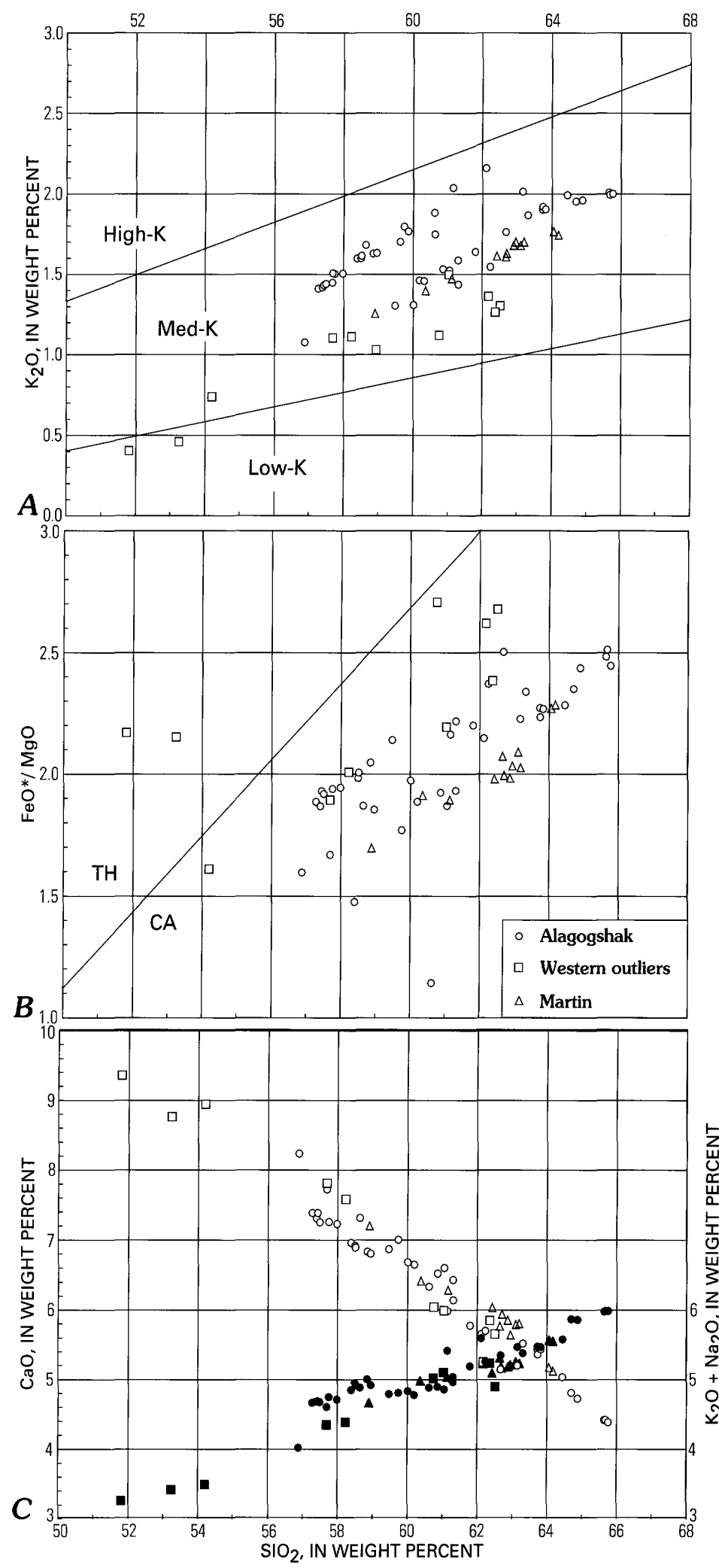

Figure 5. Whole-rock compositional data for Alagogshak and Martin volcanoes and the western outliers: $A, \mathrm{~K}_{2} \mathrm{O}$ vs. $\mathrm{SiO}_{2} ; B, \mathrm{FeO} / \mathrm{MgO}$ vs. $\mathrm{SiO}_{2} ; C_{r}\left(\mathrm{~K}_{2} \mathrm{O}+\mathrm{Na}_{2} \mathrm{O}\right)$ (closed symbols) and $\mathrm{CaO}$ (open symbols) vs. $\mathrm{SiO}_{2}$. $\mathrm{TH} / \mathrm{CA}$ is conventional boundary between tholeiitic and calcalkaline suites in panel $B$. $\mathrm{FeO}^{*}$ is total iron calculated as $\mathrm{FeO}$. 
Table 2. Chemical analyses of eruptive products.

[The ten major oxides are normalized to $\mathrm{H}_{2} \mathrm{O}$-free totals of 99.6 weight percent (allowing 0.4 weight percent for trace oxides and halogens). Determinations by wavelength-dispersive X-ray fluorescence in USGS laboratory at Lakewood, Colo.; D.F. Siems, analyst. "FeO*" is total iron calculated as FeO. "Original total" is the volatile-free sum of the ten oxides, as analyzed, before normalization, with total iron calculated as $\mathrm{Fe}_{2} \mathrm{O}_{3}$. LOI, weight percent loss on ignition at $\left.900^{\circ} \mathrm{C}\right]$

\begin{tabular}{|c|c|c|c|c|c|c|c|c|c|c|c|c|}
\hline $\begin{array}{c}\text { Sample } \\
\text { no. }\end{array}$ & $\mathrm{SiO}_{2}$ & $\mathrm{TiO}_{2}$ & $\mathrm{Al}_{2} \mathrm{O}_{3}$ & $\mathrm{FeO}{ }^{*}$ & MnO & $\mathrm{MgO}$ & $\mathrm{CaO}$ & $\mathrm{Na}_{2} \mathrm{O}$ & $\mathrm{K}_{2} \mathrm{O}$ & $\mathrm{P}_{2} \mathrm{O}_{5}$ & LOI & $\begin{array}{l}\text { Original total } \\
\text { (dry) }\end{array}$ \\
\hline \multicolumn{13}{|c|}{ ALOGOGSHAK VOLCANO } \\
\hline K-2049 & 61.1 & 0.70 & 16.1 & 6.55 & 0.13 & 3.50 & 6.60 & 3.33 & 1.53 & 0.15 & 0.13 & 99.40 \\
\hline $2049-i$ & 56.9 & 0.72 & 16.7 & 7.83 & 0.15 & 4.90 & 8.24 & 2.94 & 1.08 & 0.15 & 0.56 & 98.66 \\
\hline 2074 & 60.0 & 0.73 & 17.1 & 6.63 & 0.14 & 3.36 & 6.68 & 3.52 & 1.31 & 0.17 & 0.51 & 98.58 \\
\hline 2075 & 62.2 & 0.67 & 16.5 & 6.31 & 0.12 & 2.66 & 5.70 & 3.71 & 1.55 & 0.17 & 0.31 & 98.81 \\
\hline 2076 & 62.7 & 0.70 & 16.4 & 6.44 & 0.13 & 2.57 & 5.15 & 3.58 & 1.76 & 0.15 & 0.81 & 98.43 \\
\hline 2077 & 60.2 & 0.66 & 16.4 & 6.93 & 0.14 & 3.67 & 6.65 & 3.31 & 1.46 & 0.15 & 0.24 & 98.72 \\
\hline 2078 & 60.6 & 0.64 & 14.7 & 6.46 & 0.13 & 5.65 & 6.34 & 3.00 & 1.88 & 0.17 & 1.55 & 97.77 \\
\hline 2079 & 61.3 & 0.68 & 16.6 & 6.59 & 0.13 & 2.97 & 6.14 & 3.60 & 1.44 & 0.17 & 0.56 & 98.41 \\
\hline 2080 & 61.8 & 0.67 & 16.3 & 6.60 & 0.13 & 3.00 & 5.78 & 3.55 & 1.64 & 0.17 & 0.72 & 98.49 \\
\hline 2081 & 64.5 & 0.69 & 15.4 & 5.67 & 0.11 & 2.48 & 5.04 & 3.59 & 1.99 & 0.15 & 0.12 & 98.85 \\
\hline 2082 & 63.7 & 0.69 & 15.6 & 5.86 & 0.12 & 2.62 & 5.36 & 3.55 & 1.92 & 0.16 & 0.51 & 98.39 \\
\hline 2083 & 63.8 & 0.69 & 15.6 & 5.75 & 0.11 & 2.54 & 5.44 & 3.56 & 1.90 & 0.15 & 0.02 & 99.23 \\
\hline 2087 & 62.1 & 0.76 & 16.0 & 6.27 & 0.12 & 2.92 & 5.65 & 3.43 & 2.16 & 0.19 & -0.03 & 99.31 \\
\hline 2088 & 58.6 & 0.73 & 16.4 & 7.40 & 0.14 & 3.96 & 7.31 & 3.20 & 1.68 & 0.18 & 0.02 & 99.11 \\
\hline 2089 & 63.7 & 0.69 & 15.6 & 5.83 & 0.11 & 2.56 & 5.43 & 3.57 & 1.90 & 0.16 & 0.00 & 99.18 \\
\hline 2089-A & 63.3 & 0.71 & 15.7 & 6.07 & 0.12 & 2.60 & 5.52 & 3.51 & 1.87 & 0.16 & 0.11 & 98.92 \\
\hline 2099 & 59.0 & 0.77 & 16.7 & 7.25 & 0.14 & 3.91 & 6.80 & 3.29 & 1.63 & 0.18 & 0.06 & 99.03 \\
\hline 2100 & 59.8 & 0.74 & 16.2 & 6.86 & 0.14 & 3.88 & 7.01 & 3.01 & 1.80 & 0.18 & 1.34 & 97.60 \\
\hline 2102 & 58.5 & 0.81 & 17.1 & 7.34 & 0.14 & 3.70 & 6.92 & 3.35 & 1.60 & 0.19 & 0.09 & 98.99 \\
\hline 2103 & 57.3 & 0.80 & 17.2 & 7.79 & 0.14 & 4.13 & 7.39 & 3.25 & 1.41 & 0.18 & 0.22 & 99.04 \\
\hline 2104 & 57.4 & 0.80 & 17.4 & 7.61 & 0.14 & 3.94 & 7.39 & 3.26 & 1.43 & 0.19 & 0.15 & 98.86 \\
\hline 2105 & 57.8 & 0.79 & 17.1 & 7.66 & 0.14 & 3.95 & 7.26 & 3.24 & 1.50 & 0.18 & 0.24 & 98.76 \\
\hline 2106 & 61.2 & 0.77 & 16.1 & 6.72 & 0.13 & 3.11 & 5.99 & 3.38 & 2.04 & 0.19 & 1.20 & 97.74 \\
\hline 2107 & 58.9 & 0.82 & 16.9 & 7.28 & 0.13 & 3.55 & 6.83 & 3.37 & 1.63 & 0.19 & 0.24 & 98.77 \\
\hline 2108 & 57.4 & 0.80 & 17.3 & 7.68 & 0.14 & 4.11 & 7.31 & 3.27 & 1.42 & 0.19 & 0.26 & 98.99 \\
\hline 2109 & 63.2 & 0.69 & 16.3 & 5.84 & 0.11 & 2.62 & 5.20 & 3.45 & 2.02 & 0.18 & 0.74 & 98.32 \\
\hline 2110 & 57.5 & 0.81 & 17.3 & 7.72 & 0.14 & 4.02 & 7.26 & 3.23 & 1.44 & 0.18 & 0.30 & 98.93 \\
\hline 2111 & 58.0 & 0.80 & 17.1 & 7.57 & 0.14 & 3.89 & 7.23 & 3.20 & 1.51 & 0.18 & 0.25 & 98.67 \\
\hline 2112 & 58.5 & 0.80 & 17.0 & 7.43 & 0.14 & 3.71 & 6.90 & 3.31 & 1.62 & 0.19 & 0.26 & 98.87 \\
\hline 2120 & 64.7 & 0.63 & 15.7 & 5.33 & 0.12 & 2.27 & 4.81 & 3.91 & 1.95 & 0.18 & 0.01 & 99.27 \\
\hline 2121 & 65.6 & 0.61 & 15.5 & 5.08 & 0.12 & 2.05 & 4.42 & 3.97 & 2.01 & 0.17 & 0.14 & 98.96 \\
\hline 2122 & 59.5 & 0.70 & 17.1 & 7.04 & 0.14 & 3.29 & 6.87 & 3.49 & 1.30 & 0.19 & 0.81 & 98.55 \\
\hline 2123 & 58.4 & 0.75 & 16.2 & 7.23 & 0.14 & 4.90 & 6.96 & 3.25 & 1.60 & 0.17 & 0.76 & 98.63 \\
\hline 2124 & 65.8 & 0.60 & 15.6 & 4.99 & 0.12 & 2.04 & 4.38 & 3.98 & 2.00 & 0.17 & 0.15 & 99.01 \\
\hline 2125 & 65.7 & 0.60 & 15.7 & 4.94 & 0.12 & 1.97 & 4.42 & 3.99 & 2.00 & 0.18 & 0.23 & 99.13 \\
\hline 2135 & 57.7 & 0.73 & 16.3 & 7.66 & 0.14 & 4.58 & 7.72 & 3.15 & 1.45 & 0.18 & 0.15 & 98.54 \\
\hline 2140 & 64.9 & 0.62 & 15.8 & 5.26 & 0.12 & 2.16 & 4.72 & 3.90 & 1.96 & 0.18 & 0.19 & 99.00 \\
\hline 2141 & 60.9 & 0.69 & 16.4 & 6.53 & 0.13 & 3.39 & 6.53 & 3.36 & 1.53 & 0.15 & 0.59 & 98.91 \\
\hline 2142 & 61.3 & 0.70 & 16.1 & 6.49 & 0.12 & 3.36 & 6.44 & 3.38 & 1.59 & 0.15 & 0.11 & 99.07 \\
\hline 2259 & 60.6 & 0.75 & 16.6 & 6.70 & 0.14 & 3.24 & 6.31 & 3.31 & 1.75 & 0.21 & 0.84 & 98.27 \\
\hline 2260 & 59.9 & 0.75 & 16.7 & 6.90 & 0.14 & 3.44 & 6.63 & 3.20 & 1.77 & 0.23 & 0.90 & 98.34 \\
\hline 2261 & 59.6 & 0.75 & 16.6 & 7.13 & 0.14 & 3.46 & 6.82 & 3.18 & 1.70 & 0.20 & 0.82 & 98.45 \\
\hline 2262 & 57.7 & 0.76 & 16.7 & 7.53 & 0.14 & 4.32 & 7.56 & 3.16 & 1.51 & 0.22 & 0.03 & 100.00 \\
\hline 2263 & 60.3 & 0.70 & 16.9 & 6.56 & 0.13 & 3.21 & 6.84 & 3.33 & 1.46 & 0.17 & 0.27 & 98.77 \\
\hline
\end{tabular}

quartz, or sanidine. Microdioritic blebs and clots, predominantly made up of pyroxene and plagioclase but generally containing a little glass, are common in the products of both centers. Some of the andesites that have less than 59 percent $\mathrm{SiO}_{2}$ additionally carry a little olivine, as do mafic magmatic inclusions present in some Alagogshak lavas.
Thirteen samples from Mount Martin range from 58.9 percent to 64.2 percent $\mathrm{SiO}_{2}$ and plot rather tightly (fig. 5) relative to the scattered compositional arrays for 44 samples from the long-lived Alagogshak center (54.2-65.8 percent $\mathrm{SiO}_{2}$ ). All samples from both volcanoes fall in the medium-K field (fig. $5 A$ ), and both suites define calcalkaline differentiation trends 
Table 2. Chemical analyses of eruptive products-Continued.

\begin{tabular}{|c|c|c|c|c|c|c|c|c|c|c|c|c|}
\hline $\begin{array}{c}\text { Sample } \\
\text { no. }\end{array}$ & $\mathrm{SiO}_{2}$ & $\mathrm{TiO}_{2}$ & $\mathrm{Al}_{2} \mathrm{O}_{3}$ & $\mathrm{FeO} 0^{*}$ & $\mathrm{MnO}$ & MgO & $\mathrm{CaO}$ & $\mathrm{Na}_{2} \mathrm{O}$ & $\mathrm{K}_{2} \mathrm{O}$ & $\mathrm{P}_{2} \mathrm{O}_{5}$ & LOI & $\begin{array}{l}\text { Original total } \\
\text { (dry) }\end{array}$ \\
\hline \multicolumn{13}{|c|}{ ALOGOGSHAK VOLCANO-Western outliers, north } \\
\hline $\mathrm{K}-2085$ & 61.0 & 0.69 & 16.7 & 6.71 & 0.14 & 3.06 & 5.99 & 3.60 & 1.50 & 0.17 & 0.25 & 99.12 \\
\hline $2085-\mathrm{i}$ & 54.2 & 0.63 & 19.9 & 7.48 & 0.15 & 4.65 & 8.95 & 2.75 & 0.74 & 0.14 & 0.98 & 98.45 \\
\hline 2086 & 57.7 & 0.74 & 17.1 & 7.55 & 0.16 & 3.99 & 7.81 & 3.24 & 1.10 & 0.18 & 0.78 & 98.22 \\
\hline 2253 & 58.9 & 0.64 & 17.2 & 6.98 & 0.14 & 3.67 & 7.63 & 3.16 & 1.03 & 0.17 & 0.12 & 99.53 \\
\hline \multicolumn{13}{|c|}{ ALOGOGSHAK VOLCANO - Western outliers, middle } \\
\hline K-2168 & 58.2 & 0.72 & 17.2 & 7.47 & 0.15 & 3.72 & 7.58 & 3.27 & 1.11 & 0.18 & 0.21 & 99.23 \\
\hline 2254 & 53.2 & 0.90 & 18.0 & 10.20 & 0.18 & 4.74 & 8.76 & 2.96 & 0.46 & 0.19 & 0.01 & 99.01 \\
\hline 2255 & 51.8 & 0.92 & 18.3 & 10.66 & 0.20 & 4.91 & 9.36 & 2.85 & 0.40 & 0.16 & -0.09 & 99.12 \\
\hline \multicolumn{13}{|c|}{ ALOGOGSHAK VOLCANO-Western outliers, south } \\
\hline K-2257 & 62.2 & 0.73 & 16.5 & 6.76 & 0.14 & 2.58 & 5.25 & 3.86 & 1.36 & 0.22 & 2.72 & 95.72 \\
\hline 2258 & 62.5 & 0.71 & 16.3 & 6.65 & 0.14 & 2.49 & 5.65 & 3.59 & 1.30 & 0.21 & 3.34 & 94.90 \\
\hline \multicolumn{13}{|c|}{ ALOGOGSHAK VOLCANO - Western outliers, westernmost } \\
\hline $\mathrm{K}-2169$ & 62.4 & 0.70 & 17.2 & 5.60 & 0.13 & 2.35 & 5.84 & 3.97 & 1.27 & 0.21 & 1.14 & 98.10 \\
\hline 2256 & 60.8 & 0.74 & 17.0 & 7.07 & 0.16 & 2.61 & 6.04 & 3.89 & 1.12 & 0.21 & 0.27 & 98.08 \\
\hline \multicolumn{13}{|c|}{ MOUNT MARTIN } \\
\hline K-2066 & 63.1 & 0.67 & 15.8 & 5.88 & 0.12 & 2.82 & 5.79 & 3.58 & 1.67 & 0.15 & 0.16 & 99.37 \\
\hline 2067 & 62.4 & 0.66 & 16.0 & 6.04 & 0.12 & 3.05 & 6.03 & 3.48 & 1.61 & 0.15 & 0.04 & 99.25 \\
\hline 2068 & 62.7 & 0.66 & 16.0 & 5.88 & 0.12 & 2.94 & 5.94 & 3.53 & 1.63 & 0.15 & -0.05 & 99.31 \\
\hline 2090 & 64.2 & 0.69 & 15.7 & 5.63 & 0.12 & 2.47 & 5.13 & 3.80 & 1.75 & 0.15 & 0.16 & 98.89 \\
\hline 2093 & 63.2 & 0.65 & 15.7 & 5.83 & 0.12 & 2.87 & 5.79 & 3.53 & 1.70 & 0.15 & -0.01 & 99.17 \\
\hline 2094 & 62.9 & 0.68 & 15.7 & 5.97 & 0.12 & 3.01 & 5.85 & 3.52 & 1.67 & 0.15 & 0.24 & 98.85 \\
\hline 2095 & 63.0 & 0.66 & 16.1 & 5.90 & 0.12 & 2.90 & 5.63 & 3.51 & 1.69 & 0.14 & 0.51 & 98.72 \\
\hline 2096 & 62.7 & 0.65 & 16.1 & 5.86 & 0.12 & 2.93 & 5.94 & 3.56 & 1.62 & 0.15 & 0.19 & 99.00 \\
\hline 2097 & 62.7 & 0.69 & 16.1 & 5.98 & 0.12 & 2.89 & 5.76 & 3.70 & 1.61 & 0.15 & 0.05 & 98.97 \\
\hline 2098 & 60.4 & 0.73 & 16.5 & 6.73 & 0.13 & 3.52 & 6.42 & 3.59 & 1.39 & 0.17 & -0.03 & 99.30 \\
\hline 2115 & 61.2 & 0.72 & 16.2 & 6.46 & 0.13 & 3.42 & 6.29 & 3.56 & 1.47 & 0.17 & -0.13 & 99.52 \\
\hline 2170 & 64.1 & 0.67 & 15.8 & 5.59 & 0.12 & 2.47 & 5.17 & 3.80 & 1.76 & 0.15 & 0.12 & 99.16 \\
\hline 2333 & 58.9 & 0.73 & 16.7 & 6.99 & 0.14 & 4.12 & 7.20 & 3.41 & 1.25 & 0.16 & 0.36 & 98.30 \\
\hline
\end{tabular}

(fig. 5B). Alkali-lime intersections at 63-63.5 percent $\mathrm{SiO}_{2}$ (fig. 5C) define calcic suites for both Alagogshak and Martin, similar to the zoned suite ejected at nearby Novarupta in 1912 (Hildreth, 1983).

The rocks of both volcanoes are typically low-Ti arc suites, most having $\mathrm{TiO}_{2}$ contents of only $0.60-0.82$ percent; the two most mafic samples (K-2254, K-2255, both from the middle outlier) have $0.90-0.92$ percent $\mathrm{TiO}_{2}$. Relatively primitive material has not erupted here, as all but one sample represents magma that had evolved to less than 5 percent $\mathrm{MgO}$. Contents of $\mathrm{Al}_{2} \mathrm{O}_{3}$ are mostly $<17.5$ percent, although the three samples lowest in $\mathrm{SiO}_{2}$ (fig. 5; all from the western outliers) have higher $\mathrm{Al}_{2} \mathrm{O}_{3}$ values of $18.0-19.9$ percent.

Relatively mafic magmatic inclusions, generally finer grained than the host lava and typically $1-10 \mathrm{~cm}$ across, are common in a few Alagogshak lava flows, are sparse to absent in most, and were not found in products of Mount Martin. The two such inclusions analyzed (samples K-2049-i and K-2085-i) are among the least silicic (56.9 percent; 54.2 percent $\mathrm{SiO}_{2}$ ) eruptive products sampled here.

Two subparallel trends are conspicuous for Alagogshak data in the $\mathrm{K}_{2} \mathrm{O}-\mathrm{SiO}_{2}$ panel (fig. $5 A$ ), one suite having about 0.4 weight percent more $\mathrm{K}_{2} \mathrm{O}$ than the other at any given $\mathrm{SiO}_{2}$ content. The suite higher in $\mathrm{K}_{2} \mathrm{O}$ includes (a) near-vent scoria; (b) 11 of 12 samples from the southerly (East Fork) sector; (c) 4 of 10 samples from the southwesterly (Kejulik cleaver) sector; and (d) 5 of 16 samples from the northwesterly (Angle Creek) sector. On the other hand, the trend lower in $\mathrm{K}_{2} \mathrm{O}$ includes the southeasterly (Alagogshak Creek) lobe, all lavas of the western outliers, and the entire Holocene suite from Mount Martin, as well as many lavas of the Kejulik cleaver and Angle Creek sectors (fig. 5A). No mineralogical difference is recognized between the lavas lower and higher in $\mathrm{K}_{2} \mathrm{O}$, which are interstratified without any consistent age distinction. Lavas lower in $\mathrm{K}_{2} \mathrm{O}$ include those dated at $954 \mathrm{ka}, 680 \mathrm{ka}, 389 \mathrm{ka}$, and $43 \mathrm{ka}$, as well as the crater-rim dacite (fig. 3 ) and all the postglacial products of Mount Martin.

The sample richest in $\mathrm{MgO}$ (K-2078; 5.65 percent $\mathrm{MgO}$ ), which stands out from the rest in figure $5 B$ by virtue of its low $\mathrm{FeO} / \mathrm{MgO}$ ratio, is anomalous also in its relative $\mathrm{Al}_{2} \mathrm{O}_{3}$ deficiency (14.7 percent $\mathrm{Al}_{2} \mathrm{O}_{3}$; table 2). The sample is an olivine-bearing, plagioclase-rich, two-pyroxene andesite (60.6 percent $\mathrm{SiO}_{2}$ ) belonging to the suite higher in $\mathrm{K}_{2} \mathrm{O}$ and does not appear in thin section to be olivine-accumulative. It is from the basal colonnade of a 75-m-thick flow that makes up ridge 2451 at the northwest limit of the Angle Creek sector. 
Resting on Jurassic sandstone, this flow is likely to be one of the oldest in the sector.

\section{Conclusions}

The simple central-vent Alagogshak stratovolcano produced $10-18 \mathrm{~km}^{3}$ of eruptive products, mostly andesite-dacite lava flows, in several eruptive episodes spread widely over at least 600,000 years. About two-thirds of the Alagogshak volcano has already been stripped by glacial erosion, even though substantial lava production took place as recently as $43 \pm 8 \mathrm{ka}$. The eruptive focus shifted in the Holocene to Mount Martin, $3 \mathrm{~km}$ northeast. In contrast to the coherent compositional arrays for eruptive products of Mount Martin, the variety of compositions erupted at Alagogshak probably reflects independent evolution of successive magma batches that arose at widely separated intervals in its long history.

Like Alagogshak, the adjacent volcanic-front edifice to the southwest, Kejulik volcano (fig. 1), is apparently now extinct (Riehle and others, 1993; Shew and Lanphere, 1992). This leaves a 76-km-long inactive gap along the front between the Holocene cones of Mount Martin and Mount Peulik (fig. 1). So lengthy a gap accentuates the anomaly of the adjacent Katmai cluster (Hildreth, 1983), where the recently active Martin, Mageik, Trident, Griggs, and Katmai stratovolcanoes are all closely spaced along a $25-\mathrm{km}$ reach of the volcanic arc.

\section{Acknowledgments}

Michelle Coombs and Dave Tucker contributed substantially to the fieldwork, Tracey Felger to the map preparation,
Forrest McFarland to sample preparation, and James Saburomaru to the K-Ar dating. John Paskievitch arranged and anchored the sometimes difficult logistics, and pilot Paul Walters got us where we needed to go (fig. 4). Game McGimsey and Chris Nye provided helpful reviews of the manuscript.

\section{References Cited}

Hildreth, W., 1983, The compositionally zoned eruption of 1912 in the Valley of Ten Thousand Smokes, Katmai National Park, Alaska: Journal of Volcanology and Geothermal Research, v. 18, p. 1-56.

Hildreth, W., and Lanphere, M.A., 1994, Potassium-argon geochronology of a basalt-andesite-dacite arc system: The Mount Adams volcanic field, Cascade Range of southern Washington: Geological Society of America Bulletin, v. 106, p. 1413-1429.

Riehle, J.R., and Detterman, R.L., 1993, Quaternary geologic map of the Mount Katmai quadrangle and adjacent parts of the Naknek and Afognak quadrangles, Alaska: U.S. Geological Survey Miscellaneous Investigations Series Map 1-2032, scale 1:250,000.

Riehle, J.R., Detterman, R.L., Yount, M.E., and Miller, J.W., 1993, Geologic map of the Mount Katmai quadrangle and adjacent parts of the Naknek and Afognak quadrangles, Alaska: U.S. Geological Survey Miscellaneous Investigations Series Map 1-2204, scale 1:250,000.

Shew, N., and Lanphere, M.A., 1992, Map showing potassium-argon ages from the Mount Katmai and adjacent parts of the Naknek and Afognak quadrangles, Alaska Peninsula, Alaska: U.S. Geological Survey Miscellaneous Field Studies Map MF-2021-E, scale $1: 250,000$.

Stacey, J.S., Sherrill, N.D., Dalrymple, G.B., Lanphere, M.A., and Carpenter, N.V., 1981, A five-collector system for the simultaneous measurement of argon isotope ratios in a static mass spectrometer: International Journal of Mass Spectrometry \& Ion Physics, v. 39, p. $167-180$.

Reviewers: Game McGimsey, Chris Nye. 



\title{
Gravity Changes During the 26 Years Following the 1964 Alaskan Earthquake
}

\author{
By David F. Barnes
}

\section{Abstract}

The 1964 Alaskan earthquake caused elevation changes that were measured in meters and extended over distances that were measured in hundreds of kilometers. Most of the measured gravity changes showed a Bouguer ratio to the elevation changes and thus suggested addition of mass in the uplift areas and loss of mass in the subsidence areas. However, the gravity increase measured at Anchorage was only $0.04 \mathrm{mGal}$ despite a subsidence of nearly $1 \mathrm{~m}$, which might have caused a free-air change of $0.3 \mathrm{mGal}$. This small change in gravity was probably the result of elastic expansion and a density decrease that diminished the free-air elevation effect.

Post-earthquake readjustments have caused small elevation changes during the 25 years that followed the earthquake. However, measured gravity changes are much less extensive and suggest that differing tectonic processes are now active in the earthquake area. The first change reported was an uplift of nearly $0.5 \mathrm{~m}$, shown by releveling along the highway southeast of Anchorage. No gravity change has been measured, and the uplift is interpreted to be caused by horizontal elastic compression. Later global positioning system (GPS) measurements showed that this domal uplift extends into the central Kenai Peninsula, where additional measurements are probably needed to confirm the absence of gravity change.

In contrast, there is better evidence for a post-earthquake gravity change at Cordova, where repeated visits have shown a gravity increase of $0.1 \mathrm{mGal}$ during a 25 -year period and where tide-gage data show a subsidence of nearly $0.2 \mathrm{~m}$ during the same period. The combined data indicate a free-air process with no change in underlying mass and a probable isostatic phasechange response to the broad uplift during the 1964 earthquake. At Middleton Island, on the edge of the continental shelf, a gravity decrease of perhaps $0.2 \mathrm{mGal}$ has been measured, but the large gravimeter drift and data scatter show the need for additional measurements. GPS data and barnacle observations also indicate uplift.

\section{Introduction}

The vertical crustal deformation during the 1964 Alaskan earthquake exceeded that measured in any previous earthquake
(Krauskopf, 1972) and was sufficient to cause measurable and interpretable gravity changes in at least part of the deformed region (Barnes, 1965). During the 30 years following the earthquake, evidence for further vertical movement and post-earthquake readjustment has gradually accumulated (Brown and others, 1977; Savage and Plafker, 1981; Cohen and others, 1995; Gilpin and Carver, 1994). I have also measured small gravity changes that have accompanied some of this post-earthquake readjustment. However, the gravity changes are much less extensive than the elevation changes, and the relationship between the vertical movement and the gravity changes is not uniform, suggesting that more than one physical process may be active in the post-earthquake readjustment. Furthermore, additional data collected during the summer of 1965 after the preparation of the initial paper (Barnes, 1965) provide supplemental data that support some variation in gravity change and deformation process during the 1964 coseismic event The gravity changes in that event were not as consistent as once reported. Therefore, in this paper, I reexamine some of the 1964 coseismic gravity-change data, in addition to evaluating the post-earthquake gravity-change data.

\section{Interpretation Principles}

The limited 1964 earthquake gravity-change data were interpreted by plotting gravity changes as a function of elevation changes and comparing the data with two lines derived from physical principles (fig. 1). The line with the steeper slope (fig.1) represents the free-air gradient of gravity, which is the rate of change of gravity above the Earth's surface with no change in underlying mass; its slope is determined by the distance of the measurement from the Earth's center. The gentler slope represents the Bouguer gradient, which shows how the gradient is modified by considering the additional gravitational attraction of an infinite slab of normal rock density near the Earth's surface. The Bouguer slope provided the best fit for most of the data obtained after the 1964 earthquake. However, the agreement was least satisfactory in the negative-elevation-change or subsidence portion of the graph, for which many of the points were acquired after the initial publication. Later studies have refined the analysis of gravity changes and have considered the gravity-change effects of density variation and specific dislocation models (Whitcomb, 1976; Walsh and Rice, 1979; Savage, 1984) 


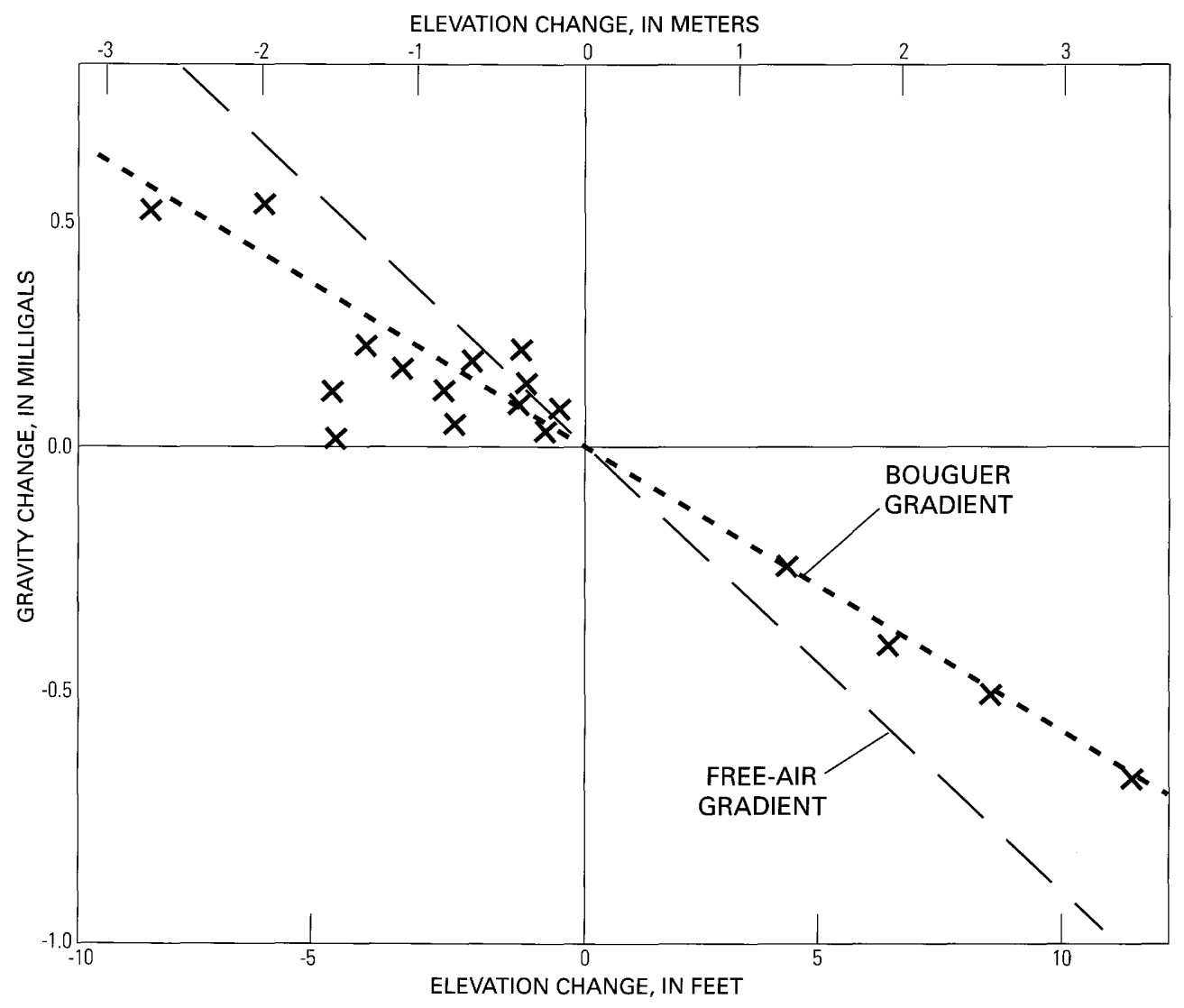

Figure 1. Gravity changes caused by the 1964 Alaskan earthquake calculated from measurements before and after the earthquake and plotted as a function of elevation change. From Barnes (1965) plus additional data collected during the summer of 1965.

\section{Data Collection}

Almost all of these Alaskan gravity-change data have been accumulated as an incidental product of the preparation of successive Alaskan State regional gravity maps (Thiel and others, 1958; Woollard and others, 1960; Barnes, 1970, 1977; Barnes and others, 1995). This regional mapping objective thus influenced both the equipment used for this study and the areal distribution of the coverage. Virtually all the data were collected with gravimeters that were primarily designed for quick measurement of gravity differences between exploration stations and previously established base stations. Absolute gravimeters may now provide better methods for measuring temporal gravity changes (Carter and others, 1997), but such equipment was in its infancy at the time of the earthquake. Furthermore the Alaskan earthquake was the first to produce measurable coseismic and postseismic gravity changes. Better studies should be possible for future earthquakes of comparable magnitude.

Measurement of temporal gravity changes requires good control of the accuracy and stability of gravimeter calibrations. The meters used in the Alaskan surveys have been carefully chosen and repeatedly checked. At the start and end of each field season the meters were tested on mountain calibration loops (Barnes and others, 1969) near Anchorage, Fairbanks, and the U.S. Geological Survey (USGS) headquarters in California. Most of the meters have also been intermittently compared with pendulum data along parts of the North America Midcontinent calibration range (Woollard and Rose, 1963), which provided the basic scale for establishing mountain calibration loops.

Base stations initially used during collection of data for the regional maps provide the primary framework for gravitychange studies. These base stations were occupied as often as possible to insure the accuracy of regional coverage. Thus multiple ties are available for many station pairs, and their data scatter provides an estimate of measurement reliability. For convenience and ease of reoccupation, most base stations were located at transportation hubs, airports, and road junctions, where they could be most easily reached after the regional survey had extended to other areas. The standard deviation of base-station ties made during the Alaskan gravity surveys is 0.02 to 0.03 $\mathrm{mGal}$, which is probably the best indicator of data precision. However, the precision of the gravity-change data was also affected by two other factors, the magnitudes of which are difficult to estimate. First, a variety of factors sometimes made reoccupation of the same spot difficult and uncertain. Second, during other ties, the drift of the gravimeter was very large and thus added measurement uncertainty.

Alaskan economic development has been rapid during the more than 30 years since the earthquake, and there have been major changes at many airports and along the primary highways. A good example is provided by the Richardson Highway (fig. 2), which extends northeast from Valdez toward Tonsina. Gravity measurements were made with a LaCoste and Romberg gravimeter at mile posts along this road in 1962, and those 


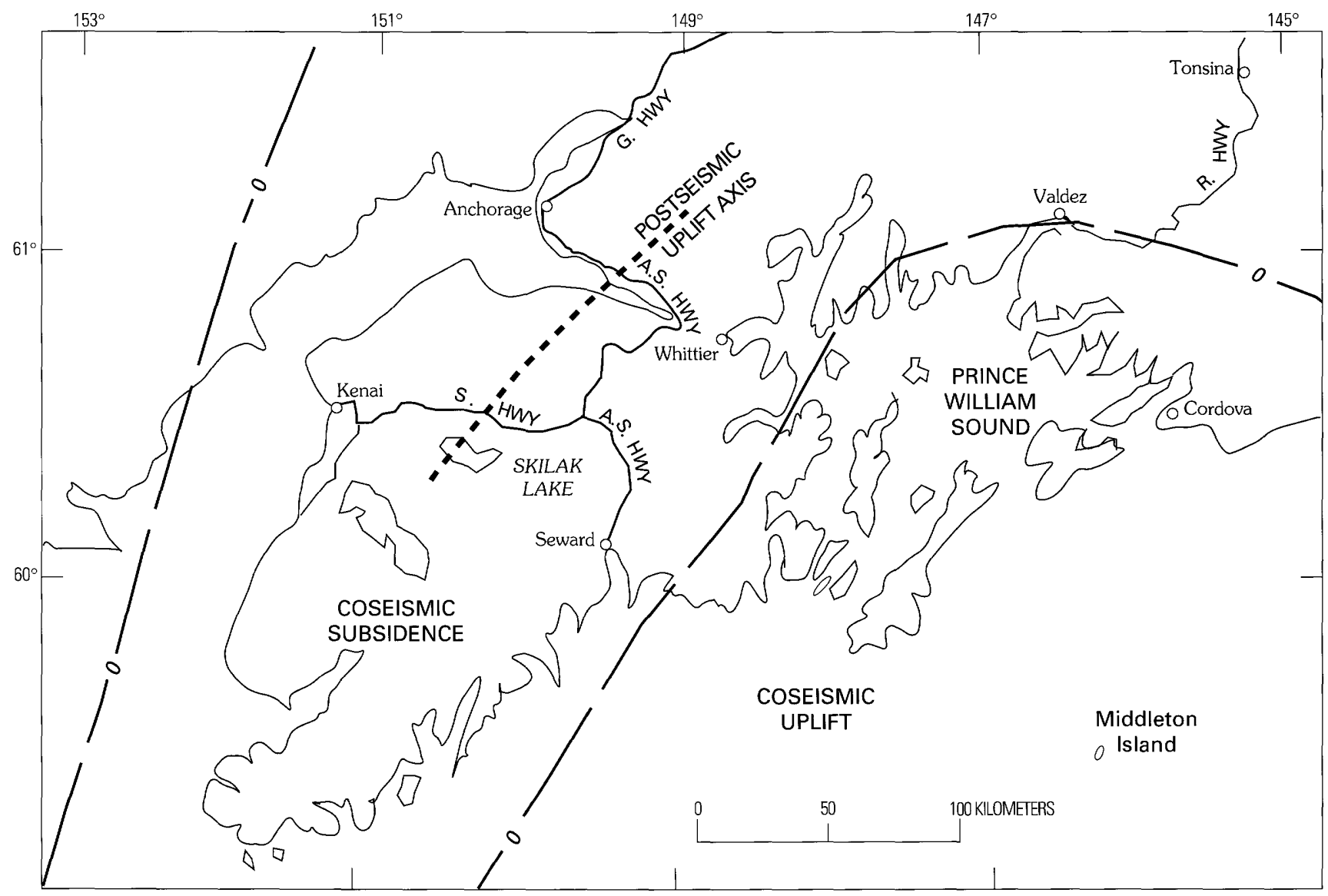

Figure 2. Map of area deformed in the 1964 Alaska eartnquake. Long dashes are zero isobase lines on borders of subsidence and uplift areas (Plafker, 1965). Dotted line within the coseismic subsidence area is axis of post-earthquake uplift (Brown and others, 1977; Cohen and Freymueller, 1997). Highways: R., Richardson; A.S., Anchorage-Seward; S., Sterling; G., Glenn.

stations were among the first stations reoccupied after the 1964 earthquake. The preliminary results indicated a small, probable free-air gravity change suggesting no loss or addition of underlying mass (Barnes, 1965). The possibilities for measuring postearthquake changes along this traverse once seemed good (Rice, 1969), although there had been some minor scatter in base ties for the early measurements. Later measurements were made in 1965,1972 , and 1981, but each of these reoccupation attempts encountered more missing base stations (Barnes, 1968, p. 9C), which thus increased the uncertainty in defining gravity changes.

During the late 1970's the Alaska pipeline was constructed along the route and a new highway was built. In 1990 only short segments of the original road could be found, and virtually all of the original mile posts that marked station locations had been destroyed. Further measurement of the gravity change was considered impossible along this road. In other areas and airports, more frequent visits have enabled us to observe the potential loss of base stations and establish new bases by ties to nearby bases - thus it was possible to keep track of gravity changes. However, establishing new satellite bases probably increases the uncertainty of gravity differences relative to distant bases. Problems associated with measurement at specific satellite bases are, thus, briefly mentioned in the following text.

\section{Anchorage Gravity Change}

The nearly 1-m subsidence of the land near Anchorage was recognized soon after the earthquake (Grantz and others, 1964). When I first returned to the city less than a month after the earthquake, I expected to be able to measure a gravity increase of 0.2 to $0.3 \mathrm{mGal}$, depending on the tectonic process that caused the subsidence. However, data collected at that time immediately suggested a much smaller increase of $<0.05 \mathrm{mGal}$. Larger gravity changes were soon measured elsewhere, and the small change at Anchorage was long considered evidence of inadequate pre-quake data. The small change was shown in the Barnes (1965) graph of gravity change versus elevation change but was ignored in the discussion. Four years later, Rice (1969) reported additional pre-earthquake data, including a 1963 Air Force gravimeter calibration effort with seven LaCoste and Romberg gravimeters, that yielded a value of pre-earthquake Anchorage gravity very close to my own and also an earthquake change of $0.05 \mathrm{mGal}$, which was less than what he considered the uncertainty of the measurements. His data thus supported my own conclusion that the coseismic gravity increase was only about $0.04 \mathrm{mGal}$. This may have been less than the uncertainty of the measurements, but even this change (as well as the uncertainty) 
was less than the magnitude of the change expected from application of either the free-air or Bouguer gradients to the measured elevation change. It was also significantly smaller than the changes measured elsewhere in the quake area, particlarly along the highway system north and east of the city of Anchorage (stations described in Barnes, 1968, p. 7-C and 8-C).

The reason for the small gravity change at Anchorage gradually became apparent after the earthquake. Less than a year after the earthquake, Plafker (1965) suggested the hypothesis that the quake was the result of an overthrust movement toward the Aleutian trench and along a shallow-dipping fault that outcropped along the trench. Anchorage is near the zone of elastic expansion that provided the push for this movement. Whitcomb (1976) showed that density changes can significantly reduce the gravity change expected from elevation changes, and, a few years later, Jachens (1979) published a graph showing that, for elastic expansion and compression with a Poisson's ratio near 0.25 , the resulting gravity change could be very small. The gravity data from the Anchorage area thus provides further support for Plafker's hypothesis, which is now a well-accepted aspect of plate tectonics.

In some ways, the small gravity change at Anchorage was very convenient because the city is the prime transportation hub for Alaska, so any measured changes in gravity differences with respect to other cities suggested that the change had probably occurred at the distant city. Furthermore, the base network could be expanded without making corrections for changes at its principal bases (Anchorage bases are described in Barnes, 1968, p. 6-C). Preparation of a State gravity map did provide multiple opportunities for measuring the gravity differences between stations at Anchorage airport and those at other airports within the State. Although the measurements show some scatter at all stations, the only evidence of a steady trend indicating a temporal gravity change was measured at other airports in the earthquake area. Post-earthquake ties to such distant cities as Fairbanks, McGrath, and Bethel show a scatter of $\pm 0.5 \mathrm{mGal}$, but no evidence of change. The importance of Anchorage as a transportation hub and reference point for temporal changes elsewhere led to an absolute gravity measurement by free-fall apparatus (Marson and Alasia, 1980). The measurement has not been repeated because there has been no other evidence of a postearthquake Anchorage gravity change.

The tide-gage data from Anchorage (Savage and Plafker, 1981) also show small, post-earthquake sea-level changes of $<3$ $\mathrm{mm} / \mathrm{yr}$ or less than a total of $5 \mathrm{~cm}$ in the 25 years since the earthquake. This would cause a free-air gravity change of $<0.2 \mathrm{mGal}$, which is less than the standard deviation of the various ties to other airports and base stations in Alaska. Thus, a detectable post-earthquake gravity change would not be expected from the tide-gage data.

\section{Gravity Change South of Anchorage}

The area of small or undetectable coseismic and postseismic gravity change seems to extend over a considerable area south and west of the city, although only limited data are available. Few pre-earthquake gravity data were available for the Seward Highway along the Turnagain Arm southeast of
Anchorage, but in 1965 a first-order level line and two sets of gravity measurements by both the U.S. Coast and Geodetic Survey (USC\&GS) and the USGS were made along the road. The USC\&GS leveling between Anchorage and Portage was repeated the following year, in 1968, and again in 1975 (some gravity measurements were also made in 1975). The leveling showed a domal uplift centered about $40 \mathrm{~km}$ southeast of Anchorage with a maximum amplitude of about $50 \mathrm{~cm}$ (Brown and others, 1977). However, the gravity changes did not show a consistent relationship to vertical movements and had the same magnitude as the scatter of the data. Hoping to reduce the uncertainty, I repeated many of the 1965 USGS gravity measurements (base stations described in Barnes, 1968, p. 3-B) along the same highway in 1977 with very similar results. Figure 3 shows a plot of distance versus uplift, measured gravity changes, and the gravity changes that might be expected by applying the Bouguer gravity gradient to the measured uplift. The measured changes are much smaller than the calculated Bouguer changes and are approximately the same magnitude as the data scatter. The postquake uplift south of Anchorage is thus accompanied by a very small gravity change similar to that which accompanied the coseismic subsidence at Anchorage during the 1964 earthquake. Brown and others (1977) used the dislocation program of Savage and Hastie (1966) to explain the uplift as the result of elastic creep and compression above a flexure of the thrust fault that ruptured in 1964. Seismic data suggest that the dip of this fault steepens significantly beneath the uplift area and thus causes compression in the overthrusting, overlying North American plate. The small gravity change is thus the result of horizontal elastic compression just as elastic expansion near Anchorage caused the small coseismic gravity change and subsidence.

\section{Kenai Peninsula Gravity Change}

Recent GPS measurements (Cohen and others, 1995; Cohen 1996; Cohen and Freymueller, 1997) along other highway level lines on the Kenai Peninsula have shown that this postearthquake uplift extends southwest from the Anchorage-Seward highway and is as much as $90 \mathrm{~cm}$ near the center of the Peninsula along the Sterling highway. No public, pre-quake gravity data were available against which to measure the coseismic gravity changes. The first-order level survey was made along the highway in 1964, and both the USGS and USC\&GS made gravity measurements along the highway in 1965. In 1981, I reoccupied six of the USGS gravity base stations (Barnes, 1968, p. 4B) and measured small gravity decreases ranging from 0.01 to $0.12 \mathrm{mGal}$ and averaging $0.05 \mathrm{mGal}$. However, only one gravimeter was used for these measurements and the drift control for the traverse was poor. Without the present evidence of uplift, the gravity decrease was not considered significant. In 1990, only one base station, at Skilak Lake near the center of the uplift, was reoccupied (within approximately $3 \mathrm{~m}$ of the original station) with two gravimeters. These readings suggest that the gravity at that station decreased by either 0.03 or $0.15 \mathrm{mGal}$, but new base stations were used for the reoccupation and the two values may not be comparable. Furthermore, the difference between the two measurements is large and shows significant scatter. The change in gravity, if any, is certainly smaller than the 


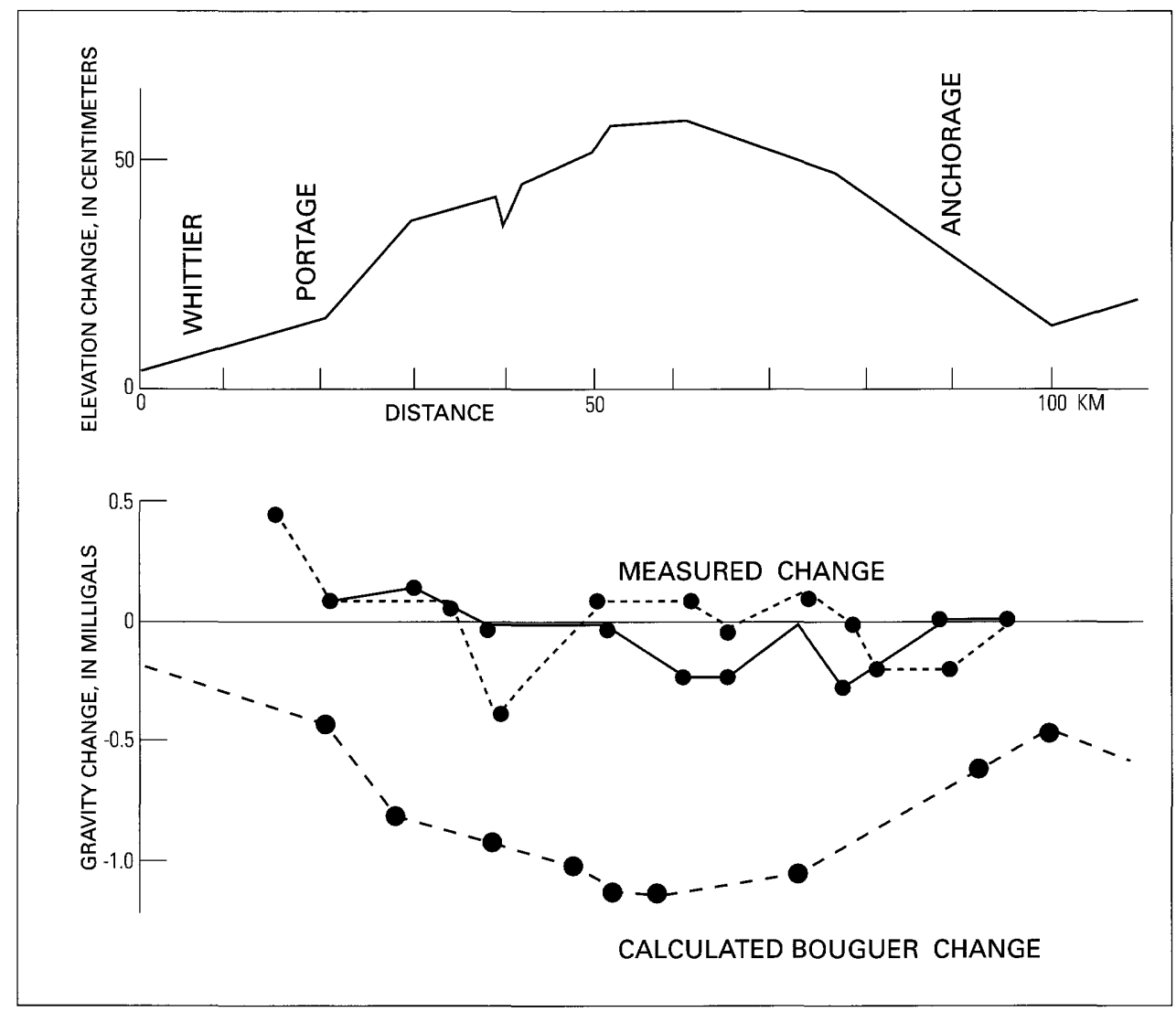

Figure 3. Plot of post-earthquake elevation change, measured gravity change, and calculated Bouguer change (calculated from the elevation change) as a function of distance along the Anchorage-Seward Highway, southeast of Anchorage. The solid and short-dashed lines are separate data sets and show the measurement scatter.

nearly $0.3 \mathrm{mGal}$ that might be expected from the measured elevation change and Bouguer gradient.

Somewhat different processes may be active on the northwest edge of this post-earthquake uplift dome. This area along the northern shore of the Kenai Peninsula and central Cook Inlet is marked by a major gravity low with a Bouguer amplitude below $-140 \mathrm{mGal}$, which represents a very large thickness of Mesozoic and Cenozoic sedimentary rock. The entire gravity low lies within the 1964 coseismic subsidence area, and the earthquake may have been one more episode in a long history of downwarping that created the sedimentary basin. However, the axis of maximum 1964 coseismic subsidence is south of the present gravity low. In 1965, the year following the earthquake, I thought I successfully reoccupied a station reported by Thiel and others (1958) at Kenai Airport. I measured, in a tie to Anchorage, a coseismic gravity increase of $0.05 \mathrm{mGal}$, which seemed consistent with the nearly $1 \mathrm{~m}$ of coseismic subsidence later reported at the nearby Nikiski tide gauge (Small and Wharton, 1969). The large gravity difference between Anchorage and Kenai may have increased the uncertainty of the measurement. Thiel had described the station as "Pacific Northern Airlines terminal on ground 40 feet from field entrance" and, after the earthquake, I decided that this spot was also the end of a concrete walkway south of the building. However, when I next returned to the airport in 1981, I wondered whether this had actually been the field entrance, so the reoccupation could have been questionable. By then the building had been converted to a gift shop and the airlines were using a new terminal building nearly a mile to the north along the single runway.

A new station, "KENT," with an aluminum marker, was established at the Kenai terminal and was tied to both the old terminal station and to Anchorage. This station was reoccupied and again retied to Anchorage in 1990. Comparison of the two ties suggests a gravity increase of $0.07 \mathrm{mGal}$ at Kenai Airport during the 9-year interval. However, different base stations had to be used at Anchorage, and the suggested change is nearly the same as the measurement scatter. The change, if any, is certainly not well established. This questionable gravity increase would suggest a small subsidence at Kenai Airport between 1981 and 1990. In contrast, a 9-year record of tide-gage data between 1970 and 1979 at nearby Nikiski (Savage and Plafker, 1981) indicated a sea-level fall or land uplift of $19 \mathrm{~mm} / \mathrm{yr}$ or nearly 17 $\mathrm{cm}$ in that 9-year period. However, Cohen and Freymueller (1997) have recently reported GPS data that make the tidal data suspect.

\section{Cordova Gravity Change}

In contrast to the Anchorage-Kenai area, there is better evidence of a post-quake gravity change at Cordova, where there were nearly $2 \mathrm{~m}$ of uplift and $0.5 \mathrm{mGal}$ of gravity decrease during the earthquake. Repetitive measurements at this city were 


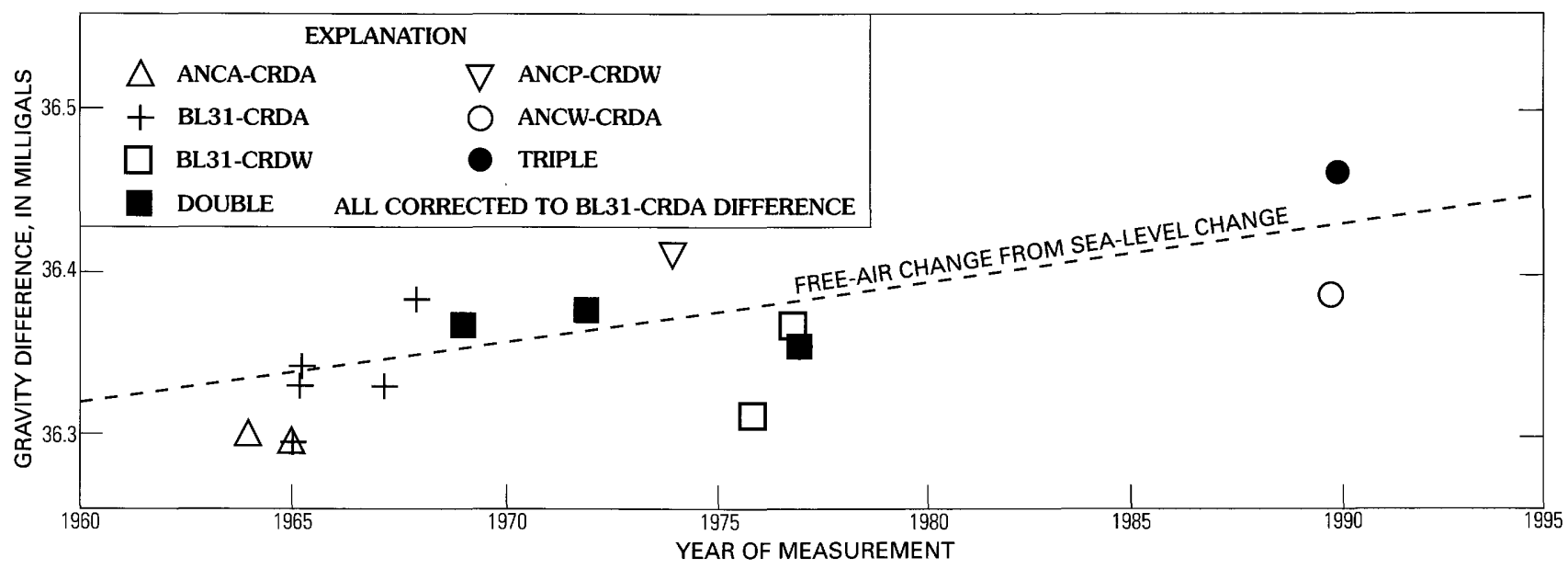

Figure 4. Graph of measured gravity differences between Anchorage and Cordova as a function of date of measurement. Symbols represent station pairs used in measurement (see text). Solid symbols indicate multiple differences. Dashed line is free-air gravity change calculated from tidal data.

easier than at most points in the earthquake area because, during different periods, Cordova was often a commercial air stop between Anchorage and the USGS California headquarters. However, frequent airport improvements and changing security regulations sometimes made reoccupations of the same base stations difficult. Although multiple bases were used at both airports, each station was either well tied to the previous base or to other known bases in these cities, so that the record of gravity change was adequately maintained. The standard deviation of these local ties, either within the airport or within the city, was $0.03 \mathrm{mGal}$. Figure 4 shows a graph of the gravity difference between Anchorage and Cordova airports plotted versus the date of measurement for the period 1964 to 1990 . The difference plotted is for the station pair BL31 in Anchorage and CRDA at Cordova airport, but the symbols show which station pair was actually measured on that date and whose differences were later converted to the BL31-to-CRDA differences. The four-character station designations are those used in the USGS Alaskan gravity base station network reports (Barnes, 1968, 1972). From 1966 to the late 1980's, BL31 was the principal USGS gravity base at Anchorage airport where the gravity meter was read above USC\&GS benchmark L31 at the base of a flagpole outside the post office. The station, flagpole, and post office were demolished in the late 1980's, but, by that time, the station had been well tied to other stations in the city (Barnes, 1968, p. 6-C). CRDA at Cordova was initially described as "on asphalt apron northwest of the field steps to $\log$ cabin air terminal and above a USGS gravity marker" (Barnes, 1968). Five years after the 1964 earthquake, a new concrete terminal was built and the old log terminal was moved closer to the new apron where it served as an annex. For several years, gravity was measured in the same place relative to the building steps, but in its new location; this station was renamed CRDW. However, increased security regulations gradually made access to this spot difficult, and another station, CRDH, was established on the road outside the new concrete terminal next to a fire hydrant. At the time of my 1990 visit, all three stations could still be reoccupied, although airport security police would only permit access to the older stations when commercial aircraft were not at the airport.
Figure 4 shows a 25 -year increase of slightly more than 0.1 $\mathrm{mGal}$ in the gravity difference between Anchorage and Cordova airports, which must be caused by a gravity increase at Cordova because we have no present evidence of a gravity change at Anchorage. Cordova tide-gage data summarized by Savage and Plafker (1981) showed a sea-level rise or land subsidence of 9.7 $\mathrm{mm} / \mathrm{yr}$ between 1965 and 1989 or $14 \mathrm{~cm}$ in the 25 -year period. The dashed line in figure 4 shows the free-air gravity change that would result from this subsidence rate if no underlying material is lost, and it provides a fairly good fit to the observed data. Thus, a post-earthquake process of subsidence involving minimal mass transfer, but probable density change, is indicated. Such processes might include a phase change at the base of the crust for isostatic compensation as discussed by Broecker (1962). Another possibility is a mechanism of elastic relaxation involving a density change without transfer of mass, but I have not found a good reference for such a process.

\section{Gravity Change at Prince William Sound Transition Area}

Available data indicate both very small coseismic and even smaller, if any, postseismic gravity changes at Anchorage in the coseismic subsidence area. However, a significantly larger gravity decrease during the earthquake at Cordova, in the coseismic uplift area, was followed from 1965 to 1990 by a gravity increase and accompanying subsidence. Both coseismic and postseismic gravity-change data between these two zones are very limited. Only a few gravity measurements had been made on the islands of Prince William Sound before the earthquake, and only one of these stations was successfully reoccupied after the earthquake (Barnes, 1965). Following the earthquake, both the USGS and the U.S. Coast and Geodetic Survey made detailed surveys to determine the gravity field in the vicinity of the epicenter (Case and others, 1966; Rice, 1969; respectively). Most of the 
USGS stations were inadequately described for accurate reoccupation, and the base-station control (Barnes, 1968, p. 2B) on this ship-supported survey was inadequate for the measurement of gravity change. The other survey (i.e., the USC\&GS survey) was made by aircraft flights from Anchorage, and the stations were better marked. However, that survey's prime base station at Anchorage airport has been destroyed, and the possibility of making an adequate repeat survey may depend on how well that station can be recovered from local ties in the Anchorage area. Access to the original data sheets would probably be necessary before evaluating the probable value of repeating parts or all of this survey.

\section{Middleton Island Gravity Change}

There may be better evidence for gravity change at Middleton Island on the northern edge of the Aleutian Trench. Here, Plafker (1969) reported a series of five marine terraces that represent successive uplifts extending over a period of more than 4,000 years. Thiel and others (1958) reported one pre-earthquake measurement made on the island in 1954 while an aircraft-weather and flight-control station was under construction. The station description by Thiel and others (1958) involved buildings that were removed after the construction, but, after a series of phone calls, I learned that the the spot could be closely relocated by the defining roadway and runway intersection (Dan Scroggs, Federal Aviation Administration, oral commun., September 1965). Commercial air flights were then available from Cordova to Middleton Island every second weekend, so a tie was made to the island and a gravity decrease of $0.6 \mathrm{mGal}$ was measured. The gravity difference from Cordova was less than $14 \mathrm{mGal}$, and the meter drift on the flight was $0.03 \mathrm{mGal}$. Plafker (1969) had used raised barnacle and vegetation lines to measure a coseismic uplift of more than $3 \mathrm{~m}$ at the island. These data were not shown on the Barnes (1965) graph of coseismic gravity change versus elevation but provided an additional point along the Bouguer line at the lower right hand corner of figure 1 (this report). Readings were also made at two of the tidal benchmarks that had been recently placed on the island.

Rice (1969) later reported that the Coast and Geodetic Survey had used a twin-engine Piper Aztec to make post-earthquake gravity ties between Anchorage and Middleton Island in 1964, 1965, and 1967. Between 1964 and 1965, a gravity decrease of $0.17 \mathrm{mGal}$ (equivalent to an uplift of $0.3 \mathrm{~m}$ ) was recorded, but the 0.03-mGal increase between 1965 and 1967 was within the uncertainty of the measurements. The uplift was believed to have stabilized, and no further measurements have been reported. The USC\&GS base station at Anchorage airport has been destroyed, and reoccupations of a cited reference station have been unsatisfactory. However, Prescott and Lisowski (1977) reported tilt measured on Middleton Island by repeated leveling in 1964, 1969, 1972, and 1975 and, thus, suggested a small continued movement of the island.

In 1990, I was anxious to repeat the 1964 gravity tie and measure any changes that might have happened since my 1965 measurement. Commercial aircraft no longer visited Middleton Island, and the only available transportation, without a prohibitively expensive charter flight, was a biweekly resupply flight for the aviation controllers and seismologists then occupying the island. Although I was able to arrange passage on the flight, I knew that its departure from Kenai instead of Cordova or Anchorage presented additional problems. I thought that the base value at Kenai air terminal could be adequately rechecked, although some of the ties to Anchorage showed evidence of change, as discussed earlier. The gravity difference between Middleton Island and Kenai is, however, much larger (more than $131 \mathrm{mGal}$ ) than the difference between the island and either Cordova (about $14 \mathrm{mGal}$ ) or Anchorage (about $42 \mathrm{mGal}$ ); therefore, calibration posed a problem. After leaving Kenai, the plane climbed to more than 4,000 $\mathrm{m}$ altitude enroute to the island-this pressure drop may have increased the gravimeter drift. The two gravimeters showed unusually high drift on the flight $(0.19 \mathrm{mGal}$ by meter G- 8 and 0.27 mGal by meter D-26). The tide- and driftcorrected gravity difference measured by G-8 was 131.57 mGal between stations KENT at Kenai and MID6 (tidal benchmark 6) at Middleton Island and $131.36 \mathrm{mGal}$ for D-26. Applying the mean of these differences to the best gravity value at Kenai Airport on the old 1960 datum, as used in 1965 , gives a gravity value at the Middleton Island benchmark of $981,968.27$ which is more than $0.3 \mathrm{mGal}$ lower than the value of $981,968.58$ measured at the same benchmark in 1965. Meter G-8, which had the smaller drift, would give an observed gravity about $0.1 \mathrm{mGal}$ higher than the mean value, but even that value suggests a gravity decrease of about 0.2 $\mathrm{mGal}$ during the 25 -year period. The gravity measurements are not directly comparable with the Coast and Geodetic Survey measurements in 1965-67 (Rice, 1969) because Rice did not identify the stations used on the island, although tidal benchmarks are presumed.

When this gravity decrease was first presented (Barnes, 1993) the uplift of the island was considered doubtful. However in 1995, Plafker (oral commun., January 1998) revisited the island and could no longer see a barnacle line visible during his earlier 1964 visit, but he estimated an uplift of at least $0.5 \mathrm{~m}$. More recently Savage and others (1998) have reported evidence from GPS measurements that the island was rising at a rate of $20 \mathrm{~mm} / \mathrm{yr}$ between 1993 and 1997. If this rate had been constant through the earlier 25-year period of 1965 to 1990 covered by the gravity measurements, the gravity change would have been only 0.10 or $0.15 \mathrm{mGal}$, depending on whether the uplift is either a Bouguer or free-air process involving the addition of underlying mass or no change in underlying mass, respectively. The fact that the measured gravity change is at least 50 percent larger makes the gravity measurements very doubtful. However, if the rate of gravity change reported by Rice (1969) for the 1964-to-1965 interval had extended over the whole 25 -year period, the gravity change might have been more than $0.40 \mathrm{mGal}$ or even higher than that measured by meter D-26, which had such a large drift during the trip to the island. The gravity-change data do suggest that the uplift of the island involves a very different geologic process than the post-earthquake uplift south of Anchorage, where no gravity change has been measured. 


\section{References Cited}

Barnes, D.F., 1965, Gravity changes during the Alaska earthquake: Journal of Geophysical Research, v. 71, no. 2, p. 451-456.

Barnes, D.F., 1968, Alaskan gravity base station network: U.S. Geological Survey Open-File Report 68-4, 44 p.

Barnes, D.F., 1970, Alaskan Bouguer gravity anomalies, in U.S. Geological Survey, The National Atlas of the United States of America, p. 69, scale $1: 38,500,000$.

Barnes, D.F., 1972, Southeast Alaska gravity base station network: U.S. Geological Survey Open-File Report 72-18, 42 p.

Barnes, D.F., 1977, Bouguer gravity map of Alaska: U.S. Geological Survey Geophysical Investigations Map GP-913, 1 sheet, scale $1: 2,500,000$.

Barnes, D.F., 1993. Small gravity changes indicate that different processes are involved in post-1964 Alaska earthquake elevation changes [abs.]: EOS-Transactions, American Geophysical Union, Fall Meeting, p. 95.

Barnes, D.F., Oliver, H.W., and Robbins, S.L., 1969, Standardization of gravimeter calibrations in the Geological Survey: EOSTransactions, American Geophysical Union, v. 50, no.10, p. 526-527.

Barnes, D.F, Mariano, John, Morin, R.L., Roberts, C.W., and Jachens, R.C., 1995, Incomplete isostatic anomaly map of Alaska, in Plafker, G.W., and Berg, H.C., eds, Geology of Alaska: Geological Society of America, Decade of North America Geology Volume, 1055 p., [plate 9 , scale $1: 2,500,000]$.

Broeker, W.L., 1962, The contribution of pressure induced phase changes to glacial rebound: Journal of Geophysical Research, v. 67, no. 10, p. 4837-4842.

Brown, L.D., Reilinger, R.E., Holdahl, S.R., and Balazs, G.I., 1977, Postseismic crustal uplift near Anchorage, Alaska: Journal of Geophysical Research, v. 67, p. 3369-3378.

Carter, W.E., Sasagawa, Glen, and Richter, Bernard, 1997, Researchers explore possibilities of microgal gravimetry: EOS-Transactions, American Geophysical Union, v. 78, no. 36, p. 383-385.

Case, J.E., Barnes, D.F., Plafker, G.W., and Robbins, S.L., 1966, Gravity survey and regional geology of the Prince William Sound epicentral region, Alaska: U.S. Geological Survey Professional Paper 543-C, $12 \mathrm{p}$.

Cohen, S.C., 1996, Time-dependent uplift of the Kenai Peninsula and adjacent regions of south-central Alaska since the 1964 Prince William Sound earthquake: Journal of Geophysical Research. v. 101, no. B4, p. 8595-8604.

Cohen, S.C., Holdahl, S.R, Caprette, Douglas, Hilla, Stephen, Safford, Robert, and Schultz, Donald, 1995, Uplift of the Kenai Peninsula since the 1964 Prince William Sound earthquake: Journal of Geophysical Research, v. 100, no. B2, p. 2031-2038.

Cohen, S.C., and Freymueller, J.T., 1997, Deformation of the Kenai Peninsula: Journal of Geophysical Research., v. 102, no. B9, p. 2047920487.

Gilpin, Lou, and Carver, Gary, 1994, Tidal benchmark readings and postseismic rebound of Kodiak Islands. SW extent of the 1964 great Alaskan earthquake rupture [abs.]: Seismological Research Letters, v. 65 , no. 1, p. 65.
Grantz, Arthur, Plafker, G.W., and Kachadoorian, Reuben, 1964, Alaska's Good Friday earthquake, March 27, 1964: A preliminary evaluation: U.S. Geological Survey Circular 491, 35 p.

Jachens, R.C, 1979, Temporal gravity changes as applied to studies of crustal deformation, in Everden, J.L., convener, Proceedings of Conference VII, Stress and Strain Measurements Related to Earthquake Prediction: U.S. Geological Survey Open-File Report 79-370, p. 222243.

Krauskopf, K.B., 1972, The great Alaska earthquake of 1994 [preface], in The Great Alaska Earthquake of 1964: Seismology and Geodesy: National Academy of Sciences, $996 \mathrm{p}$.

Marson, Iginio, and Alasia, Franco, 1980, Absolute gravity measurements in the United States of America: [Final report contract AFOSR-806157 to Air Force Geophysics Laboratory, Hanscom AFB, Mass., unpub. report].

Plafker, George, 1965, Tectonic deformation associated with the 1964 Alaska earthquake: Science, v. 148, no. 3678, p. 1675-1687.

Plafker, George, 1969, Tectonics of the March 27, 1964, Alaska earthquake. U.S. Geological Survey Professional Paper 543-I, 74 p.

Prescott, W.H., and Lisowski, Michael, 1977, Deformation of Middleton Island, Alaska during the decade after the Alaskan earthquake of 1964: Bulletin, Seismological Society of America, v. 67, no. 3, p. 579586.

Rice, D.A., 1969, Gravity Observations in Alaska, 1964-1965, including some repeat observations, in Leipold, L.E., ed., The Prince William Sound, Alaska, Earthquake of 1964 and Aftershocks: U.S. Goast and Geodetic Survey Publication 10-3, p. 5-20.

Savage, J.C., 1984, Local gravity anomalies produced by dislocation sources: Journal of Geophysical Research, v. 89, no. B3, p. 19451952.

Savage, J.C., and Hastie, L.M., 1966, Surface deformation with dip-slip faulting: Journal of Geophysical Research, v. 71, no. 20, p. 4897-4904.

Savage, J.C., and Plafker, G.W., 1981, Tide-gage measurements of uplift along the south coast of Alaska: Journal of Geophysical Research, v. 96, p. $4325-4335$.

Savage, J.C., Svarc, J.L., Prescott, W.H., and Gross, W.K., 1998, Deformation across the rupture zone of the 1964 Alaska earthquake 19931997: Journal of Geophysical Research, v. 103, no. B9, p. 2127521284.

Small, J.B., and Wharton, L.C., 1969, Vertical displacements determined by surveys after the Alaskan earthquake of March 1964, in Leipold, L.E., ed., The Prince William Sound, Alaska, earthquake of 1964 and aftershocks: U.S. Coast and Geodetic Survey Publication 10-3, p. 2133.

Thiel, Edward, Bonini, W.E., Ostenso, N.A., and Woollard, G.P., 1958, Gravity measurements in Alaska: Woods Hole, Mass., Woods Hole Oceanographic Institution Reference 58-54, 104 p.

Walsh, J.B., and Rice, J.R., 1979, Local changes in gravity resulting from deformation: Journal of Geophysical Research, v. 84, no. B1, p. 165170.

Whitcomb, T., 1976, New vertical geodesy: Journal of Geophysical Research, v. 81, no. 26, p. 4937-4944.

Woollard, G.P., Ostenso, N.A., Thiel, Edward, and Bonini, W.E., 1960, Gravity anomolies, crustal structure and geology in Alaska: Journal of Geophysical Research, v. 65, no. 3, p. 1021-1037.

Woollard, G.P., and Rose, J.C., 1963, International gravity measurements: Dallas, Society of Exploration Geophysicists, $518 \mathrm{p}$.

Reviewers: Jim Savage, Bob Jachens. 


\title{
Metal Cycling Along the Northwestern Seward Peninsula, Alaska: A Possible Natural Cause of Metal Contamination in the Arctic
}

\author{
By Cynthia C. Parnow, Richard J. Goldfarb, Karen D. Kelley, and Geoff S. York
}

\section{Abstract}

The northwestern Seward Peninsula was targeted for detailed geochemical study after evaluation of data collected during the NURE reconnaissance-level program indicated anomalously high arsenic (60-635 ppm) concentrations in stream sediments. The arsenic is associated with tin skarn, greisen, and replacement deposits in the western Seward Peninsula. Surficial sampling of waters and sediments indicate that arsenic is being transported detritally but that solution transport is insignificant. Our new data indicate that sediments downstream from these tin occurrences are characterized by anomalous values of As (85$530 \mathrm{ppm})$ and $\mathrm{Sn}(14-36 \mathrm{ppm})$, as well as consistent anomalies of $\mathrm{Ag}, \mathrm{Be}, \mathrm{Cu}, \mathrm{Sb}$, and $\mathrm{W}$. Stream sediments collected from drainages underlain by slate, but distal to the exposed tin occurrences, are characterized by background levels of As (10-60 ppm), Li (16-80 ppm), Sn (5-14 ppm), and W (5-10 ppm). These background levels for As and Sn are much higher than concentrations in typical slates and suggest a broad, weak hydrothermal alteration during mineral-deposit formation in much of the study area. A consistent pattern of $\mathrm{Ca}>>\mathrm{Mg}>\mathrm{Na}>\mathrm{K}$ and generally alkaline $\mathrm{pH}(7.2-8.2)$ characterize waters throughout the study area. Dissolved sulfate concentrations range from 10 to 40 $\mathrm{ppm}$ for waters draining slates and from 3 to $20 \mathrm{ppm}$ for water draining carbonates. The waters collected in areas of known tin occurrences in the Potato Mountain area are characterized by increased dissolved sulfate (43-75 ppm) and are generally acidic ( $\mathrm{pH}$ 4.7-6.5), but most trace metals are at or below detection limits. Dissolved arsenic concentrations in the areas of known tin occurrences are at or below $2 \mathrm{ppb}$. Our data suggest that detrital arsenic and tin from mineral occurrences may be naturally entering the nearshore marine environment.

\section{Introduction}

A geochemical study to determine natural background concentrations of metals in surficial materials was carried out on the northwestern Seward Peninsula near the village of Wales, Alaska (fig. 1). This part of the Seward Peninsula was targeted for detailed study after assessment of regional element distribution maps, which were compiled from stream- and lake-sediment geochemical data collected during the National Uranium Resource Evaluation (NURE) program (Hoffmann and Buttleman, 1996). Interpretation of the NURE data indicated that some drainages feeding Lopp Lagoon (fig. 1) had anomalously high arsenic (60-635 ppm) and tin (60-144 ppm) concentrations in sediments, compared to regional background concentrations (between 5-60 ppm for As and 7-12 ppm for Sn).

The presence of relatively metal rich sediments, soils, or waters in the northwestern Seward Peninsula may be significant for a number of reasons. The population of Wales relies heavily on the local ecosystem for food sources. Plants are harvested throughout the summer from the tundra adjacent to the village, and fish are collected from nearby Lopp Lagoon-a body of water that receives most of the surface runoff and sediment load from the northwestern corner of the Seward Peninsula. Also, the proximity of the study area to the Bering Strait allows for rapid cycling of any eroding metals into the nearshore marine ecosystem.

Locally anomalous concentrations of many trace metals occur in tin-rich granites and adjacent metasedimentary rocks at scattered locations across the northwestern Seward Peninsula. The anomalous arsenic values observed in the NURE sediments are, at least in part, erosional products of metalliferous granites and adjacent skarn and replacement bodies. Dispersion of arsenic and other genetically associated ore metals, Sn, Be, and $\mathrm{W}$, from bedrock sources into local soils, stream sediments, and surface waters is a normal part of the processes of weathering and erosion.

The aim of the present study is to better understand the element dispersion along the northwestern side of the York Mountains by sampling surface waters and stream sediments.

Resulting local geochemical background data are critical for any future environmental impact assessment, land-use planning, pollution monitoring, and mineral-resource-assessment work in the area. These data may help identify areas of the tundra that 


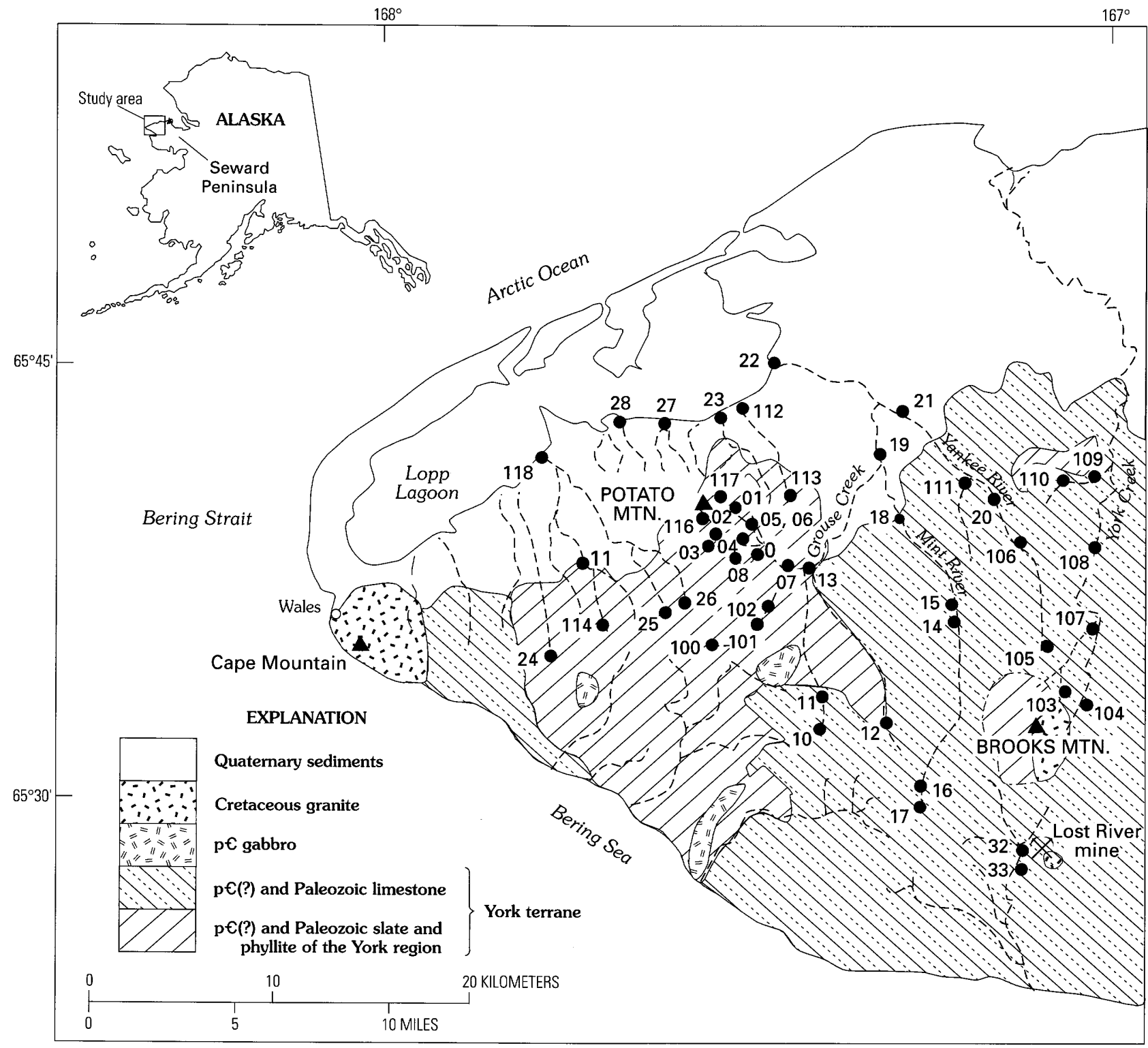

Figure 1. Site locations for stream-sediment and water samples collected in northwestern Seward Peninsula, Alaska. (Geology from Sainsbury, 1972)

should be avoided for plant harvesting. If metal levels in sediments or waters are relatively high near the mouths of the streams and rivers, then this might signal the need for detailed study of any effects on organisms in Lopp Lagoon. Certainly, at least, the possibility of biomagnification of metals within the food chain exists in the Wales area. Atmospherically transported, anthropogenic $\mathrm{As}, \mathrm{Cd}, \mathrm{Hg}$, and other contaminants in high-latitude arctic ecosystems are now widely recognized in the tissue and organs of large marine mammals and native populations (Pearce, 1997). Identification of any significant natural metal cycling into the food chain in this part of the Seward Peninsula is a first step in determining whether regional geological processes may also be contributing contaminants to the environment. Also, data from this study may prove useful in better defining new areas of tin, uranium, and other granite-related mineral resources in this part of Alaska.

\section{General Geology}

Sainsbury (1972) mapped the geology of the western portion of the Seward Peninsula at a scale of 1:250,000. The area consists mainly of Precambrian(?) and early Paleozoic argillaceous and dolomitic limestones, shales, slates, and phyllites (fig. 1), which are referred to as slate of the York region. Much of this unit was mapped by Sainsbury (1972) as Precambrian in age, but it is lithologically similar to Lower Ordovician rocks found elsewhere on the Seward Peninsula (Till and Dumoulin, 1994). The weakly metamorphosed units were deposited in shallow marine to restricted carbonate platform sedimentary environments. The York terrane may be subdivided into two major units: (1) the slate of the York region - an informal name for graphitic, slaty, or phyllitic rocks that originally consisted mainly of 
carbonaceous siltite, but also contained diverse laminated to thin-bedded mudstone, siltstone, fine-grained sandstone, calcareous sandstone, and carbonaceous limestone (Sainsbury, 1972) — and (2) a thick sequence of fossiliferous limestone, dolomitic limestone, and argillaceous limestone.

The York terrane was subsequently intruded by highly evolved, tin-rich granites and syenogranites between about 80 $70 \mathrm{Ma}$. Seven of nine tin-enriched granite plutons are exposed and two are inferred at depth in the northwestern Seward Peninsula (Hudson and Arth, 1983). Three of these, Cape Mountain, Brooks Mountain, and an inferred pluton at Potato Mountain, occur within our study area. These granites are similar to granites exposed in eastern Siberia (Swanson and others, 1990) and, therefore, are likely to occur beneath the waters of the Bering Strait.

On the Seward Peninsula, tin lodes are generally found as cassiterite in greisenized plutonic cupolas and in pyroxene-garnet-tourmaline-axinite-cassiterite skarn in adjacent marbles (Swanson and others, 1990; Hudson and Reed, 1997). Uranium, tungsten, and rare-earth-element (REE) enrichments are commonly associated with many of these evolved plutons. Hudson and Arth (1983) report as much as 20 to $30 \mathrm{ppm} U$ for representative samples of some plutons. Recognized sulfide and oxide mineral phases include pyrrhotite, arsenopyrite, fluorite, tetrahedrite, scheelite, cassiterite, stannite, wolframite, chalcopyrite, thorite, zeunerite, and many Be-bearing phases (West and White, 1952; Sainsbury, 1963; Sainsbury and others, 1968; Swanson and others, 1990).

About 3,000 metric tons ( $t$ ) of tin have been recovered from lodes and related placers of the Seward Peninsula, representing more than 90 percent of Alaska's historic production. Past production is dominated by 1,676 $\mathrm{t}$ of tin from the Cape Mountain placer deposit, 1,000 t of tin from the Potato Mountain placer deposit, and $314 \mathrm{t}$ of tin from the Lost River lode deposit (Hudson and Reed, 1997). Lode reserves remaining at the Lost River deposit include 22.3 million metric tons of 0.15 percent tin, 16.3 percent fluorite, and 0.03 percent $\mathrm{WO}_{3}$ (Bundtzen and others, 1994). In addition, beryllium-fluorite-rich veins that replaced limestone at the Lost River deposit contain 4,500 t of beryllium, one of the world's largest reserves (Sainsbury, 1988).

Sainsbury and others (1968) carried out stream-sediment, soil, and plant-geochemical surveys around tin occurrences in the Brooks Mountain and Lost River areas. Although the data were generally semiquantitative and often below analytical determination limits, some stream sediments near the Lost River mine tailings contained as much as $160 \mathrm{ppm} \mathrm{Be}, 1,100 \mathrm{ppm} \mathrm{Sn}$, 70 ppm W, 150 ppm Cu, 150 ppm Pb, 300 ppm Zn, 3,000 ppm As, $700 \mathrm{ppm} \mathrm{B}$, and 3,000 ppm Li. Concentrations for soils in the Lost River valley were of equivalent magnitude. Various tundra plants in the valley showed $\mathrm{Cu}, \mathrm{Pb}, \mathrm{B}$, and, less consistently, $\mathrm{Ag}$ and $\mathrm{Zn}$ enrichment. However, $\mathrm{As}, \mathrm{Be}$, and $\mathrm{Sn}$ were not strongly concentrated in the studied plant species.

\section{Field and Laboatory Methods}

Water samples were collected at 49 sites in the drainage basins of Potato Mountain and along and to the west of the Mint, York, and Yankee Rivers (fig. 1) during July 1997. Stream-sediment samples were collected from 48 sites, but at two of the sites there was not enough material collected for subsequent analysis. At each sample site, $\mathrm{pH}$ and conductivity of stream water were measured with portable meters. Three $60-\mathrm{mL}$ water samples were collected in high-density polyethylene (HDPE) bottles at each site. Two samples, one filtered and one unfiltered, were taken for cation analysis. The unfiltered sample was analyzed for total metals, dissolved plus suspended cations. The second sample was passed through a $0.45-$ $\mu \mathrm{m}$ filter to determine dissolved-cation concentrations. Both samples were acidified with concentrated nitric acid to a $\mathrm{pH}$ of less than 2. A third filtered, unacidified sample was taken for anion analysis. This sample was packed in ice for shipping and then refrigerated until analyzed. Stream sediments were sieved in the field to minus10 -mesh ( $2 \mathrm{~mm})$. Panned heavy-mineral-concentrate samples were collected at 29 sites.

In the laboratory, the stream sediments were dried at room temperature and sieved to minus-80-mesh $(1 \mathrm{~mm})$, pulverized, and chemically analyzed. The panned concentrates were dried and sieved to minus-18-mesh $(<1 \mathrm{~mm})$. The heavy minerals in each sample were then gravity-separated using bromoform (specific gravity $>2.86$ ). The heavy-mineral concentrates were separated into magnetic, paramagnetic, and nonmagnetic fractions. The nonmagnetic fraction was further separated into two splits. One split was pulverized and chemically analyzed; the remaining split was retained for optical mineralogic examination.

\section{Analytical Techniques}

The filtered and unfiltered water samples were analyzed for major and minor cations and trace elements by inductively coupled plasma-mass spectrometry (ICP-MS) (Meier and others, 1994). Anion concentrations were determined on the unacidified, filtered water samples using ion chromatography as described by d'Angelo and Ficklin (1996). Total alkalinity was measured by titration as bicarbonate $\left(\mathrm{HCO}_{3}^{-}\right)$.

Stream sediments were digested in a total decomposition process using a mixture of hydrochloric, nitric, perchloric, and hydrofluoric acids at low temperature (Crock and others, 1983). The resulting solution was analyzed for 40 major, minor, and trace elements by inductively coupled plasma-atomic emission spectrometry (ICP-AES). Additionally, a 10-element ICP-AES (Motooka, 1996) method was applied that utilizes a partial digestion of the sample in a hydrochloric acid-hydrogen peroxide solution. These data are useful because of lower detection limits for $\mathrm{Ag}, \mathrm{Cd}, \mathrm{Mo}$, and $\mathrm{Sb}$, whose concentrations were below the detection limit utilizing the 40-element ICP-AES method. $\mathrm{Se}, \mathrm{W}$, and $\mathrm{Hg}$ were determined using the chemical methods outlined by Arbogast (1996). The nonmagnetic heavy-mineral-concentrate samples were analyzed by semiquantitative emission spectrography (ES) for 36 elements (Grimes and Marranzino, 1968). Analytical results of the stream sediment data are listed in Parnow and others (in press).

\section{Geochemistry of Stream Sediment Samples}

A summary table of basic statistics for the 45 streamsediment samples (table 1) lists the median, minimum, and maximum values for elements that have detection ratios greater 
than 0.5 (detection ratio equals the number of unqualified results divided by the total number of samples in the data set). The data for the sample (site 32; fig. 1) collected from below the Lost River mine tailings were excluded from the basic statistics because the extremely high concentrations of many elements would bias the mean statistics. This sample was collected from the Lost River immediately downstream from the main tailings pile along the river bank and is not representative of natural background geochemistry of the area. However, this sample is useful for defining the geochemical signature representative of tin-bearing skarn deposits in the western Seward Peninsula. The metalliferous sediment sample contained 13 percent $\mathrm{Ca}, 4.3$ percent $\mathrm{Mg}, 3$ ppm Ag, 1,435 ppm As, 106 ppm Bi, 21 ppm Cd, 285 ppm Cu, 768 ppm Li, 766 ppm Pb, 14 ppm Sb, 136 ppm Sn, 210 ppm W, and 5,200 ppm Zn. Many of these values are several times greater than the maximum concentrations from the remaining data set.
The sediment samples taken in the area underlain by the slate unit of the York terrane are characterized by background levels of 10-60 ppm As, 5-14 ppm Sn, <0.1 ppm Ag, $<10$ ppm Bi, 0.06-0.8 ppm Cd, 18-40 ppm Cu, 16-80 ppm Li, 25-31 ppm $\mathrm{Pb}, 1-9 \mathrm{ppm} \mathrm{Sb}$, and 5-10 ppm W. Background values were estimated from visual evaluation of histograms of the geochemical data. The background values for $\mathrm{As}, \mathrm{Li}$, and $\mathrm{Sn}$ are quite high relative to average values for stream sediments derived from shales and slates (Levinson, 1980), reflecting a broad hydrothermal enrichment possibly associated with tin granite alteration haloes. Typical calcium concentrations of 0.2 to 0.5 percent characterize sediments derived from the slates, but calcium concentrations are locally higher due to calcareous silt and carbonaceous limestone interbedded in the slates.

Sediment samples taken from the upper reaches of streams draining Potato Mountain (fig. 1), an area of known tin occurrences, are consistently anomalous in As (85-530 ppm; fig. $2 A$ ).

Table 1. Statistical summary of selected elements from 45 stream-sediment samples (minus-80-mesh) from the western Seward Peninsula, Alaska.

[All concentrations in ppm unless otherwise noted; detection ratio is the number of samples with unqualified values divided by the total number of samples; values below detection limit were set equal to 0.7 times the lower detection limit. All elements were analyzed by 40 -element inductively coupled plasma-mass spectrometry (ICP-AES), except for Cd, Mo, and Sb, which were analyzed by 10 -element ICP-AES because of lower detection limits. Selenium was analyzed by hydride generation; tungsten was analyzed by neutron activation analysis (NAA). Samples were also analyzed for Ag and Au by 10-element ICP-AES, but more than 60 percent of the data were below the lower determination limit. All analyses done by USGS contract lab, XRAL Inc., Ontario, Canada]

\begin{tabular}{|c|c|c|c|c|c|c|c|c|}
\hline $\begin{array}{l}\text { Element } \\
\text { analyzed }\end{array}$ & $\begin{array}{c}\text { Detection } \\
\text { ratio }\end{array}$ & Median & Minimum & Maximum & $\begin{array}{c}\text { 85th } \\
\text { percentile } \\
\end{array}$ & $\begin{array}{c}\text { 90th } \\
\text { percentile }\end{array}$ & $\begin{array}{c}\text { Geometric } \\
\text { mean }\end{array}$ & $\begin{array}{c}\text { Geometric } \\
\text { deviation }\end{array}$ \\
\hline $\mathrm{Al}(\%)$ & 1 & 7 & 1.1 & 8.6 & 7.9 & 8.2 & 6.1 & 1.5 \\
\hline $\mathrm{Ca}(\%)$ & 1 & .68 & 0.18 & 22 & 11 & 16 & 1.5 & 5.4 \\
\hline $\mathrm{Fe}(\%)$ & 1 & 4.8 & 0.73 & 6.9 & 5.9 & 6 & 4.2 & 1.6 \\
\hline K (\%) & 1 & 1.9 & 0.34 & 2.5 & 2.3 & 2.3 & 1.7 & 1.5 \\
\hline $\mathrm{Mg}(\mathbf{\%})$ & 0.96 & 1.3 & 0.67 & 6.5 & 3.1 & 3.7 & 1.5 & 1.7 \\
\hline $\mathrm{Na}(\%)$ & 1 & .65 & 0.05 & 1.1 & 0.93 & 0.94 & .56 & 1.8 \\
\hline $\mathrm{P}(\%)$ & 1 & .07 & 0.01 & 0.13 & 0.11 & 0.12 & .07 & 1.7 \\
\hline $\mathrm{Ti}(\%)$ & 1 & .41 & 0.05 & 0.95 & 0.54 & 0.61 & .32 & 1.9 \\
\hline As & 0.87 & 32 & 10 & 530 & 95 & 101 & 33 & 2.9 \\
\hline $\mathrm{Ba}$ & 1 & 353 & 38 & 2510 & 995 & 1460 & 439 & 2.2 \\
\hline $\mathrm{Cd}$ & 1 & 0.2 & 0.05 & 1.6 & 0.6 & 0.8 & 0.2 & 2.4 \\
\hline $\mathrm{Ce}$ & 1 & 82 & 7 & 361 & 115 & 118 & 70 & 1.9 \\
\hline Co & 1 & 19 & 3 & 32 & 27 & 28 & 17 & 1.7 \\
\hline $\mathrm{Cr}$ & 1 & 95 & 13 & 140 & 123 & 125 & 84 & 1.6 \\
\hline $\mathrm{Cu}$ & 1 & 23 & 3 & 59 & 30 & 41 & 22 & 1.7 \\
\hline $\mathrm{Ga}$ & 1 & 21 & 14 & 31 & 26 & 26 & 21 & 1.2 \\
\hline $\mathrm{Hg}$ & 1 & 0.063 & 0.014 & 0.287 & 0.174 & 0.212 & 0.06 & 2.2 \\
\hline $\mathrm{La}$ & 1 & 38 & 4 & 132 & 51 & 54 & 32 & 1.8 \\
\hline $\mathrm{Li}$ & 1 & 61 & 9 & 132 & 80 & 96 & 57 & 1.6 \\
\hline $\mathrm{Mn}$ & 1 & 744 & 175 & 3660 & 1010 & 1180 & 726 & 1.7 \\
\hline Mo & 1 & 0.7 & 0.3 & 8.2 & 2.8 & 3.1 & 1 & 2.2 \\
\hline $\mathrm{Nd}$ & 0.96 & 36 & 10 & 199 & 56 & 59 & 34 & 1.8 \\
\hline $\mathrm{Ni}$ & 1 & 50 & 8 & 76 & 61 & 66 & 46 & 1.5 \\
\hline $\mathrm{Pb}$ & 1 & 25 & 4 & 86 & 30 & 31 & 23 & 1.6 \\
\hline $\mathrm{Sb}$ & 0.51 & 0.7 & 1 & 26 & 9 & 9 & 1.5 & 3.0 \\
\hline $\mathrm{Sc}$ & 1 & 15 & 3 & 21 & 19 & 19 & 13 & 1.5 \\
\hline $\mathrm{Se}$ & 0.91 & 0.3 & 0.2 & 1 & 0.6 & 0.8 & 0.3 & 1.7 \\
\hline Sn & 0.64 & 7 & 5 & 62 & 14 & 16 & 7 & 2.0 \\
\hline $\mathrm{Sr}$ & 1 & 86 & 53 & 470 & 302 & 337 & 119 & 1.9 \\
\hline V & 1 & 142 & 17 & 298 & 166 & 180 & 131 & 1.6 \\
\hline W & 1 & 6.7 & 4 & 17 & 10 & 10 & 6.9 & 1.4 \\
\hline$Y$ & 1 & 14 & 4 & 24 & 17 & 18 & 14 & 1.3 \\
\hline $\mathrm{Zn}$ & 1 & 100 & 15 & 155 & 120 & 133 & 90 & 1.5 \\
\hline
\end{tabular}




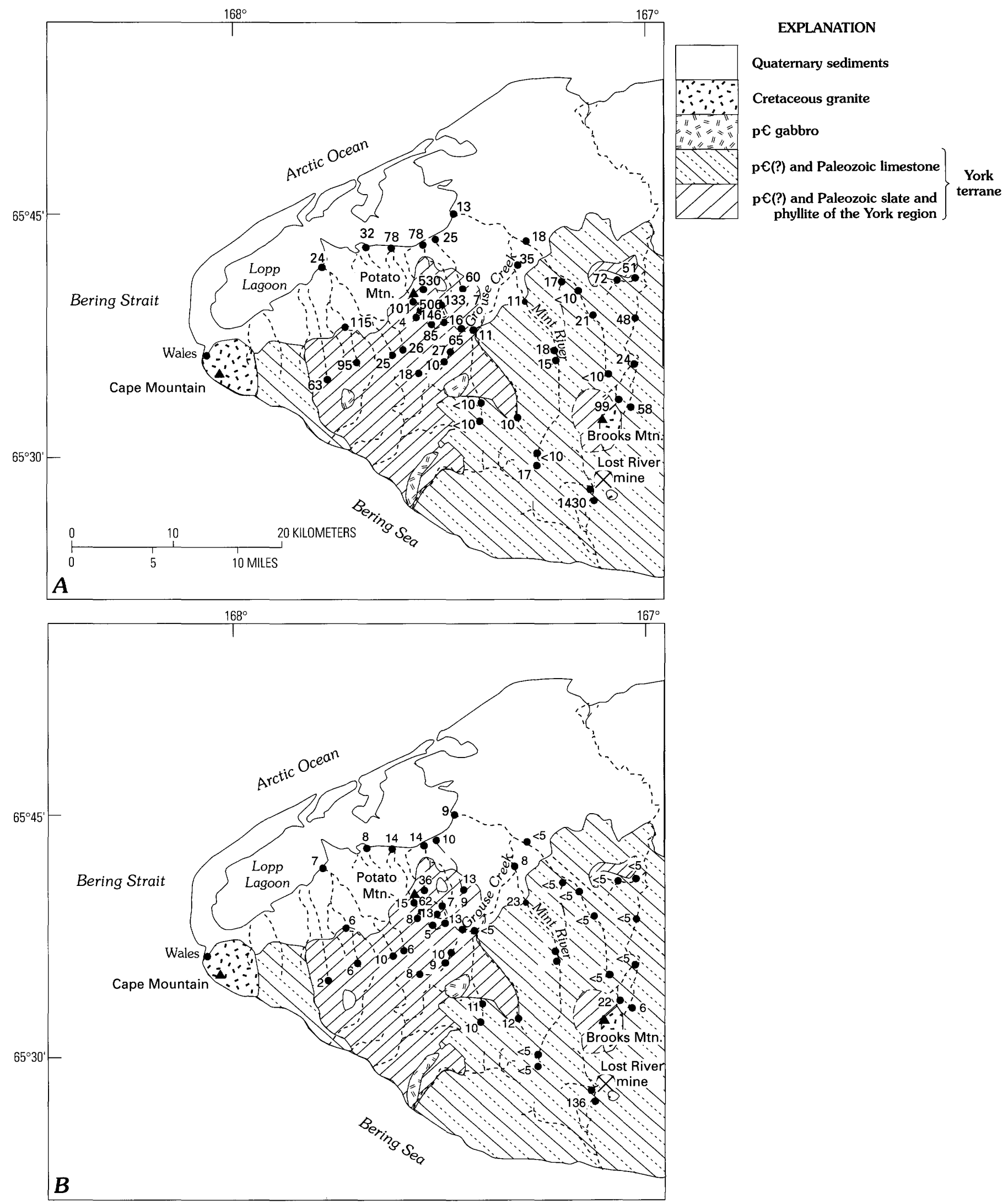

Figure 2. A, Concentrations of arsenic in ppm in stream sediments. $B$, Concentrations of tin in ppm in stream sediments.

Also, they show consistent anomalies for tin (fig. $2 B$ ), as well as enrichments of $\mathrm{Ag}, \mathrm{Be}, \mathrm{Cu}, \mathrm{Ni}, \mathrm{Sb}$, and $\mathrm{W}$. The sediment samples collected in the same drainages, but at lower elevations flowing through the Quaternary sediments directly upstream from Lopp Lagoon (sites 23, 27, 28, 112, and 118; fig. 1), generally contain background tin and arsenic concentrations. However, two of the five samples (sites 23 and 27) collected in the lower reaches contain As concentrations of $78 \mathrm{ppm}$. In addition, 
Table 2. Factor loadings for selected elements for 45 streamsediment samples collected from the western Seward

Peninsula, Alaska.

[Leaders (--) indicate loadings less than $|0.5|$; negative loadings in boldface type]

\begin{tabular}{|c|c|c|c|c|}
\hline Element & Factor 1 & Factor 2 & Factor 3 & Factor 4 \\
\hline $\mathrm{Al}$ & 0.92 & -- & -- & -- \\
\hline $\mathrm{Ca}$ & -0.81 & -- & -. & -- \\
\hline $\mathrm{Fe}$ & 0.95 & -- & -- & -- \\
\hline $\mathrm{K}$ & 0.89 & -- & -- & -- \\
\hline $\mathrm{Mg}$ & -0.82 & -- & -- & -- \\
\hline $\mathrm{Na}$ & 0.89 & -- & -- & -- \\
\hline $\mathrm{P}$ & 0.93 & -- & -- & -- \\
\hline $\mathrm{Ti}$ & 0.88 & -. & .- & -- \\
\hline $\mathrm{Mn}$ & 0.72 & -. & - & -- \\
\hline As & -- & -- & 0.84 & -- \\
\hline $\mathrm{Ba}$ & -- & 0.89 & -- & -- \\
\hline $\mathrm{Cd}$ & -- & 0.87 & -- & -- \\
\hline $\mathrm{Ce}$ & 0.86 & -- & -- & -- \\
\hline Co & 0.88 & -- & -- & -- \\
\hline $\mathrm{Cr}$ & 0.93 & -- & -- & -- \\
\hline $\mathrm{Cu}$ & 0.50 & 0.68 & -- & -- \\
\hline $\mathrm{Ga}$ & -- & -- & -- & -- \\
\hline $\mathrm{Hg}$ & -- & -- & -- & -0.66 \\
\hline $\mathrm{La}$ & 0.83 & -- & -- & -- \\
\hline $\mathrm{Li}$ & 0.79 & -- & -- & -- \\
\hline Mo & -- & 0.87 & -- & -- \\
\hline $\mathrm{Nd}$ & 0.85 & -- & .- & -- \\
\hline $\mathrm{Ni}$ & 0.82 & -- & -- & -- \\
\hline $\mathrm{Pb}$ & 0.69 & -- & -- & -- \\
\hline $\mathrm{Sb}$ & -- & -- & 0.87 & -- \\
\hline $\mathrm{Sc}$ & 0.94 & -- & -- & -- \\
\hline $\mathrm{Se}$ & -- & 0.71 & -- & -- \\
\hline Sn & -- & -- & 0.72 & -- \\
\hline $\mathrm{Sr}$ & -0.63 & 0.55 & -- & -- \\
\hline $\mathrm{V}$ & 0.83 & 0.51 & -- & -- \\
\hline W & 0.03 & -- & 0.62 & -- \\
\hline $\mathrm{Y}$ & 0.62 & 0.54 & -- & -- \\
\hline $\mathrm{Zn}$ & 0.87 & -- & -- & $\cdots$ \\
\hline
\end{tabular}

the most western of these (site 118) contains $361 \mathrm{ppm} \mathrm{Ce,} 8 \mathrm{ppm}$ $\mathrm{Eu}, 132 \mathrm{ppm} \mathrm{La}$, and $199 \mathrm{ppm} \mathrm{Nd}$, the highest concentrations of these elements from the study area.

The samples collected in the eastern portion of the study area, which is underlain by the dolomitic part of the limestone unit of the York terrane, had mainly background concentrations of $\mathrm{Ag}(<0.8 \mathrm{ppm}), \mathrm{As}(10-50 \mathrm{ppm}), \mathrm{Ba}(400-1,000 \mathrm{ppm}), \mathrm{Cu}(5-$ $30 \mathrm{ppm}), \mathrm{Cd}(0.3-0.8 \mathrm{ppm}), \mathrm{Ni}$ (30-60 ppm), Pb (15-30 ppm), Sb (<1 ppm), Sn (<5 ppm), V (80-180 ppm), Zn (56-130 ppm), and $\mathrm{W}(5-10 \mathrm{ppm})$. Background populations were again defined through visual inspection of histograms of the data for the sediments draining the limestone unit. Locally high concentrations of $\mathrm{Ba}(1,460-2,510 \mathrm{ppm}), \mathrm{Cd}(0.8-1.6 \mathrm{ppm})$, Mo (3.1-5.4 ppm), and $V(180-225 \mathrm{ppm})$ primarily reflect material weathered from argillaceous interbeds.

Two samples (sites 109 and 110; fig. 1) collected from a small tributary of York Creek along the eastern border of the study area contained arsenic concentrations of 51 and $72 \mathrm{ppm}$ (fig. $2 A$ ), respectively, suggesting possible hydrothermal mineralization even though there are no known granites in the area. The sample with $72 \mathrm{ppm}$ As also contained detectable gold and silver, further suggesting hydrothermal mineralization distal to any known granite. Anomalous Mo (6.8-8.2 ppm) and V (298$235 \mathrm{ppm}$ ) in the two samples can best be attributed to argillaceous units within the limestone-dominant unit.

One of the sediment samples collected from a stream on the northeast side of Brooks Mountain (site 103; fig. 1) had a tin value of $22 \mathrm{ppm}$. This sample also had anomalous values for As (99 ppm), Cu (49 ppm), Li (110 ppm), Ni (66 ppm), Pb (86 $\mathrm{ppm})$, and $\mathrm{Sb}(9 \mathrm{ppm})$. These enrichments reflect the known tin occurrences upstream and nearer to the peak of Brooks Mountain. This is further supported by a concentration of $26 \mathrm{ppm} \mathrm{Sb}$ at an adjacent site (site 104; fig. 1).

\section{Factor Analysis of Stream-Sediment Data}

Element associations for the 45 stream sediments (not including the Lost River site) were determined by factor analysis. R-mode factor analysis with Varimax rotation was used to identify dominant geochemical associations in the sediment data. The data were log-transformed prior to analysis, as most geochemical data are log-normally distributed (Koch and Link, 1970). The log transformation attempts to normalize the distribution so that the statistical analysis is valid. Resulting factors often delineate suites of elements that are geochemically associated with specific rock types or mineral deposits. Factor loadings may be interpreted in much the same way as correlation coefficients. A high factor loading indicates a strong association of the particular variable (element) with the given factor. The association of each sample within a given factor is analyzed by its score onto that factor. The greater the absolute score of a given sample, the stronger the correlation to the element or suite of elements defined by the factor.

A four-factor model, which accounts for 82 percent of the total variance, was chosen as the most appropriate for summarizing the data set. Table 2 lists the factor loadings for all of the elements that had a detection ratio of greater than 0.5 and were, therefore, included in the factor analysis. Gold and silver, although elements of economic interest, were among those elements deleted from the factor analysis due to low detection ratios.

Factors 1 and 2 (figs. $3 A$ and $3 B$ ) reflect geochemical signatures of the dominant lithologies of the study area. Factor 1 has high positive loadings for $\mathrm{Al}, \mathrm{Fe}, \mathrm{K}, \mathrm{Na}, \mathrm{P}, \mathrm{Ti}, \mathrm{Mn}, \mathrm{Ce}, \mathrm{Co}, \mathrm{Cr}$, $\mathrm{Cu}, \mathrm{La}, \mathrm{Li}, \mathrm{Nd}, \mathrm{Ni}, \mathrm{Pb}, \mathrm{Sc}, \mathrm{V}, \mathrm{Y}$, and $\mathrm{Zn}$, and negative loadings for $\mathrm{Ca}, \mathrm{Mg}$, and Sr. Plots of the factor scores (fig. $3 A$ ) indicate that samples with positive scores for factor 1 are located mainly in the western portion of the study area, which is underlain by slates. All of the sites having strong negative scores for factor 1 are located along the Mint River, Yankee River, and York Creek, or their tributaries, and are underlain by carbonate rocks. Factor 2 has high positive loadings for $\mathrm{Ba}, \mathrm{Cd}$, and $\mathrm{Mo},(>0.85)$, and slightly lower positive loadings $(0.5-0.71)$ for $\mathrm{Cu}, \mathrm{Se}, \mathrm{Sr}, \mathrm{V}$, and Y. The samples with highest scores onto factor 2 (fig. $3 B$ ) were collected from the streams and rivers that are underlain by carbonates; thus, the geochemical signature must reflect the 


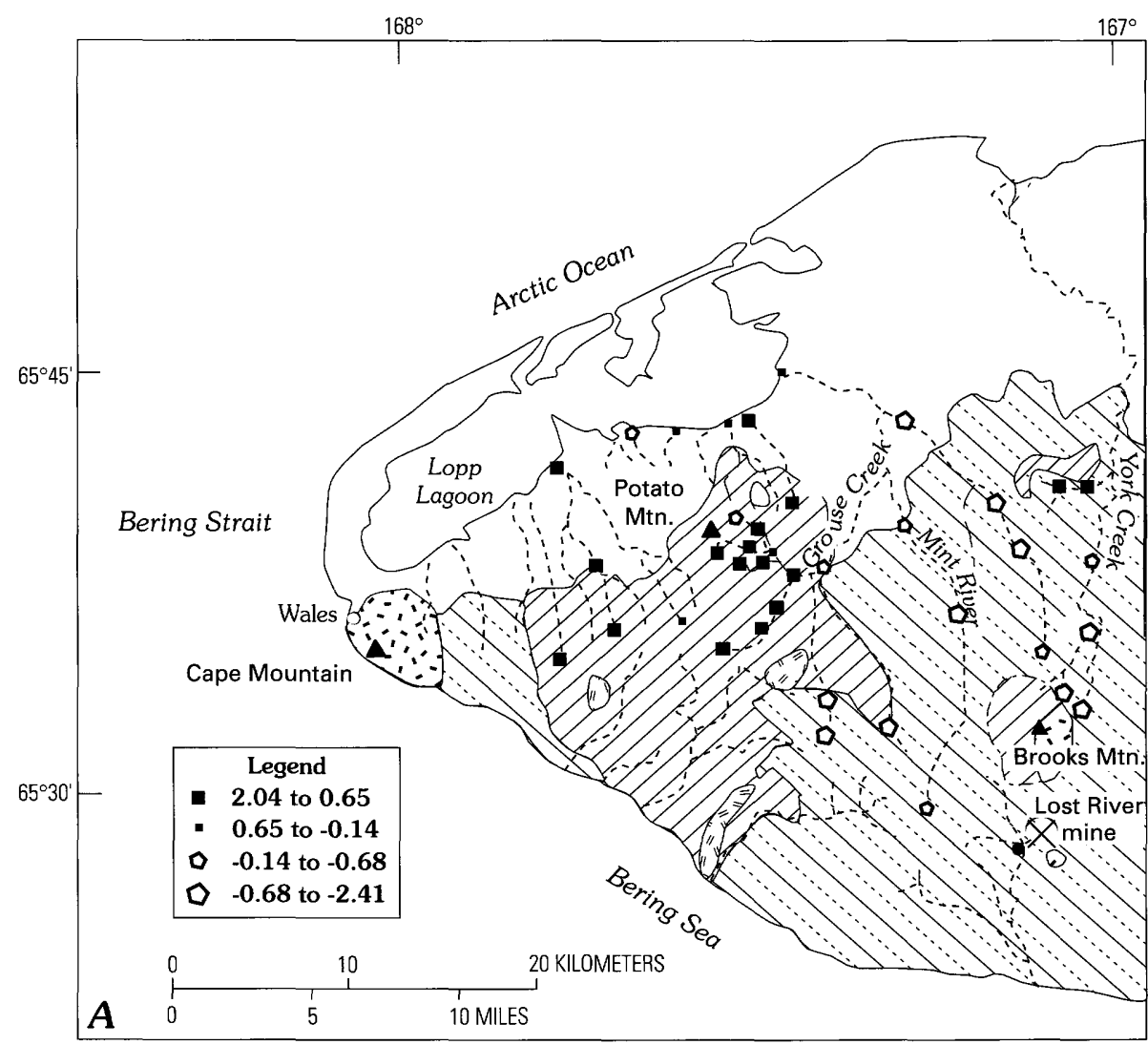

EXPLANATION

Quaternary sediments

Cretaceous granite

$p €$ gabbro

p€(?) and Paleozoic limestone

$p \in(?)$ and Paleozoic slate and phyllite of the York region

York terrane

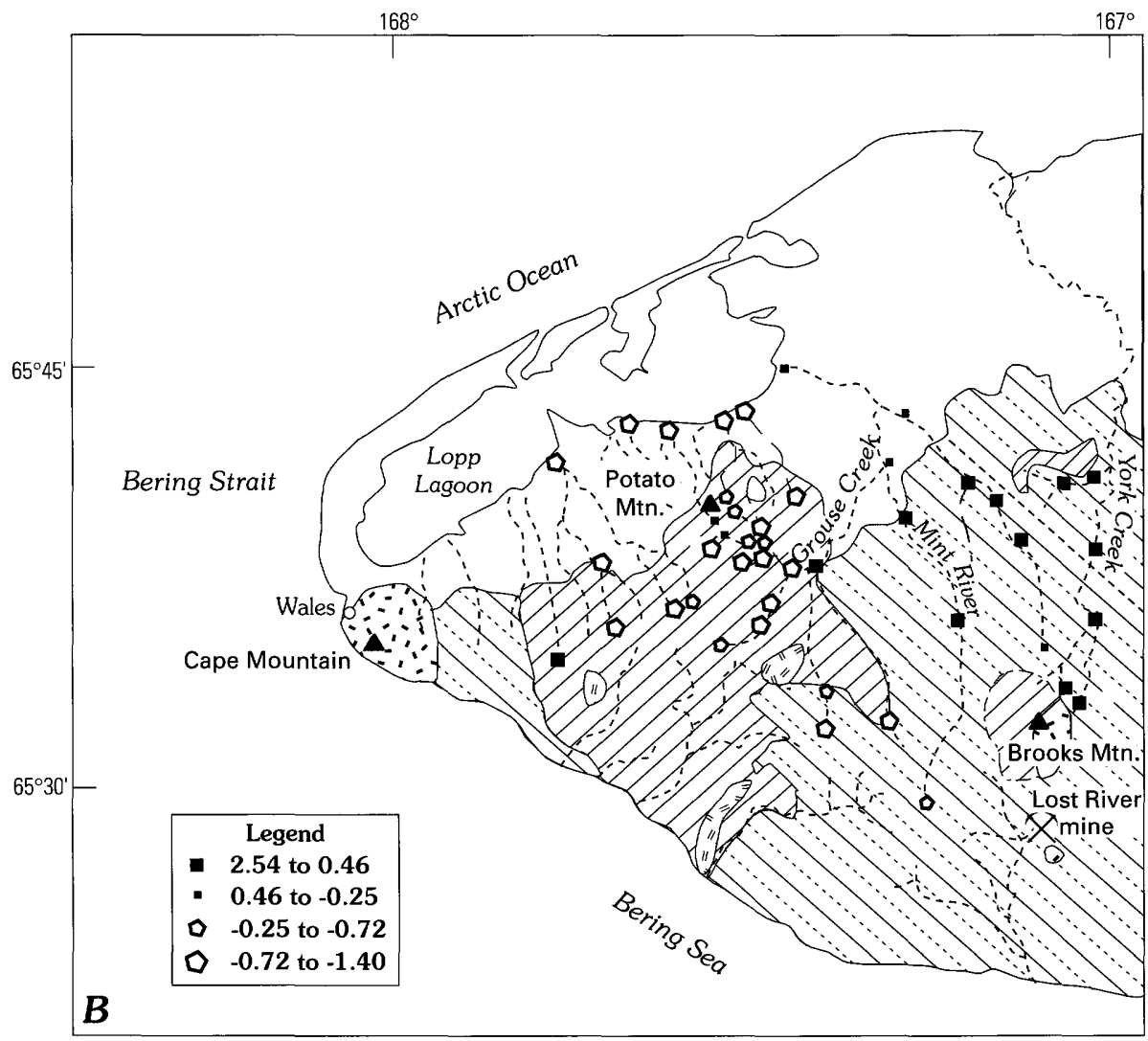

Figure 3. A, Plot of scores for factor 1 (positive loadings for $\mathrm{Al}, \mathrm{Fe}, \mathrm{K}, \mathrm{Na}, \mathrm{P}, \mathrm{Ti}, \mathrm{Mn}, \mathrm{Ce}, \mathrm{Co}, \mathrm{Cr}$, $\mathrm{Cu}, \mathrm{La}, \mathrm{Li}, \mathrm{Nd}, \mathrm{Ni}, \mathrm{Pb}, \mathrm{Sc}, \mathrm{V}, \mathrm{Y}$, and $\mathrm{Zn}$ and negative loadings for $\mathrm{Ca}, \mathrm{Mg}$, and $\mathrm{Sr}$ ). $B$, Plot of scores for factor 2 (positive loadings for $\mathrm{Ba}, \mathrm{Cd}, \mathrm{Cu}, \mathrm{Mo}, \mathrm{Se}, \mathrm{Sr}, \mathrm{V}$, and $\mathrm{Y}$ ). 
abundance of argillaceous interbeds. Sites 109 and 110 (fig. 1) have high positive scores for factor 1, but also have positive scores for factor 2 . These two samples sites reflect sediment contributions from both the slates and the carbonates in an area along the west side of York Creek.

Factor 3 shows a strong positive correlation between As, $\mathrm{Sb}, \mathrm{Sn}$, and W. This group of elements defines the geochemical signature of sediments influenced by tin occurrences of the region. Ten of the samples with high scores $(>0.4)$ for factor 3 (fig. 4) were collected in the stream drainages of Potato Mountain. Other samples with high factor-three scores were collected from drainages northeast of Brooks Mountain (sites 103 and 104; fig. 1) and from southwest of Potato Mountain (sites 24 and 114; fig. 1). Although none of these sites contained elevated tin concentrations in the stream sediments, most had anomalous As and $\mathrm{W}$, which are indicator elements for hydrothermal tin granites. Possibly, the data from sites 24 and 114 indicate additional undiscovered tin occurrences between Potato Mountain and Cape Mountain. Stream-sediment samples collected from a small tributary of York Creek (sites 109 and 110; fig. 1) had low scores for factor 3 and low concentrations of tin, although heavy-mineral-concentrate samples contained greater than 2,000 $\mathrm{ppm}$ tin and detectable tungsten $(150 \mathrm{ppm})$ collected from the same sites.

Factor 4 was defined by a loading for mercury of -0.66 . Analysis of the raw data indicates that this is due to minor variation in the mercury concentrations due to differences in lithology.

\section{Geochemistry and Mineralogy of Heavy- Mineral-Concentrate Samples}

Basic statistics for the heavy-mineral concentrates are listed in table 3. Eight of the fourteen samples collected in the drainages of the Potato Mountain area (fig. 1) contained greater than 2,000 ppm Sn. Three of these samples (sites 04, 116, and 117; fig. 1) showed significant amounts of As (500 ppm), B (1,000$2,000 \mathrm{ppm}), \mathrm{Bi}(20-2,000 \mathrm{ppm})$, and Be (2-5 ppm), but similar

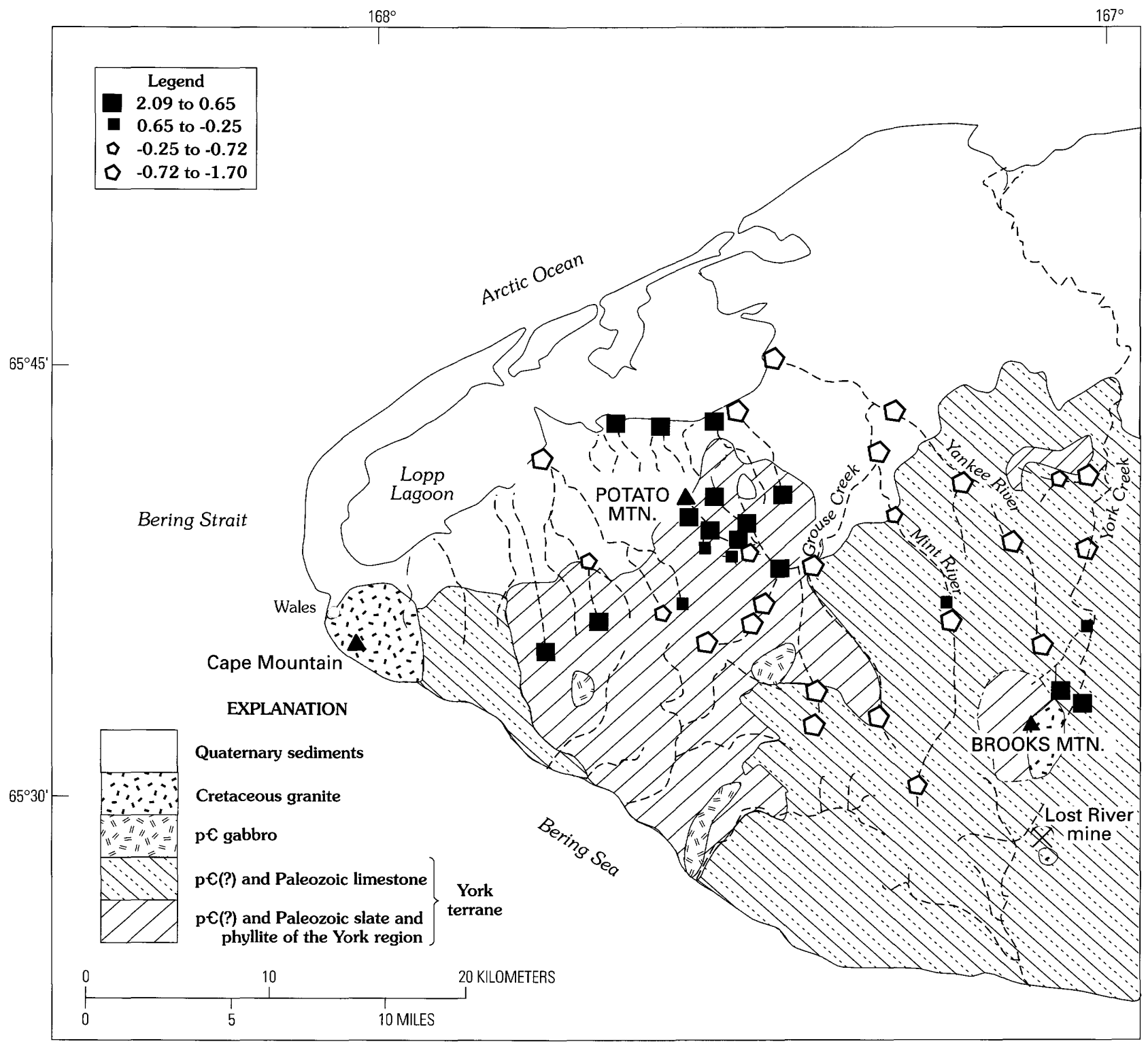

Figure 4. Plot of scores for factor 3 (positive loadings for As, Sb, Sn, and W). 
Table 3. Basic statistics for 29 heavy-mineral-concentrate samples collected from the western Seward Peninsula.

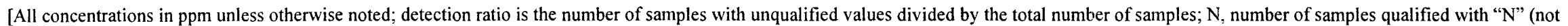
detected at lower detection limit); L, number of samples qualified with "L" (less than lower detection limit); G. number of samples qualified with "G" (greater than detection limit); geometric mean

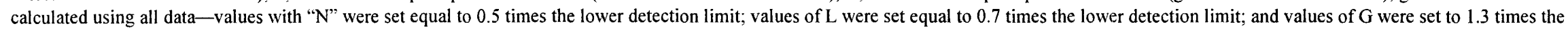
upper detection limit]

\begin{tabular}{|c|c|c|c|c|c|c|c|c|c|c|c|}
\hline Element & $\begin{array}{l}\text { Detection } \\
\text { ratio }\end{array}$ & $\mathrm{N}$ & $L$ & G & Median & Minimum & Maximum & $\begin{array}{c}\text { 85th } \\
\text { percentile }\end{array}$ & $\begin{array}{c}\text { 90th } \\
\text { percentile }\end{array}$ & $\begin{array}{c}\text { Geometric } \\
\text { mean }\end{array}$ & $\begin{array}{c}\text { Geometric } \\
\text { deviation }\end{array}$ \\
\hline $\mathrm{Ca}(\%)$ & 1 & 0 & 0 & 0 & 2 & 0.1 & 15 & 10 & 10 & 2.39 & 0.65 \\
\hline $\mathrm{Fe}(\%)$ & 1 & 0 & 0 & 0 & 0 & 0.2 & 5 & 2 & 2 & 0.94 & 0.30 \\
\hline $\mathrm{Mg}(\%)$ & 1 & 0 & 0 & 0 & 1 & 0.07 & 7 & 2 & 2 & 0.78 & 0.46 \\
\hline$P(\%)$ & 0.72 & 3 & 5 & 0 & 1 & 0.7 & 3 & 2 & 2 & 0.91 & 0.34 \\
\hline $\mathrm{Ti}(\%)$ & 0.76 & 0 & 0 & 7 & 1 & 0.03 & 2.6 & 2.6 & 2.6 & 0.90 & 0.45 \\
\hline B & 0.97 & 1 & 0 & 0 & 700 & 20 & 2000 & 1500 & 2000 & 295 & 0.11 \\
\hline $\mathrm{Ba}$ & 0.97 & 0 & 0 & 1 & 500 & 50 & 13000 & 5000 & 7000 & 705 & 0.72 \\
\hline $\mathrm{Co}$ & 0.69 & 2 & 8 & 0 & 20 & 20 & 70 & 30 & 50 & 21 & 0.67 \\
\hline $\mathrm{Cr}$ & 0.93 & 0 & 2 & 0 & 50 & 20 & 200 & 100 & 100 & 48 & 0.21 \\
\hline $\mathrm{Cu}$ & 0.93 & 0 & 2 & 0 & 15 & 15 & 100 & 50 & 50 & 20 & 0.27 \\
\hline $\mathrm{Ga}$ & 0.59 & 5 & 7 & 0 & 10 & 10 & 20 & 15 & 15 & 9 & 0.29 \\
\hline $\mathrm{La}$ & 0.55 & 2 & 4 & 7 & 200 & 100 & 2600 & 2600 & 2600 & 357 & 0.17 \\
\hline $\mathrm{Mn}$ & 1 & 0 & 0 & 0 & 500 & 50 & 700 & 500 & 700 & 325 & 0.65 \\
\hline $\mathrm{Ni}$ & 0.72 & 2 & 6 & 0 & 20 & 15 & 150 & 70 & 100 & 22 & 0.29 \\
\hline $\mathrm{Pb}$ & 0.45 & 2 & 14 & 0 & 14 & 20 & 100 & 50 & 50 & 21 & 0.35 \\
\hline $\mathrm{Sc}$ & 0.83 & 1 & 4 & 0 & 15 & 10 & 100 & 30 & 50 & 16 & 0.27 \\
\hline $\mathrm{Sn}$ & 0.66 & & & 10 & 1000 & 20 & 2600 & 2600 & 2600 & 471 & 0.77 \\
\hline $\mathrm{Sr}$ & 0.83 & 3 & 2 & 0 & 500 & 300 & 1000 & 1000 & 1000 & 480 & 0.33 \\
\hline $\mathrm{V}$ & 0.97 & 0 & 1 & 0 & 70 & 50 & 200 & 100 & 100 & 69 & 0.20 \\
\hline $\mathrm{Y}$ & 0.97 & 0 & 1 & 0 & 70 & 30 & 100 & 100 & 100 & 61 & 0.24 \\
\hline $\mathrm{Zr}$ & 1 & 0 & 0 & 0 & 300 & 50 & 2000 & 1000 & 1500 & 359 & 0.40 \\
\hline
\end{tabular}


concentrations of $\mathrm{Cu}(15-50 \mathrm{ppm}), \mathrm{Ni}(20-100 \mathrm{ppm})$, and $\mathrm{Pb}$ (20-100 ppm) when compared to the stream sediments. Two of the samples also contained 200-300 ppm tungsten.

The samples that were collected in the eastern portion of the study area, which is underlain by carbonate rocks, typically had barium concentrations of 3,000 to greater than $10,000 \mathrm{ppm}$. This is supportive of the presence of detrital barite. But, in addition, the sample collected from the stream northeast of Brooks Mountain (site 103; fig. 1) contained $500 \mathrm{ppm}$ As and $200 \mathrm{ppm}$ $\mathrm{Sn}$. The two samples from a tributary of York Creek (sites 109 and 110; fig. 1) contained greater than $2,000 \mathrm{ppm}$ Sn and detectable W $(150 \mathrm{ppm})$. These data support the existence of exposed tin-bearing occurrences in these drainage basins.

Concentrate samples with anomalous tin and arsenic values were microscopically examined for identification of associated mineral phases. Because of the highly weathered nature of the grains, it was not possible to optically determine if the dominant sulfide in the concentrates was pyrite or arsenopyrite. Emission spectrographic analyses of the sulfide grains indicate that the samples contained concentrations of greater than $20,000 \mathrm{ppm}$ As. This suggests that arsenopyrite is the dominant sulfide mineral. Metal-rich samples collected at sites in the Potato Mountain area and from streams northeast of Brooks Mountain contained cassiterite, pyrite, monazite, zircon, titanite, tourmaline, and rutile, in addition to arsenopyrite. Two of the samples also contained minor amounts of scheelite. Two samples (109 and 110; fig. 1) collected in a tributary of the York River contained cassiterite, monazite, rutile, tourmaline, sphene, and some pyrite/ arsenopyrite.
The samples that were collected in the eastern part of the study area, in the drainages underlain by argillaceous and dolomitic limestone, contained appreciable amounts of barite and zircon, and fragments of limestone and dolomite. One sample (site 12; fig. 1), collected in the upper reaches of Skookum Creek at the contact between slates and carbonates, contained 2,000 $\mathrm{ppm} \mathrm{Mo}$, which indicates an upstream molybdenite-bearing occurrence.

\section{Geochemistry of Water Samples}

Water samples were collected at 49 sites. Table 4 lists the basic summary statistics for the water samples collected during this study. Samples collected near the Lost River mine were excluded from the tabulation because they would strongly skew the data. Field $\mathrm{pH}$ measurements (table 5) indicate that most surface waters are slightly alkaline, with values ranging from 7.58.2. Conductivity, also measured in the field, varied over almost an order of magnitude, ranging between 51 and 729 micromhos/s (table 5). The lowest values reflect headwater seeps that have limited flowpaths and thus relatively little water/rock interaction (sites 02, 08, and 103; fig. 1). Analytical results for filtered water samples (Parnow and others, in press) indicate a consistent pattern of $\mathrm{Ca}>>\mathrm{Mg}>\mathrm{Na}>\mathrm{K}$ in both the areas underlain by slate and carbonate units of the York terrane. There are no appreciable differences in dissolved calcium and magnesium levels, regardless of whether the samples were collected in streams underlain by slate or limestone. This is indicative of

Table 4. Data for dissolved constituents in filtered, acidified stream-water samples collected from the western Seward Peninsula.

[All concentrations in ppb unless otherwise noted]

\begin{tabular}{ccccccccc}
\hline $\begin{array}{c}\text { Element } \\
\text { analyzed }\end{array}$ & $\begin{array}{c}\text { Detection } \\
\text { ratio }\end{array}$ & Median & Minimum & Maximum & $\begin{array}{c}\text { 85th } \\
\text { percentile }\end{array}$ & $\begin{array}{c}90 \text { th } \\
\text { percentile }\end{array}$ & $\begin{array}{c}\text { Geometric } \\
\text { mean }\end{array}$ & $\begin{array}{c}\text { Geometric } \\
\text { deviation }\end{array}$ \\
\hline $\mathrm{Ca}(\mathrm{ppm})$ & 1 & 20 & 1.8 & 84 & 38 & 46 & 18 & 0.32 \\
$\mathrm{Mg}(\mathrm{ppm})$ & 1 & 6.4 & 0.30 & 19 & 11 & 13 & 5.22 & 0.38 \\
$\mathrm{Na}(\mathrm{ppm})$ & 1 & 3.2 & 0.76 & 5.9 & 4.4 & 4.9 & 2.76 & 0.24 \\
$\mathrm{SiO}_{2}(\mathrm{ppm})$ & 0.64 & 2 & 1 & 9.6 & 4 & 5 & 1.1 & 0.33 \\
$\mathrm{~K}$ & 1 & 280 & 54 & 830 & 430 & 510 & 245 & 0.28 \\
$\mathrm{Al}$ & 0.85 & 5 & 0.83 & 1100 & 100 & 100 & 14 & 1.49 \\
$\mathrm{Ag}$ & 0.66 & 0.07 & 0.01 & 100 & 100 & 100 & 0.52 & 0.46 \\
$\mathrm{As}$ & 0.26 & 0.14 & 0.20 & 2 & 0.30 & 0.60 & 0.20 & 0.30 \\
$\mathrm{Ba}$ & 1 & 5 & 0.10 & 66 & 18 & 28 & 5.59 & 0.56 \\
$\mathrm{Co}$ & 0.62 & 0.02 & 0.01 & 8.9 & 0.07 & 0.10 & 0.03 & 0.65 \\
$\mathrm{Fe}$ & 1 & 31 & 7 & 160 & 59 & 85 & 31 & 0.33 \\
$\mathrm{Li}$ & 0.87 & 1.2 & 0.20 & 100 & 2.1 & 3.7 & 1.32 & 0.43 \\
$\mathrm{Mn}$ & 1 & 0.33 & 0.01 & 120 & 8.5 & 29 & 0.47 & 1.09 \\
$\mathrm{Mo}$ & 0.55 & 0.04 & 0.10 & 0.51 & 0.40 & 0.40 & 0.07 & 0.54 \\
$\mathrm{Ni}$ & 1 & 0.40 & 0.1 & 20 & 1.0 & 1.2 & 0.51 & 0.46 \\
$\mathrm{P}$ & 0.51 & 10 & 1 & 100 & 39 & 100 & 11.38 & 0.56 \\
$\mathrm{Rb}$ & 0.98 & 0.30 & 0.2 & 100 & 1.4 & 2.5 & 0.34 & 0.66 \\
$\mathrm{Sb}$ & 0.55 & 0.03 & 0.02 & 0.30 & 0.10 & 0.20 & 0.03 & 0.42 \\
$\mathrm{Sc}$ & 0.83 & 0.30 & 0.10 & 100 & 0.50 & 0.5 & 0.31 & 0.22 \\
$\mathrm{Se}$ & 0.53 & 0.2 & 0.2 & 0.6 & 0.3 & 0.4 & 0.22 & 0.19 \\
$\mathrm{Sr}$ & 1 & 100 & 9.2 & 320 & 170 & 210 & 83 & 0.33 \\
$\mathrm{U}$ & 0.74 & 0.04 & 0.008 & 5.80 & 1.10 & 1.30 & 0.06 & 1.05 \\
\hline
\end{tabular}


Table 5. Field-measured $\mathrm{pH}$ and conductivity for 49 streamwater samples collected from the western Seward Peninsula.

\begin{tabular}{|c|c|c|}
\hline $\begin{array}{l}\text { Site } \\
\text { no. }\end{array}$ & $\mathrm{pH}$ & $\begin{array}{l}\text { Conductivity } \\
(\mu \mathrm{S} / \mathrm{cm})\end{array}$ \\
\hline \multicolumn{3}{|c|}{ 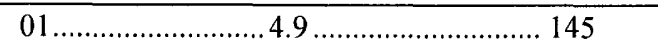 } \\
\hline \multicolumn{3}{|c|}{ 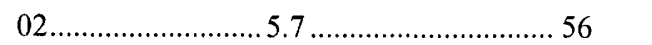 } \\
\hline \multicolumn{3}{|c|}{ 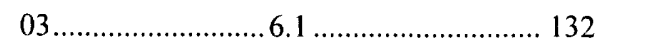 } \\
\hline \multicolumn{3}{|c|}{ 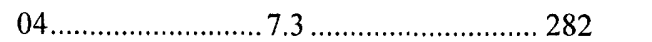 } \\
\hline \multicolumn{3}{|c|}{05} \\
\hline \multicolumn{3}{|c|}{$06 \ldots$} \\
\hline \multicolumn{3}{|c|}{ 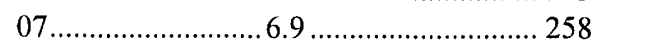 } \\
\hline \multicolumn{3}{|c|}{ 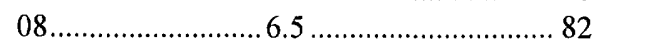 } \\
\hline \multicolumn{3}{|c|}{ 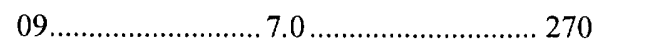 } \\
\hline \multicolumn{3}{|c|}{ 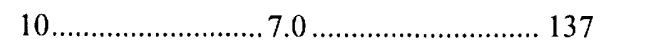 } \\
\hline \multicolumn{3}{|c|}{ 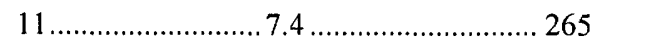 } \\
\hline \multicolumn{3}{|c|}{ 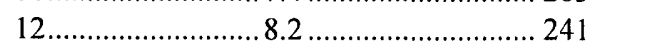 } \\
\hline \multicolumn{3}{|c|}{ 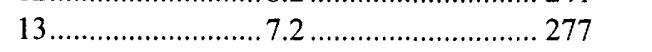 } \\
\hline \multicolumn{3}{|c|}{$14 \ldots \ldots \ldots$} \\
\hline \multicolumn{3}{|c|}{$15 \ldots \ldots$} \\
\hline \multicolumn{3}{|c|}{$16 \ldots \ldots \ldots \ldots-165$} \\
\hline \multicolumn{3}{|c|}{ 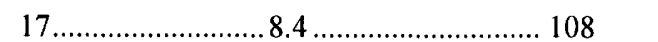 } \\
\hline \multicolumn{3}{|c|}{ 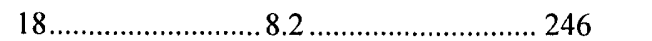 } \\
\hline \multicolumn{3}{|c|}{19} \\
\hline \multicolumn{3}{|c|}{ 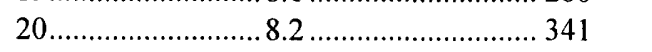 } \\
\hline \multicolumn{3}{|c|}{ 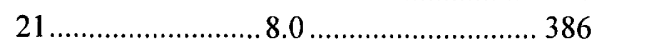 } \\
\hline \multicolumn{3}{|c|}{ 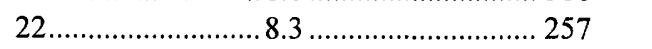 } \\
\hline \multicolumn{3}{|c|}{ 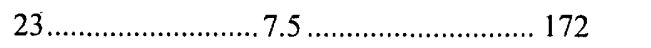 } \\
\hline 24 & ... 7.9 & $\ldots 388$ \\
\hline 25 . &.. .7 .6 & .... 231 \\
\hline 26. & $\ldots 7.2$ & .... 450 \\
\hline & $\ldots 5.5$ & ...... 99 \\
\hline 28. & $\ldots 6.7$ & $\ldots .145$ \\
\hline & ... 8.3 & $\ldots .163$ \\
\hline 33. &. .8 .0 & $\ldots .167$ \\
\hline 100 & $\ldots 6.7$ & $\ldots .322$ \\
\hline 101 & $\ldots 7.5$ & .... 243 \\
\hline 102 & $\ldots 7.4$ & .... 142 \\
\hline 103 & ... 8.7 & ...... 51 \\
\hline 104 &. .8 .4 & $\ldots 165$ \\
\hline 105 &. .8 .1 & .... 106 \\
\hline 106 & $\ldots 7.5$ & $\ldots .416$ \\
\hline 107 & ... 9.0 & ... 321 \\
\hline 108 & ... 7.9 & $\ldots .496$ \\
\hline 109 & $\ldots 7.5$ & .... 729 \\
\hline 110 & $\ldots 7.6$ & $\ldots .635$ \\
\hline 111 & $\ldots 7.8$ & ... 447 \\
\hline 112 & $\ldots 7.3$ & $\ldots .335$ \\
\hline 113 & $\ldots 7.7$ & $\ldots 190$ \\
\hline 114 & $\ldots 7.6$ & .... 159 \\
\hline 115 & $\ldots 7.4$ & $\ldots .173$ \\
\hline 116 & $\ldots 7.3$ & .... 234 \\
\hline 117 & $\ldots 4.7$ & $\ldots 165$ \\
\hline 118 & $\ldots 8.0$ & $\ldots 160$ \\
\hline
\end{tabular}

interbedded carbonates within the slates of the York terrane contributing significant amounts of calcium to dissolved loads. This is further supported by the variation of bicarbonate in the area underlain by slate (fig. $5 \mathrm{~A}$ ). Dissolved sulfate levels for waters collected in streams underlain by slates are typically in the range of $20-38 \mathrm{ppm}$, and the waters collected in the areas underlain by carbonates have sulfate concentrations $<20 \mathrm{ppm}$ (fig. $5 B$ ). Dissolved iron generally ranges between $20-40 \mathrm{ppb}$ in both areas, but most trace metals were always below analytical determination limits; these include $<0.2 \mathrm{ppb} \mathrm{As},<0.05 \mathrm{ppb} \mathrm{Be}$, $<0.02 \mathrm{ppb} \mathrm{Cd},<0.5 \mathrm{ppb} \mathrm{Cu},<10 \mathrm{ppb} \mathrm{Mn},<0.2 \mathrm{ppb} \mathrm{Mo},<1 \mathrm{ppb}$ $\mathrm{Ni},<0.05 \mathrm{ppb} \mathrm{Pb},<0.02 \mathrm{ppb} \mathrm{Sb},<0.05 \mathrm{ppb} \mathrm{Sn}$, and $<3 \mathrm{ppb} \mathrm{Zn}$.

Two water samples were collected at the Lost River mine, located south of the study area. Samples were collected from a small seep at the base of the main tailings piles (site 33; fig. 1) and from Lost River, immediately downstream from the mine workings (site 32; fig. 1). Resulting analytical data provided geochemical signatures characteristic of waters that have interacted with tin-rich mineralized rock typical of the western Seward Peninsula. Conductivity values were at background levels (167 and 163 micro-mhos/s) and were comparable to those from elsewhere in the study area. The carbonate host rock buffered the water such that the $\mathrm{pH}$ 's were alkaline despite the abundance of sulfide minerals in the tailings. The seep in the tailings, despite the alkaline $\mathrm{pH}$, does show significant enrichment of metals when compared to the waters collected from the areas of known, but undeveloped, occurrences in the Potato Mountain area. However, all absolute concentrations remain extremely low. The filtered water sample from site 33 (fig. 1) contained only $3.2 \mathrm{ppm}$ sulfate, but $180 \mathrm{ppb} \mathrm{As}, 90 \mathrm{ppt} \mathrm{Cd}, 0.7 \mathrm{ppb} \mathrm{Cu}, 8.9$ ppb Mo, $1.5 \mathrm{ppb} \mathrm{Sb}, 0.4 \mathrm{ppb} \mathrm{W}$, and $10 \mathrm{ppb} \mathrm{Zn}$. A large volume of suspended material in the water is indicated by elevated concentrations of metals in the unfiltered samples from the same site (4.4 ppm Al, 2.5 ppm Fe, 470 ppb As, 3.1 ppb Be, 2.7 ppb Cd, 38 ppb Cu, 420 ppb Mn, 5.5 ppb Mo, 2.2 ppb Ni, 270 ppb Pb, 6.2 ppb Sb, 8 ppb Sn, 23 ppb Ti, and 600 ppb Zn)

Most of the water samples collected in the drainages surrounding Potato Mountain contained background concentrations of all metals, including the same metals that were anomalous in sediments collected from these sites. However, waters at sites 04-07 and 116-117 (fig. 1) showed enrichments in As (0.2-0.7 $\mathrm{ppb})$ and $\mathrm{Sb}(0.2-0.3 \mathrm{ppb})$ concentrations, both which are indicator elements for tin deposits. Also, samples collected at sites 01 and 117, located just north of Potato Mountain (fig. 1) were acidic ( $\mathrm{pH} 4.85$ and 4.73 , respectively) and had relatively high concentrations of dissolved $\mathrm{Al}(1,100$ and $360 \mathrm{ppb}), \mathrm{Cu}$ ( 2 and 6 ppb), Mn (120 and $71 \mathrm{ppb}$ ), and Ni (20 and $12 \mathrm{ppb})$. Sites 01 and 117 had very low alkalinity ( 3.3 and $1.0 \mathrm{ppm}$ ) relative to the other samples collected near Potato Mountain, signifying a decrease in the buffering ability of the water. This may be due to weathering of sulfides, which would decrease the $\mathrm{pH}$. However, there was no significant increase in dissolved iron, although sample 117 did have measurable arsenic $(0.7 \mathrm{ppb})$. Dissolved sulfate concentrations ranged between $40-75 \mathrm{ppm}$ in the waters collected from areas of known tin occurrences. Increased sulfate concentrations may be the best hydrogeochemical indicators of the Potato Mountain tin-bearing occurrences.

The sites located in the carbonate-dominated unit of the York terrane, along the eastern margin of the studied area that had anomalous arsenic and tin in stream sediments and heavymineral concentrates, also contained slightly elevated metal concentrations in water samples. Sites 109 and 110, located within the York Creek watershed (fig. 1), had the highest conductivities of any samples (729 and 635 micro-mhos/s), but $\mathrm{pH}$ was slightly alkaline (7.5 and 7.6). Relatively high concentrations of Fe (85-160 ppb) and Mg (13-19 ppm) at these sites 


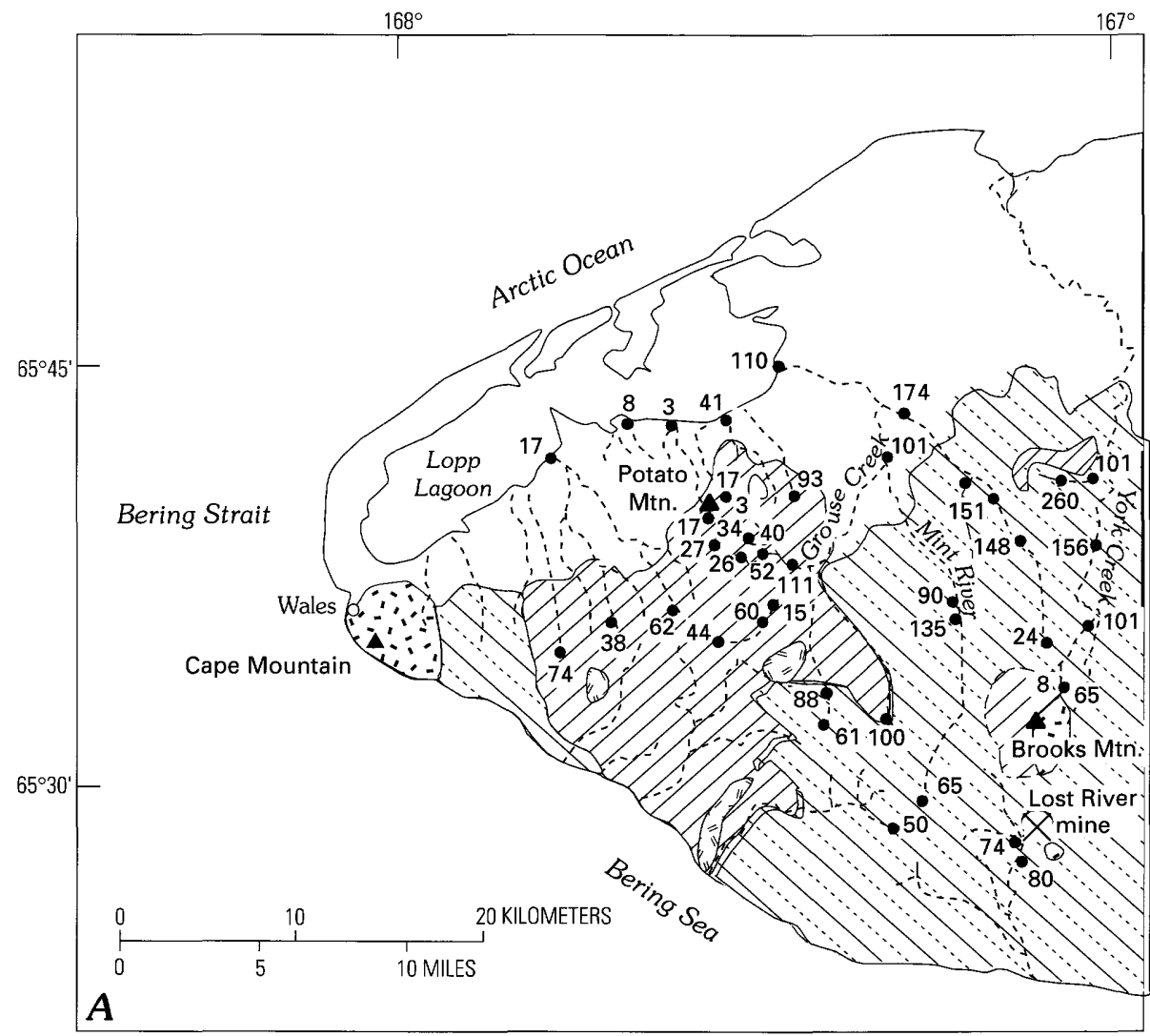

EXPLANATION
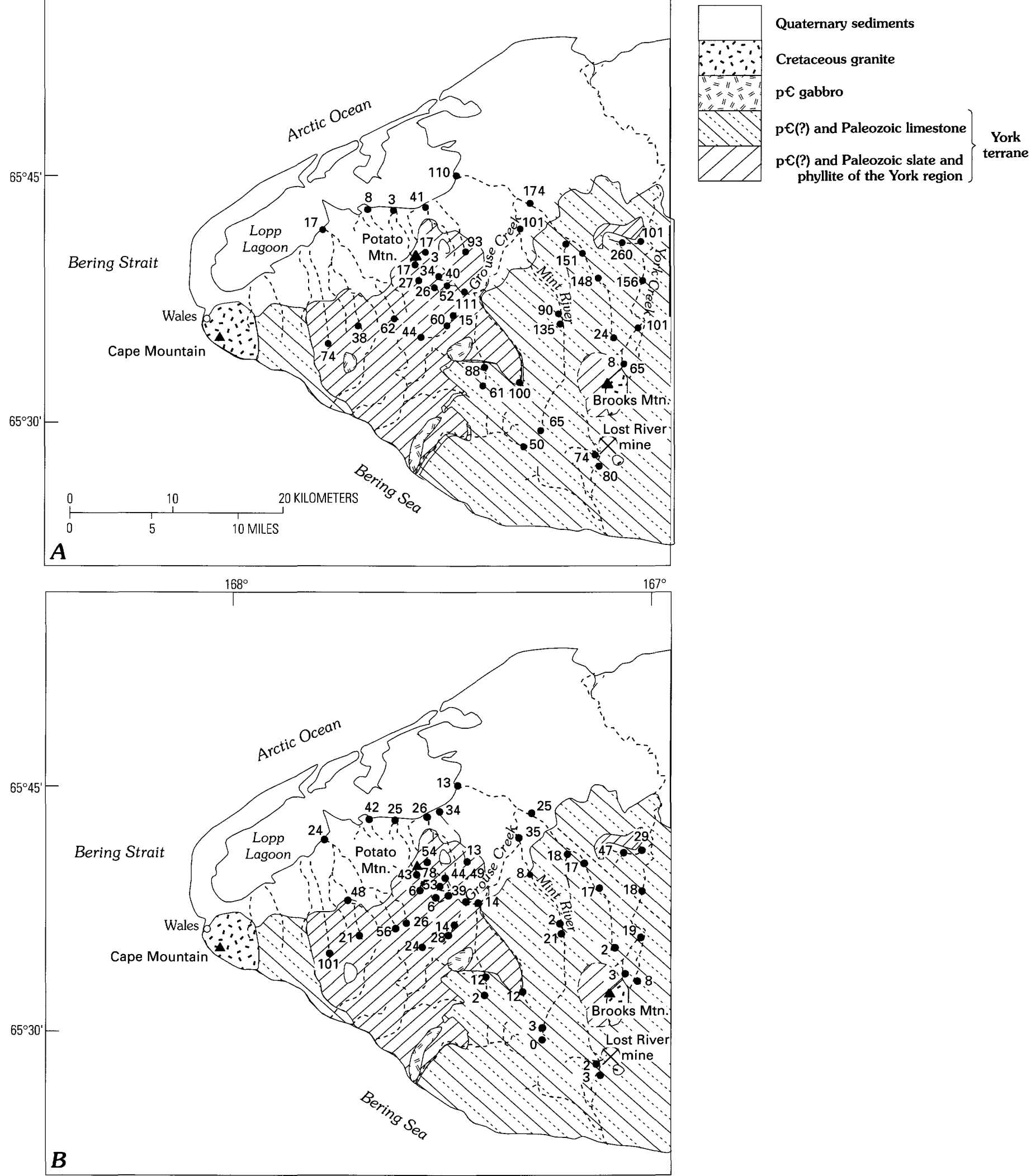

Figure 5. A, Concentrations of bicarbonate in ppm in filtered water samples. $B$, Concentrations of dissolved sulfate in ppm in filtered water samples.

could be due to lithologic variations, representative of waterrock interaction with both the slates and the carbonates, as there is a small outcrop of slate to the north of the two sites (fig. 1).
However, slight enrichments in dissolved tin $(0.1 \mathrm{ppb}$ at site $110)$ and arsenic $(0.5 \mathrm{ppb}$ at site 109$)$ are consistent with a tinmineralized source. Sites 103 and 104, in the headwaters of 
York Creek (fig. 1) and also characterized by previously described anomalies in other sample media, contained 1-2 ppb dissolved arsenic and $0.2 \mathrm{ppb}$ dissolved tin.

Six samples were collected from the mouths of streams at Lopp Lagoon. The waters had a broad variation in $\mathrm{pH}$ ranging from 5.5-8.3. Five of the six samples were collected from streams draining Potato Mountain, and that from site 22 was collected at the mouth of the Mint River. Sites 27, 28, and 118 were sulfate-dominated waters, whereas sites 22, 23, and 112 (fig. 1) were bicarbonate-dominated waters. The sulfate-dominated waters may indicate greater input from waters that have interacted with the tin occurrences and their associated sulfide minerals. Site 27 had the lowest $\mathrm{pH}$ (5.53) and had corresponding high concentrations of dissolved cations ( $99 \mathrm{ppb} \mathrm{Al}, 47 \mathrm{ppb} \mathrm{Mn}, 8.3$ ppb Ni, and $8 \mathrm{ppb} \mathrm{Zn}$ ) and detectable levels of As ( $0.3 \mathrm{ppb})$. Sites 27 and 112 contained elevated concentrations of Fe (110$150 \mathrm{ppb}$ ) and $\mathrm{Mn}(47$ and $29 \mathrm{ppb}$ ). The elevated iron and manganese at these two sites compared to the rest of the study area may be due to particulates in the water that are smaller than the 0.45 $\mu \mathrm{m}$ filter that was used. The increased particulates may be due to greater biologic activity in the lower reaches of the stream, which increases respiration and causes oxidation of the sediments. This is further supported by the total iron concentrations at these sites, which are an order of magnitude greater than the average iron concentration $(250-280 \mathrm{ppm}$, avg $=64 \mathrm{ppm})$. Only two of the water samples (sites 23 and 27) collected at the mouths of the streams contained detectable arsenic concentrations $(0.3 \mathrm{ppb})$. These sites also contained the highest arsenic concentrations $(78 \mathrm{ppm})$ in the stream sediments collected from near Lopp Lagoon.

\section{Discussion}

An understanding of the distribution of trace metals in the environment along the western Seward Peninsula is critical for a number of reasons. The tin granites and their associated mineral occurrences represent a potentially significant metal source that is directly eroded into Lopp Lagoon and the Bering Sea. Furthermore, geophysical evidence suggests that these metalliferous intrusive systems continue beneath the Bering Strait (Rick Saltus, oral commun., 1997). If correct, then additional mineralized material is exposed across this shallow part of the Bering Sea. Metals such as $\mathrm{Cd}$, As, and $\mathrm{Hg}$ have been recognized at elevated levels in marine mammals off Alaska's west coast (for example, see Taylor and others, 1989; Becker and others, 1995). This may simply be natural biomagnification of such metals by the large mammals, but these high metal concentrations may, in part, reflect more local contributions from metalliferous locations on the western Seward Peninsula. Also of concern is whether metals are being enriched in plants or in fish in Lopp Lagoon, both of which the villagers of Wales depend on as a food source.

The first step in identifying any potential environmental problems within the local ecosystem is to determine background levels for the metals. Arsenic and tin appear to be the elements with the greatest natural fluctuation in this studied area of western Alaska. Our stream-sediment data identify natural arsenic levels in stream sediments of as much as $530 \mathrm{ppm}$ downstream from known, but undeveloped, mineral occurrences in the higher elevations of Potato Mountain. To the east, concentrations of about $100 \mathrm{ppm}$ As characterize streams draining Brooks Mountain, and stream sediments with as much as $72 \mathrm{ppm}$ As occur in tributaries of lower York Creek. The former anomalies are also associated with known mineral occurrences, whereas the latter suggest the presence of exposed, but unrecognized, occurrences. To the west, in the lower elevations approaching the village of Wales, a concentration of $95 \mathrm{ppm}$ As was determined at site 114 (fig. 1), also distal from any recognized tin-bearing occurrences. The data collected in 1997 were not able to duplicate the NUREreported concentration of $635 \mathrm{ppm}$ As for stream sediments just upstream from Lopp Lagoon, but concentrations of as much as $78 \mathrm{ppm}$ As confirm that significant amounts of arsenic are reaching the lagoonal muds.

Tin, while not usually an element of environmental concern, has been found in elevated concentrations (19-35 ppm dry weight) in the livers and kidneys of walruses harvested from the Bering Sea (Warburton and Seagars, 1993). The average concentration of tin in most rock types ranges between 1 and $3 \mathrm{ppm}$ (Levinson, 1980), and sediments eroded from such rocks will typically have similar tin concentrations. Due to the tin-rich alteration of rocks throughout this part of the Seward Peninsula, resulting sediments from our studied area consistently contain greater than $5 \mathrm{ppm} \mathrm{Sn}$. Highest concentrations are again in sediments on Potato Mountain, with measured concentrations of as great as $62 \mathrm{ppm}$, and below Brooks Mountain with $22 \mathrm{ppm}$. All six sediment samples collected along Lopp Lagoon contained 7$14 \mathrm{ppm} \mathrm{Sn}$, indicating that detrital tin is likely accumulating along this part of the coastline. In addition, $15 \mathrm{ppm}$ Sn characterizes sediments from site 114 in the western part of our studied area (fig. 1).

Other elements that are commonly enriched in the tinbearing occurrences, such as $\mathrm{Ag}, \mathrm{Cu}, \mathrm{F}, \mathrm{Pb}, \mathrm{W}$, and $\mathrm{Zn}$, are not anomalous in stream sediments of the western Seward Peninsula, excluding the anthropogenic enrichments below the large Lost River mine tailings pile. Beryllium, a signature element for many of the tin occurrences in the Seward Peninsula, rarely shows significant enrichment in stream sediments. A few samples from the study area contained 1-2 ppm Be, but the remainder contained less than $1 \mathrm{ppm}$ Be. All samples contained less than $0.3 \mathrm{ppm} \mathrm{Hg}$, and all except one sample contained less than $0.8 \mathrm{ppm} \mathrm{Cd}$. The only sample with a greater cadmium concentration (1.6 ppm) was collected at site 103 below Brooks Mountain (fig. 1).

Extremely low concentrations of dissolved trace elements characterize all waters from the study area. Many trace elements become slightly more soluble in the acidic surface flow ( $\mathrm{pH} 5-6$ ) near the top of Potato Mountain. Dissolved species in the Potato Mountain samples include $1.1 \mathrm{ppm} \mathrm{Al,} 2 \mathrm{ppb}$ As, $200 \mathrm{ppb}$ Be, $300 \mathrm{ppb} \mathrm{Cd}, 6 \mathrm{ppb} \mathrm{Cu}, 71 \mathrm{ppb} \mathrm{Mn}, 20 \mathrm{ppb} \mathrm{Ni}, 300 \mathrm{ppb} \mathrm{Sb}$, and $10 \mathrm{ppb} \mathrm{Zn}$. Similar concentrations of these elements also characterize acidic streamflow just above Lopp Lagoon at site 27. The reason for the $\mathrm{pH}-5.5$ water at this low elevation, far from any exposed bedrock, is uncertain. Perhaps it identifies (a) a large influx of ground water from higher elevation recharge zones to the south, or (b) a possible acidic input from the breakdown of organics. Many of the unfiltered water samples from throughout the study area contain 2-5 times more iron than filtered water samples from the same sites. Concentrations of 100-250 ppb Fe in these unfiltered waters reflect minor amounts of colloidal 
oxyhydroxides. Arsenic is not soluble at the $\mathrm{pH}$ range of our study area, but may adsorb onto oxyhydroxides. However, the data shows no correlation between increased total iron and increased arsenic.

\section{Conclusions}

Results from our study indicate that solution transport of metal phases from the tin-bearing occurrences of the western Seward Peninsula is extremely insignificant. Furthermore, acidic water drainage in this area poses little problem due to the natural buffering of waters by carbonate units of the York terrane. However, detrital transport of tin- and arsenic-enriched mineral grains is significant, as is indicated by relatively high concentrations of the elements in stream sediments surrounding Potato Mountain. Enrichments in sediments are traceable as far north as the edge of Lopp Lagoon. Future study should examine any further dispersion through the local ecosystem, such that arsenic or tin enrichments can be traced into lagoonal muds, fish, and seals within the lagoon or into local plant species harvested by the villagers of Wales. Other inorganic contaminants that may be of environmental concern along the Bering Sea, such as mercury and cadmium, do not have any obvious point sources within our studied area.

\section{Acknowledgments}

We would like to thank the following people for their support: Toby Anungazuk of the Wales Native Corporation, Wales, Alaska; Dale Taylor, Laurie Balistrieri, Barrett Cieutat, Pete Theodorakis, Steve Sutley, Al Meier, and Rich Wanty of the USGS.

\section{References Cited}

Arbogast, B.F., ed., 1996, Analytical methods manual for the Mineral Resource Surveys Program of the United States Geological Survey: U.S. Geological Survey Open-File Report 96-525, 184 p.

Becker, P., Mackey, E.A., Demiralp, R., Koster, B.J., Greenburg, R.R., Wise, S.A., and Muir, D.C.G., 1995, Concentrations of chlorinated hydrocarbons, heavy metals, and other elements in tissues banked by the Alaska marine mammal tissues archive project: National Institute of Standards and Technology, NISTIR 5620, 80 p.

Bundtzen, T.K., Swainbank, R.C., Clough, A.H., Henning, W.W., and Hansen, E.W., 1994, Alaska's mineral industry 1993: Alaska Division of Geological and Geophysical Surveys Special Report 48, 84 p.

Crock, J.G., Lichte, F.E., and Briggs, P.H., 1983, Determination of elements in National Bureau of Standards' Geological Reference Materials SRM278 Obsidian and SRM 688 Basalt by inductively coupled argon plasma-atomic emission spectrometry: Geostandards Newsletter, v. 7, p. 335-340.

d'Angelo, W.M., and Ficklin, W.M., 1996, Fluoride, chloride, fluoride, nitrate, and sulfate in aqueous solution by chemically suppressed ion chromatography, in Arbogast, B.F., ed., Analytical Methods Manual for the Mineral Resource Surveys Program of the United States Geological Survey: U.S. Geological Survey Open-File Report 96-525, p. 149-153.

Grimes, D.J., and Marranzino, A.P., 1968, Direct-current arc and alternating-current spark emission spectrographic field methods for quantitative analysis of geologic materials: U.S. Geological Survey Circular 591, 6 p.

Hoffmann, J.D., and Buttleman, K., 1996, National geochemical database: 1. National Uranium Resource Evaluation (NURE) hydrogeochemical and stream sediment reconnaissance (HSSR) data for Alaska: U.S. Geological Survey Digital Data Series DDS-18-B, CD-ROM.

Hudson, T.L., and Arth, J.G., 1983, Tin granites of Seward Peninsula, Alaska: Geological Society of America Bulletin, v. 94, p. 768-790.

Hudson, T.L., and Reed, B.L., 1997, Tin deposits in Alaska: Economic Geology Monograph 9, p. 450-465.

Koch, G.S., Jr., and Link, R.F., 1970, Statistical analysis of geological data: New York, Dover Publications, $438 \mathrm{p}$.

Levinson, A.A., 1980, Introduction to exploration geochemistry: Wilmette, Illinois, Applied Publishing, $613 \mathrm{p}$.

Meier, A.L., Grimes, D.J., and Ficklin, W.H., 1994, Inductively coupled plasma-mass spectrometry-A powerful analytical tool for mineral resource potential and environmental studies [abs.], in Carter, L.M.H., Toth, M.I., and Day, W.C., eds., U.S. Geological Survey Research on Mineral Resources-1994, Part A-Program and Abstracts, V. E. McKelvey Forum on Mineral and Energy Resources, 9th, Tucson, Ariz., February 22-25, 1993: U.S. Geological Survey Circular 1103-A, p. 67-68.

Motooka, J.M., 1996, Organometallic halide extraction for 10 elements by inductively coupled plasma-atomic emission spectrometry, in Arbogast, B.F., ed., Analytical methods manual for the Mineral Resource Surveys Program of the United States Geological Survey: U.S. Geological Survey Open-File Report 96-525, p. 102-108.

Parnow, C.C., Kelley, K.D., Goldfarb, R.J., Meier, A.L, Sutley, S.J, and Theodorakos, P.M., in press, Analytical results of stream sediment, heavy-mineral concentrates, and water data collected from northwestern Seward Peninsula, Alaska: U.S. Geological Survey OpenFile Report.

Pearce, F., 1997, Why is the apparently pristine Arctic full of toxic chemicals that started off thousands of kilometers away?: New Scientist, v. 29 , no. 2, p. 24-27.

Sainsbury, C.L., 1963, Beryllium deposits of the western Seward Peninsula, Alaska: U.S. Geological Survey Circular 479, 18 p.

Sainsbury, C.L., 1972, Geologic map of the Teller quadrangle, western Seward Peninsula, Alaska: U.S. Geological Survey Miscellaneous Investigations Series Map I-685, 4 p., scale 1:250,000.

Sainsbury, C.L., 1988, Vertical and horizontal zoning from tin to beryllium deposits, Lost River district, Alaska, in Kisvarsanyi, G., and Grant, S.K., eds., North American Conference on Tectonic Control of Ore Deposits and the Vertical and Horizontal Extent of Ore Systems, Proceedings Volume: University of Missouri-Rolla, p. 80-91.

Sainsbury, C.L., Hamilton, J.C., and Huffman, C., Jr., 1968, Geochemical cycle of selected trace elements in the tin-tungsten-beryllium district, western Seward Peninsula, Alaska-A reconnaissance study: U.S. Geological Survey Bulletin 1242-F, 41 p.

Swanson S.E., Newberry, R.J., Coulter, G.A., and Dyehouse, T.M., 1990, Mineralogical variation as a guide to the petrogenesis of the tin granites and related skarns, Seward Peninsula, Alaska: Geological Society of America Special Paper 246, p. 143-159.

Taylor, D., Schliebe, S., and Metsker, H., 1989, Contaminants in blubber, liver, and kidney tissues of Pacific walruses: Marine Pollution Bulletin, v. 20 , no. 9 , p. $465-468$.

Till, A.B., and Dumoulin, J.A., 1994, Geology of the Seward Peninsula and Saint Lawrence Island, in Plafker, G., and Berg, H.C., eds., The Geology of Alaska: Boulder, Colo., Geological Society of America, The Geology of North America, v. G-1, p. 141-152. 
Warburton, J., and Seagars, D., 1993, Metal concentrations in liver and kidney tissues of Pacific walrus: Continuation of a baseline study: U.S. Fish and Wildlife Service Technical Report R7/MMM 93-1, 23 p.
West, W.S., and White, M.G., 1952, The occurrence of zuenerite at Brooks Mountain, Seward Peninsula, Alaska: U.S. Geological Survey Circular 214, $7 \mathrm{p}$.

Reviewers: Helen W. Folger, James G. Crock. 



\title{
Major-Element, Trace-Element, and Strontium-Isotope Systematics of Natural Waters in the Fairbanks Mining District: Constraints from Local Geology
}

\author{
By Richard J. Goldfarb, G. Lang Farmer, Barrett A. Cieutat, and Allen L. Meier
}

\section{Abstract}

The chemical characteristics of natural waters were studied over a large area north of Fairbanks, Alaska, within the YukonTanana terrane. Waters dominated by calcium, bicarbonate, and sulfate from seeps and streams within areas underlain by rocks of the Fairbanks Schist typically had $\mathrm{pH}$ levels of 7.2-8.2, conductivities of 100-200 $\mu \mathrm{S} / \mathrm{cm}$, and concentrations of 13-20 ppm $\mathrm{Ca}, 4-7 \mathrm{ppm} \mathrm{Mg}, 0.7-1.2 \mathrm{ppm} \mathrm{K}$, and 8-36 ppm sulfate. In contrast, waters that interacted with mid-Cretaceous granitoids intruding the schist were characterized by more acidic $\mathrm{pH}$ 's of 6.8-7.1, much lower conductivities, and $<9 \mathrm{ppm} \mathrm{Ca}, 0.6-0.7$ $\mathrm{ppm} \mathrm{Mg}, 0.3-0.4 \mathrm{ppm} \mathrm{K}$, and $\leq 2 \mathrm{ppm}$ sulfate. These waters were also enriched in dissolved $\mathrm{Si}, \mathrm{F}^{-}$, and $\mathrm{U}$ relative to those in contact with schist. Dissolution of relatively soluble $\mathrm{Ca}-\mathrm{Mg}$ silicates in eclogite, the dominant lithology of the Chatanika assemblage, were responsible for highly alkaline surface waters that contain as much as $61 \mathrm{ppm} \mathrm{Ca}, 22 \mathrm{ppm} \mathrm{Mg}, 2.6 \mathrm{ppm} \mathrm{K}$, and $140 \mathrm{ppm}$ sulfate. Strontium isotopic ratios varied from 0.7146 to 0.7182 for waters in contact with granites to $0.7282-0.7364$ for those in contact with schist; those draining eclogite were intermediate to these two ranges. Ground and surface waters that had contacted eolian overburden have less predictable hydrogeochemical signatures.

\section{Introduction}

Fairbanks is Alaska's second largest city, with a 1997 population of about 80,000 . Many inhabitants rely on ground water from private domestic wells for their drinking supply. Therefore, background hydrogeochemical data for surface and ground water in the Fairbanks region are critical for land-use planning, regulatory considerations, and prioritization. In the mid-1970's, extremely high concentrations of dissolved arsenic were found to be present locally near Fairbanks, both in ground water near Ester dome and in a belt extending northeast along the Goldstream Creek valley (fig. 1A). Dissolution of widespread arsenopyrite, and its oxidation product scorodite, within many aquifers was shown to contribute to natural background concentrations of dissolved arsenic in ground water at as much as $10 \mathrm{ppm}$ (Hawkins and others, 1982), 200 times greater than the $50 \mathrm{ppb}$ maximum contaminant level recommended by the State of Alaska (Alaska Department of Environmental Conservation, 1996). This has caused many homeowners in affected areas to use distillation, reverse osmosis, or activated alumina to remove much of the arsenic

Despite the abundance of information on dissolved arsenic content, published data for other low-level trace metals in ground water across the area are relatively uncommon. Some multielement hydrogeochemical data have been recently collected from areas proximal to specific ore deposits, due to renewed interest in lode gold mining in the Fairbanks area. For example, Fairbanks Gold Mining, Inc., has collected a wealth of such data from wells and streams surrounding the Fort Knox gold mine (i.e., Fairbanks Gold Mining, Inc., Water Quality Data, 1992-1994, unpub. company report). In addition, government agencies have begun to collect background data for waters surrounding active placer (Ray and others, 1992) and abandoned lode (Goldfarb and others, 1997) gold mines. However, as additional commercial and residential development takes place in the Fairbanks area, detailed hydrogeochemical data will be needed for areas other than those directly adjacent to present or past mining activities.

The aim of this investigation is to obtain major- and traceelement hydrogeochemical and $\mathrm{Sr}$ isotopic data that will serve as a baseline for Fairbanks ground and surface waters. In addition, these data will provide constraints on the source lithologies of specific ground-water chemical characteristics (e.g., high arsenic content). Such information will be important for future assessments of water supply and quality in the Fairbanks area, as well as for human health studies. Furthermore, bedrock exposures in central Alaska are limited, due to low relief and thick loess cover, and, as a result, the subsurface geology of the region is difficult to constrain. However, because the chemical and isotopic compositions of the ground waters are mainly a function of the underlying geology and the amount of water-rock interaction, hydrogeochemical signatures in the region could help identify unexposed rock units, including felsic igneous rocks that are spatially associated with many of the Fairbanks gold deposits. 

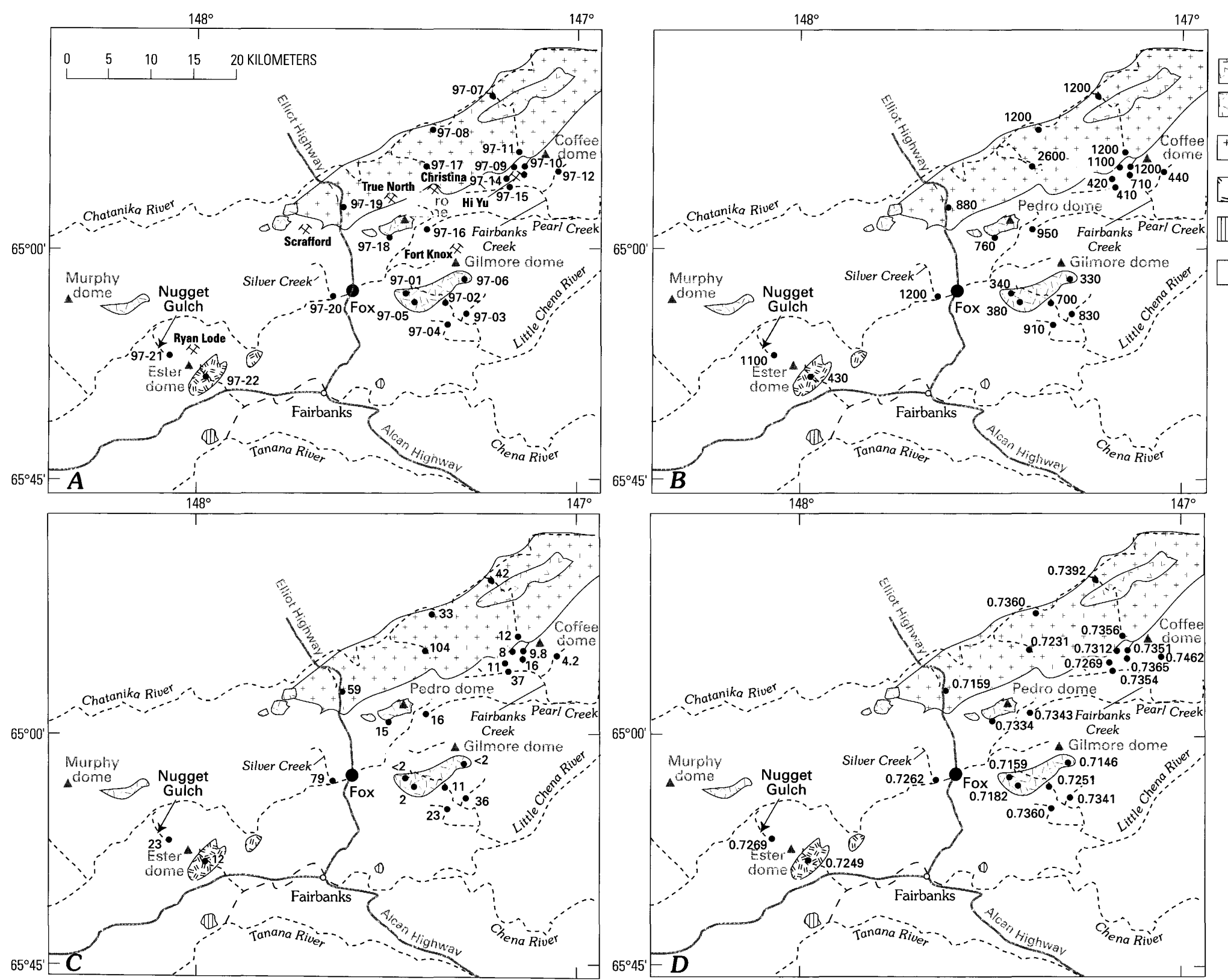

EXPLANATION

Tertiary basalt

Mid-Cretaceous granite

Devonian-Mississippian Chatanika assemblage

\section{Late Devonian}

Muskox Sequence

Devonian Birch

\section{Hill Sequence}

Proterzoic Fairbanks

Schist locally intruded

by Mississippian

orthogneiss

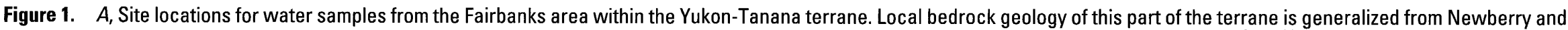

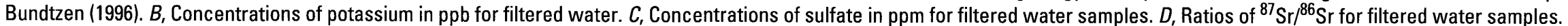




\section{Regional Geology}

The Fairbanks area (fig. $1 A$ ) is underlain by metamorphosed rocks of the Yukon-Tanana terrane, which have been described in detail most recently by Robinson and others (1990) and Newberry and Bundtzen (1996). The area's structurally highest rocks, belonging to the more northerly Chatanika assemblage, include Devonian to Mississippian quartzite, schist, marble, and amphibolite. A calc-silicate mineral assemblage is common throughout these oceanic rocks, which have been regionally metamorphosed to eclogite facies. The underthrust parautochthonous metasedimentary rocks of the Proterozoic Fairbanks Schist are mainly composed of quartzite and quartz-muscovite schist, with lesser amphibolite and rare marble. They have been locally intruded by Mississippian granodioritic orthogneiss. The entire Fairbanks Schist package was metamorphosed to amphibolite facies at the end of the Paleozoic, followed by greenschist-grade retrograde metamorphism in the Early Cretaceous (Newberry and Bundtzen, 1996). A few local lithologic sequences have been distinguished in fault contact with the Fairbanks Schist near the Fairbanks city limits. Greenschist-facies phyllites of the Ordovician(?) to Devonian Birch Hill Sequence crop out along the northern and western borders of the city limits. Amphibolite-facies metavolcanic rocks and schists of the Upper Devonian Muskox Sequence are mainly recognized about $10 \mathrm{~km}$ west of the city.

Mid-Cretaceous intrusive bodies and early Tertiary olivine basalts are scattered throughout the Chatanika assemblage and the Fairbanks Schist (Robinson and others, 1990; Newberry and Bundtzen, 1996). The intrusive rocks are granitic, granodioritic, or tonalitic in composition; range in age from 94-88 Ma; and comprise the topographic domes that are responsible for much of the local relief (e.g., Pedro, Ester, and Gilmore domes). Many of the epigenetic gold-bearing quartz veins and stockworks of the Fairbanks mining district are spatially associated with these igneous bodies. The olivine basalts are tholeiitic in composition, range in age from about 56-50 $\mathrm{Ma}$, and represent subaerial and lacustrine extrusions (Newberry and Bundtzen, 1996).

More than 8 million ounces of gold have been recovered historically from the placer fields of the Fairbanks region. More recently, low-grade bulk-tonnage deposits, the probable source for much of the alluvial gold, have become important new mining targets in the district. Mining has commenced at the Fort Knox deposit (>7 million oz gold), and additional large targets exist at the True North and Ryan Lode properties. Deposits are hosted by porphyritic granitic bodies (i.e., Fort Knox), the Fairbanks Schist (i.e, Hi-Yu, Christina, and Scrafford), and the Chatanika assemblage (i.e., True North). In addition to gold, most deposits contain an average of one percent arsenopyrite, variable pyrite, common stibnite, and local minor scheelite, molybdenite, pyrrhotite, loellingite, sulfosalts, and base-metal sulfides (McCoy and others, 1997). Consequently, hydrothermally altered rocks are irregularly enriched in $\mathrm{Fe}, \mathrm{As}$, and (or) $\mathrm{Sb}$, with grab samples of sedimentary rocks commonly containing many tens to hundreds of parts-per-million arsenic and antimony and finely disseminated sulfides (Metz, 1991). The widespread sulfidation of all lithologic units within the Fairbanks area is of concern with regard to ground-water quality, given that oxidation of the sulfide minerals can result in production of acidic waters with high contents of arsenic and other metals.

\section{Methods}

\section{Description of Studied Sites}

We visited 22 sites within the Fairbanks area between July $1-4,1997$. Weather during this period was exceptionally warm and dry, except for a few brief thunderstorms. Sites were selected such that samples would be representative of waters in contact with various lithologic units within the Fairbanks region (fig. 1A). Our sampling was restricted to ground-water seeps and stream headwater regions north and east of Fox and, to a lesser extent, near Ester dome. We did not sample the lower elevations along the Goldstream Creek valley because private wells in this location had been already sampled by previous workers such as Hawkins and others (1982) and Weber (1986). Also, we wanted to develop a better understanding of the more regional hydrogeochemical patterns over a broader region north of Fairbanks. Resulting data could then be compared with the arsenic-rich ground waters already recognized along the Goldstream Creek valley.

Because the Fairbanks Schist composes much of the bedrock geology in the area, about half of the selected sites were from areas partly or totally underlain by this unit. Discharge derived from the metasedimentary schist and metavolcanic amphibolite of the Fairbanks Schist (Newberry and Bundtzen, 1996) was not distinguished because these two lithologies are complexly interbedded throughout the study area. Five samples were collected from streams within the Chatanika assemblage (sites 97-07, -08, -11, -17, -19); one of these also partly drains Tertiary basalt (site 97-07). Samples from sites 97-01, -05, and -06 represent flow discharging from areas exclusively underlain by mid-Cretaceous granites, whereas five other sites in the Fairbanks Schist (sites 97-02, -03, -04, -18, -21) contain some granite outcrops upstream from the sample locations. Site 97-16 was selected because waters there exclusively drain Mississippian orthogneiss intruding the Fairbanks Schist. Sample 97-22 is representative of waters interacting solely with rocks of the Muskox Sequence.

Whenever possible, we attempted to place sites as high in elevation as possible within a given catchment. Therefore, many of the samples are essentially springs, located by tracing flowing streams up to their headwaters where the initial discharge was seeping from the ground. In other cases, we collected samples farther downstream either because of difficulty of access (sites 97-08, 97-18 to 97-22), or to examine the impact on the water compositions of changes in rock composition within a given basin ( sites 97-02 to 97-04, -07, -13, -15). In all of these cases, sample sites were located on the upstream side of access roads.

\section{Field Methods}

Water temperature, $\mathrm{pH}$, and conductivity were measured at each of the sampled sites (table 1) using standard meters and 
probes, and calibrating instruments at each site. Three filtered water samples were collected in polyethylene bottles at each location using a $0.45-\mu \mathrm{m}$ filter and a portable battery-operated pump. An acid-rinsed 60-mL bottle was filled with filtered water for dissolved-cation analyses, and the sample was acidified in the field to a $\mathrm{pH}<2$ with ultrapure concentrated nitric acid. An unacidified, filtered $125-\mathrm{mL}$ sample was collected for determination of anions. A 1-L acid-rinsed bottle was filled with sample for isotopic analysis, and it too was acidified with ultrapure concentrated nitric acid. All samples were kept cool with ice until they were returned to Denver, where they were then refrigerated prior to analysis.

\section{Analytical Methods}

Concentrations for more than 60 trace, minor, and major elements were determined by inductively coupled plasma-mass spectrometry (ICP-MS) using the methodology of Meier and others (1994). This analytical method is characterized by analytical determination limits down to the parts-per-trillion level for most elements. Laboratory quality control was confirmed by analysis of one duplicate sample for every ten samples, as well as a number of U.S. Geological Survey Water Resources Division quality-control water standards. Anions were determined by ion chromatography as described in d'Angelo and Ficklin (1996). Alkalinity as $\mathrm{CaCO}_{3}$ was measured in the laboratory by titration. These cation and anion data are shown in tables 2 and 3 , respectively.

For the $\mathrm{Sr}$ isotopic analyses, five 50-mL aliquots of each water sample were dried in a Teflon beaker in a class-100 cleanroom environment, and the residues were passed through conventional cation-exchange columns to separate Sr. Total procedural blanks for $\mathrm{Sr}$ were approximately $1 \mathrm{ng}$, with ${ }^{87} \mathrm{Sr} /$ ${ }^{86} \mathrm{Sr}=0.720$. Because an average of $1,200 \mathrm{ng}$ of $\mathrm{Sr}$ was processed for each sample, the blank contribution to each measured ${ }^{87} \mathrm{Sr} /$ ${ }^{86} \mathrm{Sr}$ ratio was inconsequential $(<0.00002)$. The Sr isotopic compositions were obtained using a 6-collector Finnigan MAT 261 thermal ionization mass spectrometer at the University of Colorado, Boulder. Repeated measurements of the NBS-987 Sr standard during the study period produced a mean ratio of $0.710312 \pm 2(2 \sigma ; n=8)$, which is $\sim 0.00003$ higher than accepted values for this standard. As a result, the $\mathrm{Sr}$ isotopic data reported in table 4 have been corrected downward by this increment.

\section{Interpretation of Water Data}

\section{Hydrogeochemistry in Areas of Cretaceous Granite}

Waters in contact with mid-Cretaceous granitic rocks at Gilmore dome have a characteristic and consistent hydrogeochemical signature. Seeps in granite at the head of Nugget (site 97-01), Rex (site 97-05), and Pearl (site 97-06) Creeks were observed in the field to have relatively low conductivities (40-65 $\mu \mathrm{S} / \mathrm{cm}$ ) compared to waters draining metasedimentary units (table 1). The $\mathrm{pH}$ values for these seeps were essentially neutral, ranging between 6.8 and 7.1, with relatively low alkalinities between 27 and $32 \mathrm{mg} / \mathrm{L}$ (table 3 ). Rainwater is slightly acidic in the Fairbanks area as noted from the $\mathrm{pH}$ measurement of 6.27 at site 95-20 from Goldfarb and others (1997), and, therefore, we interpret slightly more alkaline measurements for the seeps on Gilmore dome to reflect waters that have interacted with midCretaceous granitic bedrock.

As suggested by the low conductivity measurements, many of the major cations have relatively low concentrations. Calcium concentrations of less than $9 \mathrm{ppm}, \mathrm{Mg}$ of about $0.6-0.7 \mathrm{ppm}$, and $\mathrm{K}$ between 330 to $380 \mathrm{ppb}$ are relatively low compared to most samples in the Fairbanks area (table 2; fig. 1B). These waters also have distinctively low sulfate levels ( 2 ppm or less; fig. 1C) but have the highest levels of dissolved silica observed in the study area, with concentrations as high as $17 \mathrm{ppm} \mathrm{Si}$ in the headwaters of Nugget Creek (site 97-01; table 2). In addition, two of the three "granite" seeps (sites 97-01 and -06) have relatively high fluoride concentrations of $0.6-0.9 \mathrm{ppm}$, compared to typical background levels over much of the study area of $0.1-0.2$ $\mathrm{ppm}$. Uranium concentrations of 3.9 and $4.7 \mathrm{ppb}$ at these two sites are 1 to 2 orders of magnitude greater than background levels of most waters collected away from granite, which have uranium concentrations in the parts-per-trillion range.

With increasing distance south of the crest of Gilmore dome (sites 97-02, -03, -04), Ca, Mg, and $\mathrm{K}$ concentrations in surface waters increase dramatically, presumably reflecting the fact that these waters have interacted considerably with metasedimentary units of the Fairbanks Schist, the predominant bedrock in the lower elevations of these catchments. As a result, these major-element data provide little indication that the headwaters of each watershed are underlain by granitic rocks. However, surface waters at site 97-02, several kilometers down Nugget Creek from site $97-01$, do have high $\mathrm{U}(7.8 \mathrm{ppb})$ and $\mathrm{F}^{-}$contents $(0.4$ ppm), suggesting that these elements may provide a fingerprint of the upstream granitic rocks, which can be recognized even after the addition of significant dissolved material from the Fairbanks Schist.

Ground water that has interacted with the granitic rocks does not, however, invariably have elevated $\mathrm{U}$ and $\mathrm{F}^{-}$concentrations. Water sampled at the crest of Gilmore dome at site 97-05 (Rex Creek) lacks $\mathrm{U}$ and $\mathrm{F}^{-}$enrichments, despite its low $\mathrm{Mg}$ and Ca contents (and low ${ }^{87} \mathrm{Sr} /{ }^{86} \mathrm{Sr}$; see below) that we consider characteristic of ground waters that have interacted with granitic rocks. Most likely, given the similar neutral $\mathrm{pH}$ and low conductivity values for the waters from sites $97-01,-05$ and -06 , the lower concentrations of the $U$ and $\mathrm{F}^{-}$at site 95-05 may reflect local changes in granite chemical characteristics. Alternatively, the seep at Rex Creek may simply reflect waters that have more limited flow distances through the bedrock at this location and, therefore, have interacted less with the granitic rocks. It is also interesting to note that the Rex Creek seep contains 48 ppb As, a value much higher than any other seep in the study area (table 2). This may be an indication that igneous rocks at depth are more highly sulfidized at Rex Creek (site 97-05) than in other portions of Gilmore dome, and, therefore, this region represents a potential target for precious-metal mineralization. We also note that surface waters within Nugget Gulch, on the northwest side of Ester dome (site 97-21), contain only $7 \mathrm{ppm} \mathrm{Ca}$ and $3 \mathrm{ppm} \mathrm{Mg}$. This suggests that much of the flow may be derived from areas underlain by the poorly exposed, upstream mid-Cretaceous granite. 
Table 1. Field measurements and site descriptions for hydrogeochemical sampling sites in the Fairbanks area.

[Leaders (--) signify no measurement at a given site]

\begin{tabular}{|c|c|c|c|c|c|}
\hline $\begin{array}{l}\text { Site } \\
\text { no. }\end{array}$ & $\mathrm{pH}$ & $\begin{array}{l}\text { Temp. } \\
\left({ }^{\circ} \mathrm{C}\right)\end{array}$ & $\begin{array}{c}\text { Conductivity } \\
(\mu \mathrm{S} / \mathrm{cm})\end{array}$ & $\begin{array}{c}\text { Site } \\
\text { description }\end{array}$ & Geology \\
\hline $97-01$ & 7.1 & 5 & 40 & Spring at head of Nugget Creek & Mid-Cretaceous granite \\
\hline $97-02$ & 7.3 & 8 & 133 & Nugget $\mathrm{Ck}, 0.5 \mathrm{~km}$ above junction with Smallwood $\mathrm{Ck}$ & Fairbanks Schist; granitic headwaters \\
\hline $97-03$ & 7.5 & 5 & 200 & Gay Gulch, $0.5 \mathrm{~km}$ above junction with Smallwood $\mathrm{Ck}$ & Fairbanks Schist; granitic headwaters \\
\hline $97-04$ & 7.3 & 7 & 161 & Rex Ck, $0.8 \mathrm{~km}$ above junction with Smallwood Ck & Fairbanks Schist; granitic headwaters \\
\hline $97-05$ & 6.9 & 10 & 65 & Spring at head of Rex Creek & Mid-Cretaceous granite \\
\hline $97-06$ & 6.8 & 3 & 55 & Spring at head of Pearl Creek & Mid-Cretaceous granite \\
\hline $97-07$ & 6.9 & 12 & 201 & Alder Ck on upstream side of Steese Highway & Chatanika assemblage and Tertiary basalt \\
\hline $97-08$ & 7.6 & 10 & 181 & Pilot Ck on upstream side of Steese Highway & Chatanika eclogite \\
\hline $97-09$ & 8.2 & 6 & 150 & Spring at head of Alder Creek & Fairbanks Schist \\
\hline $97-10$ & 7.7 & 5 & 165 & Spring at head of Ginger Creek & Fairbanks Schist \\
\hline $97-11$ & 7.4 & 7 & 183 & Spring at head of Kokomo Creek & Chatanika assemblage \\
\hline $97-12$ & 6.8 & 10 & 52 & Spring at head of Deep Creek & Fairbanks Schist \\
\hline $97-13$ & 7.2 & 3.5 & 165 & Ginger Creek at 1,300 - $\mathrm{ft}$ elevation & Fairbanks Schist; marble in headwaters \\
\hline $97-14$ & 7.7 & 3 & 115 & Moose Creek above Hi-Yu mill tailings; same as 96-15 & Fairbanks Schist \\
\hline $97-15$ & 7.3 & 9 & 179 & Moose $\mathrm{Ck}$ at base of $\mathrm{Hi}-\mathrm{Yu}$ mill tailings; same as $96-16$ & Fairbanks Schist \\
\hline $97-16$ & 7.2 & 2 & 132 & Tributary on south side of Pedro Creek & Orthogneiss \\
\hline $97-17$ & -- & 3 & 555 & Dora Ck on upstream side of Steese Highway & Chatanika assemblage \\
\hline $97-18$ & 7.2 & 7 & 143 & Flume Creek at 1,200 -ft elevation & Fairbanks Schist; minor granite in headwaters \\
\hline $97-19$ & 7.4 & 15 & 392 & Creek at about Mile 7.5 along Dome Ck Road & Chatanika assemblage \\
\hline $97-20$ & 7.7 & 9 & 395 & Silver Creek on upstream side of Goldstream Rd & Fairbanks Schist \\
\hline $97-21$ & 7.0 & 3 & 108 & Nugget Creek at $1,000-\mathrm{ft}$ elevation & Fairbanks Schist \\
\hline $97-22$ & 7.0 & 4 & 69 & Eastern tributary to Ready Bullion $\mathrm{Ck}$ at 1,050 -ft elevation & Muskox Sequence \\
\hline
\end{tabular}


Table 2. Concentrations of dissolved cations in filtered, acidified waters collected in the Fairbanks region.

[All data in ppb except for $\mathrm{Ca}, \mathrm{Mg}$, and $\mathrm{Na}$, which are in ppm]

\begin{tabular}{|c|c|c|c|c|c|c|c|c|c|c|c|c|c|c|c|c|c|}
\hline $\begin{array}{l}\text { Sample } \\
\text { site }\end{array}$ & $\mathrm{Ca}$ & $\mathrm{Mg}$ & $\mathrm{Na}$ & $K$ & $\mathrm{Si}$ & $\mathrm{Fe}$ & $\mathrm{Al}$ & $\mathrm{Mn}$ & Co & $\mathrm{Ni}$ & $\mathrm{Cu}$ & $\mathrm{Pb}$ & $\mathrm{Zn}$ & $\mathrm{Cd}$ & As & $\mathrm{Sb}$ & U \\
\hline $97-01$ & 5.6 & 0.61 & 2.5 & 340 & 17 & 150 & 13 & 11 & 0.1 & 0.4 & 0.5 & $<0.05$ & 2 & $<0.02$ & 2 & 0.1 & 3.9 \\
\hline $97-02$ & 16 & 2.9 & 3.2 & 700 & 13 & 150 & 17 & 73 & 0.2 & 0.8 & 0.6 & $<0.05$ & 1 & $<0.02$ & 2 & 0.1 & 7.8 \\
\hline $97-03$ & 17 & 6.8 & 2.4 & 830 & 10 & 300 & 8.6 & 120 & 0.4 & 0.7 & 0.6 & $<0.05$ & 1 & $<0.02$ & 4 & 0.2 & 0.21 \\
\hline $97-04$ & 11 & 5.5 & 2.6 & 910 & 12 & 200 & 8.0 & 62 & 0.2 & 0.8 & 0.5 & $<0.05$ & 0.9 & $<0.02$ & 3 & 0.1 & 0.08 \\
\hline $97-05$ & 7.3 & 0.72 & 1.7 & 380 & 14 & 88 & 9.9 & 17 & 0.08 & 0.3 & $<0.5$ & $<0.05$ & 1 & $<0.02$ & 48 & 0.4 & 0.57 \\
\hline $97-06$ & 8.9 & 0.67 & 1.8 & 330 & 13 & 15 & 8.6 & 0.41 & $<0.02$ & 0.1 & $<0.5$ & $<0.05$ & 1 & $<0.02$ & 0.6 & 0.02 & 4.7 \\
\hline $97-07$ & 17 & 6.6 & 3.1 & 1200 & 10 & 1400 & 15 & 230 & 1.1 & 1.6 & 0.8 & $<0.05$ & 2 & $<0.02$ & 1 & 0.2 & 0.13 \\
\hline $97-08$ & 17 & 6.5 & 1.8 & 1200 & 10 & 300 & 8.6 & 38 & 0.1 & 1.0 & 0.7 & $<0.05$ & 1 & $<0.02$ & 1 & 0.2 & 0.33 \\
\hline $97-09$ & 16 & 3.9 & 0.58 & 1100 & 6 & 25 & 1.9 & 0.06 & $<0.02$ & 0.2 & $<0.5$ & $<0.05$ & 0.5 & $<0.02$ & 5 & 0.1 & 0.15 \\
\hline $97-10$ & 18 & 4.8 & 0.63 & 1200 & 5 & 27 & 1.2 & 0.06 & $<0.02$ & 0.2 & $<0.5$ & $<0.05$ & 0.7 & $<0.02$ & 9 & 0.3 & 0.36 \\
\hline $97-11$ & 20 & 4.3 & 0.59 & 1200 & 5 & 29 & 6.4 & 0.13 & 0.02 & 0.3 & $<0.5$ & $<0.05$ & 0.5 & $<0.02$ & 1 & 0.4 & 0.27 \\
\hline $97-12$ & 3.8 & 1.4 & 0.61 & 440 & 8 & 11 & 9.5 & 0.09 & $<0.02$ & 0.6 & $<0.5$ & $<0.05$ & 0.9 & $<0.02$ & 1 & 0.05 & 0.01 \\
\hline $97-13$ & 17 & 5.8 & 0.83 & 710 & 6 & 27 & 5.7 & 0.22 & 0.02 & 0.3 & $<0.5$ & $<0.05$ & $<0.5$ & 0.02 & 4 & 0.50 & 0.47 \\
\hline $97-14$ & 13 & 2.7 & 0.85 & 410 & 8 & 19 & 4.9 & 0.13 & $<0.02$ & 0.4 & $<0.5$ & $<0.05$ & 2 & 0.78 & 12 & 0.86 & 0.04 \\
\hline $97-15$ & 14 & 4.6 & 1.1 & 420 & 10 & 170 & 11 & 220 & 0.4 & 9.6 & 1.0 & 0.3 & 140 & $<0.02$ & 78 & 8.0 & 0.04 \\
\hline $97-16$ & 14 & 2.9 & 1.8 & 950 & 10 & 22 & 7.0 & 0.08 & $<0.02$ & 0.3 & $<0.5$ & $<0.05$ & 0.5 & $<0.02$ & 0.4 & 0.06 & 0.41 \\
\hline $97-17$ & 61 & 22 & 1.9 & 2600 & 11 & 90 & 6.5 & 1.0 & 0.04 & 2.8 & 0.9 & $<0.05$ & 1 & $<0.02$ & 0.5 & 0.07 & 7.3 \\
\hline $97-18$ & 14 & 3.0 & 2.8 & 760 & 12 & 68 & 10 & 13 & 0.2 & 1.1 & $<0.5$ & $<0.05$ & 0.5 & $<0.02$ & 2 & 0.1 & 0.29 \\
\hline $97-19$ & 32 & 13 & 2.9 & 880 & 9 & 730 & 20 & 130 & 0.3 & 2.0 & 1.0 & $<0.05$ & 0.7 & $<0.02$ & 1 & 0.06 & 0.18 \\
\hline $97-20$ & 38 & 14 & 5.5 & 1200 & 9 & 300 & 6.4 & 30 & 0.1 & 1.3 & 1.0 & $<0.05$ & 0.9 & $<0.02$ & 1 & 0.2 & 2.4 \\
\hline $97-21$ & 7.0 & 4.1 & 1.9 & 1100 & 13 & 16 & 5.5 & 1.9 & 0.03 & 1.2 & $<0.5$ & $<0.05$ & 1 & $<0.02$ & 2 & 0.1 & 0.01 \\
\hline $97-22$ & 4.3 & 2.3 & 1.8 & 430 & 13 & 26 & 18 & 0.46 & 0.02 & 0.8 & 0.5 & $<0.05$ & 0.9 & $<0.02$ & 5 & 0.73 & 0.01 \\
\hline
\end{tabular}


Table 3. Concentrations of dissolved anions in filtered water from the Fairbanks region.

[All samples were also analyzed for $\mathrm{Br}^{-}$and $\mathrm{HPO}_{4}{ }^{2-}$, with all values below lower determination limits of $0.2 \mathrm{ppm}$ and $1 \mathrm{ppm}$, respectively. All data in ppm; alkalinities in $\mathrm{mg} / \mathrm{L} \mathrm{CaCO}_{3}$ ]

\begin{tabular}{ccrccc}
\hline $\begin{array}{c}\text { Sample } \\
\text { site }\end{array}$ & $\mathrm{F}^{-}$ & $\mathrm{NO}_{3}{ }^{-}$ & $\mathrm{Cl}^{-}$ & $\mathrm{SO}_{4}{ }^{2-}$ & $\begin{array}{c}\text { Measured } \\
\text { alkalinity }\end{array}$ \\
\hline $97-01$ & 0.6 & $<0.4$ & 0.2 & $<2.0$ & 27 \\
$97-02$ & 0.4 & $<0.4$ & 0.5 & 11 & 61 \\
$97-03$ & 0.2 & 0.7 & 0.3 & 36 & 55 \\
$97-04$ & 0.2 & 1.0 & 0.3 & 23 & 45 \\
$97-05$ & 0.2 & $<0.4$ & 0.2 & 2.0 & 30 \\
$97-06$ & 0.9 & 3.4 & 0.2 & $<2.0$ & 32 \\
$97-07$ & 0.1 & $<0.4$ & 0.3 & 42 & 47 \\
$97-08$ & 0.2 & 0.6 & 0.3 & 33 & 52 \\
$97-09$ & 0.1 & 1.3 & 0.3 & 8.0 & 67 \\
$97-10$ & 0.7 & 0.8 & 0.3 & 9.8 & 74 \\
$97-11$ & 0.1 & 0.8 & 0.3 & 12 & 74 \\
$97-12$ & 0.1 & 1.7 & 0.2 & 4.2 & 17 \\
$97-13$ & 0.1 & 1.0 & 0.3 & 16 & 67 \\
$97-14$ & 0.1 & 2.7 & 0.3 & 11 & 44 \\
$97-15$ & 0.1 & 1.6 & 0.2 & 37 & 33 \\
$97-16$ & 0.1 & 1.3 & 0.3 & 16 & 46 \\
$97-17$ & 0.4 & 0.9 & 0.4 & 140 & 166 \\
$97-18$ & 0.1 & 0.8 & 0.3 & 15 & 51 \\
$97-19$ & 0.2 & 0.4 & 0.2 & 59 & 100 \\
$97-20$ & 0.2 & 0.9 & 0.4 & 79 & 122 \\
$97-21$ & 0.2 & 3.3 & 0.4 & 23 & 21 \\
$97-22$ & 0.1 & 1.3 & 0.3 & 12 & 15 \\
\hline
\end{tabular}

Waters that have interacted with the granites at Gilmore dome also have a unique strontium isotope signature (table 4). Measured ${ }^{87} \mathrm{Sr} /{ }^{86} \mathrm{Sr}$ values from the three seeps in the granites (sites 97-01, -05, and -06) range between 0.7146 and 0.7182 . These values are much less radiogenic than waters draining metasedimentary rock lithologies (figs. $1 D, 2$ ). Water collected at site 97-02, noted above to contain high concentrations of $U$ and $\mathrm{F}^{-}$that may have been inherited from upstream granitic rocks, has a high strontium concentration $(174 \mathrm{ppm})$ and a ${ }^{87} \mathrm{Sr} /$ ${ }^{86} \mathrm{Sr}$ ratio of 0.7251 . The strontium isotopic composition is intermediate between that of waters that interacted exclusively with granites and those that have interacted exclusively with schist (fig. 1D), which suggests that the higher strontium concentration in water from Nugget Creek was derived from the schist. Farther downstream in this drainage, at sites 97-03 and -04, isotopic ratios are more radiogenic and waters no longer retain an isotopic signature characteristic of the upstream igneous units (fig. $1 D$ ).

\section{Hydrogeochemistry in Areas of Fairbanks Schist}

Water samples collected from springs and streams within the Fairbanks Schist typically have $\mathrm{pH}$ values of 7.2-8.2 and conductivities of 100-200 $\mu \mathrm{S} / \mathrm{cm}$. Concentrations of dissolved major ions are high compared to those of seeps in granites, with typical concentrations from areas within the schist of 13-20 ppm Ca, 4-7 ppm Mg, 700-1,200 ppb K (fig. 1B), and 8-36 ppm sulfate (fig. $1 C$ ). Trace-element concentrations vary little from those that characterize waters in contact with granites,
Table 4. Sr elemental and isotopic data for surface waters from the Fairbanks area.

\begin{tabular}{|c|c|c|}
\hline Site & $\operatorname{Sr}(p p b)$ & ${ }^{87} \mathrm{Sr} /{ }^{86} \mathrm{Sr}$ \\
\hline \multicolumn{3}{|c|}{ 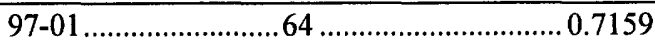 } \\
\hline \multicolumn{3}{|c|}{ 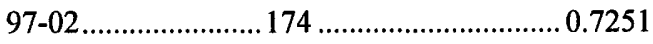 } \\
\hline \multicolumn{3}{|c|}{ 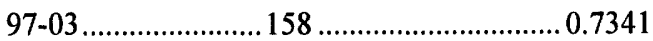 } \\
\hline \multicolumn{3}{|c|}{ 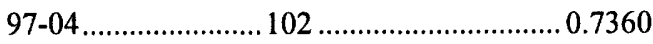 } \\
\hline \multicolumn{3}{|c|}{ 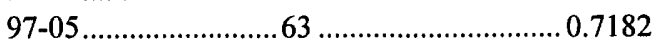 } \\
\hline \multicolumn{3}{|c|}{ 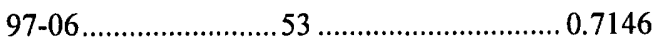 } \\
\hline \multicolumn{3}{|c|}{ 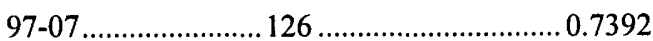 } \\
\hline \multicolumn{3}{|c|}{ 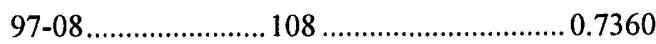 } \\
\hline \multicolumn{3}{|c|}{ 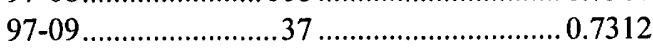 } \\
\hline \multicolumn{3}{|c|}{ 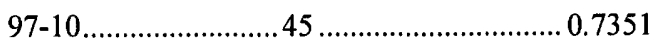 } \\
\hline \multicolumn{3}{|c|}{ 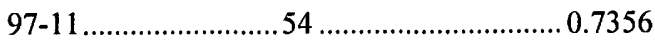 } \\
\hline \multicolumn{3}{|c|}{ 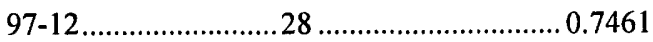 } \\
\hline \multicolumn{3}{|c|}{ 97-13 } \\
\hline \multicolumn{3}{|c|}{ 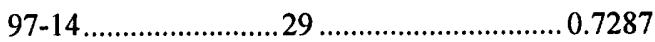 } \\
\hline \multicolumn{3}{|c|}{ 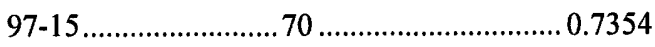 } \\
\hline \multicolumn{3}{|c|}{ 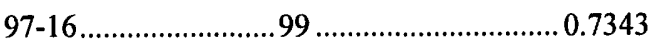 } \\
\hline \multicolumn{3}{|c|}{ 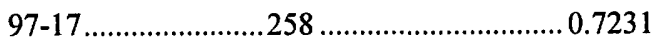 } \\
\hline \multicolumn{3}{|c|}{ 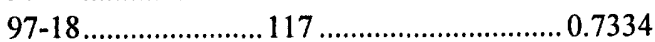 } \\
\hline \multicolumn{3}{|c|}{ 97-19 } \\
\hline \multicolumn{3}{|c|}{ 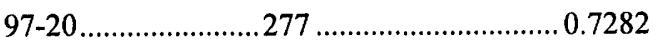 } \\
\hline \multicolumn{3}{|c|}{ 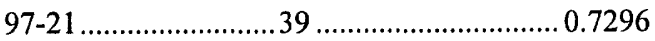 } \\
\hline \multicolumn{3}{|c|}{ 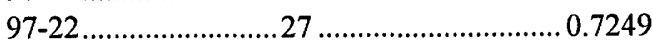 } \\
\hline
\end{tabular}

except for the consistent relatively lower $\mathrm{U}$ and $\mathrm{F}^{-}$contents. Strontium-isotope compositions are typically much more radiogenic than those of waters that have interacted with granitic rocks. The ${ }^{87} \mathrm{Sr} /{ }^{86} \mathrm{Sr}$ values range between 0.7282 and 0.7364 , with the one spring on the south side of Coffee dome having the highest value measured from any surface water or seep in the study area $(0.7461$; fig. $1 D)$.

In spite of some similar characteristics, there is significant variability in waters draining the Fairbanks Schist. For instance, a comparison of data from the Smallwood Creek watershed (sites 97-03 and -04) with those from the north side of Fairbanks Creek (sites 97-09, -10, -12, -13, -14) indicates that, although similar in $\mathrm{Ca}, \mathrm{Mg}$, and $\mathrm{K}$ concentrations, the waters in the Fairbanks Creek area are significantly lower in dissolved Fe, Al, Mn, $\mathrm{Si}$, and sulfate. One possibility could be more limited interaction between ground water and the Fairbanks Schist in the Fairbanks Creek area. However, because conductivities and many of the major elements show similar concentrations in waters from both areas, we favor local mineralogical differences in the schist as the most likely cause of the hydrogeochemical variability.

It is noteworthy that there is little difference in the hydrogeochemistry between water from site 97-10, at the head of Ginger Creek, and site 97-13, located $1.5 \mathrm{~km}$ downstream (fig. 3). A large lens of limestone within the Fairbanks Schist on the western side of the basin (Newberry and Bundtzen, 1996) has no obvious affect on the dissolved load or ${ }^{87} \mathrm{Sr} /{ }^{86} \mathrm{Sr}$ value; in fact, the measured $\mathrm{pH}$ of the stream decreased by one-half a $\mathrm{pH}$ unit below the limestone. Most likely, ground waters within the Ginger Creek watershed have not interacted to any significant degree with the limestone.

There are anomalous concentrations of metals in waters from some sites underlain by the schist. As expected and observed in a previous study (Goldfarb and others, 1997), Moose 


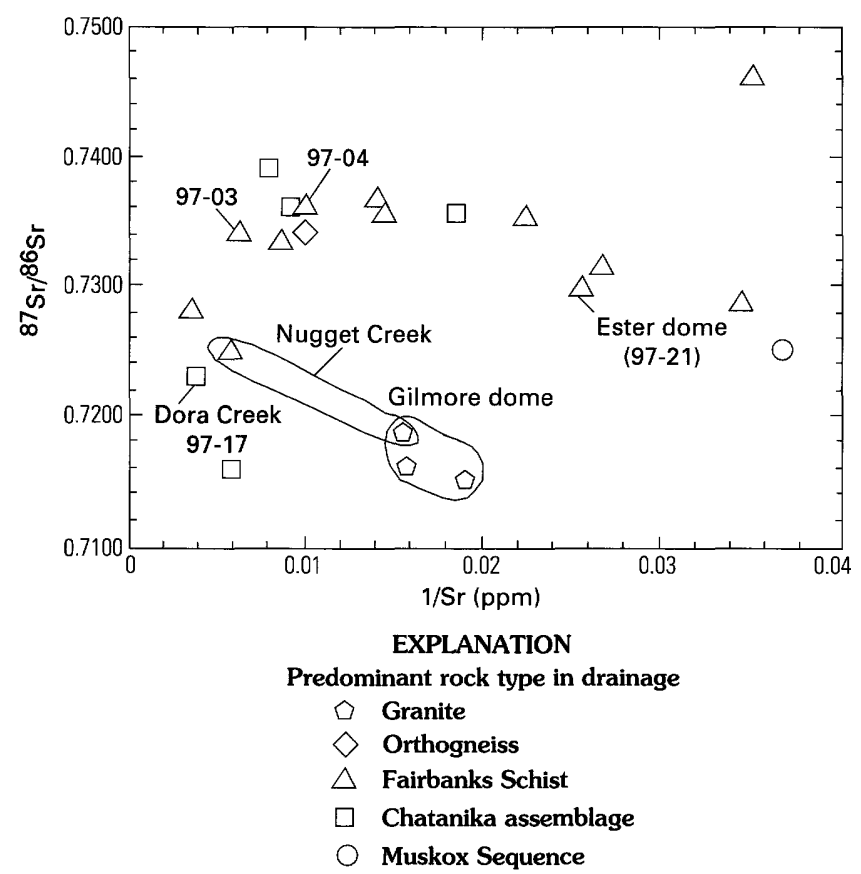

Figure 2. Plot of ${ }^{87} \mathrm{Sr} /{ }^{86} \mathrm{Sr}$ vs. $1 / \mathrm{Sr}$ for surface waters in the Fairbanks area. Note the isotopically low values for waters draining granite and the possible mixing relationship downstream of granites along Nugget Creek. Even though the strontium isotope data vary with lithology, absolute strontium concentrations do not show a similar relationship.

Creek below the Hi-Yu mill tailings is enriched in many dissolved components including $\mathrm{As}, \mathrm{Cd}, \mathrm{Co}, \mathrm{Fe}, \mathrm{Mn}, \mathrm{Ni}, \mathrm{Pb}, \mathrm{Sb}$, and $\mathrm{Zn}$. The sampled water was significantly less acidic in 1997 (site 97-15, pH 7.3) than in 1995 (site 95-16, pH 5.18; Goldfarb and others, 1997), and the absolute concentrations of the dissolved metal species are also significantly lower. A few hundred meters upstream from the tailings (site 97-14), where seep discharge into Moose Creek represents the initial surface-water flow in the creekbed during this time of year, concentrations of dissolved $\mathrm{K}$ were only $410 \mathrm{ppb}$. This is the lowest value measured for waters interacting with the schist. Once again, this is thought to reflect local mineralogical variations within the schist.

Two samples hint at the presence of nearby, unexposed granite. The spring at the head of Ginger Creek (site 97-10) contained $0.7 \mathrm{ppm} \mathrm{F}^{-}$, a level consistent with measurements for waters from samples draining granitic rocks (sites 97-01 and 06). In addition, the spring on the south side of Coffee dome (site 97-12) had low concentrations of $\mathrm{Ca}, \mathrm{Mg}, \mathrm{K}$ (fig. $1 B$ ), and sulfate (fig. $1 C$ ) relative to all other samples collected from the Fairbanks Schist. But the relatively high ${ }^{87} \mathrm{Sr} /{ }^{86} \mathrm{Sr}$ ratio $(0.7461)$ for this latter water (fig. $1 D$ ) is atypical of those that have interacted exclusively with mid-Cretaceous granites (fig. 3).

Water at site 97-20, from Silver Creek immediately above its junction with Goldstream Creek, is characterized by a relatively low $\mathrm{pH}$ and high levels of $\mathrm{Mg}, \mathrm{Ca}, \mathrm{Na}, \mathrm{Cu}, \mathrm{U}, \mathrm{Sr}$, and sulfate. It also has a ${ }^{87} \mathrm{Sr} /{ }^{86} \mathrm{Sr}$ ratio of 0.7282 , a value marking the low end of the range for waters collected from areas underlain by the Fairbanks Schist. The sampled site is notable in that it is downstream from an extensive accumulation of eolian silt that overlies bedrock throughout most of the Silver Creek watershed. Contributions from eolian deposits appear to significantly
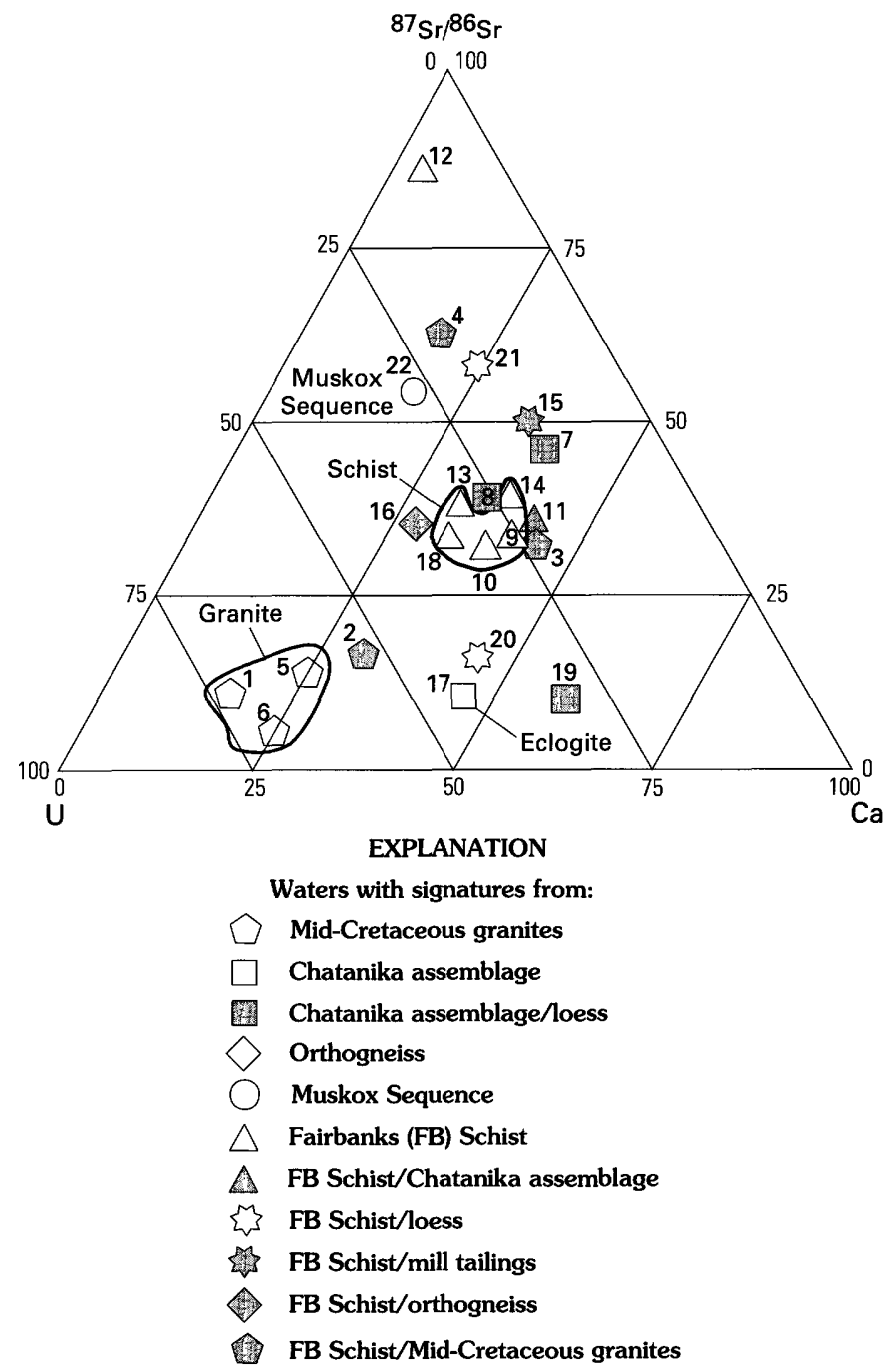

Figure 3. Triangular plot showing fields that best characterize waters that have interacted with granite (sites 1, 5, and 6), schist (sites 9, 10, 13, 14, and 18), and eclogite (site 17), based on uranium and calcium concentrations (in percent) and strontium isotopic values. Mixed signatures reflect waters that have also interacted with loess, mining waste, or multiple lithologies, and specific sites are described throughout the text.

increase dissolved loads such that water chemistry will not simply reflect local bedrock lithology, and, thus, data for site 9720 plot far from the field typical of waters that have interacted with the schist (fig. 3).

Site 97-16 was purposely located where a body of orthogneiss underlies the lower part of a small drainage basin on the south side of Pedro Creek within the Fairbanks Schist. Hydrogeochemical data measured for the sample are indistinguishable from those collected elsewhere within the schist (fig. 3). The ${ }^{87} \mathrm{Sr} /{ }^{86} \mathrm{Sr}$ ratio of 0.7343 is typical of waters from the schist (figs. $1 D, 2)$. Because of the present limited data, we cannot determine what, if any, hydrogeochemical differences distinguish waters that have interacted with the orthogneiss from those that have interacted with the schist. Perhaps most of the discharge at site 97-16 actually reflects ground water that has been in contact with upstream schist and had only limited flow through the orthogneiss. 


\section{Hydrogeochemistry in Areas of Muskox Sequence}

A large area throughout the southeast side of Ester dome is underlain by interbedded schist, amphibolite, and metarhyolite of the Muskox Sequence (Newberry and Bundtzen, 1996). In the center of the area, we collected a water sample (site 97-22) on a small tributary to Ready Bullion Creek. Similar to the samples in equilibrium with the granites, this water was characterized by relatively low levels of potassium $(430 \mathrm{ppb})$ and calcium (4.3 ppm) and a low alkalinity of $15 \mathrm{mg} / \mathrm{L}$. In addition, the sample had extremely low concentrations of strontium (27 $\mathrm{ppb})$ and uranium $(0.01 \mathrm{ppb})$. Higher magnesium $(2.3 \mathrm{ppm})$ and sulfate $(12 \mathrm{ppm})$ concentrations for the water were in sharp contrast to the extremely low levels for waters in equilibrium with granites. The ${ }^{87} \mathrm{Sr} /{ }^{86} \mathrm{Sr}$ value of 0.7249 is partly between that of waters that have interacted with the Fairbanks Schist and those that drain the granites (fig. 2). Although we only obtained one water sample from the area underlain by this rock sequence, initial interpretation of the resulting data suggests that waters that interacted with this sequence exhibit a unique geochemical signature (site 97-22; fig. 3).

\section{Hydrogeochemistry in Areas of Chatanika Assemblage}

The generally nonresistant nature of eclogite of the Chatanika assemblage results in the low-relief topography in the northern part of the study area (Newberry and Bundtzen, 1996).

Because of the resulting topography, many of the stream valleys underlain by this lithology are filled with eolian loess. Waters from sites 97-07, -08 , and -19 , although all clearly collected from streams draining rocks of the Chatanika assemblage, have chemistries that are influenced by dissolution of material from the unconsolidated silt. The sampled spring at the headwaters of Kokomo Creek (site 97-11) was located within an area underlain by eclogite, but a minority of the recharge area is also underlain by Fairbanks Schist. The sample collected on Dora Creek at site 97-17 represents the one sample we feel reflects waters that interacted solely with eclogite.

The relatively soluble $\mathrm{Ca}-\mathrm{Mg}$ silicate assemblage of eclogite has led to elevated dissolved loads in the waters of Dora Creek. At site 97-17, the conductivity was $555 \mu \mathrm{S} / \mathrm{cm}$, and major ion concentrations included $61 \mathrm{ppm} \mathrm{Ca}, 22 \mathrm{ppm} \mathrm{Mg}$, 2,600 ppm K, and $140 \mathrm{ppm}$ sulfate. In addition, $\mathrm{Ba}, \mathrm{Cs}, \mathrm{F}^{-}, \mathrm{Li}$, $\mathrm{Rb}, \mathrm{Re}, \mathrm{Ti}$, and $\mathrm{U}$ were detected at relatively elevated levels (table 2) (Goldfarb, unpub. data). A ${ }^{87} \mathrm{Sr} /{ }^{86} \mathrm{Sr}$ ratio of 0.7231 lies between that for waters that have interacted with the granites and the Fairbanks Schist (fig. 2). This was the most alkaline of the waters from the Fairbanks area, with a measured alkalinity of $166 \mathrm{mg} / \mathrm{L}$.

The other four samples collected from streams draining rocks of the Chatanika assemblage are difficult to interpret. The spring at the headwaters of the Kokomo Creek drainage (site 9711) has chemical attributes, including a strontium isotope signature, that are nearly identical to nearby springs that discharge from rocks of the Fairbanks Schist. Schist underlies only a small part of the upstream reaches of the watershed for site 97-11, but the hydrochemistry suggests a major influence by schist along the water flow path. Kokomo (site 97-07) and Pilot (site 97-08) Creeks also drain eclogite, but these waters flowed for a number of kilometers through loess, and the resulting hydrogeochemical signatures lack the characteristics of the Dora Creek water. A tributary to Dome Creek (site 97-19) drains a large area of loess, but concentrations of $13 \mathrm{ppm} \mathrm{Mg}, 730 \mathrm{ppb} \mathrm{Fe}$, and $32 \mathrm{ppm} \mathrm{Ca}$, as well as elevated Al, Ba, P, REE, and Ti, seem to be indicative of the underlying eclogite.

Site 97-07 on Kokomo Creek is only $1 \mathrm{~km}$ downstream from a large Tertiary basalt flow that seems to influence local water chemistry. Concentrations of $1,400 \mathrm{ppb} \mathrm{Fe}, 230 \mathrm{ppb} \mathrm{Mn}$, and $1.1 \mathrm{ppb}$ Co are apparently a function of water-basalt interaction, although an enriched ${ }^{87} \mathrm{Sr} /{ }^{86} \mathrm{Sr}$ ratio of 0.7392 (table 4) hints that interaction with the loess overwhelms the isotopic signature characterizing the relatively nonradiogenic basalt.

\section{Discussion}

Natural waters in the Fairbanks region may be fingerprinted by unique chemical signatures that, in large part, are a function of the underlying bedrock geology (fig. 3). Throughout the study area, calcium is consistently the dominant cation species (table 2, this paper; table 2, sites 11-24, Goldfarb and others, 1997; fig. 4 , this paper), probably reflecting dissolution of calcite and plagioclase in metasedimentary rocks. Bicarbonate, defining most of the measured alkalinity, and sulfate are both significant anion species (table 3 and fig. 4, this paper; table 4, sites 11-24, Goldfarb and others, 1997). Bicarbonate concentrations in the Fairbanks area are equal to or two to three times greater than sulfate concentrations. Because much of the sulfidization of country rocks near gold deposits was accompanied by coeval and spatially overlapping carbonate alteration, sulfate does not notably predominate as the major anion species even where waters drain abandoned mine workings (site 97-15, this study; Goldfarb and others, 1997). Nevertheless, absolute sulfate concentrations are relatively high for most water samples from the Fairbanks area (fig. 1C), indicative of the extensive sulfidization of the rocks.

The greater solubility of plagioclase, relative to microcline or orthoclase, in addition to the well-defined cleavage in plagioclase, lead to its greater susceptibility to weathering (Ollier, 1969). As a result, potassium levels are relatively low compared to calcium for all water samples from the study area (fig. 4). Typically, dissolved calcium concentrations are an order of magnitude greater than those for sodium in waters draining metasedimentary rock units, suggesting the predominance of anorthite over albite and (or) significant dissolution of calcite from the schist.

Waters draining the granites are only about one-half of an order of magnitude enriched in calcium relative to sodium; absolute sodium concentrations are similar to those of waters from the metasedimentary rocks; and dissolved silica is notably elevated. We interpret this to indicate a relatively increased amount of dissolution of Na-rich feldspars. The amount of dissolved silica released into natural waters per hydrogen ion is much greater for Na-rich feldspars than for other major mineral phases in felsic to intermediate igneous rocks (Hem, 1992). The low conductivity and calcium concentrations for these waters indicates, however, that overall rock-dissolution rates are lower 


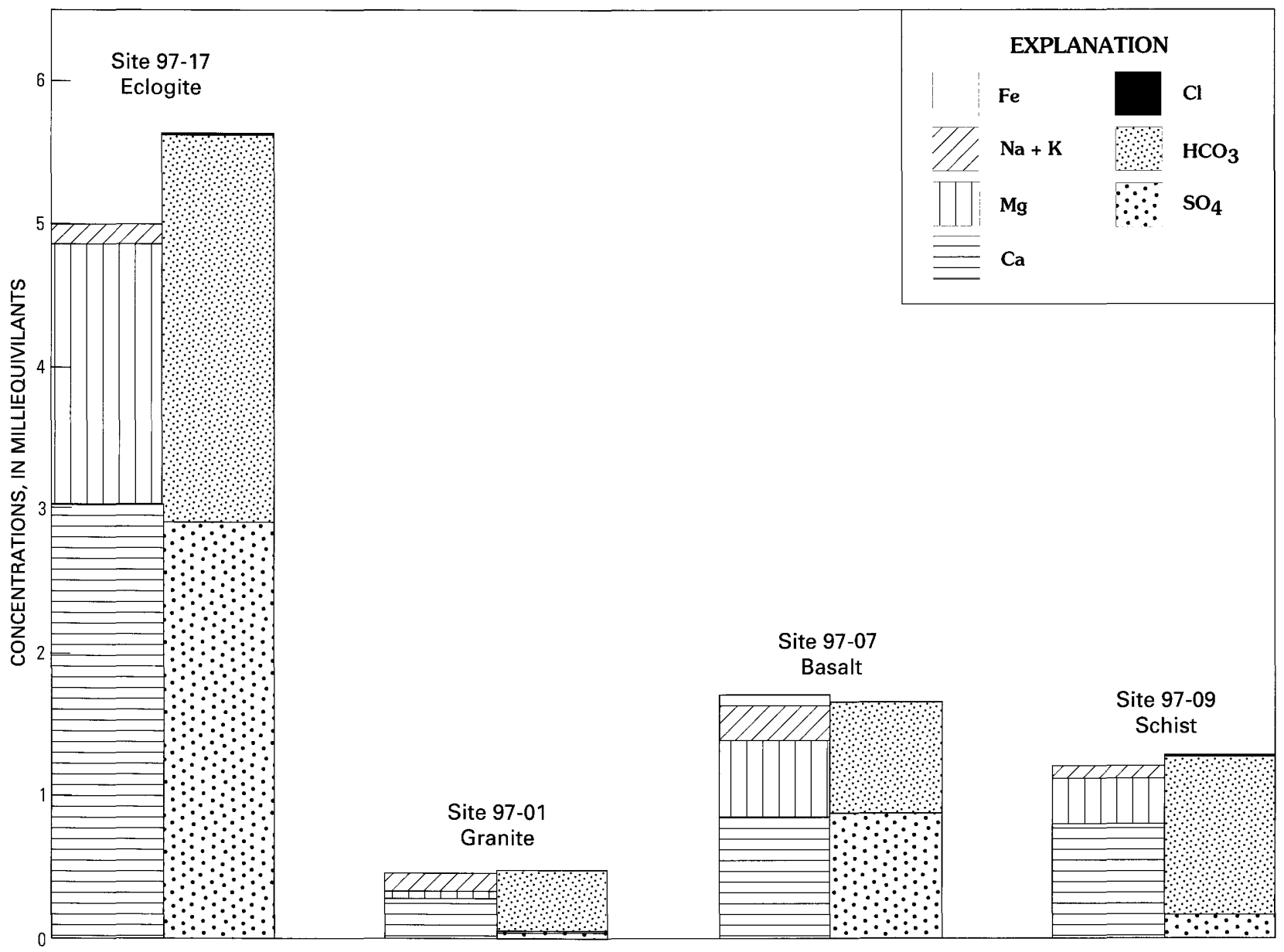

Figure 4. Sum of cations vs. anions for waters that have interacted with the various rock types in the Fairbanks area.

than in the schists. This is not surprising because the relatively low permeability and coarse-grained nature of the granitic rocks, compared to the schists, hinder rapid weathering of mineral grains.

Other chemical species that are enriched in waters that flowed through granites are unlikely to be derived from feldspar weathering. Weathering of biotite to vermiculite, for example, may have contributed fluorine to the ground waters. This possibility is consistent with the fact that biotite is the most abundant mafic mineral in the mid-Cretaceous granitic rocks of Pedro and Gilmore domes (Blum, 1985) and the fact that biotite has been demonstrated to have a significantly higher weathering rate $(4 \times$ to $6 \times)$ than plagioclase (Blum and others, 1994). It is even possible that the somewhat higher ${ }^{87} \mathrm{Sr} /{ }^{86} \mathrm{Sr}$ ratio in our ground-water seeps (average $=0.7163$ ), relative to the average equigranular and porphyritic facies of the Gilmore and Pedro domes granitic rocks ( 0.7135 ; Blum, 1985), also reflects the preferential weathering of biotite. This is because biotite generally has high $\mathrm{Rb} / \mathrm{Sr}$ ratios and, as a result, highly radiogenic strontium (Blum and others, 1994).

The greatest dissolved load in a water sample from this study, mainly reflected by elevated calcium, magnesium, sulfate, and bicarbonate concentrations, is for the sample from Dora Creek (site 97-17). The relatively abundant mafic silicate phases within the underlying Chatanika eclogite are easily broken down by circulating ground waters. Elevated trace-element concentrations in the waters at site $97-17$, compared to those flowing through all other lithologies, clearly are the result of more extensive irreversible dissolution of bedrock. It is noteworthy that the major-element chemistry of the sample from Dora Creek is very similar to that of waters collected below mine-waste rock from the Scrafford mine (site 96-23, Goldfarb and others, 1997). The local geology around the Scrafford mine is mapped as Fairbanks Schist, but the hydrogeochemistry suggests an abundance of $\mathrm{Ca}$ - and $\mathrm{Mg}$-rich mineral phases similar to those that characterizes the eclogite.

The one sample with a high dissolved-iron concentration is along Kokomo Creek (site 97-07), which was collected downstream from a large outcrop of basalt. This fine-grained mafic unit is relatively easily weathered and provides a unique fingerprint to waters that interact with it. Iron concentrations of 300$730 \mathrm{ppb}$ for sites on Pilot Creek (site 97-08), a tributary to Dome Creek (site 97-19), and on Silver Creek (site 97-20) are also higher than those from any other waters in this study. Upstream parts of these watersheds all contain significant amounts of loess, suggesting that some of the iron may be derived from loess rather than bedrock. This implies that significant loess accumulations along Kokomo Creek may also be contributing some of the iron in sample 97-07.

Absolute trace-metal concentrations are extremely low (i.e., see table 2-1 in Levinson, 1974), even from Dora Creek (site 97-17), for waters collected from all units, and we interpret these concentrations to mainly reflect local background levels. As expected, an arsenic concentration of $78 \mathrm{ppb}$ in Moose 
Creek below the Hi-Yu mill tailings (site 97-15) likely represents an anthropogenic contribution. Above the mine workings, surface flows are above background levels, but only contain 12 ppb As (site 97-14). Values of 410-420 ppb K for waters at these two sites are much lower than those of any other samples draining the Fairbanks Schist. This could be indicative of the likely broad-scale alteration of rock units in the vicinity of the mesothermal gold-type mineralization. Sericitization of the schist would convert any K-bearing feldspars in rocks surrounding the auriferous quartz veins to muscovite, generally a more resistent mineral to chemical weathering (Ollier, 1969). Only the sample from near the head of Rex Creek (site 97-05), with $48 \mathrm{ppb}$ As, contains a very high natural arsenic concentration and may define an unexposed zone of hydrothermal mineralization in granite on the southeast side of Gilmore dome.

The samples in this study were collected over a broad region, mostly a few tens of kilometers north of Fairbanks, and, typically, waters contained less than $10 \mathrm{ppb}$ As. This is consistent with the findings of Weber (1986) who, in a detailed study of well waters, showed those waters with $>10 \mathrm{ppb}$ arsenic were confined to a 5-km-wide zone between Ester and Gilmore domes (location of site 97-05) and centered along Goldstream Creek. Despite the concentration of gold occurrences to the north of Fox, the lower arsenic concentrations in natural waters in this area indicates that widespread dissolution of sulfides in local aquifers is much less severe than for lithologies along Goldstream Creek.

\section{Summary}

The chemical composition of natural waters in the Fairbanks mining district is strongly influenced by the local geology. Dissolved species in all parts of the studied area are dominated by calcium, bicarbonate, and sulfate. However, calcium concentrations are consistently lower $(<10 \mathrm{ppm})$ in waters draining granites and rocks of the Muskox Sequence, intermediate (13-20 ppm) throughout the Fairbanks Schist, and high (as much as $61 \mathrm{ppm}$ ) for waters that have interacted with the eclogite of the Chatanika assemblage. Sulfate varies from low $(<2 \mathrm{ppm})$ in waters draining granite, to intermediate $(8-36$ $\mathrm{ppm}$ ) in waters from the metasedimentary rocks, to high (140 $\mathrm{ppm}$ ) for waters in contact with the eclogite. Similarly, dissolved $\mathrm{Mg}$ and $\mathrm{K}$ concentrations increase from granite to schist to eclogite.

The presence of unexposed granite within the Fairbanks area may be indicated by the chemical characteristics of surface discharge that has interacted with such buried rock. Uranium at the ppb level, fluorine concentrations of at least 0.5 $\mathrm{ppm}$, or strontium isotopic ratios below about 0.720 within surface waters are good indicators of granitic rocks somewhere along the ground-water flow path. Except for the water collected below the Hi-Yu mill tailings at site 97-15, none of our samples contained dissolved arsenic concentrations above the State of Alaska maxium contaminant level (50 ppb; Alaska Department of Environmental Conservation, 1996). This confirms the fact that soluble and widespread arsenopyrite is mainly confined to aquifers in the area previously described by others (Hawkins and others, 1982; Weber, 1986) between Fairbanks and Fox.

\section{Acknowledgments}

Cynthia Parnow's assistance with measurement of anions and graphics is very much appreciated. We thank Lisa Campbell and Seth Mueller, University of Colorado, for their help in obtaining the Sr-isotope data.

\section{References Cited}

Alaska Department of Environmental Conservation, 1996, Drinking water regulations: State of Alaska, Department of Environmental Conservation Document 18 ACC 80, 199 p.

Blum, J.D., 1985, A petrologic and Rb-Sr isotopic study of intrusive rocks near Fairbanks, Alaska: Canadian Journal of Earth Sciences, v. 22, p. 1314-1321.

Blum, J.D., Erel, Y., and Brown, K., $1994,{ }^{87} \mathrm{Sr} /{ }^{86} \mathrm{Sr}$ ratios of Sierra Nevada stream waters: Implications for relative mineral weathering rates: Geochimica et Cosmochimica Acta, v. 58, p. 5019-5025.

d'Angelo, W.M., and Ficklin, W.H., 1996, Fluoride, chloride, nitrate, and sulfate in aqueous solution by chemically suppressed ion chromatography, in Arbogast, B.F., ed., Analytical Methods Manual for the Mineral Resource Surveys Program, U.S. Geological Survey: U.S. Geological Survey Open-File Report 96-525, p. 149 153.

Goldfarb, R.J., Taylor, C.D., Jr., Meier, A.L., d'Angelo, W.M., and O'Leary, R.M., 1997, Hydrogeochemistry of mine-drainage waters associated with low-sulfide, gold-quartz veins in Alaska, in Dumoulin, J.A., and Gray, J.D., eds., Geologic Studies in Alaska by the U.S. Geological Survey, 1995: U.S. Geological Survey Professional Paper 1574, p. 317.

Hawkins, D.B., Forbes, R.B., Hok, C.I., and Dinkel, D., 1982, Arsenic in water, soil, bedrock, and plants of the Ester dome area of Alaska: Institute of Water Resources, University of Alaska, Fairbanks, Report IWR-103, $82 \mathrm{p}$.

Hem, J.D., 1992, Study and interpretation of the chemical characteristics of natural water: U.S. Geological Survey Water-Supply Paper 2254, $263 \mathrm{p}$.

Levinson, A.A., 1974, Introduction to exploration geochemistry: Wilmette, Illinois, Applied Publishing, $614 \mathrm{p}$.

McCoy, D., Newberry, R.J., Layer, P., DiMarchi, J.J., Bakke, A., Masterman, J.S., and Minehane, D.L., 1997, Plutonic-related gold deposits of interior Alaska, in Goldfarb, R.J., and Miller, L.D., eds., Mineral Deposits of Alaska: Economic Geology Monograph 9, p. 191-241.

Meier, A.L., Grimes, D.J., and Ficklin, W.H., 1994, Inductively coupled plasma mass spectrometry-A powerful analytical tool for mineral resource and environmental studies [abs.], in Carter, L.M.H., Toth, M.I., and Day, W.C., eds., USGS Research on Mineral Resources1994, Part A-Program and Abstracts, Ninth V.E. McKelvey Forum on Mineral and Energy Resources: U.S. Geological Survey Circular 1103-A, p. 67-68.

Metz, P.A., 1991, Metallogeny of the Fairbanks district, Alaska, and adjacent areas: University of Alaska, School of Mineral Engineering, Minerals Research Industry Laboratory Report 90, 370 p. 
Newberry, R.J., and Bundtzen, T.K., 1996, Preliminary geologic map of the Fairbanks mining district, Alaska: Alaska Division of Geological and Geophysical Surveys, Public-Data File 96-12, 14 p., 2 sheets.

Ollier, C.D., 1969, Weathering: New York, American Elsevier, 304 p.

Ray, S.R., Vohden, J., and Morgan, W., 1992, Investigation of trace metals related to placer mining on Fairbanks and Porcupine Creeks: Alaska Division of Geological and Geophysical Surveys, Public-Data File 92$13,17 \mathrm{p}$.

Reviewers: P. Briggs, R. Eppinger.
Robinson, M.S., Smith, T.E., and Metz, P.A., 1990, Bedrock geology of the Fairbanks mining district, Alaska: Alaska Division of Geological and Geophysical Surveys Professional Report 106, 2 sheets, scale $1: 63,360$.

Weber, E.F., 1986, A stochastic model and risk analysis of arsenic, well depth, and well yield in the Fairbanks area, Alaska: University of Alaska, Fairbanks, unpub. M.S. thesis, $196 \mathrm{p}$. 


\title{
U.S.Geological Survey Reports on Alaska Released in 1997
}

\author{
Compiled by John P. Galloway and Susan Toussaint
}

[Some reports dated 1994, 1995, or 1996 did not become available until 1997; they are included in this listing]

Ager, Thomas, 1997, How does climate change influence Alaska's vegetation? Insights from the fossil record: U.S. Geological Survey Fact Sheet FS-071-97, 2 p.

Bailey, E.A., Hines, M.E., and Gray, J.E., 1997, Mercury speciation in soils and vegetation, southwest Alaska, in Wanty, R.B., Marsh, S.P., and Gough, L.P., eds., 4th International Symposium on Environmental Geochemistry Proceedings: U.S. Geological Survey Open-File Report 97-496, p. 8.

Bailey, E.A., and Gray, J.E., 1997, Mercury in the terrestrial environment, Kuskokwim Mountains region, southwestern Alaska, in Dumoulin, J.A., and Gray, J.E., eds., 1997, Geologic Studies in Alaska by the U.S. Geological Survey, 1995: U.S. Geological Survey Professional Paper 1574 , p. $41-56$.

Bailey, E.A., Keith, W.J., Bickerstaff, Damon, Dempsey, David, and Miller, M.L., 1996, Analytical results and sample locality maps of streamsediment, panned concentrate, stream-water, and soil samples from the Stuyahok study area, part of Holy Cross A-4 and A-5 quadrangles, Alaska : U.S. Geological Survey Open-File Report 96-505-C, 44 p., 1 diskette.

Bie, S.W., Arbogast, B.F., Light, T.D., and Weber, F.R., 1997, Analytical results of rock samples from the Livengood $1^{\circ} \times 3^{\circ}$ quadrangle, Alaska: U.S. Geological Survey Open-File Report 97-446, 131 p., 3 maps, scale 1:250,000.

Brabets, T.P., 1997, Geomorphology of the lower Copper River, Alaska: U.S. Geological Survey Professional Paper 1581, 89 p.

Brabets, T.P., 1997, Regional streamflow information in Alaska-An investment in the future: U.S. Geological Survey Open-File Report 96-630, $2 \mathrm{p}$.

Brew, D.A., 1997, Description and regional setting of the Silver Bay segment of the Sitka fault zone, southeastern Alaska, and evidence for possible sinistral separation, in Dumoulin, J.A., and Gray, J.E., eds., 1997, Geologic Studies in Alaska by the U.S. Geological Survey, 1995: U.S. Geological Survey Professional Paper 1574, p. 307-316.

Brew, D.A., 1997, Reconnaissance geologic map of the Petersburg A-2 quadrangle, southeastern Alaska: U.S. Geological Survey Open-File Report 97-156-A, 20 p., 1 map, scale 1:63,360.

Brew, D.A., 1997, Reconnaissance geologic map of the Petersburg A-3 quadrangle, southeastern Alaska: U.S. Geological Survey Open-File Report 97-156-B, 24 p., 1 map, scale 1:63,360

Brew, D.A., 1997, Reconnaissance geologic map of the Petersburg B-1 quadrangle, southeastern Alaska: U.S. Geological Survey Open-File Report 97-156-C, 20 p., 1 map, scale 1:63,360.
Brew, D.A., 1997, Reconnaissance geologic map of the Petersburg B-2 quadrangle, southeastern Alaska: U.S. Geological Survey Open-File Report 97-156-D, 21 p., 1 map, scale 1:63,360.

Brew, D.A., 1997, Reconnaissance geologic map of the Petersburg B-3 quadrangle, southeastern Alaska: U.S. Geological Survey Open-File Report 97-156-E, 23 p., 1 map, scale 1:63,360.

Brew, D.A., 1997, Reconnaissance geologic map of the Petersburg B-4 quadrangle, southeastern Alaska: U.S. Geological Survey Open-File Report 97-156-F, 20 p., 1 map, scale 1:63,360.

Brew, D.A., 1997, Reconnaissance geologic map of the Petersburg B-5 quadrangle, southeastern Alaska: U.S. Geological Survey Open-File Report 97-156-G, 19 p., 1 map, scale 1:63,360.

Brew, D.A., 1997, Reconnaissance geologic map of the Petersburg C-1 quadrangle, southeastern Alaska: U.S. Geological Survey Open-File Report 97-156-H, 23 p., 1 map, scale 1:63,360.

Brew, D.A., 1997, Reconnaissance geologic map of the Petersburg C-3 quadrangle, southeastern Alaska: U.S. Geological Survey Open-File Report 97-156-I, 18 p., 1 map, scale 1:63,360.

Brew, D.A., 1997, Reconnaissance geologic map of the Petersburg C-4 quadrangle, southeastern Alaska: U.S. Geological Survey Open-File Report 97-156-J, 21 p., 1 map, scale 1:63,360.

Brew, D.A., 1997, Reconnaissance geologic map of the Petersburg C-5 quadrangle, southeastern Alaska: U.S. Geological Survey Open-File Report 97-156-K, 18 p., 1 map, scale 1:63,360.

Brew, D.A., 1997,Reconnaissance geologic map of the Petersburg D-4 quadrangle, southeastern Alaska: U.S. Geological Survey Open-File Report 97-156-L, 21 p., 1 map, scale 1:63,360.

Brew, D.A., 1997, Reconnaissance geologic map of the Petersburg D-5 quadrangle, southeastern Alaska: U.S. Geological Survey Open-File Report 97-156-M, 22 p., 1 map, scale 1:63,360.

Brew, D.A., compiler, 1997, Geologic bibliography for the Atlin, Mount St. Elias, Skagway, and Yakutat quadrangles and adjacent areas of the Yukon and British Columbia: U.S. Geological Survey Open-File Report 97-159, $23 \mathrm{p}$.

Brew, D.A., and Grybeck, D.J., 1997, Combined description of map units and correlation of map units for the Petersburg-Wrangell area 1:63,360-scale geologic maps, southeastern Alaska: U.S. Geological Survey Open-File Report 97-156-0, 108 p.

Brew, D.A., Grybeck, D.J., Taylor, C.D., Jachens, R.C., Cox, D.P., Barnes, D.F., Koch, R.D., Morin, R.I., and Drinkwater, J.L., 1996, Undiscovered mineral resources of southeastern Alaska-Revised 
mineral-resource-assessment-tract descriptions: U.S. Geological Survey Open-File Report 96-716, 131 p., 1 map, scale 1:1,000,000.

Brew, D.A., and Koch, R.D., 1997, Reconnaissance geologic map of the Bradfield Canal B-6 quadrangle, southeastern Alaska: U.S. Geological Survey Open-File Report 97-156-N, 22 p., 1 map, scale 1:63,360.

Campbell, D.L., and Nokleberg, W.J., 1997, Interpretation of aeromagnetic map and related geophysical data for Mount Hayes $1^{\circ} \times 3^{\circ}$ quadrangle, Alaska: U.S. Geological Survey Open-File Report 97-280, 64 p., 2 maps, scale 1:250,000.

Carlson, P.R., Kvenvolden, K.A., Hostettler, F.D., Rosenbauer, R.J., and Warden, Augusta, 1997, Fate of spilled oil in Prince William Sound: Diary of a forensic geology study: U.S. Geological Survey Open-File Report 97-518, $88 \mathrm{p}$.

Celebi, M., Page, R.A., and Seekins, Linda, 1995, Building safer structures: U.S. Geological Survey Fact Sheet FS-167-95, 2 p.

Dorava, J.M., and Liepitz, G.S., 1996, Balancing the three R's (regulation, research, and restoration) on the Kenai River, Alaska: U.S. Geological Survey Fact Sheet FS-160-96, 2 p.

Dorava, J.M., and Moore, G.W., 1997, Effects of boatwakes on streambank erosion Kenai River, Alaska: U.S. Geological Survey Water Resources Investigations Report 97-4105, 84 p.

Dover, James, 1997, Alaska resource data file: Arctic quadrangle: U.S. Geological Survey Open-File Report 97-292, $10 \mathrm{p}$.

Dover, James, 1997, Alaska resource data file: Table Mountain quadrangle: U.S. Geological Survey Open-File Report 97-293, 14 p.

Dover, James, 1997, Alaska resource data file: Christian quadrangle: U.S. Geological Survey Open-File Report 97-294, 6 p.

Dover, James, 1997, Alaska resource data file: Coleen quadrangle: U.S. Geological Survey Open-File Report 97-295, 39 p.

Dover, James, 1997, Alaska resource data file: Howard Pass quadrangle: U.S. Geological Survey Open-File Report 97-296, 47 p.

Dover, James, 1997, Alaska resource date file: Misheguk Mountain quadrangle: U.S. Geological Survey Open-File Report 97-297, 18 p.

Dumoulin, J.A., and Gray, J.E., eds., 1997, Geologic studies in Alaska by the U.S. Geological Survey, 1995: U.S. Geological Survey Professional Paper 1574, 328 p.

Dumoulin, J.A., and Harris, A.G., 1997, Kinderhookian (Lower Mississippian) calcareous rocks of the Howard Pass quadrangle, west-central Brooks Range, in Dumoulin, J.A., and Gray, J.E., eds., 1997, Geologic Studies in Alaska by the U.S. Geological Survey, 1995: U.S. Geological Survey Professional Paper 1574, p. 243-268.

Eppinger, R.G., Sutley, S.J., and McHugh, J.B., 1997, Environmental geochemical study of the Nabesna gold skarn and Kennecott stratabound copper deposits, Alaska, in Dumoulin, J.A., and Gray, J.E., eds., 1997, Geologic Studies in Alaska by the U.S. Geological Survey, 1995: U.S. Geological Survey Professional Paper 1574, p. 19-39.

Evans, K.R., 1997, Offshore geologic framework and sedimentology of the Gulf of Alaska: Selected bibliography of U.S. Geological Survey studies (1970-present): U.S. Geological Survey Open-File Report 97$26,15 \mathrm{p}$.

Finch, W.I., 1996, Uranium provinces of North America-Their definition, distribution, and models: U.S. Geological Survey Bulletin 2141, 18 p., 2 plates.

Flores, R.M., Stricker, G.D., and Stiles, R.B., 1997, Tidal influence on deposition and quality of coals in the Miocene Tyonek Formation, Beluga coal field, upper Cook Inlet, Alaska, in Dumoulin, J.A., and Gray, J.E., eds., 1997, Geologic Studies in Alaska by the U.S. Geological Survey, 1995: U.S. Geological Survey Professional Paper 1574, p. 137-156.
Flowers, Chris, 1997, Observations of geothermal activitiy near Pavlof Volcano on the Alaska Peninsula during March and April of 1996: U.S. Geological Survey Open-File Report 97-146, 13 p.

Fountain, A.G., Krimmel, R.M., and Trabant, D.C., 1997, A strategy for monitoring glaciers: U.S. Geological Survey Circular 1132, $19 \mathrm{p}$.

Frenzel, S.A., 1997, Cook Inlet basin, Alaska, National Water-Quality Assessment Program: U.S. Geological Survey Fact Sheet 97-153, 4 p.

Galloway, J.P., and Carter, L.D., 1997, A selected bibliography of sand and gravel resources Arctic Coastal Plain, Alaska: U.S. Geological Survey Open-File Report 97-702, 12 p.

Galloway, J.P., and Toussaint, Susan, 1997, Reports about Alaska in nonUSGS publications released in 1995 that include USGS authors, in Dumoulin, J.A., and Gray, J.E., eds., 1997, Geologic Studies in Alaska by the U.S. Geological Survey, 1995: U.S. Geological Survey Professional Paper 1574, p. 323-328.

Galloway, J.P., and Toussaint, Susan, 1997, U.S. Geological Survey reports on Alaska released in 1995, in Dumoulin, J.A., and Gray, J.E., eds., 1997, Geologic Studies in Alaska by the U.S. Geological Survey, 1995: U.S. Geological Survey Professional Paper 1574, p. 317-321.

Glass, R.L., 1996, Alaska wetland resources in Fretwell, J.D., Williams, J.S., and Redman, P.J., compilers, National Water Summary on Wetland Resources: U.S. Geological Survey Water-Supply Paper 2425, p. 107-114.

Goldfarb, R.J., Taylor, C.D., Meier, A.L., d'Angelo, W.M., and O'Leary, R.M., 1997, Hydrogeochemistry of mine-drainage waters associated with low-sulfide, gold-quartz veins in Alaska, in Dumoulin, J.A., and Gray, J.E., eds., 1997, Geologic Studies in Alaska by the U.S. Geological Survey, 1995: U.S. Geological Survey Professional Paper 1574, p. 3-17.

Gough, L.P., and Crock, J.G., 1997, Distinguishing between natural geologic and anthropogenic trace element sources, Denali National Park and Preserve, in Dumoulin, J.A., and Gray, J.E., eds., 1997, Geologic Studies in Alaska by the U.S. Geological Survey, 1995: U.S. Geological Survey Professional Paper 1574, p. 57-71.

Gough, L., Day, W., Crock, J., Gamble, B., and Henning, M., 1997, Placergold mining in Alaska-Cooperative studies on the effect of suction dredge operations on the Fortymile River: U.S. Geological Survey Fact Sheet FS-155-97, 4 p.

Grauch, V.J.S., and Castellanos, Esther, 1995, Revised digital aeromagnetic data for areas in and adjacent to the National Petroleum Reserve Area (NPRA), North Slope, Alaska: U.S. Geological Survey Open-File Report 95-835, $11 \mathrm{p}$.

Gray, J.E., 1997, Environmental geochemistry and mercury speciation of abandoned mercury mines in southwestern Alaska, in Wanty, R.B., Marsh, S.P., and Gough, L.P., eds., 4th International Symposium on Environmental Geochemistry Proceedings: U.S. Geological Survey Open-File Report 97-496, p. 31.

Gray, J.E., Folger, P.F., and Hageman, P.L., 1997, Map showing the distribution of anomalous concentrations of mercury, antimony, and arsenic in stream sediment, heavy-mineral concentrate, and aquatic moss in the Iditarod quadrangle, Alaska: U.S. Geological Survey Miscellaneous Field Studies Map MF 2219-C, 1 map, scale 1:250.000.

Gray, J.E., Kelley, K.D., Goldfarb, R.J., and Taylor, C.D., 1997, Environmental studies of mineral deposits in Alaska, in Wanty, R.B., Marsh, S.P., and Gough, L.P., eds., 4th International Symposium on Environmental Geochemistry Proceedings: U.S. Geological Survey Open-File Report 97-496, p. 31.

Gray, J.E., Motooka, J.M., and O'Leary, R.M., 1997, Map showing the distribution of anomalous concentrations of gold and silver in stream sediment, heavy-mineral concentrate, and aquatic moss in the 
Iditarod quadrangle, Alaska: U.S. Geological Survey Miscelllaneous Field Studies Map MF 2219-D, 1 map, scale 250,000.

Gray, J.E., Ryder, J.L., and Taylor, C.D., 1997, Map showing the distribution of anomalous concentations of copper, lead, zinc, tin, and tungsten in stream sediment and heavy-mineral concentrate in the Iditarod quadrangle, Alaska: U.S. Geological Survey Miscellaneous Field Studies Map MF 2219-E, 1 map, scale 1:250,000.

Gray, J.E., and Theodorakos, P.M., 1997, Areas favorable for metallic mineral resources and newly discovered mineral occurrences in the Buckstock Mountains area, southwestern Alaska, in Dumoulin, J.A., and Gray, J.E., eds., 1997, Geologic Studies in Alaska by the U.S. Geological Survey, 1995: U.S. Geological Survey Professional Paper 1574, p. 111-123.

Gray, J.E., Theodorakos, P.M., Lee, G.K., Hageman, P.L., and Sutley, S.J., 1997, Analytical data of stream-sediment and heavy-mineral-concentrate samples collected from the Buckstock Mountains and surrounding areas, Sleetmute quadrangle, southwest Alaska: U.S. Geological Survey Open-File Report 97-743-A, 147 p., plus 97-743-B, 1 diskette.

Grommé, Sherman, and Hillhouse, J.W., 1997, Paleomagnetic results from Devonian and Permian rocks at Saginaw Bay, Kuiu Island, southeastern Alaska, in Dumoulin, J.A., and Gray, J.E., eds., 1997, Geologic Studies in Alaska by the U.S. Geological Survey, 1995: U.S. Geological Survey Professional Paper 1574, p. 295-306.

Haeussler, P.J., and Anderson, R.S., 1997, The "Twin Peaks fault:" Not a tectonic or seismogenic structure, in Dumoulin, J.A., and Gray, J.E., eds., 1997, Geologic Studies in Alaska by the U.S. Geological Survey, 1995: U.S. Geological Survey Professional Paper 1574, p. 93-99.

Harris, A.G., Brenckle, P.L., Baesemann, J.F., Krumhardt, A.P., and Gruzlovic, P.D., 1997, Comparison of conodont and calcareous microfossil biostratigraphy and lithostratigraphy of the Lisburne Group (Carboniferous), Sadlerochit Mountains, northeast Brooks Range, Alaska, in Dumoulin, J.A., and Gray, J.E., eds., 1997, Geologic Studies in Alaska by the U.S. Geological Survey, 1995: U.S. Geological Survey Professional Paper 1574, p. 195-219.

Kaufman, D.S., and Stilwell, K.B., 1997, Preliminary evaluation of emergent postglacial shorelines, Naknek and Iliamna Lakes, southwestern Alaska, in Dumoulin, J.A., and Gray, J.E., eds., 1997, Geologic Studies in Alaska by the U.S. Geological Survey, 1995: U.S. Geological Survey Professional Paper 1574, p. 73-81.

Keith, W.J., and Miller, M.L., 1997, Alaska resource data file: Sleetmute quadrangle: U.S. Geological Survey Open-File Report 97-32, 38 p.

Kelley, J.S., 1997, Alaska resource data file: Demarcation Point quadrangle: U.S. Geological Survey Open-File Report 96-678, 14 p.

Kelley, J.S., 1997, Alaska resource data file: Umiat quadrangle: U.S. Geological Survey Open-File Report 96-679, 6 p.

Kelley, J.S., 1997, Alaska resource data file: Mount Michelson quadrangle: U.S. Geological Survey Open-File Report 96-681, 21 p.

Kelley, Karen, 1997, Alaska resource data file: Chandler Lake quadrangle: U.S. Geological Survey Open-File Report 97-265, 24 p.

Kelley, Karen, 1997, Alaska resource data file: Killik River quadrangle: U.S. Geological Survey Open-File Report 97-266, 20 p.

Kelley, K.D., 1997, Natural acid drainage associated with shale-hosted $\mathrm{Ag}-\mathrm{Pb}-\mathrm{Zn}$ massive sulfide deposits in the Brooks Range, Northern Alaska, USA, in Wanty, R.B., Marsh, S.P., and Gough, L.P., eds., 4th International Symposium on Environmental Geochemistry Proceedings: U.S. Geological Survey Open-File Report 97-496, p. 44.

Kelley, K.D., Bailey, E.A., Briggs, P.H., Motooka, J.M., and Meier, A.L., 1996, Digital release of stream-sediment, heavy-mineralconcentrate, soil, water, and rock geochemical data collected in the
Howard Pass $1^{\circ} \times 3^{\circ}$ quadrangle, Alaska: U.S. Geological Survey Open-File Report 96-711, 31/2"-diskette for IBM PC or compatible.

Kelley, K.D., Taylor, C.D., and Cieutat, B.A., 1997, Silver-lead-zinc mineral occurrences in the Howard Pass quadrangle, Brooks Range, Alaska, in Dumoulin, J.A., and Gray, J.E., eds., 1997, Geologic Studies in Alaska by the U.S. Geological Survey, 1995: U.S. Geological Survey Professional Paper 1574, p. 101-110.

Kennedy, B.W., Mayo, L.R., Trabant, D.C., and March, R.S., 1997, Air temperature and precipitation data, Gulkana Glacier, Alaska, 1968-96: U.S. Geological Survey Open-File Report 97-358, 144 p.

Kleinman, J.W., Iwatsubo, E.Y., Power, J.A., and Endo, E.T., 1997, Geodetic studies in the Novarupta area, Katmai National Park, Alaska, 1990 to 1995, in Dumoulin, J.A., and Gray, J.E., eds., 1997, Geologic Studies in Alaska by the U.S. Geological Survey, 1995: U.S. Geological Survey Professional Paper 1574, p. 83-92.

Light, T.D., Barnwell, C.E., and Andrade, Julio, 1997, Livengood quadrangle GeoExplorer: U.S. Geological Survey Open-File Report 97-484-E, 1 CD-ROM.

Light, T.D., and Lee, G.K., 1997, Map showing distribution and occurrence of gold-bearing samples from the Livengood quadrangle, Alaska: U.S. Geological Survey Open-File Report 97-484-C, 21 p., 1 map, scale 1:250,000.

Lilly, M.R., DePalma, K.L., and Benson, S.L., 1995, Selected environmental and geohydrologic reports for the Fort Wainwright and Fairbanks areas, Alaska, as of July 1995: U.S. Geological Survey Open-File Report 95-420, $62 \mathrm{p}$.

Lorenson, T.D., and Kvenvolden, K.A., 1997, Methane in coastal sea water, sea ice, and bottom sediments, Beaufort Sea, Alaska--Results from 1995: U.S. Geological Survey Open-File Report 97-54, 77 p.

March, R.S., Mayo, L.R., and Trabant, D.C., 1997, Snow and ice volume on Mount Spurr Volcano, Alaska, 1981: U.S. Geological Survey WaterResources Investigations Report 97-4142, 36 p., 2 maps, scale $1: 50,000$.

March, R.S., and Trabant, D.C., 1997, Mass balance, meteorological, ice motion, surface altitude, and runoff data at Gulkana Glacier, Alaska, 1993 balance year: U.S. Geological Survey Water Resources Investigations Report 96-4299, $30 \mathrm{p}$.

McCammon, R.B., Light, T.D., Rinehart, C.D., Weber, F.R., Lee, G.K., and Bie, S.W., 1997, Map showing mineral resource potential of the Livengood quadrangle, Alaska: U.S. Geological Survey Open-File Report 97-484-B, 47 p., 1 map, scale 1:250,000.

McGimsey, R.G., and Neal, C.A., 1996, 1995 volcanic activity in Alaska and Kamchatka: Summary of events and response of the Alaska Volcano Observatory: U.S. Geological Survey Open-File Report 96-738, $22 \mathrm{p}$.

Miller, L.D., Goldfarb, R.J., Snee, L.W., McClelland, W.C., and Klipfel, P.D., 1997, Paleocene molybdenum mineralizaiton in the eastern Coast batholith, Taku River region, and new age constraints on batholith evolution, in Dumoulin, J.A., and Gray, J.E., eds., 1997, Geologic Studies in Alaska by the U.S. Geological Survey, 1995: U.S. Geological Survey Professional Paper 1574, p. 125-135.

Moore, T.E., Hemming, Sidney, and Sharp, W.D., 1997, Provenance of the Carboniferous Nuka Formation, Brooks Range, Alaska: A multicomponet isotope provenance study with implications for age of cryptic crystalline basement, in Dumoulin, J.A., and Gray, J.E., eds., 1997, Geologic Studies in Alaska by the U.S. Geological Survey, 1995: U.S. Geological Survey Professional Paper 1574, p. 173-194.

Morin, R.L., 1997, Gravity models of Abby Creek and Bion barite deposits, Howard Pass quadrangle, northwestern Brooks Range, Alaska:U.S. Geological Survey Open-File Report 97-704, 15 p. 
Morin, R.L., 1997, Gravity and magnetic maps of part of the Drenchwater Creek stratiform zinc-lead-silver deposit, Howard Pass quadrangle, northwestern Brooks Range, Alaska: U.S. Geological Survey OpenFile Report 97-705, $9 \mathrm{p}$.

Mull, C.G., Harris, A.G., and Carter, J.L., 1997, Lower Mississippian (Kinderhookian) biostratigraphy and lithostratigraphy of the western Endicott Mountains, Brooks Range, Alaska, in Dumoulin, J.A., and Gray, J.E., eds., 1997, Geologic Studies in Alaska by the U.S. Geological Survey, 1995: U.S. Geological Survey Professional Paper 1574, p. 221-242.

Neal, Christina, 1997, What is the Alaska Volcano Observatory?: U.S. Geological Survey Open-File Report 97-443, $2 \mathrm{p}$.

Neal, C.A., Casadevall, T.J., Miller, T.P., Hendley, J.W., II, and Stauffer, P.H., 1997, Volcanic ash-Danger to aircraft in the north Pacific: U.S. Geological Survey Fact Sheet 030-97, 2 p.

Neal, Christina, and McGimsey, Robert, 1996, Volcanoes of the Wrangell Mountains and Cook Inlet region, Alaska-Selected photographs: U.S. Geological Survey Digital Data Series DDS-39, 1 CD-ROM.

Neal, Christina, and McGimsey, Robert, 1997, Volcanoes of the Alaska Peninsula and Aleutian Islands, Alaska-Selected photographs: U.S. Geological Survey Digital Data Series DDS-40, 1 CD-ROM.

Neal, C.A., and McGimsey, R.G., 1997, 1996 volcanic activity in Alaska and Kamchatka: Summary of events and response of the Alaska Volcano Observatory: U.S. Geological Survey Open-File Report 97-433, 34 p.

Neal, E.G., 1998, Hydrologic investigation of the Ophir Creek watershed near Yakutat, Alaska: U.S. Geological Survey Open-File Report 98$199,33 \mathrm{p}$.

Nelson, Steven, 1997, Alaska resource data file: Ambler River quadrangle: U.S. Geological Survey Open-File Report 97-856, 98 p.

Newberry, R.J., and Brew, D.A., 1997, The upper Triassic Greens Creek VMS (volcanogenic massive sulfide) deposit and Woewodski Island VMS prospects, southeastern Alaska: Chemical and isotopic data for rocks and ores demonstrate similarity of these deposits and their host rocks: U.S. Geological Survey Open-File Report 97-539, 49 p.

Nokleberg, W.J., Bundtzen, T.K., Dawson, K.M., Eremin, R.A., Goryachev, N.A., Koch, R.D., Ratkin, V.V., Rozenblum, I.S., Shpikerman, V.I., Frolov, Yu.F., Gorodinsky, M.E., Melnikov, V.D., Ognyanov, N.V., Petrachenko, E.D., Petrachenko, R.I., Pozdeev, A.I., Ross, K.V., Wood, D.H., Grybeck, D., Khanchuck, A.I., Kovbas, L.I., Nekrasov, I.Ya., and Sidorov, A.A., 1996, Significant metalliferous and selected non-metalliferous lode deposits and placer districts for the Russian Far East, Alaska, and the Canadian Cordillera: U.S. Geological Survey OpenFile Report 96-513-A, $385 \mathrm{p}$.

Nokleberg, W.J., Parfenov, L.M., Monger, J.W.H., Baranov, B.V., Byalobzhesky, S.G., Bundtzen, T.K., Feeney, T.D., Fujita, K., Gordey, S.P., Grantz, A., Khanchuk, A.I., Natal'in, B.A., Natapov, L.M., Norton, I.O., Patton, W.W., Jr., Plafker, G., Scholl, D.W., Sokolov, S.D., Sosunov, G.M., Stone, D.B., Tabor, R.W., Tsukanov, N.V., and Vallier, T.L., 1997, Summary Circum-North Pacific tectonostratigraphic terrane map: U.S. Geological Survey Open-File Report 96-727, 1 map, scale 1:10,000,000.

O'Sullivan, P.B., Plafker, George, and Murphy, J.M., 1997, Apatite fissiontrack thermotectonic history of crystalline rocks in the northern Saint Elias Mountains, Alaska, in Dumoulin, J.A., and Gray, J.E., eds., 1997, Geologic Studies in Alaska by the U.S. Geological Survey, 1995: U.S. Geological Survey Professional Paper 1574, p. 283-293.

Patton, W.W., Jr., and Moll-Stalcup, E.J., 1996, Geologic map of the Unalakleet quadrangle, west-central Alaska: U.S. Geological Survey Miscellaneous Investigations Map I-2559, 1 map, scale 1:250,000, $39 \mathrm{p}$.
Richter, D.H., Moll-Stalcup, Elizabeth, Duffield, W.A., and Shew, Nora, 1997, Geologic map of the Nabesna A-6 quadrangle, Alaska: U.S. Geological Survey Open-File Report 97-475, 1 map, scale 1:63,360, 1 pamphlet, variously paged.

Rickman, R.L., 1996, Effect of ice formation and streamflow on salmon incubation habitat in the lower Bradley River, Alaska: U.S. Geological Survey Water Resources Investigations Report 96-4202, 63 p.

Rickman, R.L., and Rosenkrans, D.S., 1997, Hydrologic conditions and hazards in the Kennicott River basin, Wrangell-St. Elias National Park and Preserve, Alaska: U.S. Geological Survey Water Resources Investigations Report 96-4296, variously paged.

Riehle, J.R., Fleming, M.D., Molnia, B.F., Dover, J.H., Kelley, J.S., Miller, M.L., Nokleberg, W.J., Plafker, George, and Till, A.B., 1997, Digital shaded-relief image of Alaska: U.S. Geological Survey Miscellaneous Investigations Series Map 2585, 1 sheet, scale l:2,500,000, plus explanatory pamphlet, $11 \mathrm{p}$.

Rinehart, C.D., Light, T.D., and Shew, N.B., 1997, Petrography and radiometric ages for selected rocks from the Livengood quadrangle, Alaska: U.S. Geological Survey Open-File Report 97-484-D, 22 p.

Roush, James, and Brease, Phil, 1997, Glacier monitoring in Denali National Park and Preserve, Alaska: Integrating field study and remote sensing in Williams, R.S., Jr., and Ferrigno, J.G., compilers, Final Report of the Workshop on Long-Term Monitoring of Glaciers of North America and Northwestern Europe: U.S. Geological Survey Open-File Report 98-31, p. 91.

Rowan, E.L., 1997, Basin evolution, and the timing and extent of oil generation, Canning River region, North Slope, Alaska: Preliminary Basin2 calculations assuming a conductive thermal history: U.S. Geological Survey Open-File Report 97-711,61 p.

Saltus, R.W., and Simmons, G.C., 1997, Composite and merged aeromagnetic data for Alaska: A web site for distribution of gridded data and plot files: U.S. Geological Survey Open-File Report 97-520, $15 \mathrm{p}$.

Saltus, R.W., Meyer, J.F., Jr., Barnes, D.F., and Morin, R.L., 1997, Tectono-geophysical domains of interior Alaska as interpreted from new gravity and aeromagnetic data compilations, in Dumoulin, J.A., and Gray, J.E., eds., 1997, Geologic Studies in Alaska by the U.S. Geological Survey, 1995: U.S. Geological Survey Professional Paper 1574, p. 157-171.

Schneider, J.L, ed., 1997, 1996 annual report on Alaska's mineral resources: U.S. Geological Survey Circular 1142, $70 \mathrm{p}$.

Silberling, N.J., Grant-Mackie, J.A., and Nichols, K.M., 1997, The late Triassic bivalve Monotis in accreted terranes of Alaska: U.S. Geological Survey Bulletin 2151, $32 \mathrm{p}$.

Snyder, E.F., 1997, Water-resources activities of the U.S. Geological Survey in Alaska, 1997: U.S. Geological Survey Fact Sheet 028-97, 4 p.

St. John, J.M., and Babcock, L.E., 1997, Late Middle Cambrian trilobites of Siberian aspect from the Farewell terrane, southwestern Alaska, in Dumoulin, J.A., and Gray, J.E., eds., 1997, Geologic Studies in Alaska by the U.S. Geological Survey, 1995: U.S. Geological Survey Professional Paper 1574, p. 269-281.

Symonds, R.B., Ritchie, B.E., McGimsey, R.G., Ort, M.H., Poreda, R.J., Evans, W.C., and Janik, C.J., 1997, Investigations of gas seeps and springs in the vicinity of the Gas Rocks, south shore Becharof Lake, Alaska: U.S. Geological Survey Open-File Report 97-127, 27 p.

Trabant, D.C., and Hawkins, D.B., 1997, Glacier ice-volume modeling and glacier volumes on Redoubt Volcano, Alaska: U.S. Geological Survey Water-Resources Investigations Report 97-4187, $29 \mathrm{p}$.

Trabant, D.C., with contributions from workshop participants Benson, C.S., Echelmeyer, Keith, Brease, Phil, and Roush, James, 1997, Data base and geographical perspective for monitoring glaciers of 
Alaska in Williams, R.S., Jr., and Ferrigno, J.G., compilers, Final Report of the Workshop on Long-Term Monitoring of Glaciers of North America and Northwestern Europe: U.S. Geological Survey Open-File Report 98-31, p. 28-37.

U.S. Geological Survey, 1994, National Marine and Coastal Geology Program: A plan for geologic research on environmental hazards and resources issues affecting the Nation's coastal realms and marine federal lands: U.S. Geological Survey, $65 \mathrm{p}$.

U.S. Geological Survey, 1996, U.S. Geological Survey programs in Alaska: U.S. Geological Survey Fact Sheet FS-001-96, 4 p.

U.S. Geological Survey, Volcano Hazards Team, 1997, Volcano Hazards Program five-year science plan-1998 to 2002: U.S. Geological Srvey Open-File Report 97-680, 20 p.

Waitt, R.B., and Begét, J.E., with contributions from Juergen Kienle, 1996, Provisional geologic map of Augustine Volcano, Alaska: U.S. Geological Survey Open-File Report 96-516, 44 p., 1 map, scale 1:25,000.

Wang, Bronwen, 1997, Trace elements in the Kuskokwim River, Alaska, in Wanty, R.B., Marsh, S.P., and Gough, L.P., eds., 4th International Symposium on Environmental Geochemistry Proceedings: U.S. Geological Survey Open-File Report 97-496, p. 95.

Wanty, R.B., Wang, B., and Vohden, J., 1997, Studies of suction dredge gold-placer mining operations along the Fortymile River, eastern Alaska: U.S. Geological Survey Fact Sheet FS-154-97, 2 p.
Waythomas, C.F., Miller, T.P., McGimsey, R.G., and Neal, C.A., 1997, Preliminary volcanic-hazard assessment for Pavlof Volcano, Alaska: U.S. Geological Survey Open-File Report 97-135, 1 map, scale $1: 250,000$.

Weber, F.R., Wheeler, K.L., Rinehart, C.D., and Light, T.D., 1997, Generalized geologic map of the Livengood quadrangle, Alaska: U.S. Geological Survey Open-File Report 97-484-A, 1 map, scale 1:250,000.

Williams, R.S., Jr., and Ferrigno, J.G., workshop coordinators, 1997, Final report of the workshop on long-term monitoring of glaciers of North America and northwestern Europe: U.S. Geological Survey OpenFile Report 98-31, $144 \mathrm{p}$.

Wilson, F.H., 1997, Alaska resource data file: Cold Bay quadrangle: U.S. Geological Survey Open-File Report 97-168, 17 p.

Wilson, F.H., 1997, Alaska resource data file: False Pass quadrangle: U.S. Geological Survey Open-File Report 97-169, 16 p.

Wilson, F.H., Weber, F.R., Dochat, T.M., Miller, T.P., and Detterman, R.L., 1997, Revised geologic map of the Cold Bay and False Pass quadrangles, Alaska Peninsula: U.S. Geological Survey Open-File Report $97-$ 866, 34 p., 1 sheet, scale 1:250,000.

Yeend, Warren, 1997, Reconnaissance geologic map of surficial deposits for the Mount McKinley quadrangle, Alaska: U.S. Geological Survey Open-File Report 97-138, 1 map, scale 1:250,000. 



\title{
Reports About Alaska in Non-USGS Publications Released in 1997 that Include USGS Authors
}

\author{
Compiled by John P. Galloway and Susan Toussaint
}

[Some reports dated 1995 or 1996 did not become available until 1997; they are included in this listing. USGS authors are marked with asterisks $\left.\left(^{*}\right)\right]$

*Adams, K.E., Mull, C.G., and Crowder, R.K., 1997, Permian deposition in the north central Brooks Range, Alaska: Constraints for tectonic reconstructions: Journal of Geophysical Research, v. 102, no. B9, p. 20727-20748.

Ashleman, J.C., *Taylor, C.D., and Smith, P.R., 1997, Porphyry molybdenum deposits of Alaska, with emphasis on the geology of the Quartz Hill deposit, southeastern Alaska, in Goldfarb, R.J., and Miller, L.D., eds., 1997, Mineral Deposits of Alaska: Economic Geology Monograph 9, p. 334-354.

*Bacon, C.R., *Neal, C.A., Nye, C.J., and *McGimsey, R.G., 1997, Preeruptive temperatures for postcaldera magmas of Aniakchak Volcano, Alaska [abs.]: Eos (American Geophysical Union Transactions), v. 78, no. 46, supplement, p. 792.

Bahr, D.B., Meier, M.F., and *Peckham, S.D., 1997, The physical basis of glacier volume-area scaling: Journal of Geophysical Research, v. 102, no. B9, p. 20355-20362.

Bence, A.E., *Kvenvolden, K.A., and Kennicutt, M.C., II, 1996, Organic geochemistry applied to environmental assessments of Prince William Sound, Alaska, after the Exxon Valdez oil spill-A review: Organic Geochemistry, v. 24, p. 7-42.

*Brew, D.A., 1997, "The coast range megalineament" in southeastern Alaska-How does it differ from the "coast shear zone?" [abs.]: Geological Society of America, Abstracts with Programs, v. 29, no. 6, p. 81.

*Brew, D.A., 1997, Geologic mapping in the Wrangell-Petersburg area, southeastern Alaska-A case history illustrating evolution of geologic studies [abs.]: Geological Society of America, Abstracts with Programs, v. 29, no. 6, p. 425.

${ }^{*}$ Bufe, C.G., *Varnes, D.J., and Nishenko, S.P., 1996, Time-to-failure in the Alaska-Aleutian region: An update [abs.]: Eos (American Geophysical Union Transactions), v. 77, no. 46, supplement, p. 456.

Bundtzen, T.K., and *Miller, M.L., 1997, Precious metals associated with Late Cretaceous-early Tertiary igneous rocks of southwestern Alaska, in Goldfarb, R.J., and Miller, L.D., eds., 1997, Mineral Deposits of Alaska: Economic Geology Monograph 9, p. 242-286.

Cai, Jinkui, Powell, R.D., Cowan, E.A., and *Carlson, P.R., 1997, Lithofacies and seismic-reflection interpretation of temperate glacimarine sedimentation in Tarr Inlet, Glacier Bay, Alaska: Marine Geology, v. 143, p. 5-37.
Cai, Jinkui, Powell, R.D., Cowan, E.A., and *Kayen, R.E., 1996, Lithofacies, physical properties and seismic-reflection characteristcs of temperate glaciomarine deposits in Glacier Bay, Alaska [abs.]: Eos (American Geophysical Union Transactions), v. 77, no. 46, supplement, p. 332.

*Carlson, P.R., and *Kvenvolden, K.A., 1996, Tracking Exxon Valdez oil from beach to deepwater sediments of Prince William Sound, Alaska, in Exxon Valdez Oil Spill Symposium, Anchorage, Alaska, 1993, Proceedings: American Fisheries Society Symposium 18, p. 109120.

*Carlson, P.R., ${ }^{*}$ Stevenson, A.J., *Bruns, T.R., *Mann, D.M., and Huggett, Quentin, 1996, Sediment pathways in Gulf of Alaska from beach to abyssal plain in Gardner, J.V., Field, M.E., and Twichell, D.C., eds., Geology of the United States' Seafloor-The View from GLORIA: New York, Cambridge University Press, p. 255-277.

*Chouet, B.A., 1997, Hydrothermal pressure instabilites related to magmatic steam injection and reflected in long-period seismicity [abs.]: Eos (American Geophysical Union Transactions), v. 78, no. 46, supplement, p. 764-765.

*Cole, Frances, ${ }^{*}$ Bird, K.J., Toro, Jaime, Roure, François, O'Sullivan, P.B., Pawlewicz, Mark, and *Howell, D.G., 1997, An integrated model for the tectonic development of the frontal Brooks Range and Colville Basin $250 \mathrm{~km}$ west of the Trans-Alaska Crustal Transect: Journal of Geophysical Research, v. 102, no. B9, p. 20685-20708.

Cole, F., Toro, J., *Bird, K.J., and Roure, F., 1996, A forward model for thrusting and sedimentation in the north-central Brooks Range, Alaska [abs.]: Eos (American Geophysical Union Transactions), v. 77 , no. 46, supplement, p. 642.

*Collett, T.S., 1996, Sources of surficial methane flux associated with natural gas hydrate accumulations in northern Alaska [abs.]: Eos (American Geophysical Union Transactions), v. 77, no. 46, supplement, p. 184.

${ }^{*}$ Collett, T.S., 1997, Gas hydrate resources of northern Alaska: Bulletin of Canadian Petroleum Geology, v. 45, p. 317-338.

Dobson, M.R., *Karl, H.A., and *Vallier, T.L., 1996, Sedimentation along the fore-arc region of the Aleutian Island Arc, Alaska in Gardner, J.V., Field, M.E., and Twichell, D.C., eds., Geology of the United States' Seafloor-The View from GLORIA: New York, Cambridge University Press, p. 279-304. 
Dreher, S.T., Faust, J.L., ${ }^{*}$ Miller, T.P., and ${ }^{*}$ Bacon, C.R., 1997, Chemical characteristics of the Aniakchak Caldera ash-flow sheet [abs.]: Eos (American Geophysical Union Transactions), v. 78, no. 46, supplement, p. 180.

*Dumoulin, J.A., Watts, K.F., and *Harris, A.G., 1997, Stratigraphic contrasts and tectonic relationships between Carboniferous successions in the Trans-Alaska Crustal Transect corridor and adjacent areas, northern Alaska: Journal of Geophysical Research, v. 102, no. B9, p. 20709-20726.

Eichelberger, J.C., *Keith, T.C., and Nye, C.J., 1996, New monitoring and geological investigations in the central Aleutian Arc, Alaska [abs.]: Eos (American Geophysical Union Transactions), v. 77, no. 46, supplement, p. 771.

Elias, S.A., Short, S.K., and *Waythomas, C.F., 1996, Late Quaternary environments, Denali National Park and Preserve, Alaska: Arctic, v. 49, no. 3, p. 292-305.

*Evans, K.R., *Stevenson, A.J., *Barnes, P.W., *Carlson, P.R., *Hampton, M.A, and *Marlow, M.S., 1996, Sea-floor sediments in the Gulf of Alaska: New map compilation for studies of benthic biohabitats [abs.]: Eos (American Geophysical Union Transactions), v. 77, no. 46, supplement, p. 409.

*Fierstein, J., Houghton, B.F., Wilson, C.J.N., and *Hildreth, W., 1997, Complexities of Plinian fall deposition at vent: An example from the 1912 Novarupta eruption (Alaska): Journal of Volcanology and Geothermal Research v. 76, p. 215-227.

Foley, J.Y., *Light, T.D., *Nelson, S.W., and Harris, R.A., 1997, Mineral occurrences associated with mafic-ultramafic and related alkaline complexes in Alaska, in Goldfarb, R.J., and Miller, L.D., eds., 1997, Mineral Deposits of Alaska: Economic Geology Monograph 9, p. 396-449.

*Fuis, G.S., *Murphy, J.M., *Lutter, W.J., *Moore, T.E., *Bird, K.J., and Christensen, N.I., 1997, Deep seismic structure and tectonics of northern Alaska: Crustal-scale duplexing with deformation extending into the upper mantle: Journal of Geophysical Research, v. 102, no. B9, p. 20873-20896.

*Goldfarb, R.J., 1997, Metallogenic evolution of Alaska, in Goldfarb, R.J., and Miller, L.D., eds., 1997, Mineral Deposits of Alaska: Economic Geology Monograph 9, p. 4-34.

*Goldfarb, R.J., and Miller, L.D., eds., 1997, Mineral deposits of Alaska: Economic Geology Monograph 9, 482 p.

*Goldfarb, R.J., Miller, L.D., *Leach, D.L., and *Snee, L.W., 1997, Gold deposits in metamorphic rocks of Alaska, in Goldfarb, R.J., and Miller, L.D., eds., 1997, Mineral Deposits of Alaska: Economic Geology Monograph 9, p. 151-190.

*Gray, J.E., *Gent, C.A., * ${ }^{*}$ Snee, L.W., and *Wilson, F.H., 1997, Epithermal mercury-antimony and gold-bearing vein lodes of southwestern Alaska, in Goldfarb, R.J., and Miller, L.D., eds., 1997, Mineral Deposits of Alaska: Economic Geology Monograph 9, p. 287-305.

Greninger, M.L., Klemperer, S.L., and *Nokleberg, W.J., 1996, Geographic information system (GIS) database of the geology, geophysics, deep-crustal structure, and tectonics of the Russian Far East, Alaska, Canadian Cordillera, and adjacent offshore regions [abs.]: Eos (American Geophysical Union Transactions), v. 77, no. 46, supplement, p. 669.

*Guffanti, Marianne, and *Ewert, J.W., 1997, Improvements in real-time monitoring of US volcanoes [abs.]: Eos (American Geophysical Union Transactions), v. 78, no. 46, supplement, p. 37.

*Haeussler, P.J., and Bruhn, R.L., 1996, Evidence for Holocene or late Pleistocene folding in Cook Inlet, Alaska [abs.]: Eos (American Geophysical Union Transactions), v. 77, no. 46, supplement, p. 686.
*Hammond, W.R., ${ }^{*}$ Lockhart, A.B., ${ }^{*}$ Neal, C.A., ${ }^{*}$ McGimsey, R.G., and *Paskievitch, J.F., 1997, Seismic monitoring at Aniakchak Volcano, Alaska [abs.]: Eos (American Geophysical Union Transactions), v. 78, no. 46, supplement, p. 816.

*Hammond, W.R., *Paskievitch, J.F., *Power, J.A., *Lockhart, A.B., Estes, S.A., Tytgat, G., and Benevento, J., 1996, The AVO central Aleutian expansion: Seismic monitoring and instrumentation [abs.]: Eos (American Geophysical Union Transactions), v. 77, no. 46, supplement, p. 451-452.

*Harris, A.G., Brenckle, P.A., Baesemann, J.F., and Krumhardt, A.P., 1997, Conodont and calcareous microfossil biostratigraphy of the Lisburne Group (Carboniferous), Sadlerochit Mts., NE. Brooks Range, Alaska [abs.]: Geological Society of America, Abstracts with Programs, v. 29, no. 2, p. 12-13.

${ }^{*}$ Healy, J.H., *Dewey, J.W., Kossobokov, V.G., and Romashkova, L.L., 1996, Intermediate-term changes of seismicity in advance of the 10 June 1996 Delarof Islands earthquake [abs.]: Eos (American Geophysical Union Transactions), v. 77, no. 46, supplement, p. 502.

${ }^{*}$ Heinrichs, T.A., ${ }^{*}$ Mayo, L.R., Echelmeyer, K.A., and Harrison, W.D., 1996, Quiescent-phase evolution of a surge-type glacier: Black Rapids Glacier, Alaska, U.S.A.: Journal of Glaciology, v. 42, p. 110-122.

*Hildenbrand, T.G., *Blakely, R.J., *Jachens, R.C., and *Saltus, R.W., 1997 , Insights on crustal structures based on new regional magnetic anomaly data in Western U.S.A.: Eos (American Geophysical Union Transactions), v. 78, no. 17, supplement, p. 118.

*Hill, D.P., and *Guffanti, Marianne, 1997, Issues in volcano hazards mitigation: Eos (American Geophysical Union Transactions), v. 78, no. 17 , supplement, p. 47.

${ }^{*}$ Hodge, S.M., 1997, Glacier mass balances suggest recent changes in Pacific hemisphere teleconnections [abs.]: Eos (American Geophysical Union Transactions), v. 78, no. 46, supplement, p. 101.

Hudson, T.L. and *Reed, B.L., 1997, Tin deposits in Alaska, in Goldfarb, R.J., and Miller, L.D., eds., 1997, Mineral Deposits of Alaska: Economic Geology Monograph 9, p. 450-465.

Jolly, A.D., McNutt, S.R., Coombs, M.L., Sthiler, S.D., and *Paskievitch, J., 1997, Seismicity in the vicinity of the Katmai group of volcanoes, Katmai National Park, Alaska; July 1995-March 1997 [abs.]: Eos (American Geophysical Union Transactions), v. 78, no. 46, supplement, p. 442.

Jolly, A.D., McNutt, S.R., Wiener, S., and *Lahr, J.C., 1996, An evaluation of $b$-value spatial mapping techniques based on an analysis of seismicity at Mt. Spurr, Alaska, and synthetic data [abs.]: Eos (American Geophysical Union Transactions), v. 77, no. 46, supplement, p. 514.

*Karl, H.A., *Carlson, P.R., and *Gardner, J.V., 1996, Aleutian Basin of the Bering Sea: Styles of sedimentation and canyon development in Gardner, J.V., Field, M.E., and Twichell, D.C., eds., Geology of the United States' Seafloor-The View from GLORIA: New York, Cambridge University Press, p. 305-332.

*Karl, H.A., and *Carlson, P.R., 1996, Alaskan EEZ in Gardner, J.V., Field, M.E., and Twichell, D.C., eds., Geology of the United States' Seafloor-The View from GLORIA: New York, Cambridge University Press, p. 251-254.

*Kayen, R.E., *Lee, H.J., *Kvenvolden, K.A., and *Lorenson, T.D., 1997, Glacial episodes, gas hydrate, and mass wasting of the contrinental slope: Eos (American Geophysical Union Transactions), v. 78, no. 17, supplement, p. 189.

*Keith, T.E.C., 1997, Real-time monitoring and eruption warning responses by the Alaska Volcano Observatory [abs.]: Eos (American Geophysical Union Transactions), v. 78, no. 46, supplement, p. 37. 
*Keith, T.E.C., Nye, C.J., Eichelberger, J.C., *Miller, T.P., and *Power, J.A., 1996, March 1996 seismic crisis at Akutan Volcano, central Aleutian Arc, Alaska [abs.]: Eos (American Geophysical Union Transactions), v. 77, no. 46, supplement, p. 815.

*Kelley, K.D., and *Taylor, C.D., 1997, Environmental geochemistry of shale-hosted $\mathrm{Ag}-\mathrm{Pb}-\mathrm{Zn}$ massive sulfide deposits in northwest Alaska: Natural background concentrations of metals in water from mineralized areas: Applied Geochemistry, v. 12, p. 397-409.

Kusky, T.M., *Bradley, D.C., ${ }^{*}$ Haeussler, P.J., and *Karl, Sue, 1997, Controls on accretion of melange and flysch belts, Chugach terrane, south-central Alaska: Eos (American Geophysical Union Transactions), v. 78, no. 17, supplement, p. 320.

Kusky, T.M., ${ }^{*}$ Bradley, D.C., ${ }^{*}$ Haeussler, P.J., and ${ }^{*}$ Karl, Sue, 1997, Controls on accretion of flysch and mélange belts at convergent margins: Evidence from the Chugach Bay thrust and Iceworm mélange, Chugach accretionary wedge, Alaska: Tectonics, v. 16, p. 855-878.

*Lilly, M.R., Hinzman, L.D., and Wegner, M.A., 1997, Characteristics of ground-water and surface-water interactions in a subarctic basin in central Alaska [abs.]: Eos (American Geophysical Union Transactions), v. 78, no. 46, supplement, p. 311.

*Lorenson, T.D., and *Kvenvolden, K.A., 1996, Nonmethane hydrocarbon gases in permafrost [abs.]: Eos (American Geophysical Union Transactions), v. 77, no. 46, supplement, p. 184.

*Lorenson, T.D., *Kvenvolden, K.A., ${ }^{*}$ Collett, T.S., and *Kayen, R.E., 1997, The search for gas hydrate in northern Alaska and the Beaufort Sea: Eos (American Geophysical Union Transactions), v. 78, no. 17, supplement, p. 189.

*MacKevett, E.M. Jr., ${ }^{*}$ Cox, D.P., Potter, R.W., II, and *Silberman, M.L., 1997, Kennecott-type deposits in the Wrangell Mountains, Alaska: High-grade copper ores near a basalt-limestone contact, in Goldfarb, R.J., and Miller, L.D., eds., 1997, Mineral Deposits of Alaska: Economic Geology Monograph 9, p. 66-89.

*McCarthy, K.A., Hinzman, L.D., Braddock, J.F., *Lilly, M.R., Richmond, S.A., and Wegner, M.A., 1997, A hydrological, geochemical, and microbiological characterization of a trichloroethylene-contaminated alluvial aquifer in subarctic Alaska [abs.]: Eos (American Geophysical Union Transactions), v. 78, no. 46, supplement, p. 211.

McLaughlin, E.A., Lilley, M.D., Olson, E.J., ${ }^{*}$ Kvenvolden, K.A., and *Lorenson, T.D., 1996, Methane oxidation in the Beaufort Sea [abs.]: Eos (American Geophysical Union Transactions), v. 77, no. 46, supplement, p. 187-188.

McNutt, S.R., Benoit, J., Christensen, D., Estes, S., Tytgat, G., Stihler, S., Weimer, S., Jolly, A., Robinson, M., Hansen, R., Lindquist, K., Garces, M., *Lahr, J., *Hammond, R., *Power, J., and *Paskievitch, J., 1997, Broadband seismology at the Alaska Volcano Observatory, 19931997 [abs.]: Eos (American Geophysical Union Transactions), v. 78, no. 46, supplement, p. 429.

Miller, L.D., and *Goldfarb, R.J., 1997, Preface, in Goldfarb, R.J., and Miller, L.D., eds., 1997, Mineral Deposits of Alaska: Economic Geology Monograph 9, p. 1-3.

*Miller, T.P., Begét, J.E., * Stephens, C.D., and *Moore, R.B., 1996, Geology and hazards of lliamna Volcano, Alaska [abs.]: Eos (American Geophysical Union Transactions), v. 77, no. 46, supplement, p. 815.

${ }^{*}$ Miller, T.P., *Richter, D.H., ${ }^{*}$ Smith, R.L., and Dreher, S.T., 1997, Calderaforming events at Aniakchak Volcano, Alaska [abs.]: Eos (American Geophysical Union Transactions), v. 78, no. 46, supplement, p. 816.

*Molnia, B.F., 1996, Kettle formation in 1994 flood deposits -Bering Glacier, Alaska [abs.]: Eos (American Geophysical Union Transactions), v. 77 , no. 46 , supplement, p. 194.
Monger, J.W.H., and *Nokleberg, W.J., 1996, Evolution of the northern North American Cordillera: Generation, fragmentation, displacement and accretion of successive North American plate-margin arcs, in Coyner, A.R., and Fahey, P.L., eds., Geology and Ore Desposits of the American Cordillera: Geological Society of Nevada Symposium Proceedings, Reno/Sparks, Nevada, April 1995, p. 1133-1152.

*Moore, T.E., *Aleinikoff, J.N., and *Harris, A.G., 1997, Stratigraphic and structural implications of conodont and detrital zircon $\mathrm{U}-\mathrm{Pb}$ ages from metamorphic rocks of the Coldfoot terrane, Brooks Range, Alaska: Journal of Geophysical Research, v. 102, no. B9, p. 2079720820.

*Moore, T.E., Wallace, W.K., Mull, C.G., *Adams, K.E., *Plafker, George, and *Nokleberg, W.J., 1997, Crustal implications of bedrock geology along the Trans-Alaska Crustal Transect (TACT) in the Brooks Range, northern Alaska: Journal of Geophysical Research, v. 102, no. B9, p. 20645-20684.

*Morrissey, M.M., 1997, Long-period seismicity at Redoubt Volcano, Alaska, 1989-1990 related to magma degassing: Journal of Volcanology and Geothermal Research v. 75, p. 321-335.

*Morrissey, M.M., and *Chouet, B.A., 1997, A numerical investigation of choked flow dynamics and its application to the triggering mechanism of long-period events at Redoubt Volcano, Alaska: Journal of Geophysical Research, v. 102, p. 7965-7983.

Mortera-Gutierrez, C.A., and *Geist, E., 1996, The subducted high relief of Rat fracture zone beneath the Aleutian accretionary prism [abs.]: Eos (American Geophysical Union Transactions), v. 77, no. 46, supplement, p. 659.

${ }^{*}$ Neal, C.A., ${ }^{*}$ McGimsey, R.G., *Miller, T.P., Nye, C.J., *Bacon, C.R., and *Felger, T.J., 1997, New geologic map of Aniakchak Caldera, Alaska [abs.]: Eos (American Geophysical Union Transactions), v. 78, no. 46, supplement, p. 816.

Newberry, R.J., Crafford, T.C., Newkirk, S.R., Young, L.E., ${ }^{*}$ Nelson, S.W., and Duke, N.A., 1997, Volcanogenic massive sulfide deposits of Alaska, in Goldfarb, R.J., and Miller, L.D., eds., 1997, Mineral Deposits of Alaska: Economic Geology Monograph 9, p. 120-150.

Nishenko, S.P., *Bufe, C., *Dewey, J., *Varnes, D., *Healy, J., Jacob, K., and Kossobokov, V., 1996, 1996 Delarof Islands earthquake-A successful earthquake forecast/prediction? [abs.]: Eos (American Geophysical Union Transactions), v. 77, no. 46, supplement, p. 456.

*Nokleberg, W.J., Parfenov, L.M., Monger, J.W.H., Norton, I.O., Khanchuk, A.I., Stone, D.B., *Scholl, D.W., and Fujita, Kazuya, 1997, Tectonic evolution of the Russian Northeast and mainland Alaska [abs.]: Eos (American Geophysical Union Transactions), v. 78, no. 46, supplement, p. 664.

Nye, C.J., * Miller, T.P., and Layer, P.W., 1997, Chemically and temporally distinct magma series at Aniakchak Volcano and the role of crustal mixing [abs.]: Eos (American Geophysical Union Transactions), v. 78, no. 46, supplement, p. 816.

*Oppenheimer, D., *Bittenbinder, A., *Bogaert, B., *Dietz, L., *Ellsworth, W., *Jensen, E., *Kohler, W., *Van Schaack, J., ${ }^{*}$ Buland, R., ${ }^{*}$ Benz, H., Hansen, R., *Weaver, C., Malone, S., and ${ }^{*}$ Okubo, P., 1997, CREST: Consolidated reporting of earthquakes and tsunamis [abs.]: Eos (American Geophysical Union Transactions), v. 78, no. 46, supplement, p. 46.

*O'Sullivan, P.B., Murphy, J.M., and Blythe, A.E., 1997, Late Mesozoic and Cenozoic thermotectonic evolution of the central Brooks Range and adjacent North Slope foreland basin, Alaska: Including fission track results from the Trans-Alaska Crustal Transect (TACT): Journal of Geophysical Research, v. 102, no. B9, p. 20821-20845.

*Phillips, R.L., and *Grantz, Arthur, 1997, Quaternary history of sea ice and paleoclimate in the Amerasia Basin, Arctic Ocean, as recorded 
in the cyclical strata of Northwind Ridge: Geological Society of America Bulletin, v. 109, p. 1101-1115.

*Plafker, George, and *Mooney, W.D., 1997, Introduction to special section: The Trans-Alaska Crustal Transect (TACT) across Arctic Alaska: Journal of Geophysical Research, v. 102, no. B9, p. 2063920643.

*Power, J.A., Jolly, A.D., Harbin, M.L., and Nye, C.J., 1997, Deep longperiod events associated with the 1992 eruptions of Crater Peak vent, Mount Spurr, Alaska [abs.]: Eos (American Geophysical Union Transactions), v. 78, no. 46, supplement, p. 438.

*Power, J.A., *Paskievitch, J.F., *Richter, D.H., *McGimsey, R.G., Stelling, P., Jolly, A.D., and Fletcher, H.J., 1996, 1996 seismicity and ground deformation at Akutan Volcano, Alaska [abs.]: Eos (American Geophysical Union Transactions), v. 77, no. 46, supplement, p. 514.

Queen, L.K., Nye, C.J., Benoit, J.P., Dean, K.G., Engle, K., Lindquist, K.G., Servilla, M.S., and ${ }^{*}$ Hammond, W.R., 1997, Near-real time volcano monitoring via the Internet at the Alaska Volcano Observatory [abs.]: Eos (American Geophysical Union Transactions), v. 78, no. 46, supplement, p. 46.

${ }^{*}$ Riehle, J.R., *Waitt, R.B., *Meyer, C.E., and *Calk, L.C., 1996, Age of Kaguyak caldera, eastern Aleutian arc, Alaska, estimated by tephrochronology [abs.]: Eos (American Geophysical Union Transactions), v. 77 , no. 46, supplement, p. 772.

*Rosenbaum, J.G., *Reynolds, R.L., *Muhs, D.R., *Harlan, S.S., and Beget, J.E., 1997, Geochemical constraint on the interpretation of magnetic property variations in loess/paleosol sequences from central Alaska [abs.]: Eos (American Geophysical Union Transactions), v. 78, no. 46, supplement, p. 171.

Scarpa, R., and *Tilling, R.I., eds., 1996, Monitoring and mitigation of volcano hazards: New York, Springer-Verlag, 841 p.

${ }^{*}$ Schmidt, J.M., 1997, Shale-hosted $\mathrm{Zn}-\mathrm{Pb}-\mathrm{Ag}$ and barite deposits of Alaska, in Goldfarb, R.J., and Miller, L.D., eds., 1997, Mineral Deposits of Alaska: Economic Geology Monograph 9, p. 35-65.

${ }^{*}$ Schmidt, J.M., 1997, Strata-bound carbonate-hosted $\mathrm{Zn}-\mathrm{Pb}$ and $\mathrm{Cu}$ deposits of Alaska, in Goldfarb, R.J., and Miller, L.D., eds., 1997, Mineral Deposits of Alaska: Economic Geology Monograph 9, p. 90-119.

*Schneider, D.J., Dean, K.G., Engle, K., and Worley, S.L., 1997, Monitoring and analyses of volcanic activity using remote sensing data at the Alaska Volcano Observatory [abs.]: Eos (American Geophysical Union Transactions), v. 78, no. 46, supplement, p. 777.

Seramur, K.C., Powell, R.D., and *Carlson, P.R., 1997, Evaluation of conditions along the grounding line of temperate marine glaciers: An example from Muir Inlet, Glacier Bay, Alaska: Marine Geology, v. 140, p. 307-327.

Snyder-Conn, Elaine, * Garbarino, J.R., *Hoffman, G.L., and Oelkers, Alan, 1997, Soluble trace elements and total mercury in arctic Alaskan snow: Arctic, v. 50, no. 3, p. 201-215.
*Starratt, S.W., 1995, Latest Quaternary foraminifers and sediment transport in Pervenets Canyon, Bering Sea: Marine Micropaleontology, v. 26, p. 233-243.

Stevens, C.H., Davydov, V.I., and *Bradley, Dwight, 1997, Permian Tethyan Fusulinina from the Kenai Peninsula, Alaska: Journal of Paleontology, v. 71, p. 985-994.

*Svarc, J.L., *Savage, J.C., *Prescott, W.H., *Lisowski, M., and *Gross, W.K., 1997, Deformation across the Alaska collision zone near Prince William Sound, Alaska 1993-1997 [abs.]: Eos (American Geophysical Union Transactions), v. 78, no. 46, supplement, p. 162.

*Trabant, D.C., and *Mayo, L.R., 1997, Predicted responses of glaciers to climate change along the Gulf of Alaska [abs.]: Eos (American Geophysical Union Transactions), v. 78, no. 46, supplement, p. 250.

Truffer, M., Harrison, W.D., Echelmeyer, K.A., Gorda-DeMallie, J, and ${ }^{*}$ Heinrichs, T.A., 1996, Velocity variations of surge type Black Rapids Glacier [abs.]: Eos (American Geophysical Union Transactions), v. 77, no. 46, supplement, p. 213.

*Vallier, T.L., Mortera-Gutierrez, C.A., ${ }^{*}$ Karl, H.A., Masson, D.G., Prueher, Libby, and ${ }^{*}$ Chase, T.E., 1996, Geology of the Kula paleo-plate, North Pacific Ocean in Gardner, J.V., Field, M.E., and Twichell, D.C., eds., Geology of the United States' Seafloor-The View from GLORIA: New York, Cambridge University Press, p. 333-353.

*von Huene, R., and Klaeschen, D., 1997, Strain partitioning across the Kodiak margin [abs.]: Eos (American Geophysical Union Transactions), v. 78, no. 46, supplement, p. 627.

Wallace, W.K., *Moore, T.E., and *Plafker, George, 1997, Multistory duplexes with forward dipping roofs, north central Brooks Range, Alaska: Journal of Geophysical Research, v. 102, no. B9, p. 2077320796.

*Waythomas, C.F., and *Neal, C.A., 1997, Tsunami generation during the $3500 \mathrm{yr}$ B.P. caldera-forming eruption of Aniakchak Volcano [abs.]: Eos (American Geophysical Union Transactions), v. 78, no. 46, supplement, p. 816.

White, J.M., *Ager, T.A., ${ }^{*}$ Adam, D.P., Leopold, E.B., Liu, G., Jette, H., and Schweger, C.E., 1997, An 18 million year record of vegetation and climate change in northwestern Canada and Alaska: Tectonic and global climatic correlates: Palaeogeography, Palaeoclimatology, Palaeoecology, v. 130, p. 293-306.

Wolf, L.W., Stone, D.B., Fujita, K., *Brocher, T., and Klemperer, S., 1997, A crustal model of the Bering Strait: Implications for tectonic reconstructions [abs.]: Eos (American Geophysical Union Transactions), v. 78 , no. 46 , supplement, p. 628.

Young, L.E., St. George, Phil, and Bouley, B.A., 1997, Porphyry copper deposits in relation to the magmatic history and palinspastic restoration of Alaska, in Goldfarb, R.J., and Miller, L.D., eds., 1997, Mineral Deposits of Alaska: Economic Geology Monograph 9, p. 307-333. 